\title{
EXPERIMENTAL AND ANALYTICAL MODELING STUDIES OF STEAM INJECTION WITH HYDROCARBON ADDITIVES TO ENHANCE RECOVERY OF SAN ARDO HEAVY OIL
}

\author{
A Thesis \\ by \\ ROLY SIMANGUNSONG \\ Submitted to the Office of Graduate Studies of \\ Texas A\&M University \\ in partial fulfillment of the requirements for the degree of \\ MASTER OF SCIENCE
}

August 2005

Major Subject: Petroleum Engineering 


\title{
EXPERIMENTAL AND ANALYTICAL MODELING STUDIES OF STEAM INJECTION WITH HYDROCARBON ADDITIVES TO ENHANCE RECOVERY OF SAN ARDO HEAVY OIL
}

\author{
A Thesis \\ by \\ ROLY SIMANGUNSONG

\begin{abstract}
Submitted to the Office of Graduate Studies of
Texas A\&M University

in partial fulfillment of the requirements for the degree of

MASTER OF SCIENCE
\end{abstract}

Approved by:

Chair of Committee, Daulat D. Mamora

Committee Members, Maria A. Barrufet

Yalchin R. Efendiev

Head of Department, Stephen A. Holditch

August 2005

Major Subject: Petroleum Engineering 


\begin{abstract}
Experimental and Analytical Modeling Studies of Steam Injection with Hydrocarbon Additives to Enhance Recovery of San Ardo Heavy Oil. (August 2005)

Roly Simangunsong, B.S., Bandung Institute of Technology, Indonesia Chair of Advisory Committee: Dr. Daulat D. Mamora
\end{abstract}

Experimental and analytical studies have been carried out to better understand production mechanisms of heavy oil under steam injection with propane and petroleum distillate as steam additives. The studies have been conducted for heavy oil from San Ardo field $\left(12^{\circ} \mathrm{API}, 2800 \mathrm{cp}\right.$ at $\left.53.3^{\circ} \mathrm{C}\right)$, under current reservoir conditions.

The experiments consist of injecting pure steam, steam-propane, and steampetroleum distillate into a vertical cell containing a mixture of sand, water and San Ardo oil. The injection cell $(68.58 \mathrm{~cm}$ long with an ID of $7.376 \mathrm{~cm})$ is placed inside a vacuum jacket, set at the reservoir temperature of $53.3^{\circ} \mathrm{C}$. Superheated steam at $230^{\circ} \mathrm{C}$ is injected at $5.5 \mathrm{ml} / \mathrm{min}$ (cold-water equivalent) simultaneously with propane or a petroleum distillate slug. The cell outlet pressure is maintained at 260 psig. Six runs were performed, two runs using pure steam, two steam-propane runs using 5:100 propane:steam mass ratio, and two steam-petroleum distillate runs using 5:100 petroleum distillate:steam mass ratio.

We develop a simplified analytical model that describes steam front advancement and oil production for the 1D displacement experiments. The model incorporates heat and material balance, fillup time and Darcy's law pertaining to the injection cell. The analytical model results are compared against the experimental data to verify the validity of the model.

The main results of the study are as follows. First, experimental results indicate that compared to pure steam injection, oil production was accelerated by 30\% for 5:100 propane:steam injection and $38 \%$ for 5:100 petroleum distillate:steam injection 
respectively. Second, steam injectivity with steam-propane and steam-petroleum distillate increases to 1.4 and 1.9 times respectively, compared with pure steam injection. Third, steam front advancement and oil production data are in good agreement with results based on the new analytical model. The analytical model indicates that the oil production acceleration observed is due to oil viscosity reduction resulting from the addition of propane and petroleum distillate to the steam. Oil viscosity at the initial temperature with pure steam injection is $2281 \mathrm{cp}$, which is reduced to $261 \mathrm{cp}$ with steam-propane injection and $227 \mathrm{cp}$ with steam-petroleum distillate injection. 


\section{DEDICATION}

This thesis is humbly dedicated to my parents, Wesley Simangunsong and Rospita Simanjuntak, who are both models of the person I hope to become, who have supported me with their boundless love and wisdom throughout my life, and who have rejoiced with me in the good times and encouraged me to move forward through the bad. 


\section{ACKNOWLEDGEMENTS}

I wish to express my greatest respect and appreciation to my thesis advisor, Dr. Daulat D. Mamora, for his excellent guidance and funding throughout this research. He has shown nothing less than perfect patience and has kept me on the right path to complete this work.

I am also grateful to Drs. Maria A. Barrufet and Yalchin R. Efendiev for serving on my committee.

Special thanks go to Thomas Nesse and Jose Antonio Rivero who patiently explained the workings of the equipment in the Ramey Lab to me when I first started my experiments. This was time consuming work, and I really appreciate the effort. The hours spent in the lab would not have been so much fun if it was not for their pleasant company.

My sincere thanks also go to my good friend, Zuher Syihab, who, possibly without realizing it, taught me that learning is more than just a grade.

This research was conducted under the Ramey Laboratory Research Program (2004) and the Crisman Institute for Reservoir Management (2005). Sponsors included US DOE, ChevronTexaco, ConocoPhillips, and Total S.A. Their support is gratefully acknowledged. 


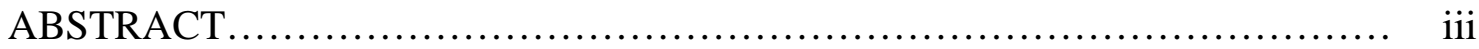

DEDICATION............................................................ v

ACKNOWLEDGEMENTS................................................ vi

TABLE OF CONTENTS ....................................................... vii

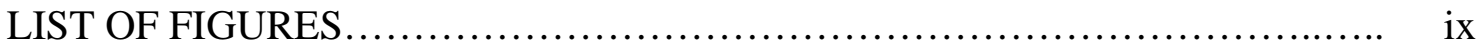

LIST OF TABLES............................................................. xiv CHAPTER

I INTRODUCTION................................................... 1

1.1 Research Objectives........................................... 3

II LITERATURE REVIEW ............................................ 4

III EXPERIMENTAL APPARATUS AND PROCEDURE................... 8

3.1 Experimental Apparatus ............................................. 8

3.1.1 Fluid Injection System ........................................ 8

3.1.2 Injection Cell .............................................. 10

3.1.3 Fluid Production System .................................... 13

3.1.4 Gas Measurement System ..................................... 13

3.1.5 Data Measurement and Recording System ..................... 13

3.2 Experimental Procedure................................................. 22

IV EXPERIMENTAL RESULTS ..................................... 25

4.1 Overview..................................................... 25

4.2 Run 3 (5:100 Propane:Steam Mass Ratio).............................. $\quad 27$

4.3 Run 4 (Pure Steam).............................................. 39

4.4 Run 5 (Pure Steam) .............................................. 48

4.5 Run 6 (5:100 Propane:Steam Mass Ratio)............................. 56

4.6 Run 7 (5:100 Petroleum Distillate:Steam Mass Ratio)................ 67

4.7 Run 8 (5:100 Petroleum Distillate:Steam Mass ratio)...................... 76

4.8 Comparison and Discussion of Experimental Results .................... 84 
CHAPTER $\quad$ Page

V ANALYTICAL MODEL …........................................ 100

5.1 Review of Analytical Models for Steamflooding ..................... 100

5.2 Predictive Models for Steamflooding................................... 103

5.3 New Analytical Model for Injection with Steam Additives........... 109

5.4 Comparison and Discussion of Experimental and Analytical

Model Results............................................. 118

VI SUMMARY, CONCLUSIONS AND RECOMMENDATIONS ......... 126

6.1 Summary ........................................................ 126

6.2 Conclusions................................................... 126

6.3 Recommendations ................................................................. 128

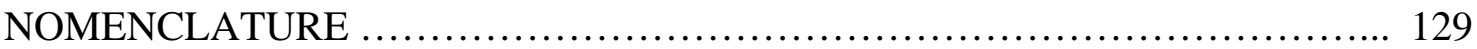

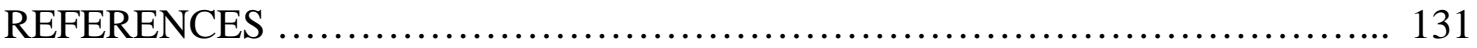

APPENDIX A CALCULATION OF FLUID SATURATIONS AND PORE VOLUME ................................................... 136

APPENDIX B TEMPERATURE AND PRESSURE DATA....................... 140

APPENDIX C PRODUCTION DATA ...................................... 203

APPENDIX D DENSITY AND VISCOSITY DATA........................... 213

APPENDIX E VISUAL BASIC SOURCE CODE FOR STEAM FRONT ADVANCEMENT AND CUMULATIVE OIL PRODUCTION CALCULATION BASED ON ONE DIMENSIONAL ANALYTICAL MODEL...................................... 214

APPENDIX F DATA INPUT FOR STEAM FRONT ADVANCEMENT AND CUMULATIVE OIL PRODUCTION BASED ON ONE DIMENSIONAL ANALYTICAL MODEL PROGRAM.

VITA ............................................................................ 297 


\section{LIST OF FIGURES}

FIGURE Page

3.1 Schematic diagram of experimental apparatus............................................

3.2 Photograph of injection cell (cell outlet at the left).......................................11

3.3 Positions of the thermocouples in the injection cell......................................... 11

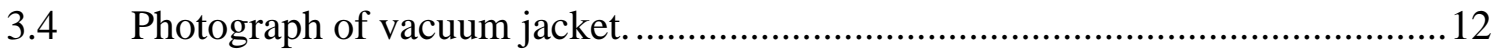

3.5 Photograph of first separator (right) and second separator (left) .......................14

3.6 Photograph of back pressure valve and wet test meter. ...................................14

3.7 Photograph of gas chromatograph........................................................... 15

3.8 Photograph of data logger, personal computer and temperature controllers........15

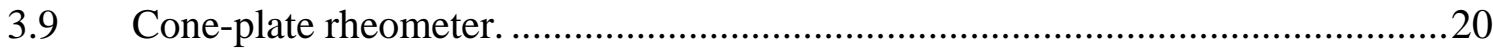

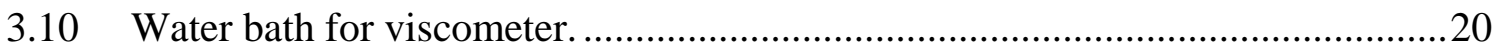

3.11 Photograph of insulated centrifuge with heater gun to keep samples warm ........21

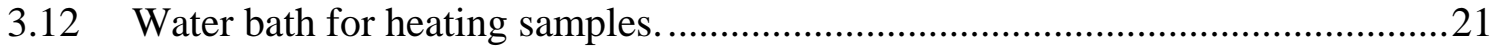

3.13 Photograph showing overview of experimental apparatus. ..............................22

4.1 Temperature profile versus time for Run 3 (5:100 propane:steam)..................28

4.2 Cumulative oil and water volumes versus time for Run 3 (5:100 propane:steam)

4.3 Cumulative oil and water volumes versus pore volume of steam injected for Run 3 (5:100 propane:steam) ......................................................30

4.4 Oil and water rates versus time 3 for Run 3 (5:100 propane:steam) ...................31

4.5 Oil and water rates versus pore volume injected for Run 3

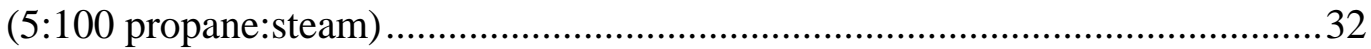

4.6 Temperature profiles at 20-minute intervals - from $\mathrm{t}=0$ to $\mathrm{t}=200 \mathrm{~min}-$ for run 3 (5:100 propane:steam)

4.7 Injection, production and differential pressures for run 3 (5:100 propane:steam) 34

4.8 Oil viscosity and API gravity for run 3 (5:100 propane:steam) ........................35

4.9 Propane injection, production, and dissolution rates after the third separator for run 3 (5:100 propane:steam). 
FIGURE

4.10 Cumulative gas production and composition after the third separator for Run 3 (5:100 propane:steam)....

4.11 Cumulative propane injection and production for Run 3

(5:100 propane:steam)

4.12 Temperature profile versus time for Run 4 (pure steam)....

4.13 Cumulative oil and water volumes versus time for Run 4 (pure steam)

4.14 Cumulative oil and water volumes versus pore volume injected for Run 4 (pure steam)

4.15 Oil and water rates versus time for Run 4 (pure steam). .43

4.16 Oil and water rates versus pore volume injected for Run 4 (pure steam)

4.17 Temperature profiles at 20-minute intervals - from $\mathrm{t}=0$ to $\mathrm{t}=240 \mathrm{~min}$ - for Run 4 (pure steam)

4.18 Injection, production and differential pressures for Run 4 (pure steam).............46

4.19 Oil viscosity and API gravity for Run 4 (pure steam) ....................................47

4.20 Temperature profile versus time for Run 5 (pure steam) .................................49

4.21 Cumulative oil and water volumes versus time for Run 5 (pure steam) .............50

4.22 Cumulative oil and water volumes versus pore volume of steam injected for Run 5 (pure steam)

4.23 Oil and water rates versus time for Run 5 (pure steam) ...................................52

4.24 Oil and water rates versus pore volume of steam injected for Run 5 (pure steam) 53

4.25 Temperature profiles at 20-minute intervals - from $\mathrm{t}=0$ to $\mathrm{t}=240 \mathrm{~min}-$ for Run 5 (pure steam) 54

4.26 Injection, production and differential pressures for Run 5 (pure steam).............55

4.27 Temperature profile versus time for Run 6 (5:100 propane:steam) ....................57

4.28 Cumulative oil and water volumes versus time for Run 6 (5:100 propane:steam) 58

4.29 Cumulative oil and water volumes versus pore volume of steam injected for Run 6 (5:100 propane:steam) 59

4.30 Oil and water rates versus time for Run 6 (5:100 propane:steam) 60 
FIGURE Page

4.31 Oil and water rates versus pore volume of steam injected for Run 6 (5:100 propane:steam)

4.32 Temperature profiles at 10 -minute intervals - from $\mathrm{t}=0$ to $\mathrm{t}=240 \mathrm{~min}-$ for Run 6 (5:100 propane:steam) .............................................................62

4.33 Injection, production and differential pressures for Run 6 (5:100 propane:steam)

4.34 Propane injection, production, and dissolution rates after the third separator for Run 6 (5:100 propane:steam)

4.35 Cumulative gas production and composition after the third separator

for Run 6 (5:100 propane:steam) ....

4.36 Cumulative propane injection and production for Run 6 (5:100 propane:steam) .66

4.37 Temperature profile versus time for Run 7 (5:100 petroleum distillate:steam) ...68

4.38 Cumulative oil and water volumes versus time for Run 7 (5:100 petroleum distillate:steam)

4.39 Cumulative oil and water volumes versus pore volume of steam injected for Run 7 (5:100 petroleum distillate:steam) .70

4.40 Oil and water rates versus time for Run 7 (5:100 petroleum distillate:steam).....71

4.41 Oil and water rates versus pore volume of steam injected for Run 7 (5:100 petroleum distillate:steam)

4.42 Temperature profiles at 20-minute intervals - from $\mathrm{t}=0$ to $\mathrm{t}=240 \mathrm{~min}-$ for Run 7 (5:100 petroleum distillate:steam)

4.43 Injection, production and differential pressures for Run 7 (5:100 petroleum

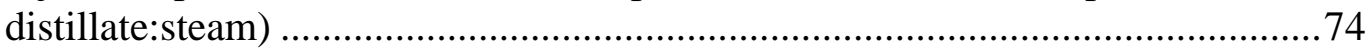

4.44 Oil viscosity and API gravity for Run 7 (5:100 petroleum distillate:steam) .......75

4.45 Temperature profile versus time for Run 8 (5:100 petroleum distillate:steam) ...77

4.46 Cumulative oil and water volumes versus time for Run 8 (5:100 petroleum

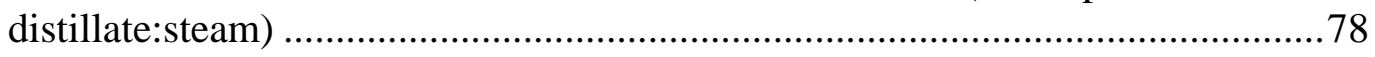

4.47 Cumulative oil and water volumes vs. pore volume of steam injected for Run 8 (5:100 petroleum distillate:steam) .79

4.48 Oil and water rates versus time for Run 8 (5:100 petroleum distillate:steam).....80

4.49 Oil and water rates versus pore volume of steam injected for Run 8 (5:100 petroleum distillate:steam) 
4.50 Temperature profiles at 10 -minute intervals - from $\mathrm{t}=0$ to $\mathrm{t}=240 \mathrm{~min}-$ for Run 8 (5:100 petroleum distillate:steam)

4.51 Injection, production and differential pressures for Run 8 (5:100 petroleum distillate:steam)

4.52 Oil rates versus time for Runs 3 to 8

4.53 Oil rates versus pore volume of steam injected (CWE) for Runs 3 to 8 .86

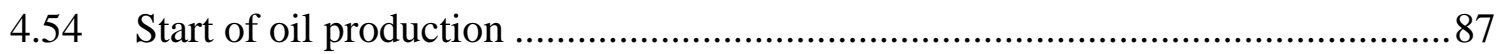

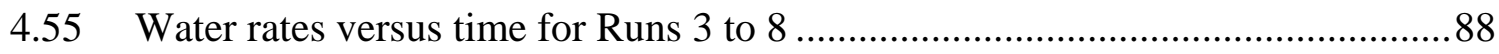

4.56 Water rates versus pore volume of steam injected (CWE) for Runs 3 to 8 ........89

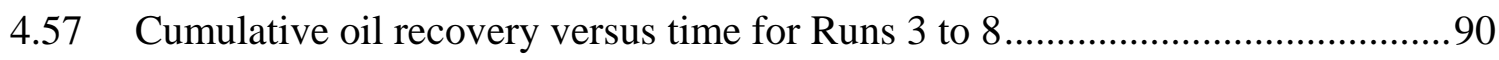

4.58 Cumulative oil recovery versus pore volume of steam injected (CWE) for Runs 3 to 8

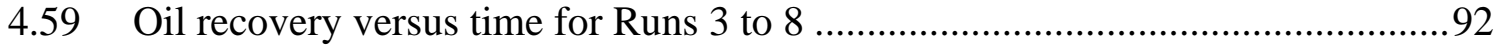

4.60 Oil recovery versus pore volume of steam injected (CWE) for Runs 3 to $8 \ldots . . . .93$

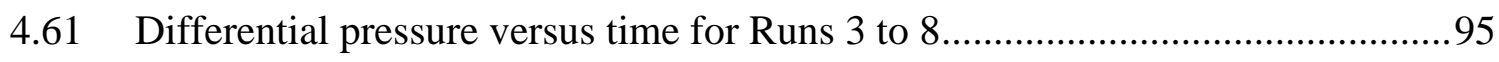

4.62 Differential pressure versus pore volume of steam injected (CWE)

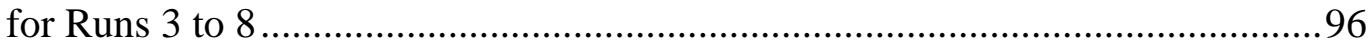

4.63 Oil density $\left(@ 50^{\circ} \mathrm{C}\right)$ vs. time for Runs 3 to 8 .....................................................97

4.64 Oil viscosity $\left(@ 50^{\circ} \mathrm{C}\right)$ vs. time for Runs 3 to 8 .....................................................98

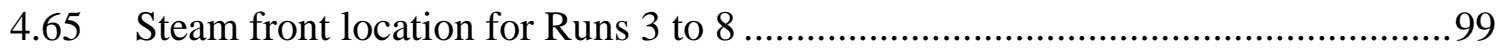

5.1 Frontal advanced displacement (reproduced from Myhill and Stegemeier) ......104

5.2 Vertical or gravity override displacement (reproduced from Neuman) ..............105

5.3 One dimensional heat balance for 1D displacement experiments.....................110

5.4 Schematic diagram of model and actual temperature profiles in $1 \mathrm{D}$ displacement experiments

5.5 Flow chart of analytical model and experimental data history-matching process.....

5.6 Steam front position for Run 3 (propane:steam = 5:100).

5.7 Cumulative oil production for Run 3 (propane:steam $=5: 100$ )

5.8 Steam front position for Run 4 (pure steam) 
FIGURE Page

5.9 Cumulative oil production for Run 4 (pure steam) ......................................121

5.10 Steam front position for Run 5 (pure steam) ..............................................122

5.11 Cumulative oil production for Run 5 (pure steam) .......................................122

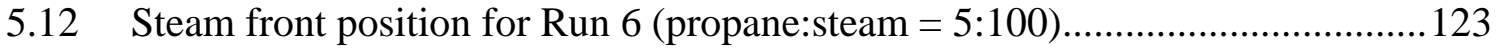

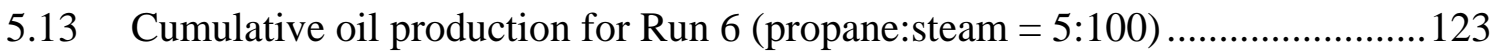

5.14 Steam front position for Run 7 (petroleum distillate:steam $=5: 100$ ) $\ldots \ldots \ldots \ldots \ldots . . . .124$

5.15 Cumulative oil production for Run 7 (petroleum distillate:steam $=5: 100$ ) $\ldots \ldots . .124$

5.16 Steam front position for Run 8 (petroleum distillate:steam $=5: 100$ ) $\ldots \ldots \ldots \ldots \ldots . . . . .125$

5.17 Cumulative oil production for Run 8 (petroleum distillate:steam $=5: 100$ ) $\ldots \ldots . .125$ 


\section{LIST OF TABLES}

Page

Table 3.1 List of main components of the apparatus. ..............................................18

Table 4.1 Sand mix properties from Runs 3 to 8 ...................................................26 


\section{CHAPTER I}

\section{INTRODUCTION}

The San Ardo field is located in the Central Coastal region of California, about 217 km (135 miles) roughly due west of Bakersfield. This giant oil field (OOIP in excess of 1 billion STB) contains heavy oil with oil gravity of $11^{\circ}-12^{\circ} \mathrm{API}^{1-3}$. The field has been steamflooded since 1968. The field contains two main heavy oil reservoirs ${ }^{3}$. The shallower Lombardi reservoir lies about $2100 \mathrm{ft}$ with an average net pay thickness of 115 $\mathrm{ft}$ in the Main Area (40 ft in North Area), oil gravity of $11^{\circ}$ API and in-situ oil viscosity of $3000 \mathrm{cp}$. The deeper Aurignac reservoir lies at about $2350 \mathrm{ft}$ with an average net pay thickness of $100 \mathrm{ft}$, oil gravity of $12^{\circ} \mathrm{API}$ and in-situ oil viscosity of $300 \mathrm{cp}$.

In fields like San Ardo, where extra-heavy oil is produced, continuous steam injection or steam flooding has been used for years to improve recovery. Several mechanisms operate in steam injection, mainly oil viscosity reduction and steam distillation of the oil. When steam is injected into a reservoir, the resulting phase distribution forms five distinct zones. Each zone has different characteristics with respect to the distance from the injection well. The first zone -nearest to the injectorcorresponds to the steam zone, where water in liquid and vapor phase and mainly residual oil are present. The light fractions of the oil are vaporized and condense ahead of the steam front creating a solvent bank, which comprises the second zone. The solvent bank is miscible with the oil, thereby reducing its interfacial tension and viscosity. The third zone consists of the hot water zone where steam and volatile oil condense upon contact with the cold matrix. As a result of oil viscosity reduction and displacement in the first three zones, an oil bank (fourth zone) is formed. The fifth zone (farthest away from the injector) is composed of the original oil.

This thesis follows the style of Journal of Petroleum Technology. 
Since it was first implemented, the principles of steam flooding have remained basically unchanged. In order to improve the process, investigations have been made to determine the viability of injecting steam along with other additives with the purpose of enhancing recovery. Additives like carbon dioxide and light hydrocarbons have been tested and showed to improve the recovery of heavy oils in the laboratory. However, the combined injection of steam and hydrocarbon additives (solvent) is often too costly and economically unattractive due to cost of the solvent. Therefore, the need exists to better understand the oil recovery mechanisms associated with steam-hydrocarbon injection (e.g. steam-propane) in order to possibly improve the technical and economical feasibility of these processes.

In the last five years, experimental and simulation studies ${ }^{4-19}$ have been carried out in the Ramey Laboratory of the Petroleum Engineering Department at Texas A\&M University to investigate the effects of the combined injection of steam and propane on heavy oil recovery. These experiments have shown encouraging results, specifically accelerated oil recovery when compared to pure steam injection.

The proposed research is intended to obtain a more complete understanding of the production mechanisms involved in steam-propane and steam-petroleum distillate injection using crude oil from the San Ardo field. The first aspect of the research is to perform a series of experiments to evaluate the effect of propane and petroleum distillate as steam additives in production acceleration and recovery.

The second aspect of the research is to model analytically the reservoir temperature propagation and cumulative oil production during the experimental runs. The results of the analytical model will be compared against experimental data to verify the validity of the model. The model will be based on heat, material balance and Darcy's Law assuming one-dimensional displacement of oil by steam of steam with additives during the experimental runs. 


\subsection{Research Objectives}

The main research objective is to evaluate the effect of propane and petroleum distillate as steam additives to enhance injectivity and to accelerate production of San Ardo oil. To achieve these objectives, steam-propane and steam-petroleum distillate displacement experiments will be conducted using propane:steam and petroleum distillate: steam mass ratio of 5:100.

The parameters to be recorded during the experiments include injection rate, injection and production pressures, cell temperatures, and produced volumes of liquids and gases. Density and viscosity measurements are carried out for the produced oil samples. Final oil recovery will be measured in addition to the production rates that will be compared between the different runs.

An analytical model of temperature propagation within the sand mix and cumulative oil production during the experimental runs will be developed. This model will be based on heat and material balance and Darcy's Law assuming one dimensional displacement of oil by steam in the injection cell. The results of the analytical model will be compared against data to verify the validity of the model. 


\section{CHAPTER II}

\section{LITERATURE REVIEW}

Several studies have been carried out to test the effects of injecting steam along with other gaseous additives. In this section, a literature review covering previous experiences with the combined use of steam and gaseous additives will be presented.

Redford (1982) ${ }^{21}$ conducted experiments to study the effect of adding carbon dioxide, ethane and/or naphtha in combination with steam. His results showed that the addition of carbon dioxide or ethane improved the recovery. Further recovery was reached when naphtha was added.

Harding et al. (1983) ${ }^{22}$ presented both experimental and simulation results suggesting that the co-injection of carbon dioxide or flue gas with steam yielded higher recoveries when compared to pure steam injection.

Stone and Malcolm (1985) ${ }^{23}$ performed several tests to study the benefits of injecting carbon dioxide along with steam. Higher production rates were obtained for the case of steam-carbon dioxide injection. Good agreement was found when comparing the experimental results with a numerical simulation also conducted in the study.

Stone and Ivory (1987) ${ }^{24}$ carried out further investigations using the model from Stone and Malcolm. ${ }^{22}$ This time, experiments with $\mathrm{CO}_{2}$ presoak and $\mathrm{CO}_{2}$ co-injection with a solvent were conducted. They found that under certain conditions, carbon dioxide pre-soaking increased recovery above the conventional $\mathrm{CO}_{2}$-steam injection.

Nasr et al. (1987) ${ }^{25}$ presented results of experiments conducted to test the effects of injecting $\mathrm{CO}_{2}, \mathrm{~N}_{2}$ and flue gas with steam. Both continuous and cyclic injections were tested. The addition of gases increased bitumen recovery. The use of $\mathrm{CO}_{2}$ resulted in higher recoveries when compared to $\mathrm{N}_{2}$ and flue gas.

Frauenfeld et al. (1988) ${ }^{26}$ presented results showing that for oils without an initial gas content, co-injection of $\mathrm{CO}_{2}$ with steam was capable of improving oil recovery over 
that obtained with pure steam injection. On the other hand, when an initial non-zero gas saturation was present, co-injection of $\mathrm{CO}_{2}$ was not beneficial.

Metwally (1990) ${ }^{27}$ employed cores from the Lindbergh Field to investigate the effects of carbon dioxide and methane on the performance of steam processes. The experiments were carried out to determine the differences in performance of simultaneous injection of steam and a gaseous additive and an injection of a gas slug prior to steam injection. The results showed that injecting a $\mathrm{CO}_{2}$ slug prior to the steam improved injectivity. However, the presence of a non-condensable gas with steam did not improve steam drive recovery and resulted in higher residual oil saturation compared to pure steam injection.

Gumrah and Okandan (1992) ${ }^{28}$ performed linear and 3-D displacement experiments to evaluate the performance of $\mathrm{CO}_{2}$ addition to steam on the recovery of $24^{\circ} \mathrm{API}, 12^{\circ} \mathrm{API}$ and $10.6^{\circ} \mathrm{API}$ oils. The $1 \mathrm{D}$ tests indicated that the oil recovery increased with increasing $\mathrm{CO}_{2}$ /steam ratios until an optimum value was reached. The addition of $\mathrm{CO}_{2}$ did not produce a significant increase in the recovery of the lighter oil. However, for the heavier oils, the oil production rate was increased considerably.

Bagci and Gumrah (1998) ${ }^{29}$ performed experiments with both linear and 3D models to investigate the effects of injecting methane and carbon dioxide along with steam in a $12.4^{\circ} \mathrm{API}$ heavy oil. The results showed that the use of $\mathrm{CO}_{2}$ or $\mathrm{CH}_{4}$ combined with steam yielded a higher incremental oil recovery than of with pure steam injection.

Butler and Mokrys (1991) ${ }^{30}$ described a new recovery concept related to the steam-assisted gravity drainage (SAGD) process. The process was intended to be used in thin reservoirs, where the application of SAGD alone was uneconomical due to the high heat losses to the formations above and below the reservoir. The process, called VAPEX, used a solvent, such as propane, which could form a vapor-filled chamber within the reservoir. Vapor dissolves in the oil around the chamber and the resulting solution drains, driven by gravity, to a horizontal production well placed low in the formation. A well, located at the top of the reservoir, is used to inject steam and the solvent. 
Additional work by Butler and Mokrys ${ }^{31,32,33}$ presented results of further investigations conducted on the VAPEX process. Their results showed that the process could be applied economically for heavy oil recovery. Additional advantages derived from VAPEX are a partial in situ deasphalting and a reduction of the content of heavy metals. The resulting oil can be lighter, of a higher quality and better suited for a direct refining.

Goite (1999) $)^{4,7}$ conducted several experiments to determine the influence of injecting steam with propane as an additive for $12.5^{\circ}$ API from the Morichal field, Venezuela. Results showed that the optimal concentration by weight of propane lies somewhere in the region of $5 \%$.

Ferguson (2000) ${ }^{5,7}$ continued Goite’s experiments using a constant steam mass rate. Several tests were performed to determine the optimum propane:steam mass ratio. Acceleration of oil production was found in the steam-propane runs when compared to pure steam injection. The optimum propane:steam mass ratio appeared to be around 5:100. The acceleration in oil production was thought to be due to the dry distillation process in which the lighter oil fractions are vaporized and carried by propane. On contact with the colder part of the reservoir, the light fractions condense and are miscible with the oil, thus lowering the interfacial tension and decreasing the viscosity of the oil.

Tinns (2001) $)^{8}$ carried out steam-propane experiments using 5:100 propane:steam mass ratio on $21^{\circ} \mathrm{API}$ Kulin oil from Indonesia. The same effect of production acceleration was observed in these experiments. Viscosity and density measurements indicated an increase in API gravity and a reduction of viscosity of the produced oil. Furthermore, injectivity was improved with the addition of propane to the steam. A reduction in the maximum injection pressure from 85 psig to 78 psig was observed in the experiments.

Rivero (2002) $)^{9,10,15}$ conducted a series of experiments to evaluate the effect of additive on recovery of Hamaca heavy oil. The same effect of production acceleration was observed in these experiments. Improvement in steam injectivity of up to threefold was observed even with propane:steam mass ration as low as 2.5:100. 
Plazas (2002) ${ }^{11}$ conducted a series of experiments of steam distillation and steam-propane distillation on light crude oil $\left(34.2^{\circ} \mathrm{API}\right)$ and intermediate crude oil (25.1 $\left.{ }^{\circ} \mathrm{API}\right)$. The results showed that the yield for steam-propane distillation is higher than steam distillation for the intermediate crude oil. On the other hand, propane seemed to have little effect on the light oil.

Hendroyono (2003) $)^{13,15}$ found acceleration in production with as little as 1.25:100 propane:steam mass ratio. Up to 30\% acceleration with an apparent optimum ratio of 5:100 propane:steam was observed. Injectivity was reported to be three times higher than with pure steam injection.

Ramirez-Garnica (2004) ${ }^{16}$ performed distillation experiments on synthetic oil, showing that propane effectively reduces the boiling point of hydrocarbons. Thus, yields are higher with steam-propane, followed by that of pure steam injection, and lowest under dry distillation (with nitrogen).

Nesse (2004) $)^{17}$ found steam-propane injection accelerates the start of production for $21^{\circ}$ API of Duri oil. The propane does not have the same effect when used with hot water, or water alternating steam. Pure steam injection accelerates oil production more than these two other methods. 


\section{CHAPTER III}

\section{EXPERIMENTAL APPARATUS AND PROCEDURE}

\subsection{Experimental Apparatus}

The experimental set-up is comprised of five main parts: fluid injection system, injection cell, fluid production system, gas measurement system and data recording system. A schematic diagram of the apparatus is shown in Fig. 3.1.

\subsubsection{Fluid Injection System}

This system is comprised of a High Performance Liquid Chromatography (HPLC) pump, a steam generator, an ISCO syringe pump, one accumulator, and a petroleum distillate vessel. Distilled water is injected into the steam generator using the HPLC pump at a set rate. A backpressure regulator with a gauge is mounted directly after the pump to maintain its minimum required operating pressure of $1200 \mathrm{psig}$. At ambient temperature $\left(75^{\circ} \mathrm{F}\right)$, saturated vapor pressure of propane is $135 \mathrm{psig}$. Therefore, the propane needs to be pressurized to about 600 psig before being introduced into the steam generator. Propane from the propane cylinder is flowed into the top of the accumulator above the piston. It is then pressurized by injecting water from an ISCO pump into the accumulator below the piston until the desired pressure (600 psig) is reached. At this desired pressure, usually after about two days, any propane within vapor phase will transform into liquid phase. The ISCO pump is then disconnected from the accumulator and nitrogen from a cylinder is connected to the accumulator to maintain the pressure at about 600 psig.

For steam-propane injection runs, liquid mass flow controller regulates the propane rate from the accumulator. The HPLC pump feeds water at a constant rate, which is mixed with propane and the mixture injected into the steam generator. The resulting mixture of propane and steam is then injected into the cell through an injection line around which is wrapped a band heater. The heater is used to prevent heat loss in the 


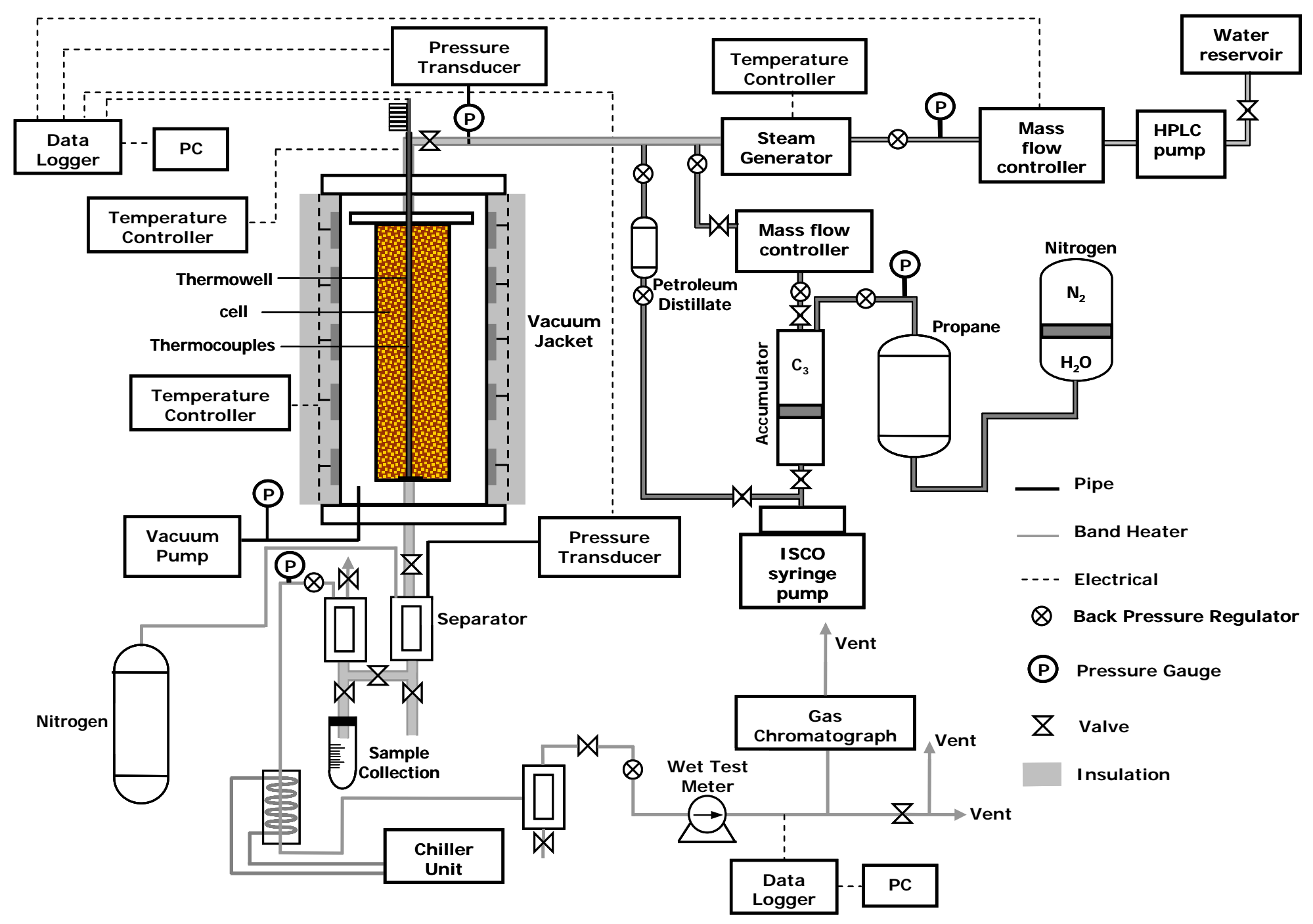

Fig. 3.1- Schematic diagram of experimental apparatus. 
injection line. A temperature controller on the band heater is used to adjust the temperature of the injected fluid, effectively allowing the user to manipulate it all the way to the entry point of the cell.

For steam-petroleum distillate injection runs, $75 \mathrm{ml}$ vertical steel vessel is mounted about six inches from the entry point of the cell. The vessel contains desired volume of petroleum distillate which will be displaced by water utilizing ISCO pump. The petroleum distillate is injected along with the steam into the cell at about 0.275 $\mathrm{ml} / \mathrm{min}$ of injection rate resulting approximately thirteen minutes petroleum distillate injection period. The petroleum distillate slug thus moves ahead of the steam. This vessel is connected with the system using a T-connection with a valve that controls the petroleum distillate flow.

\subsubsection{Injection Cell}

The injection cell is a stainless steel cylinder (Fig. 3.2) with an internal diameter of 2.904 in. and a length of 27 in. It holds a mixture of sand, water and oil that have been carefully weighed and measured. For the purpose of measuring the temperature profile, a thermowell is placed along the longitudinal axis of the cell. Six thermocouples are placed in the thermowell. These thermocouples are spaced out at different intervals to monitor temperature propagation through the experiment (Fig. 3.3). At the bottom of the cell there is a sand screen preventing sand particles from being produced with the fluid.

The cell is placed inside a larger diameter vacuum-heater jacket (Fig. 3.4). During experimental runs, the cell-vacuum jacket annulus is evacuated using an external vacuum pump. This helps reduce heat loss. After the cell has been placed inside the vacuum jacket, the temperature is set at desired level with a temperature controller. To ensure uniform temperature throughout the cell, this heater is left on for about 12 hours to stabilize the system at "reservoir temperature". 


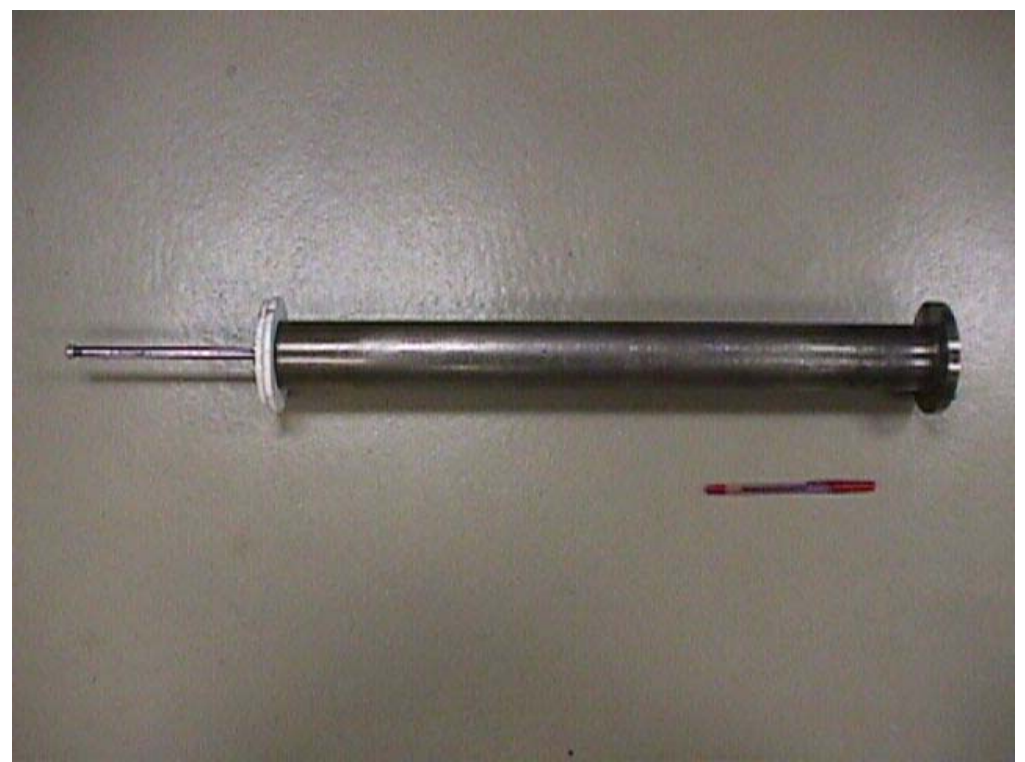

Fig. 3.2-Photograph of injection cell (cell outlet at the left).
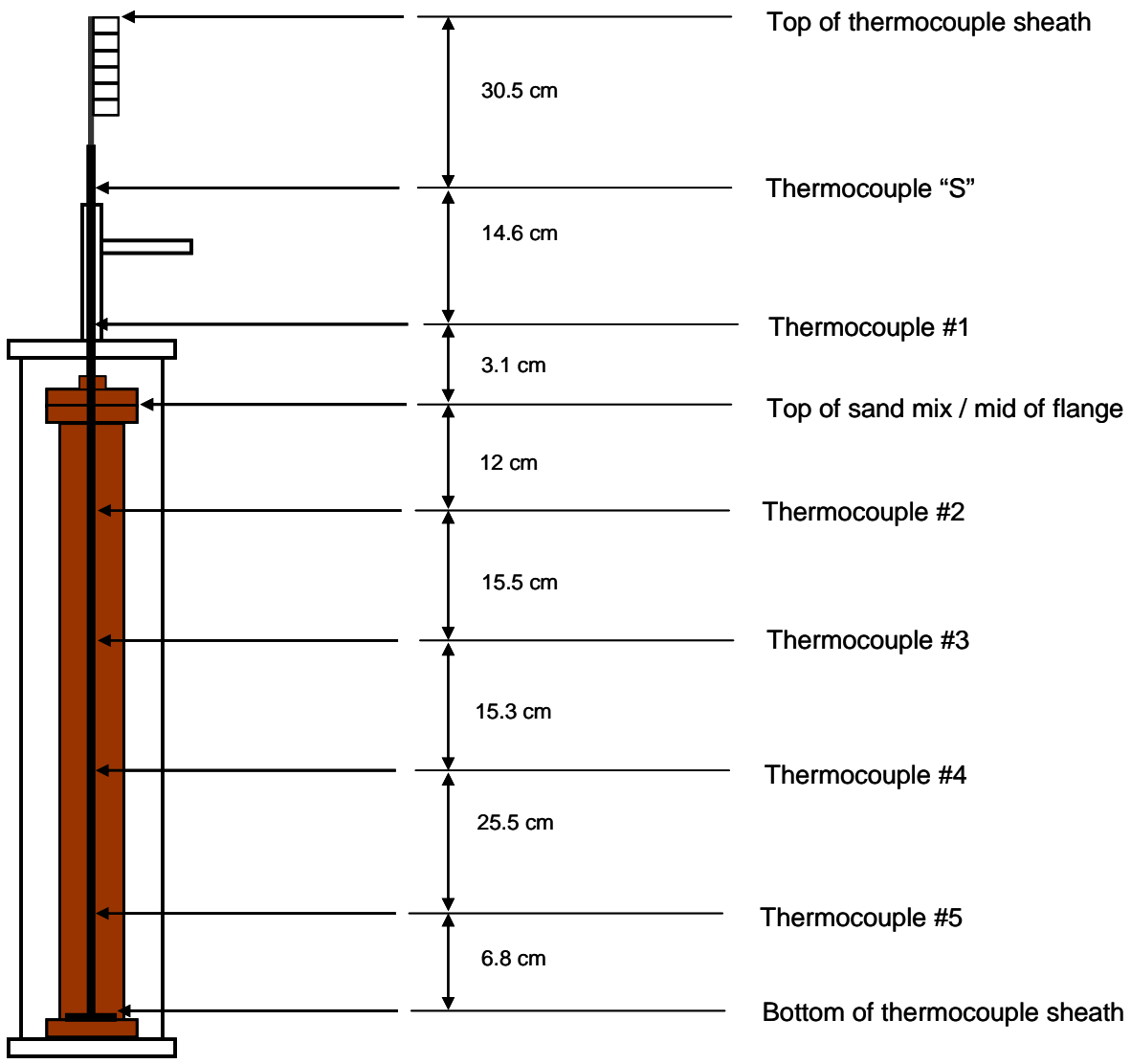

Fig. 3.3- Positions of the thermocouples in the injection cell. 


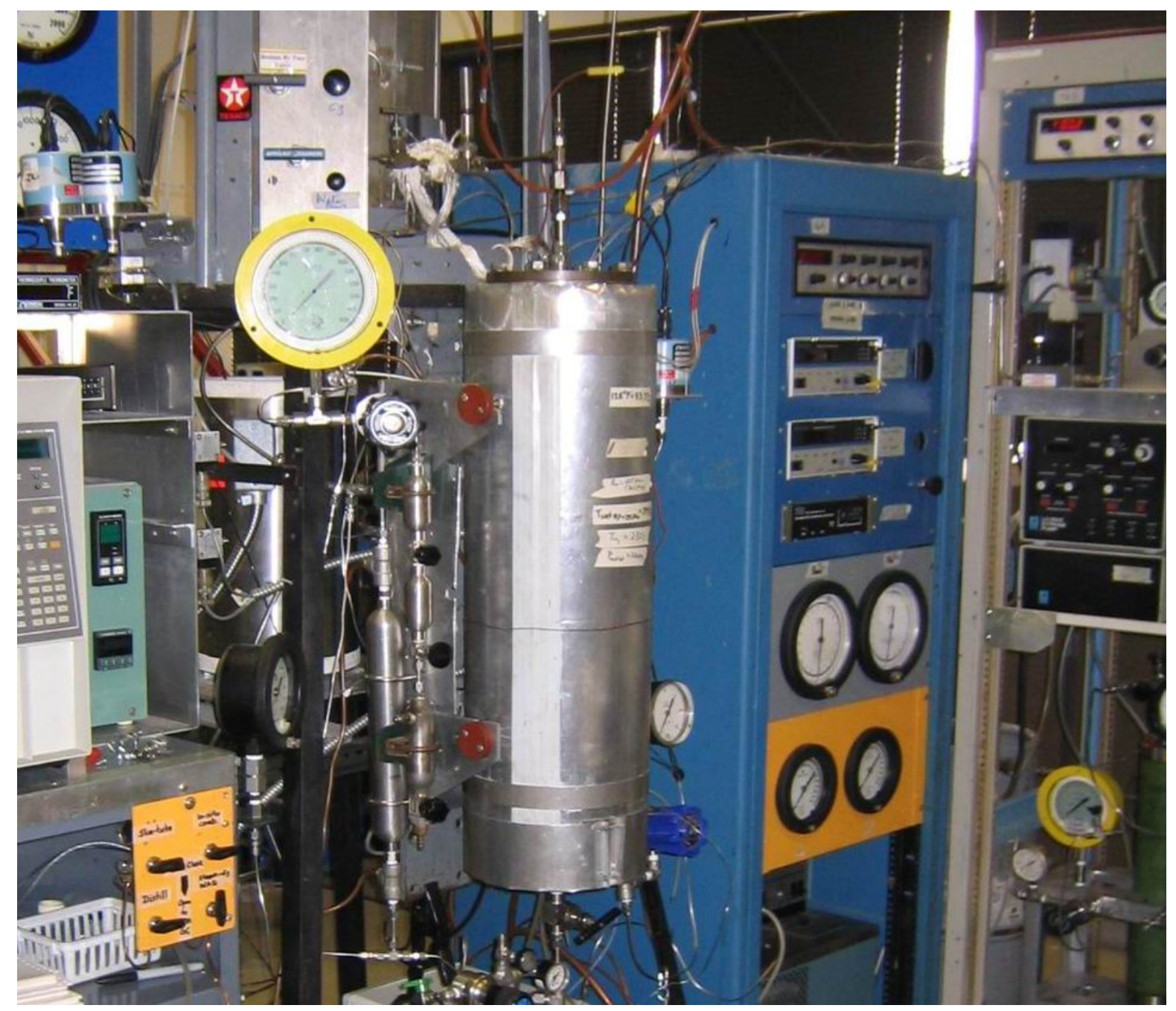

Fig. 3.4- Photograph of vacuum jacket. 


\subsubsection{Fluid Production System}

The fluids leaving the cell are produced through a heated line, which is connected to a set of three separators and a condenser (Fig. 3.5). A band heater (set at $60^{\circ} \mathrm{C}$ ) is wrapped around the production line from the cell bottom to the first separator. Two backpressure regulators are used. The first backpressure regulator (Fig 3.6) maintains the cell outlet pressure (with the help of nitrogen from a cylinder) at a constant predetermined level during the experiment (i.e. 260 psig). A second backpressure regulator maintains outlet pressure of the second separator at about 50 psig. Fluid from the first separator goes to the second separator where gas is separated from the liquid. Liquid samples are collected from the bottom of the second separator which maximizes liquid recovery. Gas from the top of the second separator is first cooled in the condenser. Cooling water for the condenser is provided by a chiller unit. To ensure no liquid carryover in the gas stream, the gas passes through a third separator and then to the gas measurement system.

\subsubsection{Gas Measurement System}

Produced gasses from the third separator flow to a wet test meter, where the cumulative gas production rate is measured. A control valve is used to redirect a portion of the produced gas to a gas chromatograph (GC), which will be used to determine its composition. Before entering the GC (Fig. 3.7), the gas is passed through a silica packed cylinder to remove any moisture. An automatic gas sampler is installed in the gas chromatograph to inject the sample. All the gas sampling, injection and measurement operations are carried out automatically at predetermined intervals.

\subsubsection{Data Measurement and Recording System}

A data logger and a personal computer (Fig. 3.8) are used to record the following parameters: injection pressure, outlet pressure, propane injection rate, produced gas rate, 


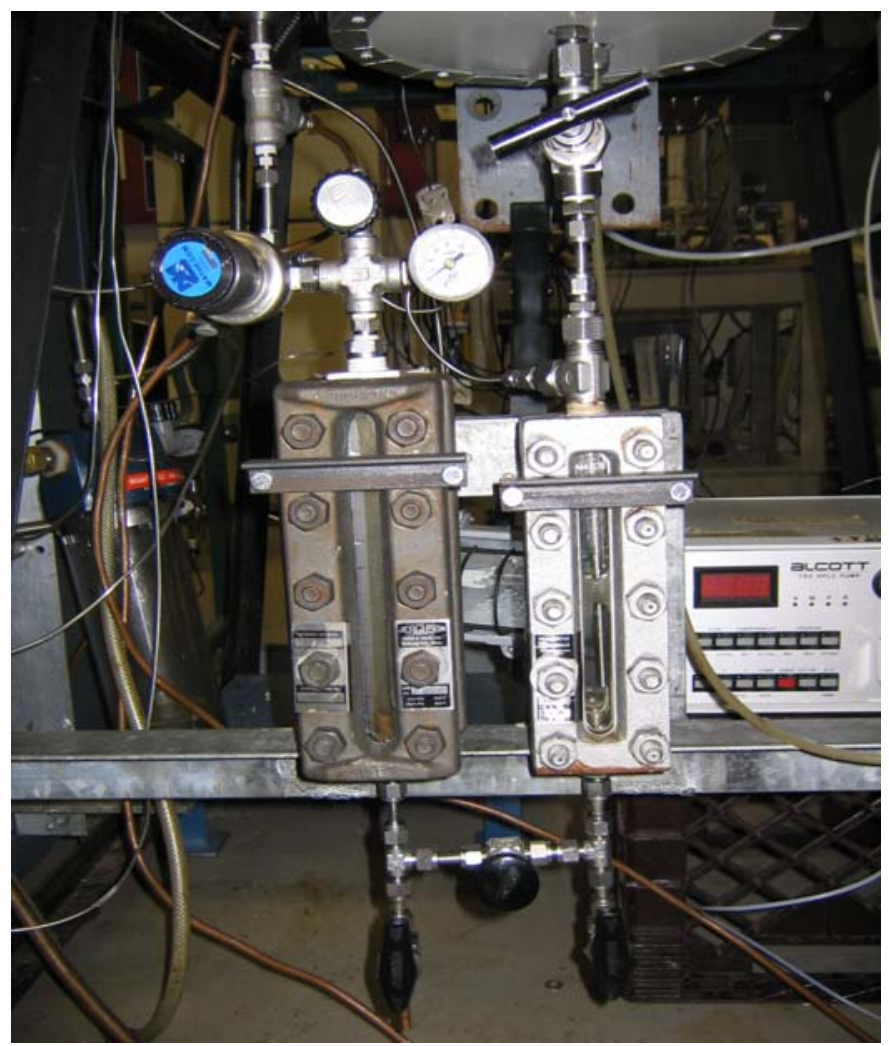

Fig. 3.5- Photograph of first separator (right) and second separator (left).

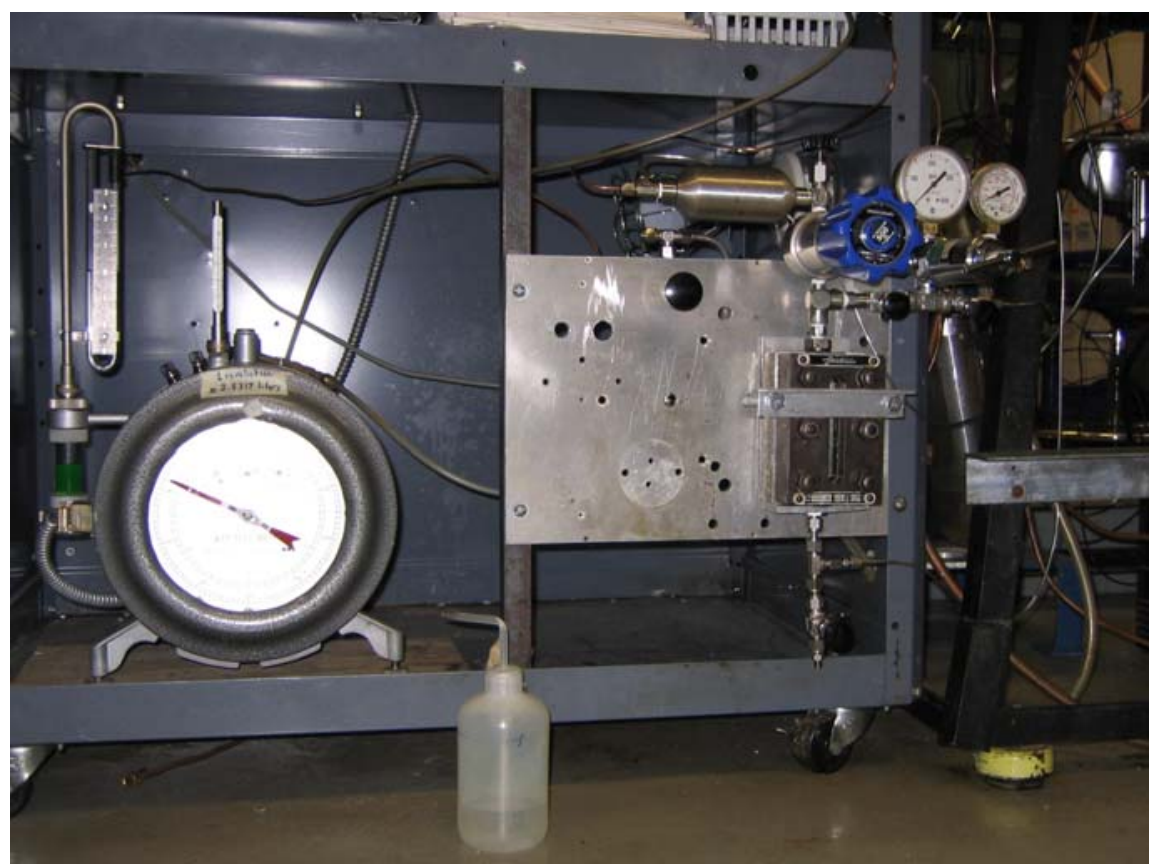

Fig. 3.6- Photograph of back pressure valve and wet test meter. 


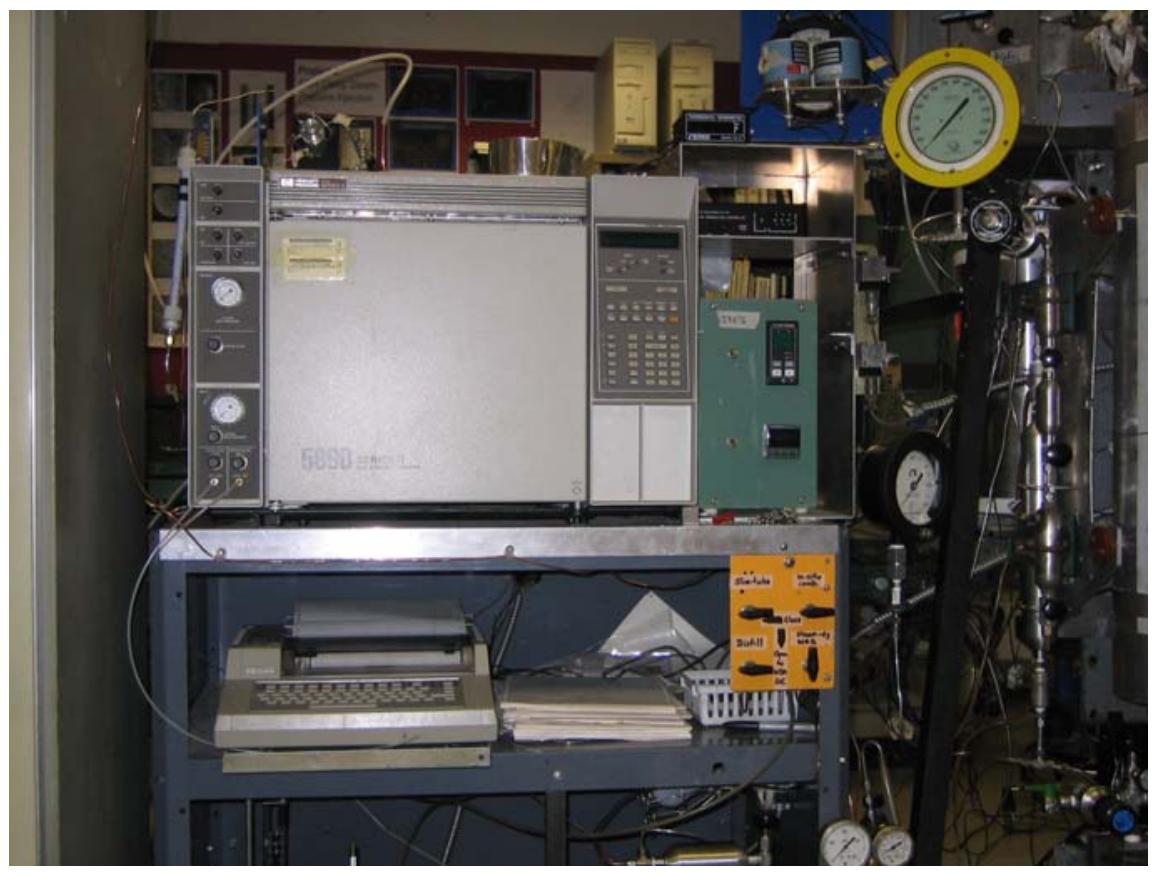

Fig. 3.7- Photograph of gas chromatograph.

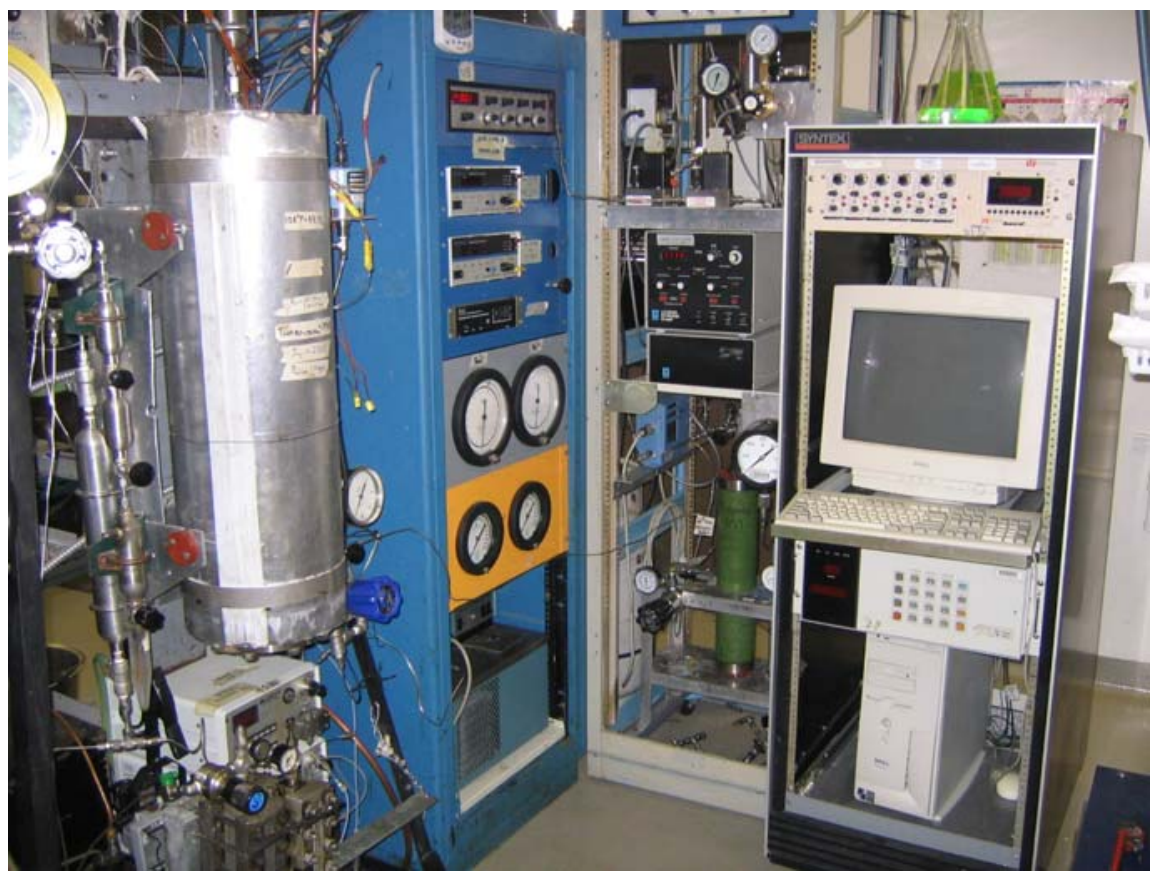

Fig. 3.8- Photograph of data logger, personal computer and temperature controllers. 
injection temperature and temperature profile along the longitudinal axis of the cell. The parameters are recorded at 30-second intervals.

A brief description of the main components of the apparatus follows.

1. HPLC pump

An Alcott High Performance Liquid Chromatograph pump supplies water to the steam generator at a very accurate rate $(5.5 \mathrm{ml} / \mathrm{min} \pm 0.1 \mathrm{ml} / \mathrm{min})$.

2. ISCO syringe pump.

An ISCO Precision High Pressure Syringe Pump supplies water to the first accumulator to pressurize the propane until the desired pressure. The pump is designed to work with aqueous and organic solvents. The pump injects water into a $950 \mathrm{ml}$ accumulator, which contains the propane that will be compressed into liquid at the desired pressure.

3. Steam generator

An electric powered steam generator of 1000 watts maximum power, sustaining a maximum pressure of 2000 psig and a maximum temperature of $1200^{\circ} \mathrm{F}$ provides the steam necessary for the experiments.

4. Temperature controller

A dual-circuit temperature controller is used to maintain a constant temperature of the steam generator. Temperature controllers are also used to control temperature of the heating jacket, and band heater around the separator system.

5. Injection cell

The stainless steel cell measures 27 inches long with an inside diameter of 2.904 inches.

6. Accumulator

The accumulator contains a piston used to compress the propane (above piston) at the desired pressure by means of water injected (below piston) with the ISCO syringe pump.

7. Mass flow controller

Regulates the mass rate of propane injected into the steam generator. 
8. Heating jacket

The heating jacket consists of 31.25 inches long, 5.6 inches inner diameter, 7.9 inches outer diameter, stainless steel cylinder with thermal insulation on the outside. Five steel band heaters are attached to the outer wall of the steel cylinder. The top and bottom are sealed by stainless steel flanges, which are insulated by two caps. The injection cell is placed within the heating jacket that is evacuated to minimize heat losses.

9. Vacuum pump

Establishes minus 29 inches mercury vacuum in the annulus between the heating jacket wall and the cell to minimize the heat losses.

10. Wet test meter

The wet test meter measures the volumetric flow of the produced gas from the first separator. It is equipped with a ten diode counter that enables automatic recording of produced gas rate.

11. Three stage separation and collection system

It is used to separate the produced gas from the produced liquid (water and oil). Nitrogen line for backpressure control is connected to the first separator, while a gas sampling lines is connected the outlet of the third separator.

12. A vessel for petroleum distillate

A vessel (inner volume of $153.77 \mathrm{ml}$ ) that contains petroleum distillate is mounted at about one foot to from the injection point. This vessel contains $66 \mathrm{ml}$ of petroleum distillate at the top layer (equivalent to 5:100 petroleum distillate:steam mass ratio and assuming 4 hours of experimental run is conducted) and 87 of water (at the bottom layer, connected to the ISCO syringe pump). The petroleum distillate is injected along with the steam for steampetroleum distillate runs. 
13. Temperature controller

A dual-circuit temperature controller is used to maintain a constant temperature of the steam generator. Temperature controllers are also used to control temperature of the heating jacket, and band heater around the separator system.

14. Gas chromatograph

The gas chromatograph analyzes the composition of the gas samples collected in the first separator and the production outlet.

15. Data logger/recording system

Takes measurements and records pressure, temperature, produced and injection gas rates and propane injection rate data every 30 seconds.

A list of the principal components of the experiment setup can be observed in

Table 3.1.

Table 3.1- List of main components of the apparatus.

\begin{tabular}{|c|c|}
\hline Water reservoir & 4-liter plastic container \\
\hline HPLC pump & Alcott 760 HPLC \\
\hline Steam generator & $\begin{array}{l}\text { Custom-made by Texaco. Max. pressure: } 2000 \text { psig. Max. } \\
\text { temperature: } 1200^{\circ} \mathrm{F}\end{array}$ \\
\hline Injection cell & $\begin{array}{l}\text { Stainless steel tube. Length: } 27 \text { in. } \\
\text { I.D.: } 2.904 \text { in. }\end{array}$ \\
\hline Temperature controller & Digi-Sense. Model 2186-10A, 20 Amp peak \\
\hline $\begin{array}{l}\text { Temperature controller- } \\
\text { band heater }\end{array}$ & VICI. Model ITCK10. 10 Amp max \\
\hline $\begin{array}{l}\text { Temperature controller- } \\
\text { steam generator }\end{array}$ & Eurotherm Digital Controller. Model 808 \\
\hline Vacuum Pump & Welch director II, model 8811 \\
\hline Mass Flow Controller & Brooks. Model 5850E series. Max. flow $1000 \mathrm{~cm}^{3} / \mathrm{min}$ \\
\hline Wet test meter & GCA/Precision Scientific, capacity $0.1 \mathrm{ft}^{3}$ per revolution. \\
\hline Gas chromatographs (GC) & Hewlett Packard 5890 Series II \\
\hline
\end{tabular}


Table 3.1- Continued.

\begin{tabular}{|c|c|}
\hline Data logger & $\begin{array}{l}\text { Hewlett Packard data acquisition unit. Model 3497A } \\
\text { with 44422A T-couple acquisition assembly. }\end{array}$ \\
\hline Back-pressure regulator & $\begin{array}{l}\text { Tescom Corporation. Max. pressure } 500 \text { psig (for } \\
\text { nitrogen), } 1500 \text { psig for HPLC backpressure control. }\end{array}$ \\
\hline Rheometer & $\begin{array}{l}\text { Brookfield. Model DV-III with cone and plate } \\
\text { assembly. (Fig. 3.9) }\end{array}$ \\
\hline Chiller unit & Hasskriss Co. Model R100 \\
\hline Pressure transducer & Omega. Model PX 621. Max pressure 1000 psig. \\
\hline Tubing & $\begin{array}{l}\text { 1/4-in., 1/8-in. and 1/16-in. stainless steel tubing with } \\
\text { Swagelok and Autoclave connections. }\end{array}$ \\
\hline Control valves & Autoclave Engineers 1/4-in. Withey 1/4-in., 1/8-in. \\
\hline Thermocouples & Omega JMQSS-020. Type J. Sheath diameter .020-in. \\
\hline Gauges & $\begin{array}{l}\text { HEISE, CM-105620 and CM-105618, Bourdon tube } \\
403 \text { ST-ST. Max. pressure } 500 \text { psig. }\end{array}$ \\
\hline Temperature bath & GCA/Precision Scientific. Model TC 500. (Fig. 3.10) \\
\hline Electronic balance & METTLER PM 4600 Delta Range. Capacity 10.45 kg. \\
\hline Balance & OHAUS Heavy duty. Capacity 20 kg. \\
\hline Thermometer & Kessler. ASTM 40C \\
\hline Industrial sand & 100 mesh supplied by Baker Oil Tools \\
\hline San Ardo oil & Circa $12^{\circ} \mathrm{API}, 2800 \mathrm{cp}$ at $50^{\circ} \mathrm{C}$ \\
\hline Paint thinner & Commercial paint thinner \\
\hline Nitrogen tank & Botco, Nitrogen compressed, 1500 psig. \\
\hline Centrifuge & $\begin{array}{l}\text { IEC HN-II Benchtop centrifuge, 0-3000 RPM (Fig. } \\
\text { 3.11) }\end{array}$ \\
\hline
\end{tabular}

Figs. 3.9 - 3.12 show various components of the experimental apparatus, while Fig. 3.13 shows the entire set up. 


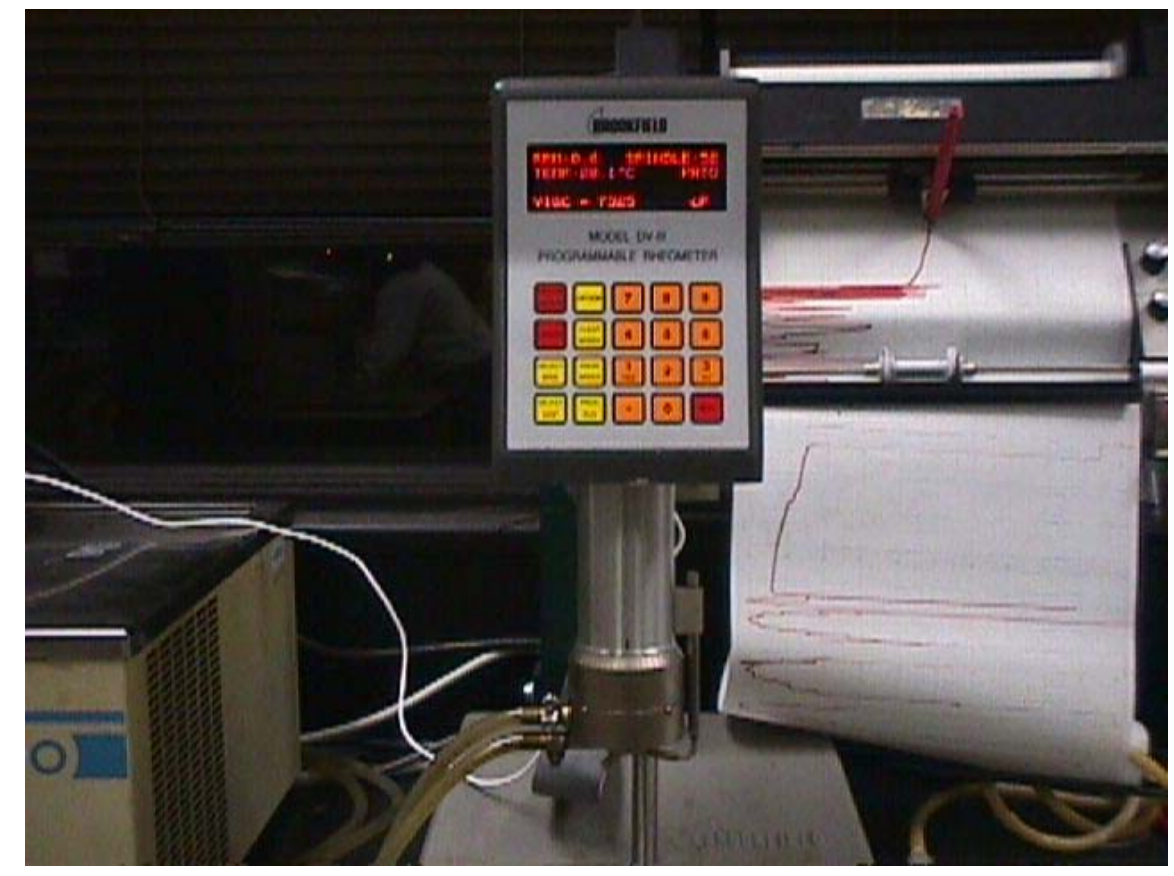

Fig. 3.9- Cone-plate rheometer.

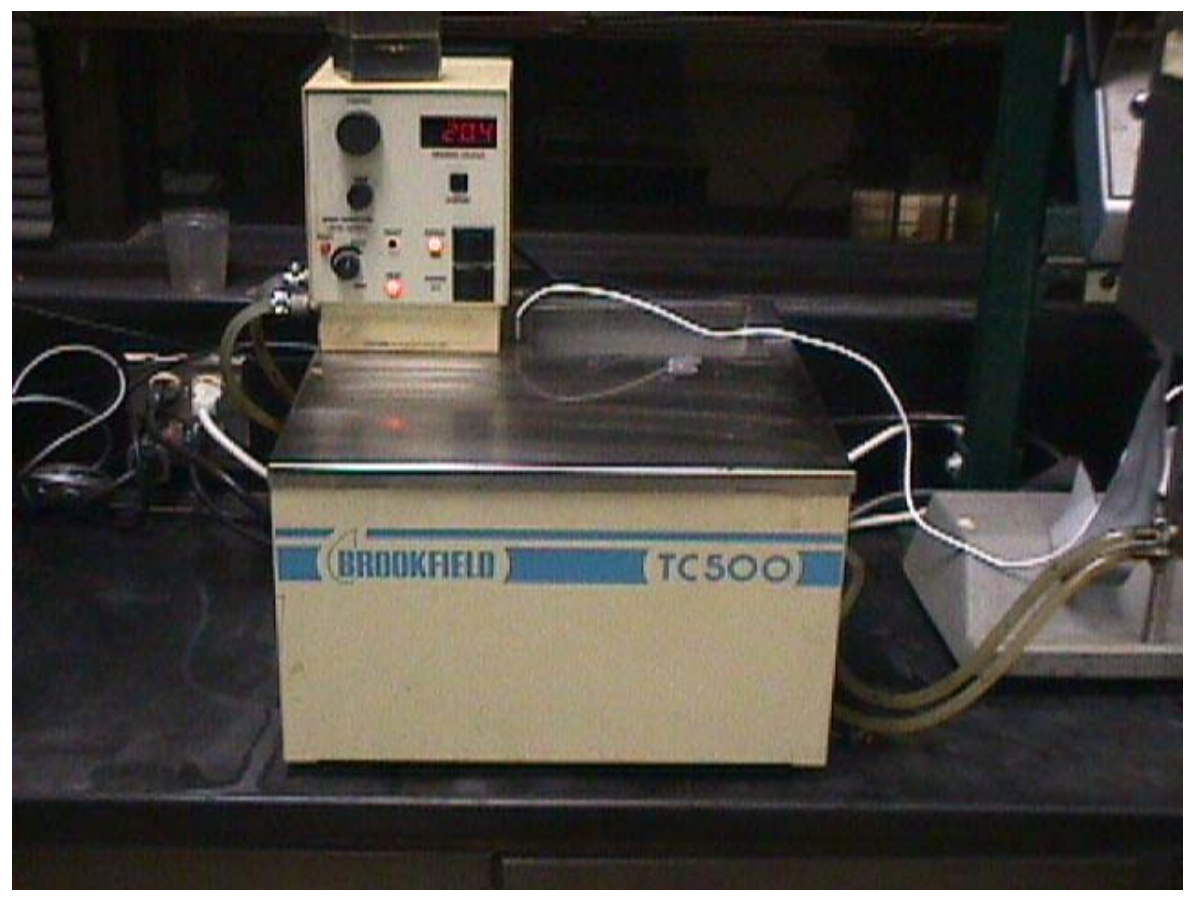

Fig. 3.10- Water bath for viscometer. 


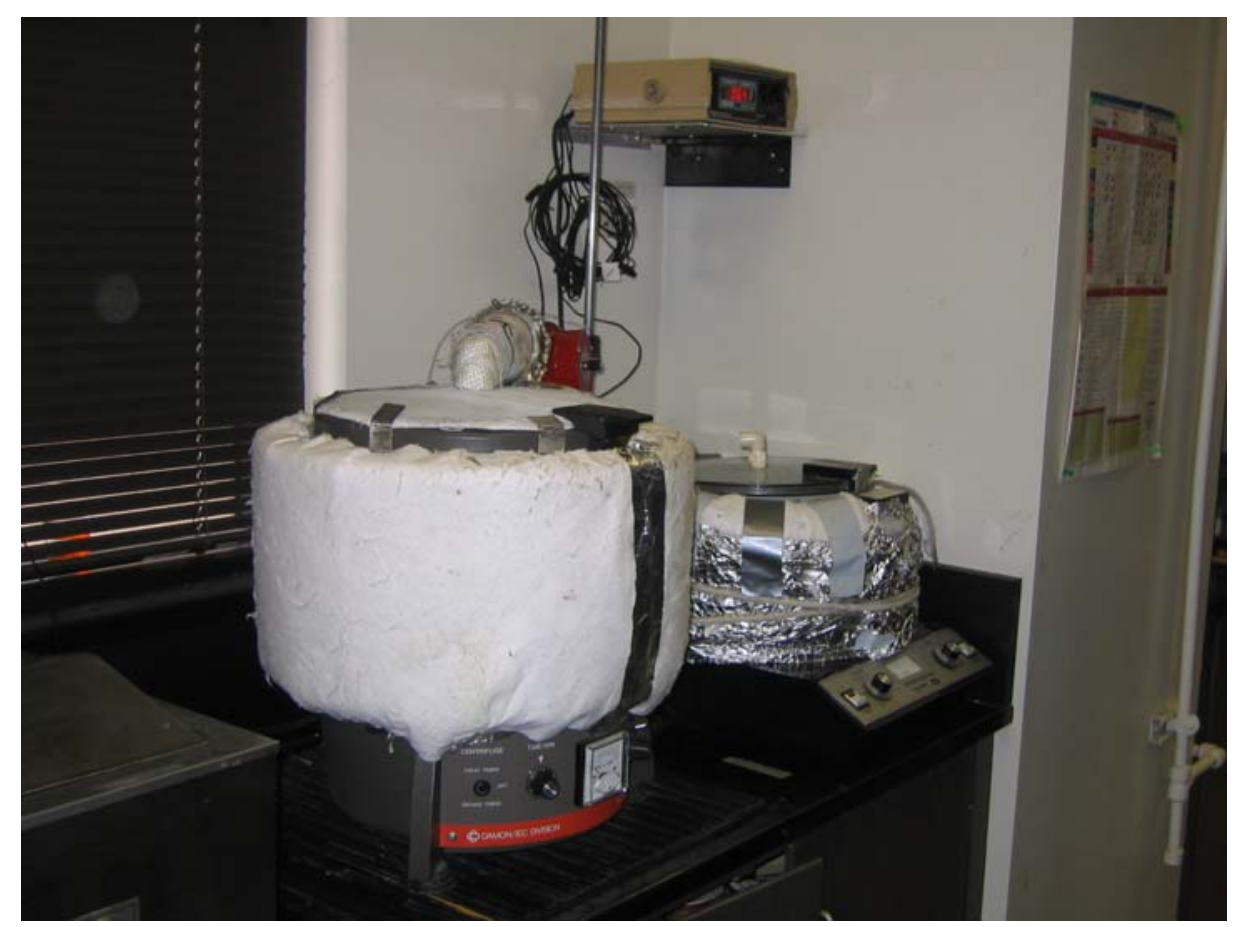

Fig. 3.11- Photograph of insulated centrifuge with heater gun to keep samples warm.

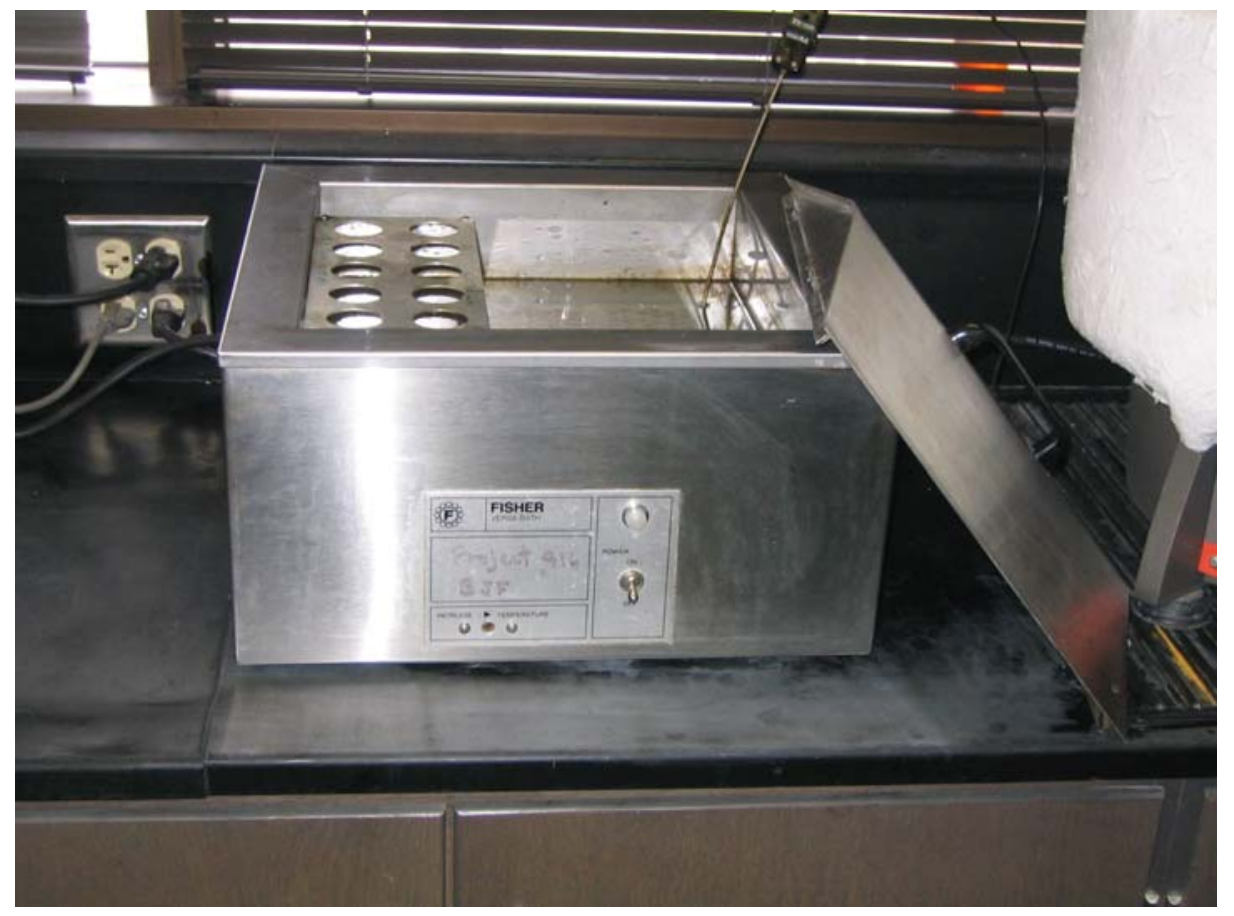

Fig. 3.12- Water bath for heating samples. 


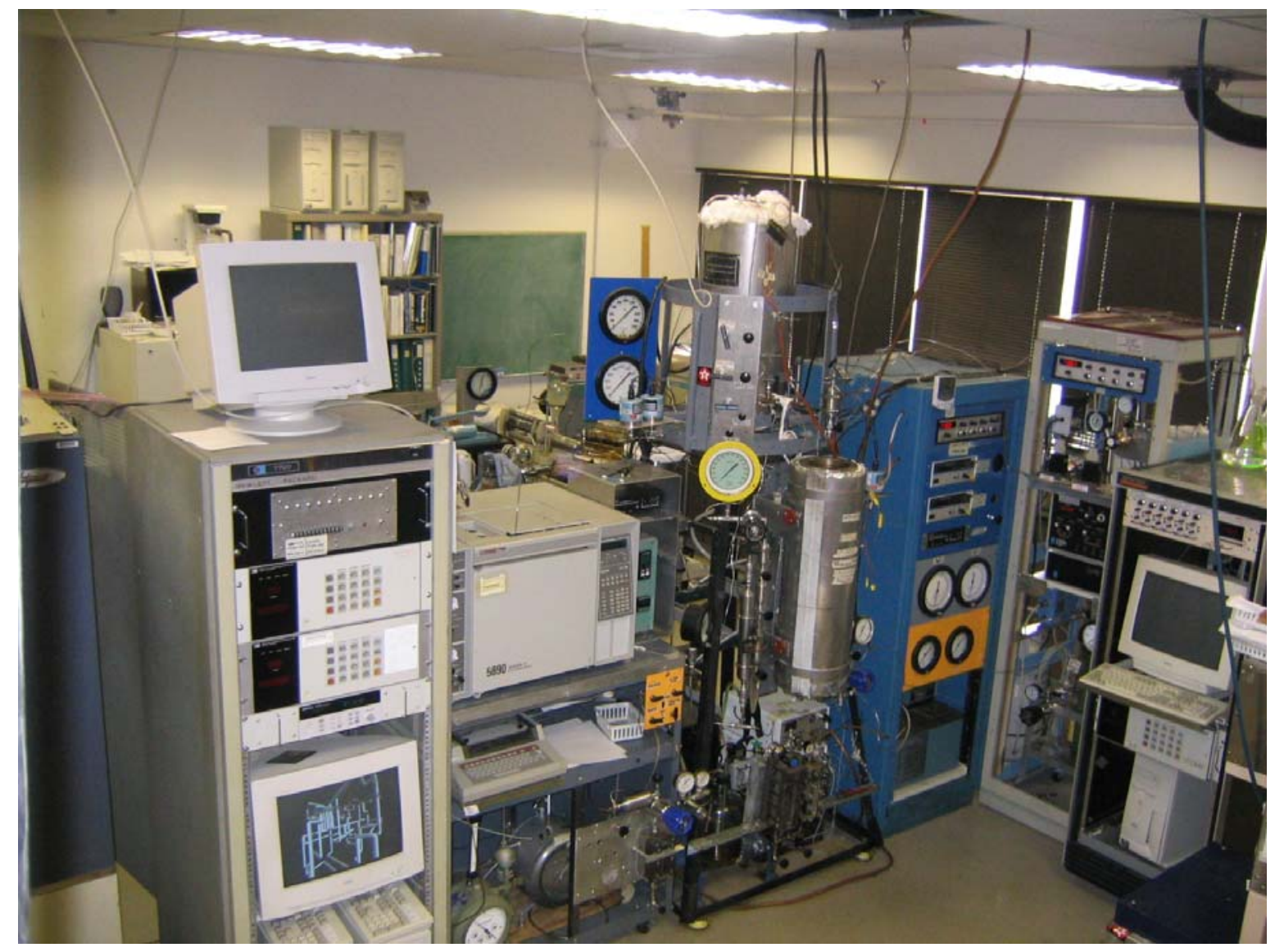

Fig. 3.13- Photograph showing overview of experimental apparatus.

\subsection{Experimental Procedure}

The physical model consists of a cylindrical stainless steel cell, which is packed with a mixture of sand, oil and water. The mixture is prepared in a mixing bowl prior to the run with constant amounts of fluids and sand (see Appendix A for details). Because of the extra-heavy oil used, heating the mixture at about $53^{\circ} \mathrm{C}$ is required to assure homogeneity. A small amount of the mixture at a time is then manually tamped into the cell. By applying a mass balance between the initial amount of mixture and the remaining left after the tamping process, the exact amount of mixture inside the cell is 
calculated. This information is used to compute the pore volume, fluid saturations and original oil in place (OOIP) as shown in Appendix A.

The cell is placed in the vacuum jacket where it is left overnight with a constant temperature of $53^{\circ} \mathrm{C}$. Before the run starts, vacuum is applied on the annulus between the jacket and the cell to ensure minimal heat loss during the run.

The HPLC pump is set to feed a constant rate $5.5 \mathrm{ml} / \mathrm{min}$ of water to the steam generator. Using a mass flowmeter, propane is injected at a fixed mass rate into the generator. The steam or propane-steam or petroleum distillate-steam mixture is first produced, bypassing the cell until superheated steam is obtained. Once the steam has reached the desired temperature $\left(230^{\circ} \mathrm{C}\right)$, injection into the cell begins and the system pressure is set at a predetermined level (260 psig) using nitrogen and a backpressure valve. The mass rate of propane and petroleum distillate is set to the value corresponding to the propane:steam or petroleum distillate:steam mass ratio to be used.

The injection starts when the temperature of the steam reaches $230^{\circ} \mathrm{C}$ and the injection pressure reaches 260 psig.

Once the injection starts, the injection temperature is maintained throughout the run by means of a temperature controller connected to a band heater wrapped around the injection line. Production pressure is controlled by means of a backpressure valve, which regulates the nitrogen pressure to the first separator and subsequently to the bottom of the cell.

Sampling is carried out every three minutes by allowing the flow from the first separator to the second. Once the produced fluids pass from the first separator to the second, the valve that connects both separators is closed. Then, the fluids are collected from the bottom of the second separator in the sampling bottles to be treated and analyzed. This procedure ensures that the cell and the first separator are never open to atmospheric pressure. The gas produced from the first separator passes through a wet test meter where the cumulative volume is measured at 30-seconds intervals. 
Gas samples from the fluid separator are collected automatically every five minutes by the means of an autosampler. These samples are then automatically analyzed using a GC.

San Ardo oil tends to smear the surface of the sample bottles. It was decided to add paint thinner to the produced fluids. The paint thinner decreases the interfacial tension and helps to create clear separation between oil and water in the sample bottles. This separation ensures correct measurement of the produced oil and water. The samples are spun for 30 minutes at $2300 \mathrm{RPM}$ inside a centrifuge heated at $140^{\circ} \mathrm{F}$ (Fig. 3.11 ) to achieve a more accurate reading of the oil and water production. This procedure is applied only for one run out of two runs conducted in the same type of additive that is used along with the steam.

Given the very low sample volumes obtained, the oil density was measured using a $2 \mathrm{~cm}^{3}$ pycnometer. Viscosity was measured using a digital rheometer with a cone-plate assembly (Fig. 3.9). A heating bath (Fig. 3.10) maintained the sample temperature at $50^{\circ} \mathrm{C}$ during the viscosity measurements.

A data logger and a personal computer were used to record at 30-seconds intervals the following parameters: injection and cell temperatures; injection and production pressures; and gas injection and production rates. These values were displayed in real time and helped us maintain the injection temperature and production pressure at constant levels throughout the run. 


\section{CHAPTER IV}

\section{EXPERIMENTAL RESULTS}

\subsection{Overview}

A total of six successful runs were carried out under steam injection, steampropane injection, and steam-petroleum distillate injection. The runs were repeated to confirm the validity of the experiments and the consistency of the results. To allow fair comparison between these runs some parameters are kept constant for all experiments. The sand mix properties are also kept as constant as possible (Table 4.1).

The experimental runs conditions and additives used are as follows:

Run 3 5:100 propane:steam mass ratio

Run 4 pure steam

Run 5 pure steam

Run 6 5:100 propane:steam mass ratio

Run 7 5:100 petroleum distillate:steam mass ratio

Run 8 5:100 petroleum distillate:steam mass ratio

The following parameters were kept constant for all the runs:

- Steam injection temperature: $230^{\circ} \mathrm{C}$

- Steam injection rate: $5.5 \mathrm{~cm}^{3} / \mathrm{min}$ cold water equivalent (CWE)

- First separator backpressure (production pressure): 260 psig

- $\quad$ Second separator backpressure (production pressure): 50 psig

- Vacuum jacket pressure: -29 in. mercury

- Initial cell temperature: $53.3^{\circ} \mathrm{C}$

The raw data is presented in Appendix A. The calculations used to process the data are also showed in the appendix.

The properties of the sand mixture inside the cell for each run are shown in Table 4.1. 
Table 4.1- Sand mix properties for Runs 3 to 8.

\begin{tabular}{|l|c|c|c|c|c|c|}
\hline & Run 3 & Run 4 & Run 5 & Run 6 & Run 7 & Run 8 \\
\hline Porosity, \% & 41.8 & 41.2 & 41.1 & 42.1 & 41.2 & 42.5 \\
\hline Pore volume, cm & 1163.2 & 1164.7 & 1162.3 & 1164.4 & 1265.7 & 1261.8 \\
\hline $\begin{array}{l}\text { Water volume inside cell, } \\
\text { cm }^{3}\end{array}$ & 190.8 & 191.2 & 190.1 & 193.0 & 192.1 & 191.5 \\
\hline $\begin{array}{l}\text { Oil volume inside cell } \\
\text { (OOIP), cm }\end{array}$ & 860.3 & 861.5 & 862.8 & 860.3 & 863.9 & 860.7 \\
\hline Oil saturation, \% & 65.6 & 65.6 & 74.1 & 65.6 & 72.6 & 71.6 \\
\hline Water saturation, \% & 15.4 & 15.4 & 16.5 & 15.4 & 16.5 & 15.7 \\
\hline Air saturation, \% & 10.1 & 10.4 & 9.1 & 9.8 & 10.2 & 9.4 \\
\hline
\end{tabular}

The data analyses and interpretations are presented individually for each run. In addition, a global comparison between the various cases is also made. 


\subsection{Run 3 (5:100 Propane:Steam Mass Ratio)}

The temperature profiles for this run are shown in Fig. 4.1. The average steam injection temperature is $230^{\circ} \mathrm{C}$ and very little variation is observed throughout the run. Figs. 4.2 and 4.3 show the cumulative volumes of oil and water versus time and pore volume of steam injected respectively. By the end of the run, when the amount of steam injected is around $95 \%$ of the pore volume, the oil recovery is $407.5 \mathrm{~cm}^{3}(47.3 \%$ of OOIP).

The oil and water rates are plotted both as a function of time and pore volume of steam injected (Figs. 4.4 and 4.5). The oil production rate peak is about $15 \mathrm{~cm}^{3} / \mathrm{min}$ and it lasts from 56 to 71 minutes. Fig. $\mathbf{4 . 6}$ shows the temperature profiles inside the cell at 20-minute intervals. It can be observed that by 141 minutes the temperature of the whole cell reaches a value around $210^{\circ} \mathrm{C}$.

The injection and production pressures are shown in Fig. 4.7. The differential pressure (injection pressure minus production pressure) is also plotted in Fig. 4.7. Production pressure is maintained at 260 psig during the whole run. On the other hand, the injection pressure started increasing at the beginning of the run to eventually decrease at around 77. The maximum differential pressure reached in this run was around 121 psig.

The oil viscosity and API gravity of the produced oil is shown in Fig. 4.8. The API gravity tends to increase with respect to time. Most of the values recorded were around the original API gravity of 12 . The oil viscosity shows an increasing trend as time increases.

The compositional analysis of the gas produced after the third separator is plotted in Fig. 4.9. The cumulative gas volume produced after the third separator is presented in Fig. 4.10. Fig. 4.11 shows the propane cumulative injection mass and propane cumulative mass production for the third separator. A total of $66 \mathrm{~g}$ propane is injected inside the cell and propane produced from the third separator is $4.43 \mathrm{~g}$ 


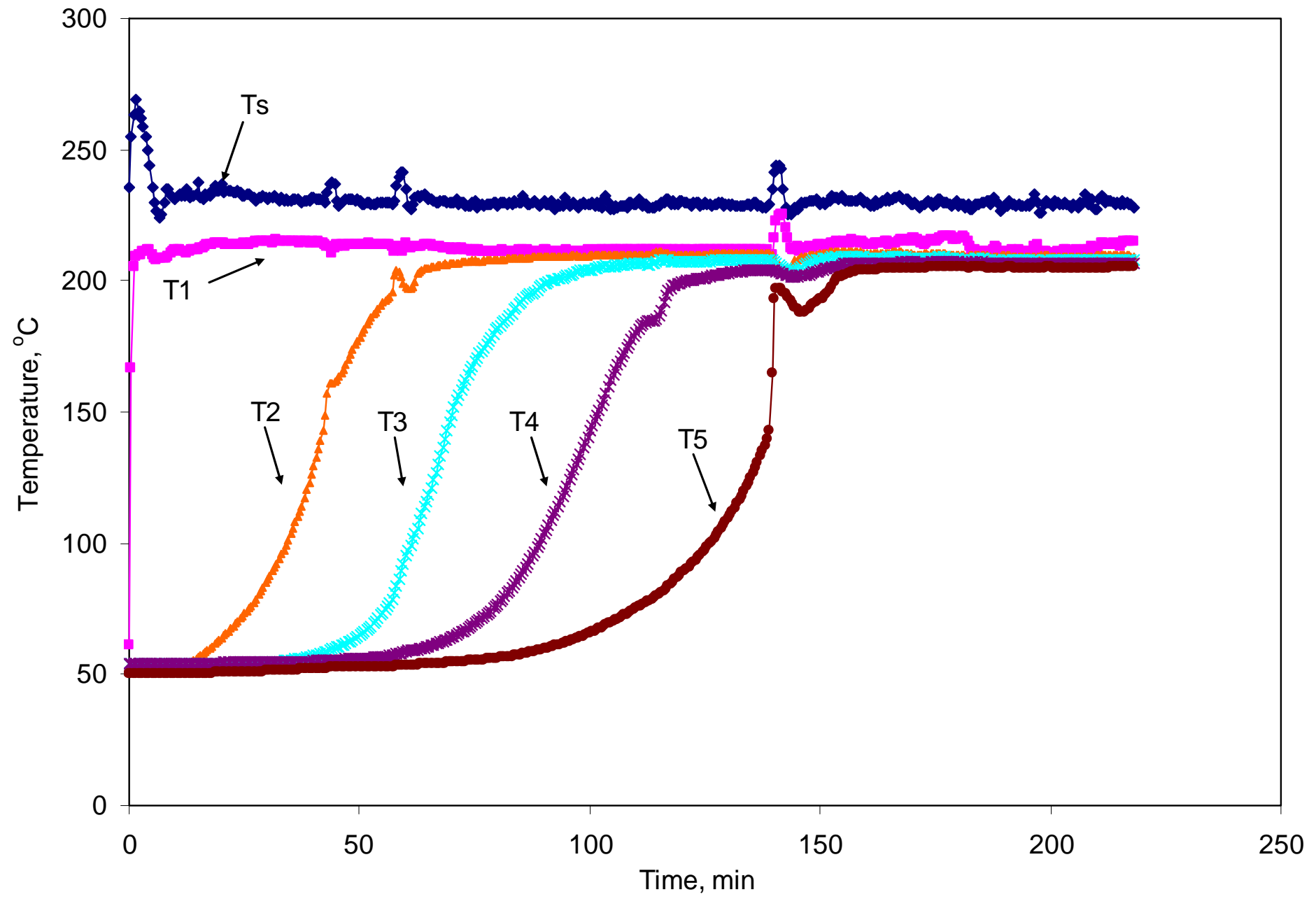

Fig. 4.1-Temperature profile versus time for Run 3 (5:100 propane:steam). 


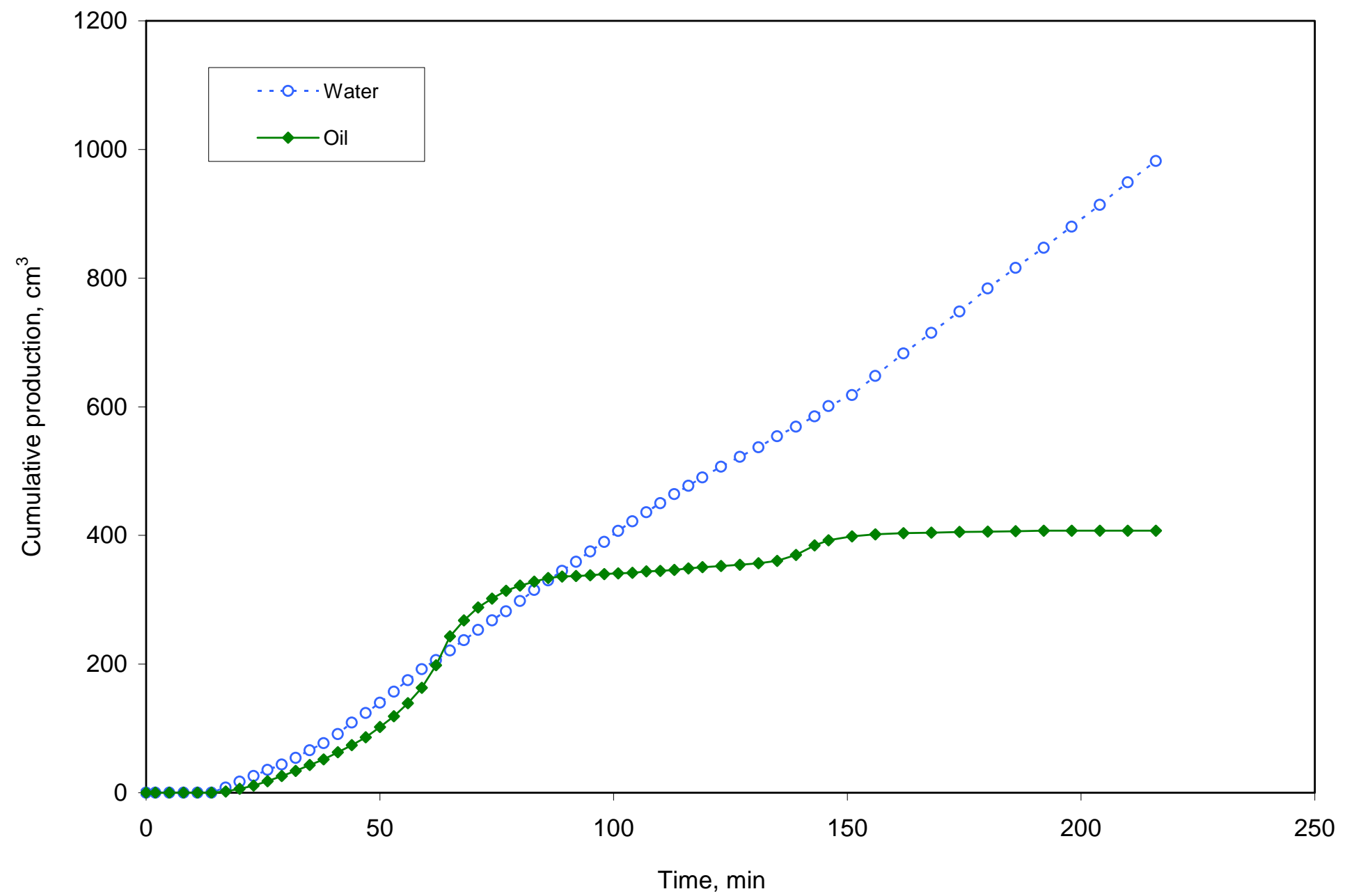

Fig. 4.2- Cumulative oil and water volumes versus time for Run 3 (5:100 propane:steam). 


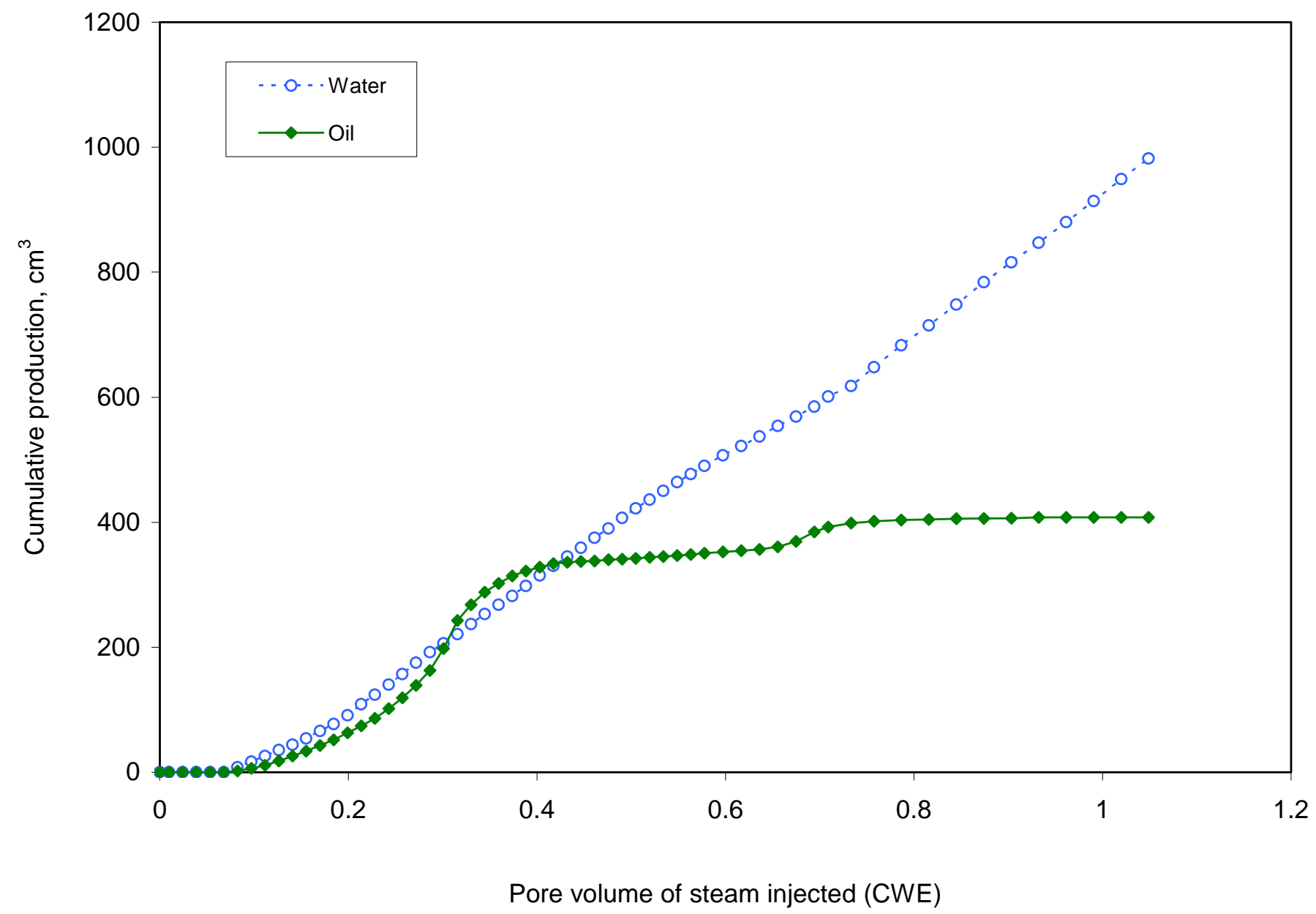

Fig. 4.3- Cumulative oil and water volumes versus pore volume of steam injected for Run 3 (5:100 propane:steam). 


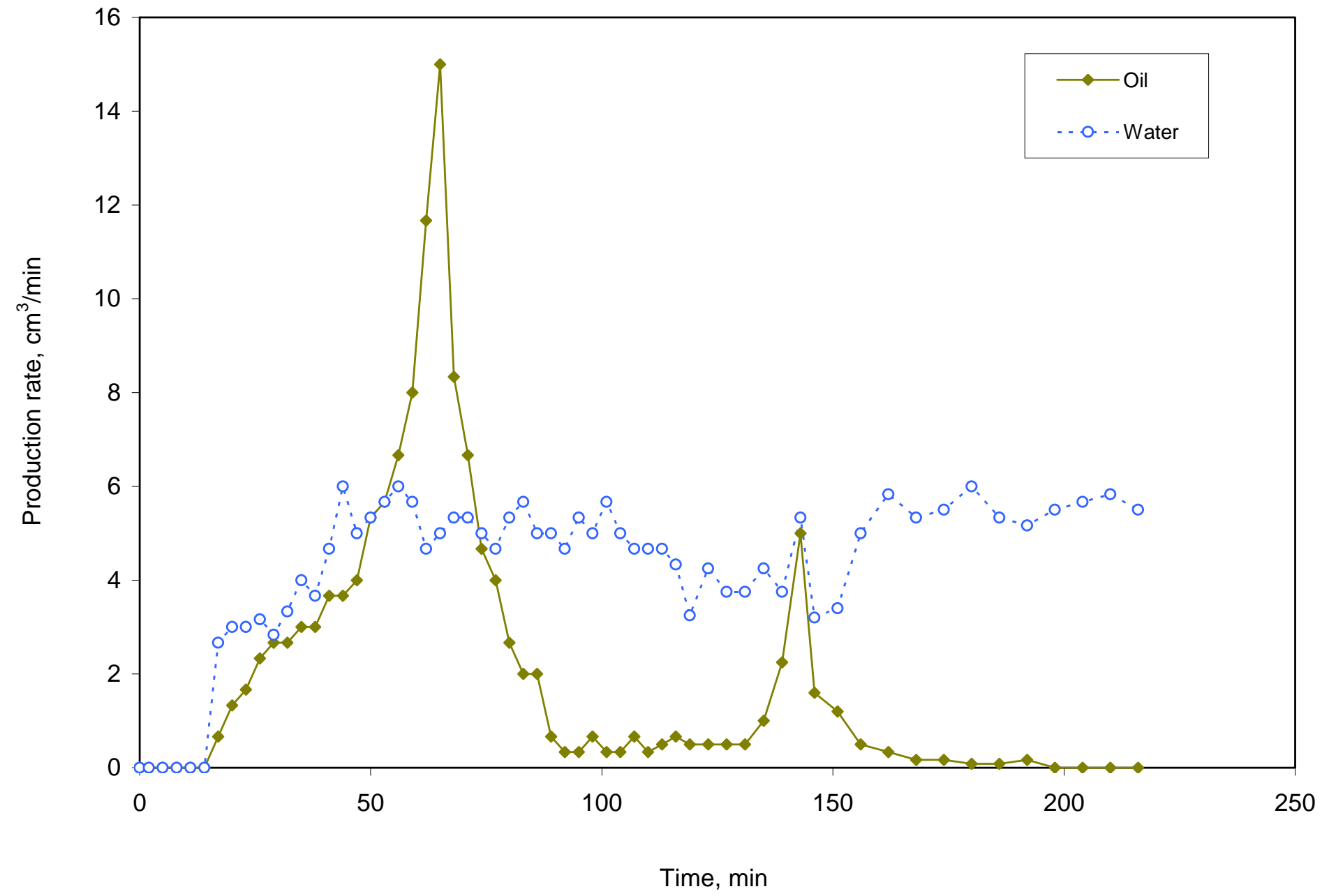

Fig. 4.4- Oil and water rates versus time for Run 3 (5:100 propane:steam). 


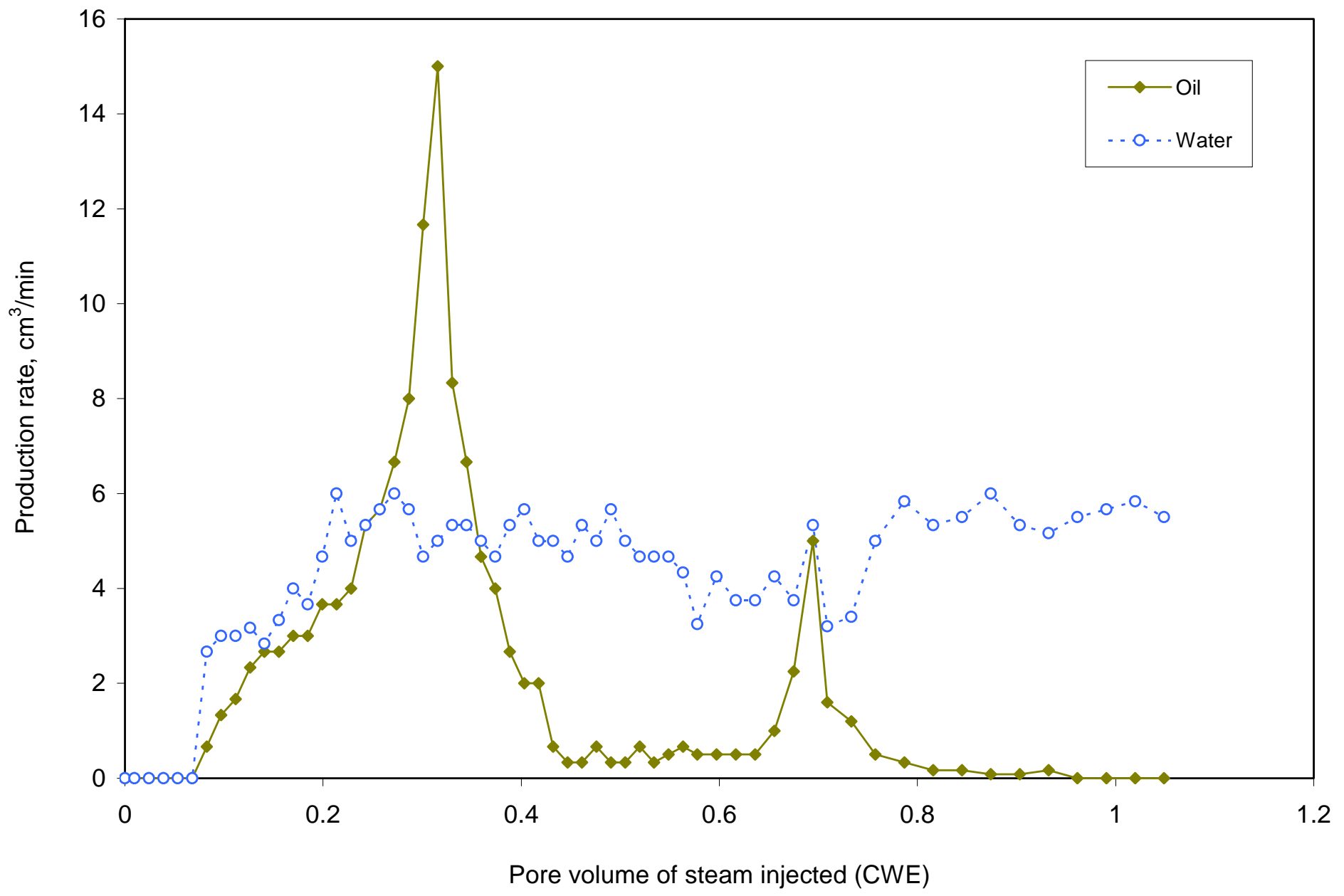

Fig. 4.5- Oil and water rates versus pore volume injected for Run 3 (5:100 propane:steam). 


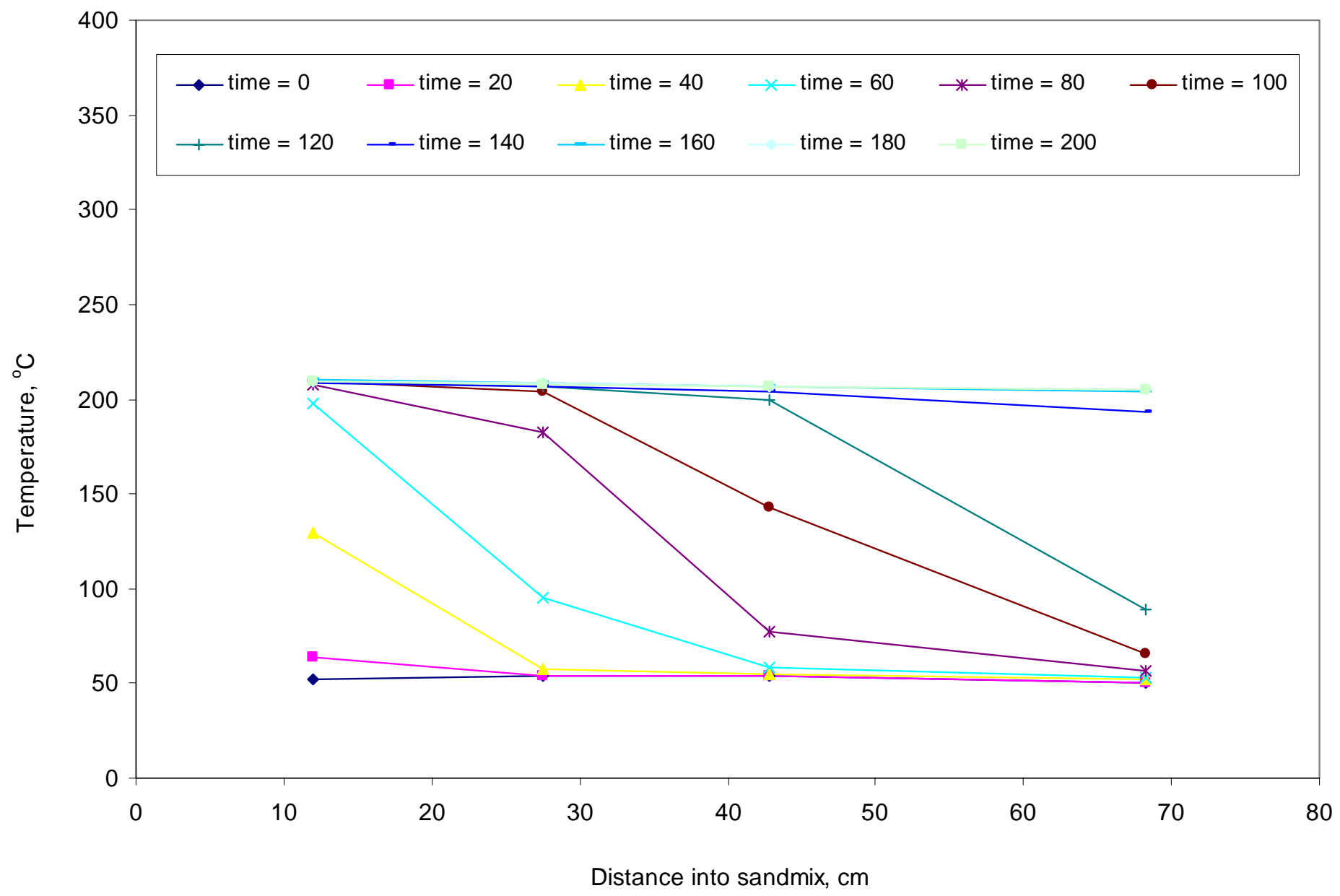

Fig. 4.6- Temperature profiles at 20-minute intervals - from $t=0$ to $t=200 \mathrm{~min}-$ for Run 3 (5:100 propane:steam). 


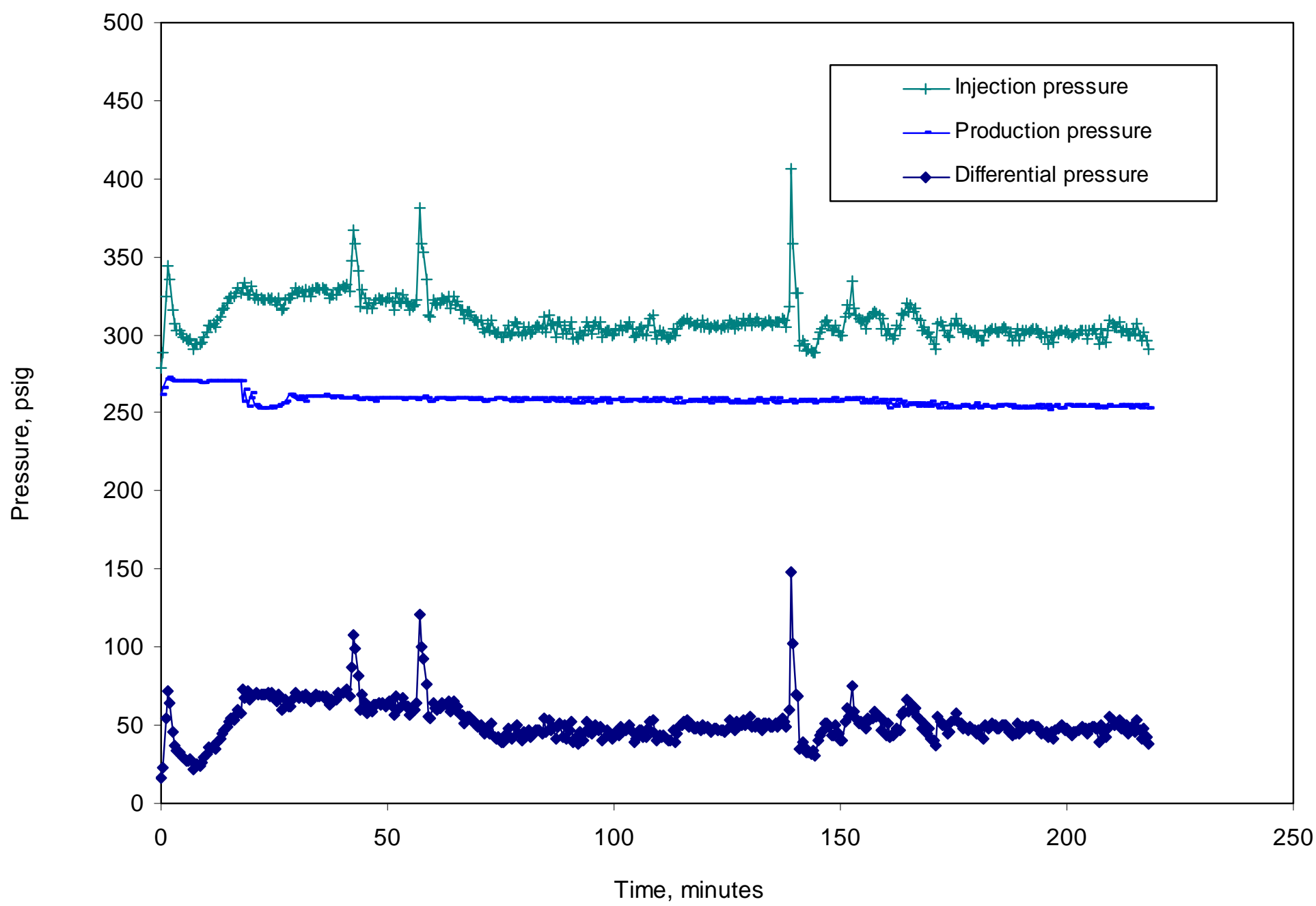

Fig. 4.7- Injection, production and differential pressures for Run 3 (5:100 propane:steam). 


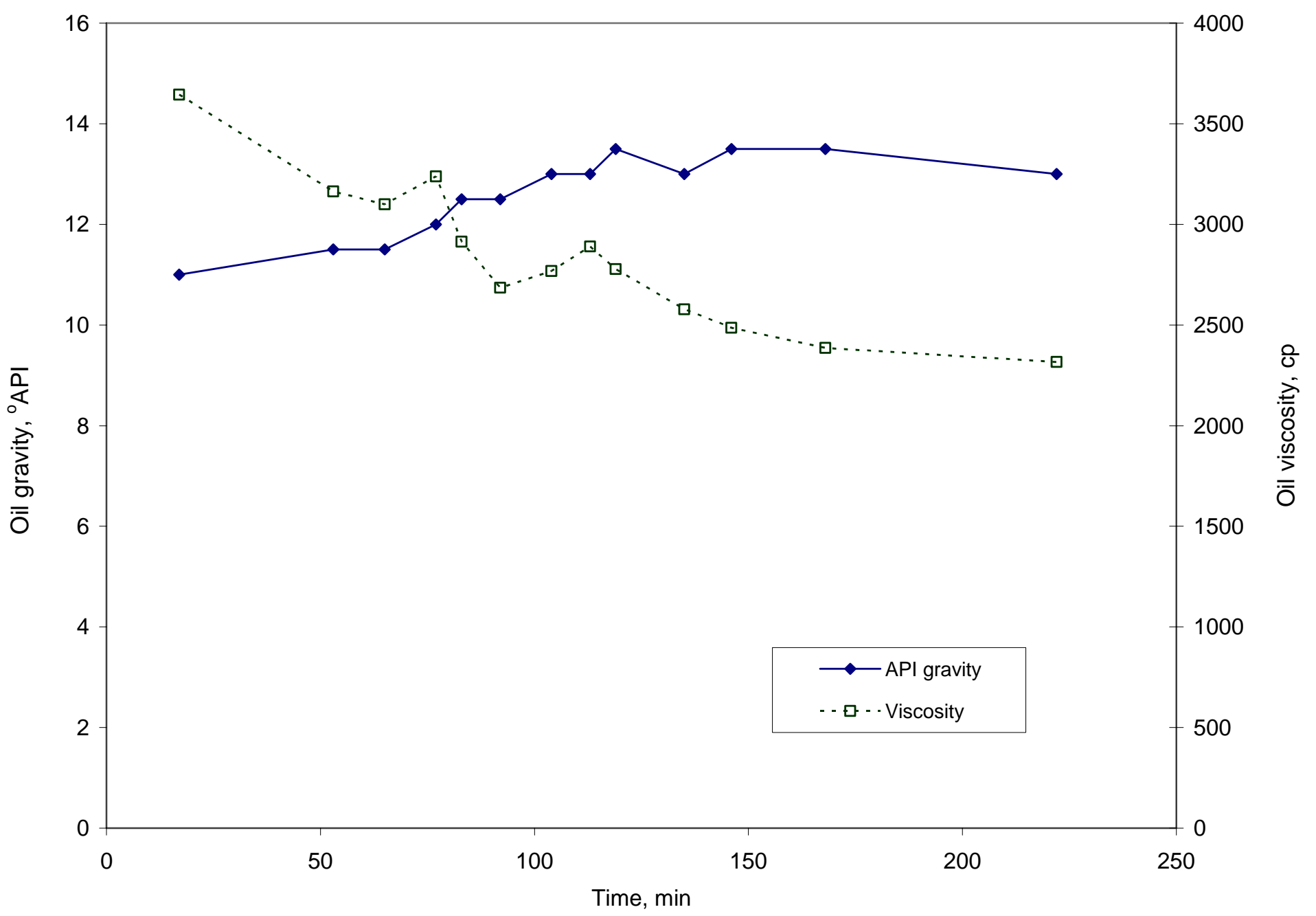

Fig. 4.8- Oil viscosity and API gravity for Run 3 (5:100 propane:steam). 


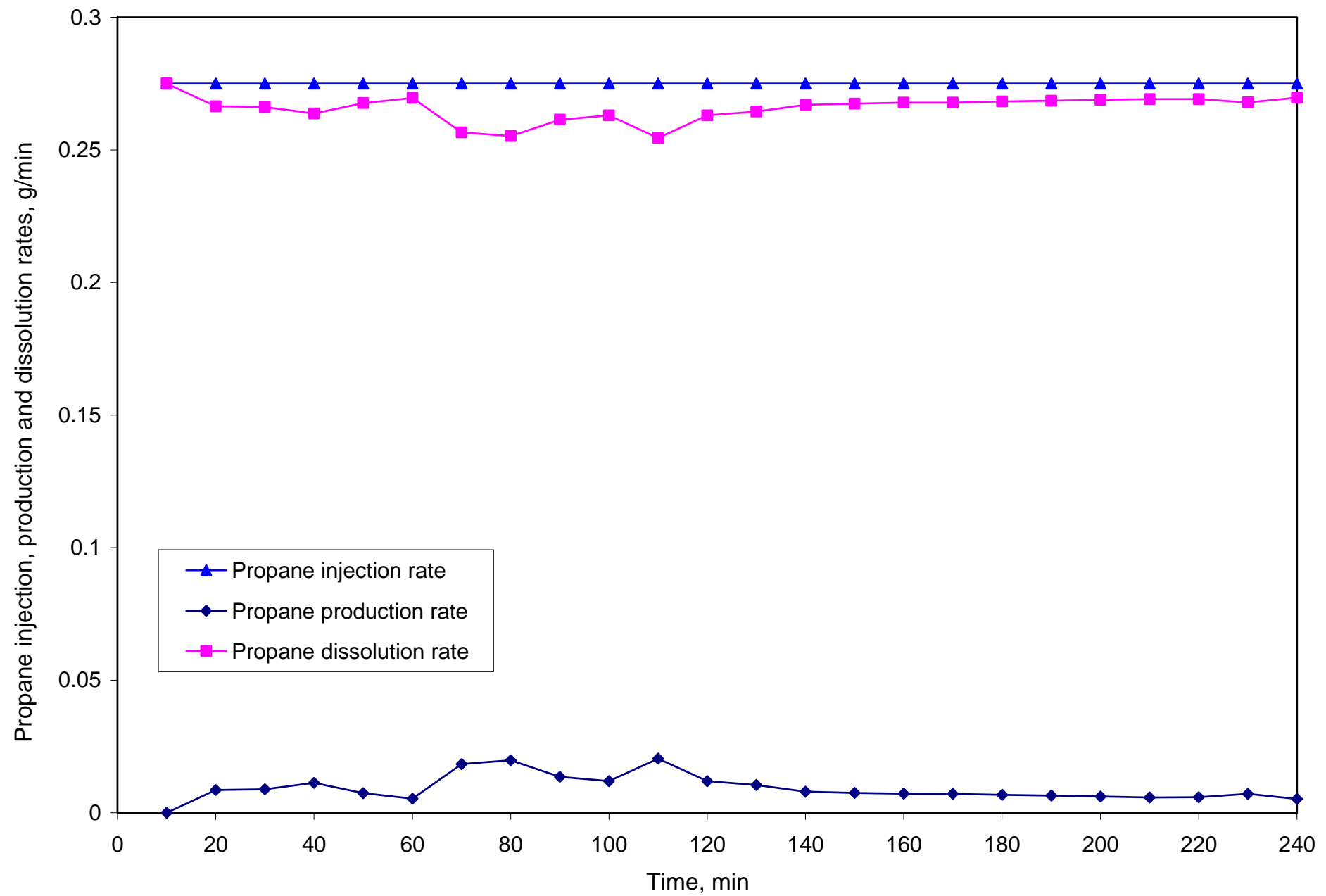

Fig. 4.9- Propane injection, production, and dissolution rates after the third separator for Run 3 (5:100 propane:steam). 


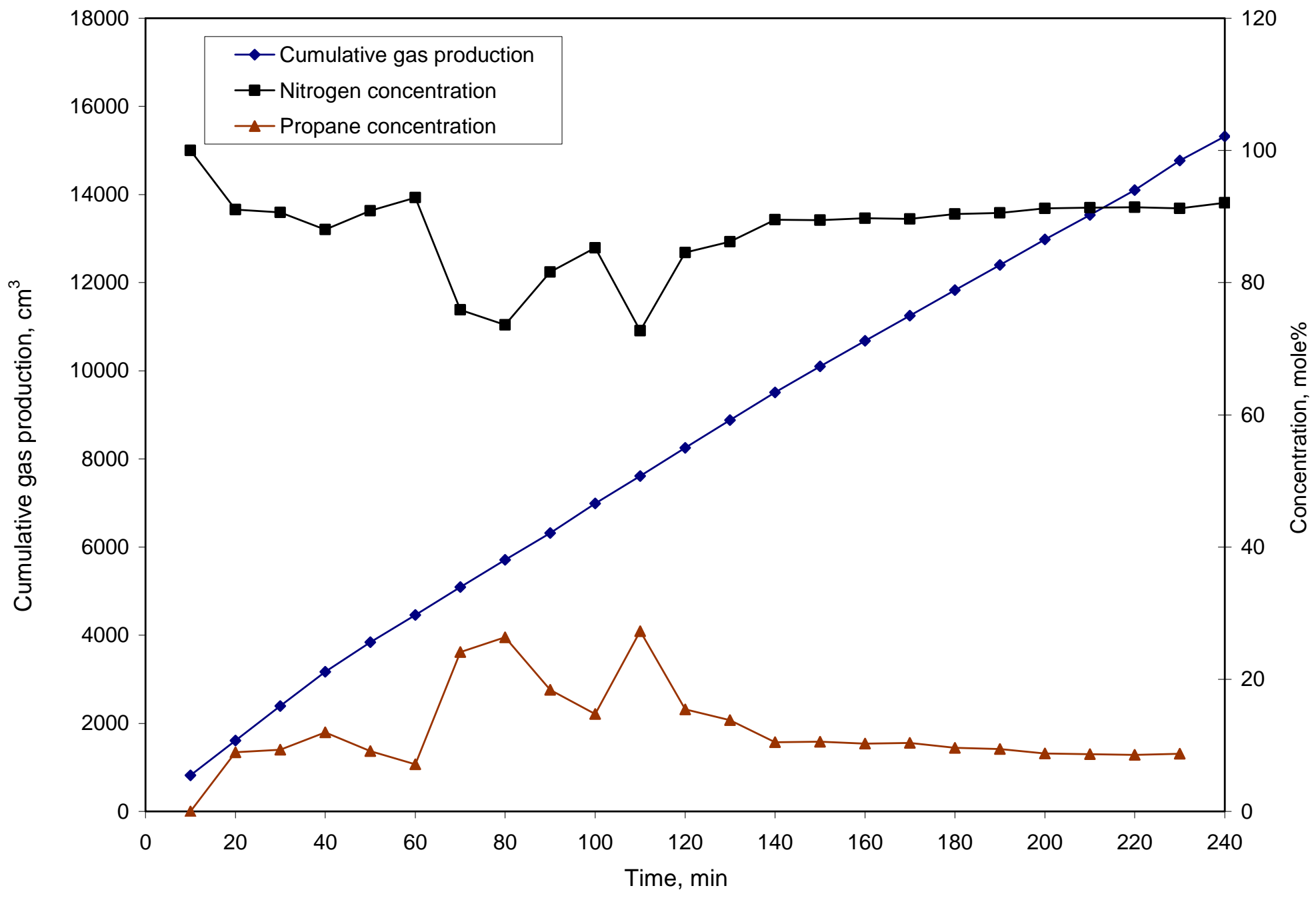

Fig. 4.10- Cumulative gas production and composition after the third separator for Run 3 (5:100 propane:steam). 


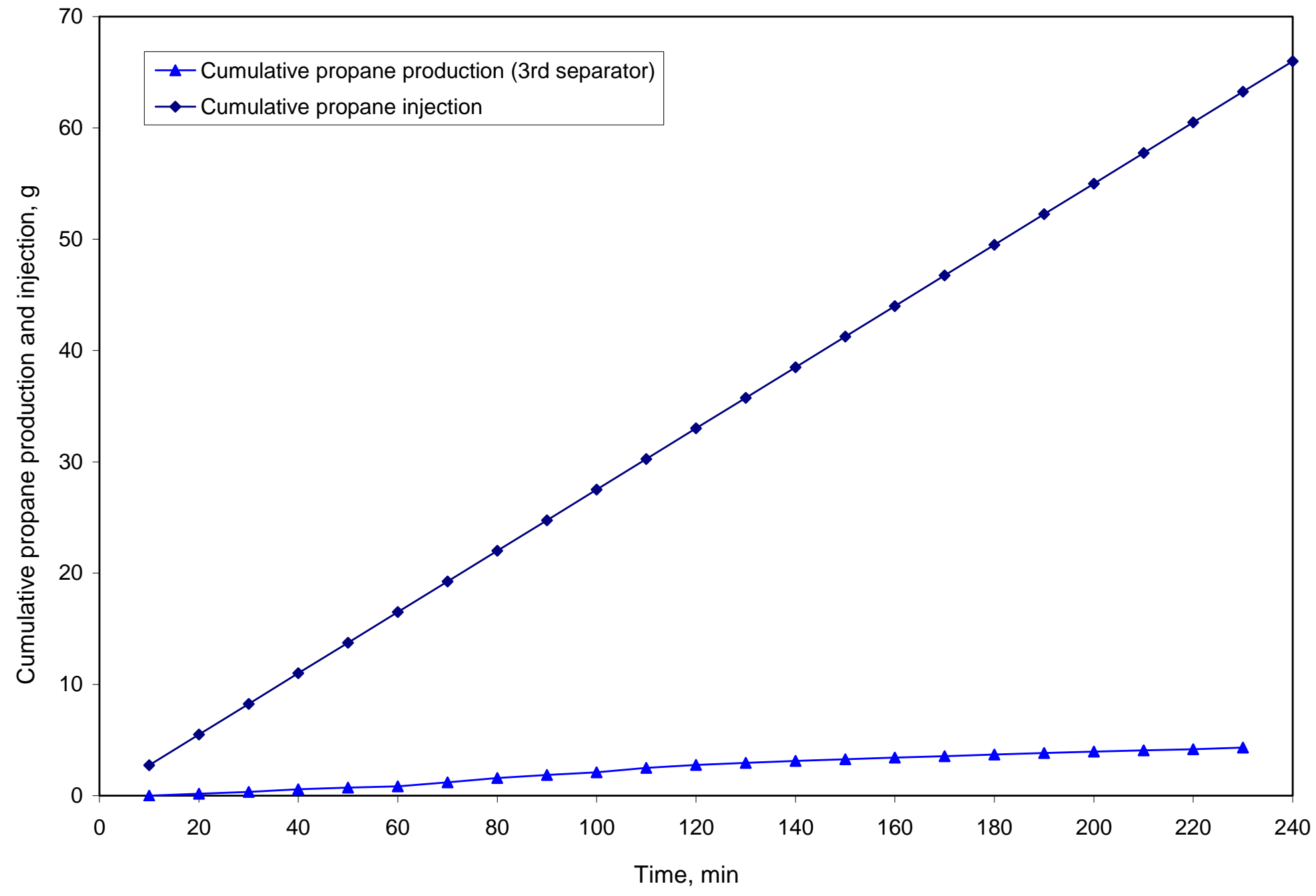

Fig. 4.11- Cumulative propane injection and production for Run 3 (5:100 propane:steam). 


\subsection{Run 4 (Pure Steam)}

The temperature profiles for run 4 are shown in Fig. 4.12. The initial injection temperature is around $200{ }^{\circ} \mathrm{C}$, however, the temperature quickly stabilizes to the preset level for all the runs $\left(220^{\circ} \mathrm{C}\right)$. Figs. 4.13 and $\mathbf{4 . 1 3}$ show the oil cumulative production plotted as a function of time and pore volume injected respectively. The final oil recovery at 273 minutes (132\% of pore volume injected) was $394 \mathrm{~cm}^{3}$, which corresponds to $40.9 \%$ of OOIP.

The oil production rates vs. time and pore volume injected are plotted in Figs. 4.15 and 4.16 respectively. The start of oil production occurs at 24 minutes, and the oil rate peak is $15 \mathrm{~cm}^{3} / \mathrm{min}$. Fig. 4.17 shows temperature profiles at 20-minute intervals. . It takes about 198 minutes for the whole cell to reach a more or less constant temperature of $210^{\circ} \mathrm{C}$

The injection, production and differential pressures are plotted in Fig. 4.18. The differential pressure peak is 31 psig. API gravity tends to increase as time increases (Fig. 4.19). Oil viscosity shows a decreasing trend with time. The viscosity values measured in run 4 shows significant upgrading as compared to the original oil viscosity. 


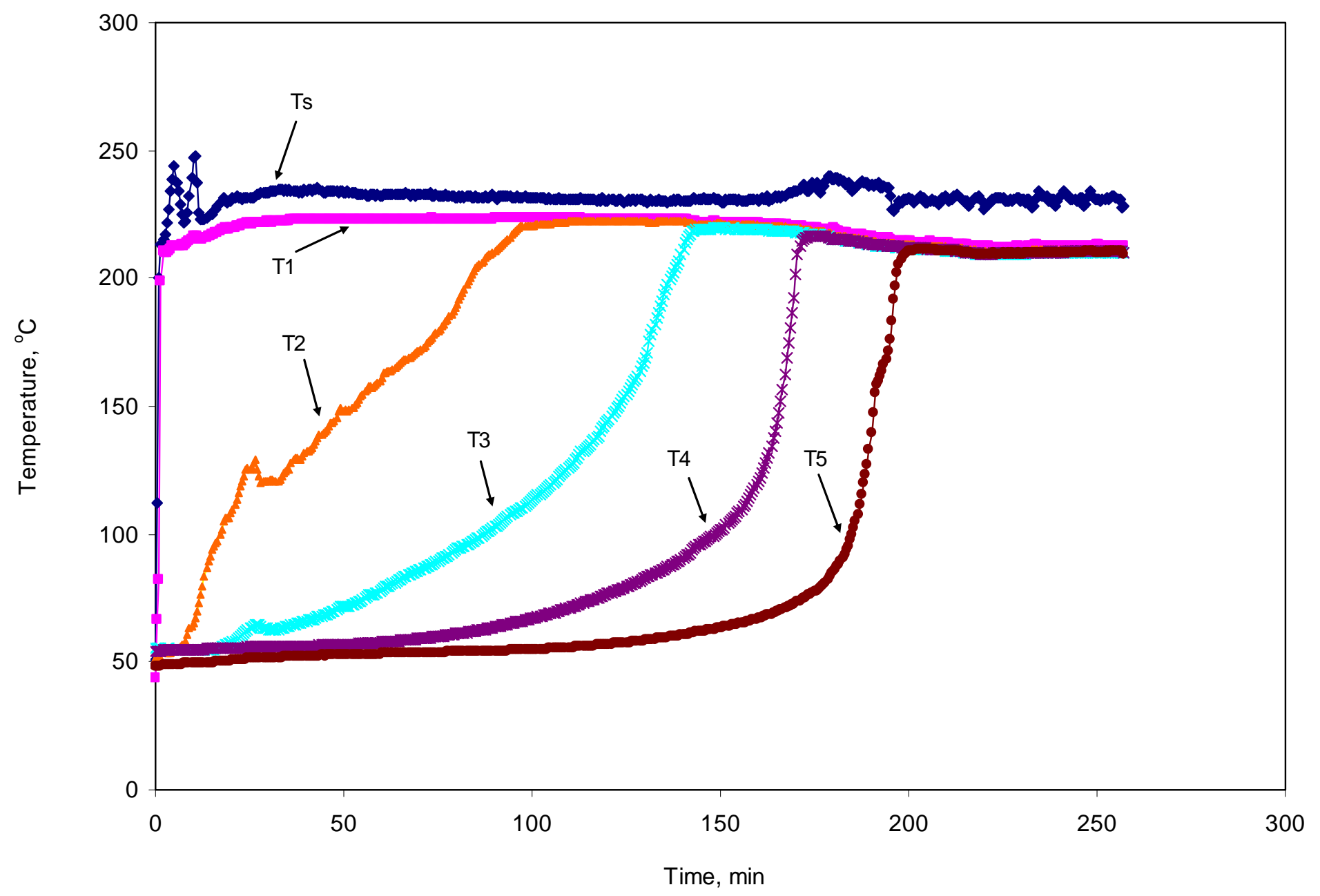

Fig. 4.12- Temperature profile versus time for Run 4 (pure steam). 


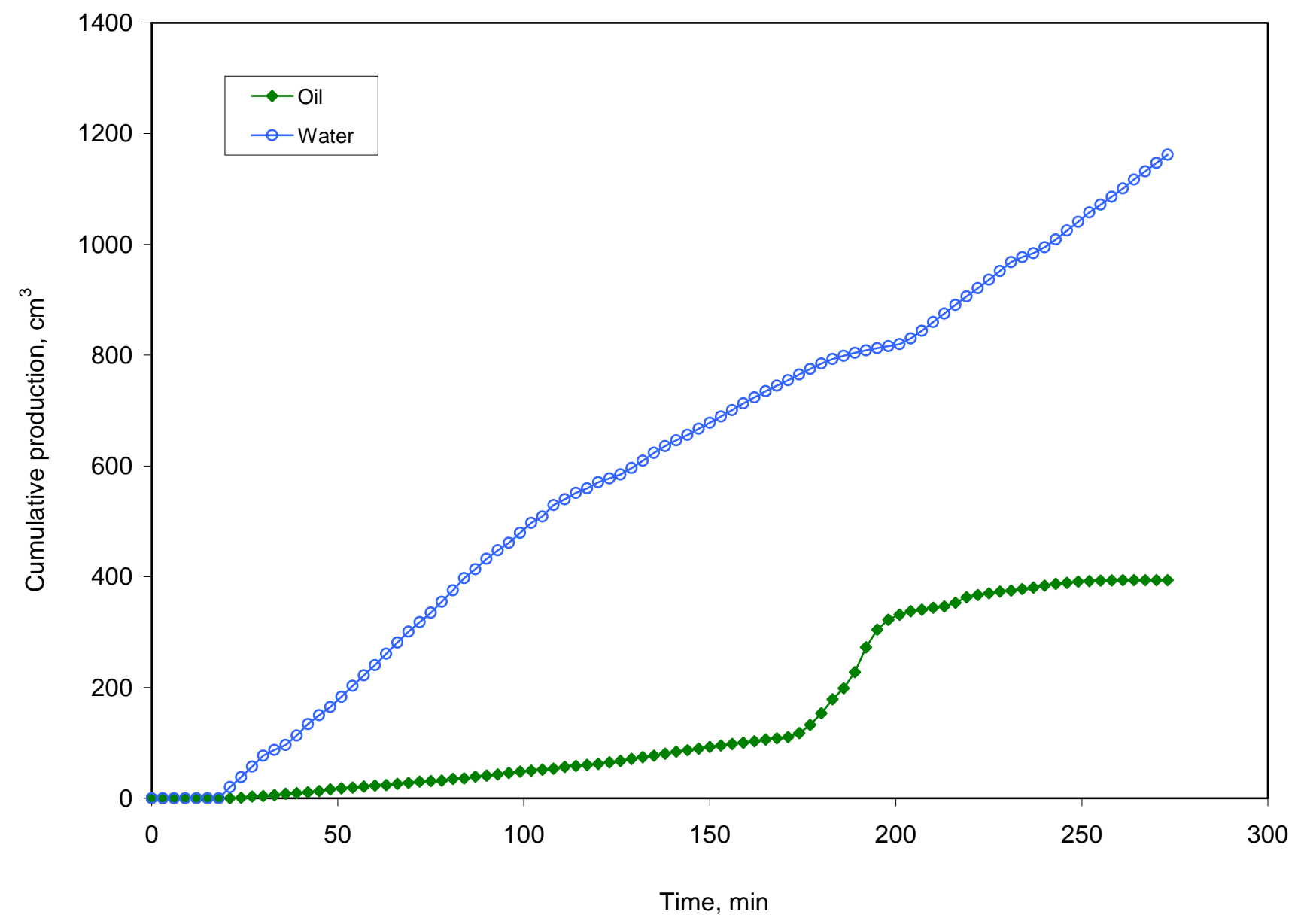

Fig. 4.13- Cumulative oil and water volumes versus time for Run 4 (pure steam). 


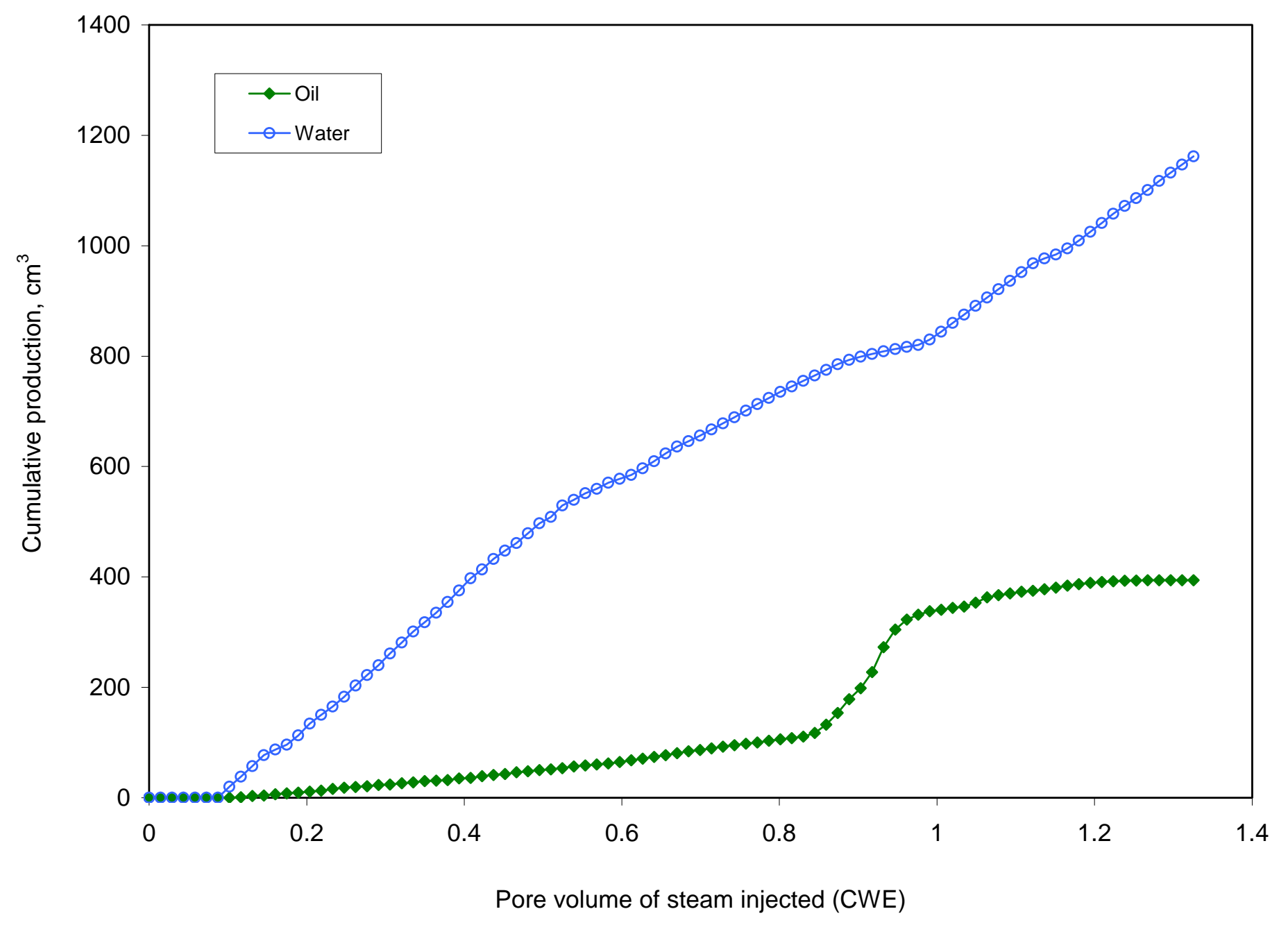

Fig. 4.14- Cumulative oil and water volumes versus pore volume injected for Run 4 (pure steam). 


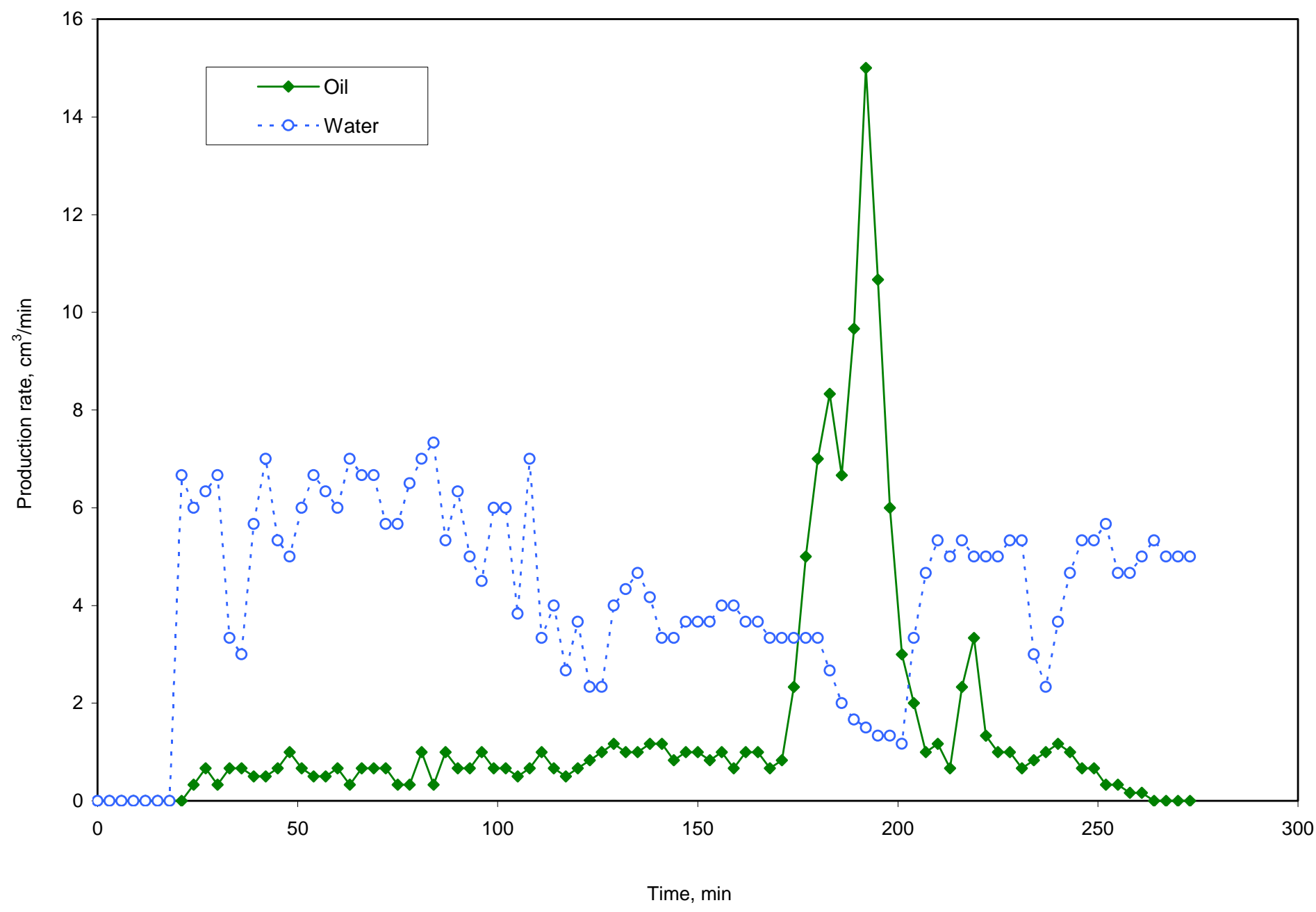

Fig. 4.15- Oil and water rates versus time for Run 4 (pure steam). 


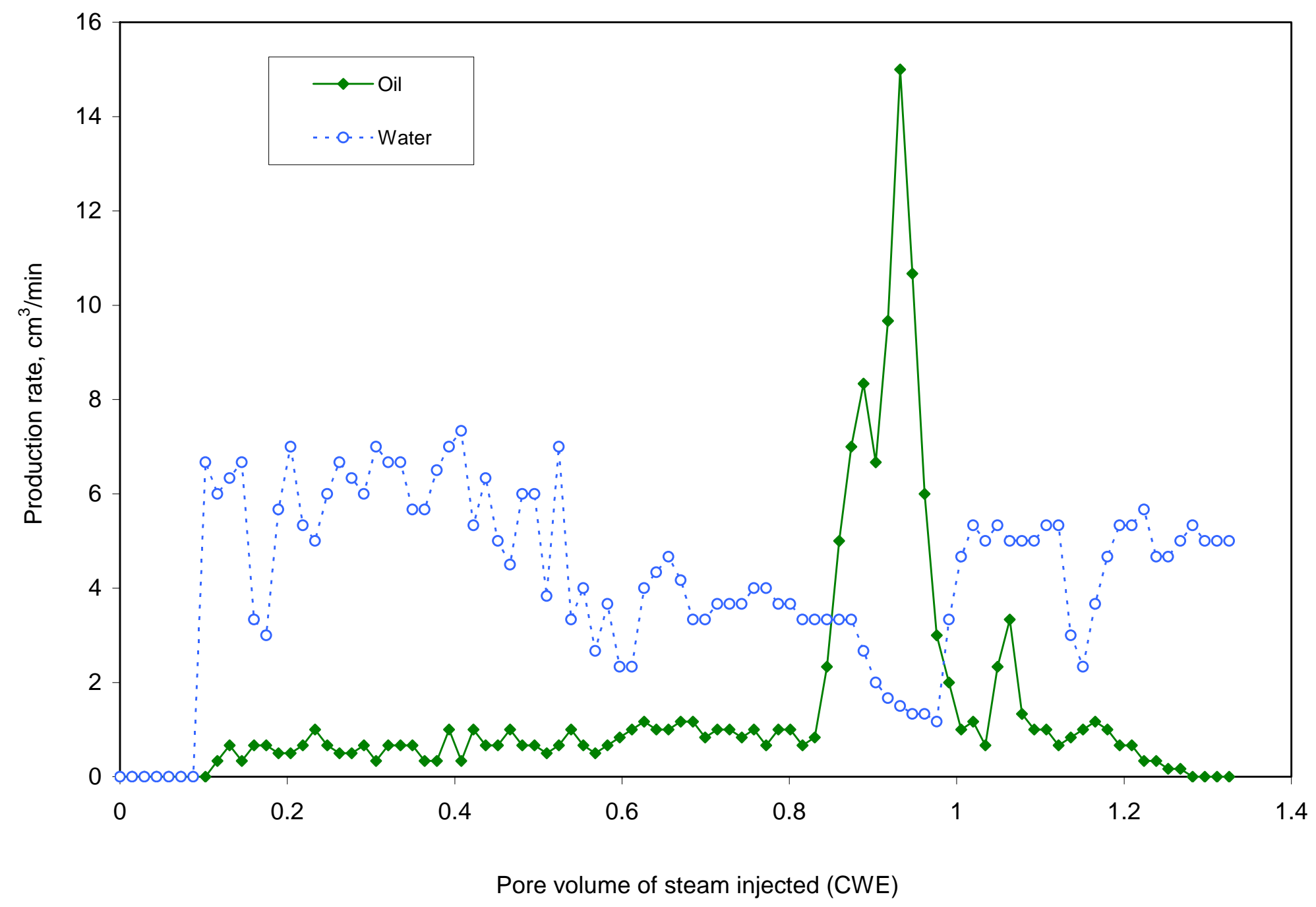

Fig. 4.16- Oil and water rates versus pore volume injected for Run 4 (pure steam). 


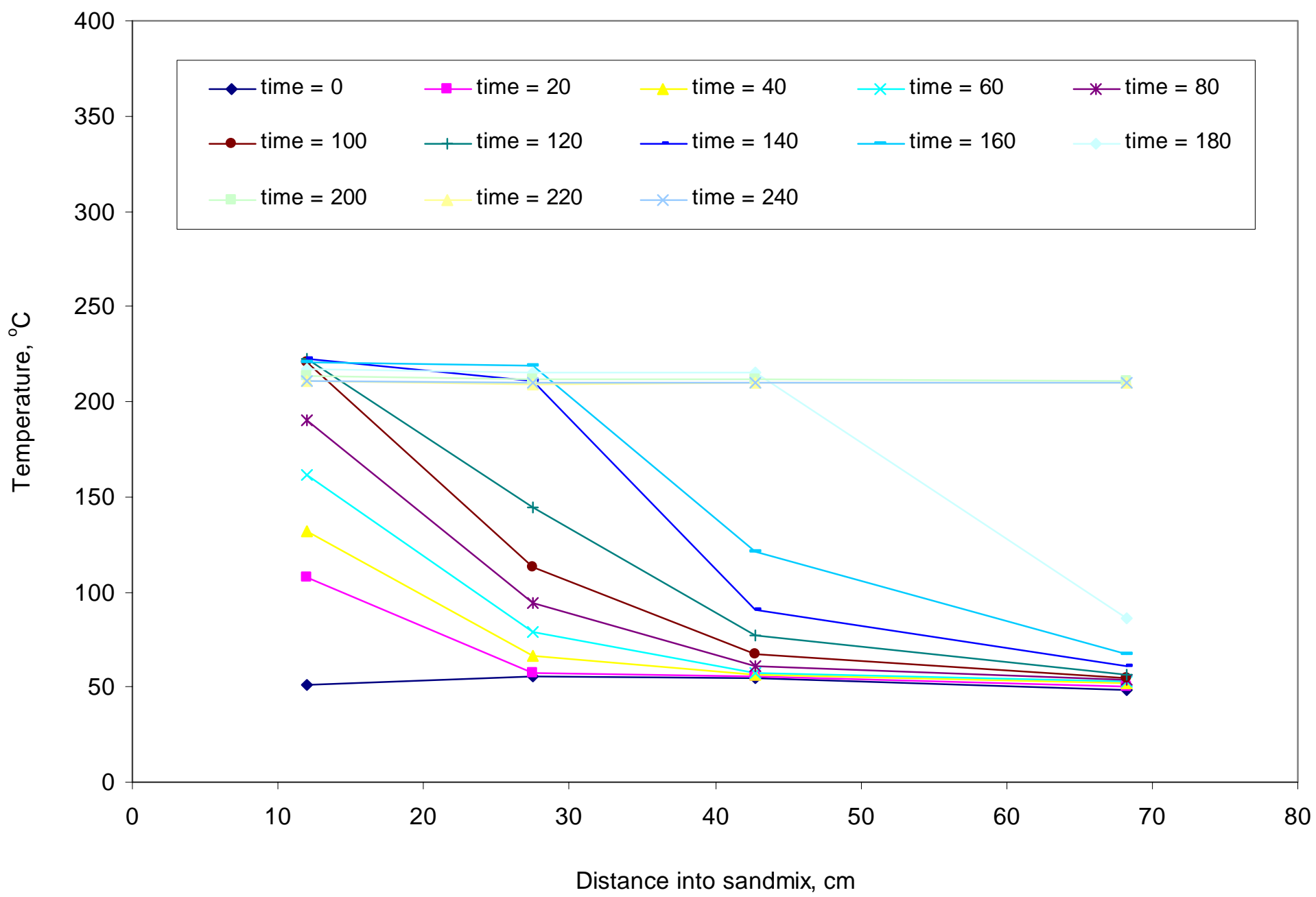

Fig. 4.17- Temperature profiles at 20-minute intervals - from $t=0$ to $t=240 \mathrm{~min}-$ for Run 4 (pure steam). 


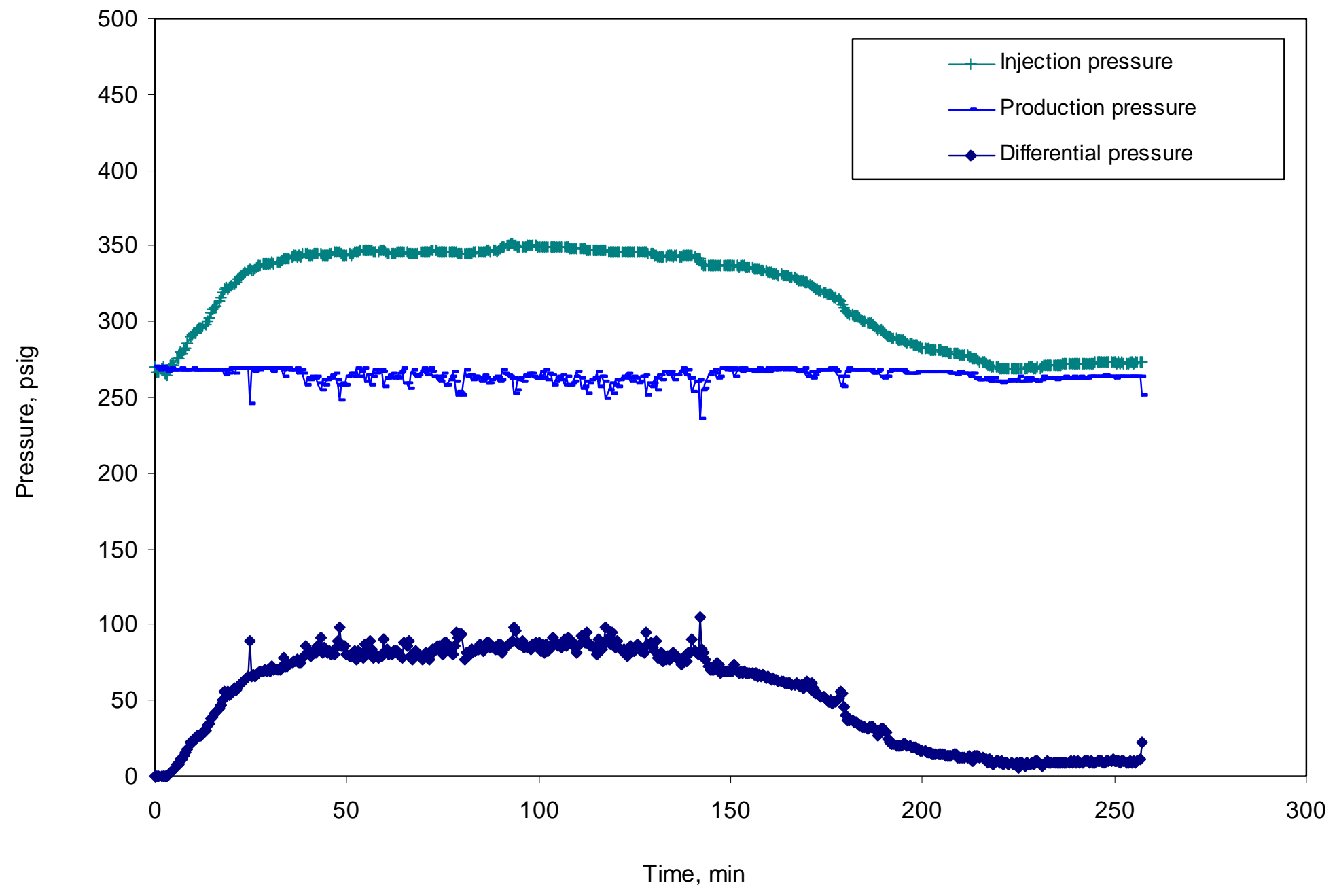

Fig. 4.18- Injection, production and differential pressures for Run 4 (pure steam). 


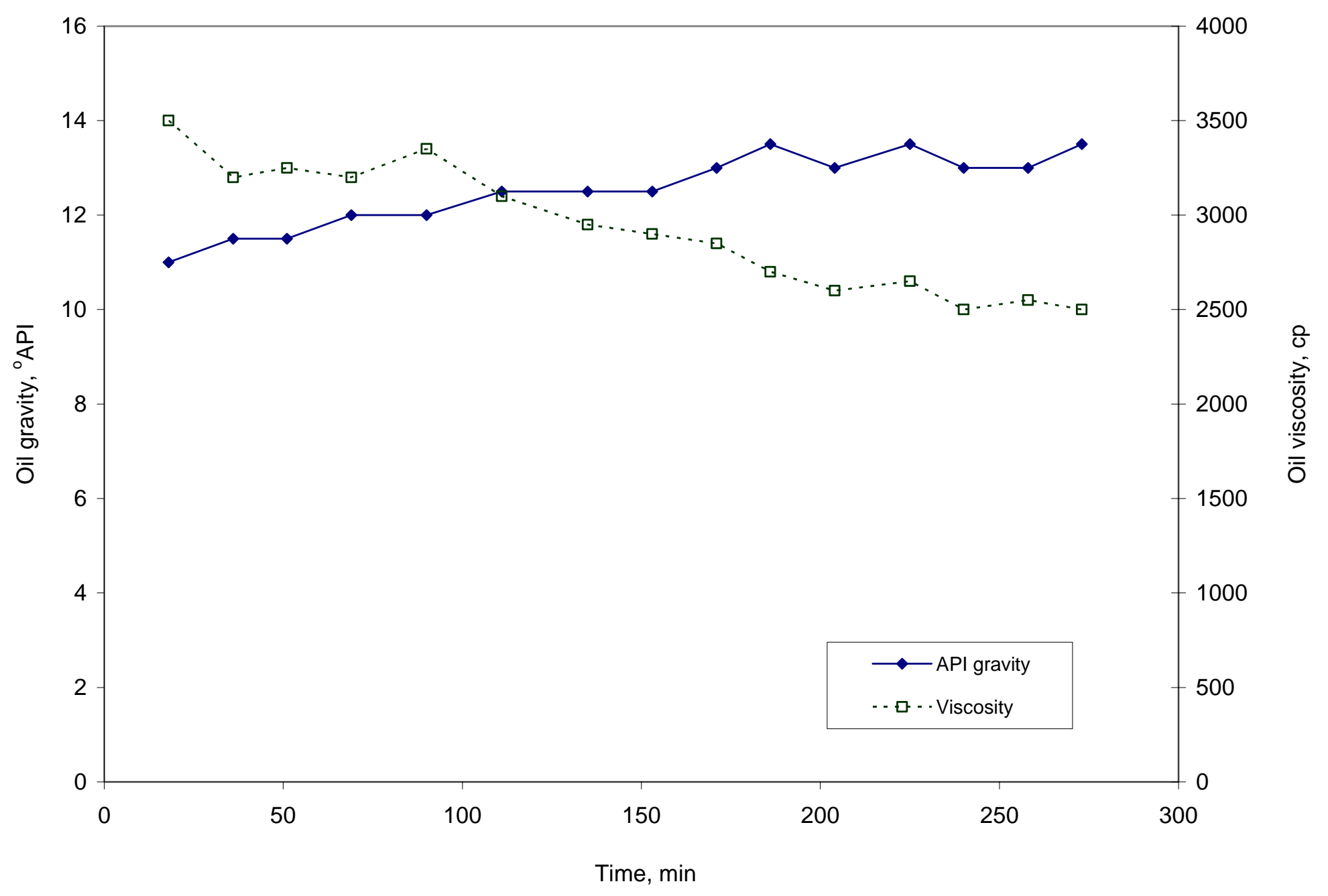

Fig. 4.19- Oil viscosity and API gravity for Run 4 (pure steam). 


\subsection{Run 5 (Pure Steam)}

The temperature profile as a function of time is presented in Fig. 4.20. A constant injection temperature is observed throughout most of the run. Some fluctuation occurs at the beginning of the run, but after 15 minutes, it stabilizes at $220^{\circ} \mathrm{C}$.

The cumulative oil and water volumes and pore volume injected versus time are depicted in Figs. 4.21 and 4.22. A recovery of 40.53\% of OOIP is achieved by the end of the run. Figs. 4.23 and 4.24 show the oil and water rates as a function of time and pore volume injected. In this run, most of the oil is produced at 177 and 277 minutes and the oil rate peak is around $15 \mathrm{~cm}^{3} / \mathrm{min}$.

Fig 4.25 shows temperature profiles at 20-minute intervals. It can be noted that at 188 minutes, the temperature in the whole cell is stabilized around $210{ }^{\circ} \mathrm{C}$. The injection and production pressure along with the differential pressure are shown in Fig. 4.26. The differential pressure peak is around 105 psig at 70 minutes and decreases when the oil

start to be produced at 60 minutes or 0.25 pore volume steam injected (CWE) and stabilizes until 140 minutes and decreases rapidly the end of the run. 


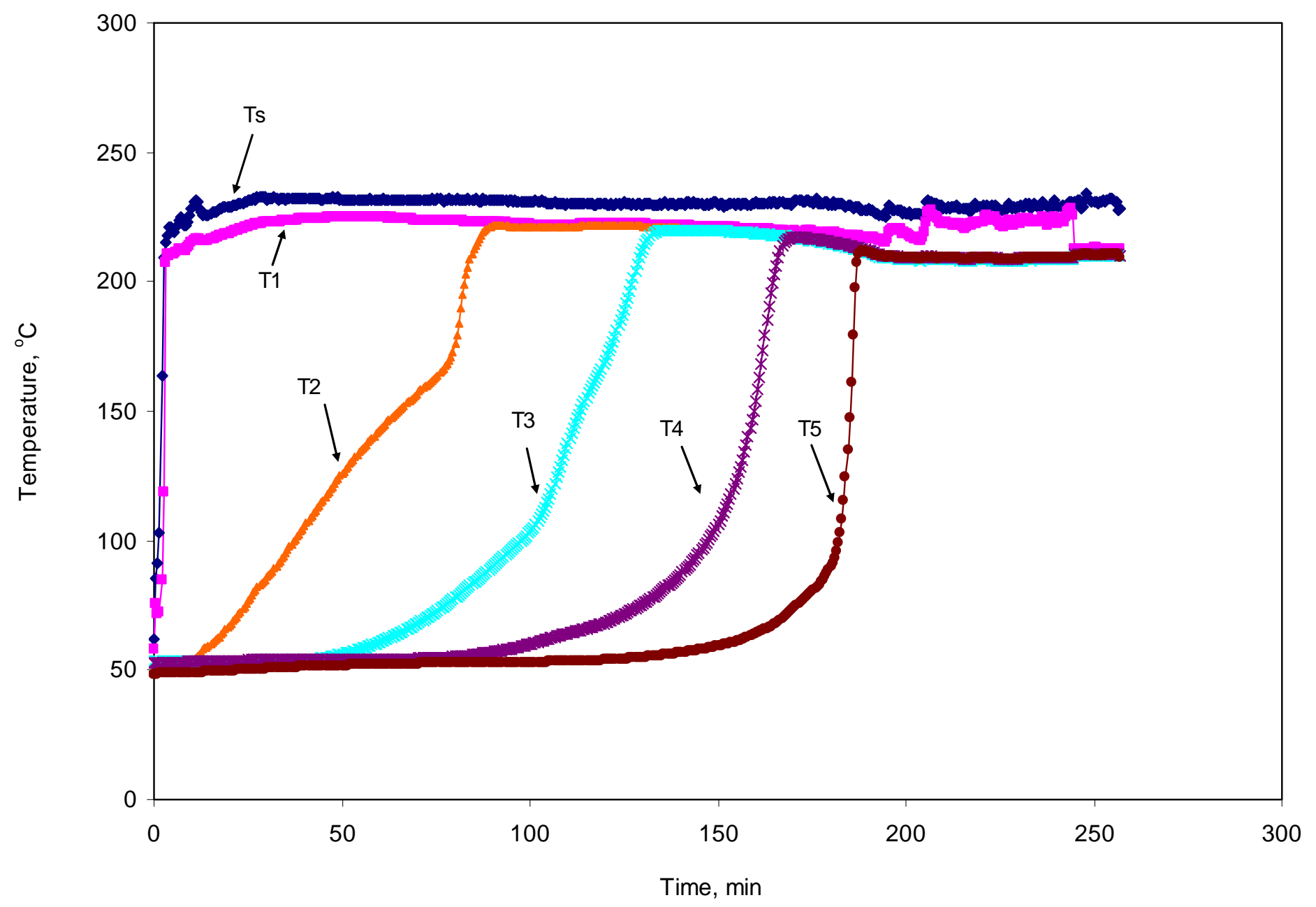

Fig. 4.20- Temperature profile versus time for Run 5 (pure steam). 


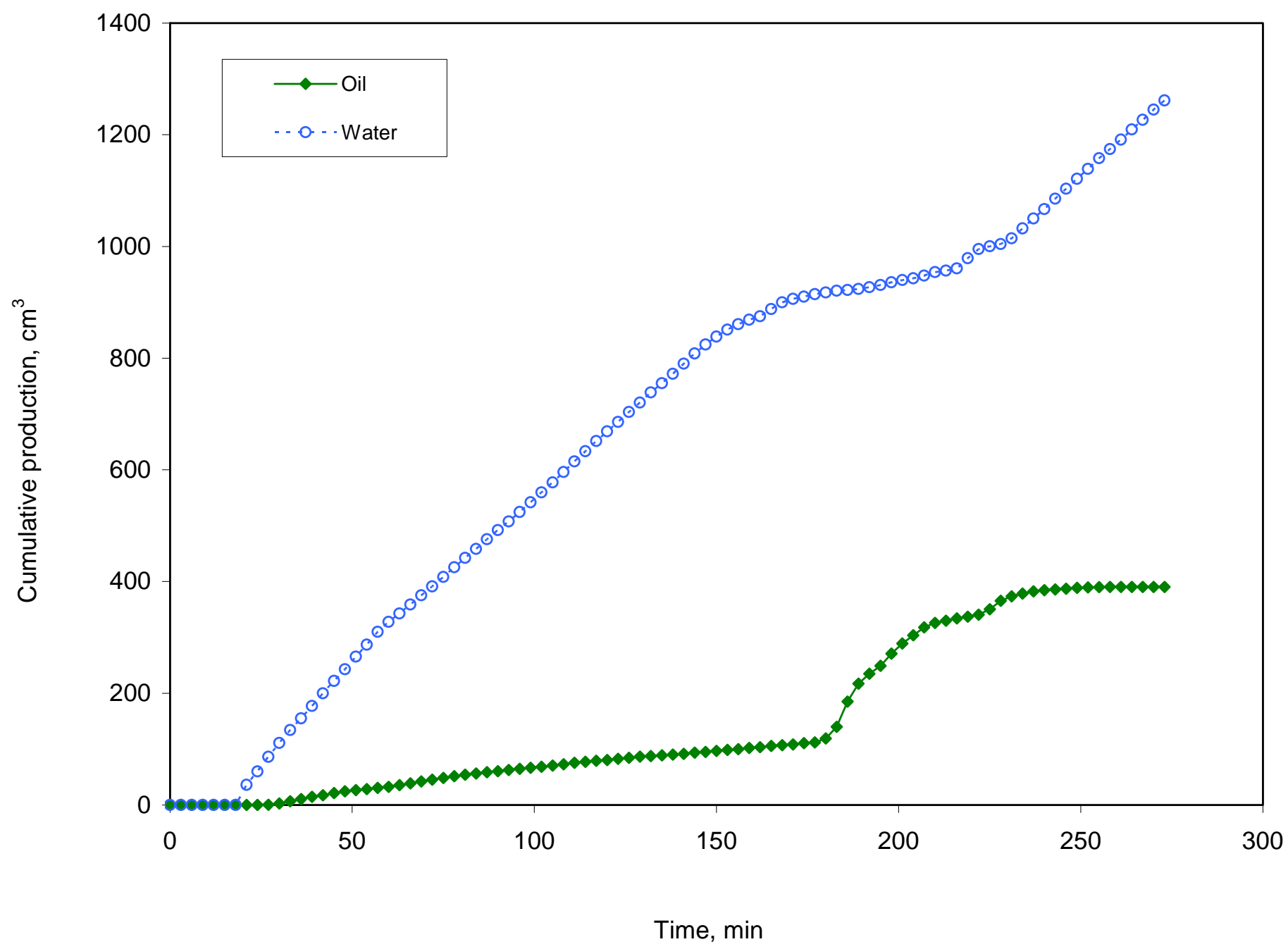

Fig. 4.21- Cumulative oil and water volumes versus time for Run 5 (pure steam). 


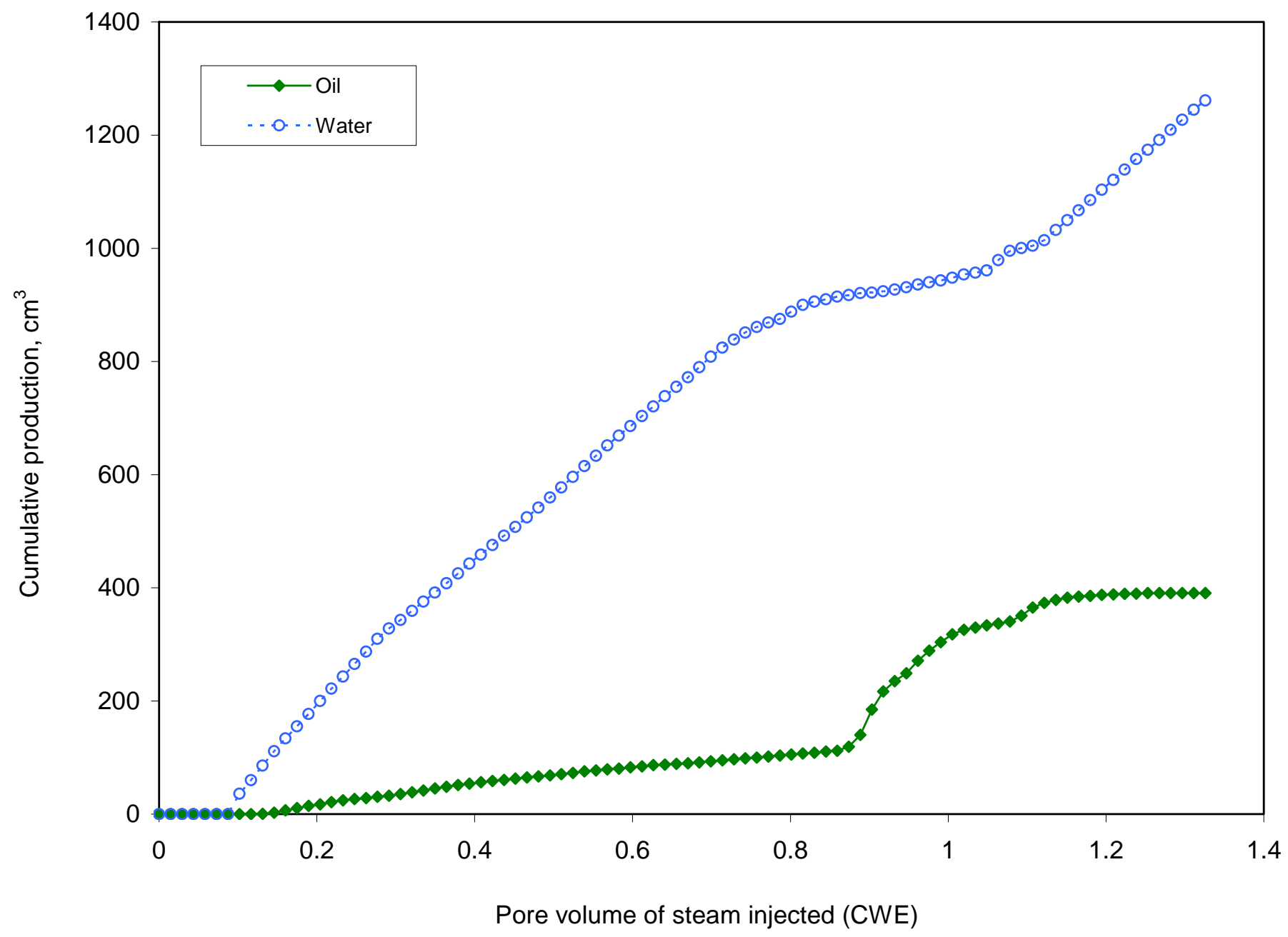

Fig. 4.22- Cumulative oil and water volumes versus pore volume of steam injected for Run 5 (pure steam). 


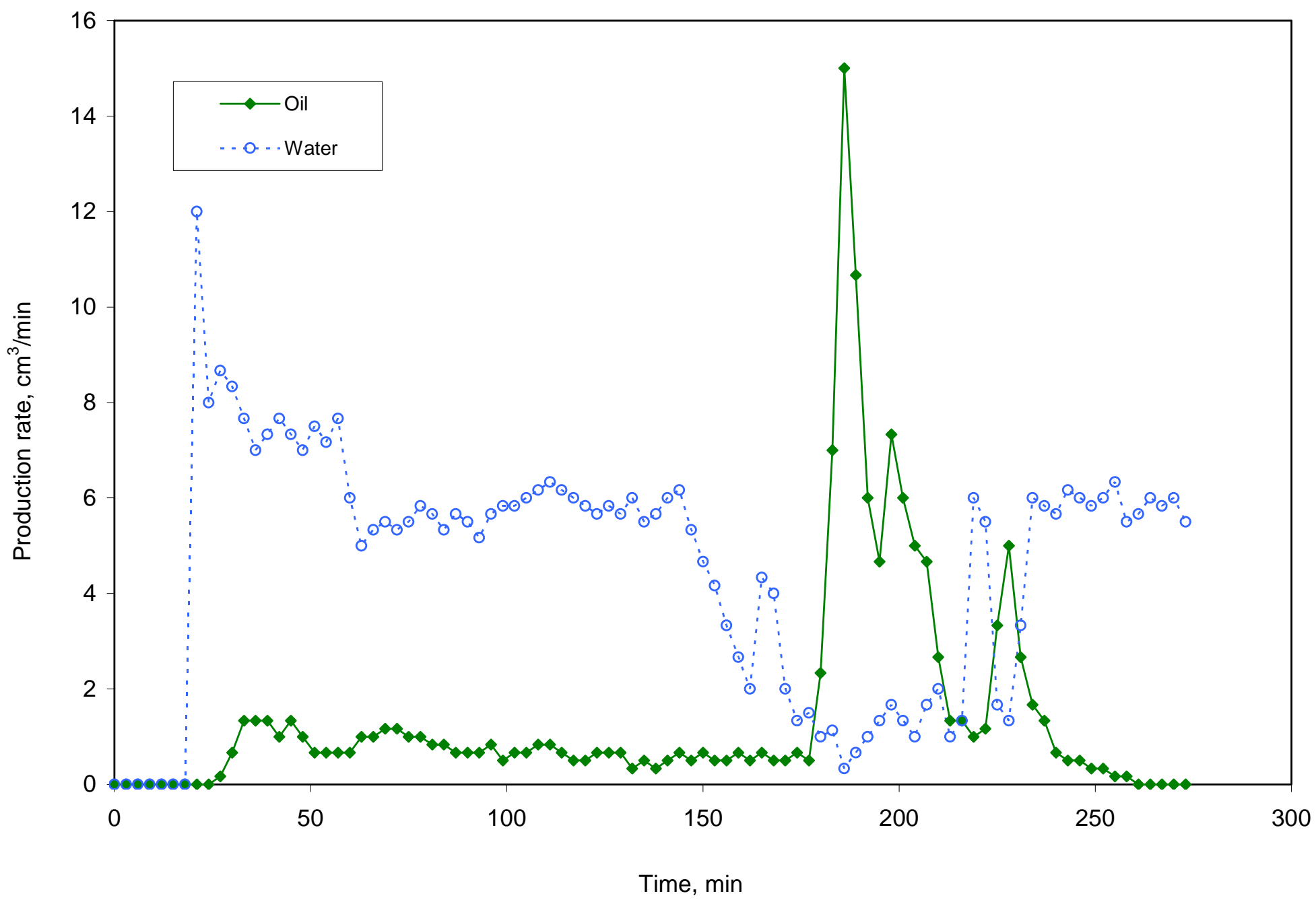

Fig. 4.23- Oil and water rates versus time for Run 5 (pure steam). 


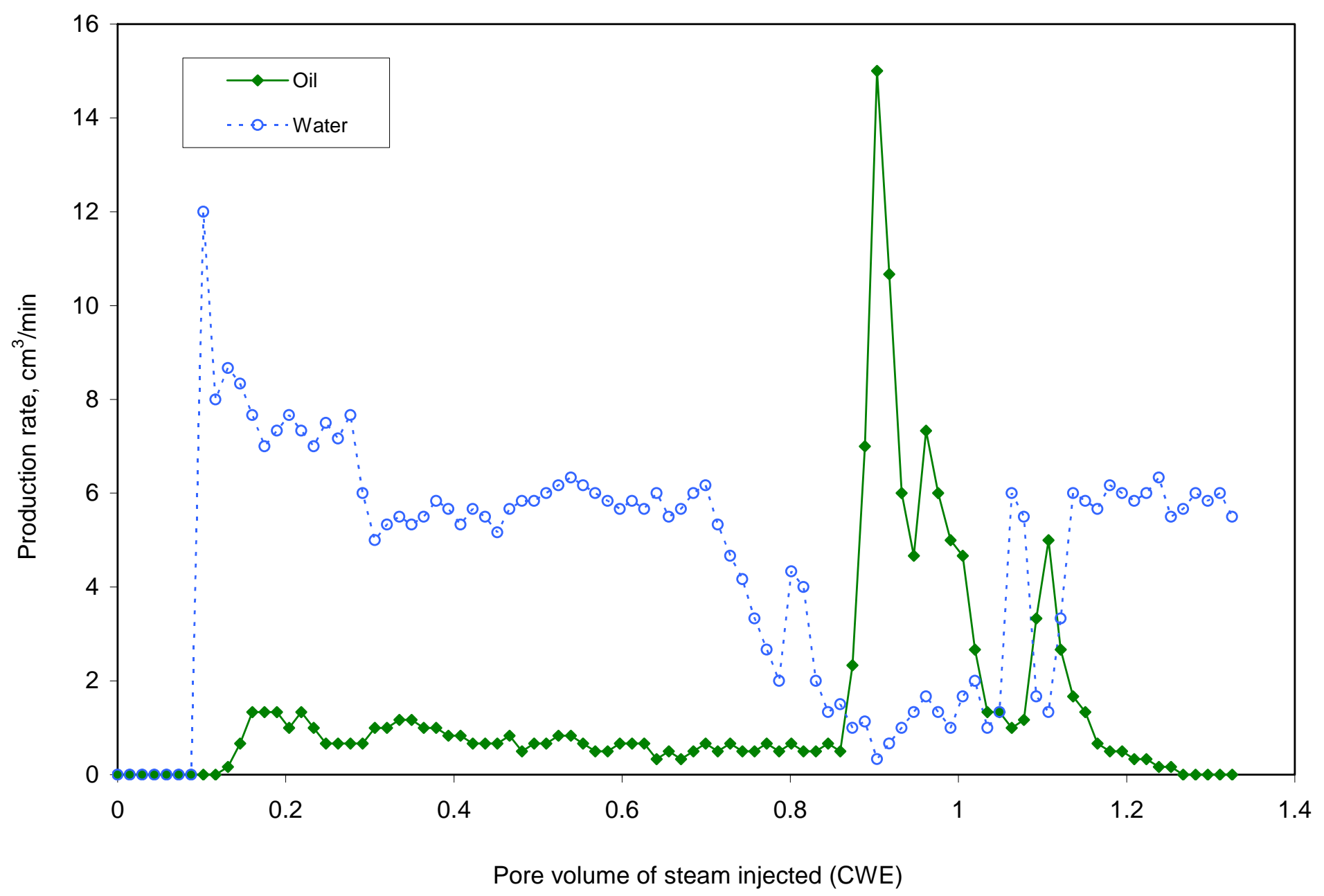

Fig. 4.24- Oil and water rates versus pore volume of steam injected for Run 5 (pure steam). 


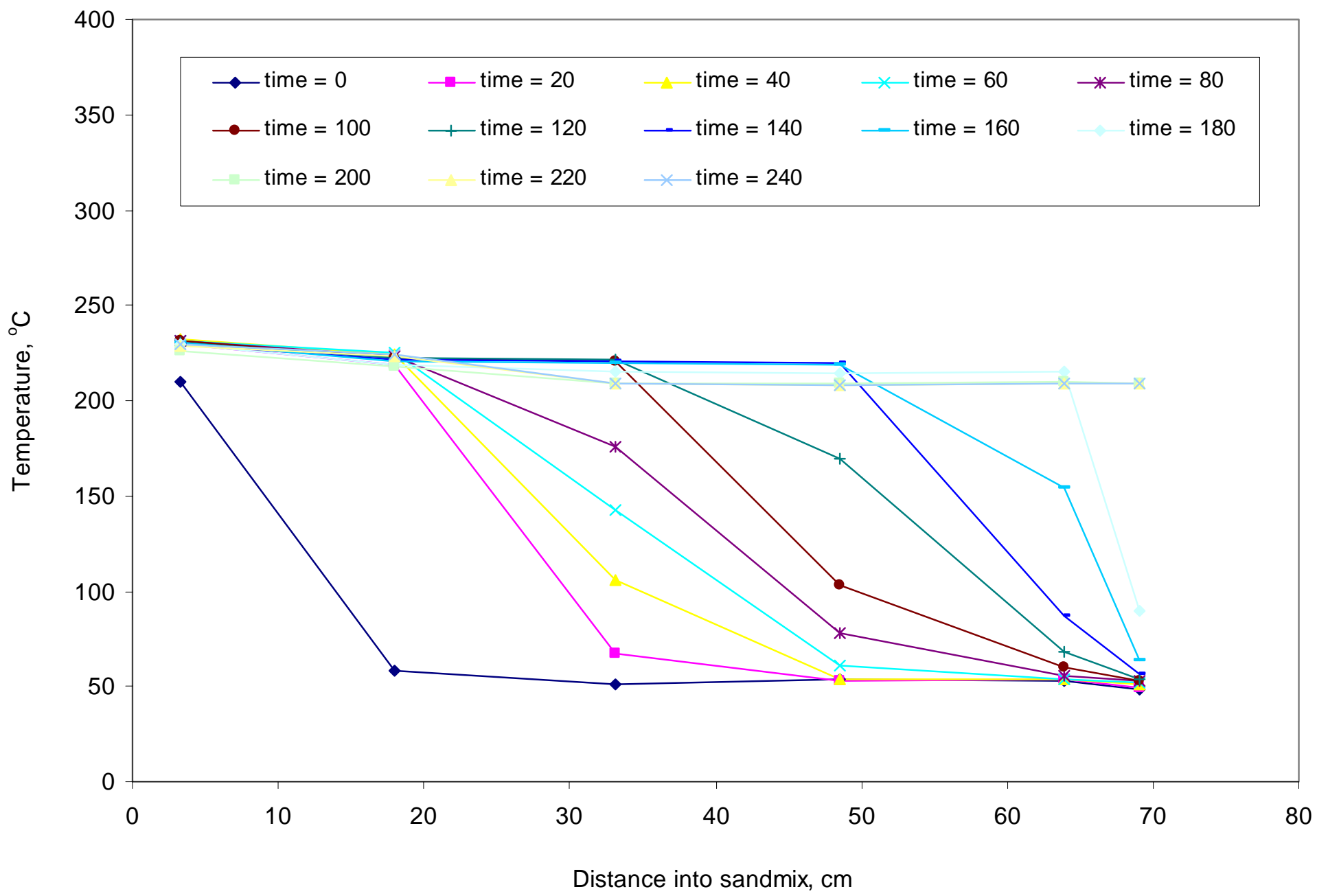

Fig. 4.25-Temperature profiles at 20-minute intervals - from $\mathrm{t}=0$ to $\mathrm{t}=240 \mathrm{~min}-$ for Run 5 (pure steam). 


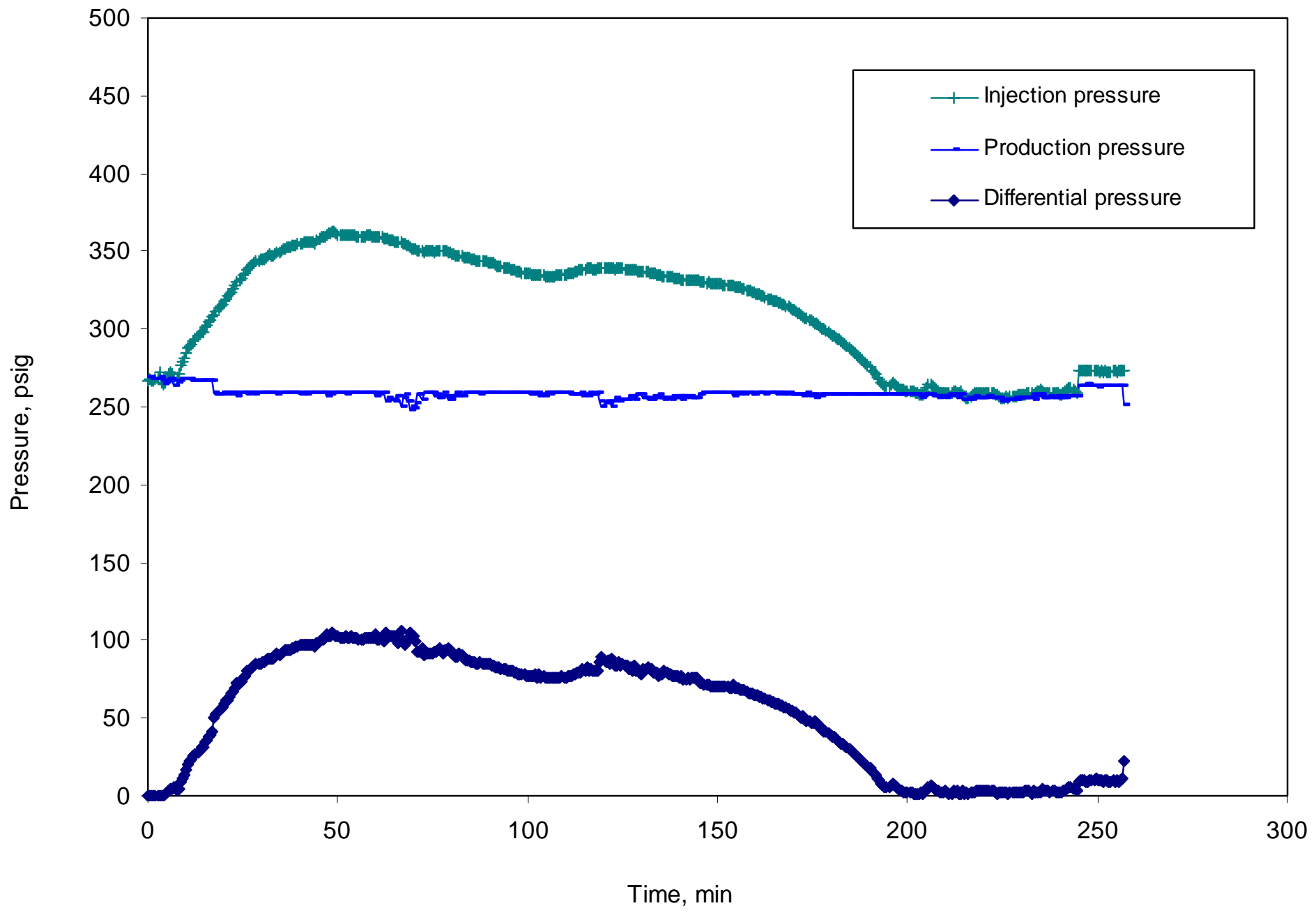

Fig. 4.26- Injection, production and differential pressures for Run 5 (pure steam). 


\subsection{Run 6 (5:100 Propane:Steam Mass Ratio)}

This is a repeat run with the same conditions as run no. 3. The movement of the steam front can be followed in Fig. 4.27. The injection temperature is kept steady at $230^{\circ} \mathrm{C}$ throughout the run, and little fluctuation is observed.

The cumulative oil and water volumes, and pore volume injected plotted versus time are depicted in Figs. 4.28 and 4.29. Ultimate recovery at the end of the 216 minutes run is about $403.5 \mathrm{~cm}^{3}$, or $42 \%$ of OOIP, as can be seen in Figs. $\mathbf{4 . 3 0}$ and $\mathbf{4 . 3 1}$ shows the oil and water production rates as a function of time. The first oil is produced after 17 minutes of injection, with a peak rate of about $15.33 \mathrm{~cm}^{3} / \mathrm{min}$ after 62 minutes.

Fig. 4.32 shows how the temperature propagation at 20 minute intervals for the different thermocouples. It takes about 100 minutes for the whole cell to reach a more or less constant temperature of $142^{\circ} \mathrm{C}$.

The injection pressure, outlet pressure, and differential pressure are shown in Fig. 4.33. The outlet pressure is kept steady at 260 psig. As the oil bank is building up injection pressure increases, and as a result of that so does the differential pressure. From an initial differential pressure of close to zero it reaches highs of about 89 psig before oil production starts.

The compositional analysis of the gas produced after the third separator is plotted in Fig. 4.34. The cumulative gas volume produced after the third separator is presented in Fig. 4.35. Fig. 4.36 shows the propane cumulative injection mass and propane cumulative mass production for the third separator. A total of $66 \mathrm{~g}$ propane is injected inside the cell and propane produced from the third separator is $4.63 \mathrm{~g}$. The balance of the propane, $61.37 \mathrm{~g}$, is produced at the second separator during sample collection. 


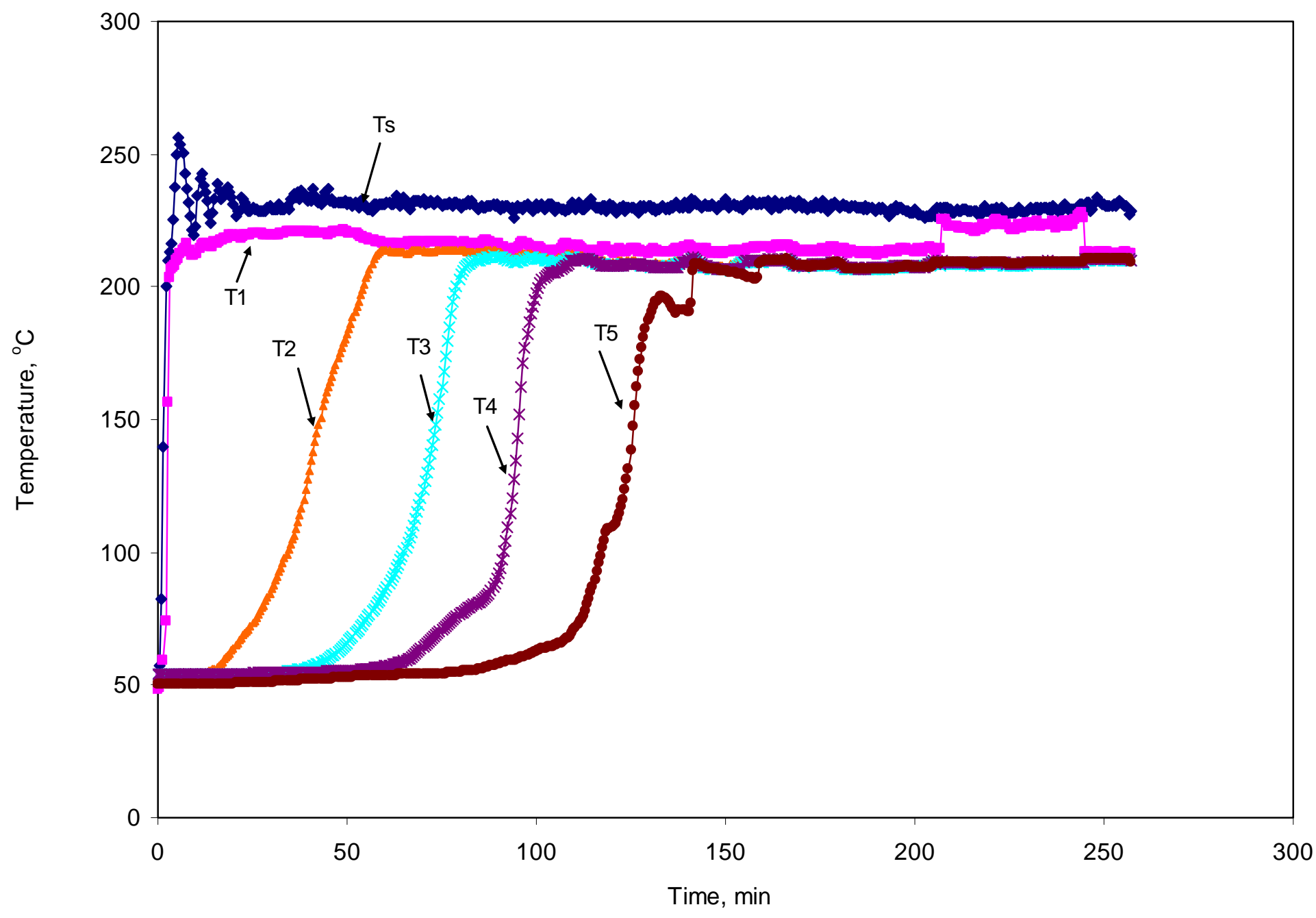

Fig. 4.27- Temperature profile versus time for Run 6 (5:100 propane:steam). 


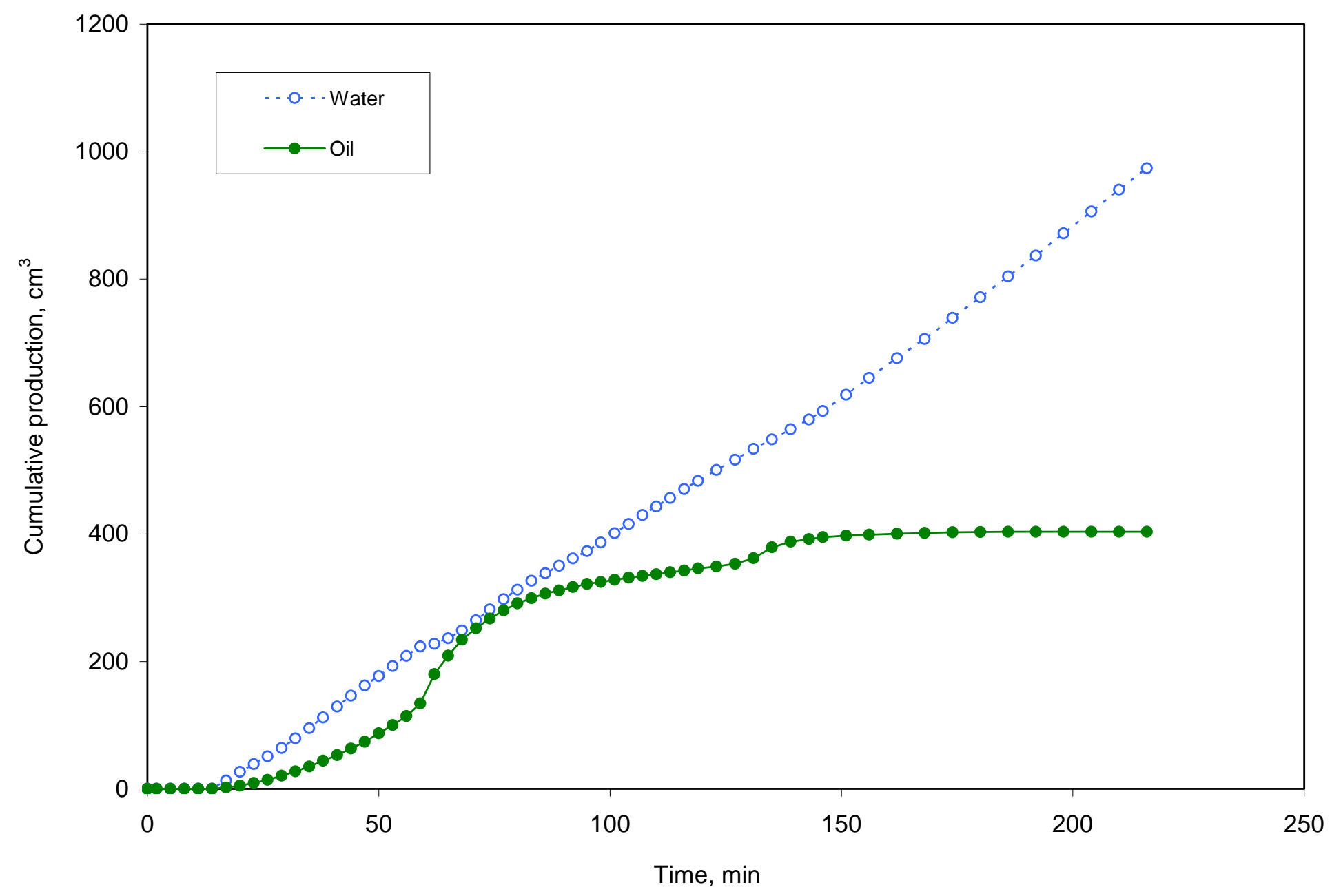

Fig. 4.28- Cumulative oil and water volumes versus time for Run 6 (5:100 propane:steam). 


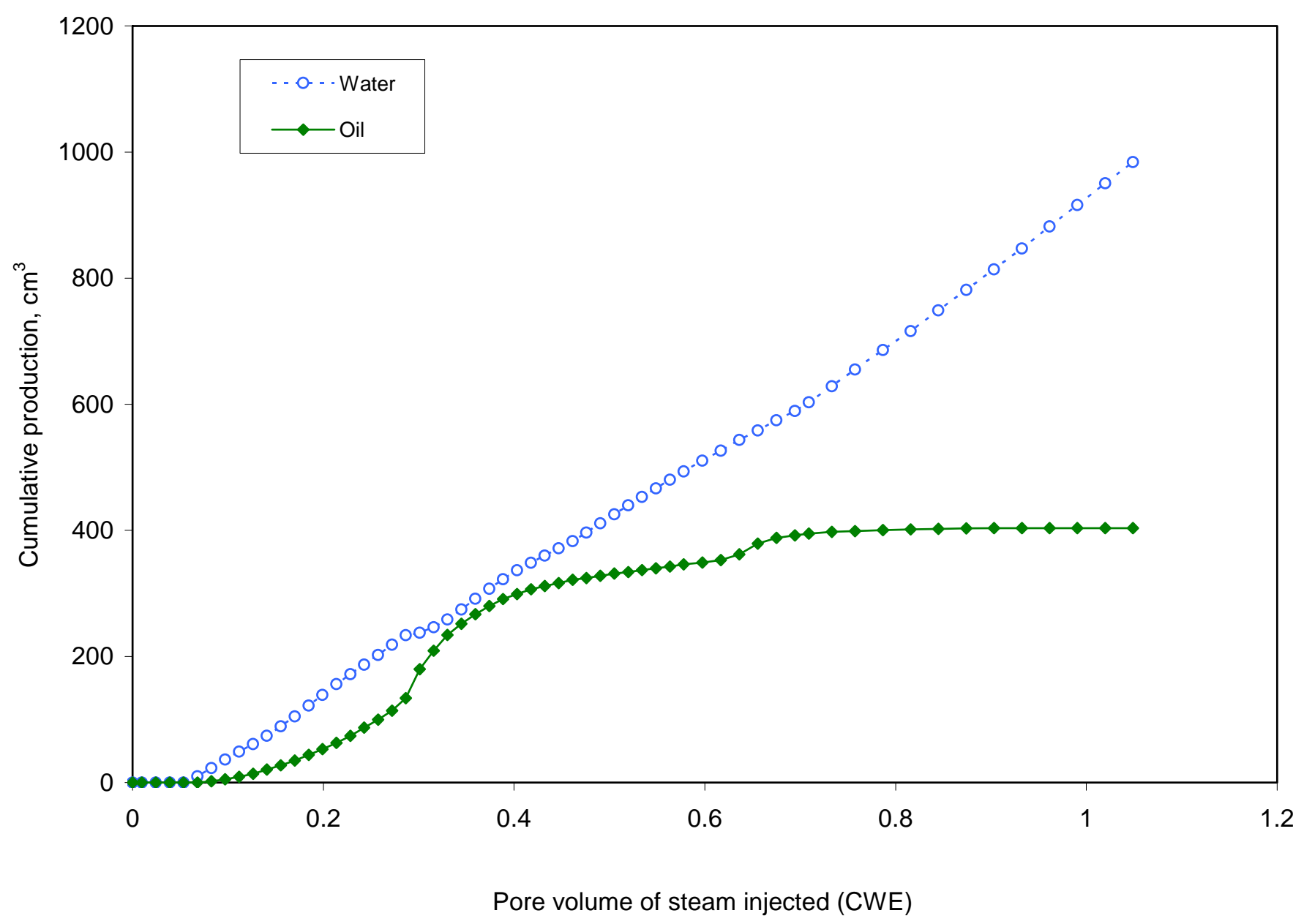

Fig. 4.29- Cumulative oil and water volumes versus pore volume of steam injected for Run 6 (5:100 propane:steam). 


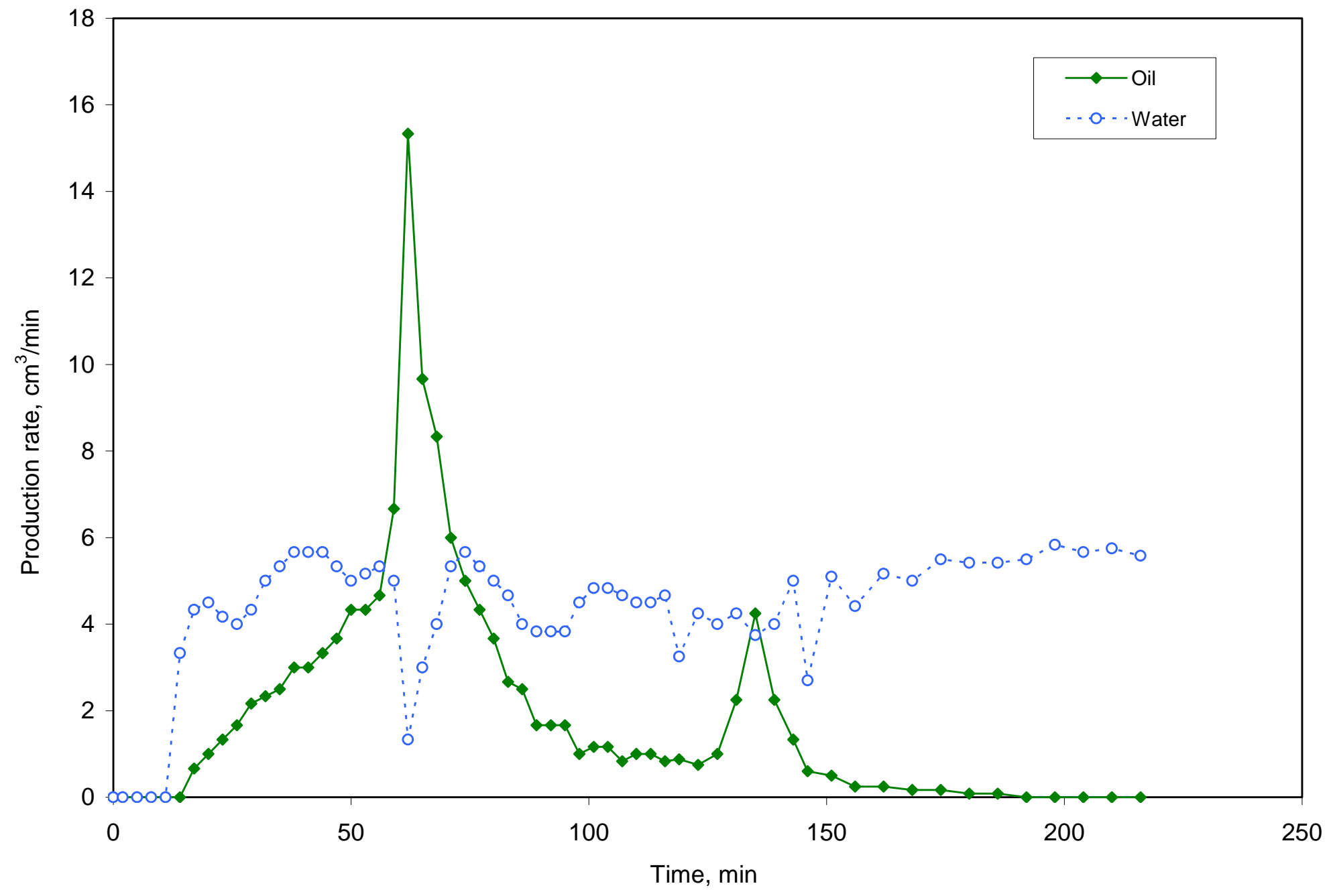

Fig. 4.30- Oil and water rates versus time for Run 6 (5:100 propane:steam). 


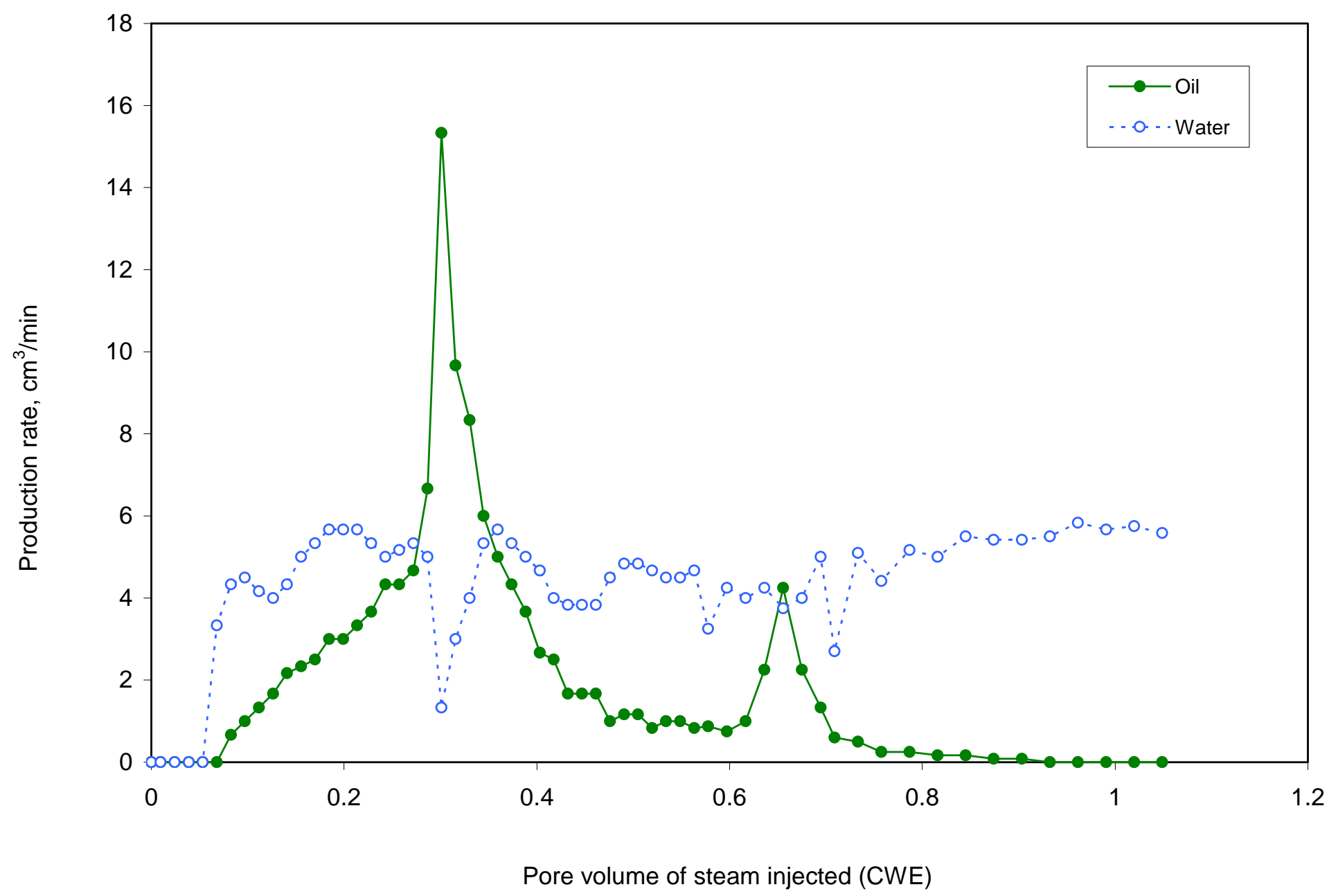

Fig. 4.31- Oil and water rates versus pore volume of steam injected for Run 6 (5:100 propane:steam). 


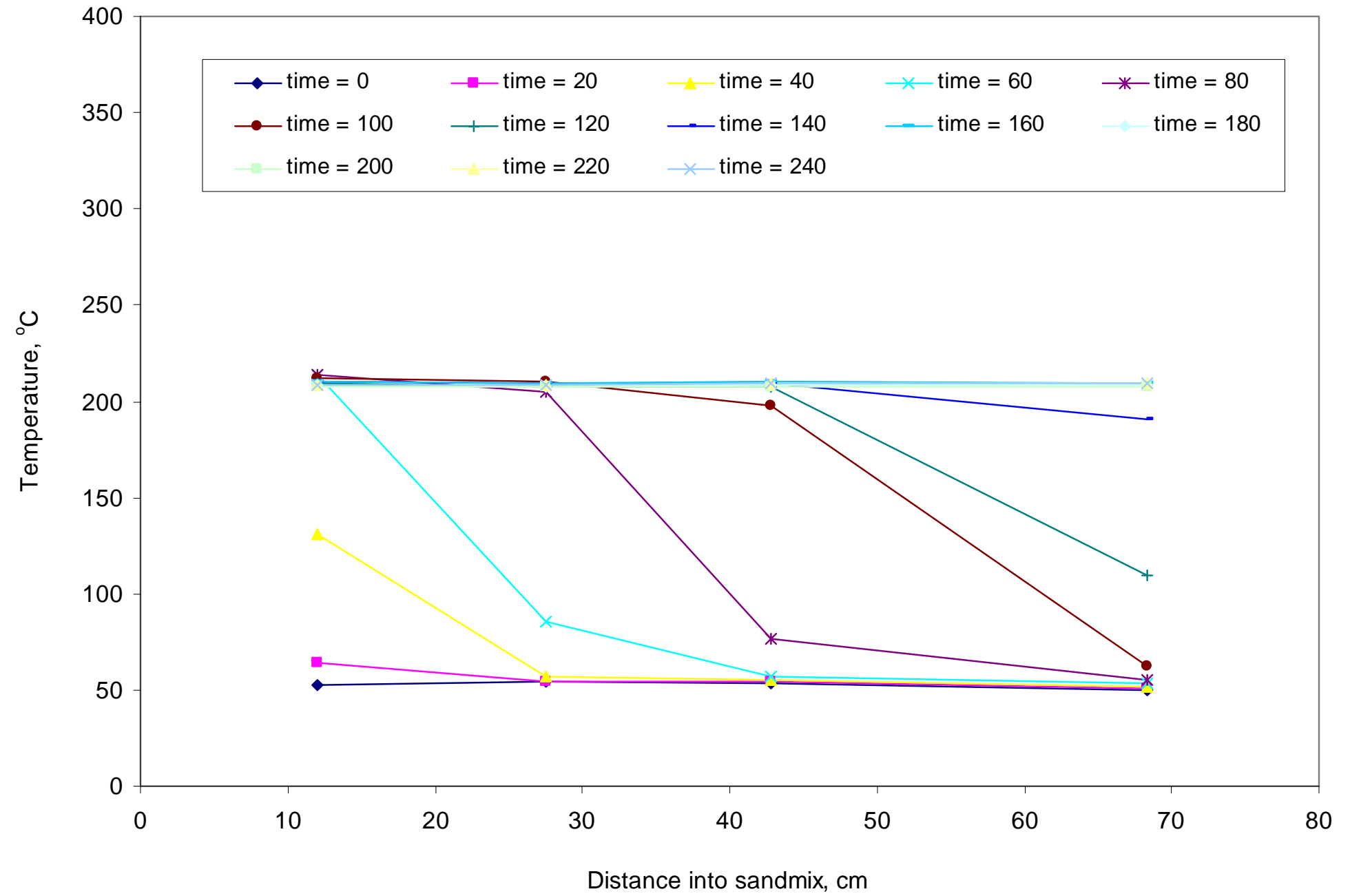

Fig. 4.32- Temperature profiles at 10-minute intervals - from $t=0$ to $t=240 \mathrm{~min}-$ for Run 6 (5:100 propane:steam). 


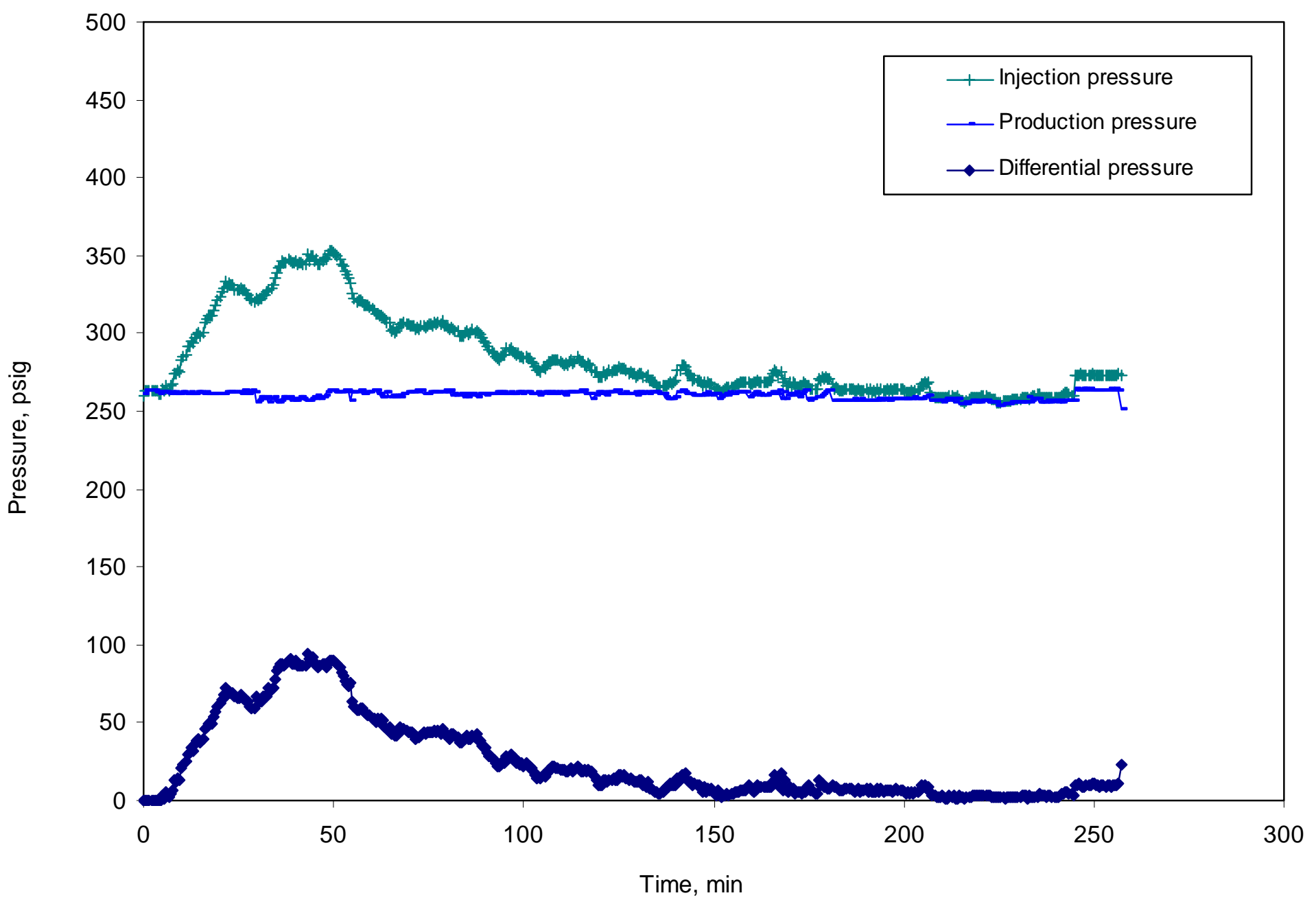

Fig. 4.33- Injection, production and differential pressures for Run 6 (5:100 propane:steam). 


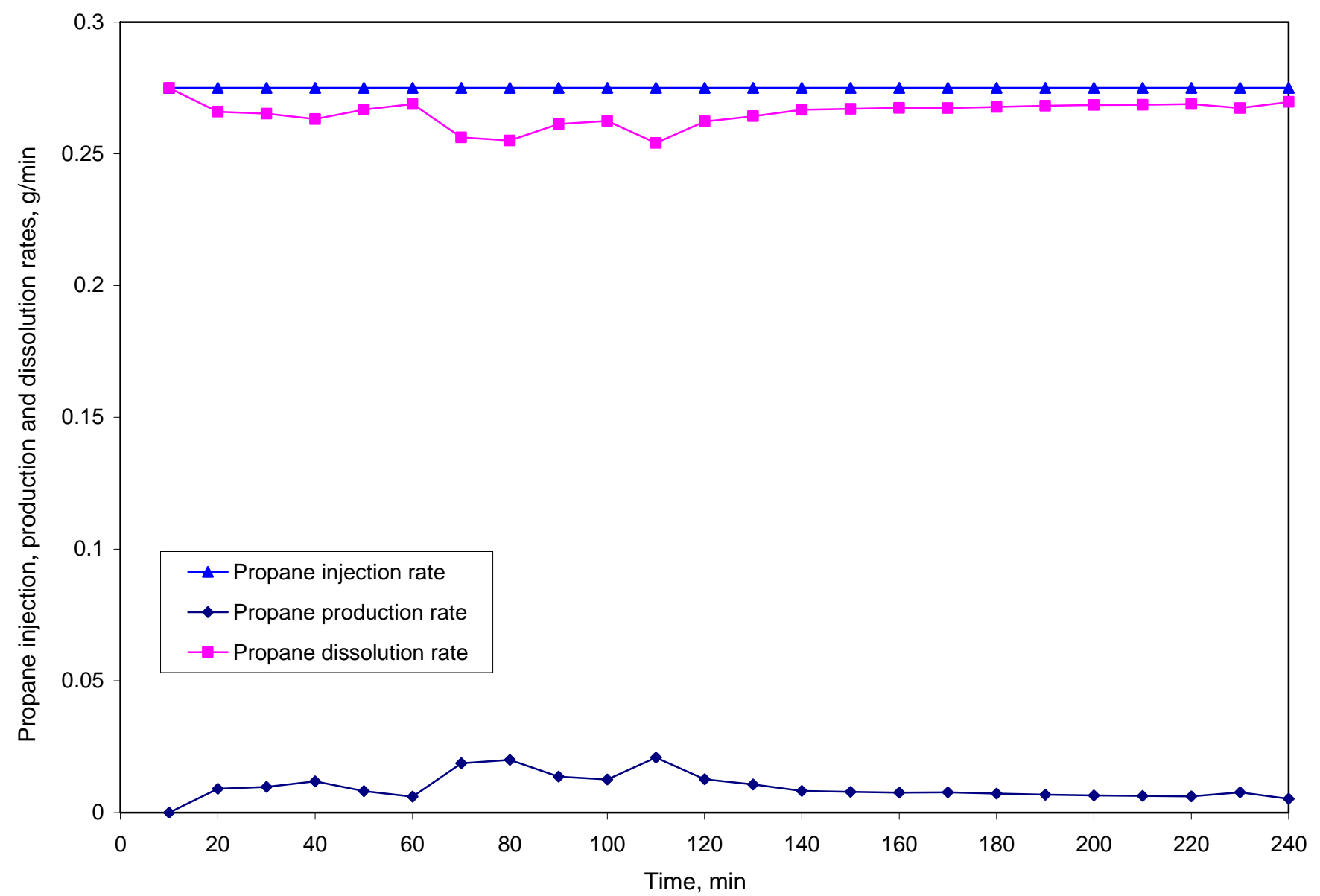

Fig. 4.34- Propane injection, production, and dissolution rates after the third separator for Run 6 (5:100 propane:steam). 


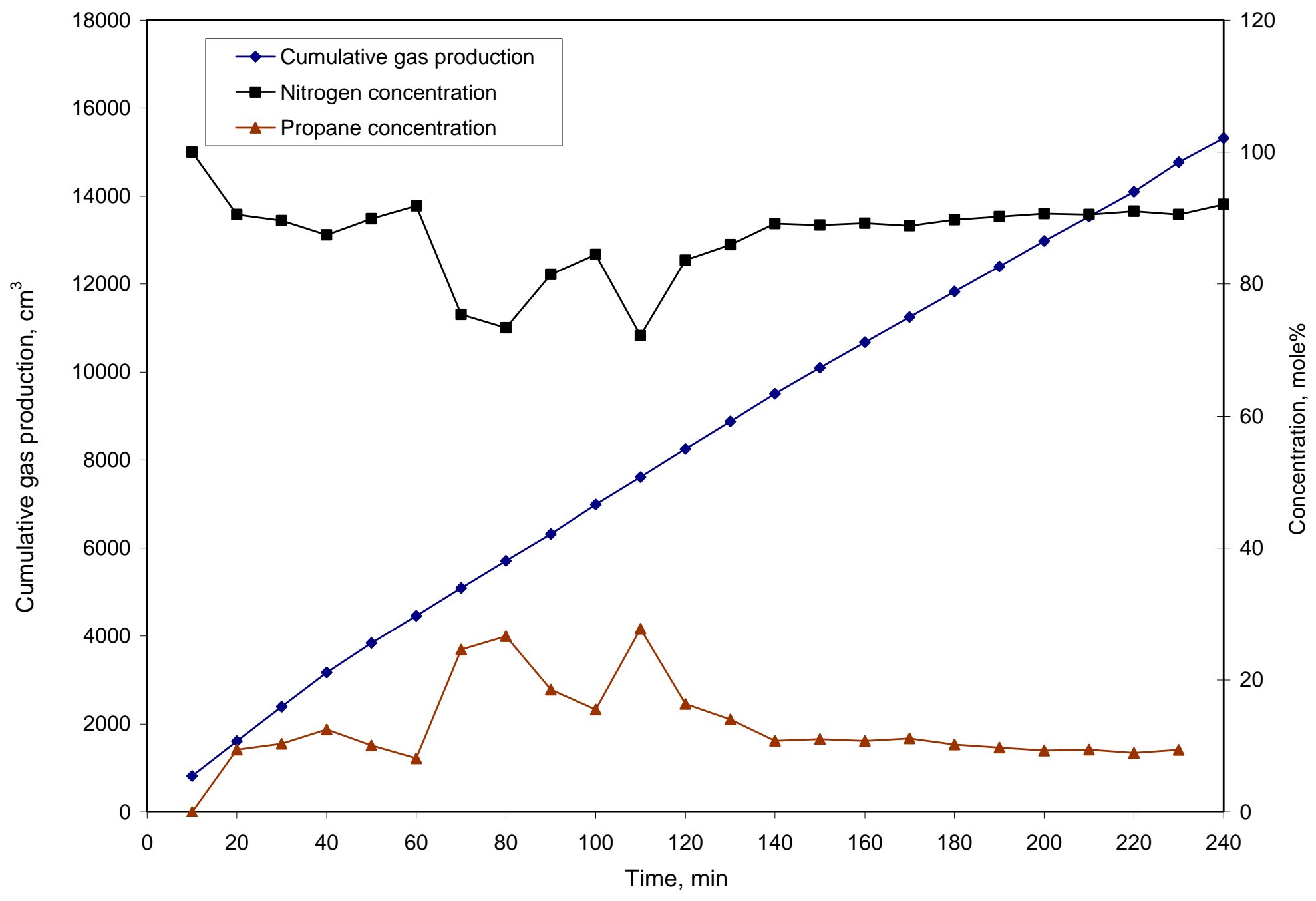

Fig. 4.35- Cumulative gas production and composition after the third separator for Run 6 (5:100 propane:steam). 


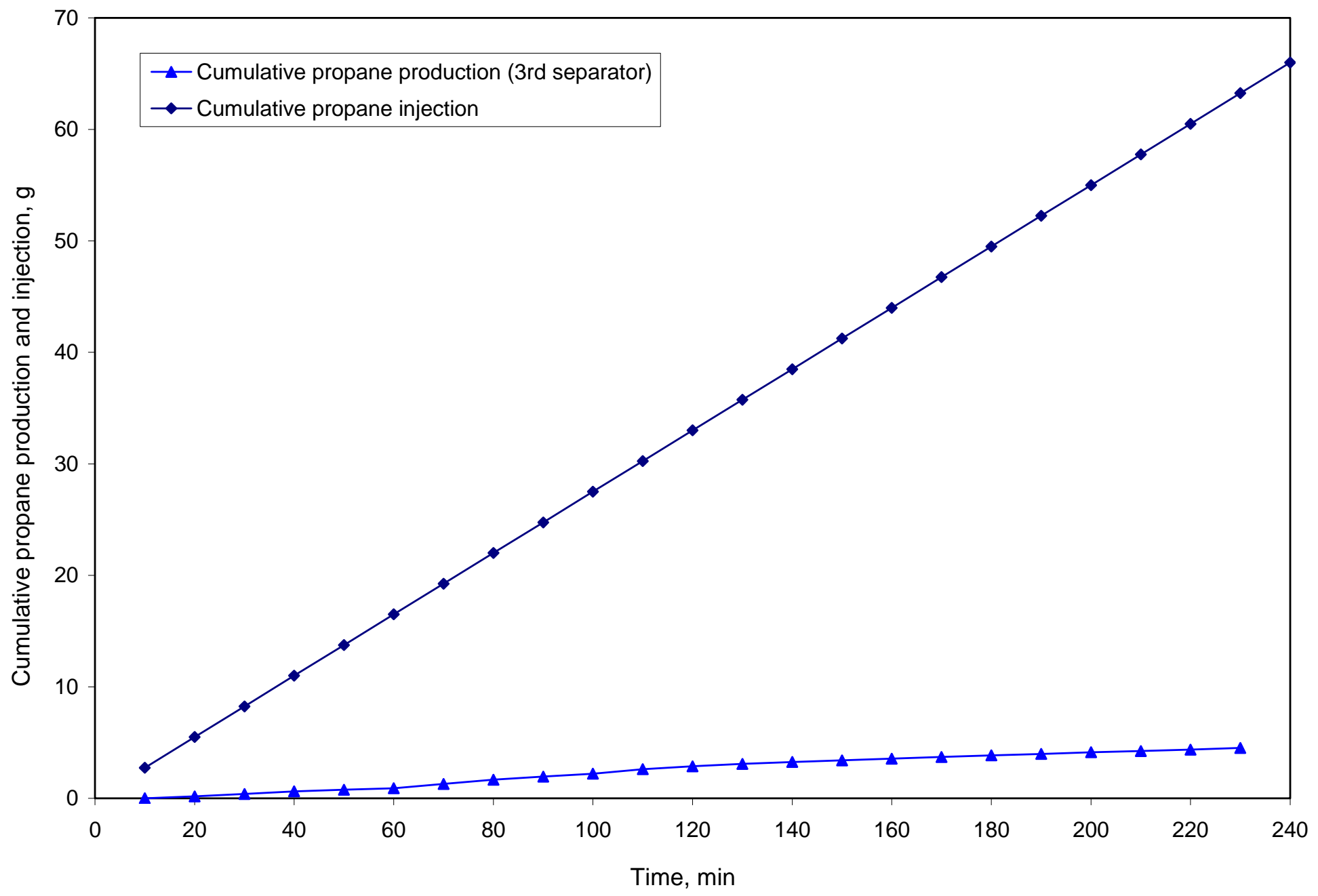

Fig. 4.36- Cumulative propane injection and production for Run 6 (5:100 propane:steam). 


\subsection{Run 7 (5:100 Petroleum Distillate:Steam Mass Ratio)}

Fig. 4.37 shows the temperature profile within the cell. Figs. 4.38 and $\mathbf{4 . 3 9}$ show the cumulative volumes of oil and water vs. time and pore volume of steam injected respectively. At the end of the run, the oil recovery is $441.5 \mathrm{~cm}^{3}$ (45.8\% of OOIP).

The oil and water rates are plotted both as a function of time and pore volume of steam injected (Figs. 4.40 and 4.41). Oil production rate peak is around $15 \mathrm{~cm}^{3} / \mathrm{min}$ and most of the oil is produced from 15 to 123 minutes. Fig. 4.42 shows the temperature profiles inside the cell at 20-minute intervals. It can be observed that by 124 minutes the temperature of the whole cell reaches a value around $210^{\circ} \mathrm{C}$.

The injection, production pressures and the differential pressure are depicted in Fig. 4.44. The injection pressure started increasing at the beginning of the run to eventually decrease at around 126 minutes. The maximum differential pressure reached in this run was around 70 psig.

The oil viscosity and API gravity of the produced oil are shown in Fig. 4.45. The API gravity shows an increasing trend. The oil viscosity shows a decreasing trend as time increases. 


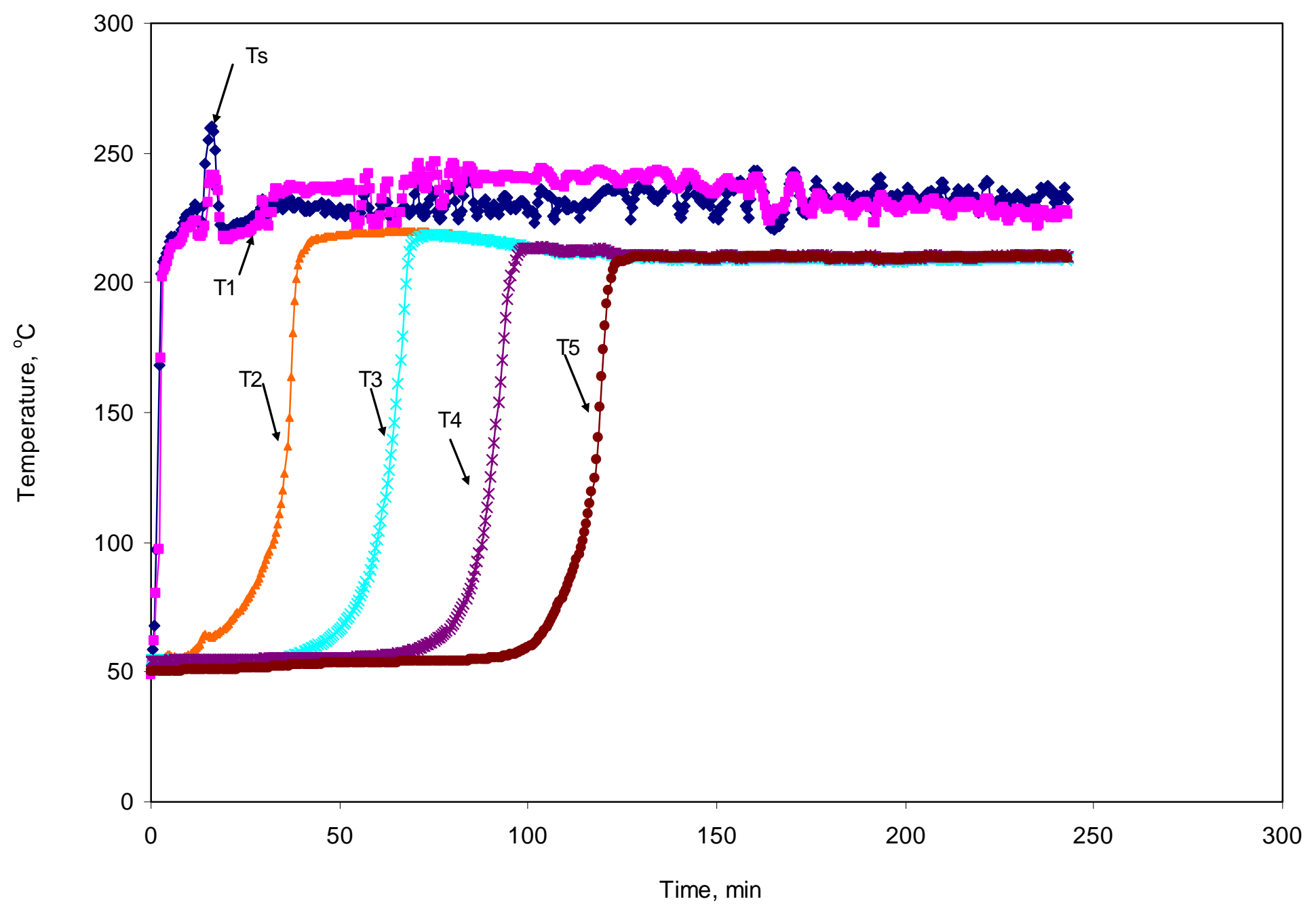

Fig. 4.37- Temperature profile versus time for Run 7 (5:100 petroleum distillate:steam). 


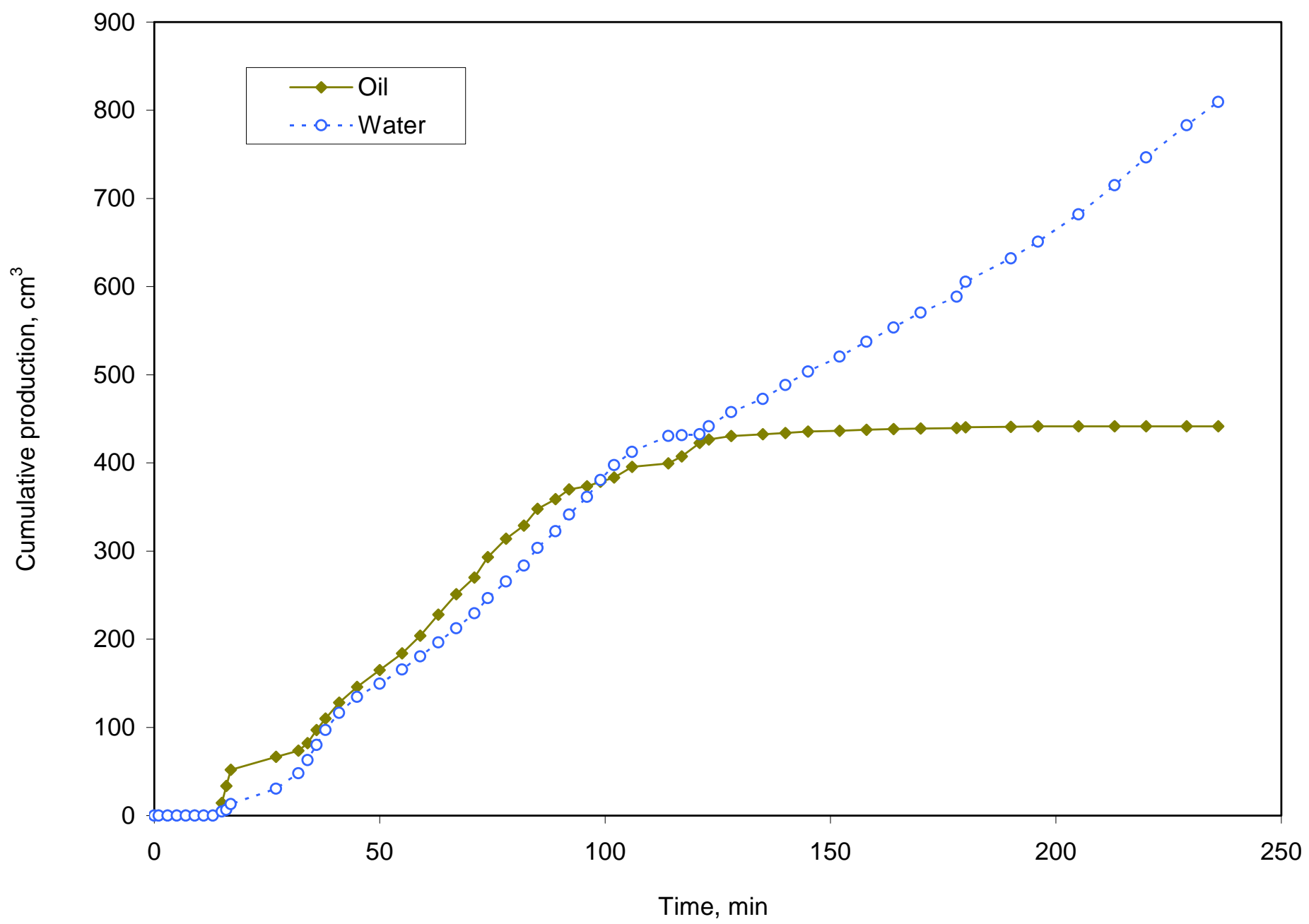

Fig. 4.38- Cumulative oil and water volumes versus time for Run 7 (5:100 petroleum distillate:steam). 


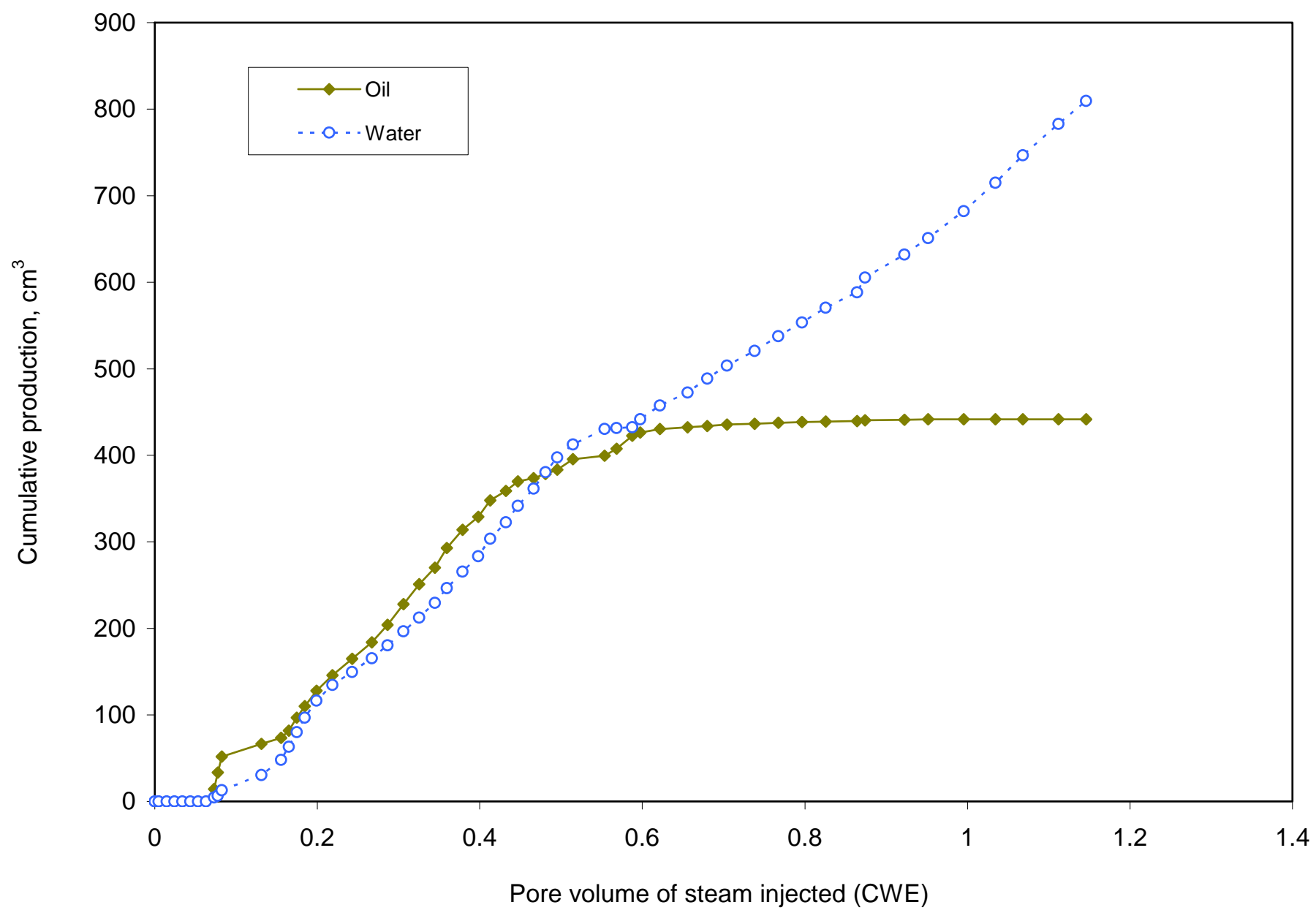

Fig. 4.39- Cumulative oil and water volumes versus pore volume of steam injected for Run 7 (5:100 petroleum distillate:steam). 


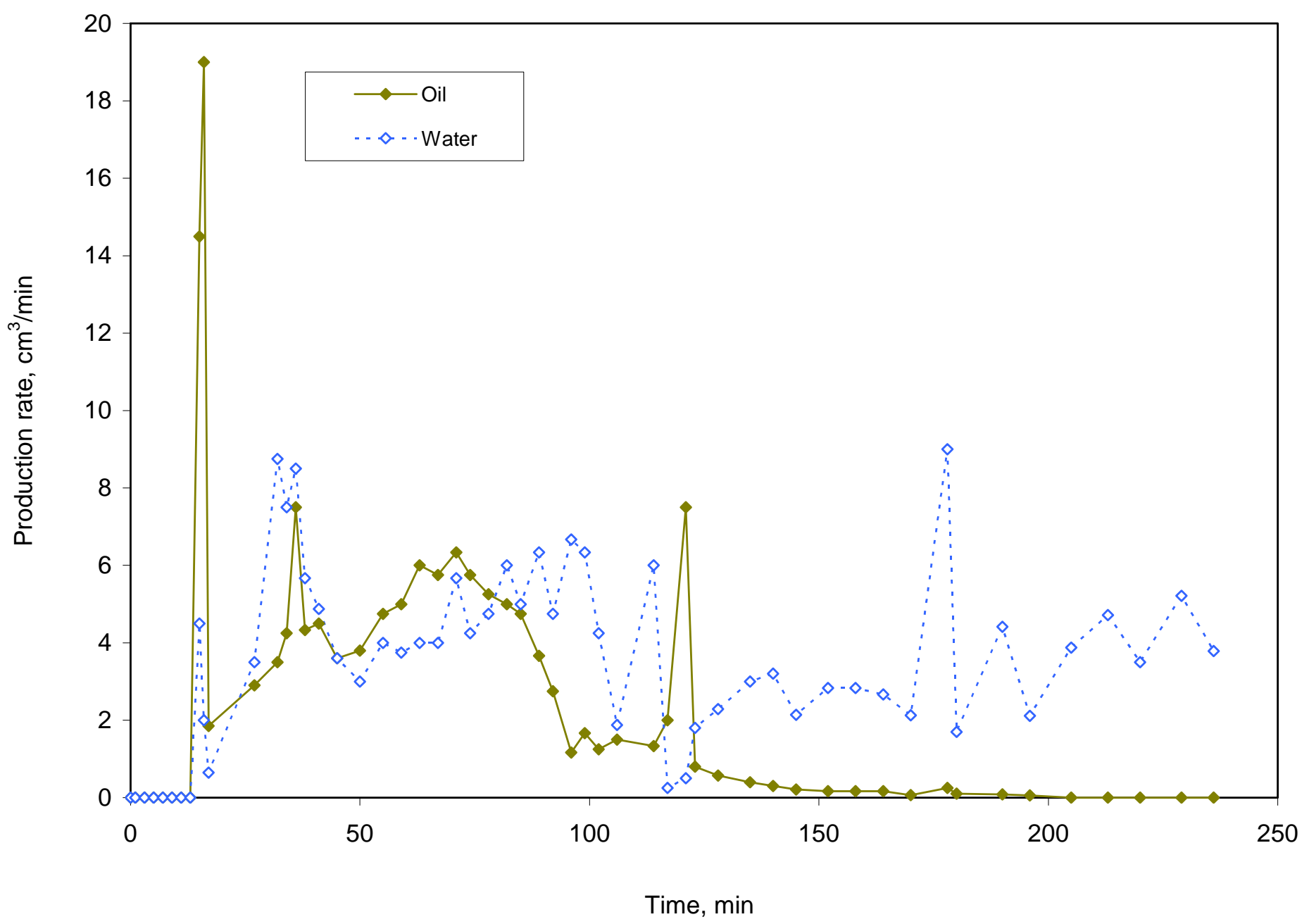

Fig. 4.40- Oil and water rates versus time for Run 7 (5:100 petroleum distillate:steam). 


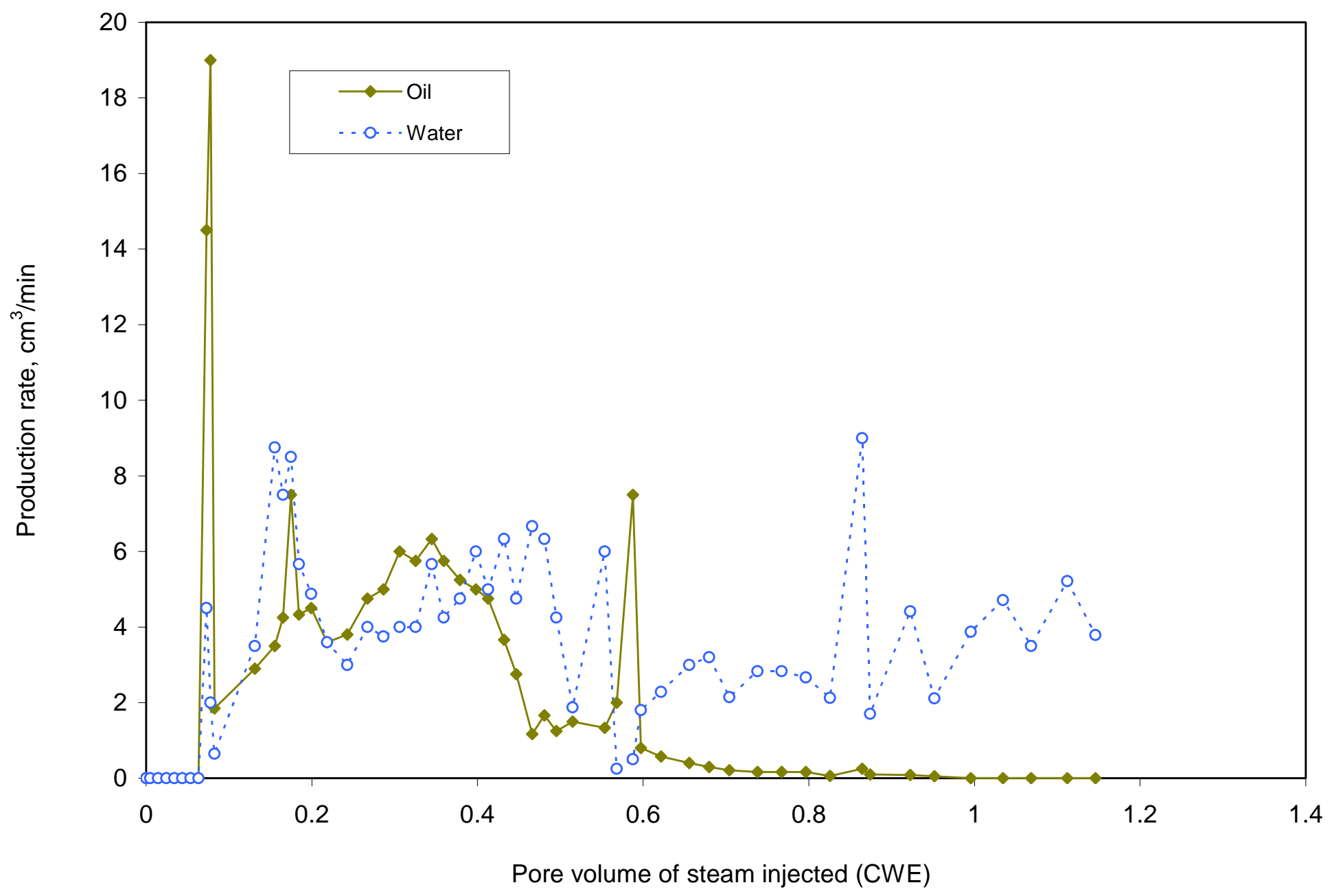

Fig. 4.41- Oil and water rates versus pore volume of steam injected for Run 7 (5:100 petroleum distillate:steam). 


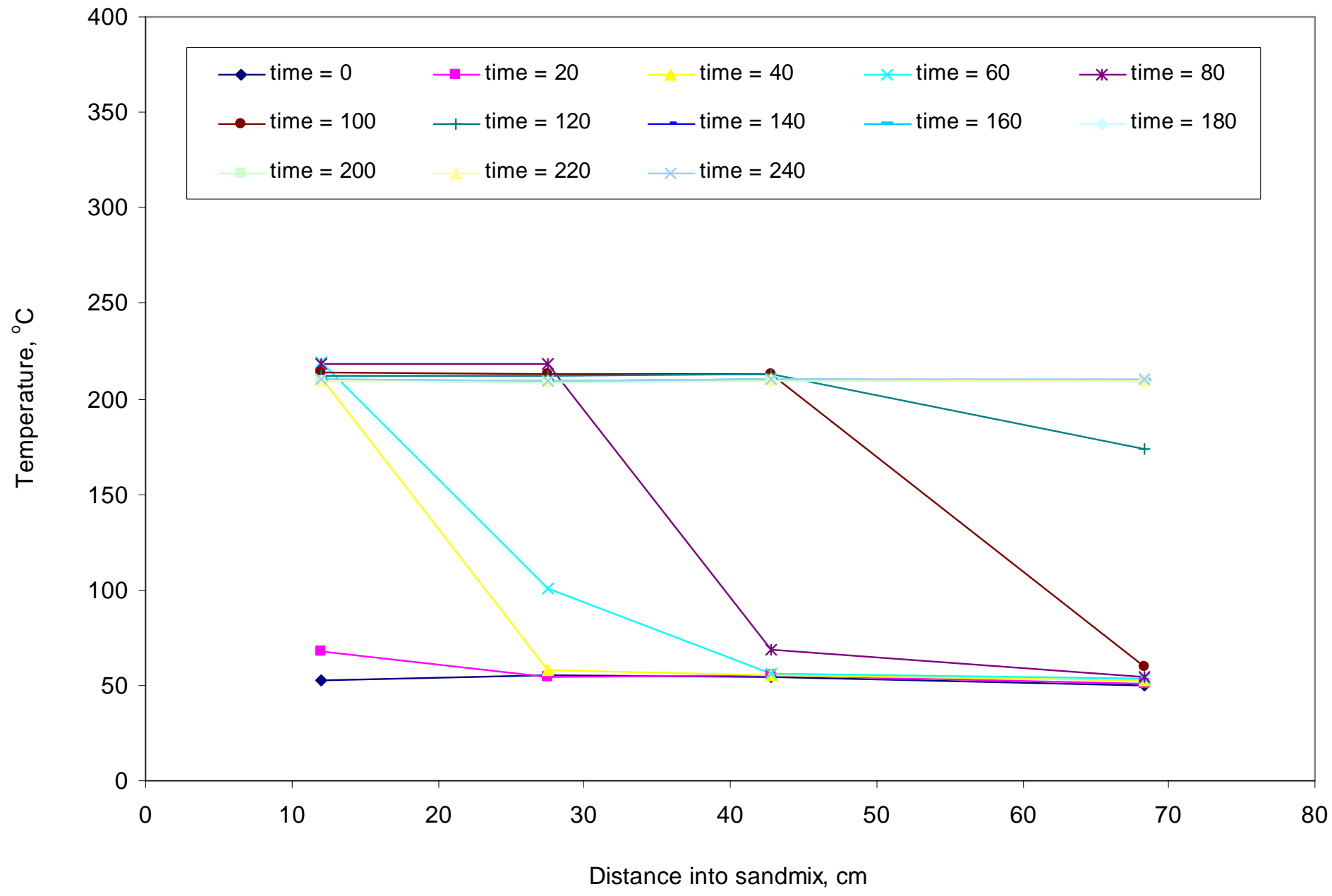

Fig. 4.42- Temperature profiles at 20-minute intervals - from $t=0$ to $t=240 \mathrm{~min}-$ for Run 7 (5:100 petroleum distillate:steam). 


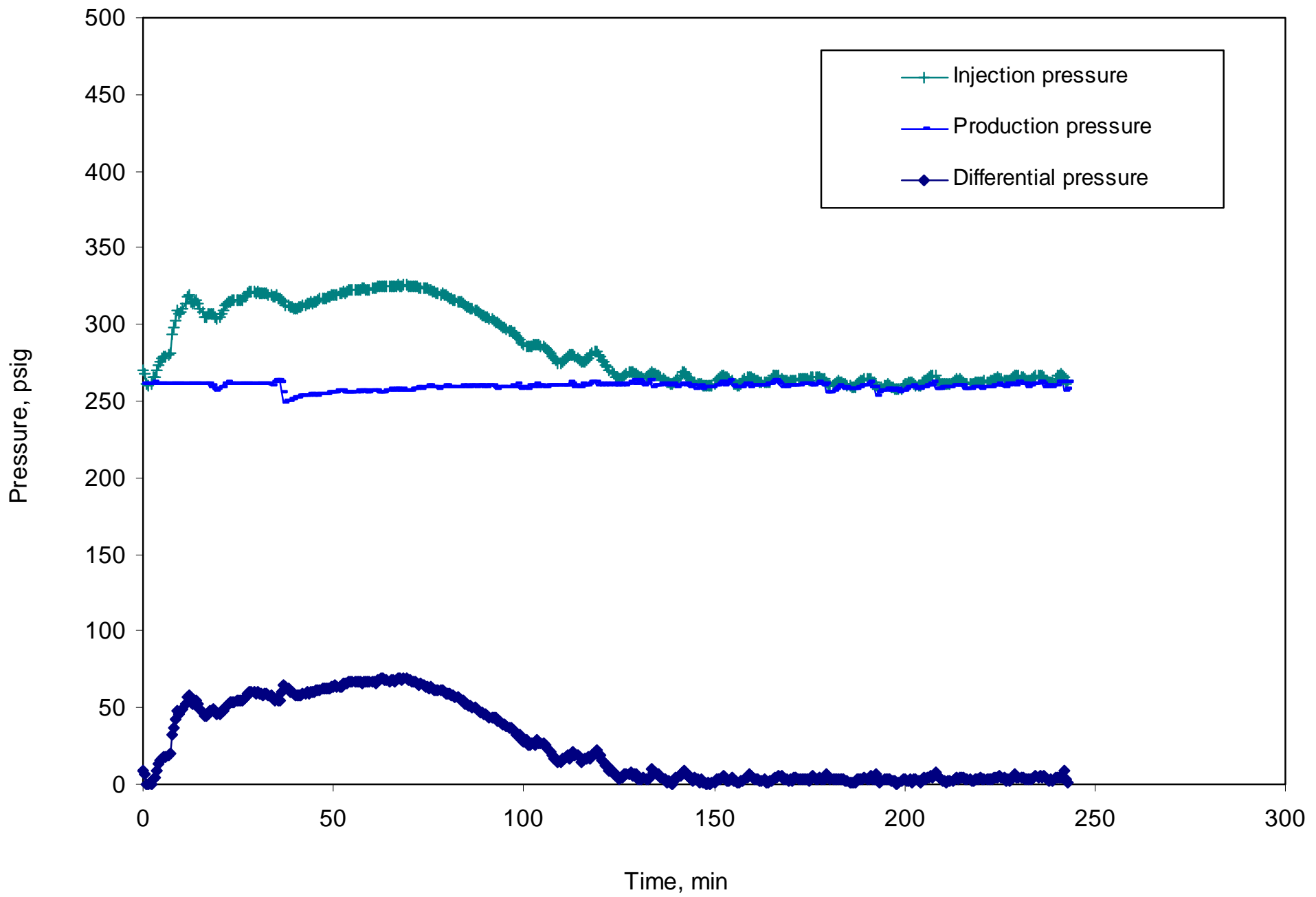

Fig. 4.43- Injection, production and differential pressures for Run 7 (5:100 petroleum distillate:steam). 


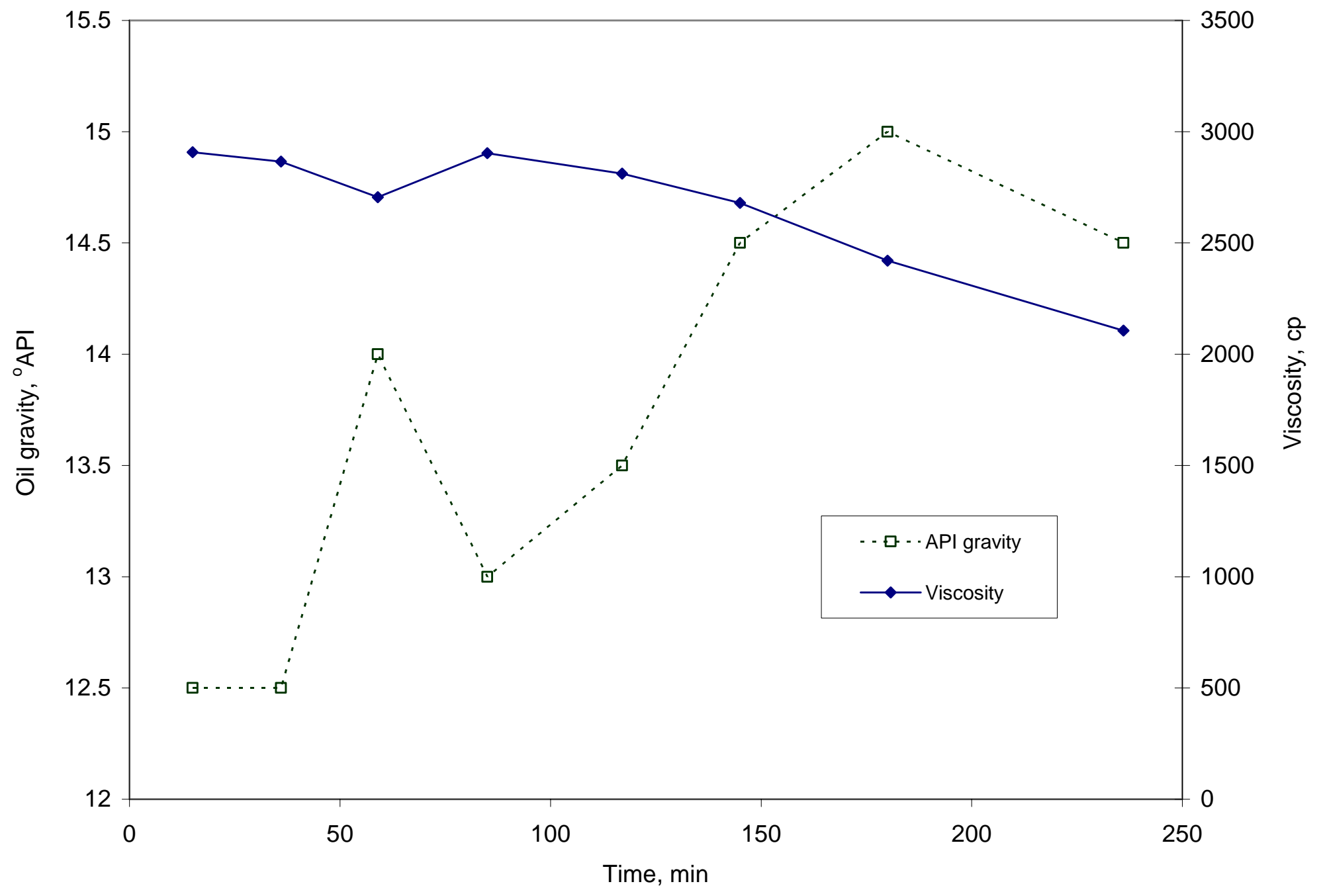

Fig. 4.44- Oil viscosity and API gravity for Run 7 (5:100 petroleum distillate:steam). 


\subsection{Run 8 (5:100 Petroleum Distillate:Steam Mass Ratio)}

This is a repeat run with the same conditions as run no. 7. The injection temperature is kept steady at $230^{\circ} \mathrm{C}$ throughout the run and outlet pressure is set to be 260 psig.

The temperature profile within the cell is shown in Fig. 4.45. Some fluctuations are found in the injection temperature throughout the run. Figs 4.46 and 4.47 show the cumulative oil and water production as a function of time and pore volume injected. At the end of the run, the oil recovery was $46.8 \%$ of OOIP.

The oil and water rates plotted versus time and pore volume injected are shown in Figs. 4.48 and 4.49 respectively. Most of the oil is produced between 15 and 121 minutes, and the oil rate peak is $16 \mathrm{~cm}^{3} / \mathrm{min}$.

Fig. 4.50 shows temperature profiles within the cell at twenty-minute intervals. The temperature in the entire cell is around $210^{\circ} \mathrm{C}$ at 132 minutes.

The injection and production pressures are shown in Fig. 4.51. The production pressure is maintained at 260 psig during the entire run. The differential pressure peaks at around 72 psig. 


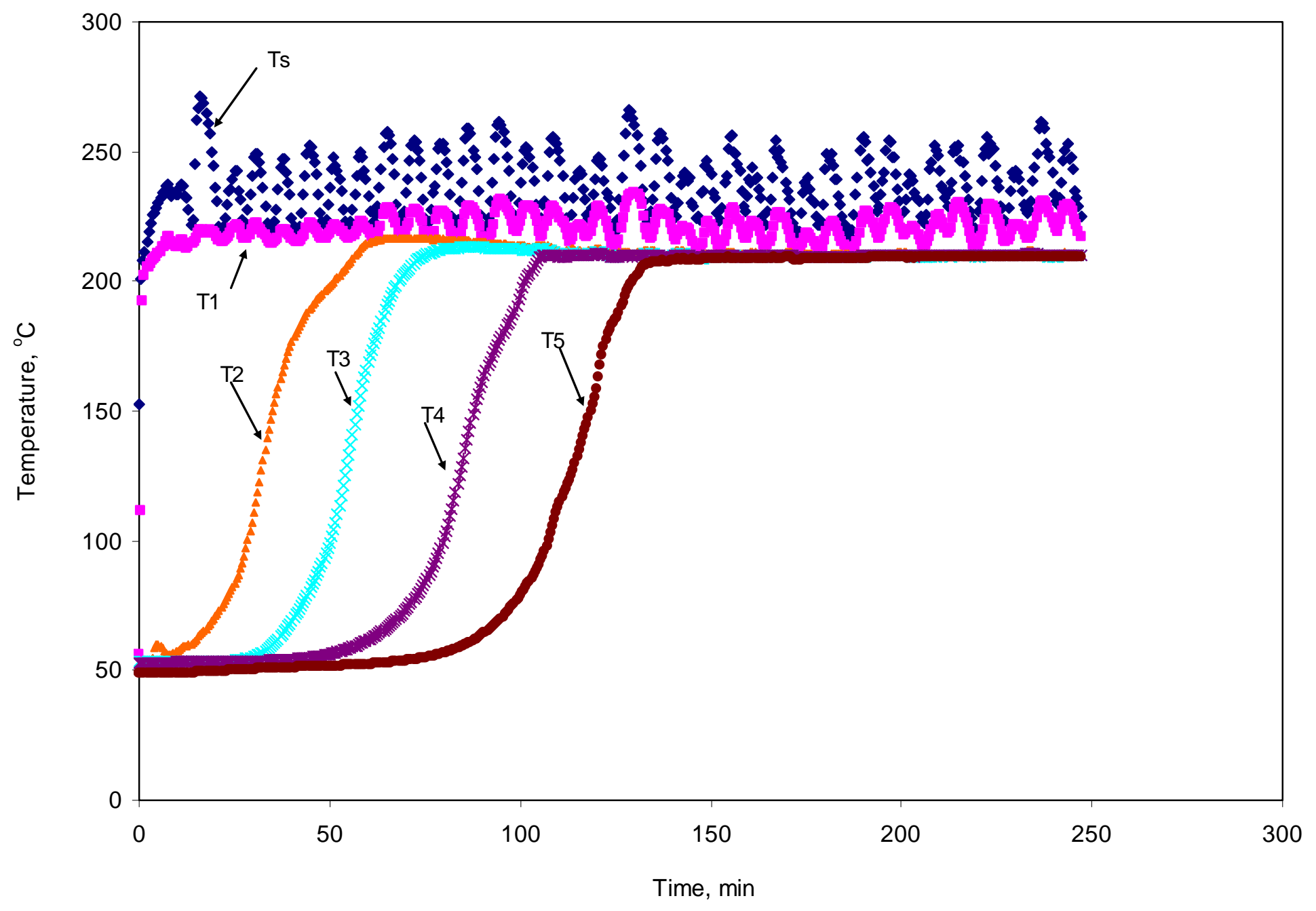

Fig. 4.45- Temperature profile versus time for Run 8 (5:100 petroleum distillate:steam). 


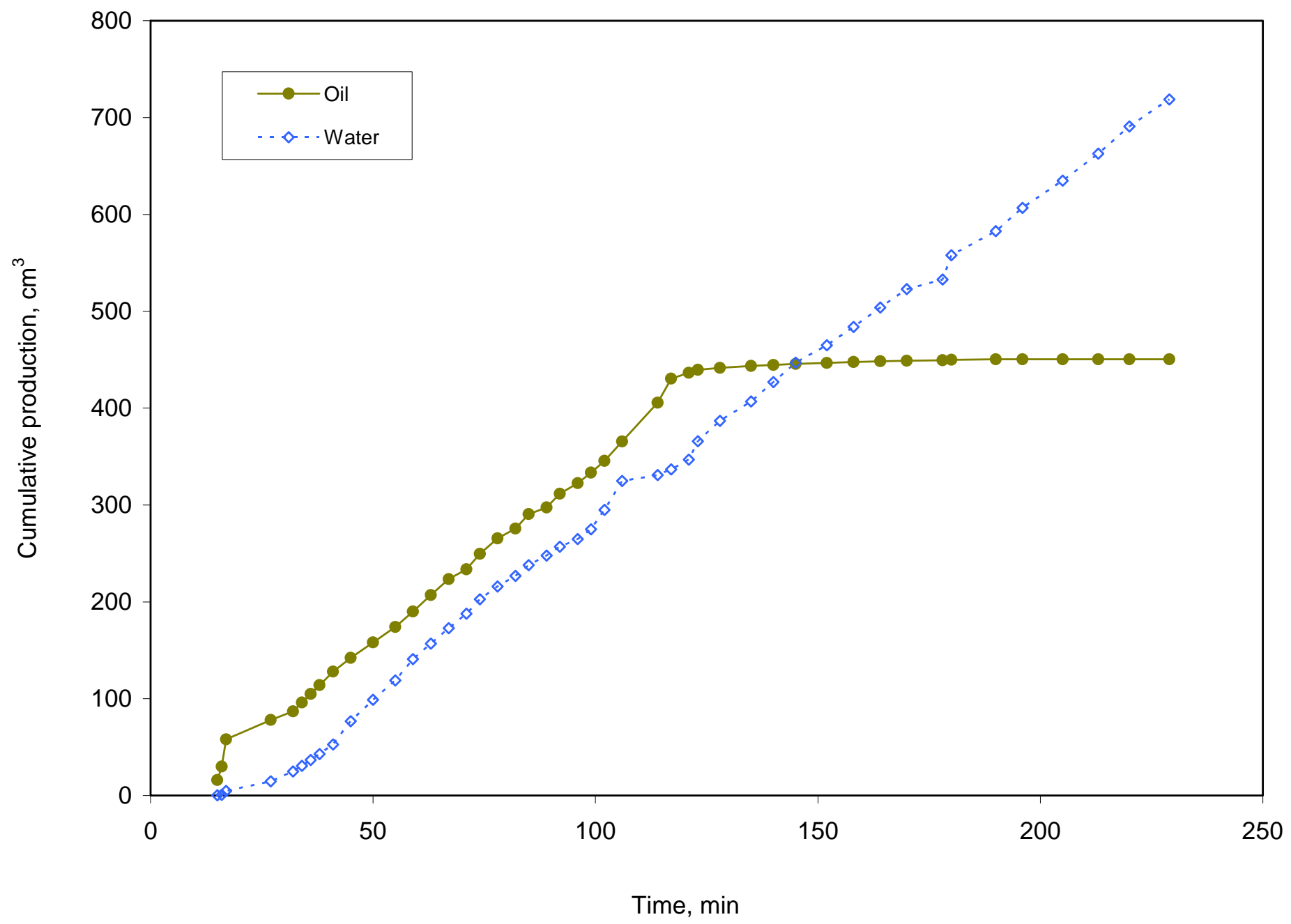

Fig. 4.46- Cumulative oil and water volumes versus time for Run 8 (5:100 petroleum distillate:steam). 


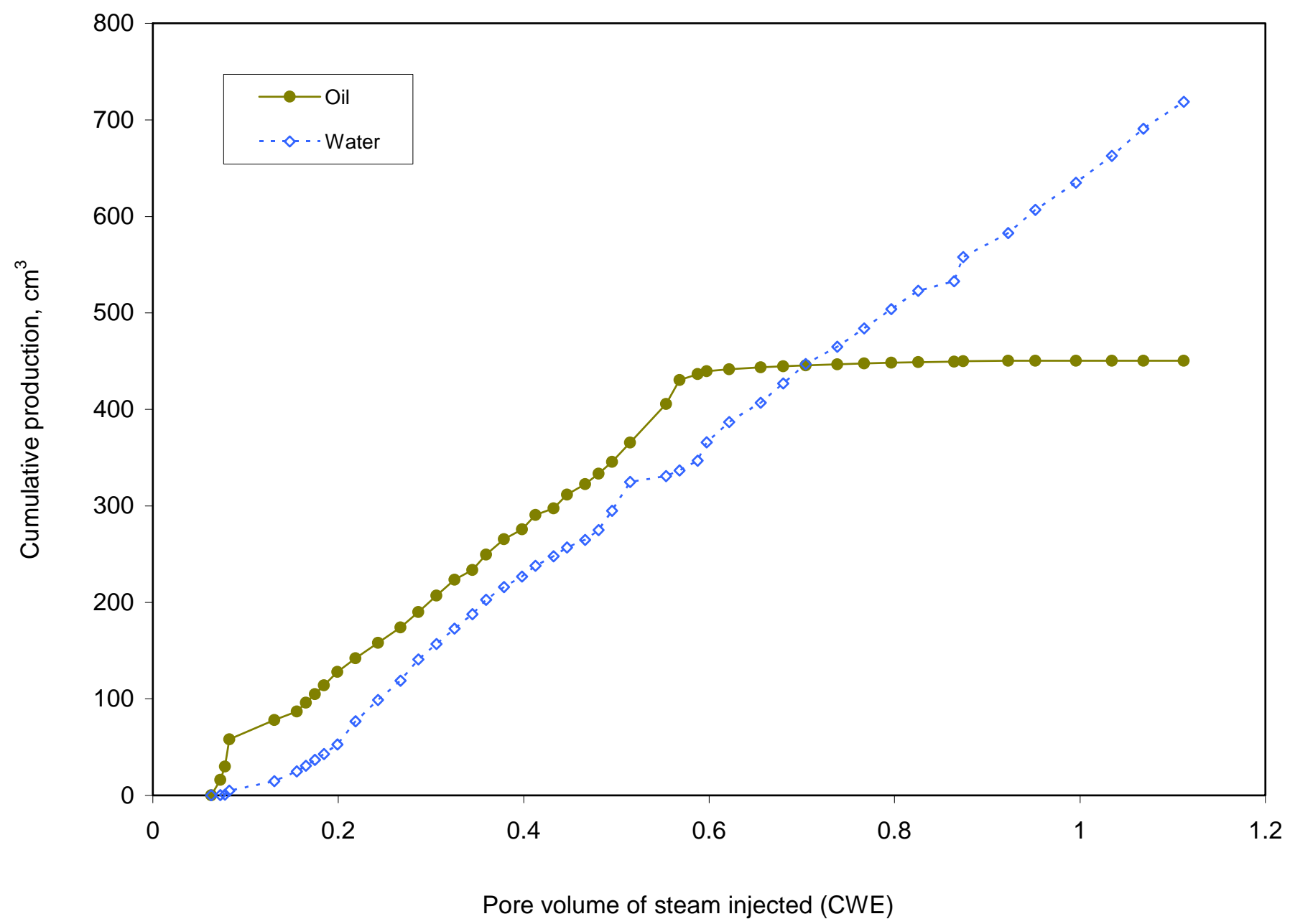

Fig. 4.47- Cumulative oil and water volumes vs. pore volume of steam injected for Run 8 (5:100 petroleum distillate:steam). 


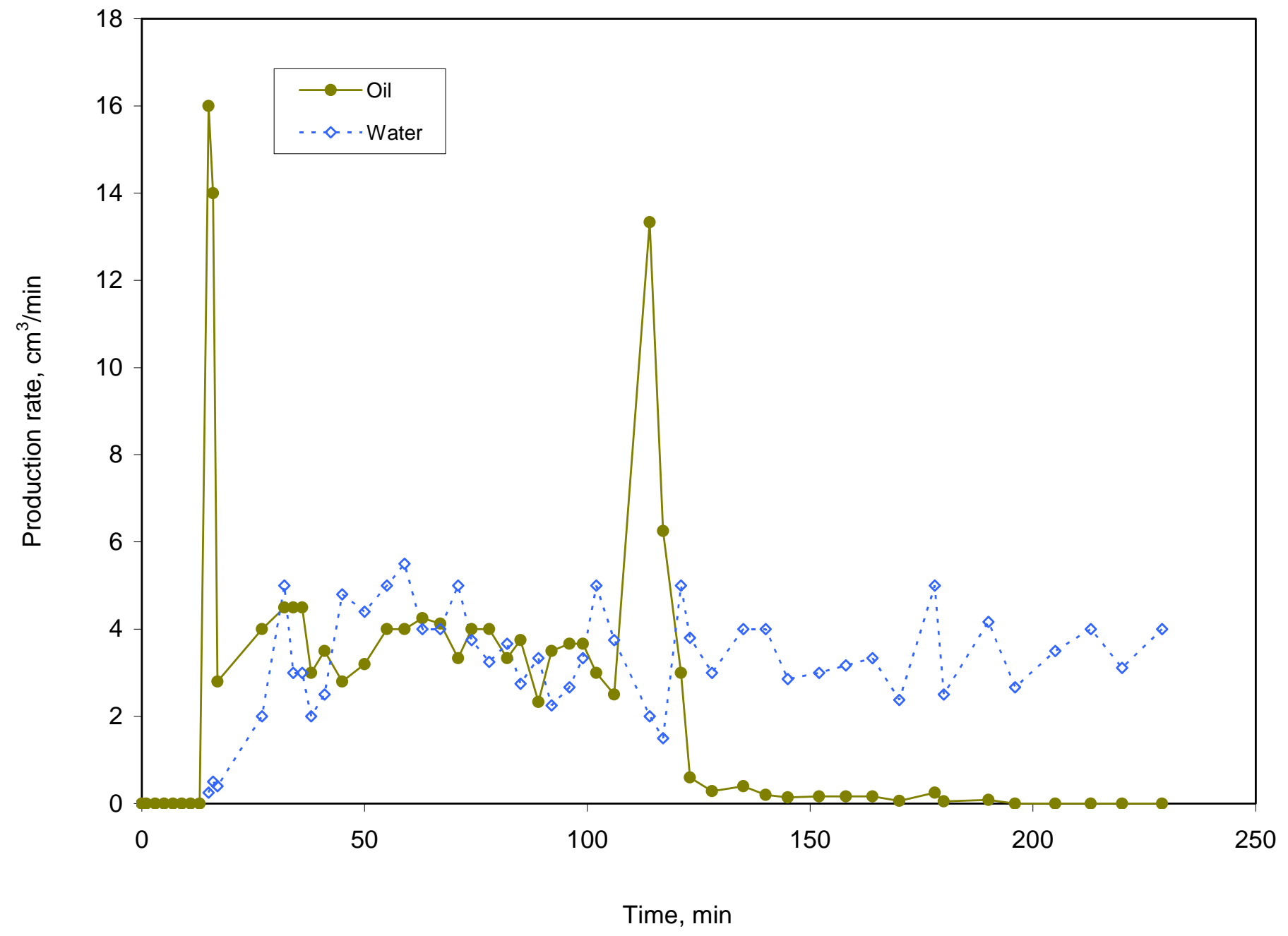

Fig. 4.48- Oil and water rates versus time for Run 8 (5:100 petroleum distillate:steam). 


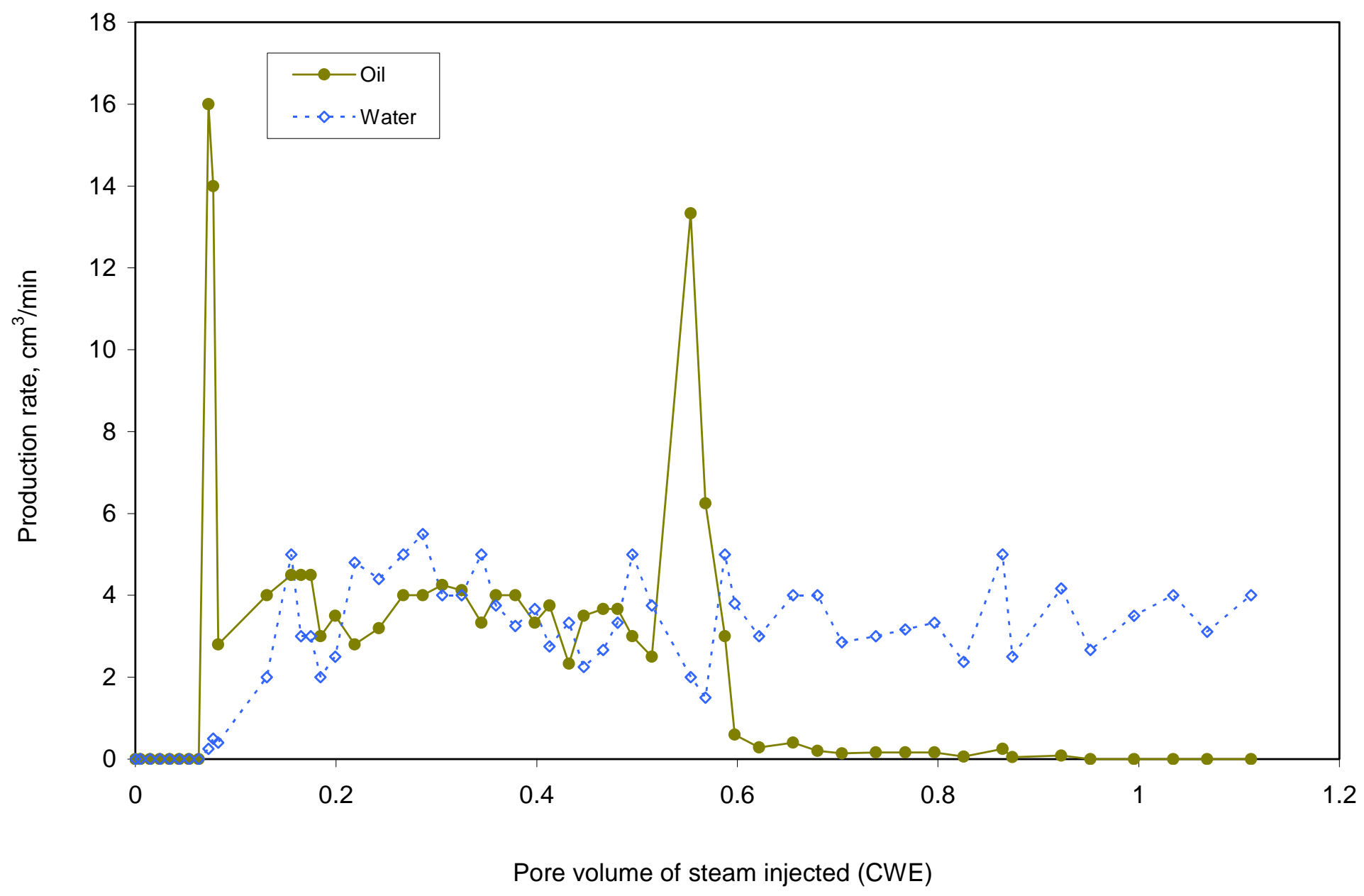

Fig. 4.49- Oil and water rates versus pore volume of steam injected for Run 8 (5:100 petroleum distillate:steam). 


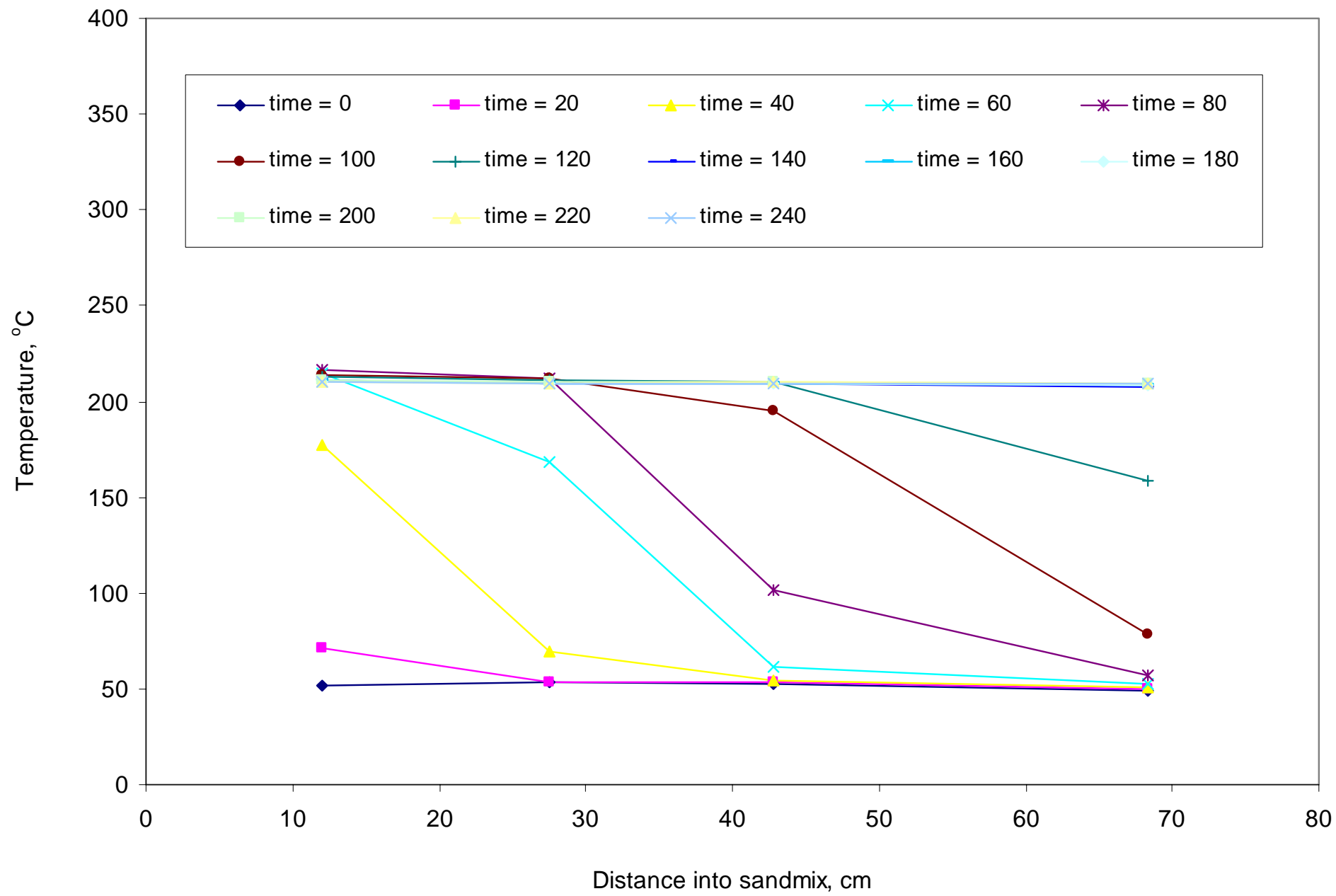

Fig. 4.50- Temperature profiles at 10-minute intervals - from $t=0$ to $t=240$ min - for Run 8 (5:100 petroleum distillate:steam). 


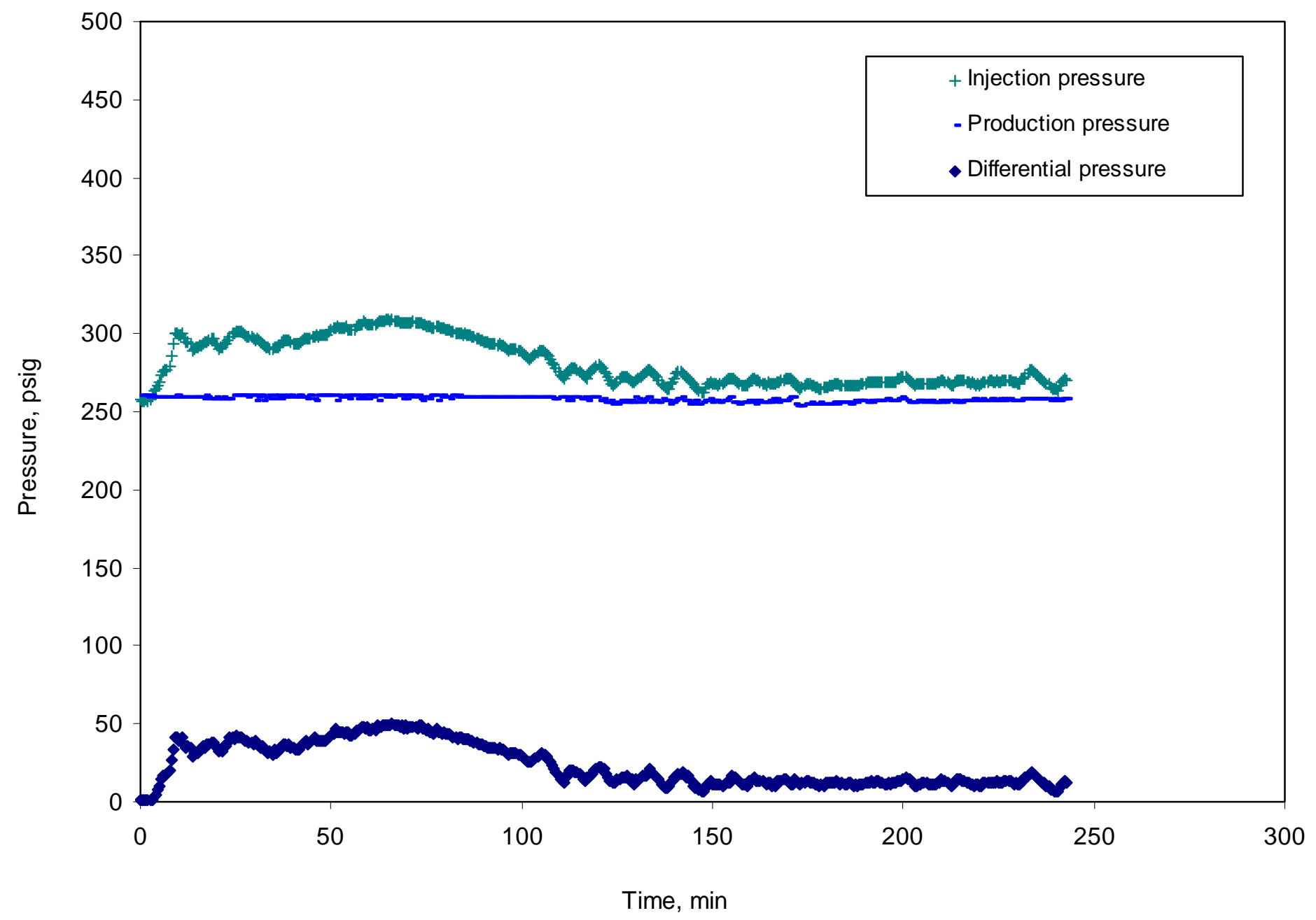

Fig. 4.51- Injection, production and differential pressures for Run 8 (5:100 petroleum distillate:steam). 


\subsection{Comparison and Discussion of Experimental Results}

Figs. 4.52 and 4.53 show a plot of oil rate vs. time and pore volume of water injected respectively for all runs. It can be observed that oil production starts earlier in the steam-petroleum distillate runs, followed by steam- propane runs as compared to pure steam runs. On average, steam-petroleum distillate and steam-propane oil production starts 14 and 17 minutes respectively, while oil production starts at 24 minutes in pure steam runs (average), this represents an acceleration of using petroleum distillate and propane as additives of $34 \%$ and $46 \%$ respectively in terms of production time. Fig. 4.54 shows each start of production runs. In Fig. 4.53, the oil rate is plotted vs. pore volume injected (CWE). For the steam-petroleum distillate and steam-propane runs, oil production starts when $7 \%$ and $9 \%$ (on average) respectively of the pore volume has been injected. On the other hand, the average pore volume injected before starting production in a pure steam run is $12.5 \%$. The oil rate peaks of the steam-petroleum distillate are the tallest, followed by steam-propane and pure steam runs. And as a result, oil production in the steam-petroleum distillate runs last shorter as compared to steampropane runs, followed by steam-propane runs that last shorter than pure steam runs.

Water rates (shown in Figs. 4.55 and 5.56) are not affected by the addition of propane and petroleum distillate. Although for one steam-petroleum distillate run (run 8), higher rates are found, but another steam-petroleum distillate run (run 8) does not indicate the same trend. Hence, suffice to say that the role of petroleum distillate in water production can not be established based on the observed experimental results. For all runs, water production starts around the same time (average of 14 minutes), then the rate fluctuates and finally decrease and stabilize around the water injection rate of 5.5 $\mathrm{cm}^{3} / \mathrm{min}$, cold water equivalent.

The cumulative volume of oil produced is shown in Figs. 4.57 and 4.58. Figs. 4.59 and 4.60 show the oil recovery as a percentage of Original Oil in Place. Recovery for the steam-petroleum distillate and steam-propane runs is on average $51 \%$ and $47 \%$ respectively while the average for the pure steam runs is $41 \%$. By evaluating all runs, higher recoveries are found when petroleum distillate or propane is added. The effect of 


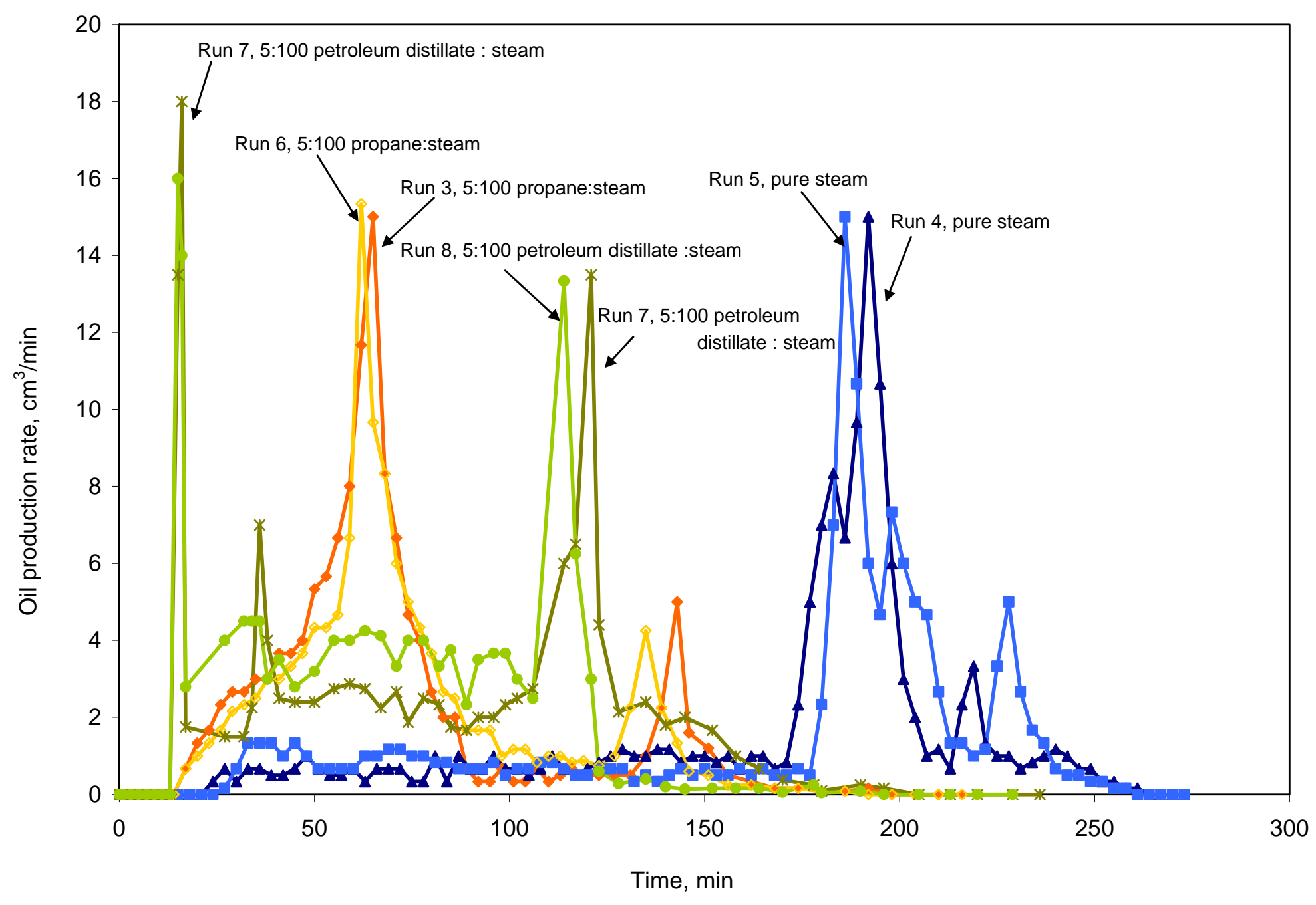

Fig. 4.52- Oil rates versus time for Runs 3 to 8. 


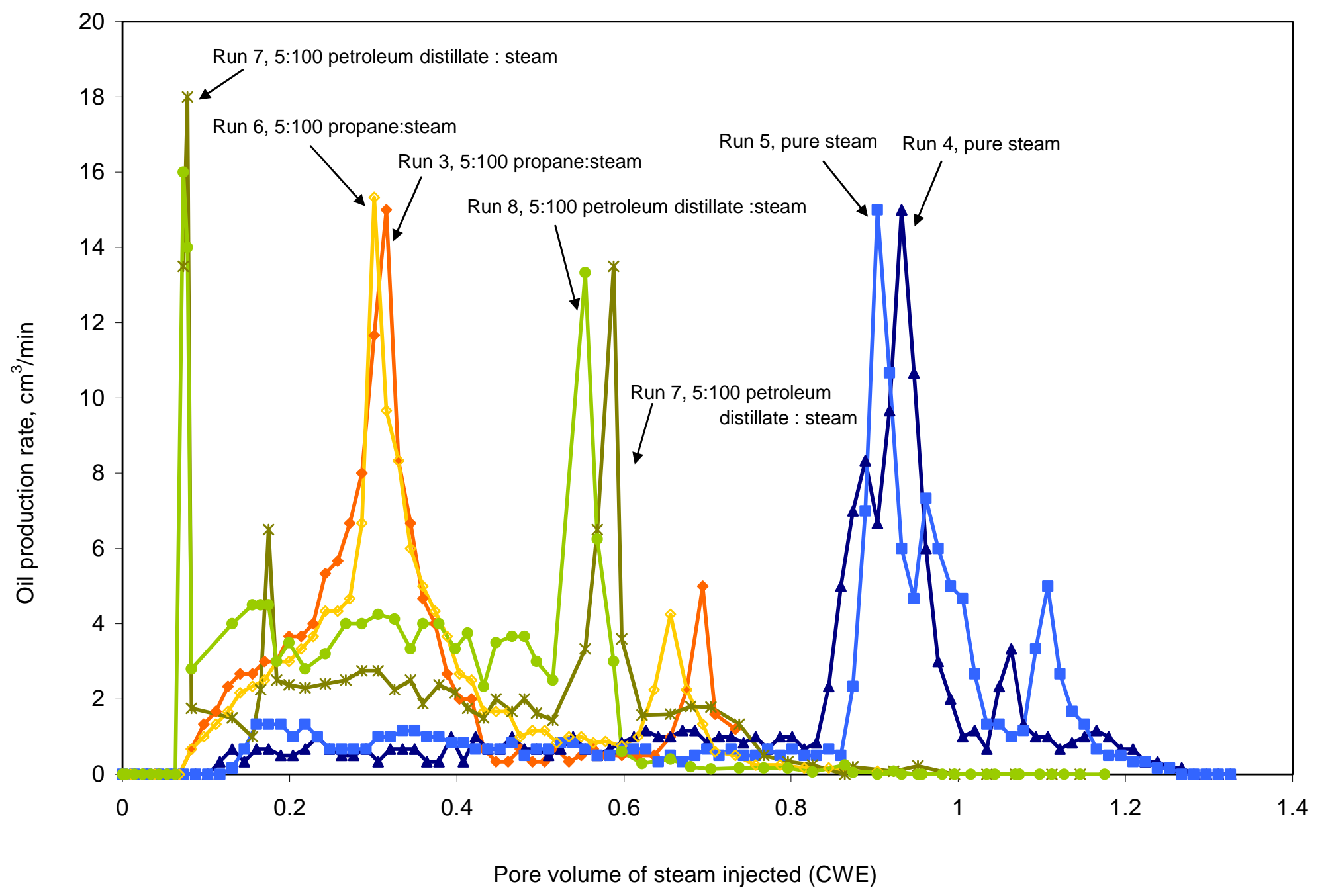

Fig. 4.53- Oil rates versus pore volume of steam injected (CWE) for Runs 3 to 8. 


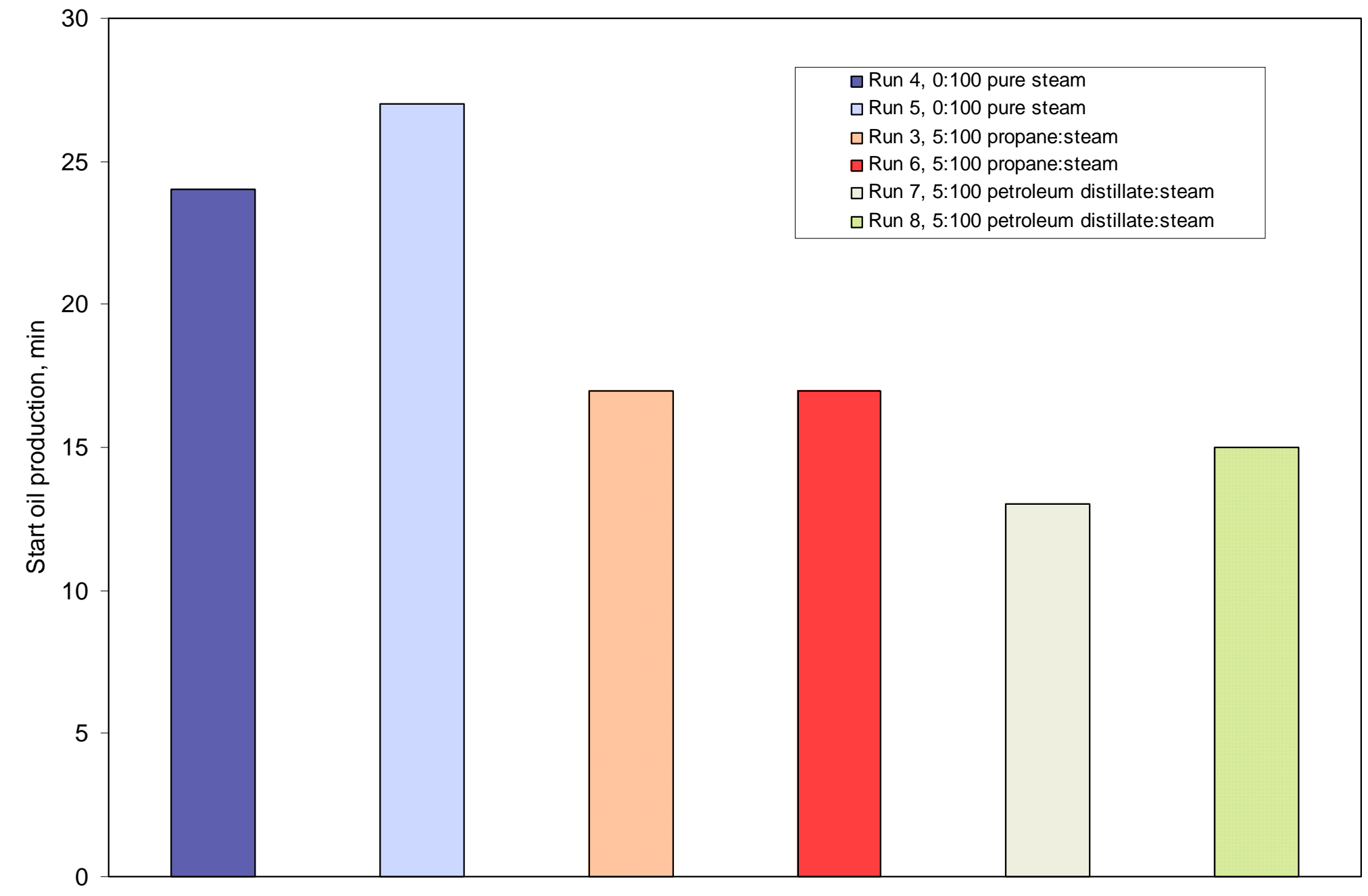

Fig. 4.54- Start of oil production. 


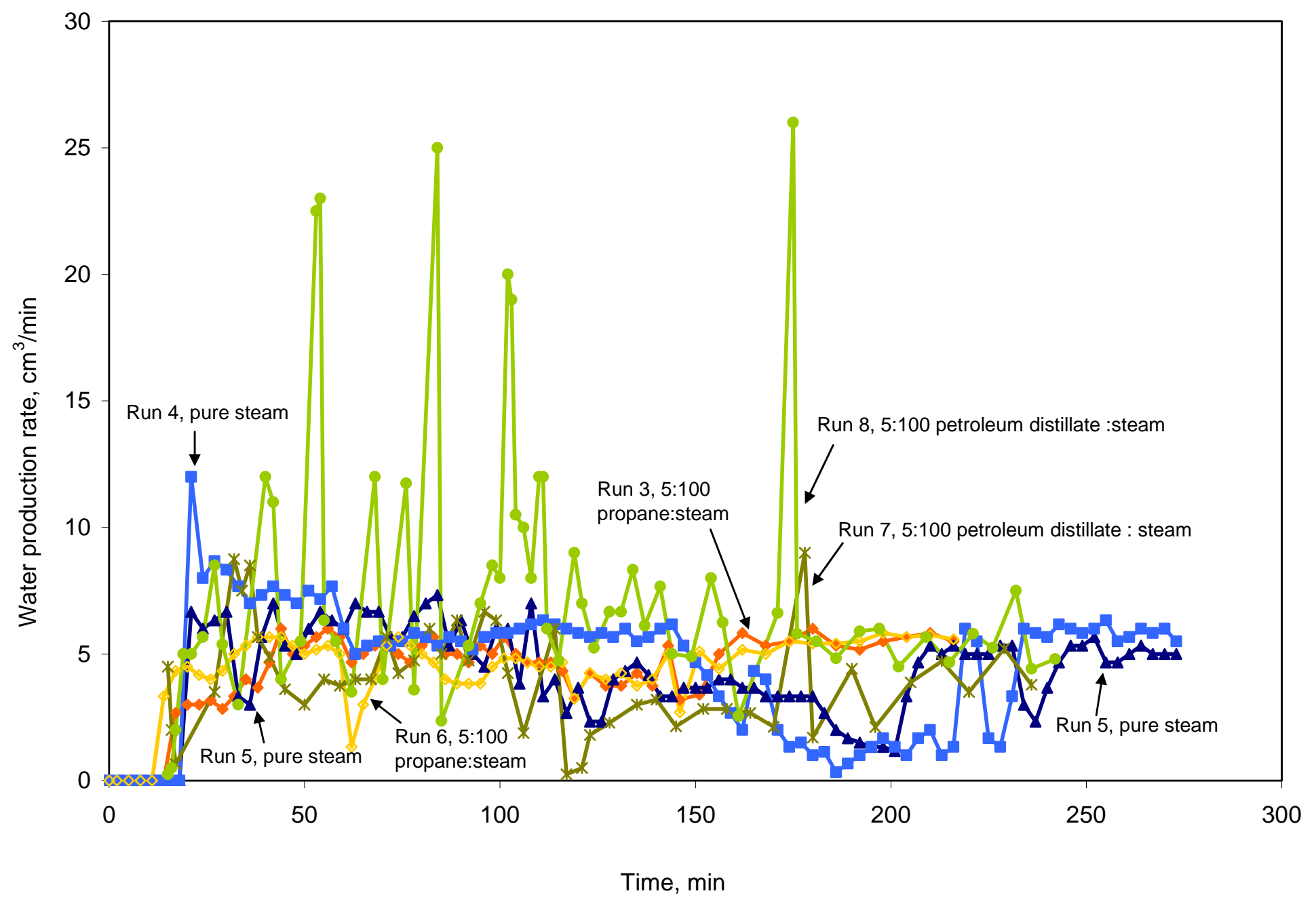

Fig. 4.55- Water rates versus time for Runs 3 to 8. 


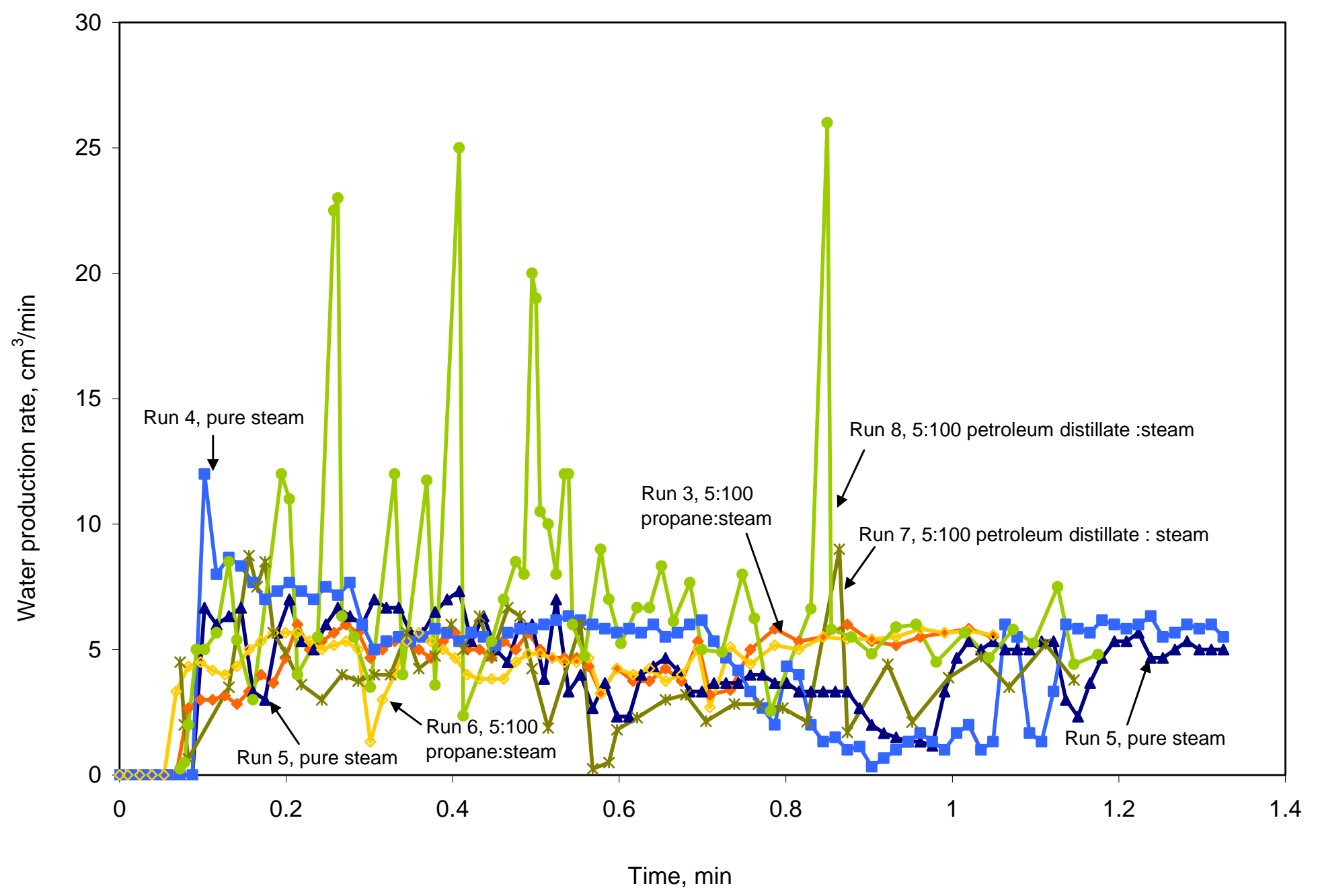

Fig. 4.56- Water rates versus pore volume of steam injected (CWE) for runs 3 to 8. 


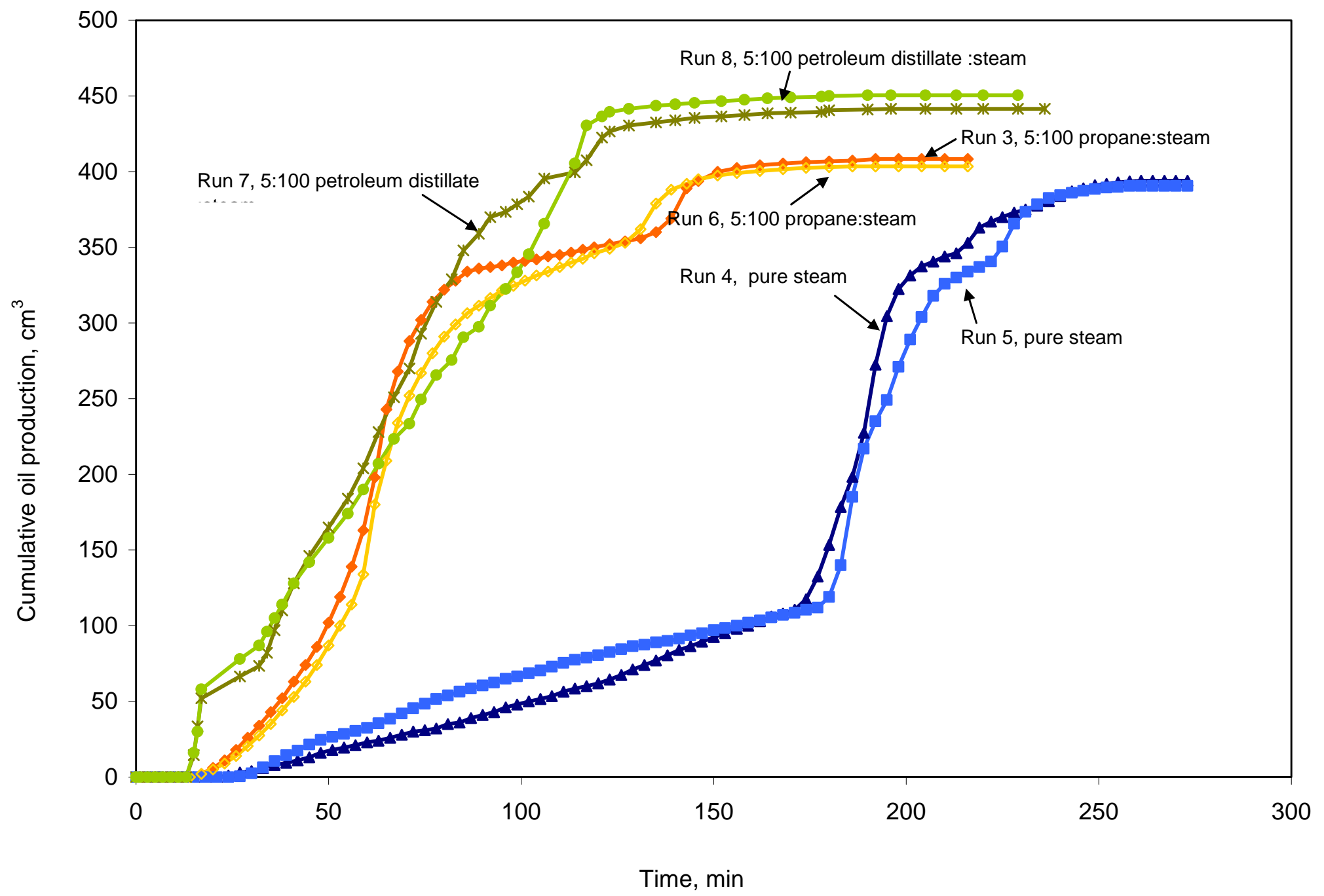

Fig. 4.57- Cumulative oil recovery versus time for Runs 3 to 8. 


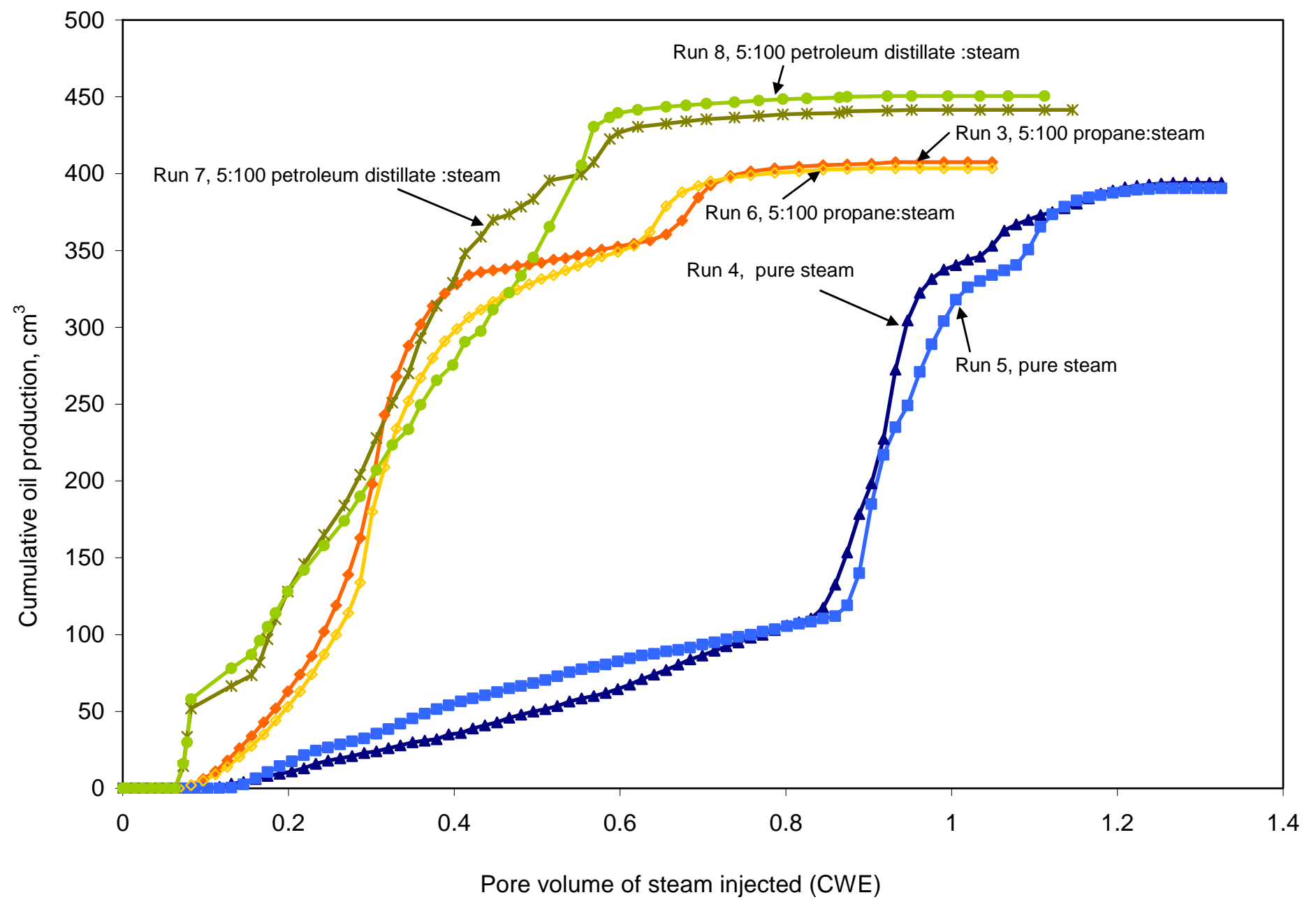

Fig. 4.58- Cumulative oil recovery versus pore volume of steam injected (CWE) for Runs 3 to 8. 


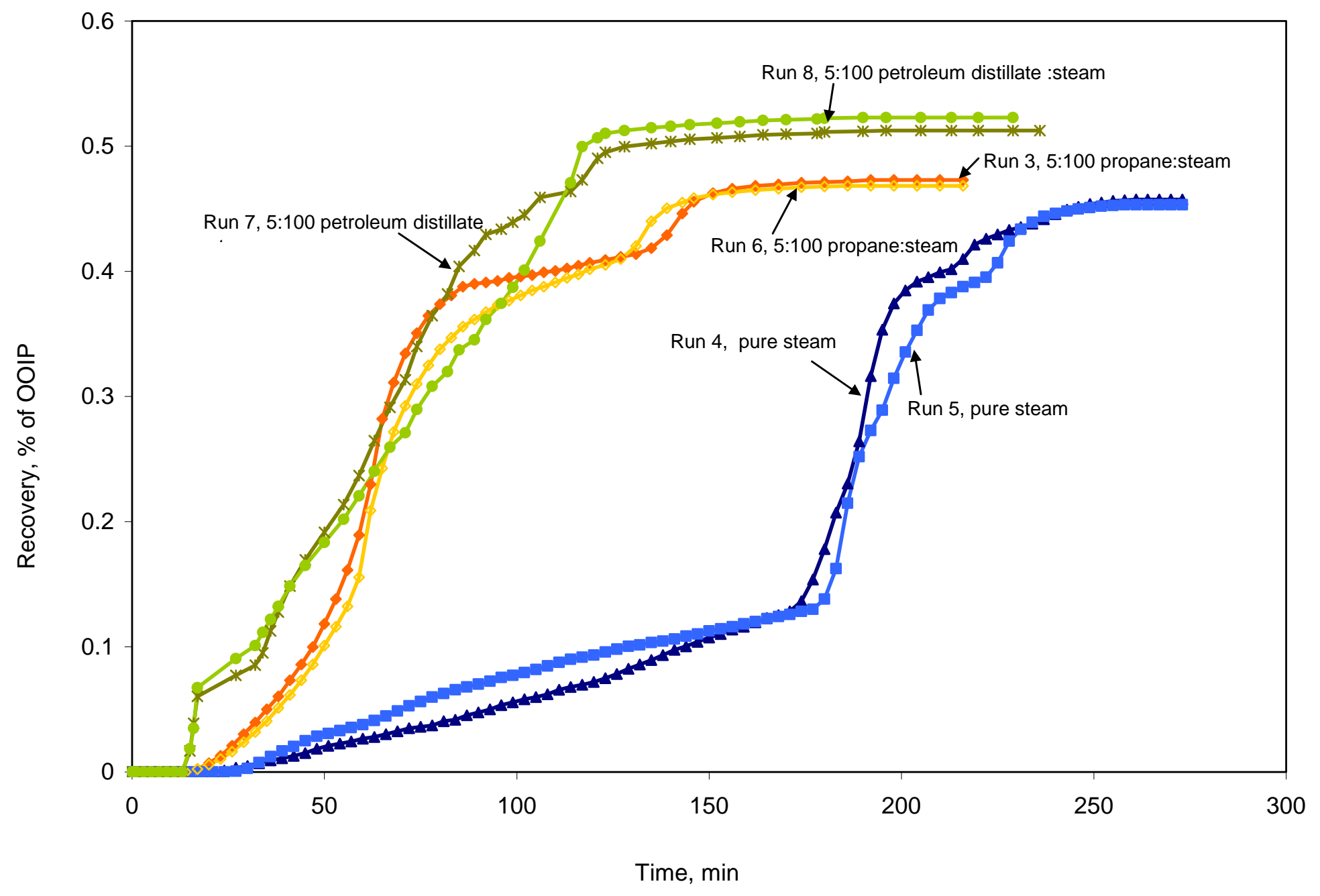

Fig. 4.59- Oil recovery versus time for Runs 3 to 8. 


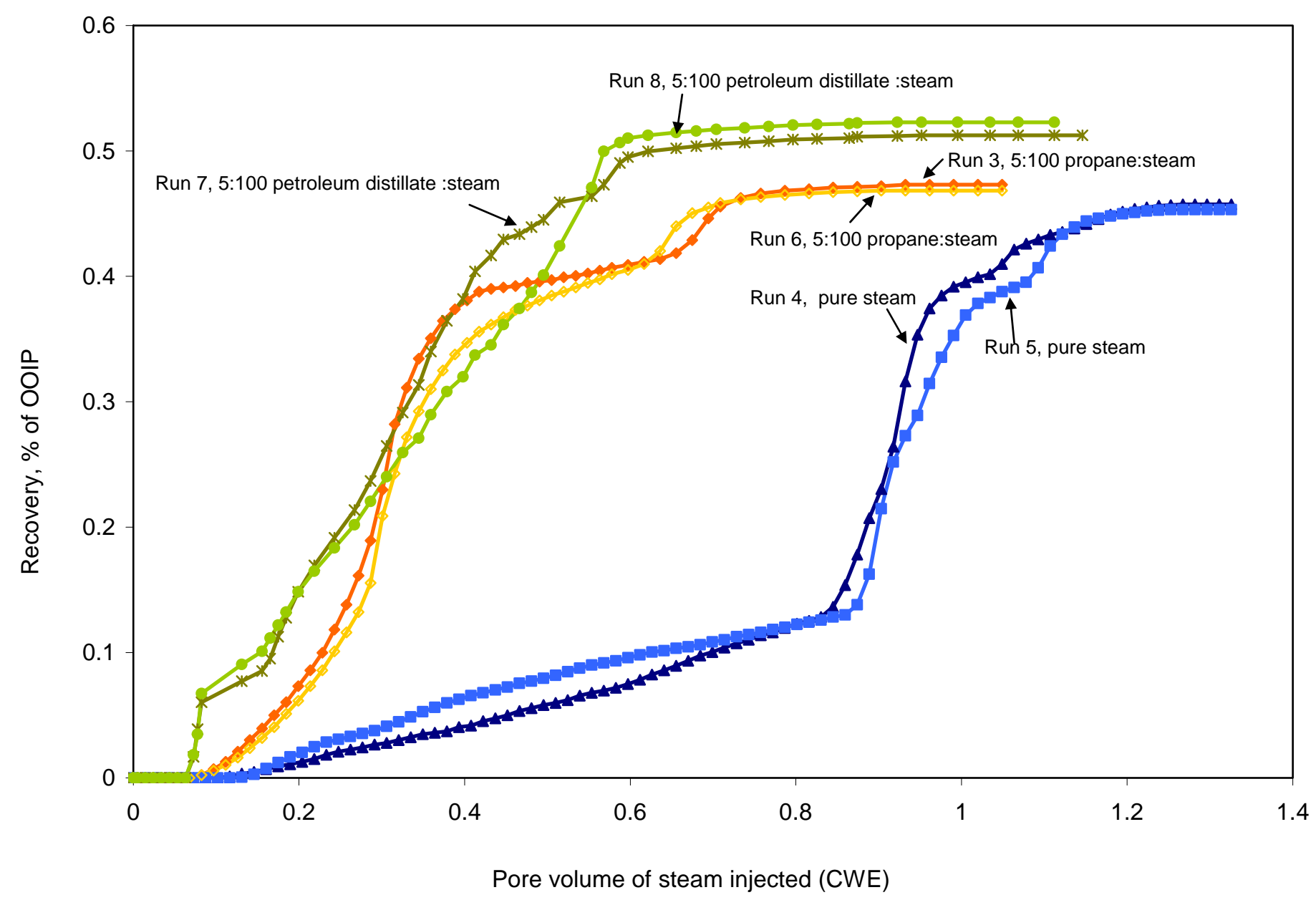

Fig. 4.60- Oil recovery versus pore volume of steam injected (CWE) for Runs 3 to 8. 
petroleum distillate to enhance oil production has been recognized quite recently in the oil industry and supported by some the field experiences in heavy oil productions such one as described by Léautét6 .

Figs. 4.61 and 4.62 show the differential pressure as a function of time for all runs. The differential pressure is the difference between the injection and production pressure (production pressure is held constant at 260 psig). The injection pressure starts increasing just after the run begins. On average, the maximum differential pressure for the steam-petroleum distillate and steam-propane runs are 26 and 40 psig. In contrast, the pure steam runs have a maximum differential pressure (average) of 62 psig. Thus, petroleum distillate and propane as additives improve the injectivity by reducing the pressure differential to $150 \%$ and $240 \%$.

Oil density, expressed as API gravity, is plotted in Fig. 4.63. Since only a small amount of oil was available in every sample, a pycnometer with a volume of $2 \mathrm{~cm}^{3}$ was used to obtain these density measurements. Given the size of the pycnometer, even a small measurement error is magnified causing the scattering observed in the plot. No specific trend was observed in the plots; however, API values for the steam-propane runs are in most cases higher than the corresponding value for the original oil (12 $\mathrm{API})$. On the other hand, pure steam runs yielded API gravities equal to or slightly lower than the original API gravity.

Fig. 4.64 depicts the oil viscosity vs. time measured at $50^{\circ} \mathrm{C}$. Though some scatter is present, all runs (pure steam, steam-propane and steam-petroleum distillate) show an increasing trend with time. Viscosity values for the steam-petroleum distillate and steam-propane runs are generally higher than those corresponding to the pure steam runs. Most of the viscosity values measured are below the original oil viscosity (2800 cp at $50^{\circ} \mathrm{C}$ ). The steam front location for each run is plotted in Fig. 4.65. Five thermocouples were placed inside a thermowell within the cell. In this plot, the slope of the curve indicates the steam front velocity. The steam front is faster in the steampropane runs, this may explain the fact that oil production starts earlier when petroleum distillate and propane are added. 


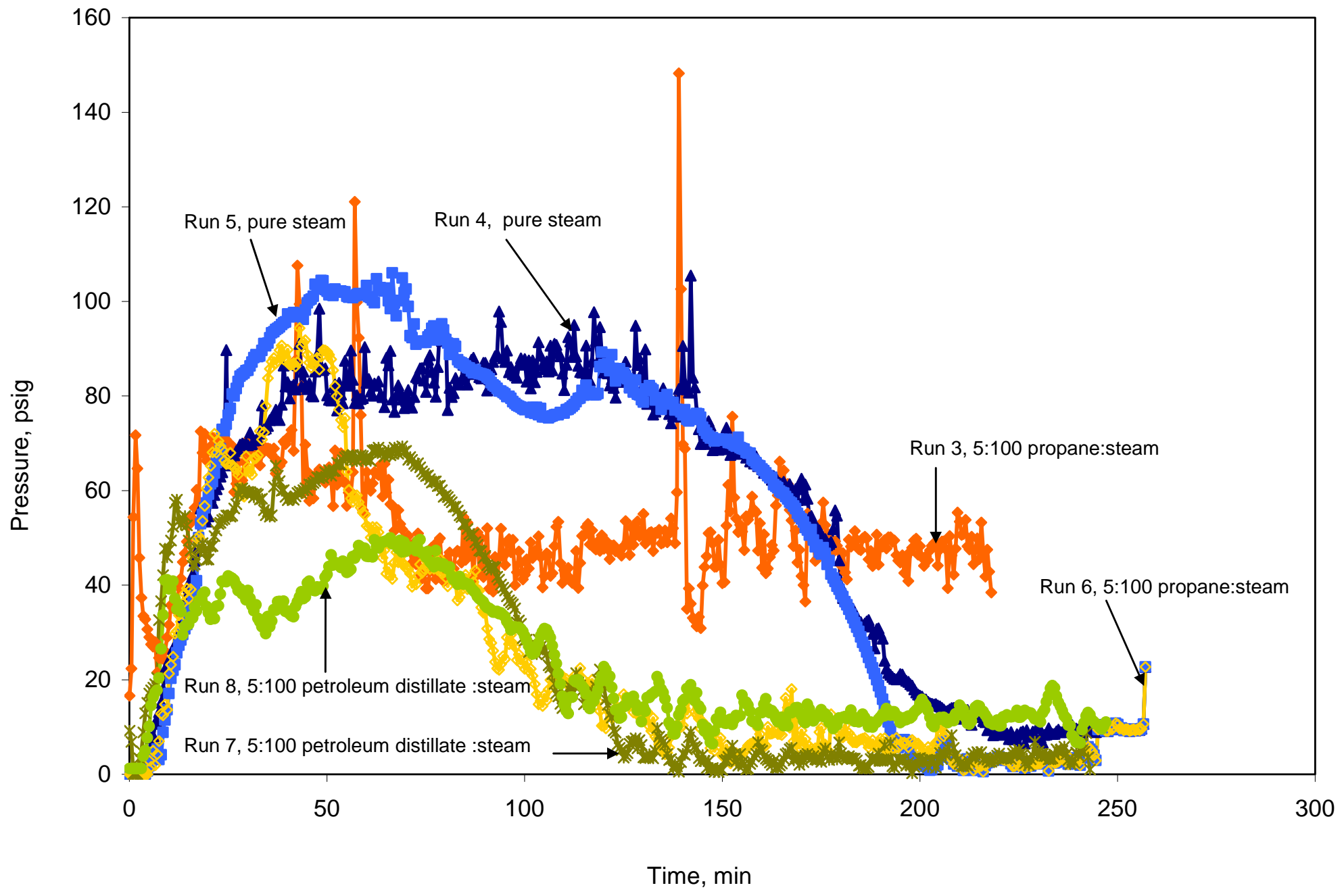

Fig. 4.61- Differential pressure versus time for Runs 3 to 8. 


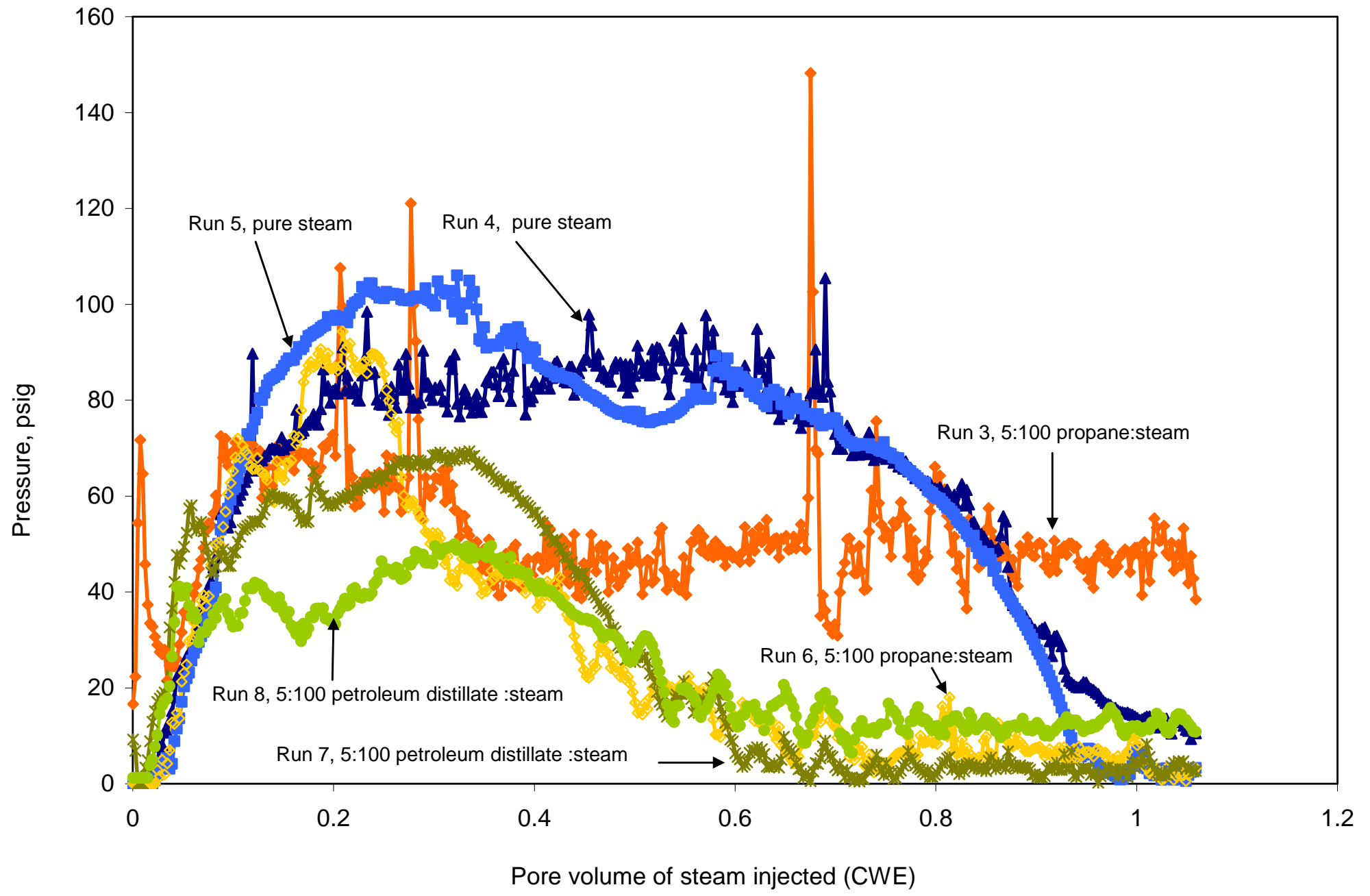

Fig. 4.62- Differential pressure versus pore volume of steam injected (CWE) for Runs 3 to 8 . 


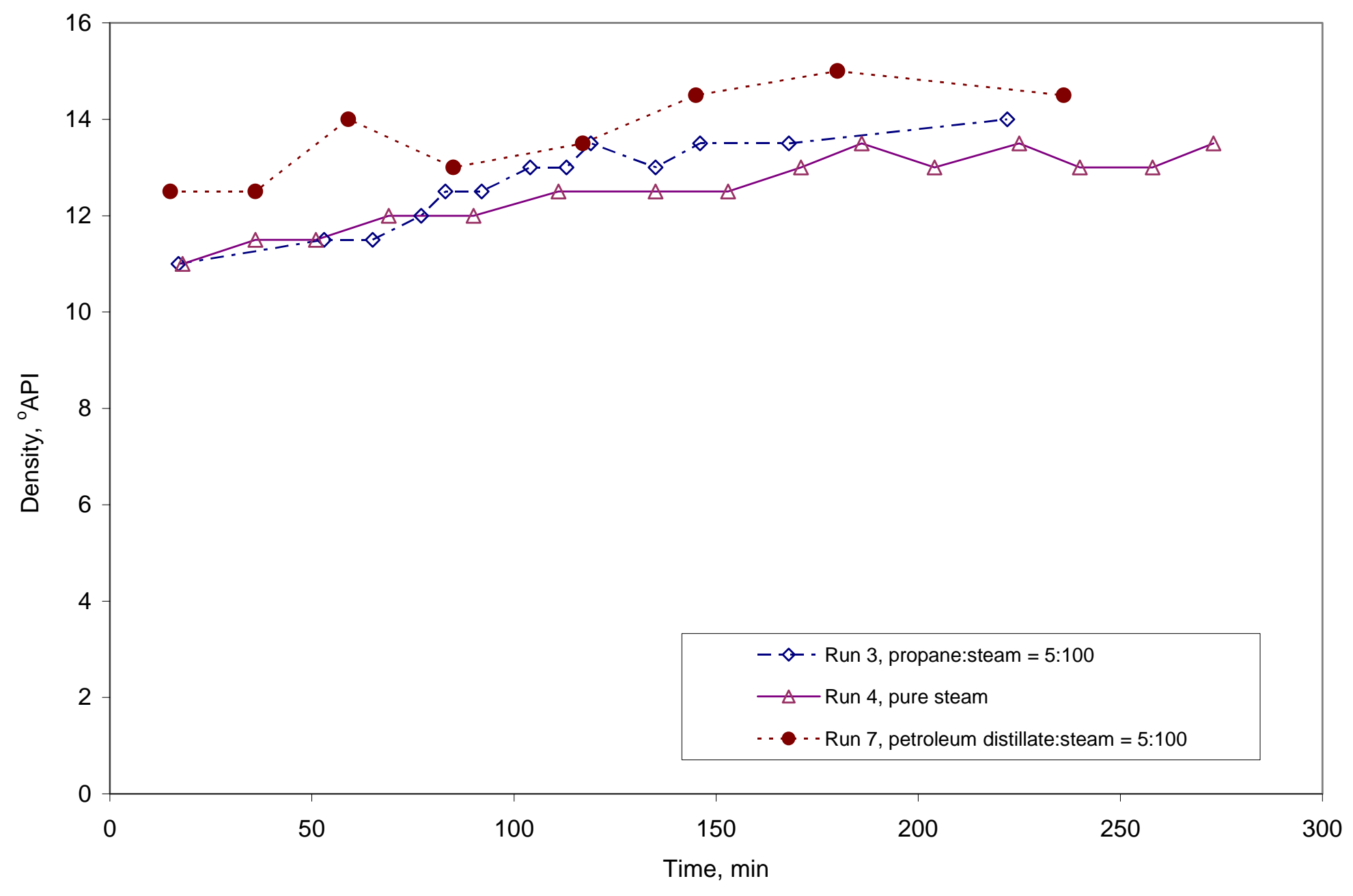

Fig. 4.63- Oil density (@50ㄷ) vs. time for Runs 3 to 8. 


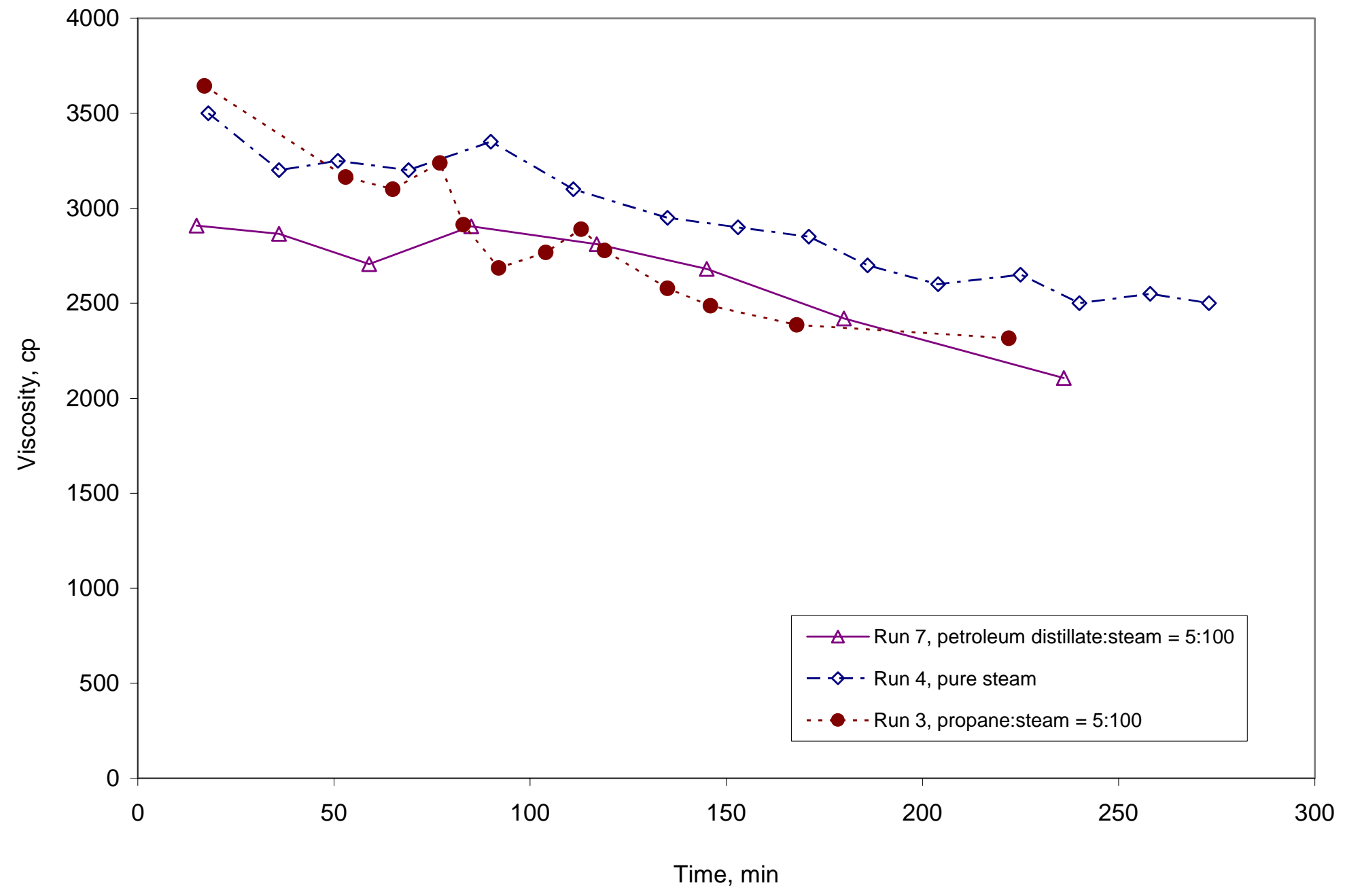

Fig. 4.64- Oil viscosity $\left(@ 50^{\circ} \mathrm{C}\right)$ vs. time for Runs 3 to 8. 


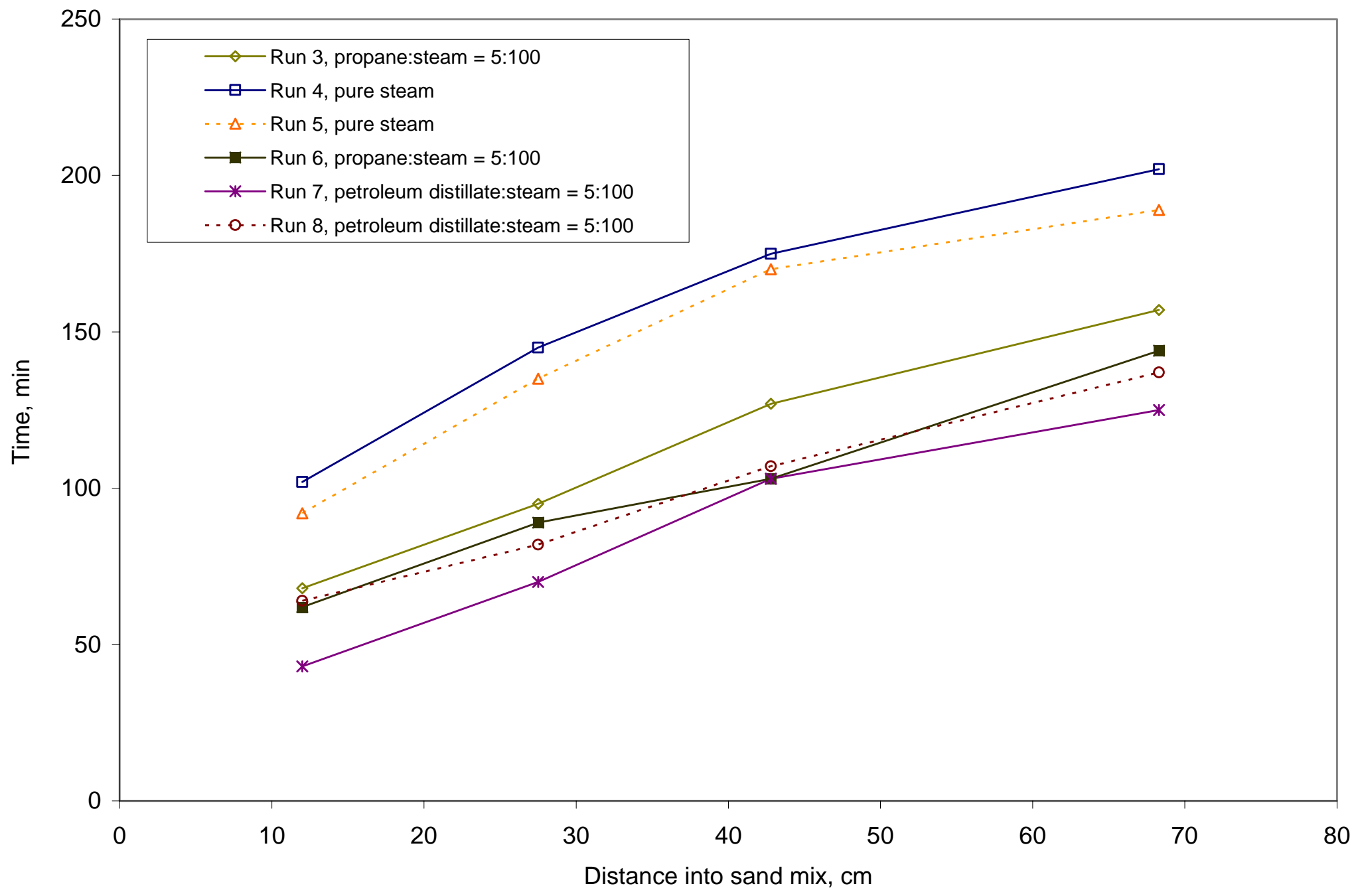

Fig. 4.65- Steam front location for Runs 3 to 8. 


\section{CHAPTER V}

\section{ANALYTICAL MODEL}

\subsection{Review of Analytical Models for Steamflooding}

Analytical models have dealt with the basic problems of heat and mass transport in an oil reservoir. Analytical solutions are possible after many assumptions are made. The first study of heat transport within a reservoir due to hot fluid injection was presented by Lauwerier ${ }^{35}$. Lauwerier considered the temperature decrease along the flow direction due to the heat loss to the adjacent strata. He derived the temperature distribution both in the reservoir and adjacent formation under several assumptions.

Marx and Langenheim ${ }^{36}$ presented a model for estimating thermal invasion rate, cumulative heated area (Eq. 5.1) and theoretical economic limits for sustained heat injection at constant rate into an idealized reservoir.

$$
R_{h}^{2}=\frac{h_{n} M_{s}\left(f_{s d h} L_{v d h}+h_{f s}-h_{f r}\right) \xi_{s}}{4 \pi K_{h}\left(T_{s}-T_{R}\right) t_{i n j} N_{s}},
$$

where

$$
\begin{aligned}
& \xi_{s}=e^{\tau} \operatorname{erfc}(\sqrt{\tau})+\frac{2}{\sqrt{\pi}} \sqrt{\tau}-1, \\
& \tau=\frac{4 K_{h} t_{i n j}}{h_{n}(\rho C)_{t}},
\end{aligned}
$$

$K_{h}=$ thermal conductivity of overburden, in Btu/ft- ${ }^{\circ} \mathrm{F}$

$h_{n}=$ reservoir thickness, $\mathrm{ft}$

$(\rho C)_{t}=$ volumetric heat capacity of reservoir material, BTU $/ \mathrm{ft}^{3}$

$t_{\text {inj }}=$ injection time, $\mathrm{D}$

$h_{f s}=$ enthalpy of liquid water at $T_{R}$, BTU/lbm

$\tau=$ lag time, $\mathrm{D}$ 
$T_{s} \quad=$ steam temperature, in ${ }^{\circ} \mathrm{F}$

$T_{R} \quad=$ original reservoir temperature in ${ }^{\circ} \mathrm{F}$

$\alpha \quad=$ the ratio of oil displaced from the steam zone below to that displaced from the steam zone itself

$M_{s} \quad=$ the heat capacity of the steam zone, in Btu $/ \mathrm{ft}^{3}-{ }^{\circ} \mathrm{F}$

$L_{v d h}=$ heat of vaporization of water at reservoir pressure in Btu/lb

$f_{s d h}=$ downhole steam quality, fraction.

They assumed that the injected heat was retained by the formation within a constant temperature steam zone and lost to the overlying and the underlying strata by conduction and that there was no heat flow ahead of the steam front. The model has been found to be applicable to thick formations, high steam qualities, low pressures, and high steam injection rates. Ramey ${ }^{37}$ extended the Marx-Langenheim work to permit a variable injection rate.

Willman et al. ${ }^{38}$ presented an equation to estimate the heated radius, $R_{h}$ (Eq. 5.5) for a constant injection rate, $i_{s t}$ :

$$
R_{h}^{2}=\frac{14.6 i_{s t} L_{v d h}}{K_{h}\left(T_{s}-T_{R}\right)} \sqrt{\frac{\alpha}{\pi}}\left[\frac{\sqrt{t}}{2}-\frac{h_{n}}{8} \sqrt{\frac{\pi}{\alpha}} \frac{\left(\rho C_{p}\right)_{t}}{\left(\rho C_{p}\right)_{o b}} \ln \left[\frac{4}{h_{n}} \sqrt{\frac{\alpha}{\pi}} \frac{\left(\rho C_{p}\right)_{o b}}{\left(\rho C_{p}\right)_{t}} \sqrt{t}+1\right]\right]
$$

They also predicted the steam injection rate required for a constant volumetric rate of steam in radial flow reservoir as the following:

$$
i_{s t}=\frac{\pi R_{h}^{2}\left(T_{s}-T_{R}\right)}{14.6 t L_{v d h}}\left[h_{n}\left(\rho C_{p}\right)_{t}+4 k_{h} \sqrt{\frac{t}{\pi \alpha}}\right],
$$

where

$K_{h}=$ thermal conductivity of overburden, in Btu/ft- ${ }^{\circ} \mathrm{F}$

$h_{n}=$ reservoir thickness, $\mathrm{ft}$

$(\rho C)_{t}=$ volumetric heat capacity of reservoir material, BTU $/ \mathrm{ft}^{3}$

$t_{i n j}=$ injection time, $\mathrm{D}$

$h_{f s}=$ enthalpy of liquid water at $T_{R}$, BTU/lbm 


$$
\begin{aligned}
& T_{S}=\text { steam temperature, in }{ }^{0} \mathrm{~F} \\
& T_{R}=\text { original reservoir temperature in }{ }^{\circ} \mathrm{F} \\
& \alpha \quad= \\
& \quad \text { the ratio of oil displaced from the steam zone below to that displaced } \\
& \quad \text { from the steam zone itself } \\
& M_{S}=\text { the heat capacity of the steam zone, in Btu/ } / \mathrm{ft}^{3}{ }^{\circ} \mathrm{F} \\
& L_{v d h}=\text { heat of vaporization of water at reservoir pressure in Btu/lb } \\
& f_{s d h}=\text { downhole steam quality, fraction. }
\end{aligned}
$$

The concepts, assumptions, and restrictions of this model were similar to those of Marx and Langenheim. Mandl and Volek ${ }^{39}$ improved upon these models by noting that at a critical time the latent heat content of the injected steam was not sufficient to supply heat loss and also raise rock ahead of the steam zone to the steam temperature. As a result, convective heat transfer at the steam front cannot be neglected. Prior to the critical time, they used the same equation as Marx and Langenheim. The Mandl-Volek equation for the steam volume after the critical time is

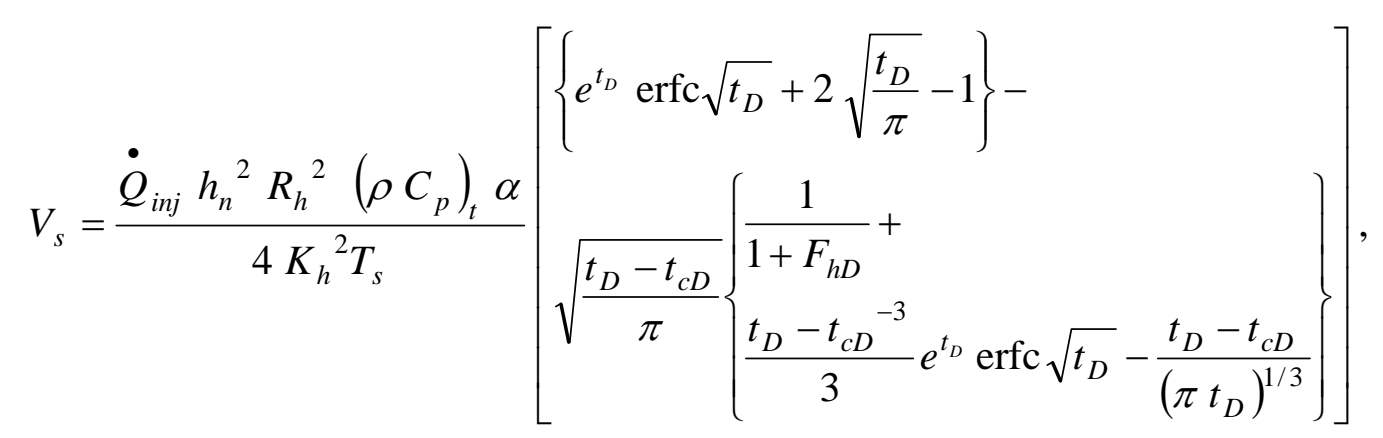

where

$$
\begin{aligned}
& F_{h D}=\frac{f_{s d h} L_{v d h}}{C_{w} T_{s}}, \\
& t_{D}=\frac{4 K_{h}(\rho C)_{o b} t}{h_{n}{ }^{2}(\rho C)_{t}{ }^{2}} .
\end{aligned}
$$


The dimensionless critical time $\left(t_{c D}\right)$ for constant injection rate, temperature and steam quality was determined by solving the relation

$$
e^{t_{c D}} \operatorname{ercf} \sqrt{t_{c D}}=\left[1+\frac{f_{s d h} L_{v d h}}{C_{w} T_{s}}\right]^{-1}
$$

An important study of steam override was published by Van Lookeren ${ }^{40}$. He presented an equation to estimate the laterally averaged steam zone thickness, $h_{s t}$, on segregated flow principles

$$
h_{s t}=0.5 h_{n} A_{R D}
$$

where

$$
\begin{aligned}
& h_{n}=\text { reservoir thickness, } \mathrm{ft}, \\
& A_{R D}=\text { shape factor for radial flow. }
\end{aligned}
$$

The treatment was similar to that isothermal, incompressible and immiscible displacement under segregated conditions presented by Dietz and Dake ${ }^{41}$. However, the conventional mobility ratio was used by Dietz while the equivalent ratio (pseudo mobility ratio) was used by Van Lookeren. An important discussion of the equivalent mobility ratio was presented by Prats ${ }^{42}$. The vertical conformance $\left(A_{R D}\right)$ can be used to calculate the optimum steam injection rate. Van Lookeren showed that the vertical seep decreased with a decreased steam injection rate, increased pressure, or decreased steam quality.

\subsection{Predictive Models for Steamflooding}

The basic concepts developed in the studies mentioned have led to the development of various models to predict steamflooding performance. The models can be divided into two categories:

1. Frontal advance models: The steam-drive mechanism was modeled as a horizontal frontal displacement (Fig. 5.1). The steam zone was assumed to 
grow horizontally and the tendency of the steam to finger beyond the front suppressed by condensation.

2. Vertical displacement or gravity override models: The problem of gravity override of the steam due to its low density has been recognized. The gravity override models (Fig. 5.2) assume principal direction of steam zone propagation is vertically downward.

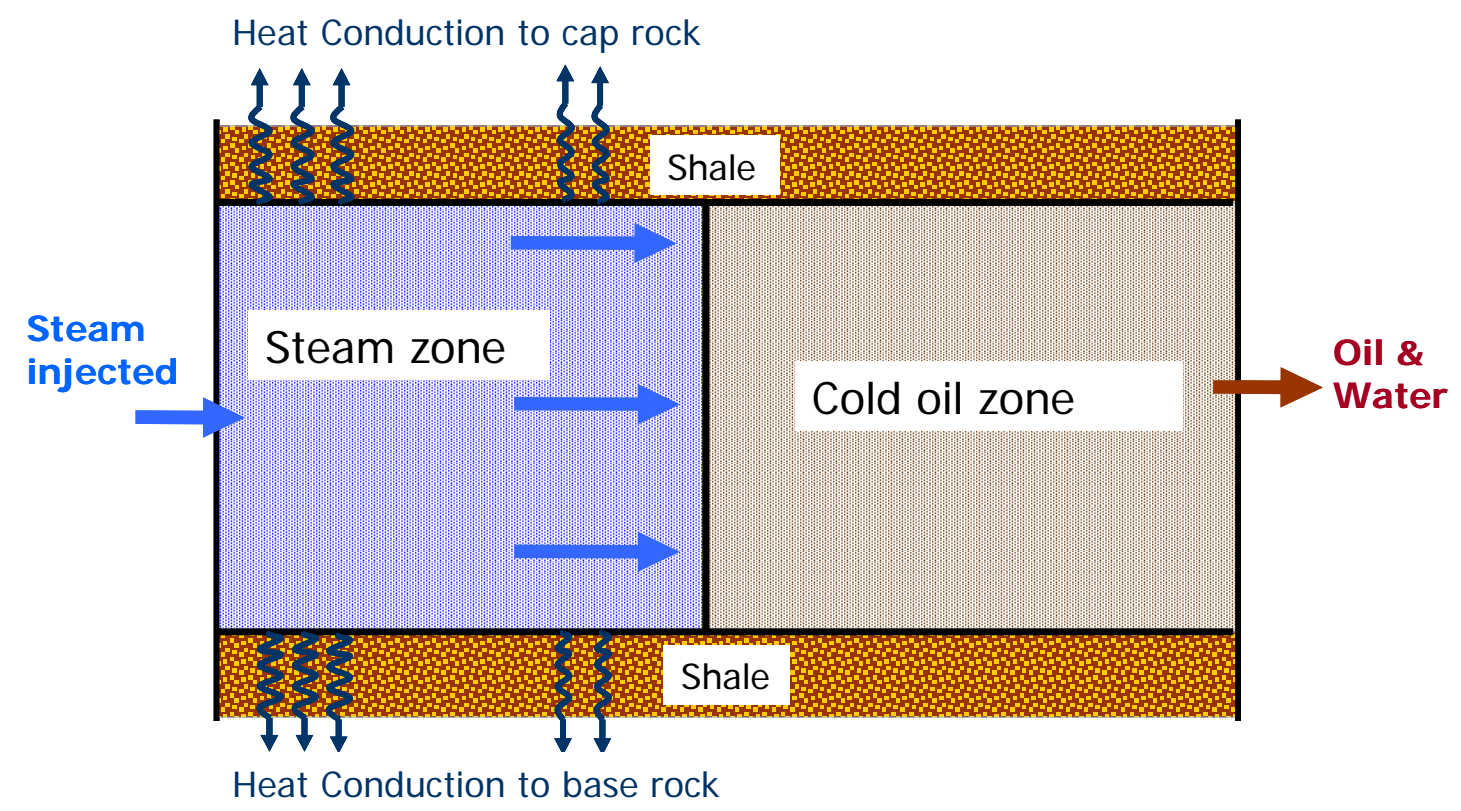

Fig. 5.1- Frontal Advanced Displacement (reproduced from Myhill and Stegemeier ${ }^{40}$ ). 


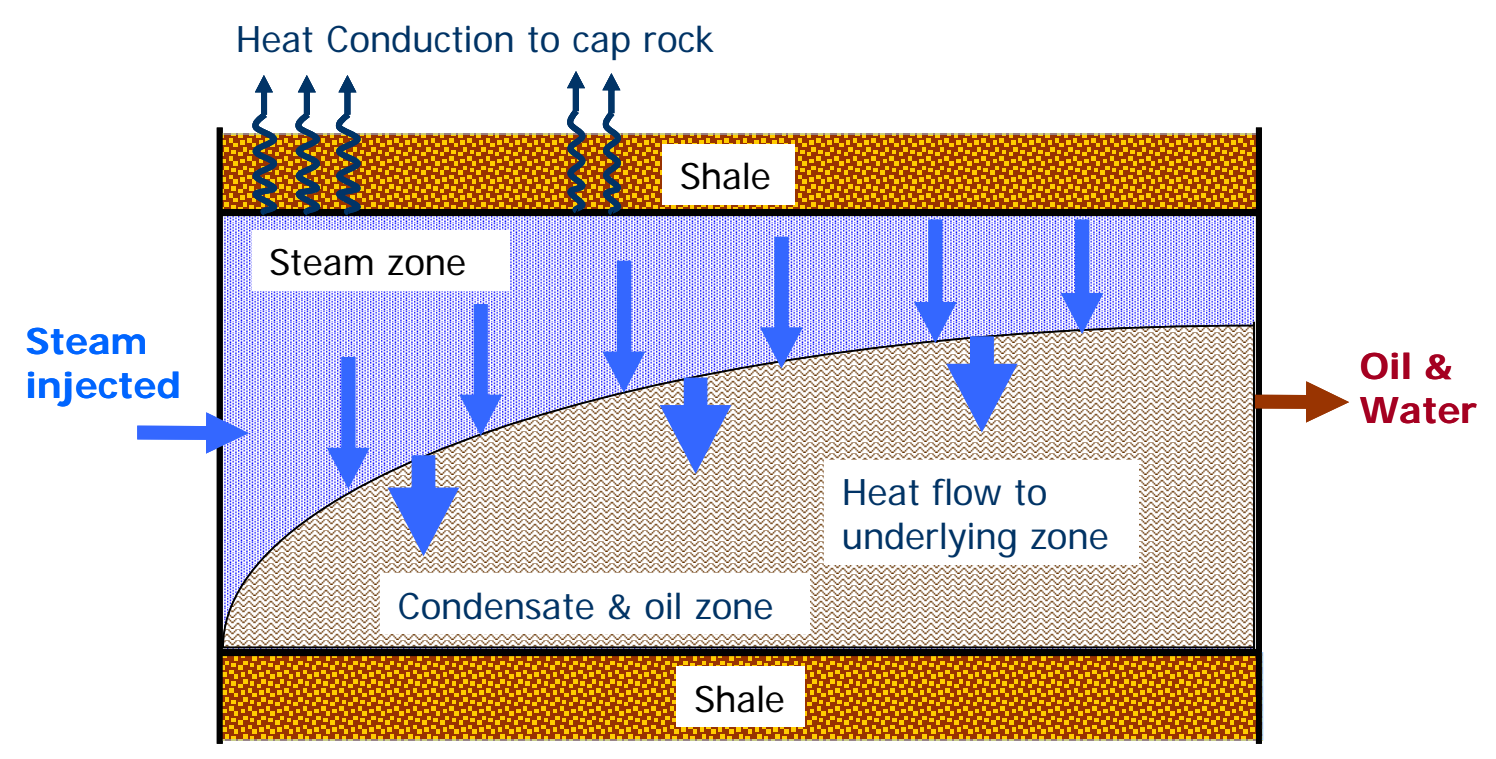

Fig. 5.2- Vertical or Gravity Override Displacement (reproduced from Neuman ${ }^{42}$ ).

Because the frontal advance model developed by Mandl and Volek neglected the higher order terms for the steam zone volume calculation, Myhill and Stegemeier ${ }^{43}$ modified the model based on the Prats solution to calculate the thermal efficiency after the critical time from the relation

$$
E_{h s}=\frac{\sqrt{t_{D}}}{\sqrt{\pi} t_{D}}-\left[\frac{\sqrt{t_{D}-t_{c D}}}{1+F_{h D}}-\int_{0}^{t_{c D}} \frac{e^{u} \operatorname{erfc} \sqrt{u}}{\sqrt{t_{D}^{-u}}} d u\right],
$$

where

$$
\begin{aligned}
& t_{D}=\text { dimensionless time } \\
& t_{c D}=\text { critical dimensionless time, } \\
& u \quad=\text { velocity of steam-oil interface, } \mathrm{ft} / \mathrm{sec}
\end{aligned}
$$

This model assumes (a) a uniform oil concentration, formation thickness and constant petrophysical properties; (b) steam injection at constant pressure, rate and quality; and (c) no vertical temperature gradients. Jones (1981) noted that the MyhillStegemeier model often overestimates the oil production, especially in the early phase of 
a project. One of the deficiencies of the Myhill-Stegemeier model is its prediction that the oil displaced by the steam zone is immediately produced.

Neuman ${ }^{44}$ presented a gravity override model of steam drive which predicted the following:

1. Steam zone thickness, $h_{s t}$ and areal extent, $A$

$$
\begin{aligned}
h_{s t} & =\frac{4 k_{h} C_{w}\left(T_{s}-T_{R}\right)}{M_{s} L_{v d h}} \sqrt{\frac{t-\tau}{\pi \alpha}} \\
A & =\frac{f_{s d h}\left(1-f_{p}\right) \rho_{w} L_{v d h}}{K_{h}\left(T_{s}-T_{R}\right)} \sqrt{\frac{\alpha t}{\pi}}
\end{aligned}
$$

where

$t-\tau=$ the time elapsed since steam first arrived where the steam zone thickness is to be calculated.

$K_{h} \quad=$ thermal conductivity of overburden, in Btu $/ \mathrm{ft}-{ }^{\circ} \mathrm{F}$

$T_{s} \quad=$ steam temperature, in ${ }^{\circ} \mathrm{F}$

$T_{R} \quad=$ original reservoir temperature in ${ }^{\circ} \mathrm{F}$

$\alpha \quad=$ the ratio of oil displaced from the steam zone below to that displaced from the steam zone itself.

$M_{s} \quad=$ the heat capacity of the steam zone, in Btu/ $/ \mathrm{ft}^{3}-{ }^{\circ} \mathrm{F}$.

$L_{v d h}=$ heat of vaporization of water at reservoir pressure in Btu/lb

$f_{\text {sdh }} \quad=$ downhole steam quality, fraction

$f_{p} \quad=$ fraction of injected heat that is produced, fraction. 
2. The volume of oil displaced from the steam zone and the heated reservoir beneath it.

$$
V_{o}=\frac{\left(1+f_{b}\right) \phi\left(S_{o i}-S_{o r s}\right) f_{s d h}\left(1-f_{p}\right) i_{s t} \rho_{w} C_{w} t}{M_{s}},
$$

and $f_{b}=F \frac{T^{*}-T_{R}}{T_{s}-T_{R}}$ is obtained from Neuman's graphical solution.

3. The reduced injection rate $\left(i_{m}\right)$ and the downhole steam quality that sustains the steam zone can be determined by solving Eq. 5.15 .

$$
i_{m} f_{d m}\left(1-f_{p m}\right)=i_{b} f_{d b}\left(1-f_{p b}\right) \frac{2}{\pi} \tan ^{-1}\left(\frac{t^{*}}{1-t^{*}}\right)
$$

The subscript "b" and " $m$ " represent the injection conditions before and after the heat reduction and $\mathrm{t}^{*}$ is the time at which reduction is begun.

4. The additional oil displaced $\left(V_{O T}\right)$ after steam injection is stopped is given by

$$
V_{o T}=\frac{2\left(T_{s l}-T_{R}\right)-\left(T_{s 2}-T_{R}\right) V_{o}}{T_{s l}-T_{R}}
$$

where $T_{o}$ is the oil displaced from Eq. 5.15 using $t^{*}$ and subscript 1 and 2 refer to the conditions before and after steam injection is stopped. It is noted that Neuman did not suggest a way to evaluate the value of the fraction injected that is produced $\left(f_{p}\right)$ in Eqs. 5.13 and 5.14. Thus, this value is an adjustable parameter. 
Rhee and Doscher ${ }^{45}$ also proposed that the principal direction of steam zone growth is vertically downward. They developed a semi-analytical predictive model based on this approach. The model includes some of the concept of Marx and Langenheim $^{34}$ and Van Lookeren ${ }^{38}$. In the model, the heated zone volume and shape were calculated by a rigorous heat balance, the volume and composition were calculated by approximate enthalpy and material balances, and the results were combined with a fluid flow model similar to that of Higgins and Leighton (1960). Although the model showed good agreement with the available experimental data, it is not easy to use due to the sophisticated distillation mechanism and the fluid flow model used.

Jones $^{46}$ developed a simplified predictive model based on Van Lookeren's calculation for optimum steam injection rate and a modification of the Myhill and Stegemeier model for oil production rate. Empirical factors based upon field performance were included to simulate the oil production history at a given steam injection rate. 


\subsection{New Analytical Model for Injection with Steam Additives}

The methods that have been previously discussed involved steam injection growth in radial direction and developed for three dimensional model. A one-dimensional model of steam front velocity and oil production under pure steam injection, and steam injection with additives (propane and petroleum distillate) has been developed to describe the experimental data. The model is used to predict the steam front advancement with respect to time. In this new model, the complex temperature and phase distribution profiles actually present in the cell during steam injection are approximated, as depicted in Figs. 5.3 and 5.4. Fig. 5.3 depicts the new model.

The following simplifying assumptions are made in this present model:

(1) The sand mix in the injection cell is homogenous.

(2) The steam zone movement is piston-like outward from the injection point.

(3) The sand mix temperature profile follows a one-step temperature distribution: steam temperature, $T_{s}$, from the injection point until the steam front, where it drops to the original reservoir temperature, $T_{o}$.

(4) Heat loss to the adjacent formation is only by convection and radiation normal to the steam injection path direction.

(5) Constant heat injection rate, $H_{s}$, BTU/D

(6) Constant injected fluid phase throughout the production.

(7) Pressure drop in the steam zone is negligible. 


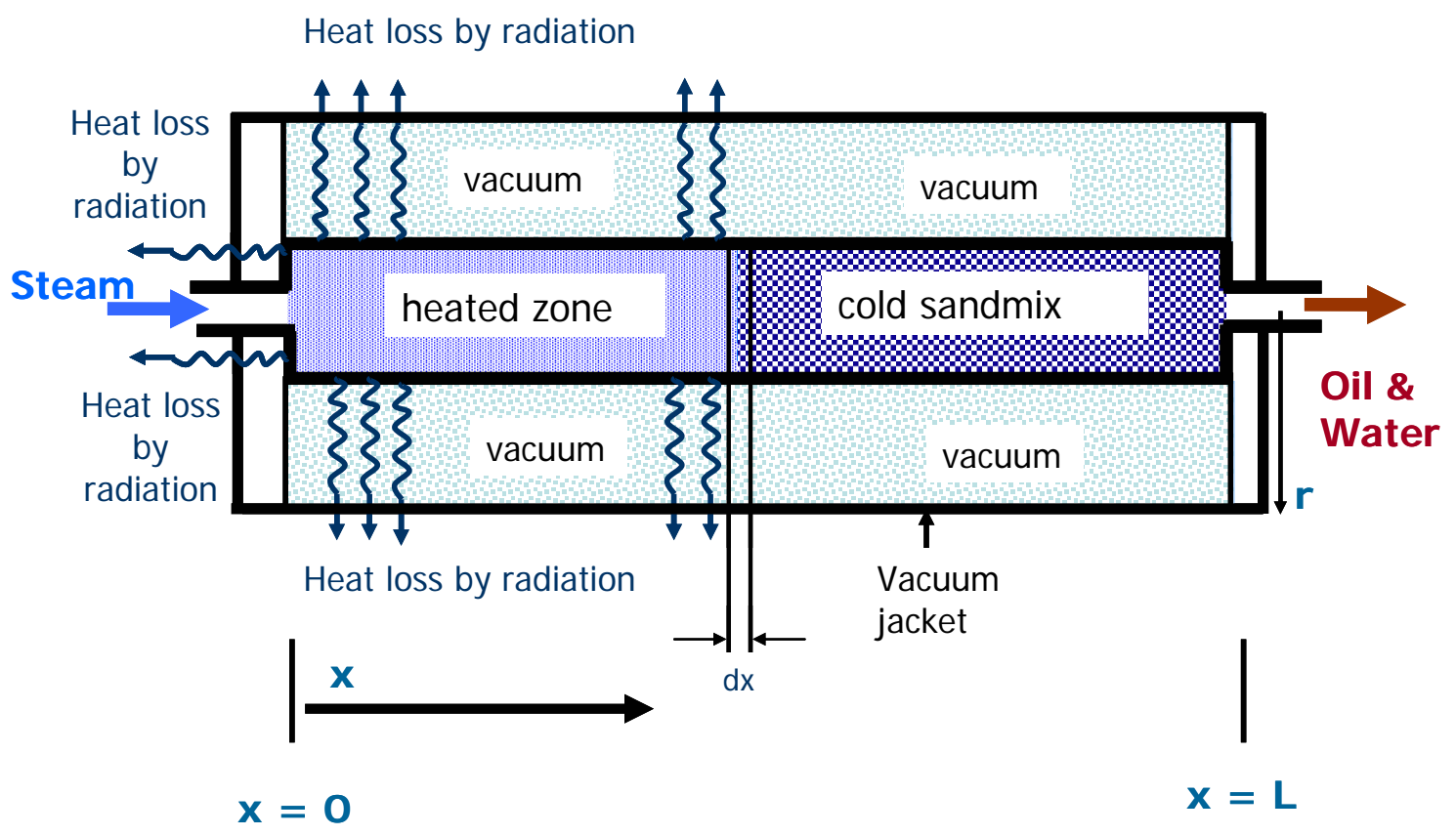

Fig. 5.3- One dimensional heat balance for $1 \mathrm{D}$ displacement experiments.

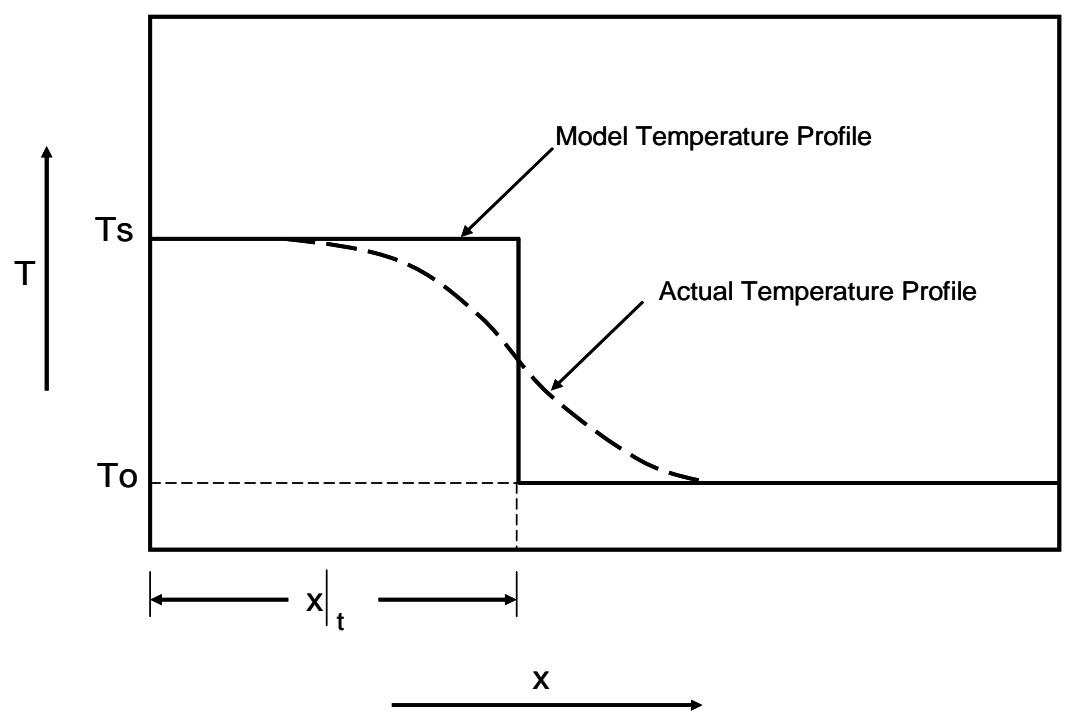

Fig. 5.4- Schematic diagram of model and actual temperature profiles in 1D displacement experiments. 
The heat balance based on process as depicted in Fig. 5.3. can be expressed as the following:

Heat in - heat loss by radiation/convection =

heat to raise temperature of element $d x$ of sandmix,

Evaluating the heat balance for time period $d t$ gives:

Heat in $=H_{s} d t$,

heat loss by radiation for outer wall of cell $=\left(2 \pi r\left(T_{s}-T_{o}\right)\left(x+L_{\text {void }}\right)\right) U$,

heat loss by radiation for top flange $=\left(\pi r^{2}\right) U d t$,

heat to raise temperature of element $d x$ of sandmix $=$ heat loss by radiation for outer wall of cell + heat loss by radiation for top flange.

Thus, $H_{s} d t-\left(2 \pi r\left(T_{s}-T_{o}\right)\left(x+L_{\text {void }}\right)+\pi r^{2}\right) U d t=\pi r^{2} M_{R}\left(T_{s}-T_{o}\right) d x$.

where:
$U=$ overall coefficient of heat loss, in BTU/(ft-D- $\left.{ }^{\circ} \mathrm{F}\right)$,
$H_{s} \quad=$ heat injected into the injection cell, in BTU/D,
$L_{\text {void }}=$ length of void space in the top of sandmix (measured after the end of experiment run), cm,
$M_{R}=(1-\phi) C_{r} \rho_{r}+\phi S_{o} C_{o} \rho_{o}+\phi S_{w} C_{w} \rho_{w}$.

Multiplying through Eq. 5.22 by $\frac{1}{d t}$ gives:

$$
H_{s}-\left(2 \pi r\left(T_{s}-T_{o}\right)\left(x+L_{v o i d}\right)+\pi r^{2}\right) U=\pi r^{2} M_{r}\left(T_{s}-T_{o}\right) \frac{d x}{d t}
$$

Grouping variables which are independent upon time gives us: 


$$
\begin{aligned}
& A=H_{s}, \\
& B=-2 \pi r\left(T_{s}-T_{o}\right) U, \\
& C=-\pi r^{2} U, \\
& D=\pi r^{2} M_{R}\left(T_{s}-T_{o}\right) .
\end{aligned}
$$

Hence Eq. 5.24 can be written as:

$$
A+B\left(x+L_{\text {void }}\right)=D \frac{d x}{d t}
$$

Rearranging Eq. 5.29 gives us:

$$
\frac{d x}{d t}=\frac{A+B\left(x+L_{\text {void }}\right)+C}{D}
$$

Rearranging Eq. 5.30 gives us:

$$
d t=\frac{D d x}{A+B\left(x+L_{v o i d}\right)+C}
$$

Integrating Eq. 5.31 gives:

$$
t=\int_{0}^{t} d t=D \int_{0}^{x} \frac{d x}{A+B\left(x+L_{\text {void }}\right)+C}
$$

Assigning the limit of the integer in Eq. 5.32 yields:

$$
t=D \ln [A+B(x+\text { Lvoid })]+E,
$$

where $E$ is a constant of integration. 
Evaluating at the boundary condition:

$$
t=0 \text { at } x=L_{v o i d}
$$

then Eq. 5.33 can be rearranged as:

$E=-D \ln \left(A+2 B L_{\text {void }}+C\right)$.

Substituting Eq. 5.34 into Eq. 5.33 yields:

$t=\frac{C}{B} \ln (A+B x)-\frac{C}{B} \ln A$.

Rearranging Eq. 5.35 gives:

$\ln \left(\frac{A+B\left(x+L_{\text {void }}\right)+C}{A+2 B L_{\text {void }}+C}\right)=\frac{t}{D}$.

Rearranging Eq. 5.36 gives:

$A+B\left(x+L_{v o i d}\right)+C=\left(A+2 B L_{v o i d}+C\right) e^{t / D}$.

Rearranging Eq. 5.37 gives:

$x=\frac{\left(A+2 B L_{\text {void }}+C\right) e^{t / D}-(A+C)}{B}-L_{\text {void }}$.

Substituting Eqs. 5.25 to 5.28 into 5.38 yields: 


$$
x=L_{\text {void }}+\frac{\left(H_{s}-\pi r^{2} U\right)-\left[H_{s}-4 \pi r\left(T_{s}-T_{o}\right) U L_{v o i d}-\pi r^{2} U\right] e^{t / \pi r^{2} M_{R}\left(T_{s}-T_{o}\right)}}{2 \pi r\left(T_{s}-T_{o}\right) U}
$$

Although Eq. 5.24 can be derived and takes final form as expressed in Eq. 5.39, in calculation routine however, Eq. 5.24 is used by simply discretizing it. The analytical solution of Eq. 5.24 as expressed in Eq, 5.39 is restricted by a critical assumption which is constant injection temperature $T_{s}$ (and $H_{s}$ ) throughout the entire run. It is decided to directly apply the Eq. 5.24 rather than Eq. 5.39 due to the fact that in reality, $H_{s}$ (the amount heat injected to the cell) depends upon time, $t$. This causes Eq. 5.24 to be nonlinear equation. The second reason is that the small time interval (30 seconds) recorded for the experimental run is sufficiently small to be used as $d t$. Thus, the interval time can be treated as $d t$, hence Eq, 5.24 can be used to solve for $d x$. The distance from the top of sand mix to the steam front, $x$, is then computed by performing summation of the elemental form of $x, d x$. Performing direct calculation by discretizing Eq. 5.24 avoids the numerical fail criteria. Furthermore, $H_{s}$ can be computed independently using table lookup of steam table, and the injected fluid phase of the current time step can also be determined. The table lookup routine to obtain the value of $H_{s}$ and current fluid phase involves interpolation programming procedure of large steam table. In applying Eq. 5.24, the backward difference scheme is used to obtain $d t$ based on the experimental data. From Eq. 5.24, the following expression for $x_{j}$ at time index $j$ is used to calculate steam front location:

$$
x_{j}=\sum_{i=1}^{j} \frac{\left\{H_{s_{i}}-\left[2 \pi r\left(T_{s_{i}}-T_{o}\right)\left(x_{i}+L_{\text {void }}\right)+\pi r^{2}\right] U-\pi r^{2} M_{R}\left(T_{s_{i}}-T_{o}\right)\right\}\left(t_{i+1}-t_{i}\right)}{\pi r^{2} M_{R}\left(T_{s_{i}}-T_{o}\right)}
$$

The total fill-up time ( $t_{\text {futotal }}$ ) occurs during the experimental work is incorporated to the calculation using the analytical model. The total fill-up time comprises in two parts. The first part is due to the void space created during high pressure difference in the top part sand mix, $t_{\text {fusandmix }}$. The void space is translated into time required for the steam 
to travel from the mid of the flange to the top of the sand mix that has been pushed during the experimental run due to the high pressure difference mainly occurring during the early production time. Thus, $t_{\text {fusandmix }}$ can be expressed as the following:

$$
t_{\text {fusandmix }}=\frac{V_{\text {sandpack }} \times \phi \times S_{g}{ }^{\prime}}{i_{s}},
$$

where:

$$
S_{g}{ }^{\prime}=S_{g} \times \frac{P_{a t m}}{P_{i n j}} \times \frac{T_{i n j}}{T_{a t m}} .
$$

The second part of the fill-up time is caused by the time required to warm up the top of the flange cap $\left(t_{\text {flange }}\right)$. And $t_{\text {flange }}$ is modeled using this following relation:

$$
\int_{o}^{t} H_{s} d t=M_{f}\left(T_{s}-T_{o}\right)
$$

where $M_{f}=\pi r_{f}^{2} h_{f} \rho_{f} c_{f}$, then the total fill-up time is computed as:

$$
t_{\text {futotal }}=t_{\text {fusandmix }}+t_{\text {flange }} \text {. }
$$

Steam front calculation is started in the total fill-up time.

The cumulative oil production can be calculated using two approaches. The first approach is to apply material balance (assuming a piston-like displacement occurs as steam is injected through the porous media) and the second approach is to apply Darcy's Law and manipulate it to obtain cumulative oil production.

The first approach is used by applying the steam front distance, $x$, to calculate, the cumulative oil production. The material balance equation that couples the present steam velocity model is explained in details as the following:

$$
N_{p}=V_{s w p}\left(S_{o i}-S_{o r}\right)
$$


where $V_{\text {swp }}=$ area swept by steam and can be expressed as the following:

$V_{s w p}=\pi r^{2} \times \phi$

Substituting Eqs. 5.46 into 5.45 gives:

$N_{p}=\pi r^{2} \phi \times\left(1-S_{w c}-S_{g i}-S_{o r}\right)$.

The distance of steam front from sand mix top, $x$, is obtained by using Eq. 5.16 hence we can directly predicted $N_{p}$.

The second approach is to use Darcy's Law such that $N_{p}$ can be expressed as the following relationship:

$N_{p}=J \int_{0}^{t}\left(p_{i}-p_{o}\right) d t$,

numerically,

$N_{p_{j}}=\sum_{i=1}^{j} J\left(p_{i}-p_{o}\right)$

where $J$ is productivity index and given by the following:

$J=\frac{\pi r^{2} k k_{r o}}{\mu_{o}\left(L-x-L_{\text {void }}\right)}$.

In the model, pressure drop in the steam zone is negligible and is only across the oil zone. Thus $J$ is inversely proportional to $\mu_{o}$, that is 
$J=\frac{C}{\mu_{o}\left(L-x-L_{\text {void }}\right)}$,

where $C$ is a constant found by history-matching cumulative oil production for pure steam injection runs, knowing $\mu_{o}$ at initial cell temperature.

Combining Eqs. 5.49 and 5.51 gives us:

$N_{p_{j}}=C \sum_{i=1}^{j} \frac{\left(p_{i}-p_{o}\right)_{i}}{\left(L-x-L_{\text {void }}\right)}$.

Later, by applying both method to calculate $N_{p}$, it is decided to use Eq 5.52 (Darcy's Law approach) to model $N_{p}$ with respect to time, $t$. The pseudo productivity index $(C)$ is obtained by history-match the $N_{p}$ calculated by analytical model with the $N_{p}$ obtained from the experimental data. After the "matched" $C$ is achieved, the same value of $C$ will be used for the rest of cumulative oil production calculation under steampropane and steam-petroleum distillate injection conditions. However, in order to obtain a good match for steam with additives runs, $\bar{\mu}_{0}$ is modified to get a match between analytical model and experimental results. Fig. 5.5 depicts the flow chart of analytical model and experimental data history-matching process.

The analytical model requires a rather tedious computation, hence, a program written in Visual Basic is developed to generate the result of steam front advancement and cumulative oil production based on the analytical model, and display that composite plot of analytical model results and experimental data. The program and data input for the program is attached in Appendices $\mathbf{E}$ and $\mathbf{F}$ respectively. 


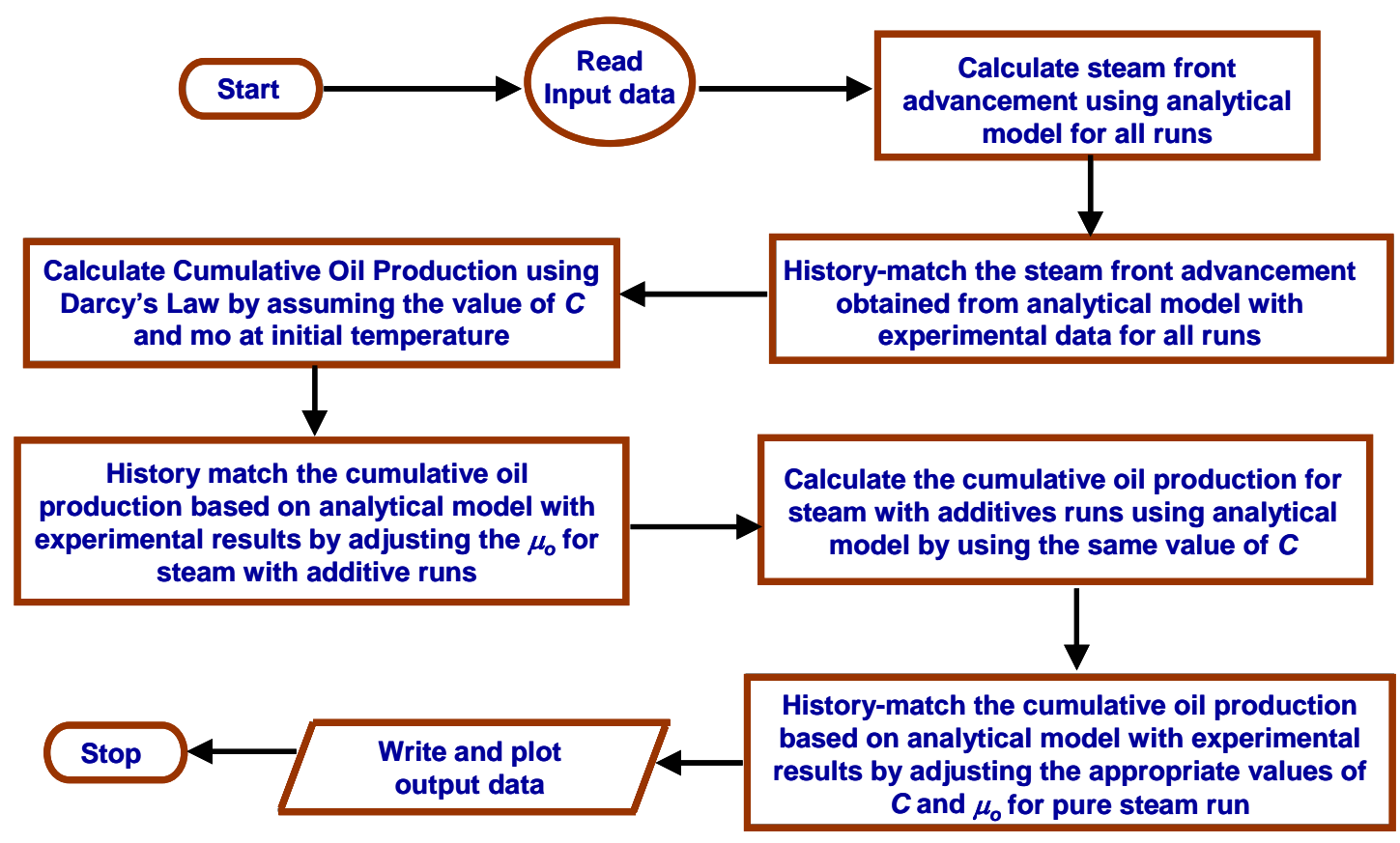

Fig. 5.5- Flow Chart of Analytical Model and Experimental Data History-Matching Process.

\subsection{Comparison and Discussion of Experimental and Analytical Model Results}

Figs. 5.6-5.7 shows plots of the distance of steam front from sand mix and cumulative oil production, respectively for each runs.

The steam front position for run 3 (propane:steam $=5: 100$ ) using analytical model shows an excellent agreement to analytical model, as depicted in Fig. 5.6. The analytical model trend for middle time tends to deviate and give higher prediction of steam front position as compared to experimental data. The cumulative oil production plot for both experimental and predictive analytical model for run 3 is shown in Fig. 5.7. The analytical model trend agrees with the experimental data for early and late time.

The experiments using pure steam (run 4 and 5) yield similar trends for steam front position. These results are expected since both runs are using pure steam, and performed in the same conditions. Cumulative oil production calculated using analytical 


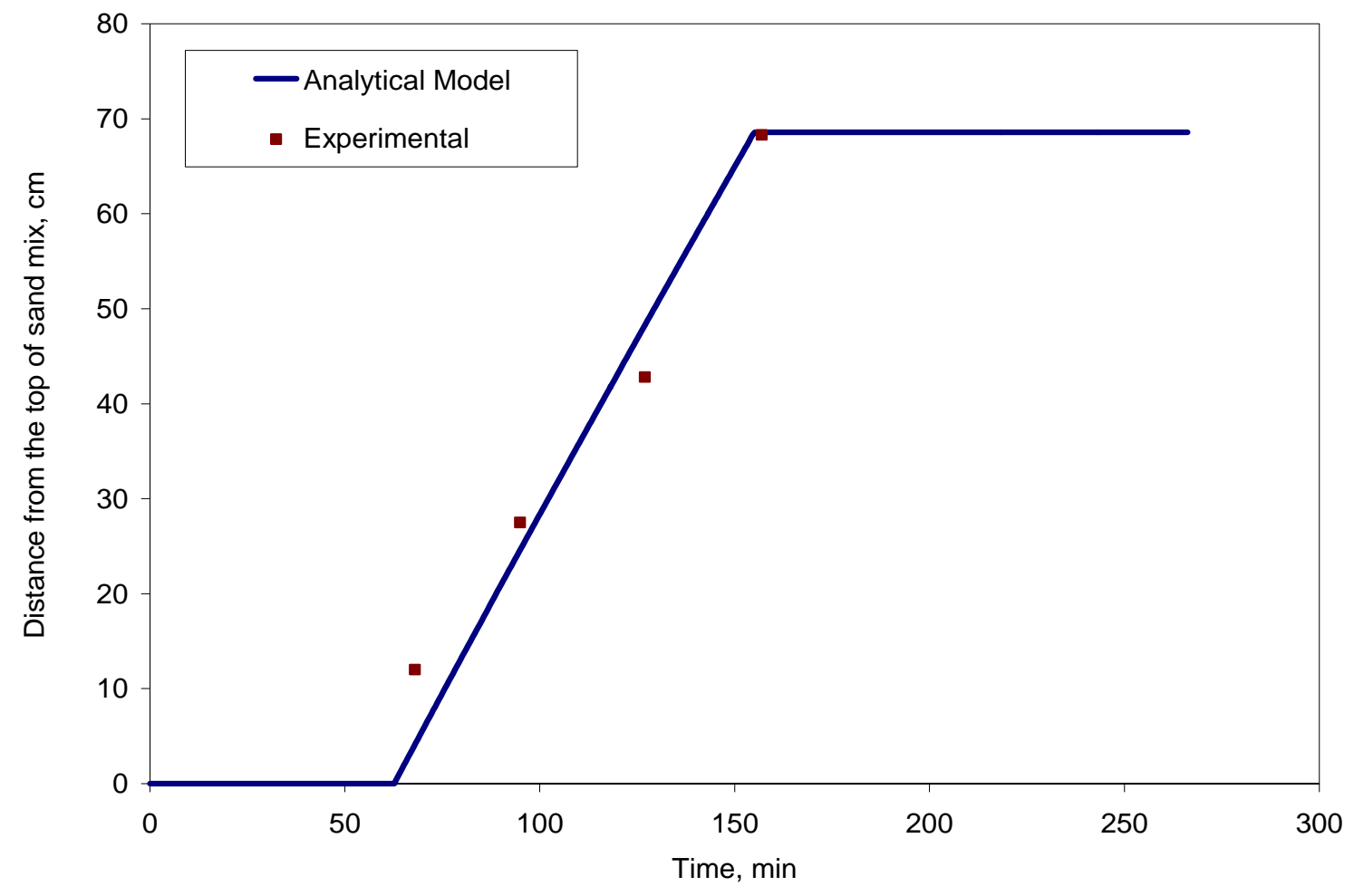

Fig. 5.6- Steam front position for Run 3 (propane:steam = 5:100).

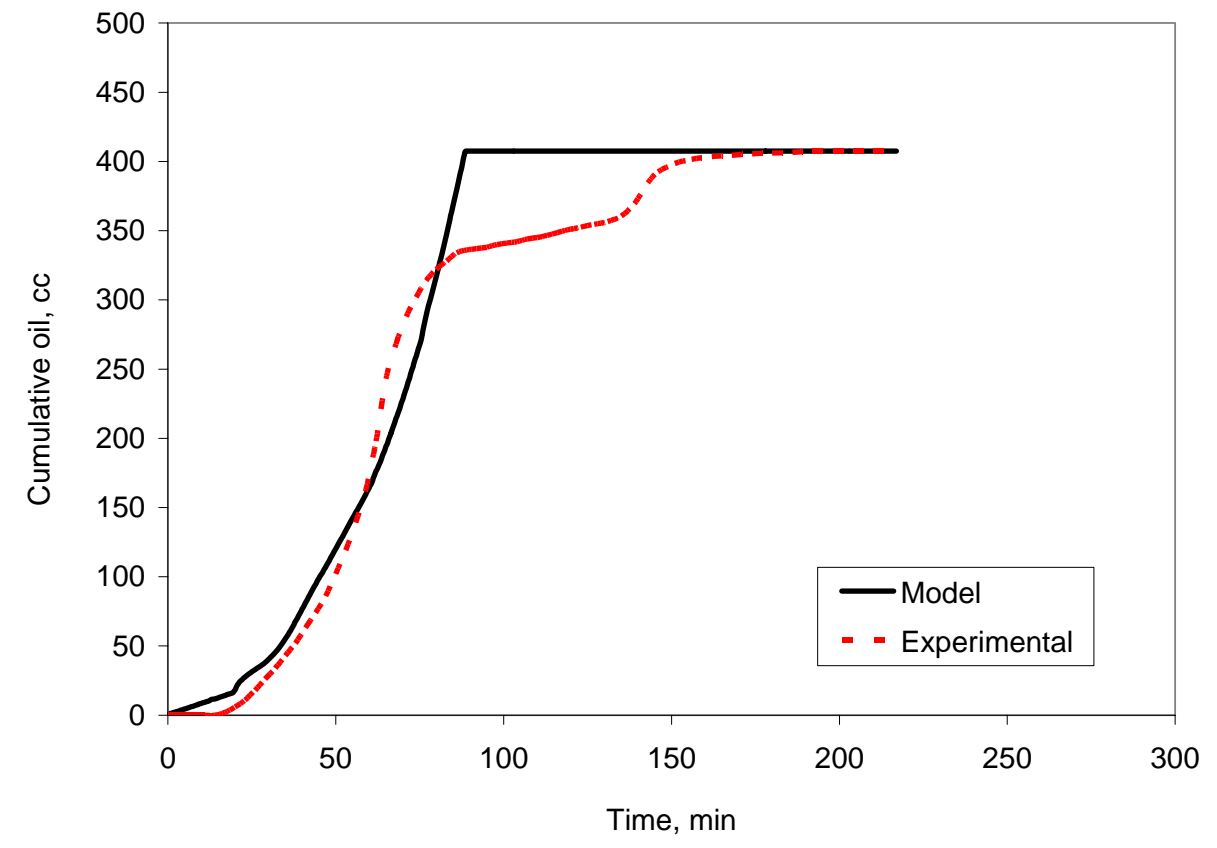

Fig. 5.7- Cumulative oil production for Run 3 (propane:steam = 5:100). 
model and experimental data show excellent matches for the entire production time run no. 4 and 5. The plots are depicted in Figs. 5.7-5.11. In terms of steam front advancement, the analytical models for both runs yield 25\% higher in average for those data in early production time. However, this overestimated trend of steam front position is vanishing at approximately 110 minutes of production time.

Run 6 (5:100 petroleum distillate:steam) is a repeat of run 3. As it is expected, the data for both analytical model and experimental runs show a similar trend for both runs. Both steam front advancement and cumulative oil production give an excellent match as depicted in Figs. 5.12 and 5.13.

The experiments runs utilizing petroleum distillate along with the steam (run 7 and 8) yield an excellent match for both steam front position and cumulative oil production profile. These results are expected since both runs are using petroleum distillate as an additive due both were performed using the same conditions. The plots are depicted in Fig. 5.14-5.17. In terms of steam front advancement, the analytical models for run 7 yield 12\% higher in average for those data in early and middle production time. However, this overestimated trend of steam front position is vanishing at approximately 120 minutes of production time. For run 7, the analytical model shows an excellent of cumulative oil production as compared to experimental data. Run 8 gives yields $18 \%$ higher steam front advancement values compared to experimental data. Nevertheless, analytical model for cumulative oil production profile meets a good agreement with the experimental data for run 8. 


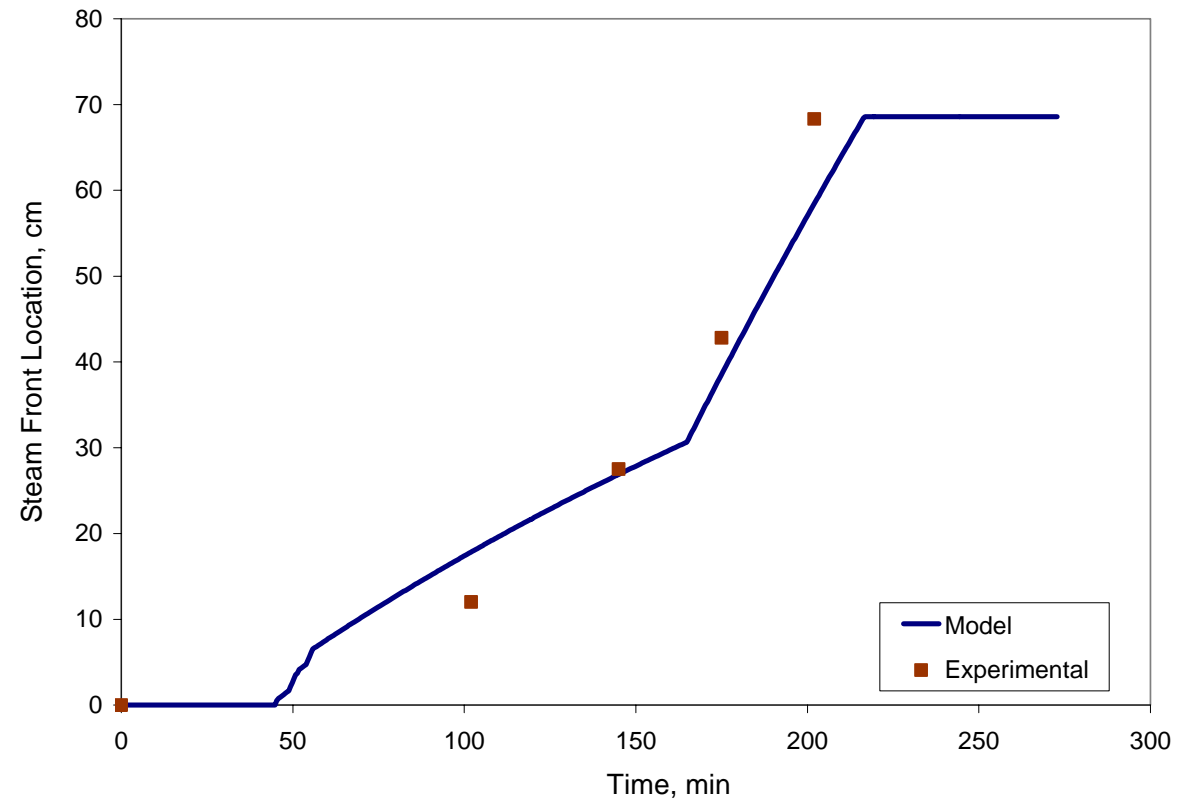

Fig. 5.8- Steam front position for Run 4 (pure steam).

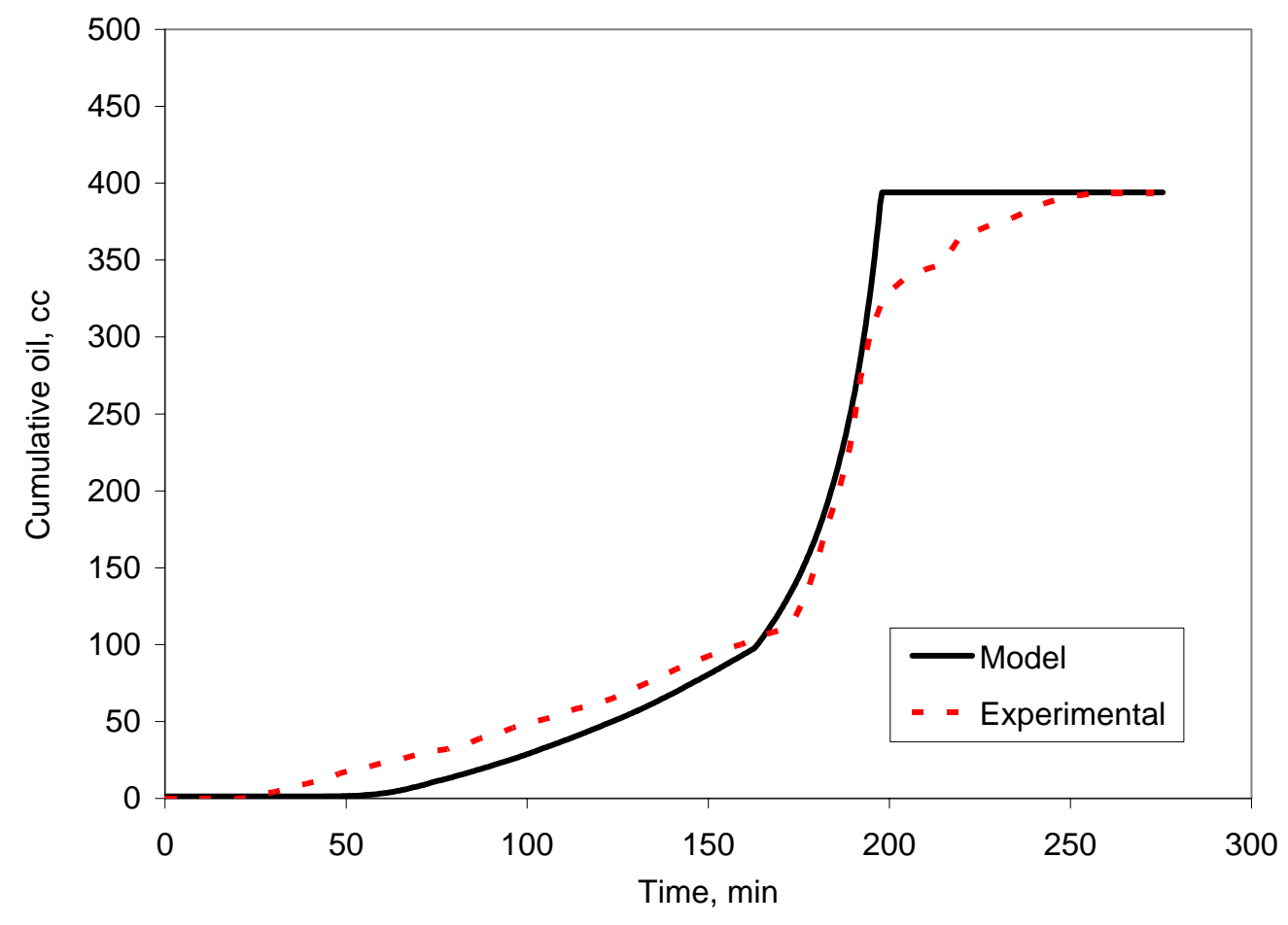

Fig. 5.9- Cumulative oil production for Run 4 (pure steam). 


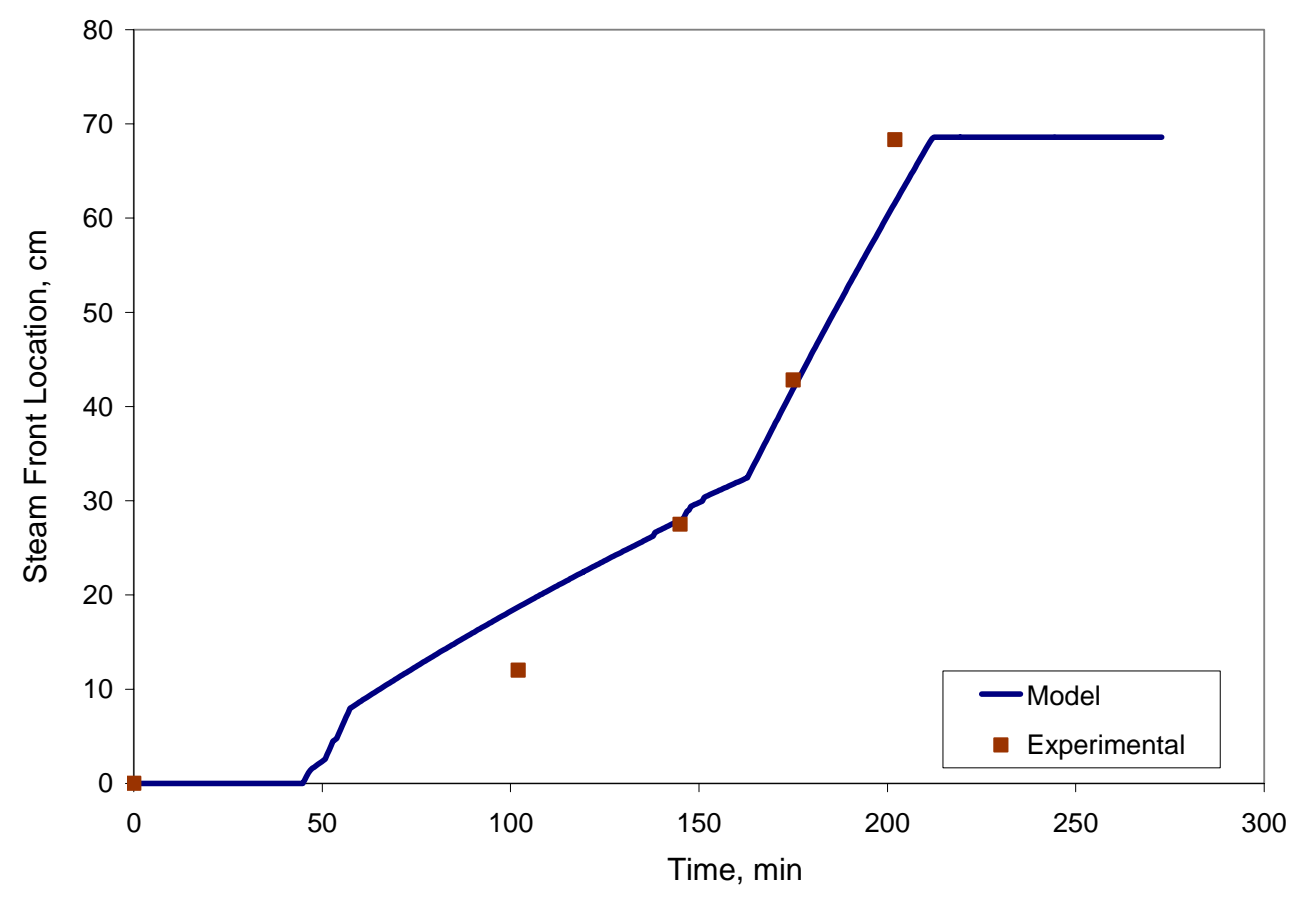

Fig. 5.10- Steam front position for Run 5 (pure steam).

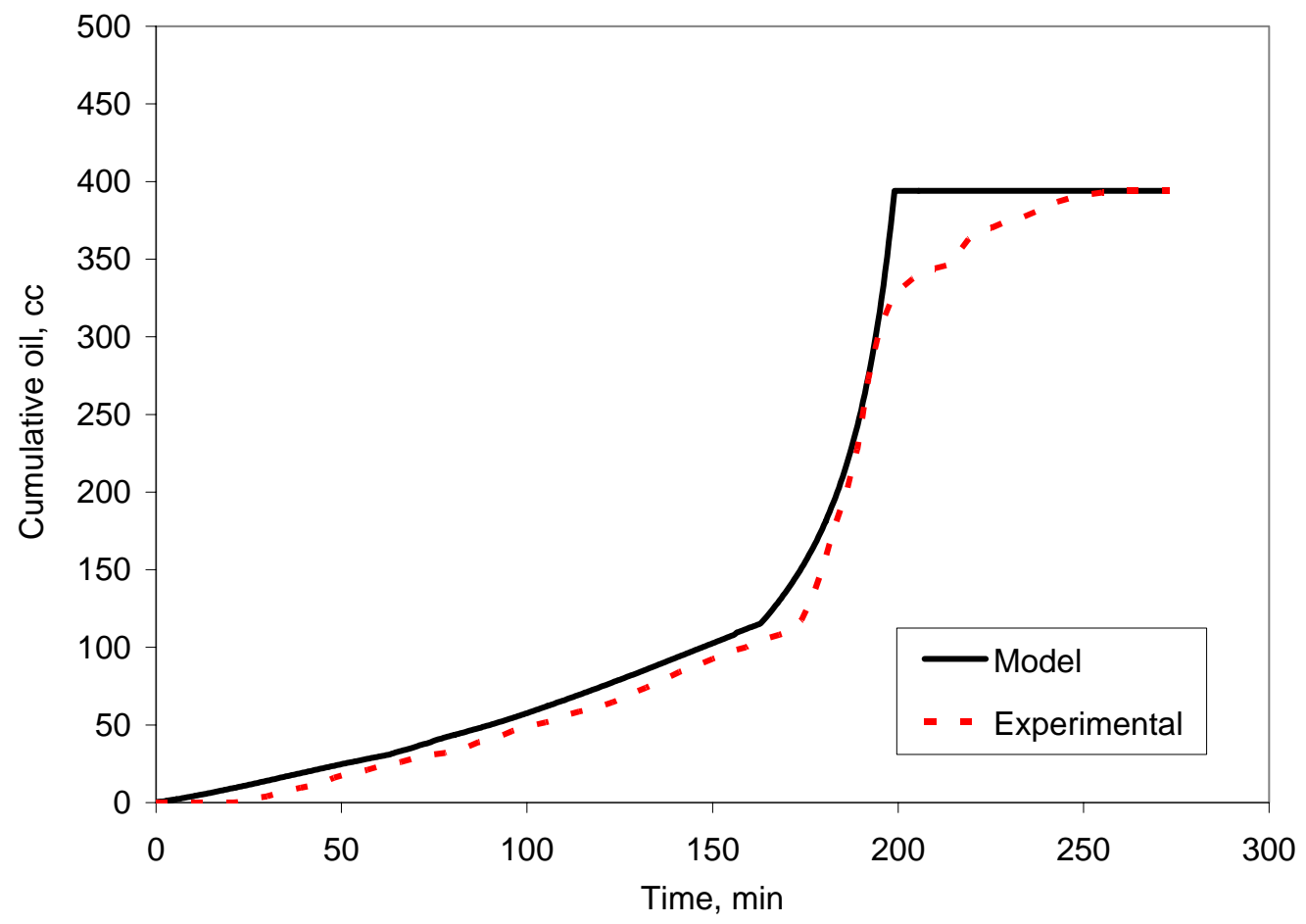

Fig. 5.11- Cumulative oil production for Run 5 (pure steam). 


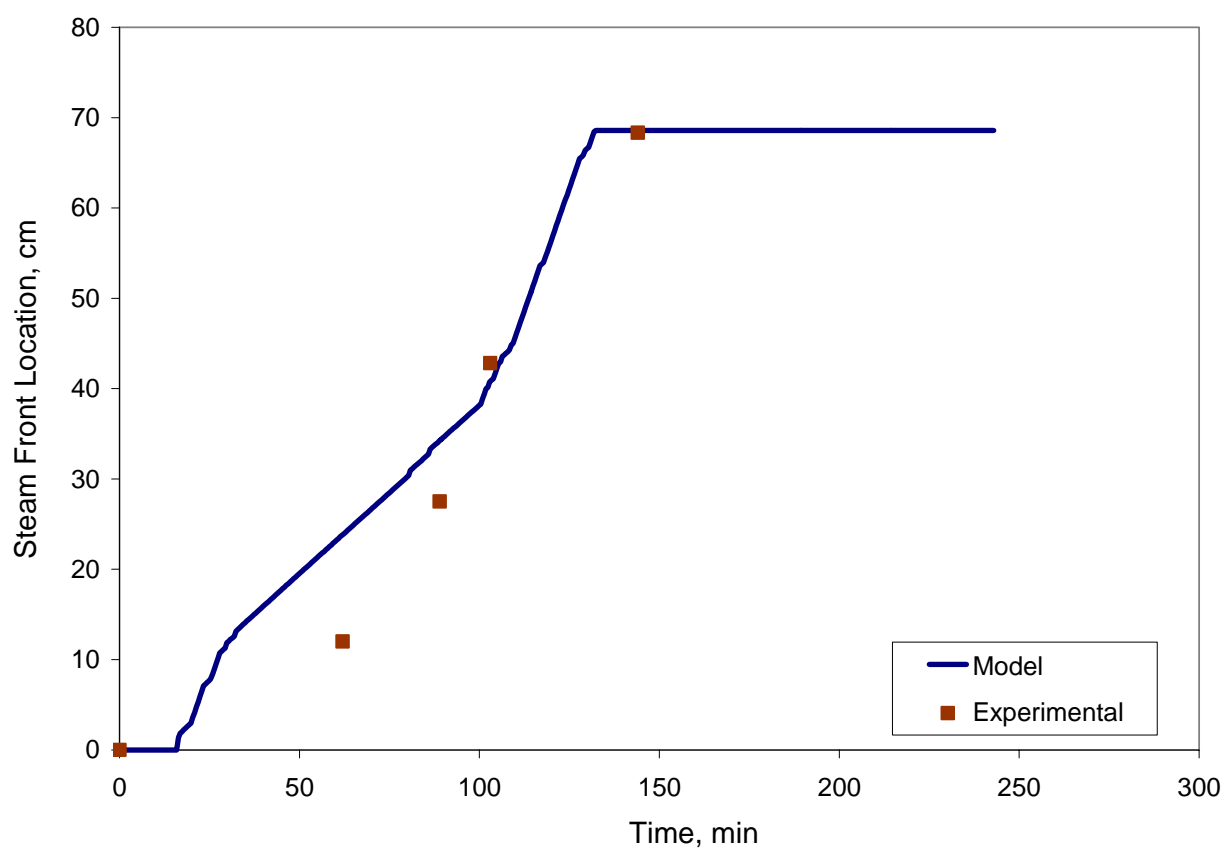

Fig. 5.12- Steam front position for Run 6 (propane:steam = 5:100).

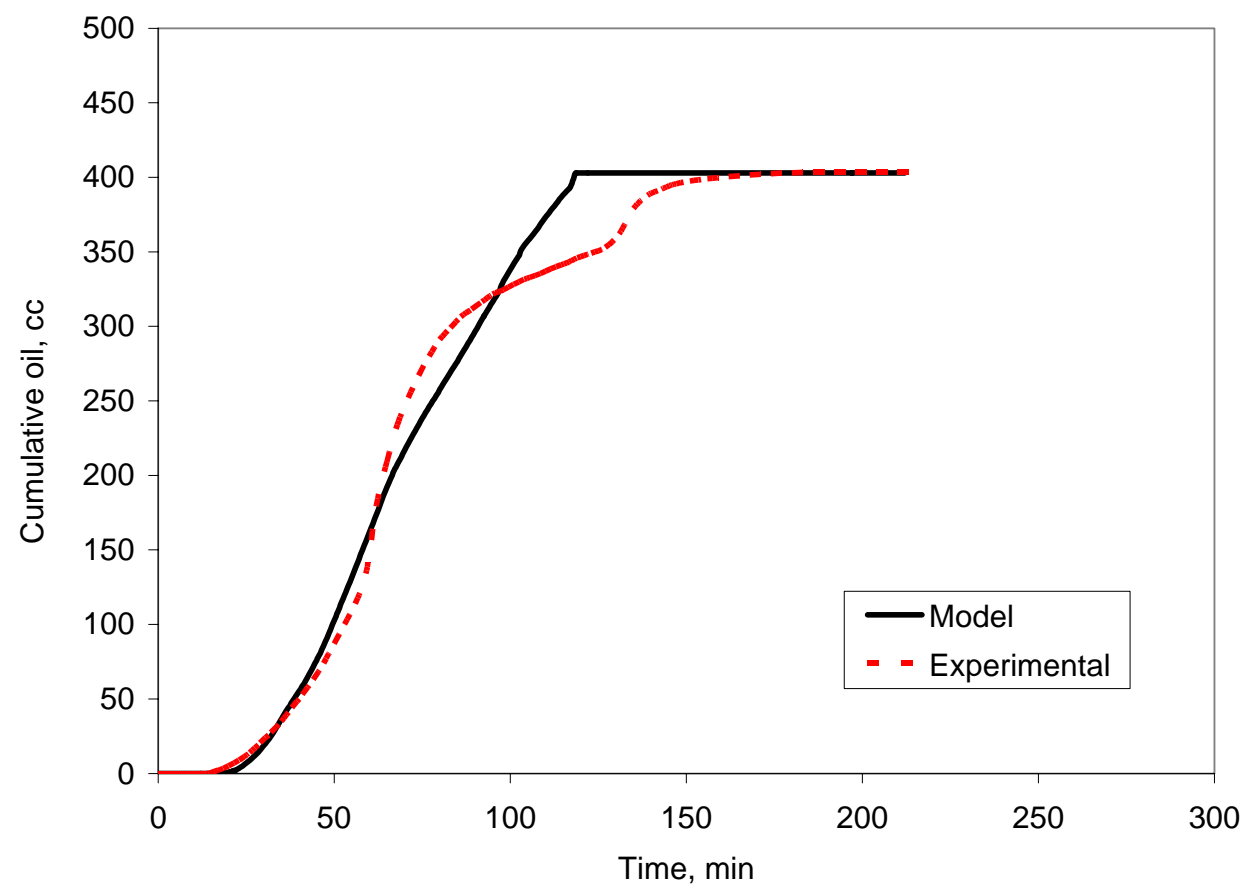

Fig. 5.13- Cumulative oil production for Run 6 (propane:steam = 5:100). 


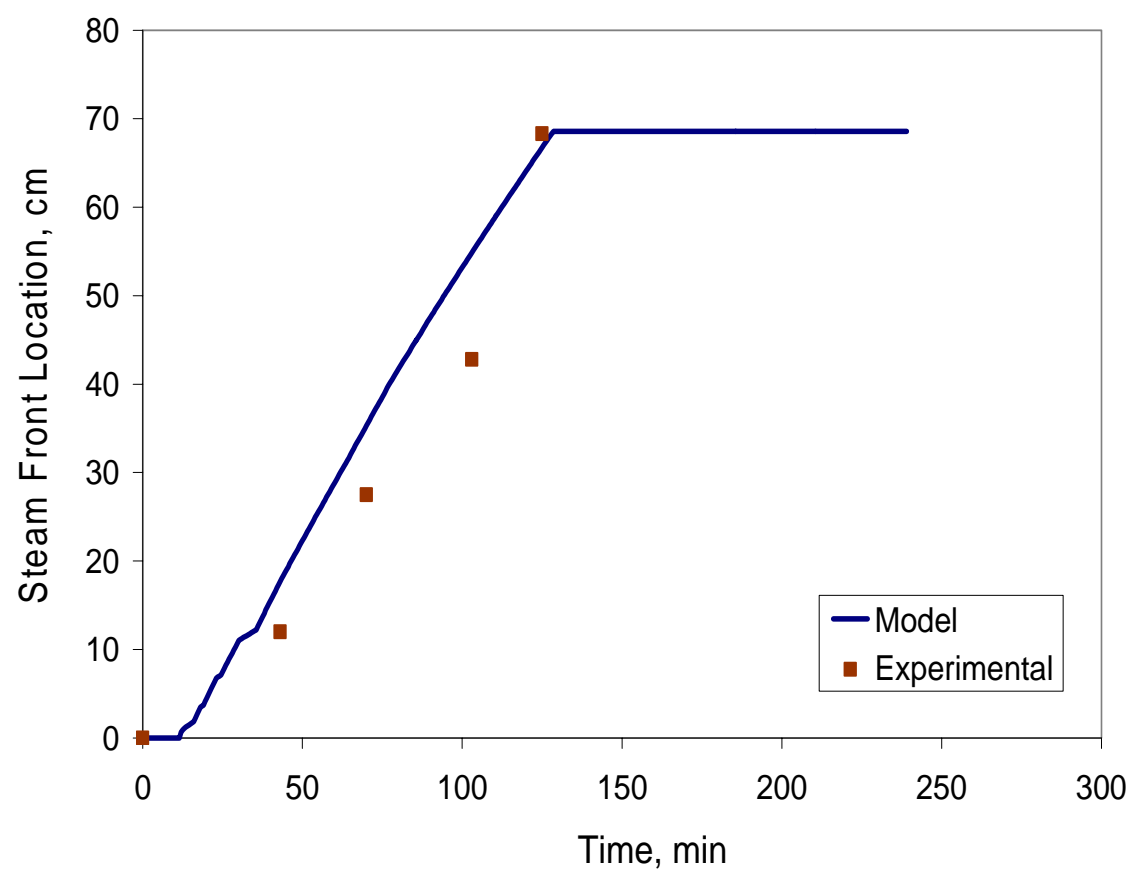

Fig. 5.14- Steam front position for Run 7 (petroleum distillate:steam = 5:100).

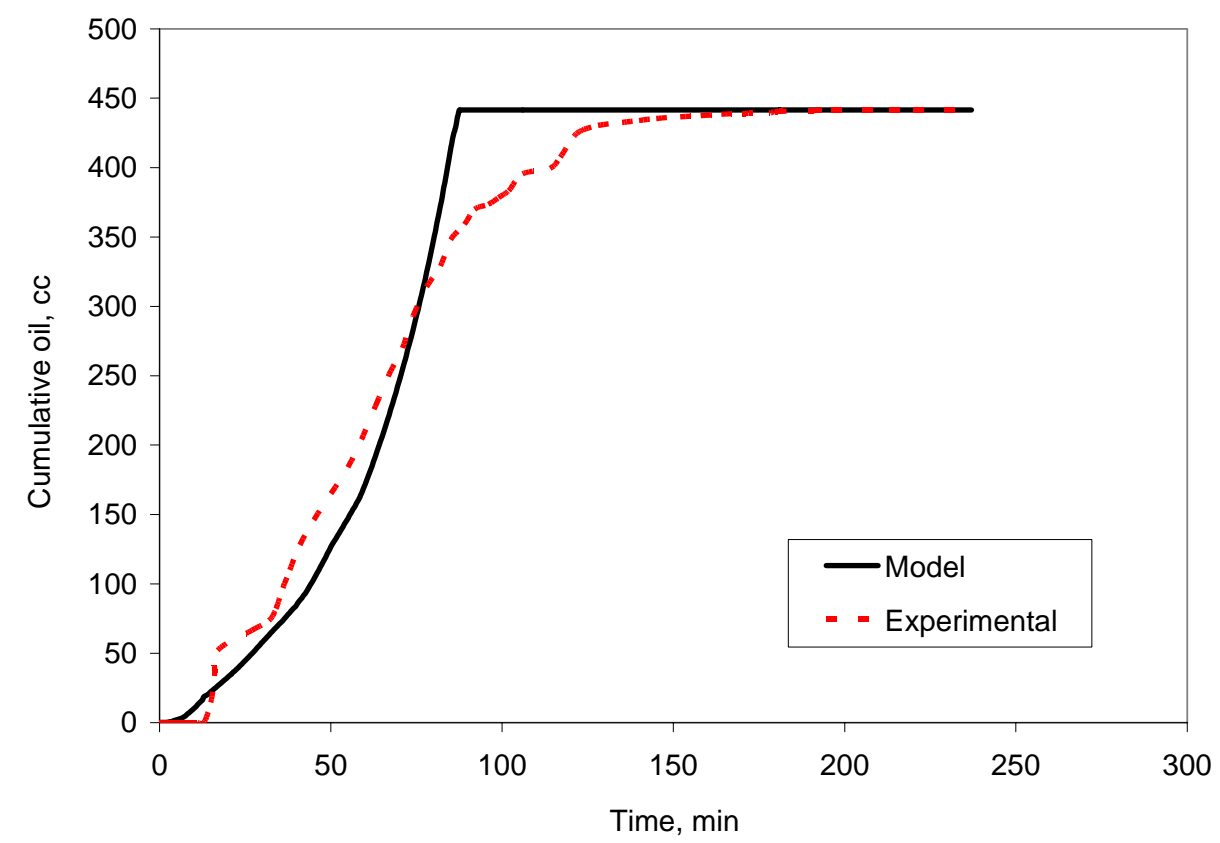

Fig. 5.15- Cumulative oil production for Run 7 (petroleum distillate:steam = 5:100). 


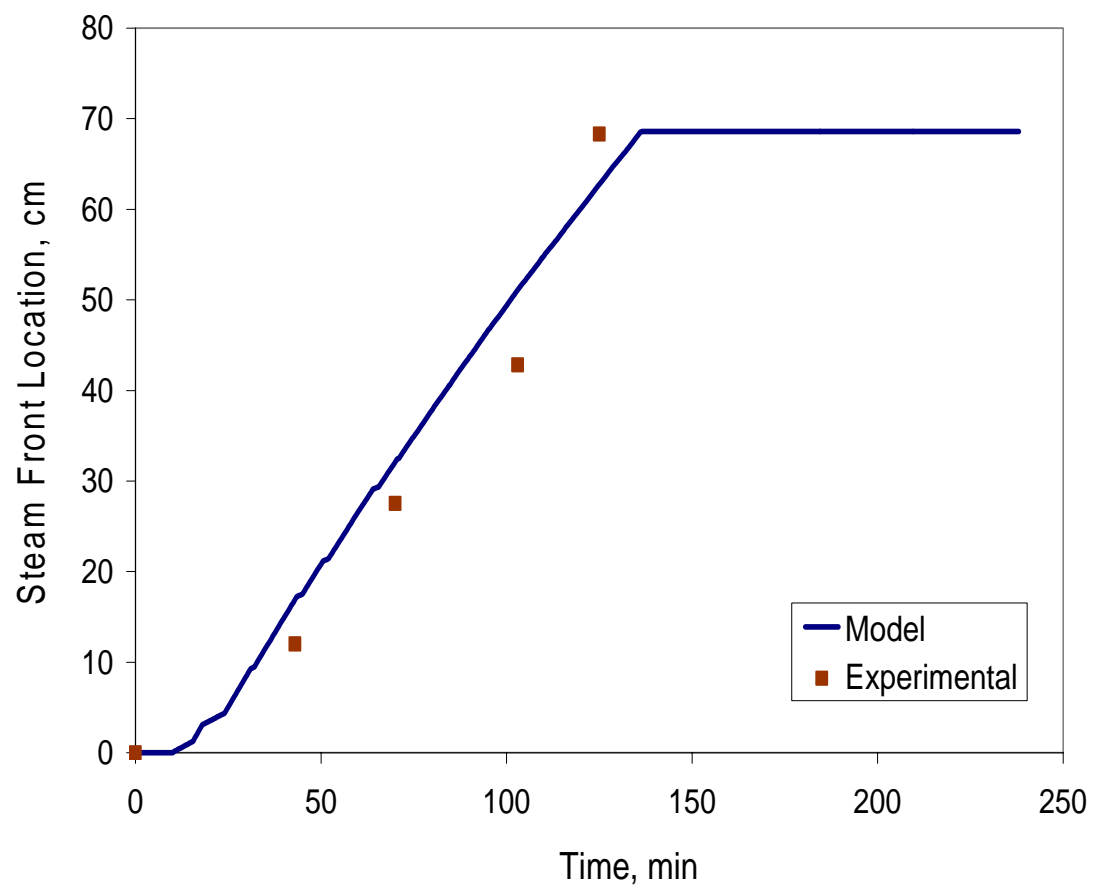

Fig. 5.16- Steam front position for Run 8 (petroleum distillate:steam = 5:100).

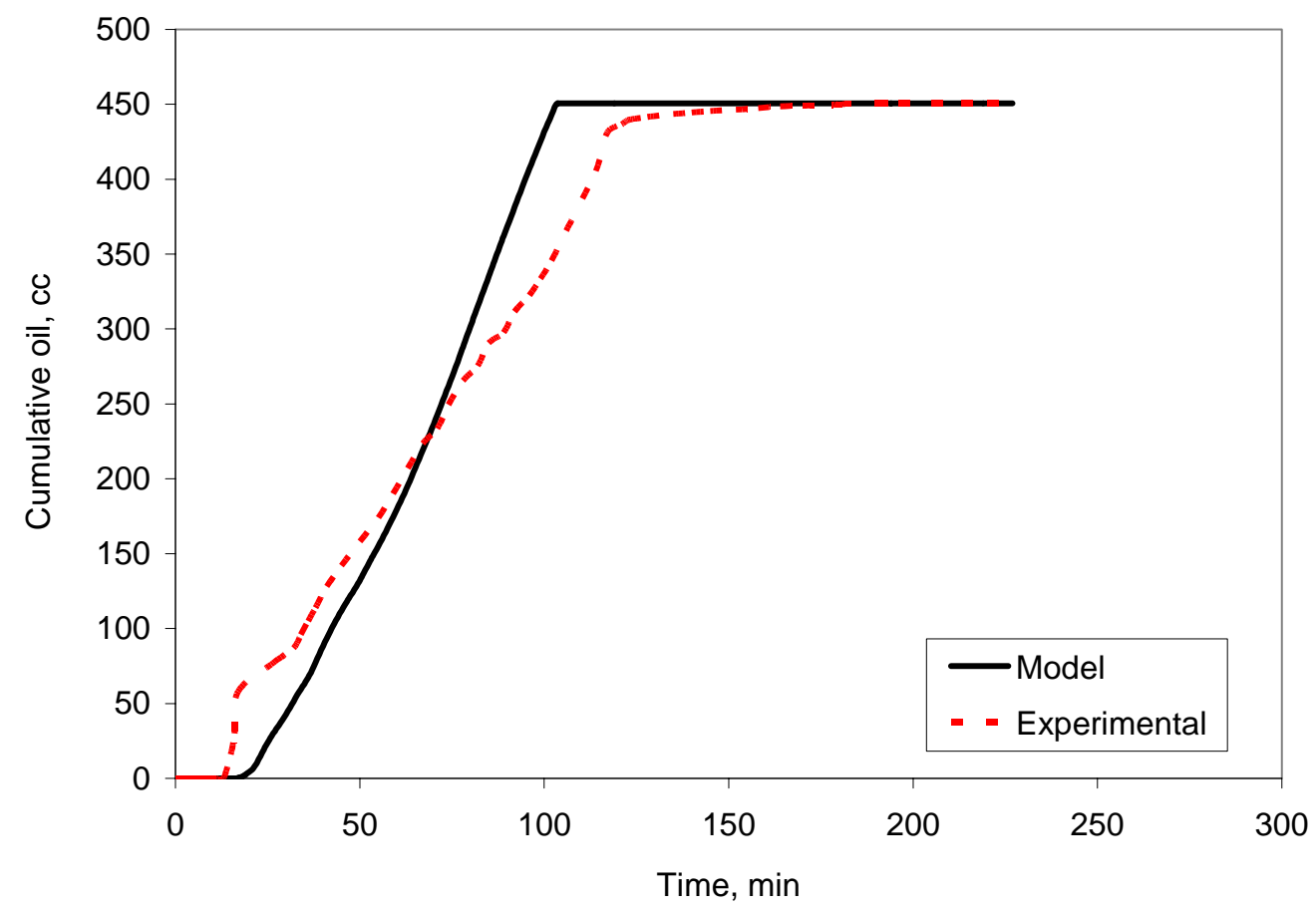

Fig. 5.17- Cumulative oil production for Run 8 (petroleum distillate:steam = 5:100). 


\section{CHAPTER VI}

\section{SUMMARY, CONCLUSIONS AND RECOMMENDATIONS}

\subsection{Summary}

Six successful runs were performed to investigate the feasibility of using propane and petroleum distillate as steam additives to improve injectivity and accelerate oil recovery for San Ardo oil. The injection rate was kept constant at $5.5 \mathrm{ml} / \mathrm{min}$ (cold water equivalent), along with a constant backpressure of 260 psig. Propane:steam and petroleum distillate:steam mass ratios of 5:100 were used in the experiments.

An analytical model to describe steam front advancement and cumulative oil production with respect time was developed. The model is based on heat and material balance and Darcy's Law in one dimensional displacement of oil. The analytical model results and experimental data show very satisfactory history match, verifying validity of the new model.

\subsection{Conclusions}

1. The use of propane as an additive to steam resulted in injection pressures lower than those of pure steam injections. On average, the differential pressure for the steam propane runs is $69 \mathrm{psig}$. In contrast, the pure steam runs have a differential pressure (average) of 97 psig. This represents an increase of steam injectivity of up to $140 \%$ with steam-propane injection.

2. Improvement of injectivity is also found for runs using petroleum distillate as an additive to steam. The average differential pressure for the steam-petroleum distillate runs is 51 psig. This yields an increase of steam injectivity up to $190 \%$ with steampetroleum distillate injection.

3. Ultimate oil recovery is found to be higher for experimental runs using petroleum distillate as an additive (51\% OOIP) followed by propane as the additive (46\% OOIP) compared to that with pure steam (41\% OOIP). 
4. Start of oil production is accelerated when propane and petroleum distillate is added to steam. Average start of oil production of 17 minutes and 14 minutes are observed in the steam-propane and steam-petroleum distillate runs respectively. On the other hand, the average start of oil production for the pure steam runs is 26 minutes.

5. API gravity of the produced oil in all runs tended to increase. For pure steam runs the API gravity increases from $12^{\circ} \mathrm{API}$ for the original oil to $13.5^{\circ} \mathrm{API}$, followed by increases up to 14 and $14.5^{\circ} \mathrm{API}$ for steam-propane and steam-petroleum distillate runs respectively.

6. The produced oil viscosity decreased for all runs. For pure steam runs, oil viscosity decreases at $50^{\circ} \mathrm{C}$ from $2800 \mathrm{cp}$ for the original oil to $2100 \mathrm{cp}$. The produced oil viscosity decreases to 2000 cp and 1800 cp for steam-propane and steam-petroleum distillate respectively.

7. The fastest steam front propagation occurs in steam-petroleum distillate runs, followed by steam-propane and pure steam injection runs. This fact can explain the acceleration in oil production that is observed when petroleum distillate and propane is added to steam. The analytical model of steam front propagation also indicates the same result.

8. The new analytical model of steam front propagation yields a good match with experimental data. The cumulative oil production prediction using the analytical model also gives a good history match. In the model, the average viscosity at initial cell temperature, $2281 \mathrm{cp}$, is reduced to $261 \mathrm{cp}$ for steam propane runs and $227 \mathrm{cp}$ for steam-petroleum distillate runs. This confirms that these steam additives are effective carrier gas.

9. Petroleum distillate is cheaper (116.6 cents per gallon) as compared to propane (128.6 cents per gallon) ${ }^{45}$. Considering the same weight of additive that will be used during the experiment, injecting petroleum distillate as a slug is easier than injecting liquid propane. Thus, using petroleum distillate as an additive appears to be more economical than propane. 


\subsection{Recommendations}

1. Repeat experiments, using another amount of petroleum distillate to decide the optimum ratio that gives the best oil rate and cumulative production.

2. In the analytical model, apply relative permeability data to accurately model the rate profile and effect of multiphase flow in porous media.

3. The use of petroleum distillate slug as an additive is promising in the sense that operationally it is easier to handle as compared to propane and may lead to more economical operations considering it is cheaper than propane.

4. It has been established that steam-petroleum distillate injection accelerates the oil production and slightly increases the oil recovery. In the field, injecting petroleum distillate continuously may lead to a better upgrading of oil production and yield a higher recovery. 


\section{NOMENCLATURE}

$A=$ cross sectional area, $\mathrm{L}^{2}$

$C_{r}=$ specific heat of rock, BTU/lb- ${ }^{\circ} \mathrm{F}$

$C_{o}=$ specific heat of oil, BTU $/ \mathrm{lb}-{ }^{0} \mathrm{~F}$

$C_{w}=$ specific heat of water, BTU/lb- ${ }^{0} \mathrm{~F}$

$d t=$ time interval, day

$d x=$ incremental steam front distance, $\mathrm{ft}$

$\Delta P=$ differential pressure, $\mathrm{m} / \mathrm{Lt}^{2}$

$f_{\text {sdh }}=$ steam quality, fraction

$f_{p}=$ fraction of injected heat that is produced, fraction

$\phi=$ porosity, fraction

$H_{s}=$ heat injected into the injection cell, BTU/D

$I=$ injectivity, $\mathrm{L}^{3} / \mathrm{m} / \mathrm{Lt}^{2}$

$i_{m}=$ steam injection rate, $\mathrm{BTU} / \mathrm{lb}$

$\mathrm{K}=$ effective permeability, $\mathrm{L}^{2}$

$L_{\text {void }}=$ length of void space in the top of sandpack, $\mathrm{cm}$

$L=$ length of porous media, $\mathrm{L}^{2}$

$M_{R}=$ volumetric heat capacity of the reservoir, $\mathrm{BTU} /\left(\mathrm{ft}^{3}-{ }^{\circ} \mathrm{F}\right)$

$\mu=$ fluid viscosity, $\mathrm{m} / \mathrm{Lt}$

$N_{p}=$ cumulative oil production, standard $\mathrm{cm}^{3}$

$p_{a t m}=$ pressure at atmospheric condition $=14.7 \mathrm{psia}$

$\rho_{r}=$ rock grain density, $\mathrm{lb} / \mathrm{ft}^{3}$

$\rho_{o}=$ oil density, $\mathrm{lb} / \mathrm{ft}^{3}$

$\rho_{w}=$ water density, $\mathrm{lb} / \mathrm{ft}^{3}$

$T_{a t m}=$ temperature at atmospheric condition $=460+70=530^{\circ} \mathrm{R}$

$p_{i}=$ injection pressure, $\mathrm{psig}$

$p_{o}=$ outlet pressure, psig 
$Q_{i n j}=$ injection rate, $\mathrm{L}^{3} / \mathrm{t}$

$r=$ radius of the injected cell, $\mathrm{ft}$

$S_{w c}=$ water connate saturation, fraction

$S_{\mathrm{g}}=$ gas saturation, fraction

$S_{g i}=$ initial gas saturation, fraction

$T_{s}=$ injection temperature, ${ }^{\circ} \mathrm{F}$

$T_{o}=$ original reservoir temperature, ${ }^{0} \mathrm{~F}$

$U=$ overall coefficient of heat loss, BTU/(ft-D- $\left.{ }^{\circ} \mathrm{F}\right)$

$x=$ distance of steam front from the top of sand mix, $\mathrm{ft}$ 


\section{REFERENCES}

1. Christianson, B.A., and Berger, E.L.: "San Ardo Field Production Testing System Upgrade,” paper SPE 21533 presented at the 1991 SPE International Thermal Operations Symposium, Bakersfield, CA, 7-8 February.

2. Lolley, C.S., and Richardson, W.C.: "Compositional Input for Reservoir Simulation of Heavy Oils with Application to the San Ardo Field,” paper SPE 37538 presented at the 1997 SPE International Thermal Operations and Heavy Oil Symposium, Bakersfield, CA, 10-12 February.

3. Piper, E.M., Richardson, T.J., and Riddell, A.W.: "Heavy Oil Mining Technical and Economic Analysis,” paper SPE 12788 presented at the 1984 California Regional Meeting, Long Beach, CA, 11-13 April.

4. Goite, J.G.: "Experimental Study of Morichal Heavy Oil Recovery Using Combined Steam and Propane Injection” M.S. Thesis, Texas A\&M University, College Station, TX (1999).

5. Ferguson, M.A.: "Further Experimental Studies of Steam-propane Injection to Enhance Recovery of Morichal Oil,” M.S. Thesis, Texas A\&M University, College Station, TX (2000).

6. Goite, J. G. and Mamora, D. D.: "Experimental Study of Morichal Heavy Oil Recovery Using Combined Steam and Propane Injection," paper SPE 69566 presented at the 2001 SPE Latin American and Caribbean Petroleum Engineering Conference, Buenos Aires, Argentina, 25-28 March.

7. Ferguson, M. A., Mamora, D. D., and Goite, J. G.: "Steam-Propane Injection for Production Enhancement of Heavy Morichal Oil," paper SPE 69689 presented at the 2001 SPE International Thermal Operations and Heavy Oil Symposium, Margarita Island, Venezuela, 12-14 March. 
8. Tinns, J.C.: "Experimental Studies of Steam-Propane Injection to Enhance Recovery of an Intermediate Crude Oil,” M.S. Thesis, Texas A\&M University, College Station, TX (2001).

9. Rivero, J.A.: “Experimental Studies of Enhancement of Injectivity and In-situ Oil Upgrading by Steam Propane Injection for the Hamaca Oil Field,” M.S. Thesis, Texas A\&M University., College Station, TX (2001).

10. Rivero, J.A., and Mamora D.D.: "Production Acceleration and Injectivity Enhancement Using Steam-Propane Injection for Hamaca Extra-Heavy Oil,” paper SPE 75129 presented at the 2002 SPE/DOE Improved Oil Recovery Symposium, Tulsa, OK, 13-17 April.

11. Plazas, J.V.: "Experimental Studies of Oil Yield and Properties of Light and Medium Venezuelan Crude Oils Under Steam and Steam-Propane Distillation,” M.S. Thesis, Texas A\&M University, College Station, TX (2002).

12. Venturini, G.J.: "Simulation Studies of Steam-propane Injection for the Hamaca heavy Oil Field,” M.S. Thesis, Texas A\&M University, College Station, TX (2002).

13. Hendroyono, A.: "Experimental Studies of Steam-Propane Injection for the Duri Intermediate Crude Oil,” M.S. Thesis, Texas A\&M University, College Station, TX (2003).

14. Venturini, G.J., and Mamora, D.D.: "Simulation Studies of Steam-Propane Injection for the Hamaca Heavy Oil Field,” paper 2003-056 presented at the 2003 Petroleum's Society’s Canadian International Conference, Calgary, Alberta, Canada, 10-12 June.

15. Mamora, D. D., Rivero, J.A., Hendroyono, A., and Venturini, G.J.: "Experimental and Simulation Studies of Steam-Propane Injection for the Hamaca and Duri Field," paper SPE 84201 presented at the 2003 SPE Annual Technical Conference and Exhibition, Denver, Colorado, 5-8 October. 
16. Ramirez-Garnicas, M. A.: “Experimental and Analytical Studies of Hydrocarbon Yields under Dry-, Steam-, and Steam with Propane- Distillation,” Ph.D. Dissertation, Texas A\&M University, College Station, TX (2004).

17. Nesse, T.: "Experimental Comparison of Hot Water/Propane Injection to Steam/Propane Injection for Recovery of Heavy Oil,” M.S. Thesis, Texas A\&M University, College Station, TX (2004).

18. Venturini, G.J., and Mamora, D.D.: “Simulation Studies Steam-Propane Injection for the Hamaca Heavy Oil Field,” paper 2003-056, JCPT (Sept. 2004) 10-20.

19. Rivero, J.A., and Mamora, D.D.: "Production Acceleration and Injectivity Enhancement Using Steam-Propane Injection for Hamaca Extra-Heavy Oil,” paper 2003-10, JCPT (Feb. 2005) 1-7.

20. Prats, M.: Thermal Recovery, Monograph Series 7, SPE, Richardson, TX (1982) 20-40.

21. Redford, D.A.: "The Use of Solvents and Gasses with Steam in the Recovery of Bitumen from Oil Sands,” JCPT (Jan.-Feb. 1982) 45-53.

22. Harding, T.G., Farouq-Ali, S.M. and Flock, D.L.: "Steam Performance in the Presence of Carbon Dioxide and Nitrogen,” JCPT (Sep.-Oct. 1983) 30-37.

23. Stone, T. and Malcolm, J.D.: "Simulation of a Large Steam- $\mathrm{CO}_{2}$ Coinjection Experiment,” JCPT (Nov.-Dec. 1985) 51-59.

24. Stone, T. and Ivory, J.: “An Examination of Steam- $\mathrm{CO}_{2}$ Processes,” JCPT (MayJune 1987) 54-61.

25. Nasr, T.N., Prowse, D.R. and Frauenfeld. T.W.J.: “The Use of Flue Gas with Steam in Bitumen Recovery from Oil Sands,” JCPT (May-June 1987) 62-69. 
26. Fraunfeld, T.W.J., Ridley, R.K. and Nguyen, D.M.: "Effect of an Initial Gas Content on Thermal EOR as Applied to Oil Sands,” JCPT (March 1988) 333-338.

27. Metwally, M.: "Effect of Gaseous Additives on Steam Processes for Lindbergh Field, Alberta,” JCPT (Nov.-Dec. 1990) 26-30.

28. Gumrah, F. and Okandan, E.: "Steam- $\mathrm{CO}_{2}$ Flooding: An Experimental Study," InSitu, 16, (1992), No.2, 89-108.

29. Bagci, S. and Gumrah, F.: "Steam-Gas Drive Laboratory Tests for Heavy-Oil Recovery,” In-Situ, 22, (1998), No.3, 263-289.

30. Butler, R.M. and Mokrys, I.J.: “A New Process (VAPEX) for Recovering Heavy Oils Using Hot Water and Hydrocarbon Vapour,” JCPT (Jan.-Feb. 1991) 97-106.

31. Butler, R.M. and Mokrys, I.J.: “In-Situ Upgrading of Heavy Oils and Bitumen by Propane Deasphalting: The Vapex Process,” SPE paper No. 25452 presented at the 1993 Production and Operations Symposium held in Oklahoma City, OK, March 2123.

32. Butler, R.M. and Mokrys, I.J.: "Recovery of Heavy Oils Using Vapourized Hydrocarbon Solvents: Further Development of the Vapex Process,” JCPT (June 1993) 56-62.

33. Butler, R.M. and Mokrys, I.J.: “Cloded Loop Extraction Method for the Recovery of Heavy Oils and Bitumens Underlain by Aquifers: The Vapex Process,” JCPT (June 1993) 56-62.

34. Léauté, R.P.: “Liquid Addition to Steam for Enhancing Recovery (LASER) of Bitumen with CSS: Evolution of Technology from Research Concept to A Field Pilot at Cold Lake,” paper SPE 79011 presented at the 2002 SPE International Thermal Operations and Heavy Oil Symposium and International Well Technology Conference, Calgary, Alberta, 4-7 November, 2002. 
35. Lauwerier, H.A.:”'The Transport of Heat in an Oil Layer Caused by the Injection of Hot Fluid,” Applied Scientific Research, (1955), Section A (2-3), 145-150.

36. Marx, J.W. and Langenheim, R.H.: "Reservoir Heating by Hot Fluid Injection,” JPT (Jan.-Feb. 1992), 302-312.

37. Ramey, H.J., Jr.: “Discussion on the Paper by Marx and Langenheim,” JPT (MarApr. 1959), 312-316.

38. Willman, B.T, Valleroy, V.V., Rumberg, G.W. and Cornelius, A.J.: “Laboratory Studies of Oil Recovery by Steam Injection,” JPT (July 1961), 681-690.

39. Mandl, C. and Volek, C.W.: "Heat and Mass Transport in Steam-Drive Processes," SPEJ (March 1969), 59-79.

40. Van Lookeren, J.: “Calculation Methods for Linear and Radial Steam Flow in Oil Reservoirs,” SPEJ (June 1983), 427-439.

41. Dake, L.P.: Fundamental of Reservoir Engineering, Elsevier Scientific Publishing Company, New York (1978).

42. Prats, M.: “A Current Appraisal of Thermal Recovery,” JPT (Aug. 1978), 11291136.

43. Myhill, N.A. and Stegemeier, G.L.: “Steam-Drive Correlation and Prediction,” JPT (Feb. 1978), 173-182.

44. Neuman, C.H.: “A Mathematical Model of the Steam Drive Process-Application,” SPE paper 4757, presented at the 1975 Annual California Regional Meeting of SPE, Ventura, CA, 2-4 April.

45. Rhee, S.W. and Doscher, T.M.: “A Method of Predicting Oil Recovery by Steamflooding Including the Effect of Distillation and Gravity Override,” JPT (Aug. 1980), 249-266. 
46. Jones, J.: “Steam Drive Model for Hand-held Programmable Calculators," JPT (Sept. 1981), 1583-1598.

47. “West Texas Intermediate Crude Oil Prices,” Petroleum Marketing Monthly, http://www.eia.doe.gov/oil_gas/petroleum/data_publications/petroleum_marketing_ monthly/pmm.html (2003). 


\section{APPENDIX A}

\section{CALCULATION OF FLUID SATURATIONS AND PORE VOLUME}

The following is a sample calculation of the fluid saturations and pore volume inside the cell. The calculations are made for run 4 .

1. Cell dimension:

diameter, $d=7.376 \mathrm{~cm}$

height, $h=66.08 \mathrm{~cm}$

Since the cell is cylindrical, the volume of the cell is:

$V_{\text {cell }}=2823.59 \mathrm{~cm}^{3}$

2. The total weight of mixture $\left(W_{\text {mix }}\right)$ :

Weight of sand, $W_{\text {sand }} \quad=5141 \mathrm{~g}$

Weight of water, $W_{\text {water }}=226 \mathrm{~g}$

Weight of oil, $W_{\text {oil }} \quad=1000 \mathrm{~g}$

$W_{\text {mix }}=W_{\text {sand }}+W_{\text {water }}+W_{\text {oil }}$

$W_{\text {mix }}=5141+226+1000 \mathrm{~g}=6367 \mathrm{~g}$

3. The weight of mixture inside the cell, $W_{\text {mix }}$ in cell

Weight of empty cell $=4339 \mathrm{~g}$

Weight of cell with mixture inside $=9798 \mathrm{~g}$

Weight of oil left over $24 \mathrm{hrs}$ cell heating $=50 \mathrm{~g}$

Weight of mixture inside the cell, $W_{\text {mixcell }}=9798-4339-50=5409 \mathrm{~g}$

4. Since the mixture is homogenous, the proportions of sand, water and oil remain constant before and after packing. The amount of each component inside the cell is then calculated below: 
Weight of sand inside the cell, $\mathrm{W}_{\text {sand }}=\frac{W_{\text {mixcell }}}{W_{\text {mix }}} W_{\text {sand }}=\frac{5409 \mathrm{~g}}{6317 \mathrm{~g}} \times 5141 \mathrm{~g}=4402 \mathrm{~g}$

Weight of water inside the cell, $\mathrm{W}_{\text {water }}=\frac{W_{\text {mixcell }}}{W_{\text {mix }}} W_{\text {water }}=\frac{5409 \mathrm{~g}}{6317 \mathrm{~g}} \times 226 \mathrm{~g}=192 \mathrm{~g}$

Weight of oil inside the cell, $\mathrm{W}_{\text {oil }}=\frac{W_{\text {mixcell }}}{W_{\text {mix }}} W_{\text {oil }}=\frac{5409 \mathrm{~g}}{6317 \mathrm{~g}} \times 1000 \mathrm{~g}=850 \mathrm{~g}$

5. The sand density, $\rho=2.65 \mathrm{~g} / \mathrm{cm}^{3}$. Thus,

Volume of sand inside the cell $=\frac{W_{\text {sandcell }}}{\rho_{\text {sand }}}=\frac{4402}{2.65}=1661 \mathrm{~g}$

6. Porosity inside the cell is calculated as the following:

$\phi=\frac{V_{\text {cell }}-V_{\text {sandcell }}}{V_{\text {cell }}}=\frac{2824-1661}{2824}=0.4117$

7. The original San Ardo oil has an oil gravity of $12^{\circ} \mathrm{API}$ which is equivalent $0.9861 \mathrm{~g} / \mathrm{cm}^{3}$. Water and oil volumes inside the cell are calculated by:

$$
\begin{aligned}
& \mathrm{V}_{\text {watercell }}=\frac{W_{\text {watercell }}}{\rho_{\text {water }}}=\frac{192}{1}=192 \mathrm{~cm}^{3} \\
& \mathrm{~V}_{\text {oilcell }}=\frac{W_{\text {oilcell }}}{\rho_{\text {oil }}}=\frac{862}{0.9862}=862 \mathrm{~cm}^{3}
\end{aligned}
$$

8. The pore volume of the cell is calculated as the following:

$$
V_{\text {pcell }}=V_{\text {cell }} \times \phi=2824 \times 0.4117=1162 \mathrm{~cm}^{3}
$$

9. The saturation of oil $\left(S_{o}\right)$ and water $\left(S_{w}\right)$ are calculated as the following:

$$
\begin{aligned}
& S_{w}=\frac{V_{\text {watercell }}}{V_{\text {pcell }}}=\frac{192}{1162}=0.1652 \\
& S_{o}=\frac{V_{\text {oilcell }}}{V_{\text {peell }}}=\frac{862}{1162}=0.7411
\end{aligned}
$$


10. The nitrogen saturation $\left(S_{g}\right)$ is calculated as the following:

$$
S_{g}=1-S_{w}-S_{o}=1-0.1652-0.7411=0.0937
$$

11. The nitrogen volume in cell is calculated as the following:

$$
V_{\text {aircell }}=S_{g} \times V_{\text {pcell }}=0.0937 \times 2824=109 \mathrm{~cm}^{3}
$$

12. The fill-up time, $t_{f u}$ is calculated by:

Steam injection rate, $i_{s}=5.5 \mathrm{~cm}^{3} / \mathrm{min}$

$$
t_{f u}=\frac{\text { Air pore volume }}{i_{s}}=\frac{109}{5.5}=19.8 \mathrm{~min}
$$




\section{APPENDIX B}

\section{TEMPERATURE AND PRESSURE DATA}

The data in the following table is:

T-steam: Steam injection temperature at $30.5 \mathrm{~cm}$ from the top of thermocouples sheat

T1 : Temperature at $45.1 \mathrm{~cm}$ from the top of thermocouples sheat

T2 : Temperature at $60.2 \mathrm{~cm}$ from the top of thermocouples sheat

T3 : Temperature at $75.7 \mathrm{~cm}$ from the top of thermocouples sheat

T4 : Temperature at $91 \mathrm{~cm}$ from the top of thermocouples sheat

T5 : Temperature at $116.5 \mathrm{~cm}$ from the top of thermocouples sheat

Pinj : Injection pressure

Pout : Production pressure

Qinj : Propane injection rate

Vw : water injection rate

TABLE B1. PRESSURE AND TEMPERATURE DATA FOR RUN 3 (PROPANE:STEAM = 5:100).

\begin{tabular}{|c|c|c|c|c|c|c|c|c|c|c|}
\hline $\begin{array}{c}\text { Time, } \\
\text { min }\end{array}$ & $\begin{array}{c}\text { T-steam } \\
{ }^{\circ} \mathrm{C}\end{array}$ & $\begin{array}{l}\mathrm{T} 1, \\
{ }^{\circ} \mathrm{C}\end{array}$ & $\begin{array}{l}\mathrm{T} 2, \\
{ }^{\circ} \mathrm{C}\end{array}$ & $\begin{array}{l}\mathrm{T} 3, \\
{ }^{\circ} \mathrm{C}\end{array}$ & $\begin{array}{l}\mathrm{T} 4, \\
{ }^{\circ} \mathrm{C}\end{array}$ & $\begin{array}{l}\mathrm{T} 5, \\
{ }^{\circ} \mathrm{C}\end{array}$ & $\begin{array}{l}\text { Pinj, } \\
\text { psig }\end{array}$ & $\begin{array}{c}\text { Pout, } \\
\text { psig }\end{array}$ & $\begin{array}{l}\text { Qinj, } \\
\text { g/min }\end{array}$ & $\begin{array}{c}\mathrm{Vw} \\
\mathrm{ml} / \mathrm{min}\end{array}$ \\
\hline 0.0512 & 235.59 & 60.89 & 52.06 & 53.90 & 53.87 & 49.92 & 278.55 & 261.93 & 0.2748 & 5.5347 \\
\hline 0.5455 & 254.67 & 166.49 & 52.06 & 54.02 & 53.87 & 49.99 & 288.36 & 266.00 & 0.2757 & 5.5826 \\
\hline 1.0453 & 263.17 & 205.57 & 52.10 & 54.12 & 53.97 & 50.04 & 325.14 & 270.74 & 0.2756 & 5.5617 \\
\hline 1.5452 & 269.27 & 209.03 & 52.14 & 54.18 & 54.03 & 50.05 & 344.16 & 272.44 & 0.2748 & 5.5415 \\
\hline 2.0440 & 264.85 & 210.19 & 52.16 & 54.22 & 54.05 & 50.03 & 335.65 & 270.98 & 0.2743 & 5.5314 \\
\hline 2.5438 & 262.14 & 210.14 & 52.15 & 54.22 & 54.03 & 50.01 & 316.01 & 270.24 & 0.2760 & 5.5579 \\
\hline 3.0447 & 258.76 & 210.86 & 52.09 & 54.18 & 54.03 & 50.01 & 307.27 & 269.93 & 0.2763 & 5.5705 \\
\hline 3.5453 & 255.19 & 211.26 & 52.04 & 54.20 & 54.03 & 50.02 & 303.15 & 269.70 & 0.2763 & 5.5677 \\
\hline 4.0452 & 249.87 & 211.79 & 52.09 & 54.21 & 54.05 & 50.04 & 302.49 & 269.73 & 0.2768 & 5.5338 \\
\hline 4.5450 & 243.72 & 211.60 & 52.29 & 54.23 & 54.05 & 50.04 & 300.54 & 269.88 & 0.2774 & 5.5221 \\
\hline
\end{tabular}


TABLE B1.- CONTINUED.

\begin{tabular}{|c|c|c|c|c|c|c|c|c|c|c|}
\hline $\begin{array}{l}\text { Time, } \\
\text { min }\end{array}$ & $\begin{array}{c}\text { T-steam } \\
{ }^{\circ} \mathrm{C}\end{array}$ & $\begin{array}{l}\mathrm{T} 1, \\
{ }^{\circ} \mathrm{C}\end{array}$ & $\begin{array}{l}\mathrm{T} 2, \\
{ }^{\circ} \mathrm{C}\end{array}$ & $\begin{array}{l}\mathrm{T} 3, \\
{ }^{\circ} \mathrm{C}\end{array}$ & $\begin{array}{l}\mathrm{T} 4, \\
{ }^{\circ} \mathrm{C}\end{array}$ & $\begin{array}{l}\mathrm{T} 5, \\
{ }^{\circ} \mathrm{C}\end{array}$ & $\begin{array}{l}\text { Pinj, } \\
\text { psig }\end{array}$ & $\begin{array}{l}\text { Pout, } \\
\text { psig }\end{array}$ & $\begin{array}{l}\text { Qinj, } \\
\text { g/min }\end{array}$ & $\begin{array}{c}\mathrm{Vw} \\
\mathrm{ml} / \mathrm{min}\end{array}$ \\
\hline 5.0448 & 235.41 & 210.01 & 52.40 & 54.23 & 54.06 & 50.06 & 298.98 & 270.01 & 0.2756 & 5.5433 \\
\hline 5.5455 & 229.93 & 208.65 & 52.42 & 54.27 & 54.06 & 50.04 & 297.30 & 269.82 & 0.2766 & 5.5828 \\
\hline 6.0453 & 226.60 & 208.18 & 52.42 & 54.30 & 54.04 & 50.04 & 296.81 & 269.79 & 0.2770 & 5.5521 \\
\hline 6.5452 & 224.27 & 208.42 & 52.46 & 54.30 & 54.06 & 50.04 & 297.68 & 269.76 & 0.2760 & 5.5337 \\
\hline 7.0450 & 225.10 & 208.65 & 52.48 & 54.32 & 54.04 & 50.04 & 291.35 & 269.88 & 0.5374 & 5.5291 \\
\hline 7.5448 & 229.91 & 208.86 & 52.48 & 54.31 & 54.04 & 50.02 & 294.53 & 269.91 & 0.2701 & 5.5744 \\
\hline 8.0447 & 234.68 & 208.60 & 52.50 & 54.31 & 54.04 & 50.02 & 294.26 & 269.71 & 0.2760 & 5.5872 \\
\hline 8.5453 & 235.12 & 209.31 & 52.55 & 54.29 & 54.06 & 50.02 & 294.03 & 269.56 & 0.2739 & 5.5738 \\
\hline 9.0452 & 232.65 & 210.26 & 52.63 & 54.30 & 54.04 & 50.02 & 295.52 & 269.52 & 0.2761 & 5.5451 \\
\hline 9.5450 & 231.67 & 210.92 & 52.73 & 54.25 & 54.04 & 50.02 & 298.58 & 269.58 & 0.2765 & 5.5413 \\
\hline 10.0458 & 231.09 & 211.81 & 52.86 & 54.23 & 54.06 & 50.06 & 301.84 & 270.24 & 0.2766 & 5.5335 \\
\hline 10.5447 & 232.67 & 212.01 & 53.03 & 54.23 & 54.08 & 50.08 & 306.28 & 270.52 & 0.2756 & 5.5965 \\
\hline 11.0455 & 232.88 & 211.69 & 53.26 & 54.23 & 54.07 & 50.08 & 305.71 & 270.56 & 0.2766 & 5.5799 \\
\hline 11.5453 & 232.31 & 211.44 & 53.50 & 54.21 & 54.07 & 50.06 & 307.17 & 270.50 & 0.2758 & 5.5428 \\
\hline 12.0450 & 232.70 & 210.88 & 53.86 & 54.24 & 54.07 & 50.07 & 304.84 & 270.36 & 0.2763 & 5.5274 \\
\hline 12.5448 & 235.26 & 210.66 & 54.28 & 54.24 & 54.09 & 50.05 & 309.70 & 270.11 & 0.2758 & 5.5316 \\
\hline 13.0457 & 231.77 & 211.45 & 54.54 & 54.22 & 54.11 & 50.05 & 311.76 & 270.37 & 0.2761 & 5.5575 \\
\hline 13.5455 & 232.44 & 211.86 & 54.98 & 54.22 & 54.18 & 50.07 & 315.41 & 270.54 & 0.2761 & 5.5484 \\
\hline 14.0443 & 232.53 & 211.91 & 55.55 & 54.20 & 54.24 & 50.09 & 317.24 & 270.54 & 0.2764 & 5.5162 \\
\hline 14.5452 & 232.98 & 211.77 & 56.13 & 54.18 & 54.27 & 50.08 & 319.75 & 270.54 & 0.2757 & 5.4871 \\
\hline 15.0450 & 237.26 & 211.96 & 56.72 & 54.17 & 54.27 & 50.10 & 323.12 & 270.41 & 0.2747 & 5.5375 \\
\hline 15.5457 & 232.08 & 212.55 & 57.23 & 54.15 & 54.27 & 50.08 & 324.67 & 270.33 & 0.2762 & 5.5236 \\
\hline 16.0455 & 231.32 & 212.60 & 57.93 & 54.15 & 54.25 & 50.10 & 324.22 & 270.39 & 0.2760 & 5.5252 \\
\hline 16.5453 & 232.97 & 213.01 & 58.63 & 54.13 & 54.24 & 50.11 & 326.68 & 270.33 & 0.2762 & 5.5343 \\
\hline 17.0452 & 232.50 & 213.51 & 59.24 & 54.13 & 54.24 & 50.17 & 330.48 & 270.34 & 0.2758 & 5.5559 \\
\hline 17.5440 & 232.43 & 213.67 & 60.01 & 54.14 & 54.26 & 50.34 & 327.19 & 269.98 & 0.2753 & 5.5800 \\
\hline 18.0457 & 234.40 & 213.74 & 60.77 & 54.12 & 54.27 & 50.47 & 329.79 & 257.31 & 0.2759 & 5.5625 \\
\hline 18.5447 & 236.17 & 214.58 & 61.60 & 54.10 & 54.31 & 50.56 & 332.88 & 264.90 & 0.2756 & 5.5428 \\
\hline 19.0453 & 234.46 & 214.25 & 62.45 & 54.12 & 54.34 & 50.59 & 325.91 & 253.94 & 0.2759 & 5.5299 \\
\hline 19.5452 & 234.53 & 214.20 & 63.28 & 54.08 & 54.38 & 50.63 & 325.66 & 259.36 & 0.2763 & 5.5556 \\
\hline 20.0450 & 236.80 & 214.62 & 64.05 & 54.05 & 54.38 & 50.57 & 330.65 & 262.13 & 0.2761 & 5.5807 \\
\hline 20.5448 & 233.81 & 214.19 & 64.80 & 54.05 & 54.41 & 50.55 & 324.04 & 254.73 & 0.2746 & 5.5627 \\
\hline 21.0447 & 234.06 & 213.94 & 65.71 & 54.07 & 54.45 & 50.55 & 325.41 & 254.29 & 0.2749 & 5.5597 \\
\hline 21.5453 & 234.07 & 213.86 & 66.55 & 54.05 & 54.46 & 50.54 & 322.64 & 253.12 & 0.2757 & 5.5270 \\
\hline 22.0452 & 234.23 & 213.89 & 67.40 & 54.06 & 54.48 & 50.54 & 323.39 & 253.15 & 0.2754 & 5.5205 \\
\hline 22.5450 & 233.67 & 213.87 & 68.26 & 54.06 & 54.51 & 50.57 & 322.33 & 252.75 & 0.2755 & 5.5539 \\
\hline 23.0448 & 233.99 & 213.83 & 69.26 & 54.07 & 54.55 & 50.59 & 322.75 & 252.95 & 0.2727 & 5.5698 \\
\hline 23.5455 & 234.42 & 213.90 & 70.29 & 54.07 & 54.58 & 50.66 & 323.37 & 252.38 & 0.2758 & 5.5568 \\
\hline 24.0453 & 233.46 & 214.04 & 71.15 & 54.08 & 54.60 & 50.77 & 322.89 & 254.00 & 0.2753 & 5.5187 \\
\hline 24.5452 & 232.40 & 214.05 & 72.20 & 54.10 & 54.59 & 50.79 & 323.53 & 252.86 & 0.2757 & 5.5382 \\
\hline 25.0442 & 232.56 & 214.16 & 73.27 & 54.11 & 54.59 & 50.86 & 321.75 & 254.09 & 0.7071 & 5.5726 \\
\hline 25.5448 & 233.33 & 214.03 & 74.26 & 54.15 & 54.60 & 50.87 & 319.48 & 254.08 & 0.1894 & 5.5913 \\
\hline 26.0457 & 232.77 & 214.01 & 75.25 & 54.16 & 54.62 & 50.93 & 324.05 & 254.69 & 0.2778 & 5.5628 \\
\hline 26.5453 & 232.26 & 214.27 & 76.19 & 54.20 & 54.60 & 50.98 & 315.99 & 256.32 & 0.2757 & 5.5313 \\
\hline 27.0443 & 231.47 & 214.63 & 77.25 & 54.23 & 54.63 & 50.99 & 317.36 & 255.91 & 0.2756 & 5.5325 \\
\hline 27.5450 & 230.73 & 214.77 & 78.68 & 54.28 & 54.61 & 51.05 & 322.84 & 256.83 & 0.2772 & 5.5568 \\
\hline 28.0448 & 232.04 & 214.96 & 80.25 & 54.34 & 54.62 & 51.10 & 323.31 & 261.20 & 0.2758 & 5.5745 \\
\hline 28.5457 & 231.69 & 214.87 & 81.83 & 54.39 & 54.64 & 51.13 & 323.37 & 261.22 & 0.2764 & 5.5481 \\
\hline 29.0455 & 232.58 & 214.93 & 83.33 & 54.42 & 54.63 & 51.15 & 326.15 & 260.07 & 0.2764 & 5.5279 \\
\hline 29.5453 & 231.70 & 214.96 & 84.71 & 54.47 & 54.63 & 51.22 & 329.88 & 259.54 & 0.7071 & 5.5243 \\
\hline
\end{tabular}


TABLE B1.- CONTINUED.

\begin{tabular}{|c|c|c|c|c|c|c|c|c|c|c|}
\hline $\begin{array}{l}\text { Time, } \\
\text { min }\end{array}$ & $\begin{array}{c}\text { T-steam } \\
{ }^{\circ} \mathrm{C}\end{array}$ & $\begin{array}{l}\mathrm{T} 1, \\
{ }^{\circ} \mathrm{C}\end{array}$ & $\begin{array}{l}\mathrm{T} 2, \\
{ }^{\circ} \mathrm{C}\end{array}$ & $\begin{array}{l}\mathrm{T} 3, \\
{ }^{\circ} \mathrm{C}\end{array}$ & $\begin{array}{l}\mathrm{T} 4, \\
{ }^{\circ} \mathrm{C}\end{array}$ & $\begin{array}{l}\mathrm{T} 5, \\
{ }^{\circ} \mathrm{C}\end{array}$ & $\begin{array}{l}\text { Pinj, } \\
\text { psig }\end{array}$ & $\begin{array}{l}\text { Pout, } \\
\text { psig }\end{array}$ & $\begin{array}{l}\text { Qinj, } \\
\text { g/min }\end{array}$ & $\begin{array}{c}\mathrm{Vw} \\
\mathrm{ml} / \mathrm{min}\end{array}$ \\
\hline 30.0450 & 231.25 & 215.09 & 86.18 & 54.55 & 54.66 & 51.27 & 326.76 & 258.38 & 0.3634 & 5.5693 \\
\hline 30.5448 & 231.71 & 214.96 & 87.65 & 54.62 & 54.70 & 51.29 & 327.79 & 260.21 & 0.2780 & 5.5626 \\
\hline 31.0457 & 231.44 & 214.59 & 89.32 & 54.71 & 54.73 & 51.38 & 327.80 & 259.12 & 0.2772 & 5.5440 \\
\hline 31.5455 & 232.22 & 214.95 & 90.62 & 54.78 & 54.74 & 51.39 & 324.86 & 257.50 & 0.2773 & 5.5162 \\
\hline 32.0453 & 231.75 & 215.41 & 91.94 & 54.83 & 54.74 & 51.47 & 329.15 & 259.90 & 0.2762 & 5.5296 \\
\hline 32.5460 & 230.60 & 215.08 & 93.70 & 54.94 & 54.77 & 51.48 & 328.29 & 260.64 & 0.2753 & 5.5769 \\
\hline 33.0440 & 231.49 & 215.24 & 95.63 & 55.03 & 54.81 & 51.53 & 325.16 & 259.86 & 0.2767 & 5.5700 \\
\hline 33.5457 & 230.79 & 215.18 & 97.37 & 55.14 & 54.82 & 51.55 & 328.64 & 260.83 & 0.2758 & 5.5412 \\
\hline 34.0445 & 231.00 & 215.21 & 99.29 & 55.23 & 54.83 & 51.60 & 329.77 & 260.51 & 0.2761 & 5.5153 \\
\hline 34.5453 & 230.58 & 215.17 & 101.29 & 55.36 & 54.87 & 51.63 & 329.54 & 260.59 & 0.2764 & 5.5361 \\
\hline 35.0452 & 230.33 & 215.10 & 103.40 & 55.49 & 54.86 & 51.68 & 329.50 & 260.64 & 0.2746 & 5.5467 \\
\hline 35.5450 & 230.86 & 214.90 & 105.68 & 55.62 & 54.88 & 51.74 & 329.69 & 260.89 & 0.7068 & 5.5537 \\
\hline 36.0457 & 231.22 & 214.75 & 107.84 & 55.77 & 54.93 & 51.79 & 328.72 & 261.20 & 0.2785 & 5.5409 \\
\hline 36.5455 & 231.03 & 214.80 & 109.90 & 55.91 & 54.94 & 51.82 & 329.34 & 260.44 & 0.2762 & 5.5216 \\
\hline 37.0453 & 230.54 & 214.68 & 111.87 & 56.04 & 54.96 & 51.88 & 323.51 & 259.92 & 0.2770 & 5.5453 \\
\hline 37.5452 & 229.95 & 215.07 & 114.05 & 56.19 & 54.97 & 51.91 & 325.97 & 259.78 & 0.2772 & 5.5500 \\
\hline 38.0440 & 230.43 & 214.78 & 117.03 & 56.33 & 54.97 & 51.94 & 325.45 & 260.21 & 0.2777 & 5.5079 \\
\hline 38.5448 & 230.46 & 214.76 & 120.21 & 56.50 & 54.98 & 51.98 & 325.84 & 259.81 & 0.2761 & 5.4960 \\
\hline 39.0447 & 230.76 & 214.47 & 123.25 & 56.70 & 55.01 & 52.05 & 330.18 & 259.85 & 0.2745 & 5.5389 \\
\hline 39.5453 & 231.63 & 214.38 & 126.32 & 56.89 & 55.03 & 52.10 & 329.32 & 259.49 & 0.2760 & 5.5663 \\
\hline 40.0452 & 231.52 & 214.80 & 129.42 & 57.09 & 55.06 & 52.13 & 330.80 & 259.67 & 0.2769 & 5.5452 \\
\hline 40.5442 & 231.32 & 214.53 & 132.56 & 57.32 & 55.07 & 52.18 & 330.57 & 259.21 & 0.2762 & 5.5222 \\
\hline 41.0448 & 231.06 & 214.72 & 135.69 & 57.54 & 55.13 & 52.24 & 331.92 & 259.10 & 0.2763 & 5.5509 \\
\hline 41.5447 & 230.86 & 214.50 & 139.16 & 57.76 & 55.16 & 52.27 & 327.67 & 259.29 & 0.2773 & 5.5777 \\
\hline 42.0453 & 231.18 & 214.46 & 142.82 & 58.00 & 55.20 & 52.32 & 347.01 & 259.46 & 0.7071 & 5.5878 \\
\hline 42.5452 & 229.62 & 213.54 & 148.73 & 58.30 & 55.23 & 52.36 & 367.37 & 259.81 & 0.2491 & 5.5609 \\
\hline 43.0450 & 233.91 & 212.86 & 157.28 & 58.85 & 55.28 & 52.39 & 358.66 & 259.24 & 0.2799 & 5.5264 \\
\hline 43.5448 & 236.95 & 212.82 & 160.92 & 59.35 & 55.35 & 52.48 & 340.71 & 258.70 & 0.2776 & 5.5372 \\
\hline 44.0465 & 237.55 & 210.79 & 161.08 & 59.65 & 55.40 & 52.51 & 317.97 & 258.39 & 0.2770 & 5.5678 \\
\hline 44.5453 & 236.65 & 212.72 & 161.56 & 59.97 & 55.47 & 52.54 & 329.29 & 259.61 & 0.2752 & 5.5867 \\
\hline 45.0443 & 230.77 & 213.56 & 162.37 & 60.38 & 55.51 & 52.58 & 323.32 & 259.35 & 0.2774 & 5.5503 \\
\hline 45.5450 & 228.84 & 213.28 & 163.52 & 60.76 & 55.58 & 52.63 & 317.21 & 259.40 & 0.2766 & 5.5341 \\
\hline 46.0448 & 229.98 & 213.44 & 164.65 & 61.13 & 55.65 & 52.68 & 319.97 & 257.87 & 0.2772 & 5.5386 \\
\hline 46.5447 & 230.37 & 213.61 & 165.96 & 61.47 & 55.68 & 52.66 & 317.24 & 258.88 & 0.2772 & 5.5431 \\
\hline 47.0455 & 231.27 & 213.41 & 167.74 & 61.80 & 55.75 & 52.65 & 319.96 & 256.90 & 0.2745 & 5.5622 \\
\hline 47.5453 & 230.86 & 213.73 & 169.68 & 62.18 & 55.80 & 52.69 & 322.90 & 259.32 & 0.2747 & 5.5681 \\
\hline 48.0450 & 231.19 & 213.56 & 171.76 & 62.59 & 55.82 & 52.70 & 323.75 & 259.31 & 0.2760 & 5.5259 \\
\hline 48.5448 & 231.17 & 213.74 & 173.91 & 63.06 & 55.89 & 52.75 & 321.91 & 259.21 & 0.2770 & 5.5361 \\
\hline 49.0457 & 230.52 & 213.77 & 175.43 & 63.58 & 55.92 & 52.77 & 322.71 & 258.82 & 0.2752 & 5.5448 \\
\hline 49.5463 & 230.80 & 213.87 & 176.98 & 64.12 & 55.96 & 52.78 & 321.27 & 259.59 & 0.2766 & 5.5755 \\
\hline 50.0453 & 230.19 & 213.90 & 178.59 & 64.74 & 55.99 & 52.81 & 323.42 & 259.46 & 0.2760 & 5.5713 \\
\hline 50.5452 & 229.17 & 214.01 & 180.25 & 65.40 & 56.04 & 52.81 & 323.67 & 258.75 & 0.2735 & 5.5403 \\
\hline 51.0450 & 229.27 & 213.96 & 181.54 & 66.09 & 56.09 & 52.84 & 321.12 & 259.01 & 0.2771 & 5.5339 \\
\hline 51.5457 & 230.12 & 213.53 & 183.21 & 66.88 & 56.14 & 52.86 & 315.74 & 259.06 & 0.2756 & 5.5426 \\
\hline 52.0463 & 230.10 & 214.13 & 184.49 & 67.69 & 56.22 & 52.87 & 327.31 & 258.83 & 0.2752 & 5.5892 \\
\hline 52.5443 & 229.31 & 213.70 & 186.05 & 68.55 & 56.29 & 52.90 & 320.83 & 259.61 & 0.2756 & 5.5661 \\
\hline 53.0452 & 229.45 & 213.86 & 187.12 & 69.49 & 56.38 & 52.90 & 320.80 & 258.76 & 0.2745 & 5.5290 \\
\hline 53.5450 & 228.99 & 213.96 & 188.12 & 70.43 & 56.43 & 52.91 & 325.75 & 257.90 & 0.2776 & 5.5137 \\
\hline 54.0448 & 229.69 & 213.61 & 189.32 & 71.44 & 56.52 & 52.95 & 320.85 & 258.94 & 0.2747 & 5.5561 \\
\hline 54.5455 & 229.68 & 213.63 & 190.28 & 72.52 & 56.59 & 52.94 & 320.61 & 258.93 & 0.2761 & 5.5714 \\
\hline
\end{tabular}


TABLE B1.- CONTINUED.

\begin{tabular}{|c|c|c|c|c|c|c|c|c|c|c|}
\hline $\begin{array}{c}\text { Time, } \\
\text { min }\end{array}$ & $\begin{array}{c}\text { T-steam } \\
{ }^{\circ} \mathrm{C}\end{array}$ & $\begin{array}{l}\mathrm{T} 1, \\
{ }^{\circ} \mathrm{C}\end{array}$ & $\begin{array}{l}\mathrm{T} 2, \\
{ }^{\circ} \mathrm{C}\end{array}$ & $\begin{array}{l}\mathrm{T} 3, \\
{ }^{\circ} \mathrm{C}\end{array}$ & $\begin{array}{l}\mathrm{T} 4, \\
{ }^{\circ} \mathrm{C}\end{array}$ & $\begin{array}{l}\mathrm{T} 5, \\
{ }^{\circ} \mathrm{C}\end{array}$ & $\begin{array}{l}\text { Pinj, } \\
\text { psig }\end{array}$ & $\begin{array}{c}\text { Pout, } \\
\text { psig }\end{array}$ & $\begin{array}{l}\text { Qinj, } \\
\text { g/min }\end{array}$ & $\begin{array}{c}\mathrm{Vw} \\
\mathrm{ml} / \mathrm{min}\end{array}$ \\
\hline 55.0453 & 229.87 & 213.62 & 191.58 & 73.65 & 56.70 & 52.95 & 315.99 & 259.22 & 0.2760 & 5.5508 \\
\hline 55.5452 & 229.92 & 213.62 & 192.42 & 74.84 & 56.79 & 52.99 & 318.56 & 258.97 & 0.2748 & 5.5360 \\
\hline 56.0440 & 229.79 & 213.76 & 193.01 & 76.04 & 56.92 & 53.02 & 318.98 & 259.31 & 0.2762 & 5.5543 \\
\hline 56.5448 & 230.06 & 213.75 & 193.92 & 77.22 & 57.03 & 53.02 & 322.45 & 258.57 & 0.2774 & 5.5565 \\
\hline 57.0447 & 229.44 & 213.37 & 196.03 & 78.81 & 57.17 & 53.03 & 380.84 & 259.79 & 0.7069 & 5.5749 \\
\hline 57.5453 & 230.17 & 211.29 & 202.16 & 81.11 & 57.40 & 53.04 & 358.65 & 258.81 & 0.2800 & 5.5556 \\
\hline 58.0452 & 236.06 & 211.66 & 203.84 & 83.74 & 57.66 & 53.15 & 352.48 & 260.19 & 0.2760 & 5.5286 \\
\hline 58.5450 & 239.44 & 213.27 & 203.66 & 86.56 & 57.86 & 53.19 & 335.00 & 259.03 & 0.2759 & 5.5181 \\
\hline 59.0448 & 241.66 & 212.46 & 201.43 & 89.45 & 58.16 & 53.22 & 312.45 & 256.66 & 0.2811 & 5.5452 \\
\hline 59.5455 & 241.35 & 211.33 & 199.12 & 92.23 & 58.46 & 53.29 & 311.96 & 256.98 & 0.2639 & 5.5602 \\
\hline 60.0453 & 234.91 & 214.31 & 197.93 & 95.04 & 58.70 & 53.34 & 321.90 & 258.00 & 0.2754 & 5.5704 \\
\hline 60.5452 & 228.52 & 213.21 & 196.80 & 97.33 & 58.92 & 53.43 & 320.77 & 259.27 & 0.2724 & 5.5226 \\
\hline 61.0450 & 227.31 & 212.71 & 196.81 & 99.46 & 59.12 & 53.48 & 319.59 & 259.61 & 0.2765 & 5.5251 \\
\hline 61.5440 & 229.44 & 212.83 & 197.56 & 101.49 & 59.31 & 53.55 & 320.72 & 259.21 & 0.2762 & 5.5520 \\
\hline 62.0457 & 231.87 & 213.07 & 199.59 & 103.62 & 59.42 & 53.64 & 323.11 & 259.20 & 0.2755 & 5.5350 \\
\hline 62.5445 & 232.56 & 213.16 & 202.56 & 105.76 & 59.51 & 53.71 & 322.84 & 259.26 & 0.2757 & 5.5175 \\
\hline 63.0452 & 231.93 & 213.21 & 203.63 & 108.81 & 59.58 & 53.77 & 321.53 & 258.23 & 0.2762 & 5.5180 \\
\hline 63.5450 & 232.16 & 213.29 & 204.20 & 111.50 & 59.76 & 53.80 & 324.45 & 259.05 & 0.2702 & 5.5669 \\
\hline 64.0448 & 233.00 & 213.75 & 204.61 & 113.65 & 59.97 & 53.81 & 317.41 & 258.65 & 0.2769 & 5.5792 \\
\hline 64.5457 & 232.56 & 213.98 & 204.85 & 115.85 & 60.24 & 53.83 & 324.79 & 259.19 & 0.2759 & 5.5766 \\
\hline 65.0455 & 231.05 & 213.58 & 204.99 & 118.29 & 60.52 & 53.86 & 318.84 & 259.29 & 0.2766 & 5.5367 \\
\hline 65.5453 & 231.01 & 213.58 & 205.22 & 121.00 & 60.84 & 53.93 & 321.43 & 259.13 & 0.2758 & 5.5396 \\
\hline 66.0442 & 230.72 & 213.39 & 205.32 & 123.93 & 61.16 & 54.00 & 316.03 & 258.90 & 0.2769 & 5.5416 \\
\hline 66.5440 & 230.04 & 213.17 & 205.51 & 127.03 & 61.50 & 54.08 & 315.87 & 259.01 & 0.2759 & 5.5731 \\
\hline 67.0447 & 230.05 & 213.03 & 205.74 & 130.30 & 61.87 & 54.13 & 310.43 & 258.93 & 0.2760 & 5.5887 \\
\hline 67.5455 & 230.28 & 213.02 & 205.86 & 133.54 & 62.20 & 54.20 & 314.75 & 258.83 & 0.2761 & 5.5607 \\
\hline 68.0453 & 230.58 & 212.71 & 206.25 & 136.72 & 62.58 & 54.25 & 314.55 & 258.68 & 0.2759 & 5.5304 \\
\hline 68.5452 & 230.30 & 212.49 & 206.43 & 139.97 & 62.95 & 54.32 & 312.28 & 259.34 & 0.2750 & 5.5366 \\
\hline 69.0450 & 230.15 & 212.42 & 206.30 & 143.03 & 63.36 & 54.41 & 309.46 & 257.69 & 0.2760 & 5.5541 \\
\hline 69.5457 & 229.93 & 212.32 & 206.43 & 146.06 & 63.77 & 54.46 & 309.02 & 259.09 & 0.2784 & 5.5834 \\
\hline 70.0463 & 230.00 & 211.96 & 206.75 & 148.99 & 64.24 & 54.53 & 307.91 & 259.01 & 0.2757 & 5.5827 \\
\hline 70.5453 & 229.98 & 212.11 & 206.87 & 151.72 & 64.67 & 54.60 & 309.04 & 258.63 & 0.2759 & 5.5304 \\
\hline 71.0452 & 231.12 & 212.22 & 206.85 & 154.30 & 65.16 & 54.71 & 303.77 & 256.64 & 0.2766 & 5.5345 \\
\hline 71.5450 & 229.29 & 211.82 & 206.90 & 156.57 & 65.64 & 54.80 & 302.02 & 257.33 & 0.2772 & 5.5576 \\
\hline 72.0457 & 229.57 & 212.23 & 207.11 & 158.52 & 66.13 & 54.88 & 307.43 & 258.53 & 0.2764 & 5.5782 \\
\hline 72.5455 & 229.82 & 211.86 & 207.30 & 160.43 & 66.63 & 54.93 & 302.70 & 258.28 & 0.2758 & 5.5683 \\
\hline 73.0443 & 228.59 & 211.81 & 207.38 & 162.30 & 67.14 & 55.00 & 309.54 & 258.64 & 0.2748 & 5.5513 \\
\hline 73.5452 & 228.29 & 211.69 & 207.51 & 164.21 & 67.66 & 55.09 & 301.22 & 258.84 & 0.2760 & 5.5161 \\
\hline 74.0450 & 228.46 & 211.60 & 207.43 & 165.93 & 68.20 & 55.16 & 300.48 & 259.07 & 0.2754 & 5.5315 \\
\hline 74.5448 & 229.80 & 211.13 & 207.66 & 167.60 & 68.80 & 55.25 & 301.77 & 259.14 & 0.2738 & 5.5437 \\
\hline 75.0455 & 230.49 & 211.61 & 207.65 & 169.40 & 69.40 & 55.32 & 298.16 & 258.85 & 0.2746 & 5.5570 \\
\hline 75.5453 & 227.89 & 211.08 & 207.47 & 170.74 & 70.00 & 55.41 & 297.98 & 258.69 & 0.2761 & 5.5589 \\
\hline 76.0452 & 228.90 & 211.58 & 207.45 & 172.25 & 70.60 & 55.50 & 301.25 & 258.07 & 0.2760 & 5.5318 \\
\hline 76.5442 & 229.04 & 211.38 & 207.42 & 173.46 & 71.23 & 55.57 & 306.34 & 258.37 & 0.2761 & 5.5548 \\
\hline 77.0438 & 228.22 & 211.34 & 207.63 & 174.61 & 71.94 & 55.66 & 299.93 & 257.15 & 0.2763 & 5.5545 \\
\hline 77.5455 & 228.89 & 211.10 & 207.73 & 175.81 & 72.67 & 55.75 & 300.49 & 259.23 & 0.2762 & 5.5943 \\
\hline 78.0445 & 228.90 & 211.26 & 207.73 & 177.03 & 73.45 & 55.82 & 308.00 & 258.82 & 0.2854 & 5.5722 \\
\hline 78.5452 & 229.15 & 211.38 & 207.78 & 178.42 & 74.26 & 55.95 & 306.90 & 256.98 & 0.2765 & 5.5451 \\
\hline 79.0450 & 229.66 & 211.14 & 208.02 & 179.89 & 75.10 & 56.02 & 301.59 & 257.35 & 0.2758 & 5.5281 \\
\hline 79.5448 & 229.98 & 211.42 & 208.09 & 181.23 & 76.01 & 56.13 & 299.62 & 258.84 & 0.2754 & 5.5420 \\
\hline
\end{tabular}


TABLE B1.- CONTINUED.

\begin{tabular}{|c|c|c|c|c|c|c|c|c|c|c|}
\hline $\begin{array}{l}\text { Time, } \\
\text { min }\end{array}$ & $\begin{array}{c}\text { T-steam } \\
{ }^{\circ} \mathrm{C}\end{array}$ & $\begin{array}{l}\mathrm{T} 1, \\
{ }^{\circ} \mathrm{C}\end{array}$ & $\begin{array}{l}\mathrm{T} 2, \\
{ }^{\circ} \mathrm{C}\end{array}$ & $\begin{array}{l}\mathrm{T} 3, \\
{ }^{\circ} \mathrm{C}\end{array}$ & $\begin{array}{l}\mathrm{T} 4, \\
{ }^{\circ} \mathrm{C}\end{array}$ & $\begin{array}{l}\mathrm{T} 5, \\
{ }^{\circ} \mathrm{C}\end{array}$ & $\begin{array}{l}\text { Pinj, } \\
\text { psig }\end{array}$ & $\begin{array}{l}\text { Pout, } \\
\text { psig }\end{array}$ & $\begin{array}{l}\text { Qinj, } \\
\text { g/min }\end{array}$ & $\begin{array}{c}\mathrm{Vw} \\
\mathrm{ml} / \mathrm{min}\end{array}$ \\
\hline 80.0457 & 228.27 & 211.27 & 207.96 & 182.05 & 76.93 & 56.24 & 304.64 & 259.23 & 0.2767 & 5.5812 \\
\hline 80.5445 & 229.72 & 211.10 & 207.94 & 183.02 & 77.84 & 56.34 & 300.82 & 258.01 & 0.2757 & 5.5803 \\
\hline 81.0452 & 229.68 & 211.50 & 207.95 & 183.98 & 78.81 & 56.47 & 305.32 & 258.41 & 0.2767 & 5.5369 \\
\hline 81.5450 & 230.03 & 211.37 & 208.14 & 184.69 & 79.84 & 56.58 & 300.75 & 258.43 & 0.2754 & 5.5206 \\
\hline 82.0448 & 229.37 & 211.38 & 208.35 & 185.57 & 80.85 & 56.69 & 302.24 & 258.77 & 0.2765 & 5.5579 \\
\hline 82.5457 & 229.86 & 211.45 & 208.51 & 186.59 & 81.93 & 56.82 & 304.69 & 257.89 & 0.2763 & 5.5673 \\
\hline 83.0455 & 229.37 & 211.35 & 208.61 & 187.57 & 83.03 & 56.96 & 306.44 & 259.17 & 0.2770 & 5.5269 \\
\hline 83.5443 & 230.25 & 211.47 & 208.66 & 188.98 & 84.22 & 57.09 & 305.14 & 258.15 & 0.2773 & 5.5044 \\
\hline 84.0450 & 229.72 & 211.25 & 208.69 & 190.19 & 85.48 & 57.26 & 303.75 & 258.99 & 0.2774 & 5.5632 \\
\hline 84.5448 & 230.15 & 211.64 & 208.62 & 191.03 & 86.77 & 57.41 & 311.13 & 256.76 & 0.2757 & 5.5862 \\
\hline 85.0447 & 229.73 & 211.10 & 208.84 & 192.01 & 88.10 & 57.59 & 301.92 & 256.58 & 0.2762 & 5.6105 \\
\hline 85.5455 & 232.54 & 211.59 & 208.86 & 192.92 & 89.46 & 57.74 & 312.54 & 259.34 & 0.2757 & 5.6019 \\
\hline 86.0453 & 230.67 & 211.48 & 208.83 & 193.62 & 90.85 & 57.92 & 305.57 & 259.22 & 0.2778 & 5.5675 \\
\hline 86.5452 & 229.43 & 211.57 & 208.78 & 194.38 & 92.30 & 58.13 & 307.14 & 258.66 & 0.2765 & 5.5641 \\
\hline 87.0450 & 229.37 & 211.31 & 208.90 & 194.77 & 93.76 & 58.31 & 298.89 & 257.46 & 0.2764 & 5.5624 \\
\hline 87.5457 & 229.80 & 211.22 & 209.02 & 195.21 & 95.24 & 58.49 & 307.97 & 259.02 & 0.2757 & 5.5679 \\
\hline 88.0463 & 230.54 & 211.32 & 209.10 & 195.94 & 96.82 & 58.72 & 308.24 & 257.11 & 0.2765 & 5.5767 \\
\hline 88.5453 & 229.48 & 211.37 & 209.04 & 196.67 & 98.46 & 58.92 & 301.08 & 258.25 & 0.2765 & 5.5927 \\
\hline 89.0442 & 228.57 & 211.31 & 209.02 & 197.47 & 100.07 & 59.14 & 306.58 & 256.69 & 0.2762 & 5.5955 \\
\hline 89.5450 & 229.33 & 211.38 & 209.12 & 198.36 & 101.75 & 59.37 & 300.56 & 258.84 & 0.2764 & 5.5702 \\
\hline 90.0467 & 230.18 & 211.52 & 209.14 & 198.82 & 103.43 & 59.62 & 304.30 & 257.40 & 0.2757 & 5.5519 \\
\hline 90.5455 & 229.32 & 211.57 & 209.11 & 199.05 & 105.13 & 59.85 & 308.28 & 256.27 & 0.2761 & 5.5437 \\
\hline 91.0453 & 229.44 & 210.95 & 209.27 & 199.51 & 106.86 & 60.13 & 296.98 & 257.71 & 0.2767 & 5.5673 \\
\hline 91.5452 & 230.33 & 211.20 & 209.23 & 200.03 & 108.62 & 60.37 & 298.21 & 255.80 & 0.2757 & 5.5813 \\
\hline 92.0440 & 229.43 & 211.23 & 209.12 & 200.33 & 110.46 & 60.63 & 297.43 & 258.81 & 0.2765 & 5.5879 \\
\hline 92.5448 & 227.55 & 211.23 & 209.02 & 200.41 & 112.28 & 60.89 & 302.16 & 256.23 & 0.2770 & 5.5721 \\
\hline 93.0455 & 229.48 & 211.38 & 209.22 & 200.64 & 114.19 & 61.17 & 299.64 & 259.17 & 0.2766 & 5.5370 \\
\hline 93.5453 & 228.59 & 211.20 & 209.19 & 200.90 & 116.04 & 61.45 & 302.47 & 256.68 & 0.2755 & 5.5316 \\
\hline 94.0452 & 228.77 & 211.30 & 209.28 & 201.13 & 117.95 & 61.71 & 308.60 & 256.70 & 0.2760 & 5.5772 \\
\hline 94.5450 & 231.63 & 211.35 & 209.44 & 201.51 & 119.96 & 62.03 & 305.23 & 259.13 & 0.2768 & 5.5599 \\
\hline 95.0438 & 230.71 & 211.56 & 209.43 & 201.99 & 121.94 & 62.32 & 300.29 & 256.00 & 0.2755 & 5.5615 \\
\hline 95.5455 & 228.29 & 211.45 & 209.46 & 202.02 & 123.99 & 62.60 & 306.35 & 257.35 & 0.2758 & 5.5386 \\
\hline 96.0445 & 229.34 & 211.39 & 209.60 & 202.26 & 125.90 & 62.92 & 307.97 & 258.27 & 0.2750 & 5.5320 \\
\hline 96.5452 & 229.62 & 211.19 & 209.72 & 202.87 & 127.84 & 63.24 & 303.48 & 256.56 & 0.2772 & 5.5619 \\
\hline 97.0450 & 228.73 & 211.26 & 209.58 & 203.42 & 129.84 & 63.59 & 308.67 & 259.12 & 0.2752 & 5.5665 \\
\hline 97.5448 & 228.13 & 211.34 & 209.53 & 203.64 & 131.94 & 63.91 & 298.11 & 258.12 & 0.2760 & 5.5522 \\
\hline 98.0457 & 229.32 & 211.12 & 209.49 & 203.78 & 134.03 & 64.26 & 301.79 & 259.09 & 0.2754 & 5.5030 \\
\hline 98.5453 & 229.84 & 211.35 & 209.49 & 203.89 & 136.21 & 64.66 & 303.31 & 256.23 & 0.2760 & 5.5216 \\
\hline 99.0452 & 229.23 & 211.19 & 209.47 & 204.13 & 138.37 & 65.01 & 302.61 & 257.88 & 0.2764 & 5.5707 \\
\hline 99.5442 & 228.99 & 211.49 & 209.46 & 204.17 & 140.60 & 65.42 & 299.14 & 257.91 & 0.2774 & 5.5881 \\
\hline 100.0448 & 229.31 & 211.20 & 209.60 & 204.22 & 142.67 & 65.78 & 300.69 & 258.55 & 0.2762 & 5.5953 \\
\hline 100.5457 & 229.90 & 211.48 & 209.69 & 204.39 & 144.62 & 66.19 & 303.85 & 258.41 & 0.2759 & 5.5690 \\
\hline 101.0455 & 228.76 & 211.42 & 209.79 & 204.51 & 146.56 & 66.60 & 302.74 & 259.22 & 0.2787 & 5.5501 \\
\hline 101.5462 & 230.03 & 211.52 & 209.84 & 204.85 & 148.60 & 67.03 & 305.96 & 256.95 & 0.2764 & 5.5486 \\
\hline 102.0450 & 228.77 & 211.48 & 209.78 & 205.13 & 150.59 & 67.44 & 302.77 & 257.01 & 0.2769 & 5.5643 \\
\hline 102.5440 & 228.00 & 211.50 & 209.82 & 205.23 & 152.56 & 67.85 & 305.22 & 258.08 & 0.2759 & 5.6031 \\
\hline 103.0447 & 230.97 & 211.24 & 209.96 & 205.52 & 154.80 & 68.32 & 304.88 & 257.45 & 0.2777 & 5.5712 \\
\hline 103.5455 & 232.15 & 211.86 & 209.74 & 205.69 & 157.24 & 68.77 & 308.64 & 258.35 & 0.2753 & 5.5601 \\
\hline 104.0453 & 229.87 & 211.72 & 209.70 & 205.54 & 159.70 & 69.21 & 304.06 & 258.11 & 0.2766 & 5.5397 \\
\hline 104.5452 & 227.28 & 211.48 & 209.66 & 205.45 & 161.99 & 69.66 & 298.15 & 258.63 & 0.2751 & 5.5341 \\
\hline
\end{tabular}


TABLE B1.- CONTINUED.

\begin{tabular}{|c|c|c|c|c|c|c|c|c|c|c|}
\hline $\begin{array}{l}\text { Time, } \\
\text { min }\end{array}$ & $\begin{array}{c}\text { T-steam } \\
{ }^{\circ} \mathrm{C}\end{array}$ & $\begin{array}{l}\mathrm{T} 1, \\
{ }^{\circ} \mathrm{C}\end{array}$ & $\begin{array}{l}\mathrm{T} 2, \\
{ }^{\circ} \mathrm{C}\end{array}$ & $\begin{array}{l}\mathrm{T} 3, \\
{ }^{\circ} \mathrm{C}\end{array}$ & $\begin{array}{l}\mathrm{T} 4, \\
{ }^{\circ} \mathrm{C}\end{array}$ & $\begin{array}{l}\mathrm{T} 5, \\
{ }^{\circ} \mathrm{C}\end{array}$ & $\begin{array}{l}\text { Pinj, } \\
\text { psig }\end{array}$ & $\begin{array}{l}\text { Pout, } \\
\text { psig }\end{array}$ & $\begin{array}{l}\text { Qinj, } \\
\text { g/min }\end{array}$ & $\begin{array}{c}\mathrm{Vw} \\
\mathrm{ml} / \mathrm{min}\end{array}$ \\
\hline 105.0450 & 227.57 & 211.51 & 209.72 & 205.46 & 164.19 & 70.17 & 299.92 & 258.04 & 0.2759 & 5.5550 \\
\hline 105.5457 & 228.67 & 211.58 & 209.79 & 205.58 & 166.04 & 70.62 & 304.15 & 257.44 & 0.2750 & 5.5684 \\
\hline 106.0455 & 228.15 & 211.52 & 209.88 & 205.71 & 167.87 & 71.06 & 304.95 & 258.57 & 0.2758 & 5.5691 \\
\hline 106.5443 & 228.70 & 211.61 & 209.89 & 205.83 & 169.56 & 71.55 & 300.84 & 258.89 & 0.2767 & 5.5445 \\
\hline 107.0452 & 228.23 & 211.46 & 209.85 & 205.88 & 171.14 & 72.05 & 299.29 & 256.78 & 0.2762 & 5.5337 \\
\hline 107.5450 & 228.62 & 211.60 & 209.92 & 206.04 & 172.74 & 72.48 & 305.86 & 257.60 & 0.2773 & 5.5528 \\
\hline 108.0448 & 228.05 & 211.60 & 210.02 & 206.23 & 174.32 & 73.01 & 310.13 & 257.79 & 0.2761 & 5.5735 \\
\hline 108.5455 & 229.98 & 211.68 & 210.07 & 206.49 & 175.89 & 73.49 & 312.62 & 259.22 & 0.2762 & 5.5805 \\
\hline 109.0453 & 229.60 & 211.52 & 210.01 & 206.56 & 177.38 & 74.03 & 302.09 & 257.44 & 0.2778 & 5.5398 \\
\hline 109.5452 & 228.95 & 211.34 & 209.98 & 206.63 & 179.10 & 74.57 & 297.45 & 257.00 & 0.2731 & 5.5341 \\
\hline 110.0458 & 229.12 & 211.49 & 209.92 & 206.66 & 180.57 & 75.09 & 302.51 & 259.33 & 0.2789 & 5.5323 \\
\hline 110.5448 & 230.96 & 211.72 & 209.91 & 206.66 & 181.66 & 75.65 & 299.98 & 257.91 & 0.2757 & 5.5739 \\
\hline 111.0455 & 229.47 & 211.88 & 209.82 & 206.58 & 182.59 & 76.19 & 301.51 & 257.90 & 0.2751 & 5.5836 \\
\hline 111.5453 & 228.74 & 211.70 & 209.82 & 206.45 & 183.52 & 76.72 & 298.89 & 257.55 & 0.2756 & 5.5547 \\
\hline 112.0452 & 229.23 & 211.82 & 209.83 & 206.41 & 184.29 & 77.24 & 297.27 & 256.83 & 0.2753 & 5.5295 \\
\hline 112.5458 & 227.92 & 211.90 & 209.86 & 206.30 & 184.57 & 77.72 & 298.94 & 258.41 & 0.2757 & 5.5147 \\
\hline 113.0438 & 228.03 & 212.03 & 209.97 & 206.33 & 184.75 & 78.19 & 303.62 & 256.44 & 0.2755 & 5.5801 \\
\hline 113.5455 & 228.18 & 212.00 & 210.27 & 206.55 & 184.90 & 78.69 & 299.02 & 259.62 & 0.2750 & 5.5626 \\
\hline 114.0453 & 228.00 & 212.04 & 210.52 & 206.85 & 185.01 & 79.14 & 303.42 & 258.74 & 0.2757 & 5.5519 \\
\hline 114.5443 & 228.71 & 211.87 & 210.92 & 207.33 & 185.33 & 79.70 & 306.94 & 256.51 & 0.2763 & 5.5375 \\
\hline 115.0450 & 228.75 & 211.81 & 211.05 & 207.72 & 186.54 & 80.31 & 308.02 & 257.56 & 0.2758 & 5.5474 \\
\hline 115.5448 & 229.57 & 211.61 & 210.90 & 207.62 & 188.76 & 80.98 & 309.28 & 257.00 & 0.2760 & 5.5737 \\
\hline 116.0455 & 229.71 & 211.65 & 210.66 & 207.80 & 191.84 & 81.73 & 310.42 & 257.48 & 0.2762 & 5.5785 \\
\hline 116.5453 & 230.92 & 211.36 & 210.46 & 207.81 & 194.60 & 82.56 & 306.79 & 258.20 & 0.2750 & 5.5532 \\
\hline 117.0452 & 231.10 & 211.64 & 210.21 & 207.65 & 196.21 & 83.50 & 308.71 & 257.16 & 0.2756 & 5.5301 \\
\hline 117.5450 & 229.30 & 211.44 & 210.13 & 207.47 & 197.28 & 84.39 & 306.29 & 258.38 & 0.2766 & 5.5163 \\
\hline 118.0458 & 230.28 & 211.54 & 210.04 & 207.34 & 197.99 & 85.26 & 306.08 & 258.53 & 0.2767 & 5.5628 \\
\hline 118.5457 & 229.44 & 211.54 & 209.97 & 207.25 & 198.42 & 86.08 & 306.66 & 257.46 & 0.2766 & 5.5969 \\
\hline 119.0463 & 229.06 & 211.27 & 209.96 & 207.17 & 198.92 & 86.94 & 304.77 & 257.54 & 0.2747 & 5.5910 \\
\hline 119.5443 & 230.24 & 211.57 & 209.91 & 207.11 & 199.24 & 87.74 & 309.31 & 258.81 & 0.2755 & 5.5778 \\
\hline 120.0450 & 230.43 & 211.44 & 209.88 & 207.04 & 199.45 & 88.55 & 304.60 & 256.90 & 0.2756 & 5.5422 \\
\hline 120.5448 & 229.62 & 211.62 & 209.88 & 207.02 & 199.70 & 89.39 & 307.68 & 258.32 & 0.2749 & 5.5499 \\
\hline 121.0457 & 229.82 & 211.56 & 209.91 & 207.01 & 200.00 & 90.19 & 303.90 & 256.24 & 0.2746 & 5.5735 \\
\hline 121.5455 & 229.38 & 211.47 & 209.93 & 207.03 & 200.16 & 91.06 & 304.57 & 259.08 & 0.2766 & 5.5744 \\
\hline 122.0453 & 228.86 & 211.38 & 209.98 & 207.10 & 200.17 & 91.84 & 306.53 & 258.25 & 0.2738 & 5.5817 \\
\hline 122.5442 & 229.68 & 211.50 & 209.98 & 207.18 & 200.36 & 92.77 & 305.02 & 256.63 & 0.2759 & 5.5455 \\
\hline 123.0450 & 229.90 & 211.35 & 210.01 & 207.22 & 200.63 & 93.66 & 305.92 & 258.79 & 0.2755 & 5.5341 \\
\hline 123.5447 & 229.89 & 211.64 & 209.99 & 207.30 & 200.74 & 94.60 & 304.34 & 257.00 & 0.2759 & 5.5411 \\
\hline 124.0455 & 228.82 & 211.54 & 210.02 & 207.36 & 200.86 & 95.58 & 306.20 & 259.03 & 0.2755 & 5.5649 \\
\hline 124.5453 & 229.37 & 211.67 & 210.06 & 207.41 & 200.98 & 96.55 & 304.96 & 258.71 & 0.2768 & 5.5804 \\
\hline 125.0452 & 229.69 & 211.72 & 210.09 & 207.44 & 201.16 & 97.49 & 304.78 & 256.57 & 0.2760 & 5.5945 \\
\hline 125.5450 & 229.47 & 211.75 & 210.16 & 207.49 & 201.55 & 98.55 & 309.38 & 255.84 & 0.2761 & 5.5953 \\
\hline 126.0457 & 229.09 & 211.86 & 210.25 & 207.56 & 201.76 & 99.53 & 306.67 & 257.50 & 0.2764 & 5.5710 \\
\hline 126.5455 & 228.48 & 211.76 & 210.32 & 207.65 & 201.99 & 100.62 & 304.27 & 257.90 & 0.2764 & 5.5412 \\
\hline 127.0443 & 228.98 & 211.66 & 210.38 & 207.75 & 202.37 & 101.74 & 308.08 & 255.91 & 0.2757 & 5.5480 \\
\hline 127.5452 & 228.89 & 211.89 & 210.40 & 207.79 & 202.44 & 102.87 & 306.03 & 257.46 & 0.2765 & 5.5745 \\
\hline 128.0450 & 230.12 & 211.76 & 210.38 & 207.82 & 202.36 & 104.07 & 307.00 & 256.44 & 0.2764 & 5.5852 \\
\hline 128.5448 & 229.69 & 211.85 & 210.38 & 207.84 & 202.59 & 105.33 & 310.64 & 257.17 & 0.2757 & 5.5862 \\
\hline 129.0455 & 228.62 & 211.68 & 210.41 & 207.85 & 202.86 & 106.56 & 306.45 & 256.08 & 0.2765 & 5.5602 \\
\hline 129.5453 & 229.38 & 211.99 & 210.41 & 207.89 & 203.04 & 107.85 & 309.26 & 257.91 & 0.2764 & 5.5035 \\
\hline
\end{tabular}


TABLE B1.- CONTINUED.

\begin{tabular}{|c|c|c|c|c|c|c|c|c|c|c|}
\hline $\begin{array}{l}\text { Time, } \\
\text { min }\end{array}$ & $\begin{array}{c}\text { T-steam } \\
{ }^{\circ} \mathrm{C}\end{array}$ & $\begin{array}{l}\mathrm{T} 1, \\
{ }^{\circ} \mathrm{C}\end{array}$ & $\begin{array}{l}\mathrm{T} 2, \\
{ }^{\circ} \mathrm{C}\end{array}$ & $\begin{array}{l}\mathrm{T} 3, \\
{ }^{\circ} \mathrm{C}\end{array}$ & $\begin{array}{l}\mathrm{T} 4, \\
{ }^{\circ} \mathrm{C}\end{array}$ & $\begin{array}{l}\mathrm{T} 5, \\
{ }^{\circ} \mathrm{C}\end{array}$ & $\begin{array}{l}\text { Pinj, } \\
\text { psig }\end{array}$ & $\begin{array}{l}\text { Pout, } \\
\text { psig }\end{array}$ & $\begin{array}{l}\text { Qinj, } \\
\text { g/min }\end{array}$ & $\begin{array}{c}\mathrm{Vw} \\
\mathrm{ml} / \mathrm{min}\end{array}$ \\
\hline 130.0462 & 229.13 & 211.88 & 210.41 & 207.89 & 203.11 & 109.18 & 310.95 & 255.91 & 0.2767 & 5.5308 \\
\hline 130.5450 & 228.70 & 211.64 & 210.43 & 207.89 & 203.39 & 110.54 & 306.14 & 257.34 & 0.2768 & 5.5880 \\
\hline 131.0448 & 229.17 & 211.84 & 210.36 & 207.92 & 203.36 & 111.92 & 307.87 & 258.40 & 0.2756 & 5.5690 \\
\hline 131.5455 & 228.72 & 211.66 & 210.39 & 207.92 & 203.44 & 113.41 & 310.04 & 259.03 & 0.2762 & 5.5437 \\
\hline 132.0453 & 228.86 & 211.64 & 210.39 & 207.95 & 203.55 & 114.92 & 308.68 & 257.48 & 0.2740 & 5.5555 \\
\hline 132.5452 & 228.63 & 211.82 & 210.32 & 207.94 & 203.68 & 116.39 & 305.88 & 258.71 & 0.2760 & 5.5602 \\
\hline 133.0450 & 228.84 & 211.55 & 210.30 & 207.92 & 203.71 & 118.12 & 306.74 & 255.66 & 0.2762 & 5.5735 \\
\hline 133.5448 & 228.70 & 211.59 & 210.26 & 207.86 & 203.78 & 119.75 & 308.74 & 257.70 & 0.2756 & 5.6020 \\
\hline 134.0455 & 229.36 & 211.80 & 210.23 & 207.85 & 203.96 & 121.45 & 309.77 & 258.11 & 0.2754 & 5.5873 \\
\hline 134.5453 & 228.95 & 211.77 & 210.24 & 207.83 & 204.03 & 123.19 & 307.75 & 258.92 & 0.2759 & 5.5767 \\
\hline 135.0443 & 228.50 & 211.75 & 210.24 & 207.81 & 204.09 & 124.93 & 306.19 & 257.19 & 0.2759 & 5.5399 \\
\hline 135.5450 & 229.99 & 211.64 & 210.30 & 207.81 & 204.18 & 126.85 & 307.96 & 256.44 & 0.2754 & 5.5469 \\
\hline 136.0448 & 229.95 & 211.93 & 210.26 & 207.83 & 204.09 & 128.85 & 308.08 & 259.01 & 0.2764 & 5.5793 \\
\hline 136.5457 & 228.29 & 211.66 & 210.24 & 207.81 & 204.21 & 130.80 & 308.55 & 257.61 & 0.2759 & 5.5757 \\
\hline 137.0455 & 228.29 & 211.68 & 210.26 & 207.83 & 204.39 & 132.95 & 310.94 & 256.85 & 0.2767 & 5.5777 \\
\hline 137.5453 & 228.14 & 211.77 & 210.23 & 207.85 & 204.37 & 135.05 & 309.60 & 257.56 & 0.2756 & 5.5564 \\
\hline 138.0460 & 228.04 & 211.43 & 210.21 & 207.83 & 204.38 & 137.18 & 305.39 & 256.54 & 0.2766 & 5.5284 \\
\hline 138.5440 & 228.45 & 211.64 & 210.23 & 207.85 & 204.36 & 139.42 & 317.56 & 257.95 & 0.7069 & 5.5441 \\
\hline 139.0457 & 229.11 & 208.80 & 210.70 & 208.19 & 204.00 & 142.83 & 406.27 & 258.02 & 0.7068 & 5.5724 \\
\hline 139.5445 & 234.67 & 209.61 & 209.75 & 207.94 & 203.82 & 164.66 & 358.74 & 256.14 & 0.2455 & 5.5818 \\
\hline 140.0453 & 241.32 & 216.62 & 208.16 & 207.10 & 203.92 & 193.37 & 327.23 & 257.49 & 0.2762 & 5.5891 \\
\hline 140.5452 & 243.81 & 222.92 & 206.83 & 206.48 & 203.56 & 196.91 & 326.42 & 257.59 & 0.2762 & 5.5378 \\
\hline 141.0440 & 244.10 & 224.36 & 205.82 & 205.85 & 203.17 & 197.17 & 293.03 & 258.08 & 0.2762 & 5.5329 \\
\hline 141.5457 & 242.56 & 225.54 & 205.05 & 205.30 & 202.86 & 196.69 & 296.47 & 257.15 & 0.2746 & 5.5239 \\
\hline 142.0445 & 234.69 & 225.04 & 204.53 & 204.82 & 202.56 & 195.99 & 294.50 & 258.41 & 0.2760 & 5.5673 \\
\hline 142.5453 & 228.07 & 220.21 & 204.44 & 204.44 & 202.24 & 195.10 & 289.71 & 256.67 & 0.2762 & 5.5273 \\
\hline 143.0442 & 225.64 & 216.13 & 204.95 & 204.12 & 201.96 & 194.08 & 290.69 & 258.20 & 0.2760 & 5.5144 \\
\hline 143.5450 & 225.03 & 212.57 & 205.59 & 203.89 & 201.69 & 192.81 & 289.83 & 258.52 & 0.2762 & 5.5371 \\
\hline 144.0448 & 226.80 & 211.99 & 206.69 & 203.79 & 201.50 & 191.46 & 288.96 & 255.69 & 0.2761 & 5.5617 \\
\hline 144.5455 & 227.94 & 211.79 & 207.80 & 203.92 & 201.50 & 190.18 & 288.32 & 257.42 & 0.2760 & 5.5698 \\
\hline 145.0453 & 227.49 & 212.96 & 208.25 & 204.30 & 201.70 & 189.02 & 297.30 & 257.42 & 0.2759 & 5.5792 \\
\hline 145.5452 & 228.44 & 211.13 & 208.72 & 204.89 & 201.99 & 188.14 & 301.47 & 257.66 & 0.2759 & 5.5651 \\
\hline 146.0450 & 229.68 & 211.66 & 209.04 & 205.52 & 202.10 & 187.80 & 304.15 & 258.04 & 0.2766 & 5.5301 \\
\hline 146.5448 & 229.67 & 212.61 & 209.24 & 206.06 & 201.99 & 188.04 & 307.82 & 256.83 & 0.2764 & 5.5528 \\
\hline 147.0455 & 229.63 & 212.75 & 209.44 & 206.44 & 202.07 & 188.56 & 309.23 & 258.06 & 0.2742 & 5.5747 \\
\hline 147.5453 & 230.35 & 212.78 & 209.61 & 206.82 & 202.34 & 189.48 & 306.17 & 257.38 & 0.2761 & 5.5745 \\
\hline 148.0452 & 230.41 & 213.05 & 209.79 & 207.25 & 202.76 & 190.49 & 302.74 & 258.85 & 0.2755 & 5.5958 \\
\hline 148.5440 & 230.27 & 212.71 & 209.94 & 207.65 & 203.13 & 191.22 & 302.74 & 258.80 & 0.2758 & 5.5539 \\
\hline 149.0438 & 231.02 & 212.91 & 210.06 & 207.92 & 203.53 & 191.85 & 306.30 & 256.72 & 0.2754 & 5.5411 \\
\hline 149.5447 & 230.38 & 213.50 & 210.14 & 208.10 & 203.75 & 192.29 & 301.73 & 257.44 & 0.2771 & 5.5467 \\
\hline 150.0453 & 229.39 & 213.72 & 210.20 & 208.23 & 203.92 & 192.94 & 299.09 & 258.70 & 0.2750 & 5.5592 \\
\hline 150.5462 & 229.69 & 212.82 & 210.24 & 208.32 & 204.21 & 193.37 & 299.02 & 258.35 & 0.2751 & 5.5761 \\
\hline 151.0450 & 229.02 & 213.16 & 210.28 & 208.40 & 204.50 & 194.13 & 311.65 & 259.06 & 0.7067 & 5.5641 \\
\hline 151.5440 & 229.79 & 213.54 & 210.62 & 208.49 & 204.70 & 194.97 & 319.22 & 258.06 & 0.2638 & 5.5343 \\
\hline 152.0447 & 230.35 & 213.81 & 211.14 & 208.68 & 204.95 & 196.07 & 313.75 & 258.15 & 0.2756 & 5.4985 \\
\hline 152.5453 & 232.38 & 214.57 & 211.35 & 208.93 & 205.17 & 197.45 & 334.32 & 258.72 & 0.2763 & 5.5631 \\
\hline 153.0452 & 231.93 & 214.43 & 211.31 & 209.20 & 205.26 & 199.43 & 316.48 & 257.97 & 0.2774 & 5.5801 \\
\hline 153.5450 & 231.60 & 215.13 & 210.91 & 209.01 & 205.38 & 200.87 & 313.00 & 258.93 & 0.2746 & 5.5599 \\
\hline 154.0448 & 231.21 & 214.69 & 210.84 & 208.85 & 205.63 & 201.44 & 310.16 & 258.95 & 0.2761 & 5.5298 \\
\hline 154.5457 & 232.33 & 214.66 & 210.99 & 208.90 & 205.94 & 201.70 & 308.33 & 255.61 & 0.2761 & 5.4995 \\
\hline
\end{tabular}


TABLE B1.- CONTINUED.

\begin{tabular}{|c|c|c|c|c|c|c|c|c|c|c|}
\hline $\begin{array}{c}\text { Time, } \\
\text { min }\end{array}$ & $\begin{array}{c}\text { T-steam } \\
{ }^{\circ} \mathrm{C}\end{array}$ & $\begin{array}{l}\mathrm{T} 1, \\
{ }^{\circ} \mathrm{C}\end{array}$ & $\begin{array}{l}\mathrm{T} 2, \\
{ }^{\circ} \mathrm{C}\end{array}$ & $\begin{array}{l}\mathrm{T} 3, \\
{ }^{\circ} \mathrm{C}\end{array}$ & $\begin{array}{l}\mathrm{T} 4, \\
{ }^{\circ} \mathrm{C}\end{array}$ & $\begin{array}{l}\mathrm{T} 5, \\
{ }^{\circ} \mathrm{C}\end{array}$ & $\begin{array}{l}\text { Pinj, } \\
\text { psig }\end{array}$ & $\begin{array}{l}\text { Pout, } \\
\text { psig }\end{array}$ & $\begin{array}{l}\text { Qinj, } \\
\text { g/min }\end{array}$ & $\begin{array}{c}\mathrm{Vw} \\
\mathrm{ml} / \mathrm{min}\end{array}$ \\
\hline 155.0455 & 231.10 & 214.70 & 211.08 & 209.06 & 206.36 & 201.87 & 309.81 & 258.51 & 0.2754 & 5.5034 \\
\hline 155.5443 & 230.62 & 214.65 & 211.14 & 209.17 & 206.56 & 202.19 & 304.18 & 256.78 & 0.2765 & 5.5587 \\
\hline 156.0450 & 230.16 & 214.62 & 211.22 & 209.29 & 206.78 & 202.61 & 310.51 & 255.97 & 0.2768 & 5.5452 \\
\hline 156.5448 & 230.34 & 214.34 & 211.21 & 209.31 & 206.80 & 203.05 & 312.09 & 258.26 & 0.2763 & 5.5165 \\
\hline 157.0457 & 230.72 & 215.47 & 211.14 & 209.32 & 206.72 & 203.39 & 313.87 & 258.95 & 0.2760 & 5.5208 \\
\hline 157.5455 & 230.53 & 215.19 & 211.06 & 209.27 & 206.73 & 203.74 & 314.58 & 255.84 & 0.2766 & 5.5470 \\
\hline 158.0462 & 230.16 & 214.77 & 211.01 & 209.24 & 206.68 & 203.85 & 313.40 & 257.47 & 0.2758 & 5.5655 \\
\hline 158.5452 & 231.96 & 214.58 & 210.84 & 209.17 & 206.68 & 203.93 & 313.16 & 257.78 & 0.4485 & 5.5727 \\
\hline 159.0450 & 230.98 & 214.74 & 210.68 & 209.03 & 206.62 & 204.04 & 303.52 & 256.23 & 0.2760 & 5.5434 \\
\hline 159.5457 & 229.82 & 214.07 & 210.51 & 208.92 & 206.61 & 204.10 & 310.78 & 257.69 & 0.2767 & 5.5415 \\
\hline 160.0463 & 229.74 & 213.66 & 210.41 & 208.78 & 206.58 & 204.11 & 299.53 & 255.84 & 0.2753 & 5.5380 \\
\hline 160.5453 & 229.40 & 213.81 & 210.14 & 208.57 & 206.57 & 204.15 & 303.93 & 253.20 & 0.2757 & 5.5723 \\
\hline 161.0452 & 228.30 & 213.96 & 210.26 & 208.41 & 206.55 & 204.15 & 300.29 & 257.75 & 0.2762 & 5.5827 \\
\hline 161.5440 & 228.45 & 213.91 & 210.43 & 208.49 & 206.72 & 204.18 & 297.87 & 254.37 & 0.2763 & 5.5671 \\
\hline 162.0457 & 230.50 & 214.31 & 210.40 & 208.59 & 206.80 & 204.27 & 300.09 & 254.15 & 0.2760 & 5.5466 \\
\hline 162.5447 & 229.38 & 214.64 & 210.28 & 208.60 & 206.75 & 204.37 & 306.34 & 257.88 & 0.2817 & 5.5277 \\
\hline 163.0453 & 229.63 & 214.45 & 210.46 & 208.55 & 206.81 & 204.43 & 303.84 & 256.53 & 0.2752 & 5.5519 \\
\hline 163.5452 & 230.50 & 214.28 & 210.65 & 208.75 & 206.94 & 204.49 & 313.54 & 256.69 & 0.2766 & 5.5473 \\
\hline 164.0450 & 230.52 & 214.97 & 210.74 & 208.88 & 206.82 & 204.48 & 312.56 & 253.57 & 0.2756 & 5.5689 \\
\hline 164.5438 & 231.77 & 215.21 & 210.73 & 208.93 & 206.71 & 204.56 & 320.69 & 254.60 & 0.2760 & 5.5466 \\
\hline 165.0455 & 230.72 & 213.44 & 210.83 & 208.95 & 206.78 & 204.57 & 315.32 & 256.27 & 0.2759 & 5.5103 \\
\hline 165.5453 & 231.31 & 214.11 & 210.85 & 209.03 & 206.81 & 204.50 & 318.94 & 254.69 & 0.2762 & 5.5407 \\
\hline 166.0452 & 231.95 & 214.12 & 210.88 & 209.02 & 206.73 & 204.51 & 314.70 & 256.27 & 0.2762 & 5.5441 \\
\hline 166.5450 & 231.72 & 214.30 & 210.92 & 208.99 & 206.81 & 204.55 & 317.39 & 256.01 & 0.2759 & 5.5722 \\
\hline 167.0448 & 232.18 & 215.14 & 210.95 & 209.01 & 206.92 & 204.61 & 310.27 & 254.35 & 0.2759 & 5.5373 \\
\hline 167.5455 & 231.66 & 215.53 & 210.92 & 209.04 & 206.98 & 204.71 & 309.89 & 256.22 & 0.2762 & 5.5044 \\
\hline 168.0453 & 230.90 & 215.36 & 210.82 & 209.03 & 207.06 & 204.79 & 303.06 & 254.82 & 0.2764 & 5.5285 \\
\hline 168.5452 & 231.05 & 215.25 & 210.61 & 208.96 & 207.07 & 204.83 & 304.98 & 253.48 & 0.2760 & 5.5686 \\
\hline 169.0450 & 230.99 & 215.50 & 210.40 & 208.87 & 207.02 & 204.86 & 300.91 & 256.13 & 0.2753 & 5.5625 \\
\hline 169.5440 & 229.19 & 215.32 & 210.25 & 208.75 & 207.03 & 204.94 & 301.75 & 254.31 & 0.2759 & 5.5310 \\
\hline 170.0455 & 229.32 & 216.00 & 210.11 & 208.65 & 207.00 & 204.94 & 298.51 & 256.59 & 0.2755 & 5.4844 \\
\hline 170.5463 & 229.86 & 216.08 & 209.96 & 208.53 & 207.01 & 204.93 & 294.47 & 254.49 & 0.2774 & 5.5227 \\
\hline 171.0452 & 228.28 & 215.95 & 209.81 & 208.39 & 206.96 & 204.96 & 291.10 & 254.59 & 0.2757 & 5.5752 \\
\hline 171.5450 & 227.93 & 215.17 & 209.76 & 208.31 & 206.93 & 204.96 & 307.49 & 252.35 & 0.2734 & 5.5653 \\
\hline 172.0448 & 228.29 & 214.12 & 210.11 & 208.30 & 206.89 & 204.97 & 307.99 & 255.61 & 0.2768 & 5.5099 \\
\hline 172.5457 & 229.36 & 213.45 & 210.21 & 208.45 & 206.93 & 205.00 & 305.85 & 255.89 & 0.2726 & 5.5284 \\
\hline 173.0455 & 229.62 & 214.63 & 210.20 & 208.50 & 206.92 & 205.04 & 302.93 & 253.25 & 0.2758 & 5.5510 \\
\hline 173.5453 & 230.29 & 215.11 & 210.19 & 208.50 & 206.97 & 205.05 & 299.88 & 254.77 & 0.2761 & 5.5765 \\
\hline 174.0450 & 230.06 & 215.56 & 210.25 & 208.51 & 207.06 & 205.15 & 298.68 & 253.08 & 0.2763 & 5.5703 \\
\hline 174.5448 & 228.85 & 215.34 & 210.47 & 208.56 & 207.18 & 205.23 & 305.78 & 253.62 & 0.2760 & 5.5571 \\
\hline 175.0457 & 229.13 & 215.08 & 210.75 & 208.85 & 207.31 & 205.36 & 307.36 & 254.26 & 0.2766 & 5.5158 \\
\hline 175.5455 & 231.28 & 215.94 & 210.74 & 209.11 & 207.39 & 205.55 & 310.07 & 252.62 & 0.2765 & 5.5469 \\
\hline 176.0453 & 230.93 & 216.47 & 210.58 & 209.12 & 207.24 & 205.54 & 306.00 & 253.31 & 0.2755 & 5.5690 \\
\hline 176.5452 & 229.55 & 217.00 & 210.39 & 209.02 & 207.37 & 205.51 & 305.84 & 254.85 & 0.2757 & 5.5780 \\
\hline 177.0440 & 229.73 & 216.86 & 210.31 & 208.94 & 207.40 & 205.43 & 301.49 & 253.93 & 0.2758 & 5.5544 \\
\hline 177.5457 & 230.03 & 216.76 & 210.07 & 208.82 & 207.31 & 205.38 & 304.30 & 255.08 & 0.2753 & 5.5340 \\
\hline 178.0445 & 229.21 & 216.83 & 209.99 & 208.63 & 207.18 & 205.26 & 300.62 & 253.65 & 0.2762 & 5.5329 \\
\hline 178.5453 & 228.36 & 216.73 & 209.94 & 208.50 & 207.15 & 205.24 & 302.49 & 253.24 & 0.2755 & 5.5594 \\
\hline 179.0452 & 229.10 & 215.64 & 209.97 & 208.43 & 207.11 & 205.24 & 302.24 & 253.96 & 0.2767 & 5.5713 \\
\hline 179.5450 & 230.20 & 215.69 & 209.91 & 208.39 & 207.06 & 205.20 & 302.82 & 255.66 & 0.2760 & 5.5544 \\
\hline
\end{tabular}


TABLE B1.- CONTINUED.

\begin{tabular}{|c|c|c|c|c|c|c|c|c|c|c|}
\hline $\begin{array}{c}\text { Time, } \\
\text { min }\end{array}$ & $\begin{array}{c}\text { T-steam } \\
{ }^{\circ} \mathrm{C}\end{array}$ & $\begin{array}{l}\mathrm{T} 1, \\
{ }^{\circ} \mathrm{C}\end{array}$ & $\begin{array}{l}\mathrm{T} 2, \\
{ }^{\circ} \mathrm{C}\end{array}$ & $\begin{array}{l}\mathrm{T} 3, \\
{ }^{\circ} \mathrm{C}\end{array}$ & $\begin{array}{l}\mathrm{T} 4, \\
{ }^{\circ} \mathrm{C}\end{array}$ & $\begin{array}{l}\mathrm{T} 5, \\
{ }^{\circ} \mathrm{C}\end{array}$ & $\begin{array}{l}\text { Pinj, } \\
\text { psig }\end{array}$ & $\begin{array}{l}\text { Pout, } \\
\text { psig }\end{array}$ & $\begin{array}{l}\text { Qinj, } \\
\text { g/min }\end{array}$ & $\begin{array}{c}\mathrm{Vw} \\
\mathrm{ml} / \mathrm{min}\end{array}$ \\
\hline 180.0457 & 228.69 & 216.57 & 209.83 & 208.31 & 206.98 & 205.16 & 298.26 & 254.07 & 0.7075 & 5.5277 \\
\hline 180.5447 & 229.11 & 216.56 & 209.76 & 208.24 & 206.94 & 205.11 & 299.72 & 252.98 & 0.2638 & 5.5254 \\
\hline 181.0453 & 229.26 & 216.98 & 209.61 & 208.14 & 206.91 & 205.12 & 296.39 & 253.74 & 0.2760 & 5.5477 \\
\hline 181.5452 & 228.07 & 216.01 & 209.51 & 208.04 & 206.86 & 205.06 & 296.23 & 255.03 & 0.2768 & 5.5757 \\
\hline 182.0450 & 229.42 & 215.25 & 209.45 & 207.89 & 206.80 & 205.03 & 303.18 & 253.57 & 0.2765 & 5.5611 \\
\hline 182.5448 & 227.23 & 212.91 & 209.76 & 207.81 & 206.81 & 205.04 & 301.78 & 253.65 & 0.2764 & 5.5224 \\
\hline 183.0447 & 226.97 & 211.58 & 210.06 & 208.09 & 206.99 & 205.15 & 302.34 & 253.28 & 0.2757 & 5.5115 \\
\hline 183.5453 & 229.25 & 211.66 & 210.06 & 208.38 & 207.04 & 205.34 & 304.38 & 252.96 & 0.2762 & 5.5352 \\
\hline 184.0452 & 229.40 & 211.27 & 209.98 & 208.46 & 206.92 & 205.36 & 303.50 & 253.93 & 0.2760 & 5.5376 \\
\hline 184.5440 & 229.09 & 211.24 & 209.85 & 208.45 & 206.93 & 205.30 & 302.00 & 254.38 & 0.2763 & 5.5269 \\
\hline 185.0448 & 229.51 & 211.36 & 209.75 & 208.37 & 206.98 & 205.17 & 301.76 & 254.35 & 0.2764 & 5.5184 \\
\hline 185.5465 & 229.86 & 211.10 & 209.63 & 208.31 & 206.95 & 205.05 & 304.24 & 254.39 & 0.2758 & 5.5340 \\
\hline 186.0445 & 230.51 & 211.09 & 209.59 & 208.21 & 206.88 & 204.97 & 305.02 & 254.80 & 0.2751 & 5.5498 \\
\hline 186.5452 & 230.25 & 211.22 & 209.47 & 208.09 & 206.77 & 204.89 & 304.42 & 254.54 & 0.7076 & 5.5127 \\
\hline 187.0450 & 229.31 & 210.66 & 209.40 & 207.97 & 206.68 & 204.82 & 299.42 & 253.99 & 0.2754 & 5.4948 \\
\hline 187.5448 & 231.82 & 212.04 & 209.27 & 207.86 & 206.62 & 204.80 & 301.69 & 254.50 & 0.2759 & 5.5304 \\
\hline 188.0455 & 230.45 & 213.06 & 209.17 & 207.71 & 206.54 & 204.77 & 296.55 & 252.52 & 0.2763 & 5.5346 \\
\hline 188.5453 & 230.05 & 212.44 & 209.07 & 207.55 & 206.48 & 204.76 & 299.27 & 254.12 & 0.2765 & 5.5679 \\
\hline 189.0452 & 226.53 & 210.91 & 209.35 & 207.43 & 206.45 & 204.80 & 303.62 & 252.93 & 0.2755 & 5.5124 \\
\hline 189.5450 & 227.35 & 210.38 & 209.66 & 207.59 & 206.55 & 204.87 & 296.56 & 252.21 & 0.2758 & 5.5204 \\
\hline 190.0458 & 228.80 & 210.96 & 209.64 & 207.84 & 206.65 & 205.02 & 302.40 & 253.78 & 0.2762 & 5.5401 \\
\hline 190.5447 & 228.49 & 210.59 & 209.72 & 207.94 & 206.65 & 205.10 & 300.81 & 252.66 & 0.2763 & 5.5608 \\
\hline 191.0455 & 230.56 & 211.21 & 209.65 & 208.04 & 206.63 & 205.09 & 302.36 & 252.83 & 0.2766 & 5.5696 \\
\hline 191.5453 & 229.37 & 211.68 & 209.54 & 208.05 & 206.64 & 205.06 & 301.24 & 253.51 & 0.2767 & 5.5343 \\
\hline 192.0450 & 229.04 & 211.24 & 209.49 & 208.00 & 206.61 & 204.98 & 302.93 & 252.84 & 0.2763 & 5.5311 \\
\hline 192.5440 & 228.42 & 210.84 & 209.48 & 207.98 & 206.62 & 204.90 & 303.74 & 253.64 & 0.2765 & 5.5245 \\
\hline 193.0457 & 229.39 & 211.69 & 209.42 & 207.93 & 206.57 & 204.87 & 304.46 & 255.29 & 0.2762 & 5.5439 \\
\hline 193.5445 & 228.51 & 211.41 & 209.39 & 207.87 & 206.54 & 204.84 & 302.68 & 253.22 & 0.2753 & 5.5803 \\
\hline 194.0453 & 228.85 & 211.08 & 209.38 & 207.84 & 206.53 & 204.82 & 301.14 & 254.78 & 0.2755 & 5.5681 \\
\hline 194.5452 & 228.68 & 210.87 & 209.37 & 207.81 & 206.51 & 204.81 & 298.15 & 252.99 & 0.2764 & 5.5296 \\
\hline 195.0450 & 229.14 & 210.87 & 209.34 & 207.77 & 206.48 & 204.82 & 298.89 & 254.09 & 0.7077 & 5.5195 \\
\hline 195.5457 & 228.29 & 210.94 & 209.33 & 207.72 & 206.49 & 204.79 & 301.36 & 255.08 & 0.2568 & 5.5523 \\
\hline 196.0455 & 230.18 & 211.38 & 209.29 & 207.73 & 206.46 & 204.78 & 294.62 & 252.05 & 0.2746 & 5.5645 \\
\hline 196.5443 & 232.82 & 212.77 & 209.15 & 207.65 & 206.43 & 204.79 & 299.85 & 253.87 & 0.2762 & 5.5671 \\
\hline 197.0452 & 229.73 & 213.46 & 209.05 & 207.55 & 206.37 & 204.79 & 294.93 & 254.05 & 0.2762 & 5.5274 \\
\hline 197.5450 & 226.01 & 211.23 & 209.21 & 207.45 & 206.32 & 204.75 & 301.44 & 254.40 & 0.2758 & 5.5191 \\
\hline 198.0457 & 225.90 & 210.36 & 209.61 & 207.57 & 206.48 & 204.85 & 300.30 & 252.42 & 0.2757 & 5.5569 \\
\hline 198.5455 & 228.95 & 210.90 & 209.76 & 207.95 & 206.68 & 204.96 & 302.33 & 252.87 & 0.2761 & 5.5466 \\
\hline 199.0453 & 230.48 & 210.97 & 209.68 & 208.12 & 206.64 & 205.13 & 303.06 & 253.24 & 0.2763 & 5.5414 \\
\hline 199.5442 & 229.75 & 210.80 & 209.62 & 208.13 & 206.59 & 205.16 & 301.43 & 254.98 & 0.2753 & 5.5109 \\
\hline 200.0450 & 229.42 & 210.79 & 209.53 & 208.08 & 206.62 & 205.10 & 302.89 & 254.62 & 0.2761 & 5.5314 \\
\hline 200.5438 & 228.30 & 210.72 & 209.49 & 208.06 & 206.61 & 205.00 & 300.28 & 254.43 & 0.2767 & 5.5462 \\
\hline 201.0455 & 230.53 & 210.70 & 209.41 & 207.99 & 206.65 & 204.91 & 297.96 & 253.90 & 0.2761 & 5.5304 \\
\hline 201.5445 & 229.86 & 211.06 & 209.34 & 207.95 & 206.61 & 204.87 & 299.54 & 253.47 & 0.2762 & 5.4886 \\
\hline 202.0452 & 228.93 & 210.50 & 209.37 & 207.85 & 206.58 & 204.84 & 300.20 & 254.91 & 0.2760 & 5.5052 \\
\hline 202.5450 & 229.19 & 210.70 & 209.36 & 207.80 & 206.55 & 204.81 & 301.08 & 253.75 & 0.2768 & 5.5411 \\
\hline 203.0448 & 229.95 & 211.89 & 209.37 & 207.77 & 206.52 & 204.80 & 302.84 & 254.56 & 0.2763 & 5.5783 \\
\hline 203.5455 & 229.42 & 211.54 & 209.34 & 207.75 & 206.51 & 204.79 & 302.53 & 253.65 & 0.2760 & 5.5530 \\
\hline 204.0453 & 228.54 & 211.32 & 209.35 & 207.74 & 206.50 & 204.78 & 302.63 & 254.21 & 0.2757 & 5.5133 \\
\hline 204.5452 & 227.99 & 210.41 & 209.39 & 207.71 & 206.47 & 204.79 & 297.68 & 253.56 & 0.2754 & 5.5347 \\
\hline
\end{tabular}


TABLE B1.- CONTINUED.

\begin{tabular}{|c|c|c|c|c|c|c|c|c|c|c|}
\hline $\begin{array}{c}\text { Time, } \\
\text { min }\end{array}$ & $\begin{array}{c}\text { T-steam } \\
{ }^{\circ} \mathrm{C}\end{array}$ & $\begin{array}{l}\mathrm{T} 1, \\
{ }^{\circ} \mathrm{C}\end{array}$ & $\begin{array}{l}\mathrm{T} 2, \\
{ }^{\circ} \mathrm{C}\end{array}$ & $\begin{array}{l}\mathrm{T} 3, \\
{ }^{\circ} \mathrm{C}\end{array}$ & $\begin{array}{l}\mathrm{T} 4, \\
{ }^{\circ} \mathrm{C}\end{array}$ & $\begin{array}{l}\mathrm{T} 5, \\
{ }^{\circ} \mathrm{C}\end{array}$ & $\begin{array}{l}\text { Pinj, } \\
\text { psig }\end{array}$ & $\begin{array}{c}\text { Pout, } \\
\text { psig }\end{array}$ & $\begin{array}{l}\text { Qinj, } \\
\text { g/min }\end{array}$ & $\begin{array}{c}\mathrm{Vw} \\
\mathrm{ml} / \mathrm{min}\end{array}$ \\
\hline 205.0450 & 229.43 & 211.40 & 209.40 & 207.72 & 206.48 & 204.82 & 302.46 & 254.89 & 0.2765 & 5.5442 \\
\hline 205.5440 & 228.79 & 211.66 & 209.39 & 207.76 & 206.51 & 204.83 & 302.77 & 253.55 & 0.2767 & 5.5660 \\
\hline 206.0455 & 227.96 & 211.28 & 209.42 & 207.75 & 206.50 & 204.82 & 304.20 & 254.95 & 0.2756 & 5.5439 \\
\hline 206.5445 & 229.70 & 211.28 & 209.40 & 207.76 & 206.47 & 204.82 & 300.22 & 252.56 & 0.2757 & 5.5321 \\
\hline 207.0443 & 229.73 & 210.56 & 209.39 & 207.75 & 206.48 & 204.85 & 293.64 & 254.31 & 0.2754 & 5.5319 \\
\hline 207.5450 & 232.73 & 211.87 & 209.17 & 207.76 & 206.47 & 204.87 & 304.21 & 253.87 & 0.2764 & 5.5514 \\
\hline 208.0448 & 229.03 & 211.63 & 209.25 & 207.64 & 206.40 & 204.85 & 298.55 & 252.28 & 0.2760 & 5.5745 \\
\hline 208.5457 & 230.22 & 211.19 & 209.35 & 207.70 & 206.45 & 204.82 & 295.56 & 253.37 & 0.2758 & 5.5663 \\
\hline 209.0455 & 231.32 & 213.40 & 209.10 & 207.72 & 206.44 & 204.84 & 302.30 & 254.59 & 0.2759 & 5.5218 \\
\hline 209.5453 & 227.13 & 211.72 & 209.36 & 207.55 & 206.35 & 204.82 & 308.88 & 253.53 & 0.2758 & 5.5133 \\
\hline 210.0450 & 227.37 & 210.84 & 209.80 & 207.74 & 206.45 & 204.82 & 307.42 & 253.99 & 0.2755 & 5.5525 \\
\hline 210.5440 & 230.93 & 212.62 & 209.81 & 208.02 & 206.57 & 204.96 & 304.47 & 253.85 & 0.2751 & 5.5744 \\
\hline 211.0447 & 232.51 & 214.32 & 209.81 & 208.08 & 206.52 & 204.98 & 304.73 & 253.92 & 0.2760 & 5.5561 \\
\hline 211.5445 & 231.78 & 214.67 & 209.78 & 208.10 & 206.56 & 204.99 & 308.39 & 254.60 & 0.2752 & 5.5221 \\
\hline 212.0453 & 229.59 & 213.60 & 209.84 & 208.13 & 206.59 & 204.98 & 302.06 & 253.86 & 0.2756 & 5.4981 \\
\hline 212.5452 & 230.46 & 213.63 & 209.87 & 208.19 & 206.69 & 205.02 & 302.33 & 254.80 & 0.2769 & 5.5386 \\
\hline 213.0450 & 230.78 & 213.82 & 209.81 & 208.21 & 206.73 & 205.05 & 304.07 & 253.87 & 0.2728 & 5.5605 \\
\hline 213.5465 & 229.65 & 214.49 & 209.72 & 208.17 & 206.74 & 205.07 & 299.12 & 254.70 & 0.2754 & 5.5569 \\
\hline 214.0463 & 229.49 & 213.94 & 209.73 & 208.18 & 206.76 & 205.08 & 299.83 & 252.34 & 0.2758 & 5.4921 \\
\hline 214.5453 & 229.79 & 214.03 & 209.69 & 208.15 & 206.77 & 205.09 & 304.15 & 254.29 & 0.2763 & 5.5095 \\
\hline 215.0442 & 229.02 & 213.22 & 209.75 & 208.14 & 206.78 & 205.13 & 300.70 & 255.18 & 0.2764 & 5.5760 \\
\hline 215.5450 & 230.37 & 214.25 & 209.75 & 208.18 & 206.84 & 205.15 & 307.01 & 253.79 & 0.2760 & 5.5602 \\
\hline 216.0448 & 229.43 & 214.81 & 209.85 & 208.21 & 206.88 & 205.23 & 299.58 & 252.43 & 0.1692 & 5.5212 \\
\hline 216.5465 & 230.05 & 214.76 & 209.79 & 208.23 & 206.92 & 205.22 & 296.32 & 254.49 & 0.0437 & 5.4878 \\
\hline 217.0462 & 229.18 & 215.13 & 209.62 & 208.17 & 206.88 & 205.27 & 302.15 & 254.66 & 0.0435 & 5.5179 \\
\hline 217.5460 & 228.97 & 214.83 & 209.52 & 208.07 & 206.87 & 205.24 & 295.86 & 253.04 & 0.0293 & 5.5193 \\
\hline 218.0458 & 227.78 & 214.75 & 209.40 & 207.95 & 206.86 & 205.25 & 291.25 & 252.80 & 0.0334 & 5.5327 \\
\hline
\end{tabular}


TABLE B2. PRESSURE AND TEMPERATURE DATA FOR RUN 4 (PURE STEAM).

\begin{tabular}{|c|c|c|c|c|c|c|c|c|c|c|}
\hline $\begin{array}{c}\text { Time, } \\
\text { min }\end{array}$ & $\begin{array}{c}\text { T-steam } \\
{ }^{0} \mathrm{C}\end{array}$ & $\begin{array}{l}\mathrm{T} 1, \\
{ }^{\circ} \mathrm{C}\end{array}$ & $\begin{array}{l}\mathrm{T} 2, \\
{ }^{\circ} \mathrm{C}\end{array}$ & $\begin{array}{l}\mathrm{T} 3, \\
{ }^{\circ} \mathrm{C}\end{array}$ & $\begin{array}{l}\mathrm{T} 4, \\
{ }^{\circ} \mathrm{C}\end{array}$ & $\begin{array}{l}\mathrm{T} 5, \\
{ }^{\circ} \mathrm{C}\end{array}$ & $\begin{array}{l}\text { Pinj, } \\
\text { psig }\end{array}$ & $\begin{array}{c}\text { Pout, } \\
\text { psig }\end{array}$ & $\begin{array}{l}\text { Qinj, } \\
\text { g/min }\end{array}$ & $\begin{array}{c}\text { Vw } \\
\mathrm{ml} / \mathrm{min}\end{array}$ \\
\hline 0.0548 & 50.89 & 43.59 & 51.52 & 55.76 & 54.33 & 48.39 & 269.73 & 270.62 & 0.0007 & 5.5795 \\
\hline 0.5473 & 112.28 & 66.55 & 51.04 & 55.68 & 54.28 & 48.43 & 266.31 & 268.42 & 0.0004 & 5.6026 \\
\hline 1.0480 & 200.30 & 82.34 & 50.89 & 55.38 & 54.20 & 48.39 & 266.52 & 268.40 & 0.0006 & 5.5777 \\
\hline 1.5478 & 213.00 & 198.82 & 51.60 & 55.25 & 54.42 & 48.57 & 269.22 & 269.78 & 0.0000 & 5.5862 \\
\hline 2.0468 & 215.43 & 210.78 & 56.13 & 54.86 & 54.72 & 48.63 & 269.94 & 269.23 & 0.0004 & 5.5519 \\
\hline 2.5475 & 217.22 & 210.23 & 54.71 & 55.07 & 54.65 & 48.67 & 265.57 & 267.45 & 0.0004 & 5.5429 \\
\hline 3.0483 & 221.95 & 210.18 & 53.72 & 55.17 & 54.62 & 48.67 & 264.99 & 266.91 & 0.0009 & 5.5381 \\
\hline 3.5482 & 227.00 & 210.54 & 53.40 & 55.15 & 54.68 & 48.72 & 268.50 & 267.34 & 0.0003 & 5.5346 \\
\hline 4.0480 & 233.95 & 211.38 & 53.77 & 55.08 & 54.81 & 48.74 & 270.65 & 268.44 & 0.0003 & 5.5587 \\
\hline 4.5477 & 238.87 & 212.42 & 54.34 & 55.06 & 54.87 & 48.72 & 271.22 & 268.41 & 0.0001 & 5.5885 \\
\hline 5.0467 & 243.76 & 212.73 & 54.40 & 55.05 & 54.89 & 48.72 & 271.72 & 267.48 & 0.0005 & 5.5850 \\
\hline 5.5473 & 237.60 & 212.71 & 54.86 & 55.03 & 54.94 & 48.80 & 275.47 & 267.70 & 0.0005 & 5.6024 \\
\hline 6.0482 & 234.14 & 212.59 & 55.76 & 54.98 & 54.98 & 48.92 & 275.83 & 267.78 & 0.0000 & 5.5825 \\
\hline 6.5480 & 228.81 & 212.92 & 56.25 & 54.98 & 55.02 & 49.12 & 279.96 & 269.02 & 0.0005 & 5.5691 \\
\hline 7.0468 & 224.88 & 212.99 & 56.75 & 54.98 & 55.02 & 49.21 & 278.77 & 268.09 & 0.0004 & 5.5378 \\
\hline 7.5477 & 222.05 & 212.90 & 56.51 & 54.99 & 55.01 & 49.26 & 280.85 & 267.70 & 0.0002 & 5.5479 \\
\hline 8.0483 & 222.50 & 213.21 & 58.01 & 54.99 & 55.01 & 49.28 & 282.67 & 267.53 & 0.0008 & 5.5403 \\
\hline 8.5482 & 225.49 & 213.63 & 60.72 & 54.95 & 55.03 & 49.36 & 285.47 & 267.46 & 0.0002 & 5.5353 \\
\hline 9.0480 & 231.87 & 214.77 & 63.22 & 54.86 & 55.01 & 49.34 & 290.15 & 267.91 & 0.0006 & 5.5639 \\
\hline 9.5487 & 239.08 & 215.37 & 63.72 & 54.87 & 54.94 & 49.27 & 290.95 & 268.47 & 0.0006 & 5.5710 \\
\hline 10.0485 & 247.32 & 216.30 & 64.93 & 54.87 & 54.93 & 49.31 & 292.29 & 268.14 & 0.0003 & 5.5818 \\
\hline 10.5483 & 247.51 & 216.64 & 67.09 & 54.83 & 54.95 & 49.31 & 292.54 & 268.21 & 0.0002 & 5.5610 \\
\hline 11.0482 & 237.67 & 216.47 & 69.73 & 54.80 & 54.97 & 49.30 & 294.87 & 268.35 & 0.0002 & 5.5654 \\
\hline 11.5480 & 225.78 & 215.65 & 73.33 & 54.78 & 54.99 & 49.32 & 295.64 & 268.34 & 0.0002 & 5.5492 \\
\hline 12.0478 & 222.76 & 215.38 & 76.25 & 54.77 & 55.03 & 49.38 & 295.21 & 267.89 & 0.0001 & 5.5335 \\
\hline 12.5467 & 222.87 & 215.46 & 79.42 & 54.73 & 55.06 & 49.42 & 297.05 & 267.64 & 0.0007 & 5.5455 \\
\hline 13.0475 & 222.99 & 215.76 & 83.65 & 54.72 & 55.10 & 49.50 & 298.28 & 267.75 & 0.0007 & 5.5526 \\
\hline 13.5482 & 223.68 & 216.09 & 86.59 & 54.72 & 55.14 & 49.56 & 300.71 & 267.68 & 0.0012 & 5.5676 \\
\hline 14.0480 & 224.61 & 216.41 & 89.09 & 54.70 & 55.14 & 49.62 & 302.67 & 267.74 & 0.0005 & 5.5487 \\
\hline 14.5470 & 225.24 & 216.76 & 91.31 & 54.72 & 55.14 & 49.70 & 305.39 & 267.75 & 0.0008 & 5.5475 \\
\hline 15.0477 & 225.24 & 217.12 & 93.95 & 54.82 & 55.18 & 49.74 & 307.51 & 267.96 & 0.0000 & 5.5209 \\
\hline 15.5475 & 226.23 & 217.39 & 95.15 & 54.96 & 55.23 & 49.82 & 309.10 & 267.95 & 0.0007 & 5.5555 \\
\hline 16.0482 & 227.09 & 217.71 & 96.30 & 55.13 & 55.23 & 49.92 & 310.31 & 268.03 & 0.0004 & 5.5581 \\
\hline 16.5480 & 227.94 & 218.04 & 97.34 & 55.31 & 55.25 & 49.98 & 313.22 & 268.11 & 0.0002 & 5.5532 \\
\hline 17.0488 & 229.39 & 218.42 & 99.83 & 55.52 & 55.29 & 50.09 & 315.49 & 268.24 & 0.0005 & 5.5595 \\
\hline 17.5477 & 230.43 & 218.87 & 101.91 & 55.81 & 55.31 & 50.19 & 318.70 & 268.38 & 0.0005 & 5.5823 \\
\hline 18.0485 & 231.27 & 219.39 & 105.09 & 56.17 & 55.33 & 50.29 & 321.22 & 265.03 & 0.0005 & 5.6131 \\
\hline 18.5483 & 229.94 & 219.48 & 106.06 & 56.57 & 55.36 & 50.41 & 322.00 & 267.64 & 0.0007 & 5.6155 \\
\hline 19.0490 & 229.44 & 219.39 & 106.39 & 56.88 & 55.34 & 50.43 & 321.34 & 265.39 & 0.0008 & 5.5825 \\
\hline 19.5470 & 229.91 & 219.40 & 106.53 & 57.15 & 55.36 & 50.49 & 322.08 & 268.64 & 0.0007 & 5.5606 \\
\hline 20.0477 & 230.35 & 219.61 & 108.06 & 57.45 & 55.36 & 50.53 & 323.20 & 268.67 & 0.0002 & 5.5338 \\
\hline 20.5475 & 231.79 & 219.88 & 109.89 & 57.82 & 55.37 & 50.55 & 324.41 & 265.84 & 0.0006 & 5.5364 \\
\hline 21.0483 & 231.60 & 220.05 & 111.60 & 58.22 & 55.41 & 50.65 & 325.87 & 268.47 & 0.0005 & 5.5474 \\
\hline 21.5482 & 231.97 & 220.23 & 113.78 & 58.68 & 55.43 & 50.73 & 327.75 & 268.68 & 0.0003 & 5.5602 \\
\hline 22.0488 & 231.62 & 220.45 & 116.13 & 59.21 & 55.47 & 50.83 & 328.88 & 268.62 & 0.0004 & 5.5473 \\
\hline 22.5468 & 231.62 & 220.67 & 118.42 & 59.80 & 55.51 & 50.93 & 329.97 & 268.62 & 0.0004 & 5.4950 \\
\hline 23.0475 & 231.05 & 220.85 & 120.73 & 60.47 & 55.57 & 51.01 & 331.58 & 268.64 & 0.0003 & 5.4885 \\
\hline 23.5483 & 231.74 & 221.03 & 122.77 & 61.19 & 55.61 & 51.07 & 332.53 & 268.66 & 0.0002 & 5.5136 \\
\hline 24.0490 & 231.49 & 221.30 & 125.20 & 61.97 & 55.69 & 51.13 & 334.21 & 268.70 & 0.0003 & 5.5479 \\
\hline 24.5470 & 231.76 & 221.43 & 125.57 & 62.75 & 55.77 & 51.24 & 334.75 & 245.05 & 0.0000 & 5.4969 \\
\hline
\end{tabular}


TABLE B2.- CONTINUED.

\begin{tabular}{|c|c|c|c|c|c|c|c|c|c|c|}
\hline $\begin{array}{c}\text { Time, } \\
\text { min }\end{array}$ & $\begin{array}{c}\text { T-steam } \\
{ }^{\circ} \mathrm{C}\end{array}$ & $\begin{array}{l}\mathrm{T} 1, \\
{ }^{\circ} \mathrm{C}\end{array}$ & $\begin{array}{l}\mathrm{T} 2, \\
{ }^{\circ} \mathrm{C}\end{array}$ & $\begin{array}{l}\mathrm{T} 3, \\
{ }^{\circ} \mathrm{C}\end{array}$ & $\begin{array}{l}\mathrm{T} 4, \\
{ }^{\circ} \mathrm{C}\end{array}$ & $\begin{array}{l}\mathrm{T} 5, \\
{ }^{\circ} \mathrm{C}\end{array}$ & $\begin{array}{l}\text { Pinj, } \\
\text { psig }\end{array}$ & $\begin{array}{c}\text { Pout, } \\
\text { psig }\end{array}$ & $\begin{array}{l}\text { Qinj, } \\
\text { g/min }\end{array}$ & $\begin{array}{c}\mathrm{Vw} \\
\mathrm{ml} / \mathrm{min}\end{array}$ \\
\hline 25.0478 & 231.37 & 221.46 & 124.99 & 63.15 & 55.87 & 51.36 & 334.12 & 268.79 & 0.0004 & 5.5000 \\
\hline 25.5477 & 231.62 & 221.34 & 125.96 & 63.47 & 55.91 & 51.44 & 333.77 & 266.45 & 0.0005 & 5.5373 \\
\hline 26.0483 & 231.84 & 221.47 & 128.14 & 63.97 & 55.99 & 51.52 & 335.11 & 268.92 & 0.0003 & 5.5239 \\
\hline 26.5482 & 232.11 & 221.62 & 128.98 & 64.63 & 56.05 & 51.54 & 336.09 & 268.64 & 0.0008 & 5.4743 \\
\hline 27.0480 & 232.65 & 221.69 & 124.93 & 64.80 & 56.13 & 51.66 & 336.51 & 268.17 & 0.0007 & 5.4822 \\
\hline 27.5478 & 232.90 & 221.82 & 122.58 & 64.48 & 56.13 & 51.68 & 337.51 & 268.84 & 0.0007 & 5.5275 \\
\hline 28.0477 & 233.21 & 221.95 & 120.30 & 63.69 & 56.07 & 51.68 & 338.25 & 268.57 & 0.0003 & 5.5166 \\
\hline 28.5483 & 233.39 & 221.95 & 120.83 & 63.45 & 56.08 & 51.70 & 338.59 & 268.65 & 0.0005 & 5.4762 \\
\hline 29.0482 & 233.48 & 222.06 & 120.80 & 63.24 & 56.04 & 51.76 & 338.61 & 268.86 & 0.0000 & 5.4984 \\
\hline 29.5472 & 233.41 & 222.08 & 120.49 & 62.96 & 56.01 & 51.78 & 338.43 & 268.56 & 0.0000 & 5.5443 \\
\hline 30.0478 & 233.61 & 222.08 & 120.82 & 62.87 & 55.97 & 51.79 & 338.02 & 268.46 & 0.0004 & 5.5207 \\
\hline 30.5477 & 234.08 & 222.07 & 120.99 & 62.82 & 55.97 & 51.77 & 338.86 & 266.61 & 0.0011 & 5.4791 \\
\hline 31.0483 & 234.37 & 222.05 & 121.05 & 62.84 & 56.00 & 51.79 & 338.65 & 268.84 & 0.0007 & 5.5104 \\
\hline 31.5482 & 234.35 & 222.11 & 120.92 & 62.80 & 55.98 & 51.79 & 339.12 & 268.55 & 0.0005 & 5.5181 \\
\hline 32.0480 & 234.25 & 222.24 & 120.47 & 62.81 & 55.98 & 51.80 & 339.15 & 268.47 & 0.0007 & 5.5687 \\
\hline 32.5478 & 234.49 & 222.27 & 120.60 & 62.92 & 55.97 & 51.76 & 339.20 & 268.42 & 0.0005 & 5.5332 \\
\hline 33.0477 & 234.56 & 222.38 & 121.33 & 62.96 & 55.97 & 51.78 & 340.46 & 268.41 & 0.0006 & 5.5177 \\
\hline 33.5483 & 234.81 & 222.44 & 122.87 & 63.21 & 55.99 & 51.84 & 341.39 & 263.39 & 0.0006 & 5.5184 \\
\hline 34.0482 & 234.30 & 222.51 & 123.75 & 63.52 & 56.01 & 51.87 & 341.66 & 268.75 & 0.0007 & 5.5306 \\
\hline 34.5472 & 234.48 & 222.57 & 124.34 & 63.61 & 56.01 & 51.93 & 341.63 & 268.54 & 0.0006 & 5.5804 \\
\hline 35.0478 & 234.21 & 222.64 & 125.02 & 63.73 & 56.04 & 51.95 & 341.94 & 268.29 & 0.0006 & 5.5587 \\
\hline 35.5477 & 234.70 & 222.63 & 125.39 & 63.98 & 56.06 & 52.01 & 342.69 & 267.80 & 0.0007 & 5.5815 \\
\hline 36.0485 & 234.38 & 222.79 & 128.04 & 64.32 & 56.10 & 52.05 & 343.45 & 268.04 & 0.0002 & 5.5679 \\
\hline 36.5483 & 234.25 & 222.88 & 129.09 & 64.57 & 56.16 & 52.09 & 344.03 & 268.53 & 0.0006 & 5.5698 \\
\hline 37.0480 & 234.35 & 222.91 & 129.64 & 64.78 & 56.14 & 52.11 & 343.16 & 266.07 & 0.0000 & 5.5662 \\
\hline 37.5478 & 234.21 & 222.87 & 129.61 & 65.14 & 56.18 & 52.13 & 343.65 & 268.40 & 0.0005 & 5.5498 \\
\hline 38.0477 & 233.74 & 222.77 & 129.39 & 65.22 & 56.20 & 52.17 & 343.07 & 268.16 & 0.0005 & 5.5416 \\
\hline 38.5475 & 233.91 & 222.84 & 129.39 & 65.34 & 56.21 & 52.18 & 344.32 & 266.18 & 0.0006 & 5.5463 \\
\hline 39.0483 & 233.64 & 222.92 & 130.51 & 65.59 & 56.23 & 52.20 & 344.47 & 258.03 & 0.0001 & 5.5553 \\
\hline 39.5482 & 234.66 & 222.92 & 131.77 & 65.98 & 56.27 & 52.22 & 344.47 & 262.44 & 0.0005 & 5.5589 \\
\hline 40.0480 & 234.36 & 223.03 & 132.08 & 66.16 & 56.27 & 52.24 & 344.07 & 261.15 & 0.0005 & 5.5626 \\
\hline 40.5468 & 234.56 & 222.90 & 132.48 & 66.27 & 56.29 & 52.26 & 343.34 & 263.82 & 0.0001 & 5.5707 \\
\hline 41.0475 & 234.56 & 222.89 & 133.11 & 66.31 & 56.30 & 52.28 & 344.05 & 262.10 & 0.0006 & 5.5815 \\
\hline 41.5483 & 234.35 & 222.95 & 134.00 & 66.49 & 56.30 & 52.32 & 344.59 & 262.26 & 0.0003 & 5.5721 \\
\hline 42.0482 & 234.89 & 222.98 & 135.08 & 66.75 & 56.32 & 52.36 & 344.80 & 263.44 & 0.0001 & 5.5476 \\
\hline 42.5470 & 234.50 & 223.04 & 136.19 & 66.98 & 56.36 & 52.37 & 345.30 & 258.82 & 0.0003 & 5.5470 \\
\hline 43.0478 & 235.54 & 223.02 & 137.92 & 67.57 & 56.40 & 52.45 & 345.23 & 254.21 & 0.0003 & 5.5464 \\
\hline 43.5477 & 234.86 & 222.95 & 138.72 & 67.95 & 56.44 & 52.48 & 343.45 & 261.49 & 0.0003 & 5.5615 \\
\hline 44.0492 & 234.25 & 222.90 & 139.05 & 67.99 & 56.46 & 52.51 & 343.50 & 258.36 & 0.0003 & 5.5552 \\
\hline 44.5472 & 233.59 & 222.89 & 139.38 & 68.18 & 56.48 & 52.53 & 343.79 & 261.54 & 0.0004 & 5.5864 \\
\hline 45.0480 & 233.72 & 222.89 & 140.10 & 68.37 & 56.50 & 52.49 & 343.82 & 262.36 & 0.0004 & 5.5762 \\
\hline 45.5478 & 233.92 & 222.93 & 141.17 & 68.60 & 56.53 & 52.51 & 344.52 & 261.86 & 0.0002 & 5.5544 \\
\hline 46.0477 & 233.89 & 223.09 & 142.35 & 68.98 & 56.55 & 52.53 & 345.03 & 264.79 & 0.0011 & 5.5610 \\
\hline 46.5475 & 233.91 & 223.14 & 143.41 & 69.39 & 56.59 & 52.55 & 345.45 & 265.53 & 0.0004 & 5.5520 \\
\hline 47.0482 & 234.12 & 223.18 & 143.75 & 69.70 & 56.65 & 52.60 & 346.39 & 261.20 & 0.0007 & 5.5417 \\
\hline 47.5480 & 234.05 & 223.19 & 144.25 & 70.04 & 56.67 & 52.64 & 346.17 & 257.42 & 0.0009 & 5.5449 \\
\hline 48.0478 & 234.06 & 223.21 & 145.54 & 70.53 & 56.73 & 52.68 & 346.19 & 247.71 & 0.0002 & 5.5504 \\
\hline 48.5477 & 233.77 & 223.19 & 148.24 & 71.27 & 56.81 & 52.74 & 344.94 & 258.68 & 0.0007 & 5.5694 \\
\hline 49.0475 & 234.24 & 223.00 & 149.11 & 71.42 & 56.85 & 52.76 & 343.60 & 258.10 & 0.0004 & 5.5815 \\
\hline 49.5482 & 234.10 & 222.91 & 148.12 & 71.50 & 56.87 & 52.78 & 343.88 & 258.05 & 0.0004 & 5.5719 \\
\hline
\end{tabular}


TABLE B2.- CONTINUED.

\begin{tabular}{|c|c|c|c|c|c|c|c|c|c|c|}
\hline $\begin{array}{l}\text { Time, } \\
\text { min }\end{array}$ & $\begin{array}{c}\text { T-steam } \\
{ }^{\circ} \mathrm{C}\end{array}$ & $\begin{array}{l}\mathrm{T} 1, \\
{ }^{\circ} \mathrm{C}\end{array}$ & $\begin{array}{l}\mathrm{T} 2, \\
{ }^{\circ} \mathrm{C}\end{array}$ & $\begin{array}{l}\mathrm{T} 3, \\
{ }^{\circ} \mathrm{C}\end{array}$ & $\begin{array}{l}\mathrm{T} 4, \\
{ }^{\circ} \mathrm{C}\end{array}$ & $\begin{array}{l}\mathrm{T} 5, \\
{ }^{\circ} \mathrm{C}\end{array}$ & $\begin{array}{l}\text { Pinj, } \\
\text { psig }\end{array}$ & $\begin{array}{l}\text { Pout, } \\
\text { psig }\end{array}$ & $\begin{array}{l}\text { Qinj, } \\
\text { g/min }\end{array}$ & $\begin{array}{c}\mathrm{Vw} \\
\mathrm{ml} / \mathrm{min}\end{array}$ \\
\hline 50.0490 & 233.71 & 222.87 & 148.21 & 71.52 & 56.89 & 52.78 & 343.65 & 263.56 & 0.0004 & 5.5735 \\
\hline 50.5478 & 233.78 & 222.88 & 148.54 & 71.52 & 56.89 & 52.76 & 344.33 & 265.15 & 0.0007 & 5.5509 \\
\hline 51.0468 & 233.89 & 222.90 & 148.13 & 71.70 & 56.89 & 52.79 & 344.27 & 265.23 & 0.0009 & 5.5523 \\
\hline 51.5475 & 233.80 & 223.13 & 148.60 & 71.85 & 56.89 & 52.77 & 345.24 & 263.45 & 0.0009 & 5.5542 \\
\hline 52.0482 & 233.57 & 223.15 & 149.13 & 72.11 & 56.94 & 52.81 & 345.54 & 263.10 & 0.0003 & 5.5520 \\
\hline 52.5480 & 233.47 & 223.14 & 149.47 & 72.42 & 56.96 & 52.81 & 345.76 & 268.85 & 0.0003 & 5.5550 \\
\hline 53.0470 & 233.24 & 223.16 & 150.49 & 72.72 & 57.00 & 52.83 & 346.16 & 263.40 & 0.0006 & 5.5526 \\
\hline 53.5477 & 232.88 & 223.25 & 151.63 & 73.12 & 57.06 & 52.87 & 346.77 & 266.48 & 0.0006 & 5.5697 \\
\hline 54.0485 & 232.92 & 223.30 & 152.65 & 73.53 & 57.10 & 52.88 & 347.12 & 268.69 & 0.0011 & 5.5573 \\
\hline 54.5483 & 232.83 & 223.34 & 153.79 & 74.01 & 57.16 & 52.92 & 347.51 & 260.08 & 0.0005 & 5.5644 \\
\hline 55.0472 & 232.80 & 223.36 & 154.68 & 74.63 & 57.21 & 52.96 & 347.48 & 264.17 & 0.0004 & 5.5516 \\
\hline 55.5478 & 232.80 & 223.29 & 155.82 & 75.08 & 57.29 & 52.98 & 347.08 & 264.94 & 0.0002 & 5.5372 \\
\hline 56.0487 & 232.50 & 223.28 & 156.72 & 75.52 & 57.33 & 53.02 & 347.13 & 257.54 & 0.0002 & 5.5425 \\
\hline 56.5475 & 232.25 & 223.24 & 157.61 & 75.99 & 57.41 & 53.04 & 346.93 & 263.49 & 0.0002 & 5.5407 \\
\hline 57.0473 & 232.54 & 223.17 & 157.70 & 76.27 & 57.45 & 53.04 & 346.06 & 267.52 & 0.0002 & 5.5565 \\
\hline 57.5482 & 232.84 & 223.27 & 157.20 & 76.46 & 57.47 & 53.06 & 346.34 & 266.18 & 0.0008 & 5.5452 \\
\hline 58.0480 & 232.58 & 223.23 & 157.58 & 76.63 & 57.51 & 53.07 & 346.50 & 268.06 & 0.0000 & 5.5618 \\
\hline 58.5477 & 232.28 & 223.27 & 158.27 & 76.86 & 57.57 & 53.09 & 346.70 & 267.34 & 0.0003 & 5.5739 \\
\hline 59.0475 & 232.55 & 223.18 & 159.19 & 77.18 & 57.61 & 53.11 & 346.58 & 267.03 & 0.0004 & 5.5705 \\
\hline 59.5483 & 232.41 & 223.33 & 159.82 & 77.62 & 57.67 & 53.13 & 347.16 & 256.83 & 0.0005 & 5.5772 \\
\hline 60.0482 & 232.32 & 223.19 & 161.16 & 78.48 & 57.77 & 53.17 & 345.51 & 262.76 & 0.0011 & 5.5318 \\
\hline 60.5480 & 232.53 & 223.12 & 162.77 & 78.93 & 57.87 & 53.21 & 345.30 & 261.72 & 0.0007 & 5.5169 \\
\hline 61.0468 & 232.86 & 223.05 & 163.33 & 79.25 & 57.89 & 53.23 & 345.01 & 264.11 & 0.0002 & 5.5620 \\
\hline 61.5477 & 232.59 & 223.05 & 163.39 & 79.57 & 57.93 & 53.23 & 345.20 & 265.13 & 0.0001 & 5.5497 \\
\hline 62.0483 & 233.03 & 223.00 & 163.39 & 79.80 & 58.01 & 53.26 & 345.33 & 262.71 & 0.0001 & 5.5642 \\
\hline 62.5482 & 232.94 & 223.09 & 163.73 & 80.20 & 58.07 & 53.28 & 345.28 & 263.11 & 0.0008 & 5.5815 \\
\hline 63.0470 & 232.90 & 223.13 & 164.53 & 80.57 & 58.12 & 53.30 & 345.71 & 262.63 & 0.0002 & 5.5802 \\
\hline 63.5478 & 232.66 & 223.11 & 165.18 & 80.99 & 58.16 & 53.30 & 345.68 & 265.49 & 0.0006 & 5.5856 \\
\hline 64.0477 & 232.60 & 223.17 & 165.63 & 81.50 & 58.26 & 53.30 & 346.03 & 266.29 & 0.0011 & 5.5706 \\
\hline 64.5493 & 233.07 & 223.24 & 166.35 & 81.87 & 58.30 & 53.32 & 346.37 & 268.73 & 0.0006 & 5.5721 \\
\hline 65.0473 & 232.95 & 223.31 & 166.95 & 82.36 & 58.38 & 53.36 & 346.44 & 258.40 & 0.0006 & 5.5289 \\
\hline 65.5480 & 232.98 & 223.17 & 167.57 & 83.02 & 58.49 & 53.38 & 345.66 & 259.19 & 0.0006 & 5.5345 \\
\hline 66.0487 & 233.10 & 223.09 & 168.14 & 83.45 & 58.59 & 53.42 & 345.18 & 255.66 & 0.0009 & 5.5355 \\
\hline 66.5467 & 233.53 & 223.03 & 168.56 & 83.79 & 58.69 & 53.43 & 344.63 & 265.35 & 0.0001 & 5.5536 \\
\hline 67.0475 & 232.97 & 223.00 & 168.74 & 84.05 & 58.75 & 53.45 & 344.48 & 267.84 & 0.0008 & 5.5609 \\
\hline 67.5482 & 232.46 & 223.06 & 168.91 & 84.24 & 58.79 & 53.45 & 344.98 & 264.85 & 0.0000 & 5.5695 \\
\hline 68.0480 & 232.51 & 223.08 & 169.47 & 84.47 & 58.85 & 53.47 & 345.11 & 262.89 & 0.0010 & 5.5859 \\
\hline 68.5470 & 232.51 & 223.11 & 170.17 & 84.90 & 58.93 & 53.51 & 345.15 & 264.24 & 0.0008 & 5.5761 \\
\hline 69.0477 & 232.48 & 223.12 & 170.75 & 85.33 & 58.98 & 53.51 & 345.75 & 266.49 & 0.0008 & 5.5530 \\
\hline 69.5483 & 232.98 & 223.21 & 171.33 & 85.65 & 59.04 & 53.52 & 345.87 & 268.43 & 0.0006 & 5.5366 \\
\hline 70.0482 & 232.84 & 223.26 & 171.81 & 85.95 & 59.12 & 53.54 & 346.09 & 267.15 & 0.0003 & 5.5373 \\
\hline 70.5480 & 232.86 & 223.21 & 172.34 & 86.22 & 59.22 & 53.54 & 345.87 & 264.60 & 0.0010 & 5.5555 \\
\hline 71.0478 & 232.56 & 223.18 & 173.02 & 86.54 & 59.30 & 53.58 & 346.00 & 268.15 & 0.0008 & 5.5556 \\
\hline 71.5468 & 232.51 & 223.22 & 173.80 & 86.89 & 59.35 & 53.58 & 346.13 & 268.59 & 0.0002 & 5.5820 \\
\hline 72.0475 & 232.71 & 223.25 & 174.52 & 87.25 & 59.45 & 53.60 & 346.57 & 266.77 & 0.0006 & 5.5782 \\
\hline 72.5483 & 232.87 & 223.29 & 175.54 & 87.66 & 59.53 & 53.60 & 347.14 & 263.13 & 0.0006 & 5.5635 \\
\hline 73.0472 & 233.21 & 223.29 & 175.78 & 88.09 & 59.61 & 53.63 & 346.58 & 262.84 & 0.0005 & 5.5646 \\
\hline 73.5478 & 232.37 & 223.37 & 176.73 & 88.28 & 59.71 & 53.67 & 346.53 & 260.71 & 0.0007 & 5.5502 \\
\hline 74.0487 & 232.32 & 223.24 & 177.85 & 88.75 & 59.82 & 53.69 & 346.25 & 260.34 & 0.0007 & 5.5172 \\
\hline 74.5475 & 232.63 & 223.21 & 178.55 & 89.18 & 59.92 & 53.71 & 346.15 & 262.89 & 0.0006 & 5.5162 \\
\hline
\end{tabular}


TABLE B2.- CONTINUED.

\begin{tabular}{|c|c|c|c|c|c|c|c|c|c|c|}
\hline $\begin{array}{l}\text { Time, } \\
\text { min }\end{array}$ & $\begin{array}{c}\text { T-steam } \\
{ }^{\circ} \mathrm{C}\end{array}$ & $\begin{array}{l}\mathrm{T} 1, \\
{ }^{\circ} \mathrm{C}\end{array}$ & $\begin{array}{l}\mathrm{T} 2, \\
{ }^{\circ} \mathrm{C}\end{array}$ & $\begin{array}{l}\mathrm{T} 3, \\
{ }^{\circ} \mathrm{C}\end{array}$ & $\begin{array}{l}\mathrm{T} 4, \\
{ }^{\circ} \mathrm{C}\end{array}$ & $\begin{array}{l}\mathrm{T} 5, \\
{ }^{\circ} \mathrm{C}\end{array}$ & $\begin{array}{l}\text { Pinj, } \\
\text { psig }\end{array}$ & $\begin{array}{c}\text { Pout, } \\
\text { psig }\end{array}$ & $\begin{array}{l}\text { Qinj, } \\
\text { g/min }\end{array}$ & $\begin{array}{c}\mathrm{Vw} \\
\mathrm{ml} / \mathrm{min}\end{array}$ \\
\hline 75.0483 & 232.29 & 223.19 & 179.34 & 89.50 & 59.98 & 53.69 & 346.05 & 265.21 & 0.0002 & 5.5511 \\
\hline 75.5482 & 232.43 & 223.23 & 180.15 & 89.89 & 60.07 & 53.71 & 345.98 & 258.32 & 0.0002 & 5.5921 \\
\hline 76.0480 & 232.41 & 223.30 & 181.18 & 90.41 & 60.19 & 53.75 & 346.11 & 257.52 & 0.0006 & 5.5849 \\
\hline 76.5468 & 232.20 & 223.20 & 182.18 & 90.95 & 60.30 & 53.77 & 345.91 & 260.83 & 0.0006 & 5.5921 \\
\hline 77.0475 & 232.63 & 223.15 & 183.05 & 91.29 & 60.40 & 53.77 & 345.81 & 262.99 & 0.0009 & 5.5707 \\
\hline 77.5483 & 232.24 & 223.18 & 183.86 & 91.63 & 60.48 & 53.81 & 346.15 & 266.31 & 0.0009 & 5.5669 \\
\hline 78.0482 & 231.92 & 223.26 & 184.92 & 91.91 & 60.55 & 53.82 & 346.06 & 259.92 & 0.0003 & 5.5432 \\
\hline 78.5470 & 232.12 & 223.21 & 186.16 & 92.47 & 60.69 & 53.86 & 345.68 & 250.84 & 0.0003 & 5.5460 \\
\hline 79.0478 & 232.28 & 223.12 & 187.33 & 93.31 & 60.86 & 53.86 & 345.04 & 253.40 & 0.0006 & 5.5503 \\
\hline 79.5477 & 231.55 & 223.09 & 188.57 & 93.91 & 61.04 & 53.90 & 345.09 & 251.83 & 0.0002 & 5.5703 \\
\hline 80.0483 & 231.70 & 223.16 & 189.91 & 94.24 & 61.17 & 53.94 & 344.70 & 251.40 & 0.0001 & 5.5788 \\
\hline 80.5482 & 232.13 & 223.04 & 191.44 & 94.58 & 61.29 & 53.96 & 344.43 & 267.39 & 0.0003 & 5.5676 \\
\hline 81.0480 & 231.77 & 222.98 & 192.73 & 94.86 & 61.35 & 53.98 & 344.57 & 262.88 & 0.0004 & 5.5898 \\
\hline 81.5468 & 231.87 & 222.97 & 194.29 & 95.05 & 61.44 & 53.98 & 344.64 & 264.86 & 0.0006 & 5.5849 \\
\hline 82.0477 & 231.65 & 223.10 & 195.67 & 95.33 & 61.52 & 53.99 & 344.85 & 264.24 & 0.0006 & 5.5405 \\
\hline 82.5483 & 231.96 & 223.11 & 197.30 & 95.59 & 61.60 & 53.97 & 344.93 & 261.17 & 0.0011 & 5.5386 \\
\hline 83.0482 & 232.05 & 223.10 & 198.93 & 95.93 & 61.67 & 53.99 & 345.44 & 262.89 & 0.0001 & 5.5337 \\
\hline 83.5480 & 231.96 & 223.12 & 200.44 & 96.41 & 61.79 & 54.01 & 345.79 & 263.38 & 0.0006 & 5.5641 \\
\hline 84.0478 & 231.68 & 223.17 & 201.55 & 96.88 & 61.92 & 54.03 & 345.89 & 262.44 & 0.0000 & 5.5683 \\
\hline 84.5467 & 231.80 & 223.19 & 202.66 & 97.31 & 62.06 & 54.01 & 346.15 & 259.55 & 0.0001 & 5.5677 \\
\hline 85.0493 & 231.89 & 223.26 & 203.97 & 97.85 & 62.19 & 54.05 & 346.15 & 261.06 & 0.0006 & 5.5805 \\
\hline 85.5482 & 231.49 & 223.23 & 204.89 & 98.28 & 62.31 & 54.07 & 346.36 & 263.97 & 0.0006 & 5.5714 \\
\hline 86.0480 & 231.20 & 223.25 & 205.55 & 98.76 & 62.44 & 54.10 & 346.51 & 261.87 & 0.0008 & 5.5811 \\
\hline 86.5478 & 231.70 & 223.24 & 206.11 & 99.24 & 62.56 & 54.10 & 346.74 & 259.10 & 0.0003 & 5.5548 \\
\hline 87.0468 & 231.74 & 223.24 & 207.00 & 99.87 & 62.73 & 54.12 & 346.56 & 258.50 & 0.0009 & 5.5370 \\
\hline 87.5475 & 231.85 & 223.29 & 207.74 & 100.41 & 62.88 & 54.16 & 346.50 & 261.30 & 0.0000 & 5.5519 \\
\hline 88.0482 & 231.53 & 223.33 & 208.55 & 100.90 & 63.03 & 54.18 & 347.03 & 262.21 & 0.0003 & 5.5473 \\
\hline 88.5480 & 231.46 & 223.29 & 209.07 & 101.40 & 63.17 & 54.20 & 347.04 & 263.09 & 0.0001 & 5.5631 \\
\hline 89.0470 & 232.05 & 223.26 & 209.44 & 101.86 & 63.32 & 54.20 & 346.36 & 262.66 & 0.0004 & 5.5707 \\
\hline 89.5477 & 232.61 & 223.30 & 209.70 & 102.22 & 63.46 & 54.22 & 347.23 & 260.26 & 0.0004 & 5.5446 \\
\hline 90.0485 & 231.45 & 223.37 & 210.57 & 102.75 & 63.59 & 54.24 & 347.68 & 260.67 & 0.0013 & 5.5702 \\
\hline 90.5483 & 230.77 & 223.53 & 211.36 & 103.33 & 63.76 & 54.26 & 348.78 & 267.63 & 0.0003 & 5.5562 \\
\hline 91.0480 & 231.98 & 223.62 & 212.08 & 103.90 & 63.91 & 54.30 & 349.68 & 266.14 & 0.0010 & 5.5679 \\
\hline 91.5470 & 231.99 & 223.73 & 212.92 & 104.48 & 64.10 & 54.32 & 350.27 & 264.62 & 0.0004 & 5.5613 \\
\hline 92.0477 & 232.33 & 223.77 & 213.53 & 105.02 & 64.24 & 54.34 & 350.50 & 263.31 & 0.0009 & 5.5724 \\
\hline 92.5485 & 232.37 & 223.88 & 214.09 & 105.61 & 64.41 & 54.36 & 351.20 & 262.80 & 0.0005 & 5.5617 \\
\hline 93.0483 & 232.21 & 223.97 & 214.50 & 106.22 & 64.56 & 54.39 & 351.30 & 262.41 & 0.0002 & 5.5514 \\
\hline 93.5472 & 232.15 & 223.90 & 214.93 & 106.72 & 64.77 & 54.42 & 350.61 & 252.75 & 0.0002 & 5.5350 \\
\hline 94.0480 & 232.30 & 223.79 & 215.61 & 107.31 & 65.04 & 54.46 & 350.21 & 254.56 & 0.0011 & 5.5483 \\
\hline 94.5477 & 231.51 & 223.72 & 217.14 & 108.07 & 65.29 & 54.50 & 349.68 & 261.66 & 0.0002 & 5.5596 \\
\hline 95.0467 & 231.28 & 223.69 & 217.76 & 108.35 & 65.44 & 54.54 & 349.69 & 262.50 & 0.0001 & 5.5498 \\
\hline 95.5473 & 231.37 & 223.69 & 218.36 & 108.66 & 65.57 & 54.56 & 349.60 & 259.97 & 0.0006 & 5.5681 \\
\hline 96.0482 & 232.27 & 223.73 & 219.02 & 109.09 & 65.69 & 54.60 & 349.62 & 264.58 & 0.0011 & 5.5886 \\
\hline 96.5480 & 231.66 & 223.73 & 219.83 & 109.66 & 65.86 & 54.60 & 349.69 & 263.58 & 0.0006 & 5.5955 \\
\hline 97.0478 & 231.68 & 223.68 & 220.15 & 110.09 & 66.01 & 54.64 & 349.89 & 265.25 & 0.0009 & 5.5953 \\
\hline 97.5477 & 231.59 & 223.71 & 220.37 & 110.48 & 66.16 & 54.68 & 350.01 & 265.70 & 0.0004 & 5.5765 \\
\hline 98.0483 & 232.08 & 223.77 & 220.53 & 110.98 & 66.32 & 54.68 & 349.99 & 266.11 & 0.0007 & 5.5675 \\
\hline 98.5482 & 231.38 & 223.75 & 220.64 & 111.47 & 66.47 & 54.72 & 350.06 & 263.46 & 0.0002 & 5.5460 \\
\hline 99.0480 & 231.49 & 223.75 & 220.69 & 112.07 & 66.66 & 54.76 & 350.01 & 262.06 & 0.0002 & 5.5396 \\
\hline 99.5478 & 231.60 & 223.68 & 220.60 & 112.71 & 66.85 & 54.78 & 349.59 & 262.37 & 0.0007 & 5.5421 \\
\hline
\end{tabular}


TABLE B2.- CONTINUED.

\begin{tabular}{|c|c|c|c|c|c|c|c|c|c|c|}
\hline $\begin{array}{l}\text { Time, } \\
\text { min }\end{array}$ & $\begin{array}{c}\text { T-steam } \\
{ }^{\circ} \mathrm{C}\end{array}$ & $\begin{array}{l}\mathrm{T} 1, \\
{ }^{\circ} \mathrm{C}\end{array}$ & $\begin{array}{l}\mathrm{T} 2, \\
{ }^{\circ} \mathrm{C}\end{array}$ & $\begin{array}{l}\mathrm{T} 3, \\
{ }^{\circ} \mathrm{C}\end{array}$ & $\begin{array}{l}\mathrm{T} 4, \\
{ }^{\circ} \mathrm{C}\end{array}$ & $\begin{array}{l}\mathrm{T} 5, \\
{ }^{\circ} \mathrm{C}\end{array}$ & $\begin{array}{l}\text { Pinj, } \\
\text { psig }\end{array}$ & $\begin{array}{l}\text { Pout, } \\
\text { psig }\end{array}$ & $\begin{array}{l}\text { Qinj, } \\
\text { g/min }\end{array}$ & $\begin{array}{c}\mathrm{Vw} \\
\mathrm{ml} / \mathrm{min}\end{array}$ \\
\hline 100.0477 & 231.42 & 223.67 & 220.73 & 113.32 & 67.04 & 54.80 & 349.63 & 261.85 & 0.0004 & 5.5205 \\
\hline 100.5483 & 231.62 & 223.69 & 220.75 & 113.91 & 67.23 & 54.84 & 349.48 & 266.46 & 0.0012 & 5.5666 \\
\hline 101.0482 & 231.48 & 223.62 & 220.82 & 114.52 & 67.40 & 54.88 & 349.23 & 261.74 & 0.0002 & 5.5583 \\
\hline 101.5480 & 231.44 & 223.60 & 220.95 & 115.11 & 67.59 & 54.90 & 349.16 & 267.56 & 0.0004 & 5.5941 \\
\hline 102.0478 & 231.71 & 223.62 & 221.01 & 115.71 & 67.78 & 54.94 & 349.20 & 261.70 & 0.0003 & 5.5705 \\
\hline 102.5477 & 231.62 & 223.60 & 221.11 & 116.34 & 67.99 & 54.98 & 349.03 & 266.12 & 0.0011 & 5.5761 \\
\hline 103.0483 & 231.32 & 223.62 & 221.22 & 116.98 & 68.20 & 55.02 & 349.32 & 264.22 & 0.0011 & 5.5655 \\
\hline 103.5492 & 230.96 & 223.59 & 221.24 & 117.54 & 68.39 & 55.04 & 348.91 & 257.54 & 0.0005 & 5.5539 \\
\hline 104.0472 & 231.09 & 223.61 & 221.33 & 118.27 & 68.62 & 55.08 & 349.14 & 260.58 & 0.0010 & 5.5383 \\
\hline 104.5478 & 231.22 & 223.57 & 221.39 & 118.99 & 68.82 & 55.12 & 348.84 & 262.30 & 0.0010 & 5.5385 \\
\hline 105.0477 & 230.82 & 223.59 & 221.41 & 119.56 & 69.03 & 55.16 & 348.82 & 263.12 & 0.0010 & 5.5597 \\
\hline 105.5493 & 231.00 & 223.57 & 221.57 & 120.24 & 69.22 & 55.20 & 348.84 & 261.52 & 0.0000 & 5.5764 \\
\hline 106.0473 & 231.09 & 223.59 & 221.68 & 120.90 & 69.43 & 55.24 & 349.24 & 264.06 & 0.0002 & 5.5791 \\
\hline 106.5480 & 231.17 & 223.56 & 221.70 & 121.54 & 69.66 & 55.30 & 348.82 & 258.21 & 0.0006 & 5.5809 \\
\hline 107.0478 & 231.29 & 223.54 & 221.79 & 122.33 & 69.89 & 55.34 & 348.84 & 263.43 & 0.0001 & 5.5886 \\
\hline 107.5468 & 231.04 & 223.54 & 221.93 & 122.99 & 70.11 & 55.36 & 348.86 & 257.82 & 0.0007 & 5.5393 \\
\hline 108.0475 & 230.60 & 223.58 & 221.97 & 123.75 & 70.38 & 55.43 & 348.73 & 257.95 & 0.0009 & 5.5220 \\
\hline 108.5483 & 230.98 & 223.55 & 222.01 & 124.57 & 70.63 & 55.49 & 348.44 & 259.36 & 0.0007 & 5.5090 \\
\hline 109.0480 & 230.87 & 223.49 & 222.04 & 125.38 & 70.89 & 55.51 & 348.53 & 260.12 & 0.0006 & 5.5390 \\
\hline 109.5470 & 231.01 & 223.57 & 222.15 & 126.02 & 71.10 & 55.57 & 348.31 & 263.60 & 0.0000 & 5.5873 \\
\hline 110.0487 & 230.82 & 223.58 & 222.17 & 126.64 & 71.29 & 55.59 & 348.43 & 267.16 & 0.0005 & 5.5952 \\
\hline 110.5475 & 231.02 & 223.55 & 222.23 & 127.25 & 71.50 & 55.65 & 348.52 & 261.93 & 0.0006 & 5.5914 \\
\hline 111.0483 & 230.91 & 223.53 & 222.30 & 128.02 & 71.74 & 55.71 & 348.55 & 256.13 & 0.0008 & 5.5818 \\
\hline 111.5482 & 231.09 & 223.44 & 222.30 & 128.87 & 72.03 & 55.77 & 348.33 & 256.80 & 0.0004 & 5.5633 \\
\hline 112.0470 & 231.06 & 223.48 & 222.32 & 129.77 & 72.31 & 55.82 & 348.08 & 259.83 & 0.0007 & 5.5499 \\
\hline 112.5477 & 231.07 & 223.50 & 222.32 & 130.83 & 72.67 & 55.90 & 347.61 & 252.61 & 0.0001 & 5.5431 \\
\hline 113.0475 & 231.20 & 223.38 & 222.32 & 132.07 & 73.03 & 55.98 & 346.95 & 258.58 & 0.0009 & 5.5429 \\
\hline 113.5483 & 230.91 & 223.32 & 222.38 & 132.67 & 73.29 & 56.03 & 347.30 & 260.92 & 0.0000 & 5.5402 \\
\hline 114.0472 & 231.02 & 223.29 & 222.38 & 133.24 & 73.50 & 56.07 & 347.00 & 261.90 & 0.0002 & 5.5410 \\
\hline 114.5480 & 230.79 & 223.33 & 222.36 & 133.84 & 73.69 & 56.13 & 346.85 & 261.92 & 0.0010 & 5.5812 \\
\hline 115.0478 & 230.63 & 223.31 & 222.40 & 134.54 & 73.91 & 56.19 & 347.10 & 266.28 & 0.0002 & 5.5745 \\
\hline 115.5467 & 230.74 & 223.31 & 222.43 & 135.21 & 74.14 & 56.23 & 347.16 & 256.48 & 0.0004 & 5.5766 \\
\hline 116.0473 & 230.76 & 223.33 & 222.38 & 136.11 & 74.39 & 56.29 & 346.59 & 263.40 & 0.0004 & 5.5855 \\
\hline 116.5482 & 230.99 & 223.33 & 222.42 & 136.91 & 74.63 & 56.35 & 346.95 & 263.73 & 0.0003 & 5.5835 \\
\hline 117.0480 & 230.53 & 223.35 & 222.47 & 137.74 & 74.88 & 56.42 & 347.35 & 260.05 & 0.0002 & 5.5645 \\
\hline 117.5468 & 230.65 & 223.35 & 222.47 & 138.55 & 75.12 & 56.48 & 347.11 & 249.40 & 0.0002 & 5.5677 \\
\hline 118.0477 & 230.48 & 223.32 & 222.40 & 139.93 & 75.46 & 56.56 & 346.52 & 254.85 & 0.0005 & 5.5521 \\
\hline 118.5483 & 231.35 & 223.21 & 222.35 & 141.33 & 75.86 & 56.63 & 345.97 & 258.58 & 0.0000 & 5.5577 \\
\hline 119.0482 & 230.86 & 223.30 & 222.41 & 142.26 & 76.16 & 56.73 & 346.38 & 251.86 & 0.0010 & 5.5455 \\
\hline 119.5472 & 230.48 & 223.30 & 222.41 & 143.18 & 76.44 & 56.81 & 346.35 & 257.48 & 0.0007 & 5.5655 \\
\hline 120.0478 & 230.48 & 223.27 & 222.41 & 144.06 & 76.69 & 56.85 & 346.34 & 261.37 & 0.0009 & 5.5724 \\
\hline 120.5477 & 230.68 & 223.23 & 222.39 & 145.00 & 76.95 & 56.92 & 346.11 & 257.04 & 0.0003 & 5.6136 \\
\hline 121.0483 & 231.02 & 223.16 & 222.37 & 146.16 & 77.25 & 57.00 & 345.53 & 260.77 & 0.0006 & 5.6178 \\
\hline 121.5482 & 230.63 & 223.18 & 222.34 & 147.18 & 77.53 & 57.08 & 345.72 & 263.51 & 0.0005 & 5.5914 \\
\hline 122.0472 & 230.36 & 223.23 & 222.36 & 148.10 & 77.78 & 57.11 & 345.98 & 262.44 & 0.0008 & 5.6000 \\
\hline 122.5478 & 230.32 & 223.25 & 222.37 & 149.12 & 78.04 & 57.19 & 346.00 & 264.29 & 0.0006 & 5.5818 \\
\hline 123.0468 & 230.79 & 223.16 & 222.36 & 150.19 & 78.27 & 57.27 & 345.60 & 265.93 & 0.0005 & 5.5660 \\
\hline 123.5475 & 230.81 & 223.18 & 222.38 & 151.31 & 78.55 & 57.31 & 346.06 & 261.75 & 0.0006 & 5.5368 \\
\hline 124.0482 & 229.97 & 223.22 & 222.43 & 152.51 & 78.83 & 57.39 & 345.94 & 260.20 & 0.0006 & 5.5300 \\
\hline 124.5480 & 229.88 & 223.22 & 222.41 & 153.68 & 79.13 & 57.46 & 345.98 & 263.01 & 0.0007 & 5.5408 \\
\hline
\end{tabular}


TABLE B2.- CONTINUED.

\begin{tabular}{|c|c|c|c|c|c|c|c|c|c|c|}
\hline $\begin{array}{l}\text { Time, } \\
\text { min }\end{array}$ & $\begin{array}{c}\text { T-steam } \\
{ }^{\circ} \mathrm{C}\end{array}$ & $\begin{array}{l}\mathrm{T} 1, \\
{ }^{\circ} \mathrm{C}\end{array}$ & $\begin{array}{l}\mathrm{T} 2, \\
{ }^{\circ} \mathrm{C}\end{array}$ & $\begin{array}{l}\mathrm{T} 3, \\
{ }^{\circ} \mathrm{C}\end{array}$ & $\begin{array}{l}\mathrm{T} 4, \\
{ }^{\circ} \mathrm{C}\end{array}$ & $\begin{array}{l}\mathrm{T} 5, \\
{ }^{\circ} \mathrm{C}\end{array}$ & $\begin{array}{l}\text { Pinj, } \\
\text { psig }\end{array}$ & $\begin{array}{l}\text { Pout, } \\
\text { psig }\end{array}$ & $\begin{array}{l}\text { Qinj, } \\
\text { g/min }\end{array}$ & $\begin{array}{c}\mathrm{Vw} \\
\mathrm{ml} / \mathrm{min}\end{array}$ \\
\hline 125.0478 & 230.52 & 223.24 & 222.42 & 154.64 & 79.40 & 57.54 & 345.82 & 262.70 & 0.0008 & 5.5567 \\
\hline 125.5477 & 230.88 & 223.19 & 222.42 & 155.94 & 79.70 & 57.62 & 346.08 & 258.91 & 0.0008 & 5.5955 \\
\hline 126.0475 & 230.19 & 223.17 & 222.42 & 157.28 & 80.03 & 57.69 & 345.63 & 260.56 & 0.0005 & 5.6132 \\
\hline 126.5483 & 230.76 & 223.15 & 222.40 & 158.40 & 80.35 & 57.79 & 345.79 & 263.73 & 0.0005 & 5.5904 \\
\hline 127.0480 & 230.90 & 223.15 & 222.38 & 159.52 & 80.67 & 57.85 & 345.72 & 263.46 & 0.0001 & 5.5906 \\
\hline 127.5470 & 230.49 & 223.15 & 222.38 & 160.62 & 80.97 & 57.94 & 345.54 & 264.37 & 0.0009 & 5.5678 \\
\hline 128.0477 & 230.55 & 223.19 & 222.40 & 161.76 & 81.29 & 58.02 & 345.82 & 250.97 & 0.0010 & 5.5448 \\
\hline 128.5485 & 230.78 & 223.10 & 222.31 & 163.51 & 81.71 & 58.15 & 345.02 & 258.48 & 0.0008 & 5.5351 \\
\hline 129.0483 & 230.98 & 223.03 & 222.24 & 165.35 & 82.14 & 58.25 & 344.84 & 256.89 & 0.0008 & 5.5416 \\
\hline 129.5482 & 230.17 & 222.94 & 222.22 & 167.15 & 82.51 & 58.36 & 344.35 & 259.45 & 0.0003 & 5.5359 \\
\hline 130.0480 & 230.30 & 222.96 & 222.19 & 169.00 & 82.85 & 58.44 & 344.17 & 261.85 & 0.0006 & 5.5833 \\
\hline 130.5477 & 229.71 & 222.96 & 222.15 & 171.13 & 83.26 & 58.57 & 343.94 & 254.10 & 0.0002 & 5.5771 \\
\hline 131.0475 & 230.07 & 222.82 & 221.99 & 175.21 & 83.75 & 58.67 & 342.88 & 264.56 & 0.0001 & 5.5856 \\
\hline 131.5483 & 230.66 & 222.80 & 221.99 & 177.91 & 84.12 & 58.76 & 342.64 & 264.17 & 0.0003 & 5.5651 \\
\hline 132.0482 & 230.14 & 222.75 & 222.05 & 179.99 & 84.38 & 58.84 & 342.91 & 261.00 & 0.0009 & 5.5414 \\
\hline 132.5470 & 230.36 & 222.76 & 222.03 & 182.07 & 84.72 & 58.93 & 342.96 & 266.87 & 0.0008 & 5.5430 \\
\hline 133.0478 & 230.23 & 222.82 & 222.08 & 184.39 & 85.00 & 59.03 & 343.11 & 266.42 & 0.0005 & 5.5457 \\
\hline 133.5477 & 230.89 & 222.89 & 222.08 & 186.76 & 85.36 & 59.12 & 343.50 & 264.24 & 0.0007 & 5.5554 \\
\hline 134.0483 & 230.44 & 222.91 & 222.16 & 189.19 & 85.69 & 59.24 & 343.66 & 266.43 & 0.0006 & 5.5532 \\
\hline 134.5482 & 229.94 & 222.87 & 222.14 & 191.28 & 86.03 & 59.33 & 343.24 & 264.52 & 0.0006 & 5.5545 \\
\hline 135.0470 & 230.39 & 222.87 & 222.10 & 193.53 & 86.40 & 59.41 & 343.17 & 261.58 & 0.0006 & 5.5936 \\
\hline 135.5478 & 230.48 & 222.86 & 222.10 & 195.59 & 86.78 & 59.52 & 343.25 & 264.11 & 0.0014 & 5.5795 \\
\hline 136.0485 & 229.68 & 222.78 & 222.12 & 197.92 & 87.13 & 59.62 & 343.26 & 264.32 & 0.0010 & 5.6032 \\
\hline 136.5483 & 229.97 & 222.73 & 222.11 & 199.66 & 87.54 & 59.75 & 343.10 & 266.90 & 0.0001 & 5.5643 \\
\hline 137.0473 & 230.36 & 222.75 & 222.11 & 200.93 & 87.92 & 59.85 & 342.88 & 268.69 & 0.0005 & 5.5341 \\
\hline 137.5480 & 230.95 & 222.81 & 222.11 & 202.31 & 88.31 & 59.96 & 343.28 & 267.54 & 0.0002 & 5.5288 \\
\hline 138.0468 & 230.16 & 222.88 & 222.18 & 203.94 & 88.74 & 60.06 & 343.34 & 264.40 & 0.0001 & 5.5386 \\
\hline 138.5477 & 230.63 & 222.91 & 222.16 & 205.41 & 89.20 & 60.17 & 343.48 & 267.88 & 0.0005 & 5.5526 \\
\hline 139.0483 & 231.02 & 222.93 & 222.20 & 206.64 & 89.65 & 60.29 & 343.78 & 262.34 & 0.0007 & 5.5699 \\
\hline 139.5482 & 230.09 & 222.89 & 222.20 & 209.10 & 90.14 & 60.42 & 343.94 & 262.13 & 0.0006 & 5.5739 \\
\hline 140.0480 & 230.06 & 222.84 & 222.16 & 211.08 & 90.64 & 60.55 & 343.43 & 252.85 & 0.0006 & 5.5691 \\
\hline 140.5478 & 230.04 & 222.81 & 222.07 & 213.66 & 91.26 & 60.70 & 342.67 & 259.87 & 0.0004 & 5.5715 \\
\hline 141.0468 & 230.40 & 222.74 & 221.97 & 215.49 & 91.78 & 60.84 & 341.97 & 260.59 & 0.0011 & 5.5309 \\
\hline 141.5483 & 229.90 & 222.61 & 221.95 & 216.58 & 92.19 & 60.97 & 341.76 & 261.05 & 0.0008 & 5.5202 \\
\hline 142.0482 & 230.09 & 222.56 & 221.86 & 217.26 & 92.65 & 61.10 & 341.36 & 235.92 & 0.0005 & 5.5350 \\
\hline 142.5480 & 230.63 & 222.32 & 221.48 & 218.37 & 93.92 & 61.33 & 338.02 & 254.14 & 0.0008 & 5.5647 \\
\hline 143.0478 & 231.47 & 222.22 & 221.34 & 218.80 & 94.59 & 61.54 & 337.60 & 255.77 & 0.0007 & 5.5787 \\
\hline 143.5477 & 230.88 & 222.11 & 221.34 & 218.91 & 95.14 & 61.71 & 337.30 & 260.42 & 0.0000 & 5.6055 \\
\hline 144.0493 & 230.79 & 222.06 & 221.32 & 219.05 & 95.66 & 61.82 & 336.99 & 264.01 & 0.0000 & 5.6076 \\
\hline 144.5473 & 231.19 & 221.97 & 221.30 & 219.12 & 96.13 & 61.92 & 336.95 & 267.09 & 0.0003 & 5.6000 \\
\hline 145.0480 & 231.01 & 221.93 & 221.30 & 219.19 & 96.56 & 62.05 & 336.87 & 266.30 & 0.0004 & 5.5997 \\
\hline 145.5488 & 231.63 & 221.93 & 221.31 & 219.23 & 96.97 & 62.16 & 337.19 & 267.34 & 0.0005 & 5.5442 \\
\hline 146.0468 & 230.67 & 221.91 & 221.32 & 219.27 & 97.39 & 62.28 & 337.37 & 265.08 & 0.0002 & 5.5265 \\
\hline 146.5475 & 230.67 & 222.06 & 221.36 & 219.36 & 97.84 & 62.39 & 337.16 & 262.48 & 0.0001 & 5.5367 \\
\hline 147.0483 & 230.76 & 222.06 & 221.38 & 219.39 & 98.34 & 62.54 & 337.28 & 263.58 & 0.0009 & 5.5493 \\
\hline 147.5482 & 231.10 & 222.02 & 221.38 & 219.43 & 98.84 & 62.68 & 337.40 & 268.88 & 0.0006 & 5.5654 \\
\hline 148.0480 & 231.75 & 222.11 & 221.36 & 219.46 & 99.29 & 62.81 & 337.33 & 268.62 & 0.0005 & 5.5879 \\
\hline 148.5477 & 231.14 & 222.10 & 221.40 & 219.50 & 99.81 & 62.91 & 337.28 & 268.28 & 0.0004 & 5.5969 \\
\hline 149.0475 & 230.71 & 222.04 & 221.42 & 219.56 & 100.33 & 63.04 & 337.34 & 268.70 & 0.0005 & 5.5933 \\
\hline 149.5492 & 230.76 & 222.01 & 221.42 & 219.56 & 100.88 & 63.19 & 337.42 & 267.86 & 0.0005 & 5.5896 \\
\hline
\end{tabular}


TABLE B2.- CONTINUED.

\begin{tabular}{|c|c|c|c|c|c|c|c|c|c|c|}
\hline $\begin{array}{l}\text { Time, } \\
\text { min }\end{array}$ & $\begin{array}{c}\text { T-steam } \\
{ }^{\circ} \mathrm{C}\end{array}$ & $\begin{array}{l}\mathrm{T} 1, \\
{ }^{\circ} \mathrm{C}\end{array}$ & $\begin{array}{l}\mathrm{T} 2, \\
{ }^{\circ} \mathrm{C}\end{array}$ & $\begin{array}{l}\mathrm{T} 3, \\
{ }^{\circ} \mathrm{C}\end{array}$ & $\begin{array}{l}\mathrm{T} 4, \\
{ }^{\circ} \mathrm{C}\end{array}$ & $\begin{array}{l}\mathrm{T} 5, \\
{ }^{\circ} \mathrm{C}\end{array}$ & $\begin{array}{l}\text { Pinj, } \\
\text { psig }\end{array}$ & $\begin{array}{l}\text { Pout, } \\
\text { psig }\end{array}$ & $\begin{array}{l}\text { Qinj, } \\
\text { g/min }\end{array}$ & $\begin{array}{c}\mathrm{Vw} \\
\mathrm{ml} / \mathrm{min}\end{array}$ \\
\hline 150.0490 & 230.26 & 221.97 & 221.42 & 219.55 & 101.46 & 63.30 & 337.13 & 268.01 & 0.0005 & 5.5992 \\
\hline 150.5480 & 229.76 & 221.94 & 221.36 & 219.54 & 102.01 & 63.46 & 337.25 & 268.51 & 0.0006 & 5.5708 \\
\hline 151.0478 & 229.69 & 222.04 & 221.36 & 219.50 & 102.60 & 63.63 & 337.11 & 263.84 & 0.0004 & 5.5396 \\
\hline 151.5477 & 230.67 & 221.97 & 221.33 & 219.34 & 103.44 & 63.80 & 336.75 & 267.82 & 0.0008 & 5.5132 \\
\hline 152.0483 & 231.14 & 221.92 & 221.29 & 219.40 & 104.18 & 63.95 & 336.18 & 268.65 & 0.0006 & 5.5376 \\
\hline 152.5482 & 230.85 & 221.87 & 221.29 & 219.45 & 104.88 & 64.10 & 336.78 & 267.13 & 0.0002 & 5.5802 \\
\hline 153.0470 & 230.73 & 221.81 & 221.29 & 219.49 & 105.67 & 64.29 & 336.68 & 268.61 & 0.0006 & 5.6059 \\
\hline 153.5478 & 230.75 & 221.83 & 221.28 & 219.47 & 106.41 & 64.42 & 336.52 & 267.69 & 0.0006 & 5.6014 \\
\hline 154.0467 & 230.71 & 221.83 & 221.26 & 219.45 & 107.28 & 64.59 & 336.34 & 268.04 & 0.0006 & 5.5971 \\
\hline 154.5483 & 230.26 & 221.85 & 221.22 & 219.43 & 108.13 & 64.76 & 335.95 & 268.16 & 0.0006 & 5.5965 \\
\hline 155.0482 & 230.60 & 221.80 & 221.15 & 219.40 & 108.96 & 64.93 & 335.61 & 267.54 & 0.0007 & 5.6020 \\
\hline 155.5470 & 230.23 & 221.72 & 221.12 & 219.33 & 109.85 & 65.10 & 335.46 & 267.17 & 0.0006 & 5.5838 \\
\hline 156.0478 & 230.14 & 221.76 & 221.06 & 219.27 & 110.80 & 65.31 & 335.22 & 268.25 & 0.0003 & 5.5757 \\
\hline 156.5477 & 230.21 & 221.69 & 221.01 & 219.27 & 111.78 & 65.48 & 334.78 & 266.79 & 0.0005 & 5.5507 \\
\hline 157.0493 & 230.36 & 221.60 & 220.99 & 219.24 & 112.89 & 65.67 & 334.59 & 268.45 & 0.0006 & 5.5429 \\
\hline 157.5473 & 231.11 & 221.57 & 220.94 & 219.22 & 113.96 & 65.88 & 334.32 & 267.62 & 0.0005 & 5.5538 \\
\hline 158.0480 & 230.84 & 221.55 & 220.94 & 219.19 & 115.13 & 66.09 & 333.98 & 268.29 & 0.0005 & 5.5560 \\
\hline 158.5488 & 230.81 & 221.51 & 220.91 & 219.15 & 116.46 & 66.32 & 333.67 & 266.45 & 0.0007 & 5.5632 \\
\hline 159.0468 & 230.75 & 221.44 & 220.83 & 219.12 & 117.85 & 66.54 & 333.56 & 268.42 & 0.0002 & 5.5901 \\
\hline 159.5475 & 230.80 & 221.42 & 220.78 & 219.08 & 119.25 & 66.77 & 333.03 & 267.12 & 0.0007 & 5.5895 \\
\hline 160.0482 & 230.34 & 221.42 & 220.74 & 219.03 & 120.72 & 67.00 & 332.77 & 268.27 & 0.0005 & 5.5738 \\
\hline 160.5480 & 230.66 & 221.34 & 220.71 & 218.99 & 122.26 & 67.22 & 332.45 & 268.70 & 0.0004 & 5.5730 \\
\hline 161.0478 & 231.74 & 221.23 & 220.64 & 218.96 & 123.87 & 67.45 & 331.93 & 267.72 & 0.0006 & 5.5571 \\
\hline 161.5477 & 230.80 & 221.19 & 220.60 & 218.90 & 125.68 & 67.69 & 331.61 & 268.44 & 0.0008 & 5.5264 \\
\hline 162.0475 & 231.15 & 221.12 & 220.56 & 218.87 & 127.60 & 67.92 & 331.46 & 268.24 & 0.0006 & 5.5371 \\
\hline 162.5483 & 231.93 & 221.18 & 220.50 & 218.81 & 129.72 & 68.17 & 330.79 & 268.48 & 0.0004 & 5.5374 \\
\hline 163.0480 & 230.54 & 221.19 & 220.50 & 218.78 & 131.98 & 68.41 & 331.56 & 268.53 & 0.0006 & 5.5862 \\
\hline 163.5478 & 230.72 & 221.18 & 220.48 & 218.78 & 134.48 & 68.70 & 330.56 & 267.81 & 0.0004 & 5.6230 \\
\hline 164.0477 & 231.45 & 221.11 & 220.42 & 218.74 & 137.48 & 68.98 & 330.08 & 268.46 & 0.0004 & 5.6036 \\
\hline 164.5467 & 232.22 & 221.04 & 220.35 & 218.73 & 140.50 & 69.26 & 330.24 & 268.63 & 0.0005 & 5.5910 \\
\hline 165.0473 & 231.58 & 220.93 & 220.36 & 218.67 & 143.72 & 69.59 & 329.94 & 268.52 & 0.0005 & 5.5805 \\
\hline 165.5482 & 232.12 & 221.11 & 220.30 & 218.64 & 147.22 & 69.85 & 329.63 & 267.80 & 0.0007 & 5.5803 \\
\hline 166.0480 & 231.63 & 220.98 & 220.27 & 218.53 & 151.77 & 70.15 & 329.18 & 268.48 & 0.0009 & 5.5604 \\
\hline 166.5468 & 231.90 & 220.84 & 220.18 & 218.53 & 156.72 & 70.47 & 328.75 & 268.22 & 0.0007 & 5.5438 \\
\hline 167.0475 & 232.39 & 220.75 & 220.13 & 218.44 & 162.68 & 70.79 & 328.30 & 266.98 & 0.0002 & 5.5217 \\
\hline 167.5483 & 233.05 & 220.77 & 220.04 & 218.34 & 168.84 & 71.17 & 327.45 & 267.14 & 0.0010 & 5.5457 \\
\hline 168.0482 & 233.73 & 220.75 & 219.97 & 218.28 & 174.61 & 71.49 & 327.37 & 267.75 & 0.0010 & 5.5823 \\
\hline 168.5470 & 233.29 & 220.65 & 219.93 & 218.27 & 180.33 & 71.83 & 326.92 & 266.65 & 0.0007 & 5.5898 \\
\hline 169.0478 & 233.75 & 220.59 & 219.88 & 218.23 & 186.61 & 72.17 & 326.56 & 268.62 & 0.0009 & 5.5914 \\
\hline 169.5477 & 234.09 & 220.60 & 219.83 & 218.20 & 192.38 & 72.53 & 326.24 & 265.48 & 0.0000 & 5.5975 \\
\hline 170.0492 & 234.15 & 220.43 & 219.77 & 218.04 & 201.80 & 73.00 & 325.85 & 263.36 & 0.0006 & 5.5896 \\
\hline 170.5482 & 234.02 & 220.36 & 219.63 & 217.89 & 209.30 & 73.55 & 324.83 & 266.81 & 0.0001 & 5.5654 \\
\hline 171.0480 & 234.78 & 220.26 & 219.49 & 217.82 & 212.54 & 74.00 & 323.28 & 262.01 & 0.0003 & 5.5577 \\
\hline 171.5478 & 235.50 & 220.21 & 219.35 & 217.66 & 214.30 & 74.41 & 322.80 & 264.53 & 0.0010 & 5.5326 \\
\hline 172.0477 & 236.79 & 219.99 & 219.20 & 217.59 & 215.21 & 74.81 & 321.46 & 267.17 & 0.0009 & 5.5331 \\
\hline 172.5493 & 235.43 & 219.96 & 219.13 & 217.49 & 215.86 & 75.19 & 321.89 & 267.31 & 0.0002 & 5.5306 \\
\hline 173.0473 & 234.28 & 219.90 & 219.06 & 217.42 & 216.18 & 75.58 & 320.86 & 267.47 & 0.0003 & 5.5575 \\
\hline 173.5480 & 234.41 & 219.87 & 218.96 & 217.35 & 216.40 & 76.00 & 320.31 & 267.94 & 0.0004 & 5.5576 \\
\hline 174.0487 & 235.50 & 219.82 & 218.85 & 217.26 & 216.56 & 76.43 & 319.49 & 267.40 & 0.0003 & 5.5558 \\
\hline 174.5467 & 237.08 & 219.83 & 218.76 & 217.19 & 216.61 & 76.86 & 318.91 & 267.32 & 0.0006 & 5.5582 \\
\hline
\end{tabular}


TABLE B2.- CONTINUED.

\begin{tabular}{|c|c|c|c|c|c|c|c|c|c|c|}
\hline $\begin{array}{l}\text { Time, } \\
\text { min }\end{array}$ & $\begin{array}{c}\text { T-steam } \\
{ }^{\circ} \mathrm{C}\end{array}$ & $\begin{array}{l}\mathrm{T} 1, \\
{ }^{\circ} \mathrm{C}\end{array}$ & $\begin{array}{l}\mathrm{T} 2, \\
{ }^{\circ} \mathrm{C}\end{array}$ & $\begin{array}{l}\mathrm{T} 3, \\
{ }^{\circ} \mathrm{C}\end{array}$ & $\begin{array}{l}\mathrm{T} 4, \\
{ }^{\circ} \mathrm{C}\end{array}$ & $\begin{array}{l}\mathrm{T} 5, \\
{ }^{\circ} \mathrm{C}\end{array}$ & $\begin{array}{l}\text { Pinj, } \\
\text { psig }\end{array}$ & $\begin{array}{l}\text { Pout, } \\
\text { psig }\end{array}$ & $\begin{array}{l}\text { Qinj, } \\
\text { g/min }\end{array}$ & $\begin{array}{c}\mathrm{Vw} \\
\mathrm{ml} / \mathrm{min}\end{array}$ \\
\hline 175.0475 & 236.86 & 219.73 & 218.67 & 217.10 & 216.65 & 77.31 & 318.41 & 267.76 & 0.0000 & 5.5307 \\
\hline 175.5482 & 237.13 & 219.53 & 218.60 & 217.03 & 216.69 & 77.80 & 318.19 & 268.65 & 0.0007 & 5.5429 \\
\hline 176.0480 & 234.30 & 219.30 & 218.55 & 216.96 & 216.69 & 78.37 & 317.63 & 267.94 & 0.0006 & 5.5446 \\
\hline 176.5470 & 233.55 & 219.21 & 218.50 & 216.89 & 216.67 & 78.91 & 316.98 & 268.54 & 0.0004 & 5.5609 \\
\hline 177.0477 & 235.40 & 219.30 & 218.39 & 216.80 & 216.62 & 79.49 & 316.26 & 267.57 & 0.0005 & 5.5616 \\
\hline 177.5483 & 235.97 & 219.29 & 218.27 & 216.69 & 216.51 & 80.11 & 315.43 & 266.30 & 0.0003 & 5.5694 \\
\hline 178.0482 & 238.21 & 219.29 & 218.14 & 216.55 & 216.42 & 80.83 & 314.81 & 262.97 & 0.0006 & 5.5526 \\
\hline 178.5472 & 239.86 & 219.20 & 217.97 & 216.25 & 215.98 & 82.03 & 313.44 & 257.63 & 0.0007 & 5.5659 \\
\hline 179.0478 & 240.04 & 219.22 & 217.73 & 215.96 & 215.77 & 83.24 & 311.92 & 257.24 & 0.0001 & 5.5661 \\
\hline 179.5487 & 238.77 & 219.36 & 217.31 & 215.45 & 215.28 & 84.96 & 308.95 & 263.70 & 0.0004 & 5.5751 \\
\hline 180.0485 & 239.01 & 219.51 & 216.93 & 215.36 & 215.37 & 85.73 & 307.03 & 267.20 & 0.0005 & 5.5747 \\
\hline 180.5483 & 239.08 & 218.92 & 216.77 & 215.25 & 215.34 & 86.39 & 305.88 & 268.61 & 0.0011 & 5.5565 \\
\hline 181.0472 & 239.35 & 218.69 & 216.63 & 215.16 & 215.29 & 87.12 & 305.01 & 267.95 & 0.0006 & 5.5540 \\
\hline 181.5478 & 237.95 & 218.33 & 216.54 & 215.07 & 215.20 & 88.09 & 304.42 & 267.96 & 0.0002 & 5.5493 \\
\hline 182.0477 & 238.96 & 217.92 & 216.43 & 214.99 & 215.13 & 89.24 & 303.88 & 267.88 & 0.0005 & 5.5485 \\
\hline 182.5467 & 237.89 & 218.03 & 216.36 & 214.92 & 215.06 & 90.57 & 303.31 & 268.10 & 0.0004 & 5.5588 \\
\hline 183.0473 & 237.05 & 217.87 & 216.28 & 214.85 & 214.99 & 91.97 & 303.07 & 268.05 & 0.0004 & 5.5677 \\
\hline 183.5482 & 236.74 & 217.66 & 216.17 & 214.76 & 214.94 & 93.64 & 301.96 & 268.00 & 0.0000 & 5.5542 \\
\hline 184.0480 & 237.08 & 217.46 & 216.06 & 214.67 & 214.85 & 95.52 & 301.34 & 267.94 & 0.0000 & 5.6053 \\
\hline 184.5468 & 236.57 & 217.30 & 215.96 & 214.55 & 214.76 & 97.53 & 300.47 & 268.22 & 0.0005 & 5.5941 \\
\hline 185.0475 & 233.93 & 217.12 & 215.89 & 214.46 & 214.62 & 99.74 & 300.31 & 268.16 & 0.0002 & 5.5777 \\
\hline 185.5483 & 234.96 & 217.11 & 215.77 & 214.37 & 214.53 & 102.15 & 299.60 & 268.18 & 0.0010 & 5.5742 \\
\hline 186.0482 & 235.71 & 217.45 & 215.75 & 214.30 & 214.46 & 104.72 & 299.54 & 267.64 & 0.0000 & 5.5627 \\
\hline 186.5470 & 236.99 & 217.50 & 215.68 & 214.21 & 214.36 & 107.84 & 298.88 & 266.60 & 0.0003 & 5.5495 \\
\hline 187.0478 & 237.70 & 217.35 & 215.54 & 214.07 & 214.21 & 111.48 & 298.05 & 265.44 & 0.0003 & 5.5450 \\
\hline 187.5485 & 237.76 & 216.92 & 215.38 & 213.91 & 214.06 & 115.23 & 296.97 & 265.72 & 0.0011 & 5.5284 \\
\hline 188.0483 & 237.44 & 216.72 & 215.19 & 213.74 & 213.86 & 119.71 & 295.98 & 266.31 & 0.0011 & 5.5431 \\
\hline 188.5472 & 237.49 & 216.51 & 215.03 & 213.61 & 213.79 & 122.96 & 294.91 & 268.31 & 0.0001 & 5.5891 \\
\hline 189.0480 & 237.16 & 216.48 & 214.94 & 213.51 & 213.69 & 127.04 & 294.26 & 263.56 & 0.0004 & 5.6024 \\
\hline 189.5478 & 235.89 & 216.39 & 214.80 & 213.33 & 213.47 & 133.18 & 293.53 & 262.69 & 0.0005 & 5.5815 \\
\hline 190.0485 & 236.62 & 216.37 & 214.64 & 213.17 & 213.31 & 139.37 & 292.61 & 262.85 & 0.0001 & 5.5933 \\
\hline 190.5483 & 236.23 & 216.11 & 214.50 & 212.99 & 213.14 & 147.34 & 291.66 & 262.98 & 0.0005 & 5.5653 \\
\hline 191.0492 & 236.52 & 215.93 & 214.34 & 212.84 & 213.00 & 154.98 & 290.57 & 266.56 & 0.0002 & 5.5696 \\
\hline 191.5470 & 236.67 & 215.75 & 214.22 & 212.75 & 212.96 & 158.29 & 290.03 & 267.70 & 0.0005 & 5.5419 \\
\hline 192.0478 & 237.21 & 215.74 & 214.11 & 212.73 & 212.97 & 159.79 & 289.33 & 267.92 & 0.0005 & 5.5306 \\
\hline 192.5477 & 235.56 & 215.56 & 214.06 & 212.72 & 212.99 & 161.63 & 288.95 & 267.94 & 0.0000 & 5.5275 \\
\hline 193.0493 & 235.76 & 215.99 & 213.99 & 212.66 & 212.93 & 163.80 & 288.61 & 268.01 & 0.0002 & 5.5378 \\
\hline 193.5473 & 236.35 & 216.16 & 213.92 & 212.61 & 212.94 & 166.19 & 288.18 & 267.98 & 0.0000 & 5.5670 \\
\hline 194.0480 & 235.58 & 216.09 & 213.87 & 212.60 & 212.90 & 168.55 & 288.01 & 267.95 & 0.0007 & 5.5594 \\
\hline 194.5478 & 235.12 & 215.84 & 213.83 & 212.56 & 212.85 & 171.27 & 287.85 & 267.33 & 0.0004 & 5.5813 \\
\hline 195.0468 & 231.88 & 215.41 & 213.78 & 212.50 & 212.78 & 175.88 & 287.61 & 266.45 & 0.0000 & 5.5692 \\
\hline 195.5483 & 227.19 & 214.70 & 213.75 & 212.43 & 212.68 & 183.43 & 286.94 & 265.86 & 0.0002 & 5.5600 \\
\hline 196.0473 & 226.28 & 214.47 & 213.61 & 212.32 & 212.57 & 191.43 & 286.13 & 265.94 & 0.0005 & 5.5458 \\
\hline 196.5480 & 227.30 & 214.44 & 213.52 & 212.22 & 212.48 & 197.13 & 285.73 & 265.97 & 0.0005 & 5.5464 \\
\hline 197.0478 & 228.33 & 214.47 & 213.45 & 212.13 & 212.40 & 202.37 & 285.28 & 265.77 & 0.0010 & 5.5577 \\
\hline 197.5468 & 230.19 & 214.49 & 213.37 & 212.08 & 212.33 & 205.40 & 284.94 & 265.87 & 0.0008 & 5.5554 \\
\hline 198.0485 & 229.73 & 214.30 & 213.32 & 211.99 & 212.28 & 207.41 & 284.78 & 266.07 & 0.0007 & 5.5372 \\
\hline 198.5483 & 230.86 & 214.28 & 213.25 & 211.94 & 212.21 & 208.59 & 284.17 & 266.19 & 0.0002 & 5.5712 \\
\hline 199.0480 & 231.04 & 214.54 & 213.18 & 211.87 & 212.16 & 209.35 & 283.58 & 266.25 & 0.0002 & 5.5932 \\
\hline 199.5478 & 230.85 & 214.45 & 213.13 & 211.80 & 212.09 & 209.96 & 283.24 & 266.37 & 0.0003 & 5.6056 \\
\hline
\end{tabular}


TABLE B2.- CONTINUED.

\begin{tabular}{|c|c|c|c|c|c|c|c|c|c|c|}
\hline $\begin{array}{l}\text { Time, } \\
\text { min }\end{array}$ & $\begin{array}{c}\text { T-steam } \\
{ }^{\circ} \mathrm{C}\end{array}$ & $\begin{array}{l}\mathrm{T} 1, \\
{ }^{\circ} \mathrm{C}\end{array}$ & $\begin{array}{l}\mathrm{T} 2, \\
{ }^{\circ} \mathrm{C}\end{array}$ & $\begin{array}{l}\mathrm{T} 3, \\
{ }^{\circ} \mathrm{C}\end{array}$ & $\begin{array}{l}\mathrm{T} 4, \\
{ }^{\circ} \mathrm{C}\end{array}$ & $\begin{array}{l}\mathrm{T} 5, \\
{ }^{\circ} \mathrm{C}\end{array}$ & $\begin{array}{l}\text { Pinj, } \\
\text { psig }\end{array}$ & $\begin{array}{c}\text { Pout, } \\
\text { psig }\end{array}$ & $\begin{array}{l}\text { Qinj, } \\
\text { g/min }\end{array}$ & $\begin{array}{c}\mathrm{Vw} \\
\mathrm{ml} / \mathrm{min}\end{array}$ \\
\hline 200.0477 & 231.74 & 214.29 & 213.06 & 211.75 & 212.05 & 210.43 & 282.92 & 266.34 & 0.0001 & 5.5871 \\
\hline 200.5475 & 231.08 & 214.31 & 212.99 & 211.70 & 212.00 & 210.68 & 282.74 & 266.34 & 0.0001 & 5.5834 \\
\hline 201.0483 & 231.00 & 214.15 & 212.95 & 211.67 & 211.95 & 210.82 & 282.32 & 266.45 & 0.0003 & 5.5825 \\
\hline 201.5472 & 229.75 & 214.08 & 212.94 & 211.60 & 211.88 & 210.93 & 282.25 & 266.45 & 0.0003 & 5.5588 \\
\hline 202.0480 & 231.07 & 214.05 & 212.87 & 211.58 & 211.87 & 211.01 & 281.76 & 266.48 & 0.0004 & 5.5492 \\
\hline 202.5468 & 231.43 & 214.00 & 212.80 & 211.51 & 211.82 & 211.06 & 281.47 & 266.47 & 0.0005 & 5.5286 \\
\hline 203.0475 & 232.06 & 213.95 & 212.77 & 211.46 & 211.78 & 211.09 & 281.38 & 266.48 & 0.0005 & 5.5452 \\
\hline 203.5483 & 231.26 & 213.92 & 212.74 & 211.45 & 211.72 & 211.09 & 281.05 & 266.33 & 0.0008 & 5.5488 \\
\hline 204.0482 & 231.78 & 214.03 & 212.74 & 211.41 & 211.70 & 211.09 & 281.09 & 266.25 & 0.0004 & 5.5523 \\
\hline 204.5480 & 229.26 & 213.89 & 212.69 & 211.36 & 211.65 & 211.07 & 280.94 & 266.38 & 0.0009 & 5.5629 \\
\hline 205.0478 & 229.46 & 214.03 & 212.65 & 211.35 & 211.61 & 211.08 & 280.54 & 266.22 & 0.0009 & 5.5711 \\
\hline 205.5467 & 230.20 & 214.27 & 212.60 & 211.26 & 211.56 & 211.03 & 280.32 & 266.29 & 0.0002 & 5.5822 \\
\hline 206.0483 & 230.88 & 214.14 & 212.55 & 211.26 & 211.53 & 211.03 & 280.04 & 266.37 & 0.0002 & 5.5986 \\
\hline 206.5472 & 230.42 & 213.93 & 212.50 & 211.21 & 211.48 & 211.02 & 279.65 & 266.36 & 0.0002 & 5.5857 \\
\hline 207.0480 & 230.03 & 213.67 & 212.45 & 211.16 & 211.43 & 210.98 & 279.47 & 266.06 & 0.0001 & 5.5645 \\
\hline 207.5478 & 229.87 & 213.69 & 212.42 & 211.11 & 211.38 & 210.93 & 279.48 & 265.74 & 0.0003 & 5.5378 \\
\hline 208.0467 & 228.89 & 213.57 & 212.42 & 211.06 & 211.31 & 210.88 & 279.20 & 265.19 & 0.0003 & 5.5205 \\
\hline 208.5483 & 229.48 & 213.52 & 212.37 & 211.03 & 211.30 & 210.87 & 278.95 & 264.92 & 0.0012 & 5.5455 \\
\hline 209.0482 & 231.36 & 213.86 & 212.32 & 210.98 & 211.23 & 210.83 & 278.84 & 266.31 & 0.0001 & 5.5358 \\
\hline 209.5480 & 231.94 & 214.13 & 212.29 & 210.94 & 211.21 & 210.84 & 278.30 & 266.27 & 0.0005 & 5.5520 \\
\hline 210.0478 & 231.35 & 213.94 & 212.22 & 210.91 & 211.16 & 210.79 & 277.83 & 265.50 & 0.0005 & 5.5861 \\
\hline 210.5477 & 230.01 & 213.65 & 212.19 & 210.84 & 211.08 & 210.72 & 277.82 & 266.09 & 0.0005 & 5.5880 \\
\hline 211.0475 & 230.88 & 213.55 & 212.12 & 210.79 & 211.04 & 210.70 & 277.67 & 265.84 & 0.0005 & 5.5661 \\
\hline 211.5482 & 231.15 & 213.37 & 212.10 & 210.76 & 211.01 & 210.71 & 277.51 & 265.70 & 0.0005 & 5.5871 \\
\hline 212.0472 & 232.08 & 213.30 & 212.07 & 210.75 & 211.00 & 210.66 & 277.13 & 263.52 & 0.0004 & 5.5740 \\
\hline 212.5478 & 231.96 & 213.17 & 211.97 & 210.62 & 210.82 & 210.50 & 276.74 & 265.59 & 0.0002 & 5.5542 \\
\hline 213.0477 & 232.57 & 213.20 & 211.91 & 210.57 & 210.81 & 210.50 & 276.08 & 265.60 & 0.0001 & 5.5590 \\
\hline 213.5483 & 231.37 & 213.06 & 211.79 & 210.47 & 210.70 & 210.36 & 275.81 & 261.91 & 0.0002 & 5.5287 \\
\hline 214.0473 & 230.73 & 213.30 & 211.76 & 210.35 & 210.54 & 210.20 & 275.35 & 261.78 & 0.0002 & 5.5344 \\
\hline 214.5490 & 230.18 & 213.07 & 211.60 & 210.19 & 210.35 & 210.01 & 274.48 & 261.37 & 0.0004 & 5.5363 \\
\hline 215.0470 & 230.52 & 213.27 & 211.50 & 210.07 & 210.23 & 209.91 & 273.78 & 260.96 & 0.0001 & 5.5564 \\
\hline 215.5477 & 230.08 & 213.08 & 211.39 & 209.96 & 210.14 & 209.84 & 273.31 & 261.22 & 0.0006 & 5.5736 \\
\hline 216.0485 & 228.35 & 212.65 & 211.34 & 209.91 & 210.05 & 209.77 & 272.99 & 261.74 & 0.0000 & 5.5767 \\
\hline 216.5483 & 229.32 & 212.63 & 211.24 & 209.82 & 210.02 & 209.72 & 272.28 & 261.24 & 0.0000 & 5.5403 \\
\hline 217.0480 & 231.04 & 212.76 & 211.12 & 209.74 & 209.94 & 209.67 & 271.66 & 262.37 & 0.0002 & 5.5386 \\
\hline 217.5478 & 231.83 & 212.75 & 211.03 & 209.65 & 209.85 & 209.58 & 271.07 & 259.75 & 0.0004 & 5.5334 \\
\hline 218.0468 & 231.62 & 212.64 & 210.94 & 209.55 & 209.73 & 209.46 & 270.61 & 260.09 & 0.0004 & 5.5404 \\
\hline 218.5475 & 232.09 & 212.56 & 210.82 & 209.48 & 209.70 & 209.46 & 269.96 & 261.72 & 0.0003 & 5.5335 \\
\hline 219.0483 & 231.11 & 212.46 & 210.77 & 209.39 & 209.63 & 209.36 & 269.80 & 259.82 & 0.0003 & 5.5492 \\
\hline 219.5482 & 230.18 & 212.30 & 210.72 & 209.34 & 209.58 & 209.34 & 269.57 & 259.83 & 0.0003 & 5.5498 \\
\hline 220.0488 & 227.23 & 212.43 & 210.69 & 209.38 & 209.65 & 209.42 & 269.21 & 260.52 & 0.0004 & 5.5760 \\
\hline 220.5477 & 228.52 & 212.34 & 210.64 & 209.35 & 209.62 & 209.39 & 268.83 & 258.86 & 0.0004 & 5.5850 \\
\hline 221.0475 & 229.20 & 212.34 & 210.61 & 209.36 & 209.66 & 209.44 & 268.59 & 260.24 & 0.0003 & 5.5707 \\
\hline 221.5483 & 230.42 & 212.13 & 210.59 & 209.34 & 209.66 & 209.47 & 268.58 & 260.15 & 0.0003 & 5.5649 \\
\hline 222.0482 & 230.86 & 212.08 & 210.60 & 209.34 & 209.63 & 209.38 & 268.62 & 260.03 & 0.0003 & 5.5791 \\
\hline 222.5470 & 230.88 & 212.05 & 210.58 & 209.42 & 209.85 & 209.71 & 268.48 & 261.05 & 0.0002 & 5.5405 \\
\hline 223.0478 & 231.83 & 212.02 & 210.64 & 209.44 & 209.76 & 209.48 & 268.72 & 259.82 & 0.0002 & 5.5132 \\
\hline 223.5477 & 231.71 & 212.07 & 210.62 & 209.43 & 209.78 & 209.60 & 268.70 & 261.31 & 0.0002 & 5.5243 \\
\hline 224.0492 & 231.53 & 211.97 & 210.63 & 209.41 & 209.68 & 209.45 & 268.74 & 261.17 & 0.0002 & 5.5504 \\
\hline 224.5472 & 231.48 & 211.87 & 210.58 & 209.43 & 209.79 & 209.54 & 268.50 & 260.04 & 0.0002 & 5.5863 \\
\hline
\end{tabular}


TABLE B2.- CONTINUED.

\begin{tabular}{|c|c|c|c|c|c|c|c|c|c|c|}
\hline $\begin{array}{c}\text { Time, } \\
\text { min }\end{array}$ & $\begin{array}{c}\text { T-steam } \\
{ }^{\circ} \mathrm{C}\end{array}$ & $\begin{array}{l}\mathrm{T} 1, \\
{ }^{\circ} \mathrm{C}\end{array}$ & $\begin{array}{l}\mathrm{T} 2, \\
{ }^{\circ} \mathrm{C}\end{array}$ & $\begin{array}{l}\mathrm{T} 3, \\
{ }^{\circ} \mathrm{C}\end{array}$ & $\begin{array}{l}\mathrm{T} 4, \\
{ }^{\circ} \mathrm{C}\end{array}$ & $\begin{array}{l}\mathrm{T} 5, \\
{ }^{\circ} \mathrm{C}\end{array}$ & $\begin{array}{l}\text { Pinj, } \\
\text { psig }\end{array}$ & $\begin{array}{l}\text { Pout, } \\
\text { psig }\end{array}$ & $\begin{array}{l}\text { Qinj, } \\
\text { g/min }\end{array}$ & $\begin{array}{c}\mathrm{Vw} \\
\mathrm{ml} / \mathrm{min}\end{array}$ \\
\hline 225.0480 & 231.22 & 212.16 & 210.58 & 209.47 & 209.96 & 209.78 & 268.58 & 263.36 & 0.0008 & 5.5872 \\
\hline 225.5478 & 230.28 & 212.04 & 210.66 & 209.51 & 209.83 & 209.57 & 269.02 & 260.17 & 0.0008 & 5.5975 \\
\hline 226.0477 & 230.06 & 212.02 & 210.68 & 209.46 & 209.84 & 209.62 & 269.25 & 260.17 & 0.0004 & 5.5927 \\
\hline 226.5483 & 230.16 & 211.99 & 210.68 & 209.52 & 209.97 & 209.75 & 269.08 & 262.61 & 0.0000 & 5.5886 \\
\hline 227.0482 & 231.07 & 212.19 & 210.79 & 209.60 & 209.90 & 209.63 & 269.51 & 260.20 & 0.0000 & 5.5579 \\
\hline 227.5480 & 230.43 & 212.27 & 210.76 & 209.63 & 210.01 & 209.78 & 269.77 & 261.88 & 0.0001 & 5.5374 \\
\hline 228.0478 & 230.78 & 212.23 & 210.80 & 209.50 & 209.80 & 209.59 & 269.71 & 261.44 & 0.0002 & 5.5335 \\
\hline 228.5467 & 231.66 & 212.29 & 210.77 & 209.52 & 209.82 & 209.57 & 269.83 & 260.81 & 0.0007 & 5.5405 \\
\hline 229.0475 & 231.43 & 212.33 & 210.72 & 209.52 & 209.93 & 209.72 & 269.20 & 261.03 & 0.0005 & 5.5374 \\
\hline 229.5482 & 230.18 & 212.07 & 210.76 & 209.52 & 209.81 & 209.52 & 269.34 & 259.57 & 0.0001 & 5.5505 \\
\hline 230.0480 & 231.11 & 212.10 & 210.76 & 209.56 & 209.85 & 209.53 & 270.07 & 259.94 & 0.0001 & 5.5605 \\
\hline 230.5488 & 230.63 & 211.98 & 210.71 & 209.44 & 209.89 & 209.46 & 269.25 & 261.49 & 0.0000 & 5.5835 \\
\hline 231.0477 & 229.76 & 211.95 & 210.77 & 209.62 & 210.07 & 209.89 & 269.88 & 263.23 & 0.0008 & 5.5678 \\
\hline 231.5483 & 230.91 & 212.15 & 210.88 & 209.77 & 210.16 & 209.95 & 270.81 & 262.50 & 0.0005 & 5.5517 \\
\hline 232.0482 & 230.93 & 212.51 & 210.96 & 209.74 & 210.11 & 209.97 & 270.73 & 262.33 & 0.0004 & 5.5632 \\
\hline 232.5480 & 227.85 & 212.39 & 211.03 & 209.83 & 210.17 & 209.94 & 271.55 & 261.85 & 0.0002 & 5.5515 \\
\hline 233.0478 & 228.20 & 212.72 & 211.02 & 209.71 & 210.01 & 209.80 & 271.04 & 262.25 & 0.0002 & 5.5505 \\
\hline 233.5477 & 230.75 & 213.06 & 211.02 & 209.79 & 210.05 & 209.80 & 271.02 & 262.53 & 0.0009 & 5.5287 \\
\hline 234.0485 & 231.48 & 213.06 & 211.02 & 209.70 & 209.93 & 209.75 & 270.97 & 262.44 & 0.0001 & 5.5298 \\
\hline 234.5483 & 233.92 & 212.89 & 210.96 & 209.81 & 210.13 & 209.92 & 270.76 & 261.71 & 0.0001 & 5.5495 \\
\hline 235.0480 & 232.89 & 212.84 & 211.01 & 209.81 & 210.14 & 209.90 & 271.26 & 261.86 & 0.0004 & 5.5727 \\
\hline 235.5478 & 232.19 & 212.73 & 211.05 & 209.84 & 210.19 & 210.00 & 271.10 & 262.20 & 0.0004 & 5.5785 \\
\hline 236.0477 & 232.21 & 212.76 & 211.04 & 209.87 & 210.23 & 210.00 & 271.42 & 262.50 & 0.0006 & 5.5812 \\
\hline 236.5475 & 232.22 & 212.76 & 211.10 & 209.91 & 210.29 & 210.11 & 271.80 & 262.81 & 0.0006 & 5.5699 \\
\hline 237.0483 & 230.14 & 212.71 & 211.17 & 209.99 & 210.35 & 210.15 & 271.98 & 263.00 & 0.0006 & 5.5597 \\
\hline 237.5482 & 230.51 & 212.69 & 211.25 & 210.05 & 210.37 & 210.15 & 272.11 & 262.75 & 0.0004 & 5.5459 \\
\hline 238.0480 & 231.08 & 212.64 & 211.21 & 210.00 & 210.37 & 210.17 & 271.97 & 262.66 & 0.0000 & 5.5333 \\
\hline 238.5468 & 231.05 & 212.52 & 211.20 & 209.95 & 210.20 & 209.95 & 272.10 & 261.73 & 0.0005 & 5.5574 \\
\hline 239.0475 & 228.04 & 212.35 & 211.22 & 209.95 & 210.25 & 210.06 & 272.57 & 263.22 & 0.0004 & 5.5392 \\
\hline 239.5483 & 230.30 & 212.35 & 211.19 & 209.95 & 210.26 & 210.03 & 272.08 & 262.43 & 0.0004 & 5.5464 \\
\hline 240.0482 & 232.17 & 212.46 & 211.17 & 209.96 & 210.31 & 210.14 & 271.81 & 262.36 & 0.0008 & 5.5852 \\
\hline 240.5480 & 232.94 & 212.55 & 211.18 & 209.96 & 210.32 & 210.12 & 271.93 & 262.24 & 0.0008 & 5.5908 \\
\hline 241.0478 & 234.08 & 212.65 & 211.16 & 209.96 & 210.30 & 210.12 & 271.99 & 263.09 & 0.0004 & 5.6025 \\
\hline 241.5477 & 233.22 & 212.67 & 211.17 & 209.98 & 210.34 & 210.16 & 272.02 & 262.60 & 0.0004 & 5.5814 \\
\hline 242.0483 & 232.00 & 212.60 & 211.21 & 210.02 & 210.38 & 210.17 & 272.25 & 262.64 & 0.0004 & 5.5731 \\
\hline 242.5482 & 232.22 & 212.60 & 211.19 & 210.06 & 210.42 & 210.22 & 272.42 & 262.56 & 0.0005 & 5.5629 \\
\hline 243.0480 & 230.61 & 212.79 & 211.23 & 210.05 & 210.41 & 210.21 & 272.66 & 262.91 & 0.0004 & 5.5458 \\
\hline 243.5478 & 230.74 & 212.72 & 211.27 & 210.12 & 210.46 & 210.27 & 272.38 & 262.99 & 0.0004 & 5.5214 \\
\hline 244.0485 & 230.93 & 212.90 & 211.29 & 210.11 & 210.45 & 210.29 & 272.77 & 263.25 & 0.0001 & 5.5300 \\
\hline 244.5475 & 230.54 & 212.91 & 211.31 & 210.13 & 210.49 & 210.31 & 272.50 & 263.58 & 0.0001 & 5.5676 \\
\hline 245.0482 & 230.77 & 212.78 & 211.30 & 210.15 & 210.51 & 210.31 & 273.20 & 263.76 & 0.0007 & 5.5945 \\
\hline 245.5480 & 231.60 & 212.88 & 211.39 & 210.17 & 210.52 & 210.30 & 273.35 & 263.24 & 0.0007 & 5.5752 \\
\hline 246.0478 & 231.60 & 212.88 & 211.38 & 210.18 & 210.48 & 210.27 & 273.37 & 262.92 & 0.0003 & 5.5735 \\
\hline 246.5477 & 228.47 & 212.62 & 211.43 & 210.18 & 210.50 & 210.31 & 273.88 & 263.67 & 0.0006 & 5.6004 \\
\hline 247.0483 & 228.91 & 212.49 & 211.42 & 210.26 & 210.60 & 210.40 & 273.37 & 264.23 & 0.0002 & 5.5806 \\
\hline 247.5473 & 231.47 & 212.64 & 211.44 & 210.28 & 210.64 & 210.42 & 273.35 & 264.25 & 0.0002 & 5.5628 \\
\hline 248.0480 & 233.89 & 212.72 & 211.45 & 210.25 & 210.57 & 210.34 & 273.39 & 263.39 & 0.0000 & 5.5453 \\
\hline 248.5478 & 232.10 & 212.81 & 211.43 & 210.23 & 210.59 & 210.39 & 273.42 & 263.91 & 0.0005 & 5.5201 \\
\hline 249.0467 & 231.73 & 212.83 & 211.44 & 210.27 & 210.58 & 210.36 & 273.24 & 263.34 & 0.0000 & 5.5063 \\
\hline 249.5475 & 230.88 & 212.87 & 211.44 & 210.20 & 210.49 & 210.29 & 273.73 & 262.94 & 0.0000 & 5.5489 \\
\hline
\end{tabular}


TABLE B2.- CONTINUED.

\begin{tabular}{|c|c|c|c|c|c|c|c|c|c|c|}
\hline $\begin{array}{l}\text { Time, } \\
\text { min }\end{array}$ & $\begin{array}{c}\text { T-steam } \\
{ }^{\circ} \mathrm{C}\end{array}$ & $\begin{array}{l}\mathrm{T} 1, \\
{ }^{\circ} \mathrm{C}\end{array}$ & $\begin{array}{l}\mathrm{T} 2, \\
{ }^{\circ} \mathrm{C}\end{array}$ & $\begin{array}{l}\mathrm{T} 3, \\
{ }^{\circ} \mathrm{C}\end{array}$ & $\begin{array}{l}\mathrm{T} 4, \\
{ }^{\circ} \mathrm{C}\end{array}$ & $\begin{array}{l}\mathrm{T} 5, \\
{ }^{\circ} \mathrm{C}\end{array}$ & $\begin{array}{l}\text { Pinj, } \\
\text { psig }\end{array}$ & $\begin{array}{l}\text { Pout, } \\
\text { psig }\end{array}$ & $\begin{array}{l}\text { Qinj, } \\
\text { g/min }\end{array}$ & $\begin{array}{c}\mathrm{Vw} \\
\mathrm{ml} / \mathrm{min}\end{array}$ \\
\hline 250.0492 & 231.02 & 213.07 & 211.39 & 210.17 & 210.49 & 210.24 & 273.27 & 262.76 & 0.0005 & 5.5565 \\
\hline 250.5472 & 230.58 & 213.02 & 211.32 & 210.14 & 210.48 & 210.23 & 272.70 & 263.05 & 0.0004 & 5.5953 \\
\hline 251.0470 & 230.40 & 212.86 & 211.32 & 210.11 & 210.43 & 210.25 & 272.94 & 263.17 & 0.0002 & 5.5893 \\
\hline 251.5477 & 230.57 & 212.63 & 211.26 & 210.09 & 210.41 & 210.22 & 272.57 & 263.28 & 0.0007 & 5.5928 \\
\hline 252.0485 & 230.72 & 212.64 & 211.31 & 210.11 & 210.47 & 210.29 & 272.99 & 263.48 & 0.0001 & 5.5652 \\
\hline 252.5482 & 231.35 & 212.50 & 211.28 & 210.15 & 210.53 & 210.35 & 272.80 & 263.31 & 0.0002 & 5.5712 \\
\hline 253.0480 & 230.74 & 212.52 & 211.28 & 210.16 & 210.51 & 210.32 & 272.60 & 263.43 & 0.0002 & 5.5246 \\
\hline 253.5488 & 231.44 & 212.47 & 211.31 & 210.16 & 210.52 & 210.36 & 272.86 & 263.48 & 0.0002 & 5.5234 \\
\hline 254.0477 & 232.09 & 212.51 & 211.36 & 210.18 & 210.56 & 210.36 & 273.37 & 263.86 & 0.0007 & 5.5461 \\
\hline 254.5475 & 231.91 & 212.51 & 211.37 & 210.20 & 210.54 & 210.36 & 272.88 & 263.64 & 0.0001 & 5.5620 \\
\hline 255.0473 & 231.35 & 212.59 & 211.35 & 210.19 & 210.55 & 210.38 & 272.82 & 263.30 & 0.0000 & 5.5622 \\
\hline 255.5482 & 230.65 & 212.82 & 211.39 & 210.21 & 210.55 & 210.39 & 273.12 & 263.78 & 0.0004 & 5.5535 \\
\hline 256.0480 & 230.55 & 212.79 & 211.41 & 210.21 & 210.55 & 210.36 & 273.04 & 263.44 & 0.0005 & 5.5749 \\
\hline 256.5477 & 227.43 & 212.42 & 211.45 & 210.23 & 210.56 & 210.38 & 273.69 & 263.06 & 0.0007 & 5.5879 \\
\hline 257.0467 & 228.30 & 212.33 & 211.40 & 210.06 & 209.97 & 209.22 & 273.43 & 250.72 & 0.0001 & 3.1443 \\
\hline
\end{tabular}


TABLE B3. PRESSURE AND TEMPERATURE DATA FOR RUN 5 (PURE STEAM).

\begin{tabular}{|c|c|c|c|c|c|c|c|c|c|c|}
\hline $\begin{array}{c}\text { Time, } \\
\text { min }\end{array}$ & $\begin{array}{c}\text { T-steam } \\
{ }^{\circ} \mathrm{C}\end{array}$ & $\begin{array}{l}\mathrm{T} 1, \\
{ }^{\circ} \mathrm{C}\end{array}$ & $\begin{array}{l}\mathrm{T} 2, \\
{ }^{\circ} \mathrm{C}\end{array}$ & $\begin{array}{l}\mathrm{T} 3, \\
{ }^{\circ} \mathrm{C}\end{array}$ & $\begin{array}{l}\mathrm{T} 4, \\
{ }^{\circ} \mathrm{C}\end{array}$ & $\begin{array}{l}\mathrm{T} 5, \\
{ }^{\circ} \mathrm{C}\end{array}$ & $\begin{array}{l}\text { Pinj, } \\
\text { psig }\end{array}$ & $\begin{array}{l}\text { Pout, } \\
\text { psig }\end{array}$ & $\begin{array}{l}\text { Qinj, } \\
\text { g/min }\end{array}$ & $\begin{array}{c}\text { Vw } \\
\mathrm{ml} / \mathrm{min}\end{array}$ \\
\hline 0.0540 & 61.86 & 58.30 & 50.98 & 53.55 & 52.82 & 48.54 & 267.23 & 268.56 & 0.0001 & 5.4997 \\
\hline 0.5483 & 85.13 & 75.46 & 50.96 & 53.57 & 52.79 & 48.56 & 267.75 & 269.04 & 0.0000 & 5.4933 \\
\hline 1.0480 & 91.08 & 71.54 & 50.95 & 53.52 & 52.82 & 48.57 & 267.10 & 268.39 & 0.0001 & 5.5361 \\
\hline 1.5478 & 103.11 & 72.18 & 50.84 & 53.49 & 52.80 & 48.61 & 267.29 & 268.24 & 0.0001 & 5.5041 \\
\hline 2.0477 & 163.40 & 85.05 & 50.70 & 53.38 & 52.71 & 48.65 & 267.75 & 268.41 & 0.0008 & 5.4815 \\
\hline 2.5475 & 209.47 & 118.91 & 50.68 & 53.33 & 52.72 & 48.66 & 268.57 & 268.19 & 0.0001 & 5.5066 \\
\hline 3.0483 & 214.98 & 207.16 & 50.71 & 53.49 & 52.90 & 48.78 & 272.45 & 269.01 & 0.0003 & 5.5386 \\
\hline 3.5490 & 217.64 & 210.39 & 50.71 & 53.49 & 52.96 & 48.79 & 266.01 & 266.39 & 0.0002 & 5.4895 \\
\hline 4.0480 & 220.81 & 210.18 & 50.78 & 53.46 & 52.97 & 48.79 & 264.28 & 265.04 & 0.0006 & 5.4800 \\
\hline 4.5477 & 221.12 & 210.42 & 50.76 & 53.49 & 52.99 & 48.82 & 266.13 & 265.49 & 0.0004 & 5.5217 \\
\hline 5.0475 & 219.62 & 210.71 & 50.73 & 53.45 & 53.02 & 48.84 & 268.86 & 267.07 & 0.0005 & 5.5090 \\
\hline 5.5483 & 218.95 & 210.93 & 50.74 & 53.36 & 53.02 & 48.85 & 270.65 & 267.73 & 0.0004 & 5.4872 \\
\hline 6.0490 & 221.01 & 211.56 & 50.84 & 53.29 & 53.05 & 48.85 & 272.40 & 268.29 & 0.0004 & 5.5124 \\
\hline 6.5480 & 223.88 & 212.36 & 50.88 & 53.28 & 53.03 & 48.88 & 271.39 & 266.74 & 0.0004 & 5.5225 \\
\hline 7.0478 & 224.87 & 212.56 & 50.91 & 53.27 & 53.06 & 48.89 & 269.19 & 263.86 & 0.0004 & 5.4872 \\
\hline 7.5477 & 223.84 & 212.04 & 50.93 & 53.31 & 53.06 & 48.87 & 268.54 & 265.43 & 0.0003 & 5.4801 \\
\hline 8.0483 & 221.70 & 211.67 & 50.96 & 53.34 & 53.07 & 48.90 & 270.82 & 266.67 & 0.0008 & 5.5292 \\
\hline 8.5482 & 221.92 & 212.11 & 51.00 & 53.36 & 53.10 & 48.92 & 276.66 & 267.82 & 0.0004 & 5.5266 \\
\hline 9.0470 & 223.19 & 213.01 & 51.16 & 53.33 & 53.16 & 48.95 & 279.14 & 267.66 & 0.0006 & 5.4724 \\
\hline 9.5478 & 225.42 & 213.95 & 51.47 & 53.30 & 53.26 & 48.94 & 281.32 & 267.67 & 0.0001 & 5.4950 \\
\hline 10.0477 & 228.25 & 215.04 & 52.24 & 53.27 & 53.32 & 48.94 & 284.66 & 267.60 & 0.0002 & 5.5209 \\
\hline 10.5483 & 230.71 & 215.59 & 53.43 & 53.24 & 53.37 & 48.95 & 287.83 & 267.55 & 0.0004 & 5.5095 \\
\hline 11.0482 & 231.69 & 216.42 & 54.46 & 53.20 & 53.41 & 48.96 & 289.38 & 267.38 & 0.0004 & 5.4898 \\
\hline 11.5480 & 231.14 & 216.26 & 55.34 & 53.19 & 53.42 & 48.96 & 290.31 & 267.18 & 0.0002 & 5.5231 \\
\hline 12.0478 & 228.08 & 216.63 & 56.13 & 53.16 & 53.43 & 48.99 & 292.74 & 267.07 & 0.0004 & 5.5129 \\
\hline 12.5477 & 227.23 & 216.15 & 57.01 & 53.11 & 53.45 & 49.09 & 293.95 & 266.68 & 0.0002 & 5.4804 \\
\hline 13.0475 & 225.96 & 215.72 & 57.83 & 53.08 & 53.44 & 49.19 & 295.21 & 266.84 & 0.0001 & 5.4769 \\
\hline 13.5482 & 226.06 & 215.84 & 58.38 & 53.06 & 53.48 & 49.27 & 296.34 & 266.83 & 0.0000 & 5.5087 \\
\hline 14.0480 & 225.69 & 215.75 & 58.94 & 53.05 & 53.45 & 49.30 & 297.56 & 266.81 & 0.0004 & 5.5171 \\
\hline 14.5470 & 225.89 & 215.90 & 59.50 & 53.02 & 53.46 & 49.32 & 298.50 & 266.85 & 0.0004 & 5.4759 \\
\hline 15.0477 & 226.49 & 216.23 & 60.13 & 53.01 & 53.48 & 49.35 & 300.91 & 266.86 & 0.0001 & 5.4917 \\
\hline 15.5483 & 226.96 & 216.44 & 60.67 & 53.00 & 53.49 & 49.39 & 302.25 & 266.73 & 0.0006 & 5.5286 \\
\hline 16.0482 & 226.64 & 216.69 & 61.18 & 53.00 & 53.50 & 49.40 & 304.47 & 266.70 & 0.0000 & 5.5048 \\
\hline 16.5490 & 227.15 & 216.93 & 61.74 & 52.99 & 53.50 & 49.45 & 306.03 & 266.90 & 0.0004 & 5.4899 \\
\hline 17.0470 & 227.80 & 217.22 & 62.33 & 53.00 & 53.53 & 49.49 & 308.07 & 267.15 & 0.0005 & 5.5017 \\
\hline 17.5477 & 228.04 & 217.53 & 62.93 & 52.98 & 53.54 & 49.55 & 308.99 & 258.96 & 0.0004 & 5.5361 \\
\hline 18.0485 & 228.55 & 217.85 & 63.61 & 52.97 & 53.58 & 49.56 & 311.35 & 258.35 & 0.0001 & 5.4901 \\
\hline 18.5483 & 228.73 & 218.05 & 64.68 & 52.94 & 53.61 & 49.63 & 313.19 & 258.26 & 0.0003 & 5.4856 \\
\hline 19.0480 & 228.78 & 218.27 & 65.69 & 52.93 & 53.63 & 49.65 & 314.53 & 258.19 & 0.0002 & 5.5139 \\
\hline 19.5470 & 229.04 & 218.53 & 66.22 & 52.94 & 53.66 & 49.72 & 316.14 & 258.78 & 0.0004 & 5.5187 \\
\hline 20.0477 & 229.22 & 218.78 & 67.21 & 52.92 & 53.69 & 49.76 & 317.97 & 258.77 & 0.0002 & 5.4907 \\
\hline 20.5475 & 229.03 & 219.01 & 67.99 & 52.91 & 53.69 & 49.81 & 319.51 & 258.11 & 0.0000 & 5.5084 \\
\hline 21.0483 & 229.49 & 219.28 & 69.06 & 52.92 & 53.72 & 49.85 & 321.06 & 259.16 & 0.0004 & 5.5360 \\
\hline 21.5482 & 229.47 & 219.52 & 69.74 & 52.91 & 53.75 & 49.88 & 322.73 & 259.04 & 0.0004 & 5.5097 \\
\hline 22.0480 & 229.79 & 219.72 & 70.64 & 52.90 & 53.75 & 49.93 & 324.02 & 258.11 & 0.0004 & 5.4863 \\
\hline 22.5487 & 230.15 & 220.02 & 71.66 & 52.88 & 53.76 & 49.99 & 326.13 & 258.88 & 0.0004 & 5.5299 \\
\hline 23.0475 & 230.19 & 220.26 & 72.44 & 52.89 & 53.75 & 50.04 & 327.84 & 259.03 & 0.0004 & 5.5219 \\
\hline 23.5483 & 230.07 & 220.59 & 73.61 & 52.86 & 53.79 & 50.12 & 330.01 & 257.12 & 0.0000 & 5.4812 \\
\hline 24.0472 & 230.74 & 220.86 & 74.41 & 52.89 & 53.78 & 50.16 & 331.59 & 258.95 & 0.0004 & 5.4791 \\
\hline 24.5480 & 231.09 & 221.10 & 75.62 & 52.90 & 53.79 & 50.23 & 333.14 & 259.15 & 0.0001 & 5.5167 \\
\hline
\end{tabular}


TABLE B3.- CONTINUED.

\begin{tabular}{|c|c|c|c|c|c|c|c|c|c|c|}
\hline $\begin{array}{l}\text { Time, } \\
\text { min }\end{array}$ & $\begin{array}{c}\text { T-steam } \\
{ }^{\circ} \mathrm{C}\end{array}$ & $\begin{array}{l}\mathrm{T} 1, \\
{ }^{\circ} \mathrm{C}\end{array}$ & $\begin{array}{l}\mathrm{T} 2, \\
{ }^{\circ} \mathrm{C}\end{array}$ & $\begin{array}{l}\mathrm{T} 3, \\
{ }^{\circ} \mathrm{C}\end{array}$ & $\begin{array}{l}\mathrm{T} 4, \\
{ }^{\circ} \mathrm{C}\end{array}$ & $\begin{array}{l}\mathrm{T} 5, \\
{ }^{\circ} \mathrm{C}\end{array}$ & $\begin{array}{l}\text { Pinj, } \\
\text { psig }\end{array}$ & $\begin{array}{l}\text { Pout, } \\
\text { psig }\end{array}$ & $\begin{array}{l}\text { Qinj, } \\
\text { g/min }\end{array}$ & $\begin{array}{c}\mathrm{Vw} \\
\mathrm{ml} / \mathrm{min}\end{array}$ \\
\hline 25.0468 & 231.37 & 221.36 & 76.75 & 52.88 & 53.81 & 50.27 & 334.96 & 259.04 & 0.0002 & 5.5074 \\
\hline 25.5477 & 231.66 & 221.65 & 77.92 & 52.87 & 53.80 & 50.28 & 336.36 & 259.05 & 0.0005 & 5.4842 \\
\hline 26.0483 & 231.79 & 221.80 & 78.91 & 52.88 & 53.81 & 50.31 & 338.17 & 257.75 & 0.0001 & 5.5057 \\
\hline 26.5482 & 231.87 & 222.03 & 80.32 & 52.87 & 53.84 & 50.33 & 339.05 & 259.04 & 0.0004 & 5.5456 \\
\hline 27.0480 & 231.83 & 222.25 & 81.15 & 52.90 & 53.85 & 50.34 & 340.49 & 258.94 & 0.0004 & 5.5083 \\
\hline 27.5478 & 232.53 & 222.40 & 81.96 & 52.88 & 53.85 & 50.33 & 341.89 & 258.86 & 0.0004 & 5.5006 \\
\hline 28.0477 & 232.82 & 222.53 & 82.72 & 52.89 & 53.88 & 50.40 & 342.23 & 258.06 & 0.0004 & 5.5261 \\
\hline 28.5483 & 232.94 & 222.64 & 83.32 & 52.92 & 53.89 & 50.40 & 343.61 & 259.06 & 0.0000 & 5.5139 \\
\hline 29.0473 & 232.98 & 222.78 & 84.01 & 52.93 & 53.90 & 50.49 & 343.92 & 259.07 & 0.0004 & 5.5029 \\
\hline 29.5480 & 232.51 & 222.86 & 84.69 & 52.93 & 53.92 & 50.53 & 344.36 & 259.01 & 0.0004 & 5.4915 \\
\hline 30.0478 & 231.30 & 222.77 & 85.80 & 52.94 & 53.91 & 50.58 & 344.38 & 258.96 & 0.0005 & 5.5210 \\
\hline 30.5477 & 232.11 & 222.87 & 86.83 & 52.97 & 53.92 & 50.63 & 345.20 & 258.88 & 0.0004 & 5.5255 \\
\hline 31.0483 & 231.97 & 223.04 & 87.51 & 52.99 & 53.95 & 50.67 & 346.11 & 258.97 & 0.0005 & 5.4836 \\
\hline 31.5473 & 232.34 & 223.14 & 88.24 & 53.00 & 53.93 & 50.72 & 346.89 & 258.80 & 0.0001 & 5.5001 \\
\hline 32.0480 & 232.70 & 223.25 & 89.20 & 53.03 & 53.94 & 50.76 & 347.72 & 259.04 & 0.0000 & 5.5303 \\
\hline 32.5478 & 232.07 & 223.31 & 90.07 & 53.06 & 53.97 & 50.81 & 347.27 & 258.95 & 0.0003 & 5.4974 \\
\hline 33.0468 & 231.64 & 223.21 & 91.12 & 53.08 & 53.98 & 50.84 & 347.33 & 258.90 & 0.0004 & 5.4816 \\
\hline 33.5475 & 231.96 & 223.29 & 92.26 & 53.09 & 53.97 & 50.88 & 348.55 & 258.94 & 0.0004 & 5.4968 \\
\hline 34.0482 & 231.96 & 223.37 & 93.16 & 53.14 & 53.99 & 50.93 & 349.09 & 258.02 & 0.0004 & 5.5200 \\
\hline 34.5480 & 232.28 & 223.47 & 94.10 & 53.17 & 53.98 & 50.97 & 349.54 & 258.92 & 0.0004 & 5.5028 \\
\hline 35.0470 & 231.69 & 223.53 & 95.04 & 53.21 & 53.99 & 50.98 & 350.05 & 258.90 & 0.0003 & 5.4629 \\
\hline 35.5477 & 231.99 & 223.64 & 96.29 & 53.26 & 54.00 & 51.03 & 351.06 & 258.79 & 0.0004 & 5.5144 \\
\hline 36.0485 & 232.01 & 223.79 & 97.61 & 53.28 & 53.99 & 51.04 & 352.06 & 258.75 & 0.0002 & 5.5136 \\
\hline 36.5483 & 231.90 & 223.89 & 98.66 & 53.33 & 54.00 & 51.07 & 352.49 & 258.88 & 0.0006 & 5.4734 \\
\hline 37.0480 & 232.23 & 223.90 & 99.62 & 53.40 & 54.01 & 51.09 & 352.87 & 258.85 & 0.0004 & 5.4931 \\
\hline 37.5478 & 232.21 & 224.00 & 100.55 & 53.44 & 54.03 & 51.14 & 353.13 & 258.86 & 0.0001 & 5.5132 \\
\hline 38.0477 & 232.19 & 223.99 & 101.52 & 53.49 & 54.02 & 51.17 & 353.44 & 258.87 & 0.0004 & 5.4743 \\
\hline 38.5475 & 232.41 & 224.10 & 102.59 & 53.55 & 54.01 & 51.21 & 353.91 & 258.84 & 0.0004 & 5.4955 \\
\hline 39.0483 & 232.47 & 224.15 & 103.57 & 53.62 & 54.04 & 51.26 & 354.25 & 258.80 & 0.0004 & 5.5345 \\
\hline 39.5482 & 232.30 & 224.17 & 104.54 & 53.67 & 54.03 & 51.29 & 354.41 & 258.75 & 0.0004 & 5.4924 \\
\hline 40.0480 & 232.49 & 224.25 & 105.73 & 53.75 & 54.04 & 51.31 & 355.15 & 257.82 & 0.0004 & 5.4671 \\
\hline 40.5477 & 232.42 & 224.29 & 106.66 & 53.82 & 54.04 & 51.30 & 355.43 & 258.75 & 0.0004 & 5.4971 \\
\hline 41.0467 & 232.27 & 224.34 & 107.73 & 53.90 & 54.03 & 51.35 & 355.63 & 258.80 & 0.0004 & 5.4924 \\
\hline 41.5483 & 232.49 & 224.38 & 108.73 & 53.97 & 54.02 & 51.36 & 355.87 & 258.28 & 0.0005 & 5.4648 \\
\hline 42.0482 & 232.38 & 224.35 & 109.66 & 54.05 & 54.05 & 51.38 & 356.01 & 258.82 & 0.0004 & 5.4927 \\
\hline 42.5480 & 231.97 & 224.29 & 110.66 & 54.15 & 54.06 & 51.41 & 355.60 & 258.87 & 0.0004 & 5.5250 \\
\hline 43.0478 & 232.34 & 224.32 & 111.78 & 54.22 & 54.05 & 51.44 & 355.98 & 258.66 & 0.0004 & 5.4792 \\
\hline 43.5477 & 232.29 & 224.40 & 112.78 & 54.34 & 54.06 & 51.47 & 356.06 & 258.78 & 0.0002 & 5.4814 \\
\hline 44.0483 & 232.25 & 224.33 & 113.56 & 54.43 & 54.07 & 51.48 & 354.98 & 258.76 & 0.0000 & 5.5127 \\
\hline 44.5482 & 231.57 & 224.29 & 114.47 & 54.51 & 54.04 & 51.50 & 356.88 & 258.70 & 0.0003 & 5.5055 \\
\hline 45.0470 & 231.49 & 224.56 & 115.71 & 54.65 & 54.05 & 51.53 & 358.37 & 258.70 & 0.0004 & 5.4799 \\
\hline 45.5478 & 231.86 & 224.66 & 116.79 & 54.79 & 54.05 & 51.56 & 359.10 & 258.84 & 0.0001 & 5.5203 \\
\hline 46.0477 & 232.06 & 224.76 & 117.80 & 54.92 & 54.06 & 51.59 & 359.27 & 258.78 & 0.0004 & 5.5114 \\
\hline 46.5483 & 232.34 & 224.86 & 118.88 & 55.04 & 54.05 & 51.60 & 359.81 & 258.72 & 0.0004 & 5.4694 \\
\hline 47.0473 & 232.42 & 224.90 & 119.91 & 55.16 & 54.06 & 51.63 & 360.70 & 257.12 & 0.0004 & 5.4856 \\
\hline 47.5480 & 232.56 & 225.03 & 120.97 & 55.32 & 54.07 & 51.65 & 361.45 & 258.76 & 0.0002 & 5.5074 \\
\hline 48.0478 & 232.49 & 225.10 & 121.85 & 55.47 & 54.06 & 51.66 & 361.23 & 258.30 & 0.0004 & 5.4953 \\
\hline 48.5467 & 232.36 & 225.27 & 122.96 & 55.61 & 54.05 & 51.69 & 363.17 & 258.78 & 0.0004 & 5.4822 \\
\hline 49.0475 & 232.53 & 225.26 & 124.00 & 55.79 & 54.08 & 51.72 & 363.00 & 258.66 & 0.0004 & 5.5064 \\
\hline 49.5482 & 231.78 & 225.12 & 124.90 & 55.95 & 54.09 & 51.73 & 361.19 & 258.69 & 0.0002 & 5.5026 \\
\hline
\end{tabular}


TABLE B3.- CONTINUED.

\begin{tabular}{|c|c|c|c|c|c|c|c|c|c|c|}
\hline $\begin{array}{l}\text { Time, } \\
\min \end{array}$ & $\begin{array}{c}\text { T-steam } \\
{ }^{\circ} \mathrm{C}\end{array}$ & $\begin{array}{l}\mathrm{T} 1, \\
{ }^{\circ} \mathrm{C}\end{array}$ & $\begin{array}{l}\mathrm{T} 2, \\
{ }^{\circ} \mathrm{C}\end{array}$ & $\begin{array}{l}\mathrm{T} 3, \\
{ }^{\circ} \mathrm{C}\end{array}$ & $\begin{array}{l}\mathrm{T} 4, \\
{ }^{\circ} \mathrm{C}\end{array}$ & $\begin{array}{l}\mathrm{T} 5, \\
{ }^{\circ} \mathrm{C}\end{array}$ & $\begin{array}{l}\text { Pinj, } \\
\text { psig }\end{array}$ & $\begin{array}{c}\text { Pout, } \\
\text { psig }\end{array}$ & $\begin{array}{l}\text { Qinj, } \\
\text { g/min }\end{array}$ & $\begin{array}{c}\mathrm{Vw} \\
\mathrm{ml} / \mathrm{min}\end{array}$ \\
\hline 50.0490 & 231.66 & 224.99 & 125.82 & 56.11 & 54.08 & 51.77 & 360.64 & 258.48 & 0.0004 & 5.4943 \\
\hline 50.5478 & 231.58 & 224.94 & 126.76 & 56.29 & 54.08 & 51.80 & 360.16 & 258.76 & 0.0004 & 5.4721 \\
\hline 51.0468 & 231.52 & 224.89 & 127.71 & 56.47 & 54.11 & 51.83 & 360.17 & 258.76 & 0.0002 & 5.5058 \\
\hline 51.5475 & 231.44 & 224.87 & 128.63 & 56.67 & 54.10 & 51.84 & 359.98 & 258.75 & 0.0004 & 5.5139 \\
\hline 52.0482 & 231.66 & 224.91 & 129.53 & 56.88 & 54.11 & 51.86 & 360.26 & 257.65 & 0.0004 & 5.4702 \\
\hline 52.5490 & 231.60 & 224.95 & 130.43 & 57.06 & 54.14 & 51.89 & 360.69 & 258.75 & 0.0000 & 5.4765 \\
\hline 53.0478 & 231.52 & 224.98 & 131.35 & 57.26 & 54.11 & 51.92 & 360.84 & 258.72 & 0.0002 & 5.4660 \\
\hline 53.5477 & 231.49 & 224.97 & 132.31 & 57.50 & 54.12 & 51.93 & 360.80 & 258.59 & 0.0001 & 5.4402 \\
\hline 54.0485 & 231.68 & 224.98 & 133.19 & 57.72 & 54.13 & 51.94 & 360.66 & 258.62 & 0.0001 & 5.4372 \\
\hline 54.5483 & 231.54 & 224.93 & 134.06 & 57.93 & 54.13 & 51.98 & 360.61 & 258.63 & 0.0004 & 5.4340 \\
\hline 55.0480 & 231.84 & 224.91 & 134.87 & 58.17 & 54.14 & 51.99 & 359.69 & 258.63 & 0.0004 & 5.4833 \\
\hline 55.5478 & 231.63 & 224.86 & 135.64 & 58.39 & 54.13 & 52.00 & 359.57 & 258.71 & 0.0004 & 5.4540 \\
\hline 56.0477 & 231.58 & 224.83 & 136.43 & 58.64 & 54.14 & 52.03 & 359.51 & 258.54 & 0.0004 & 5.4519 \\
\hline 56.5467 & 231.61 & 224.82 & 137.24 & 58.86 & 54.15 & 52.06 & 359.39 & 258.62 & 0.0003 & 5.4934 \\
\hline 57.0473 & 231.62 & 224.87 & 138.13 & 59.13 & 54.14 & 52.05 & 359.72 & 258.58 & 0.0002 & 5.4584 \\
\hline 57.5482 & 231.89 & 224.89 & 138.91 & 59.39 & 54.15 & 52.09 & 360.05 & 258.61 & 0.0004 & 5.4361 \\
\hline 58.0480 & 231.78 & 224.92 & 139.67 & 59.68 & 54.16 & 52.08 & 360.33 & 258.67 & 0.0004 & 5.4558 \\
\hline 58.5468 & 231.70 & 224.91 & 140.44 & 59.96 & 54.17 & 52.11 & 360.14 & 258.66 & 0.0004 & 5.4788 \\
\hline 59.0475 & 231.76 & 224.90 & 141.21 & 60.25 & 54.17 & 52.14 & 359.80 & 258.46 & 0.0004 & 5.4342 \\
\hline 59.5483 & 231.44 & 224.93 & 142.00 & 60.54 & 54.20 & 52.15 & 359.93 & 258.61 & 0.0004 & 5.4440 \\
\hline 60.0490 & 231.49 & 224.92 & 142.79 & 60.87 & 54.19 & 52.17 & 359.86 & 256.53 & 0.0002 & 5.4618 \\
\hline 60.5480 & 231.64 & 224.85 & 143.58 & 61.18 & 54.20 & 52.20 & 359.61 & 258.64 & 0.0004 & 5.4581 \\
\hline 61.0468 & 231.99 & 224.81 & 144.35 & 61.51 & 54.21 & 52.21 & 358.86 & 258.08 & 0.0004 & 5.4390 \\
\hline 61.5477 & 231.65 & 224.80 & 145.10 & 61.81 & 54.22 & 52.22 & 358.95 & 258.65 & 0.0004 & 5.4610 \\
\hline 62.0483 & 231.31 & 224.73 & 145.87 & 62.12 & 54.23 & 52.25 & 358.28 & 258.55 & 0.0002 & 5.4865 \\
\hline 62.5482 & 231.44 & 224.67 & 146.62 & 62.43 & 54.21 & 52.27 & 358.37 & 253.61 & 0.0004 & 5.4644 \\
\hline 63.0470 & 231.73 & 224.70 & 147.43 & 62.80 & 54.24 & 52.28 & 357.93 & 255.04 & 0.0004 & 5.4225 \\
\hline 63.5478 & 231.76 & 224.61 & 148.20 & 63.16 & 54.25 & 52.29 & 357.24 & 254.36 & 0.0001 & 5.4549 \\
\hline 64.0477 & 231.59 & 224.57 & 148.91 & 63.49 & 54.28 & 52.32 & 357.21 & 254.93 & 0.0004 & 5.4301 \\
\hline 64.5483 & 231.61 & 224.58 & 149.59 & 63.84 & 54.27 & 52.33 & 357.03 & 254.85 & 0.0005 & 5.4212 \\
\hline 65.0482 & 231.40 & 224.51 & 150.27 & 64.21 & 54.29 & 52.35 & 356.47 & 253.66 & 0.0001 & 5.4090 \\
\hline 65.5480 & 231.48 & 224.48 & 150.93 & 64.54 & 54.30 & 52.36 & 356.34 & 256.20 & 0.0004 & 5.4444 \\
\hline 66.0478 & 231.53 & 224.45 & 151.58 & 64.89 & 54.31 & 52.37 & 355.66 & 257.20 & 0.0005 & 5.4225 \\
\hline 66.5477 & 231.57 & 224.43 & 152.26 & 65.25 & 54.32 & 52.40 & 355.65 & 249.63 & 0.0004 & 5.4147 \\
\hline 67.0483 & 231.77 & 224.38 & 153.01 & 65.68 & 54.34 & 52.40 & 355.44 & 255.83 & 0.0004 & 5.3661 \\
\hline 67.5482 & 231.67 & 224.30 & 153.63 & 66.04 & 54.35 & 52.43 & 354.60 & 257.63 & 0.0004 & 5.3930 \\
\hline 68.0480 & 231.38 & 224.22 & 154.20 & 66.39 & 54.38 & 52.44 & 354.03 & 253.84 & 0.0004 & 5.3821 \\
\hline 68.5470 & 231.48 & 224.14 & 154.82 & 66.78 & 54.41 & 52.45 & 353.31 & 253.18 & 0.0004 & 5.3663 \\
\hline 69.0477 & 231.38 & 224.00 & 155.50 & 67.24 & 54.43 & 52.47 & 352.21 & 247.27 & 0.0004 & 5.3467 \\
\hline 69.5483 & 231.72 & 223.90 & 156.19 & 67.68 & 54.44 & 52.48 & 351.43 & 249.58 & 0.0004 & 5.3735 \\
\hline 70.0492 & 231.71 & 223.89 & 156.83 & 68.08 & 54.45 & 52.49 & 351.55 & 248.98 & 0.0004 & 5.3820 \\
\hline 70.5480 & 231.56 & 223.86 & 157.53 & 68.54 & 54.48 & 52.50 & 351.05 & 252.12 & 0.0004 & 5.3700 \\
\hline 71.0470 & 231.80 & 223.80 & 158.24 & 68.91 & 54.48 & 52.52 & 350.41 & 257.69 & 0.0005 & 5.3651 \\
\hline 71.5477 & 231.49 & 223.75 & 158.88 & 69.33 & 54.51 & 52.53 & 349.95 & 257.62 & 0.0001 & 5.3703 \\
\hline 72.0485 & 231.65 & 223.74 & 159.48 & 69.74 & 54.56 & 52.54 & 349.98 & 254.77 & 0.0004 & 5.3705 \\
\hline 72.5483 & 231.39 & 223.64 & 160.08 & 70.20 & 54.57 & 52.55 & 349.64 & 258.74 & 0.0004 & 5.3650 \\
\hline 73.0472 & 231.31 & 223.61 & 160.70 & 70.62 & 54.59 & 52.54 & 349.95 & 258.56 & 0.0004 & 5.3502 \\
\hline 73.5478 & 231.76 & 223.72 & 161.36 & 71.11 & 54.61 & 52.56 & 350.30 & 258.73 & 0.0004 & 5.3434 \\
\hline 74.0477 & 231.97 & 223.77 & 162.02 & 71.57 & 54.64 & 52.59 & 350.08 & 258.75 & 0.0000 & 5.3514 \\
\hline 74.5475 & 231.61 & 223.70 & 162.66 & 72.05 & 54.67 & 52.60 & 350.28 & 258.71 & 0.0005 & 5.3460 \\
\hline
\end{tabular}


TABLE B3.- CONTINUED.

\begin{tabular}{|c|c|c|c|c|c|c|c|c|c|c|}
\hline $\begin{array}{l}\text { Time, } \\
\text { min }\end{array}$ & $\begin{array}{c}\text { T-steam } \\
{ }^{\circ} \mathrm{C}\end{array}$ & $\begin{array}{l}\mathrm{T} 1, \\
{ }^{\circ} \mathrm{C}\end{array}$ & $\begin{array}{l}\mathrm{T} 2, \\
{ }^{\circ} \mathrm{C}\end{array}$ & $\begin{array}{l}\mathrm{T} 3, \\
{ }^{\circ} \mathrm{C}\end{array}$ & $\begin{array}{l}\mathrm{T} 4, \\
{ }^{\circ} \mathrm{C}\end{array}$ & $\begin{array}{l}\mathrm{T} 5, \\
{ }^{\circ} \mathrm{C}\end{array}$ & $\begin{array}{l}\text { Pinj, } \\
\text { psig }\end{array}$ & $\begin{array}{l}\text { Pout, } \\
\text { psig }\end{array}$ & $\begin{array}{l}\text { Qinj, } \\
\text { g/min }\end{array}$ & $\begin{array}{c}\text { Vw } \\
\mathrm{ml} / \mathrm{min}\end{array}$ \\
\hline 75.0483 & 231.59 & 223.69 & 163.35 & 72.57 & 54.73 & 52.58 & 349.96 & 258.62 & 0.0007 & 5.3452 \\
\hline 75.5482 & 231.90 & 223.66 & 163.99 & 73.06 & 54.74 & 52.63 & 349.57 & 257.07 & 0.0004 & 5.3360 \\
\hline 76.0480 & 231.96 & 223.76 & 164.75 & 73.58 & 54.80 & 52.64 & 350.57 & 257.68 & 0.0001 & 5.3283 \\
\hline 76.5477 & 231.82 & 223.82 & 165.49 & 74.09 & 54.85 & 52.64 & 350.15 & 255.85 & 0.0004 & 5.3422 \\
\hline 77.0475 & 230.99 & 223.66 & 166.37 & 74.59 & 54.88 & 52.65 & 350.26 & 255.49 & 0.0002 & 5.3192 \\
\hline 77.5483 & 231.30 & 223.74 & 167.31 & 75.18 & 54.92 & 52.66 & 350.82 & 258.99 & 0.0004 & 5.2761 \\
\hline 78.0482 & 231.79 & 223.77 & 168.27 & 75.73 & 54.97 & 52.68 & 350.16 & 256.51 & 0.0004 & 5.2220 \\
\hline 78.5480 & 232.33 & 223.92 & 169.48 & 76.28 & 55.03 & 52.69 & 350.09 & 256.85 & 0.0004 & 5.2387 \\
\hline 79.0478 & 231.60 & 223.78 & 171.16 & 76.83 & 55.07 & 52.72 & 349.75 & 254.49 & 0.0004 & 5.2561 \\
\hline 79.5467 & 231.61 & 223.61 & 173.04 & 77.40 & 55.12 & 52.72 & 348.93 & 254.99 & 0.0000 & 5.2595 \\
\hline 80.0483 & 231.71 & 223.59 & 175.87 & 78.01 & 55.18 & 52.73 & 348.39 & 256.47 & 0.0002 & 5.2653 \\
\hline 80.5482 & 231.93 & 223.64 & 179.57 & 78.54 & 55.25 & 52.74 & 347.82 & 256.63 & 0.0003 & 5.2200 \\
\hline 81.0470 & 231.84 & 223.50 & 184.05 & 79.09 & 55.31 & 52.72 & 347.50 & 258.75 & 0.0004 & 5.2246 \\
\hline 81.5478 & 231.81 & 223.49 & 189.54 & 79.66 & 55.37 & 52.73 & 347.57 & 258.74 & 0.0003 & 5.2143 \\
\hline 82.0477 & 231.62 & 223.44 & 195.12 & 80.26 & 55.44 & 52.73 & 347.34 & 256.34 & 0.0004 & 5.2625 \\
\hline 82.5483 & 231.50 & 223.32 & 199.01 & 80.85 & 55.50 & 52.74 & 346.64 & 256.19 & 0.0010 & 5.2781 \\
\hline 83.0473 & 231.81 & 223.36 & 202.73 & 81.42 & 55.58 & 52.75 & 346.33 & 258.69 & 0.0004 & 5.2337 \\
\hline 83.5480 & 231.85 & 223.31 & 205.69 & 82.04 & 55.66 & 52.75 & 345.79 & 258.66 & 0.0004 & 5.2214 \\
\hline 84.0468 & 231.36 & 223.18 & 208.48 & 82.63 & 55.75 & 52.78 & 345.69 & 258.71 & 0.0004 & 5.2462 \\
\hline 84.5477 & 231.58 & 223.13 & 210.53 & 83.23 & 55.83 & 52.77 & 345.47 & 258.71 & 0.0004 & 5.2515 \\
\hline 85.0483 & 231.62 & 223.10 & 212.29 & 83.86 & 55.93 & 52.77 & 345.02 & 258.66 & 0.0006 & 5.2419 \\
\hline 85.5482 & 232.13 & 223.19 & 213.78 & 84.44 & 56.01 & 52.78 & 344.73 & 258.72 & 0.0002 & 5.2487 \\
\hline 86.0490 & 231.58 & 223.02 & 215.20 & 85.06 & 56.09 & 52.79 & 344.55 & 258.67 & 0.0004 & 5.2475 \\
\hline 86.5470 & 230.96 & 222.92 & 216.62 & 85.67 & 56.21 & 52.77 & 343.91 & 258.62 & 0.0004 & 5.2715 \\
\hline 87.0477 & 231.11 & 222.89 & 217.88 & 86.29 & 56.30 & 52.78 & 343.47 & 258.60 & 0.0004 & 5.3139 \\
\hline 87.5483 & 231.85 & 223.07 & 218.85 & 86.91 & 56.42 & 52.80 & 343.66 & 258.15 & 0.0002 & 5.2229 \\
\hline 88.0482 & 231.60 & 222.95 & 219.86 & 87.55 & 56.50 & 52.79 & 343.47 & 258.58 & 0.0005 & 5.2207 \\
\hline 88.5472 & 231.27 & 222.87 & 220.51 & 88.17 & 56.62 & 52.80 & 343.34 & 258.60 & 0.0004 & 5.2509 \\
\hline 89.0478 & 231.45 & 222.91 & 220.87 & 88.81 & 56.74 & 52.80 & 343.32 & 258.64 & 0.0004 & 5.2613 \\
\hline 89.5477 & 231.44 & 222.90 & 221.20 & 89.47 & 56.86 & 52.83 & 343.18 & 258.65 & 0.0005 & 5.2621 \\
\hline 90.0493 & 231.53 & 222.80 & 221.44 & 90.11 & 56.97 & 52.83 & 342.95 & 258.58 & 0.0004 & 5.2858 \\
\hline 90.5483 & 231.40 & 222.89 & 221.57 & 90.75 & 57.10 & 52.84 & 342.60 & 258.61 & 0.0003 & 5.2905 \\
\hline 91.0472 & 231.56 & 222.86 & 221.63 & 91.41 & 57.22 & 52.84 & 342.22 & 258.58 & 0.0004 & 5.2978 \\
\hline 91.5478 & 231.51 & 222.76 & 221.60 & 92.04 & 57.35 & 52.85 & 341.49 & 258.66 & 0.0006 & 5.2819 \\
\hline 92.0477 & 231.30 & 222.73 & 221.55 & 92.64 & 57.49 & 52.87 & 340.86 & 258.54 & 0.0002 & 5.3064 \\
\hline 92.5467 & 231.31 & 222.61 & 221.50 & 93.28 & 57.63 & 52.90 & 340.56 & 258.52 & 0.0004 & 5.3232 \\
\hline 93.0473 & 231.47 & 222.66 & 221.45 & 93.90 & 57.75 & 52.92 & 340.23 & 258.60 & 0.0004 & 5.3180 \\
\hline 93.5482 & 231.66 & 222.78 & 221.44 & 94.56 & 57.91 & 52.93 & 339.63 & 258.55 & 0.0002 & 5.3153 \\
\hline 94.0480 & 231.13 & 222.59 & 221.44 & 95.21 & 58.06 & 52.91 & 339.51 & 258.49 & 0.0006 & 5.3424 \\
\hline 94.5468 & 231.31 & 222.50 & 221.39 & 95.87 & 58.20 & 52.92 & 339.12 & 258.44 & 0.0005 & 5.3666 \\
\hline 95.0475 & 231.26 & 222.45 & 221.34 & 96.50 & 58.36 & 52.94 & 338.84 & 258.43 & 0.0009 & 5.3752 \\
\hline 95.5483 & 231.14 & 222.33 & 221.31 & 97.16 & 58.50 & 52.95 & 338.39 & 258.56 & 0.0000 & 5.3645 \\
\hline 96.0482 & 231.38 & 222.43 & 221.26 & 97.79 & 58.67 & 52.95 & 338.41 & 258.52 & 0.0004 & 5.3666 \\
\hline 96.5470 & 231.17 & 222.16 & 221.25 & 98.48 & 58.85 & 52.96 & 337.98 & 258.48 & 0.0003 & 5.4167 \\
\hline 97.0478 & 231.14 & 222.18 & 221.20 & 99.16 & 59.00 & 52.96 & 337.36 & 258.55 & 0.0001 & 5.4625 \\
\hline 97.5477 & 230.91 & 222.21 & 221.15 & 99.87 & 59.20 & 52.97 & 336.99 & 258.59 & 0.0001 & 5.5027 \\
\hline 98.0483 & 230.93 & 222.12 & 221.12 & 100.56 & 59.35 & 52.97 & 336.57 & 258.54 & 0.0004 & 5.5300 \\
\hline 98.5482 & 230.88 & 222.13 & 221.05 & 101.28 & 59.53 & 52.97 & 336.31 & 258.49 & 0.0005 & 5.5189 \\
\hline 99.0480 & 230.90 & 222.06 & 221.02 & 101.99 & 59.70 & 52.98 & 336.11 & 258.47 & 0.0004 & 5.5406 \\
\hline 99.5478 & 230.89 & 222.03 & 221.01 & 102.72 & 59.90 & 52.99 & 335.78 & 258.48 & 0.0004 & 5.5193 \\
\hline
\end{tabular}


TABLE B3.- CONTINUED.

\begin{tabular}{|c|c|c|c|c|c|c|c|c|c|c|}
\hline $\begin{array}{l}\text { Time, } \\
\text { min }\end{array}$ & $\begin{array}{c}\text { T-steam } \\
{ }^{\circ} \mathrm{C}\end{array}$ & $\begin{array}{l}\mathrm{T} 1, \\
{ }^{\circ} \mathrm{C}\end{array}$ & $\begin{array}{l}\mathrm{T} 2, \\
{ }^{\circ} \mathrm{C}\end{array}$ & $\begin{array}{l}\mathrm{T} 3, \\
{ }^{\circ} \mathrm{C}\end{array}$ & $\begin{array}{l}\mathrm{T} 4, \\
{ }^{\circ} \mathrm{C}\end{array}$ & $\begin{array}{l}\mathrm{T} 5, \\
{ }^{\circ} \mathrm{C}\end{array}$ & $\begin{array}{l}\text { Pinj, } \\
\text { psig }\end{array}$ & $\begin{array}{l}\text { Pout, } \\
\text { psig }\end{array}$ & $\begin{array}{l}\text { Qinj, } \\
\text { g/min }\end{array}$ & $\begin{array}{c}\mathrm{Vw} \\
\mathrm{ml} / \mathrm{min}\end{array}$ \\
\hline 100.0477 & 231.11 & 222.09 & 220.99 & 103.55 & 60.09 & 53.01 & 335.55 & 258.45 & 0.0005 & 5.5413 \\
\hline 100.5483 & 230.83 & 221.98 & 220.98 & 104.39 & 60.31 & 53.01 & 335.39 & 257.98 & 0.0004 & 5.5172 \\
\hline 101.0482 & 230.56 & 221.83 & 220.93 & 105.30 & 60.54 & 53.04 & 335.03 & 257.72 & 0.0008 & 5.5259 \\
\hline 101.5480 & 231.28 & 222.06 & 220.92 & 106.28 & 60.73 & 53.06 & 335.02 & 258.49 & 0.0004 & 5.5062 \\
\hline 102.0478 & 230.91 & 222.03 & 220.92 & 107.41 & 60.95 & 53.07 & 334.93 & 257.33 & 0.0002 & 5.5014 \\
\hline 102.5477 & 230.59 & 221.96 & 220.89 & 108.64 & 61.14 & 53.09 & 334.85 & 258.51 & 0.0002 & 5.5140 \\
\hline 103.0483 & 231.22 & 221.95 & 220.88 & 109.91 & 61.37 & 53.11 & 334.39 & 256.78 & 0.0004 & 5.5172 \\
\hline 103.5482 & 230.67 & 221.85 & 220.86 & 111.36 & 61.60 & 53.10 & 334.32 & 258.27 & 0.0004 & 5.5154 \\
\hline 104.0480 & 230.59 & 221.81 & 220.83 & 112.87 & 61.81 & 53.12 & 334.33 & 256.98 & 0.0006 & 5.5346 \\
\hline 104.5478 & 230.41 & 221.78 & 220.84 & 114.55 & 62.05 & 53.15 & 334.06 & 258.49 & 0.0003 & 5.5267 \\
\hline 105.0477 & 230.27 & 221.65 & 220.82 & 116.26 & 62.26 & 53.17 & 334.06 & 258.47 & 0.0009 & 5.5138 \\
\hline 105.5483 & 230.33 & 221.79 & 220.81 & 118.08 & 62.49 & 53.19 & 333.85 & 258.23 & 0.0004 & 5.5592 \\
\hline 106.0482 & 230.39 & 221.81 & 220.83 & 120.09 & 62.70 & 53.21 & 333.87 & 258.49 & 0.0006 & 5.5344 \\
\hline 106.5472 & 230.75 & 221.89 & 220.85 & 122.18 & 62.88 & 53.22 & 334.10 & 258.46 & 0.0005 & 5.5488 \\
\hline 107.0478 & 230.23 & 221.75 & 220.85 & 124.35 & 63.07 & 53.26 & 334.19 & 258.44 & 0.0003 & 5.5555 \\
\hline 107.5468 & 230.10 & 221.58 & 220.89 & 126.56 & 63.24 & 53.26 & 334.40 & 258.41 & 0.0004 & 5.5704 \\
\hline 108.0485 & 230.32 & 221.79 & 220.92 & 128.76 & 63.44 & 53.29 & 334.53 & 258.47 & 0.0007 & 5.5699 \\
\hline 108.5483 & 230.00 & 221.69 & 220.97 & 131.14 & 63.63 & 53.33 & 334.49 & 258.45 & 0.0004 & 5.5555 \\
\hline 109.0480 & 230.05 & 221.75 & 220.98 & 133.24 & 63.80 & 53.35 & 334.94 & 258.45 & 0.0001 & 5.5637 \\
\hline 109.5478 & 229.86 & 221.70 & 221.02 & 135.41 & 64.00 & 53.36 & 334.83 & 258.39 & 0.0003 & 5.5665 \\
\hline 110.0477 & 230.17 & 221.65 & 221.02 & 137.43 & 64.17 & 53.40 & 334.97 & 258.57 & 0.0003 & 5.5856 \\
\hline 110.5475 & 230.08 & 221.87 & 221.08 & 139.49 & 64.36 & 53.40 & 335.48 & 258.42 & 0.0000 & 5.5766 \\
\hline 111.0483 & 230.23 & 221.85 & 221.10 & 141.44 & 64.54 & 53.42 & 335.52 & 258.37 & 0.0010 & 5.6013 \\
\hline 111.5482 & 230.25 & 221.94 & 221.14 & 143.28 & 64.73 & 53.45 & 335.80 & 258.35 & 0.0004 & 5.5938 \\
\hline 112.0470 & 230.15 & 221.95 & 221.18 & 145.28 & 64.92 & 53.45 & 336.18 & 258.32 & 0.0007 & 5.6208 \\
\hline 112.5477 & 230.08 & 221.99 & 221.23 & 147.11 & 65.10 & 53.47 & 336.54 & 258.36 & 0.0004 & 5.5889 \\
\hline 113.0475 & 230.21 & 221.99 & 221.29 & 148.89 & 65.31 & 53.50 & 337.16 & 258.37 & 0.0004 & 5.5914 \\
\hline 113.5483 & 230.34 & 222.08 & 221.42 & 150.65 & 65.48 & 53.52 & 337.77 & 258.30 & 0.0004 & 5.6143 \\
\hline 114.0490 & 230.09 & 222.12 & 221.48 & 152.32 & 65.69 & 53.54 & 337.99 & 258.45 & 0.0004 & 5.5878 \\
\hline 114.5488 & 229.90 & 222.18 & 221.54 & 153.97 & 65.92 & 53.58 & 338.27 & 257.09 & 0.0000 & 5.5976 \\
\hline 115.0478 & 230.29 & 222.27 & 221.57 & 155.47 & 66.12 & 53.61 & 338.48 & 258.33 & 0.0004 & 5.4813 \\
\hline 115.5477 & 230.19 & 222.33 & 221.61 & 156.98 & 66.33 & 53.61 & 338.76 & 256.49 & 0.0004 & 5.4915 \\
\hline 116.0483 & 230.26 & 222.35 & 221.62 & 158.41 & 66.52 & 53.63 & 338.81 & 258.41 & 0.0001 & 5.4576 \\
\hline 116.5482 & 230.09 & 222.43 & 221.62 & 159.76 & 66.73 & 53.66 & 338.85 & 257.92 & 0.0002 & 5.4852 \\
\hline 117.0480 & 229.77 & 222.30 & 221.61 & 161.14 & 66.94 & 53.70 & 338.68 & 258.35 & 0.0007 & 5.4983 \\
\hline 117.5478 & 229.88 & 222.25 & 221.61 & 162.41 & 67.17 & 53.70 & 338.64 & 258.37 & 0.0007 & 5.4881 \\
\hline 118.0477 & 230.33 & 222.31 & 221.58 & 163.65 & 67.38 & 53.74 & 338.66 & 258.41 & 0.0002 & 5.4488 \\
\hline 118.5483 & 230.24 & 222.35 & 221.60 & 165.06 & 67.61 & 53.76 & 338.93 & 258.45 & 0.0001 & 5.4984 \\
\hline 119.0482 & 230.00 & 222.25 & 221.62 & 166.36 & 67.86 & 53.79 & 339.07 & 252.87 & 0.0001 & 5.5379 \\
\hline 119.5472 & 230.18 & 222.23 & 221.62 & 167.99 & 68.11 & 53.83 & 338.89 & 249.65 & 0.0003 & 5.5028 \\
\hline 120.0487 & 230.06 & 222.22 & 221.62 & 169.70 & 68.40 & 53.85 & 338.84 & 252.23 & 0.0005 & 5.4755 \\
\hline 120.5477 & 229.88 & 222.17 & 221.61 & 171.48 & 68.70 & 53.93 & 338.93 & 252.89 & 0.0003 & 5.4974 \\
\hline 121.0493 & 229.80 & 222.12 & 221.62 & 173.21 & 69.01 & 53.97 & 338.84 & 252.06 & 0.0007 & 5.5029 \\
\hline 121.5473 & 230.23 & 222.35 & 221.64 & 175.02 & 69.31 & 54.01 & 339.16 & 253.85 & 0.0010 & 5.4903 \\
\hline 122.0480 & 229.84 & 222.30 & 221.66 & 176.89 & 69.62 & 54.06 & 338.99 & 250.31 & 0.0009 & 5.4690 \\
\hline 122.5478 & 229.88 & 222.34 & 221.64 & 178.79 & 69.94 & 54.10 & 338.87 & 253.09 & 0.0006 & 5.4675 \\
\hline 123.0477 & 229.99 & 222.34 & 221.61 & 181.02 & 70.25 & 54.12 & 338.72 & 255.56 & 0.0013 & 5.5024 \\
\hline 123.5483 & 229.94 & 222.42 & 221.61 & 183.00 & 70.57 & 54.16 & 338.81 & 254.89 & 0.0005 & 5.4843 \\
\hline 124.0473 & 229.60 & 222.26 & 221.60 & 185.10 & 70.89 & 54.20 & 338.75 & 253.02 & 0.0004 & 5.4663 \\
\hline 124.5480 & 230.25 & 222.41 & 221.62 & 187.24 & 71.23 & 54.26 & 338.82 & 254.90 & 0.0004 & 5.5051 \\
\hline
\end{tabular}


TABLE B3.- CONTINUED.

\begin{tabular}{|c|c|c|c|c|c|c|c|c|c|c|}
\hline $\begin{array}{l}\text { Time, } \\
\text { min }\end{array}$ & $\begin{array}{c}\text { T-steam } \\
{ }^{\circ} \mathrm{C}\end{array}$ & $\begin{array}{l}\mathrm{T} 1, \\
{ }^{\circ} \mathrm{C}\end{array}$ & $\begin{array}{l}\mathrm{T} 2, \\
{ }^{\circ} \mathrm{C}\end{array}$ & $\begin{array}{l}\mathrm{T} 3, \\
{ }^{\circ} \mathrm{C}\end{array}$ & $\begin{array}{l}\mathrm{T} 4, \\
{ }^{\circ} \mathrm{C}\end{array}$ & $\begin{array}{l}\mathrm{T} 5, \\
{ }^{\circ} \mathrm{C}\end{array}$ & $\begin{array}{l}\text { Pinj, } \\
\text { psig }\end{array}$ & $\begin{array}{l}\text { Pout, } \\
\text { psig }\end{array}$ & $\begin{array}{l}\text { Qinj, } \\
\text { g/min }\end{array}$ & $\begin{array}{c}\mathrm{Vw} \\
\mathrm{ml} / \mathrm{min}\end{array}$ \\
\hline 125.0478 & 230.57 & 222.46 & 221.64 & 189.40 & 71.62 & 54.34 & 338.91 & 254.02 & 0.0000 & 5.5167 \\
\hline 125.5477 & 230.07 & 222.32 & 221.62 & 191.62 & 71.98 & 54.38 & 338.56 & 254.31 & 0.0004 & 5.4736 \\
\hline 126.0485 & 230.18 & 222.24 & 221.59 & 193.63 & 72.34 & 54.44 & 338.42 & 254.78 & 0.0004 & 5.4795 \\
\hline 126.5483 & 229.97 & 222.24 & 221.56 & 196.14 & 72.72 & 54.50 & 338.17 & 256.45 & 0.0004 & 5.5124 \\
\hline 127.0480 & 229.89 & 222.19 & 221.56 & 198.89 & 73.10 & 54.57 & 338.06 & 255.10 & 0.0004 & 5.5244 \\
\hline 127.5470 & 230.07 & 222.12 & 221.55 & 201.31 & 73.49 & 54.62 & 338.01 & 257.21 & 0.0001 & 5.4735 \\
\hline 128.0477 & 229.88 & 222.18 & 221.52 & 204.01 & 73.89 & 54.67 & 338.01 & 254.65 & 0.0003 & 5.4938 \\
\hline 128.5475 & 230.31 & 222.25 & 221.48 & 206.81 & 74.33 & 54.73 & 337.76 & 257.41 & 0.0002 & 5.5279 \\
\hline 129.0483 & 229.99 & 222.11 & 221.47 & 209.33 & 74.74 & 54.79 & 337.48 & 257.26 & 0.0004 & 5.5042 \\
\hline 129.5482 & 229.56 & 222.10 & 221.42 & 211.23 & 75.18 & 54.85 & 337.44 & 258.26 & 0.0004 & 5.4840 \\
\hline 130.0480 & 229.89 & 222.15 & 221.40 & 212.70 & 75.61 & 54.91 & 337.05 & 258.37 & 0.0004 & 5.5113 \\
\hline 130.5477 & 230.21 & 222.12 & 221.39 & 214.28 & 76.09 & 54.99 & 337.10 & 255.63 & 0.0006 & 5.5341 \\
\hline 131.0467 & 230.02 & 222.14 & 221.39 & 215.70 & 76.54 & 55.07 & 336.95 & 255.82 & 0.0009 & 5.5236 \\
\hline 131.5483 & 229.99 & 222.04 & 221.34 & 216.72 & 77.01 & 55.13 & 336.72 & 254.56 & 0.0004 & 5.4935 \\
\hline 132.0472 & 230.02 & 222.02 & 221.32 & 217.53 & 77.54 & 55.22 & 336.37 & 254.83 & 0.0004 & 5.5002 \\
\hline 132.5480 & 229.49 & 221.97 & 221.27 & 218.41 & 78.07 & 55.30 & 335.89 & 254.64 & 0.0005 & 5.5229 \\
\hline 133.0478 & 229.40 & 221.81 & 221.20 & 219.00 & 78.58 & 55.38 & 335.71 & 256.57 & 0.0003 & 5.5129 \\
\hline 133.5467 & 229.35 & 221.73 & 221.15 & 219.35 & 79.09 & 55.46 & 335.50 & 255.88 & 0.0005 & 5.4894 \\
\hline 134.0473 & 229.60 & 221.73 & 221.12 & 219.51 & 79.63 & 55.52 & 334.83 & 255.63 & 0.0002 & 5.5045 \\
\hline 134.5482 & 230.36 & 221.79 & 221.07 & 219.60 & 80.18 & 55.64 & 334.46 & 257.28 & 0.0006 & 5.5374 \\
\hline 135.0480 & 229.88 & 221.75 & 221.05 & 219.69 & 80.76 & 55.73 & 334.56 & 255.95 & 0.0004 & 5.5104 \\
\hline 135.5487 & 229.95 & 221.77 & 221.00 & 219.73 & 81.33 & 55.83 & 334.20 & 255.90 & 0.0000 & 5.4916 \\
\hline 136.0477 & 230.56 & 221.86 & 220.99 & 219.77 & 81.91 & 55.91 & 334.20 & 253.96 & 0.0005 & 5.4853 \\
\hline 136.5483 & 229.71 & 221.65 & 220.99 & 219.83 & 82.53 & 55.99 & 334.08 & 255.35 & 0.0006 & 5.5289 \\
\hline 137.0473 & 229.92 & 221.69 & 220.94 & 219.83 & 83.13 & 56.08 & 333.70 & 255.38 & 0.0008 & 5.4993 \\
\hline 137.5480 & 230.03 & 221.71 & 220.90 & 219.81 & 83.77 & 56.18 & 333.50 & 254.90 & 0.0004 & 5.4725 \\
\hline 138.0478 & 230.41 & 221.62 & 220.87 & 219.81 & 84.39 & 56.30 & 333.21 & 256.16 & 0.0001 & 5.5033 \\
\hline 138.5467 & 229.82 & 221.57 & 220.84 & 219.82 & 85.05 & 56.40 & 333.14 & 255.99 & 0.0007 & 5.5214 \\
\hline 139.0483 & 229.74 & 221.63 & 220.78 & 219.78 & 85.66 & 56.47 & 332.85 & 255.82 & 0.0004 & 5.4922 \\
\hline 139.5473 & 229.90 & 221.50 & 220.77 & 219.80 & 86.37 & 56.57 & 332.78 & 256.15 & 0.0005 & 5.4886 \\
\hline 140.0480 & 229.92 & 221.54 & 220.72 & 219.79 & 87.05 & 56.69 & 332.23 & 256.23 & 0.0006 & 5.5022 \\
\hline 140.5470 & 230.10 & 221.42 & 220.68 & 219.75 & 87.76 & 56.79 & 331.94 & 254.87 & 0.0004 & 5.5108 \\
\hline 141.0477 & 229.82 & 221.33 & 220.63 & 219.74 & 88.47 & 56.88 & 331.78 & 256.75 & 0.0006 & 5.4850 \\
\hline 141.5483 & 230.93 & 221.60 & 220.60 & 219.71 & 89.22 & 57.00 & 331.59 & 256.88 & 0.0004 & 5.4949 \\
\hline 142.0482 & 230.66 & 221.51 & 220.62 & 219.74 & 89.99 & 57.10 & 331.53 & 255.61 & 0.0004 & 5.5261 \\
\hline 142.5480 & 229.90 & 221.48 & 220.62 & 219.74 & 90.81 & 57.19 & 331.56 & 256.56 & 0.0002 & 5.5366 \\
\hline 143.0478 & 229.90 & 221.41 & 220.61 & 219.75 & 91.65 & 57.33 & 331.68 & 255.76 & 0.0003 & 5.5067 \\
\hline 143.5477 & 230.83 & 221.40 & 220.61 & 219.77 & 92.53 & 57.43 & 331.64 & 255.38 & 0.0011 & 5.4957 \\
\hline 144.0485 & 230.26 & 221.34 & 220.59 & 219.77 & 93.46 & 57.54 & 331.43 & 255.24 & 0.0006 & 5.5177 \\
\hline 144.5483 & 229.98 & 221.24 & 220.58 & 219.77 & 94.41 & 57.70 & 331.16 & 255.68 & 0.0004 & 5.5458 \\
\hline 145.0480 & 230.19 & 221.35 & 220.58 & 219.76 & 95.36 & 57.79 & 331.08 & 257.72 & 0.0006 & 5.4918 \\
\hline 145.5470 & 229.93 & 221.21 & 220.55 & 219.74 & 96.34 & 57.93 & 330.76 & 258.02 & 0.0009 & 5.4922 \\
\hline 146.0477 & 229.44 & 221.12 & 220.49 & 219.72 & 97.33 & 58.06 & 330.68 & 258.60 & 0.0004 & 5.5158 \\
\hline 146.5475 & 229.48 & 221.09 & 220.44 & 219.65 & 98.35 & 58.18 & 330.06 & 258.56 & 0.0005 & 5.5094 \\
\hline 147.0483 & 230.07 & 221.09 & 220.39 & 219.62 & 99.37 & 58.31 & 329.73 & 258.63 & 0.0007 & 5.4805 \\
\hline 147.5482 & 229.88 & 221.23 & 220.36 & 219.59 & 100.43 & 58.43 & 329.80 & 258.65 & 0.0001 & 5.4887 \\
\hline 148.0470 & 230.13 & 221.27 & 220.30 & 219.55 & 101.51 & 58.56 & 329.10 & 258.60 & 0.0003 & 5.5429 \\
\hline 148.5477 & 230.21 & 221.22 & 220.29 & 219.54 & 102.60 & 58.72 & 329.26 & 258.64 & 0.0003 & 5.5103 \\
\hline 149.0475 & 230.57 & 221.20 & 220.29 & 219.52 & 103.79 & 58.86 & 329.30 & 258.47 & 0.0005 & 5.4696 \\
\hline 149.5483 & 230.73 & 221.13 & 220.28 & 219.54 & 105.09 & 59.03 & 329.24 & 258.53 & 0.0004 & 5.4855 \\
\hline
\end{tabular}


TABLE B3.- CONTINUED.

\begin{tabular}{|c|c|c|c|c|c|c|c|c|c|c|}
\hline $\begin{array}{l}\text { Time, } \\
\text { min }\end{array}$ & $\begin{array}{c}\text { T-steam } \\
{ }^{\circ} \mathrm{C}\end{array}$ & $\begin{array}{l}\mathrm{T} 1, \\
{ }^{\circ} \mathrm{C}\end{array}$ & $\begin{array}{l}\mathrm{T} 2, \\
{ }^{\circ} \mathrm{C}\end{array}$ & $\begin{array}{l}\mathrm{T} 3, \\
{ }^{\circ} \mathrm{C}\end{array}$ & $\begin{array}{l}\mathrm{T} 4, \\
{ }^{\circ} \mathrm{C}\end{array}$ & $\begin{array}{l}\mathrm{T} 5, \\
{ }^{\circ} \mathrm{C}\end{array}$ & $\begin{array}{l}\text { Pinj, } \\
\text { psig }\end{array}$ & $\begin{array}{l}\text { Pout, } \\
\text { psig }\end{array}$ & $\begin{array}{l}\text { Qinj, } \\
\text { g/min }\end{array}$ & $\begin{array}{c}\mathrm{Vw} \\
\mathrm{ml} / \mathrm{min}\end{array}$ \\
\hline 150.0482 & 229.78 & 220.98 & 220.28 & 219.54 & 106.44 & 59.18 & 329.31 & 258.52 & 0.0004 & 5.5376 \\
\hline 150.5470 & 230.09 & 221.07 & 220.26 & 219.55 & 107.84 & 59.34 & 329.24 & 258.55 & 0.0006 & 5.4932 \\
\hline 151.0478 & 230.47 & 221.05 & 220.26 & 219.53 & 109.34 & 59.49 & 329.27 & 258.46 & 0.0004 & 5.4819 \\
\hline 151.5477 & 228.82 & 220.84 & 220.25 & 219.55 & 110.94 & 59.70 & 328.62 & 258.50 & 0.0006 & 5.5069 \\
\hline 152.0483 & 229.40 & 220.93 & 220.19 & 219.48 & 112.56 & 59.85 & 328.61 & 258.51 & 0.0012 & 5.5280 \\
\hline 152.5482 & 229.85 & 220.91 & 220.18 & 219.46 & 114.28 & 60.04 & 328.36 & 258.44 & 0.0012 & 5.4961 \\
\hline 153.0470 & 230.15 & 220.90 & 220.11 & 219.39 & 116.05 & 60.22 & 328.11 & 258.51 & 0.0004 & 5.4815 \\
\hline 153.5478 & 230.41 & 220.92 & 220.09 & 219.38 & 117.88 & 60.43 & 327.85 & 258.45 & 0.0006 & 5.5251 \\
\hline 154.0477 & 230.25 & 220.76 & 220.06 & 219.38 & 119.83 & 60.64 & 327.74 & 256.51 & 0.0005 & 5.5339 \\
\hline 154.5483 & 229.84 & 220.81 & 220.05 & 219.35 & 121.89 & 60.85 & 327.55 & 258.45 & 0.0003 & 5.4762 \\
\hline 155.0482 & 230.18 & 220.76 & 220.01 & 219.33 & 124.05 & 61.08 & 327.30 & 258.41 & 0.0007 & 5.4846 \\
\hline 155.5470 & 230.25 & 220.57 & 219.94 & 219.23 & 126.34 & 61.31 & 327.10 & 258.37 & 0.0004 & 5.5116 \\
\hline 156.0478 & 229.52 & 220.61 & 219.89 & 219.21 & 128.77 & 61.58 & 326.53 & 258.44 & 0.0004 & 5.4934 \\
\hline 156.5477 & 230.10 & 220.59 & 219.86 & 219.18 & 131.35 & 61.82 & 326.38 & 258.44 & 0.0006 & 5.4710 \\
\hline 157.0483 & 230.15 & 220.73 & 219.79 & 219.11 & 134.08 & 62.11 & 325.66 & 257.78 & 0.0004 & 5.4688 \\
\hline 157.5482 & 230.13 & 220.61 & 219.73 & 219.05 & 136.96 & 62.40 & 325.12 & 258.43 & 0.0001 & 5.5217 \\
\hline 158.0480 & 229.89 & 220.45 & 219.66 & 219.00 & 140.02 & 62.68 & 324.73 & 258.43 & 0.0004 & 5.5182 \\
\hline 158.5470 & 229.89 & 220.47 & 219.59 & 218.91 & 143.20 & 63.00 & 324.27 & 258.42 & 0.0004 & 5.4682 \\
\hline 159.0477 & 230.21 & 220.33 & 219.49 & 218.83 & 146.54 & 63.33 & 323.67 & 258.37 & 0.0005 & 5.4904 \\
\hline 159.5483 & 230.27 & 220.31 & 219.44 & 218.78 & 150.13 & 63.65 & 323.42 & 258.23 & 0.0007 & 5.5101 \\
\hline 160.0482 & 230.63 & 220.33 & 219.35 & 218.72 & 154.10 & 64.03 & 322.97 & 258.42 & 0.0006 & 5.4884 \\
\hline 160.5480 & 229.91 & 220.18 & 219.30 & 218.65 & 158.38 & 64.38 & 322.62 & 258.41 & 0.0007 & 5.4676 \\
\hline 161.0478 & 230.06 & 220.09 & 219.23 & 218.58 & 162.96 & 64.74 & 322.12 & 258.43 & 0.0001 & 5.5127 \\
\hline 161.5477 & 230.35 & 220.07 & 219.16 & 218.51 & 167.97 & 65.08 & 321.58 & 258.43 & 0.0005 & 5.5089 \\
\hline 162.0485 & 230.22 & 220.11 & 219.11 & 218.46 & 173.61 & 65.42 & 321.08 & 258.41 & 0.0004 & 5.4787 \\
\hline 162.5483 & 230.10 & 220.20 & 219.00 & 218.37 & 179.27 & 65.74 & 320.61 & 257.80 & 0.0008 & 5.5037 \\
\hline 163.0480 & 230.53 & 220.08 & 218.91 & 218.31 & 185.07 & 66.08 & 319.64 & 258.41 & 0.0001 & 5.5201 \\
\hline 163.5478 & 230.75 & 220.15 & 218.84 & 218.20 & 190.57 & 66.43 & 319.28 & 258.40 & 0.0004 & 5.4789 \\
\hline 164.0477 & 230.02 & 219.97 & 218.76 & 218.13 & 195.43 & 66.79 & 318.94 & 258.38 & 0.0004 & 5.4894 \\
\hline 164.5475 & 230.57 & 219.96 & 218.69 & 218.06 & 199.39 & 67.17 & 318.27 & 258.41 & 0.0002 & 5.5104 \\
\hline 165.0483 & 230.75 & 219.80 & 218.60 & 217.99 & 203.05 & 67.60 & 317.97 & 258.42 & 0.0000 & 5.4798 \\
\hline 165.5472 & 230.52 & 219.87 & 218.55 & 217.94 & 206.25 & 68.13 & 317.75 & 258.35 & 0.0005 & 5.4890 \\
\hline 166.0480 & 230.38 & 219.96 & 218.48 & 217.85 & 209.03 & 68.63 & 317.09 & 258.39 & 0.0004 & 5.5200 \\
\hline 166.5477 & 230.76 & 220.06 & 218.41 & 217.80 & 211.68 & 69.14 & 316.62 & 258.40 & 0.0004 & 5.5455 \\
\hline 167.0475 & 230.94 & 219.61 & 218.34 & 217.73 & 213.87 & 69.69 & 315.90 & 258.34 & 0.0006 & 5.5385 \\
\hline 167.5483 & 230.44 & 219.52 & 218.25 & 217.62 & 215.05 & 70.37 & 315.75 & 258.42 & 0.0004 & 5.4994 \\
\hline 168.0490 & 230.37 & 219.42 & 218.16 & 217.57 & 215.85 & 71.03 & 315.04 & 258.34 & 0.0004 & 5.5112 \\
\hline 168.5480 & 230.30 & 219.36 & 218.08 & 217.48 & 216.43 & 71.71 & 314.53 & 258.39 & 0.0004 & 5.5242 \\
\hline 169.0468 & 230.05 & 219.19 & 217.97 & 217.38 & 216.82 & 72.40 & 313.45 & 258.31 & 0.0004 & 5.5398 \\
\hline 169.5477 & 229.86 & 219.26 & 217.86 & 217.27 & 217.11 & 73.12 & 312.81 & 258.30 & 0.0008 & 5.5290 \\
\hline 170.0492 & 230.97 & 219.44 & 217.72 & 217.15 & 217.29 & 73.78 & 312.31 & 258.34 & 0.0010 & 5.4835 \\
\hline 170.5482 & 231.70 & 219.48 & 217.62 & 217.04 & 217.35 & 74.46 & 311.31 & 257.97 & 0.0000 & 5.4989 \\
\hline 171.0480 & 231.44 & 219.01 & 217.49 & 216.90 & 217.33 & 75.12 & 310.45 & 258.29 & 0.0003 & 5.5327 \\
\hline 171.5478 & 231.26 & 219.21 & 217.37 & 216.80 & 217.30 & 75.74 & 309.76 & 258.14 & 0.0004 & 5.5155 \\
\hline 172.0467 & 231.48 & 219.29 & 217.23 & 216.67 & 217.21 & 76.40 & 308.84 & 258.28 & 0.0002 & 5.4904 \\
\hline 172.5483 & 231.19 & 219.16 & 217.11 & 216.53 & 217.10 & 77.02 & 308.04 & 256.32 & 0.0002 & 5.4827 \\
\hline 173.0482 & 230.62 & 218.93 & 216.98 & 216.43 & 217.04 & 77.66 & 307.26 & 257.76 & 0.0006 & 5.5348 \\
\hline 173.5488 & 231.64 & 219.63 & 216.86 & 216.30 & 216.91 & 78.25 & 306.55 & 258.44 & 0.0008 & 5.5320 \\
\hline 174.0478 & 231.34 & 219.71 & 216.74 & 216.18 & 216.83 & 78.83 & 305.69 & 257.23 & 0.0003 & 5.4890 \\
\hline 174.5477 & 230.84 & 219.91 & 216.63 & 216.08 & 216.72 & 79.43 & 305.33 & 258.37 & 0.0003 & 5.4925 \\
\hline
\end{tabular}


TABLE B3.- CONTINUED.

\begin{tabular}{|c|c|c|c|c|c|c|c|c|c|c|}
\hline $\begin{array}{l}\text { Time, } \\
\text { min }\end{array}$ & $\begin{array}{c}\text { T-steam } \\
{ }^{\circ} \mathrm{C}\end{array}$ & $\begin{array}{l}\mathrm{T} 1, \\
{ }^{\circ} \mathrm{C}\end{array}$ & $\begin{array}{l}\mathrm{T} 2, \\
{ }^{\circ} \mathrm{C}\end{array}$ & $\begin{array}{l}\mathrm{T} 3, \\
{ }^{\circ} \mathrm{C}\end{array}$ & $\begin{array}{l}\mathrm{T} 4, \\
{ }^{\circ} \mathrm{C}\end{array}$ & $\begin{array}{l}\mathrm{T} 5, \\
{ }^{\circ} \mathrm{C}\end{array}$ & $\begin{array}{l}\text { Pinj, } \\
\text { psig }\end{array}$ & $\begin{array}{l}\text { Pout, } \\
\text { psig }\end{array}$ & $\begin{array}{l}\text { Qinj, } \\
\text { g/min }\end{array}$ & $\begin{array}{c}\mathrm{Vw} \\
\mathrm{ml} / \mathrm{min}\end{array}$ \\
\hline 175.0475 & 231.03 & 219.50 & 216.53 & 215.99 & 216.63 & 80.01 & 304.62 & 258.08 & 0.0004 & 5.5204 \\
\hline 175.5473 & 230.51 & 219.07 & 216.44 & 215.88 & 216.53 & 80.75 & 303.81 & 255.93 & 0.0005 & 5.5372 \\
\hline 176.0480 & 232.00 & 219.25 & 216.28 & 215.76 & 216.42 & 81.46 & 303.12 & 256.43 & 0.0002 & 5.4958 \\
\hline 176.5488 & 230.51 & 218.23 & 216.17 & 215.65 & 216.30 & 82.17 & 302.27 & 257.88 & 0.0006 & 5.5009 \\
\hline 177.0468 & 230.14 & 217.80 & 216.03 & 215.53 & 216.21 & 82.94 & 301.43 & 256.23 & 0.0005 & 5.5573 \\
\hline 177.5483 & 229.99 & 218.75 & 215.93 & 215.43 & 216.09 & 83.84 & 300.63 & 258.31 & 0.0008 & 5.5606 \\
\hline 178.0482 & 230.19 & 218.61 & 215.78 & 215.25 & 215.96 & 84.83 & 299.79 & 258.27 & 0.0010 & 5.5446 \\
\hline 178.5480 & 230.72 & 218.61 & 215.66 & 215.16 & 215.84 & 85.92 & 299.02 & 258.28 & 0.0004 & 5.4948 \\
\hline 179.0478 & 231.00 & 219.08 & 215.50 & 215.00 & 215.70 & 87.13 & 298.00 & 258.24 & 0.0004 & 5.5031 \\
\hline 179.5468 & 229.79 & 217.74 & 215.38 & 214.86 & 215.56 & 88.42 & 297.29 & 258.20 & 0.0005 & 5.5304 \\
\hline 180.0493 & 229.68 & 218.41 & 215.24 & 214.72 & 215.43 & 89.93 & 296.29 & 258.16 & 0.0006 & 5.5095 \\
\hline 180.5483 & 230.65 & 219.18 & 215.08 & 214.60 & 215.27 & 91.59 & 295.66 & 258.13 & 0.0004 & 5.4786 \\
\hline 181.0480 & 229.60 & 219.02 & 214.95 & 214.47 & 215.17 & 93.53 & 294.63 & 258.17 & 0.0010 & 5.5022 \\
\hline 181.5470 & 229.90 & 218.64 & 214.81 & 214.31 & 215.03 & 95.89 & 294.01 & 258.17 & 0.0005 & 5.5195 \\
\hline 182.0477 & 230.08 & 218.00 & 214.69 & 214.19 & 214.89 & 99.01 & 293.01 & 258.14 & 0.0001 & 5.4887 \\
\hline 182.5475 & 230.48 & 218.25 & 214.53 & 214.05 & 214.75 & 103.03 & 292.05 & 258.17 & 0.0004 & 5.4738 \\
\hline 183.0483 & 230.36 & 217.66 & 214.39 & 213.89 & 214.60 & 108.43 & 291.21 & 258.14 & 0.0006 & 5.4932 \\
\hline 183.5482 & 229.46 & 218.06 & 214.23 & 213.73 & 214.45 & 115.72 & 290.16 & 258.10 & 0.0003 & 5.5064 \\
\hline 184.0470 & 229.14 & 217.67 & 214.05 & 213.57 & 214.27 & 124.34 & 289.20 & 258.10 & 0.0004 & 5.4842 \\
\hline 184.5477 & 229.30 & 217.88 & 213.89 & 213.41 & 214.14 & 134.84 & 288.25 & 258.06 & 0.0003 & 5.4965 \\
\hline 185.0475 & 229.32 & 217.98 & 213.70 & 213.21 & 213.95 & 147.16 & 287.10 & 258.09 & 0.0002 & 5.5246 \\
\hline 185.5483 & 228.79 & 217.49 & 213.54 & 213.06 & 213.77 & 161.14 & 285.97 & 258.09 & 0.0006 & 5.5400 \\
\hline 186.0482 & 228.45 & 217.69 & 213.36 & 212.88 & 213.58 & 179.21 & 285.12 & 258.05 & 0.0008 & 5.5222 \\
\hline 186.5480 & 228.63 & 217.10 & 213.18 & 212.70 & 213.42 & 197.56 & 283.94 & 258.08 & 0.0009 & 5.4892 \\
\hline 187.0468 & 228.87 & 216.82 & 212.97 & 212.51 & 213.24 & 207.10 & 282.75 & 258.03 & 0.0003 & 5.5249 \\
\hline 187.5477 & 228.76 & 217.70 & 212.79 & 212.31 & 213.03 & 210.63 & 281.62 & 258.02 & 0.0008 & 5.5360 \\
\hline 188.0483 & 227.79 & 217.14 & 212.62 & 212.12 & 212.83 & 211.54 & 280.48 & 257.97 & 0.0004 & 5.5202 \\
\hline 188.5482 & 227.92 & 217.11 & 212.39 & 211.88 & 212.62 & 211.74 & 279.24 & 257.96 & 0.0012 & 5.4999 \\
\hline 189.0488 & 227.78 & 217.29 & 212.17 & 211.69 & 212.41 & 211.69 & 277.89 & 257.90 & 0.0004 & 5.4778 \\
\hline 189.5478 & 227.89 & 217.08 & 211.96 & 211.46 & 212.18 & 211.55 & 276.61 & 257.91 & 0.0003 & 5.5229 \\
\hline 190.0477 & 227.96 & 216.35 & 211.71 & 211.23 & 211.96 & 211.37 & 275.29 & 257.88 & 0.0009 & 5.5433 \\
\hline 190.5483 & 227.77 & 216.90 & 211.48 & 211.00 & 211.71 & 211.18 & 273.71 & 257.87 & 0.0001 & 5.5193 \\
\hline 191.0482 & 227.50 & 216.85 & 211.21 & 210.73 & 211.46 & 210.96 & 272.28 & 257.88 & 0.0004 & 5.4993 \\
\hline 191.5470 & 226.79 & 217.26 & 210.93 & 210.48 & 211.22 & 210.73 & 270.88 & 257.90 & 0.0005 & 5.5144 \\
\hline 192.0478 & 226.38 & 216.59 & 210.64 & 210.18 & 210.95 & 210.50 & 268.94 & 257.85 & 0.0004 & 5.5444 \\
\hline 192.5477 & 226.29 & 216.68 & 210.36 & 209.88 & 210.65 & 210.24 & 267.35 & 257.82 & 0.0007 & 5.5320 \\
\hline 193.0483 & 225.95 & 216.07 & 210.06 & 209.61 & 210.36 & 209.99 & 265.44 & 257.75 & 0.0006 & 5.5039 \\
\hline 193.5482 & 225.67 & 216.16 & 209.77 & 209.36 & 210.15 & 209.81 & 264.11 & 257.68 & 0.0004 & 5.5014 \\
\hline 194.0480 & 225.42 & 215.09 & 209.62 & 209.22 & 209.97 & 209.69 & 263.54 & 257.66 & 0.0002 & 5.5117 \\
\hline 194.5478 & 225.06 & 214.99 & 209.55 & 209.13 & 209.90 & 209.62 & 263.34 & 257.72 & 0.0004 & 5.5043 \\
\hline 195.0477 & 227.43 & 216.63 & 209.58 & 209.17 & 209.90 & 209.65 & 263.78 & 257.75 & 0.0010 & 5.4913 \\
\hline 195.5483 & 229.79 & 219.12 & 209.67 & 209.24 & 209.96 & 209.71 & 264.54 & 257.84 & 0.0004 & 5.4940 \\
\hline 196.0482 & 228.92 & 219.95 & 209.78 & 209.32 & 210.00 & 209.76 & 265.20 & 257.89 & 0.0008 & 5.5471 \\
\hline 196.5480 & 227.41 & 220.18 & 209.80 & 209.32 & 210.00 & 209.75 & 264.97 & 257.90 & 0.0005 & 5.5181 \\
\hline 197.0478 & 227.43 & 220.09 & 209.68 & 209.20 & 209.91 & 209.63 & 263.80 & 257.72 & 0.0004 & 5.4943 \\
\hline 197.5477 & 227.47 & 220.24 & 209.48 & 209.00 & 209.73 & 209.48 & 262.52 & 257.50 & 0.0012 & 5.4911 \\
\hline 198.0485 & 227.42 & 219.61 & 209.29 & 208.88 & 209.61 & 209.40 & 261.48 & 257.74 & 0.0004 & 5.5306 \\
\hline 198.5483 & 227.31 & 220.57 & 209.18 & 208.79 & 209.56 & 209.36 & 261.02 & 257.75 & 0.0005 & 5.5308 \\
\hline 199.0480 & 226.53 & 218.63 & 209.11 & 208.76 & 209.51 & 209.35 & 260.63 & 257.87 & 0.0006 & 5.5058 \\
\hline 199.5478 & 226.94 & 219.24 & 209.06 & 208.72 & 209.51 & 209.33 & 260.40 & 257.92 & 0.0008 & 5.4899 \\
\hline
\end{tabular}


TABLE B3.- CONTINUED.

\begin{tabular}{|c|c|c|c|c|c|c|c|c|c|c|}
\hline $\begin{array}{c}\text { Time, } \\
\text { min }\end{array}$ & $\begin{array}{c}\text { T-steam } \\
{ }^{0} \mathrm{C}\end{array}$ & $\begin{array}{l}\mathrm{T} 1, \\
{ }^{\circ} \mathrm{C}\end{array}$ & $\begin{array}{l}\mathrm{T} 2, \\
{ }^{\circ} \mathrm{C}\end{array}$ & $\begin{array}{l}\mathrm{T} 3, \\
{ }^{\circ} \mathrm{C}\end{array}$ & $\begin{array}{l}\mathrm{T} 4, \\
{ }^{\circ} \mathrm{C}\end{array}$ & $\begin{array}{l}\mathrm{T} 5, \\
{ }^{\circ} \mathrm{C}\end{array}$ & $\begin{array}{l}\text { Pinj, } \\
\text { psig }\end{array}$ & $\begin{array}{l}\text { Pout, } \\
\text { psig }\end{array}$ & $\begin{array}{l}\text { Qinj, } \\
\text { g/min }\end{array}$ & $\begin{array}{c}\text { Vw } \\
\mathrm{ml} / \mathrm{min}\end{array}$ \\
\hline 200.0477 & 226.34 & 218.33 & 209.05 & 208.72 & 209.51 & 209.35 & 260.25 & 257.97 & 0.0008 & 5.5106 \\
\hline 200.5475 & 226.46 & 218.64 & 209.03 & 208.71 & 209.50 & 209.35 & 260.11 & 257.92 & 0.0002 & 5.5262 \\
\hline 201.0502 & 226.75 & 217.98 & 209.00 & 208.67 & 209.48 & 209.34 & 259.92 & 257.94 & 0.0005 & 5.5135 \\
\hline 201.5490 & 225.86 & 217.46 & 208.96 & 208.67 & 209.48 & 209.32 & 259.61 & 257.96 & 0.0005 & 5.4986 \\
\hline 202.0480 & 226.00 & 216.41 & 208.89 & 208.61 & 209.43 & 209.29 & 259.17 & 257.86 & 0.0006 & 5.4926 \\
\hline 202.5468 & 226.42 & 216.39 & 208.80 & 208.54 & 209.40 & 209.23 & 258.69 & 257.83 & 0.0003 & 5.5258 \\
\hline 203.0475 & 226.01 & 216.93 & 208.75 & 208.41 & 209.24 & 209.02 & 257.90 & 256.90 & 0.0006 & 5.5024 \\
\hline 203.5483 & 226.33 & 215.57 & 208.54 & 208.25 & 209.08 & 208.92 & 257.28 & 255.21 & 0.0006 & 5.4781 \\
\hline 204.0482 & 225.76 & 216.04 & 208.59 & 208.31 & 209.08 & 208.95 & 258.28 & 256.67 & 0.0007 & 5.4849 \\
\hline 204.5470 & 225.35 & 217.08 & 208.81 & 208.51 & 209.24 & 209.13 & 259.97 & 257.95 & 0.0004 & 5.5375 \\
\hline 205.0478 & 226.89 & 219.10 & 209.12 & 208.76 & 209.46 & 209.35 & 261.88 & 258.19 & 0.0003 & 5.5047 \\
\hline 205.5477 & 230.60 & 222.79 & 209.48 & 209.05 & 209.74 & 209.57 & 264.14 & 258.32 & 0.0005 & 5.4871 \\
\hline 206.0483 & 231.37 & 227.25 & 209.73 & 209.17 & 209.82 & 209.59 & 265.03 & 258.11 & 0.0004 & 5.5015 \\
\hline 206.5482 & 230.56 & 227.72 & 209.61 & 209.07 & 209.73 & 209.46 & 263.23 & 257.20 & 0.0006 & 5.5221 \\
\hline 207.0480 & 229.31 & 225.61 & 209.39 & 208.93 & 209.63 & 209.39 & 262.02 & 257.08 & 0.0002 & 5.5039 \\
\hline 207.5478 & 229.96 & 225.27 & 209.07 & 208.59 & 209.32 & 209.07 & 260.07 & 256.45 & 0.0003 & 5.4928 \\
\hline 208.0467 & 230.14 & 225.08 & 208.86 & 208.45 & 209.20 & 209.04 & 259.25 & 256.08 & 0.0003 & 5.5038 \\
\hline 208.5483 & 229.73 & 224.18 & 208.74 & 208.38 & 209.17 & 209.02 & 258.55 & 256.41 & 0.0004 & 5.5442 \\
\hline 209.0473 & 229.36 & 222.75 & 208.74 & 208.44 & 209.24 & 209.12 & 258.80 & 256.20 & 0.0012 & 5.5137 \\
\hline 209.5480 & 229.32 & 222.45 & 208.76 & 208.42 & 209.21 & 209.08 & 258.58 & 256.86 & 0.0002 & 5.4846 \\
\hline 210.0478 & 228.05 & 222.68 & 208.76 & 208.44 & 209.24 & 209.10 & 258.77 & 255.71 & 0.0006 & 5.4985 \\
\hline 210.5477 & 228.13 & 223.01 & 208.76 & 208.44 & 209.26 & 209.12 & 258.83 & 257.05 & 0.0004 & 5.5276 \\
\hline 211.0483 & 229.08 & 222.71 & 208.80 & 208.50 & 209.30 & 209.19 & 258.80 & 257.97 & 0.0004 & 5.4921 \\
\hline 211.5482 & 228.26 & 222.37 & 208.82 & 208.55 & 209.38 & 209.27 & 259.24 & 258.19 & 0.0005 & 5.4592 \\
\hline 212.0480 & 228.67 & 222.17 & 208.89 & 208.64 & 209.43 & 209.32 & 259.90 & 256.68 & 0.0002 & 5.4933 \\
\hline 212.5478 & 228.98 & 221.94 & 208.95 & 208.66 & 209.49 & 209.34 & 259.76 & 257.80 & 0.0007 & 5.5274 \\
\hline 213.0477 & 229.12 & 222.16 & 208.75 & 208.41 & 209.22 & 209.09 & 258.60 & 255.80 & 0.0005 & 5.5008 \\
\hline 213.5475 & 229.47 & 221.80 & 208.72 & 208.51 & 209.33 & 209.12 & 259.00 & 258.18 & 0.0009 & 5.4825 \\
\hline 214.0482 & 228.27 & 222.11 & 208.94 & 208.69 & 209.51 & 209.39 & 259.71 & 258.16 & 0.0004 & 5.4858 \\
\hline 214.5480 & 228.45 & 222.18 & 208.76 & 208.33 & 209.14 & 208.83 & 257.53 & 254.48 & 0.0004 & 5.5287 \\
\hline 215.0478 & 228.34 & 221.29 & 208.44 & 208.12 & 208.96 & 208.80 & 256.41 & 254.74 & 0.0002 & 5.5285 \\
\hline 215.5468 & 228.43 & 220.67 & 208.34 & 208.05 & 208.86 & 208.73 & 256.09 & 254.33 & 0.0007 & 5.4851 \\
\hline 216.0475 & 228.49 & 220.81 & 208.25 & 207.96 & 208.80 & 208.69 & 255.95 & 255.39 & 0.0004 & 5.4729 \\
\hline 216.5483 & 227.97 & 221.53 & 208.29 & 208.03 & 208.88 & 208.79 & 256.30 & 254.49 & 0.0003 & 5.5007 \\
\hline 217.0480 & 226.71 & 222.37 & 208.56 & 208.24 & 208.99 & 208.88 & 257.89 & 255.48 & 0.0008 & 5.4896 \\
\hline 217.5478 & 227.60 & 221.64 & 208.65 & 208.35 & 209.11 & 209.04 & 258.79 & 256.22 & 0.0005 & 5.4701 \\
\hline 218.0468 & 229.31 & 223.22 & 208.77 & 208.42 & 209.19 & 209.04 & 259.11 & 255.83 & 0.0005 & 5.5371 \\
\hline 218.5475 & 230.27 & 222.29 & 208.80 & 208.47 & 209.24 & 209.12 & 258.98 & 256.07 & 0.0007 & 5.5258 \\
\hline 219.0483 & 228.81 & 222.65 & 208.76 & 208.40 & 209.19 & 209.07 & 258.84 & 255.79 & 0.0000 & 5.4804 \\
\hline 219.5482 & 228.70 & 223.33 & 208.75 & 208.37 & 209.16 & 209.00 & 259.04 & 255.65 & 0.0004 & 5.4884 \\
\hline 220.0488 & 228.88 & 223.30 & 208.77 & 208.41 & 209.16 & 209.00 & 258.97 & 255.28 & 0.0004 & 5.4962 \\
\hline 220.5477 & 229.31 & 223.17 & 208.80 & 208.44 & 209.23 & 209.09 & 259.37 & 256.04 & 0.0006 & 5.4575 \\
\hline 221.0485 & 228.83 & 224.96 & 208.86 & 208.46 & 209.22 & 209.07 & 259.47 & 256.37 & 0.0004 & 5.4106 \\
\hline 221.5483 & 229.53 & 225.61 & 208.84 & 208.47 & 209.22 & 209.08 & 259.38 & 256.40 & 0.0002 & 5.4142 \\
\hline 222.0482 & 229.18 & 224.07 & 208.84 & 208.47 & 209.22 & 209.06 & 259.13 & 255.83 & 0.0003 & 5.4235 \\
\hline 222.5480 & 228.96 & 223.52 & 208.77 & 208.42 & 209.20 & 209.10 & 258.66 & 255.75 & 0.0005 & 5.3977 \\
\hline 223.0478 & 229.86 & 223.74 & 208.70 & 208.35 & 209.17 & 209.03 & 258.09 & 255.89 & 0.0005 & 5.3805 \\
\hline 223.5477 & 229.42 & 224.96 & 208.58 & 208.26 & 209.10 & 208.98 & 257.53 & 255.87 & 0.0004 & 5.3764 \\
\hline 224.0483 & 228.61 & 223.38 & 208.53 & 208.26 & 209.10 & 208.98 & 257.22 & 255.46 & 0.0002 & 5.3664 \\
\hline 224.5482 & 227.54 & 223.35 & 208.35 & 208.01 & 208.85 & 208.66 & 255.83 & 253.18 & 0.0005 & 5.3153 \\
\hline
\end{tabular}


TABLE B3.- CONTINUED.

\begin{tabular}{|c|c|c|c|c|c|c|c|c|c|c|}
\hline $\begin{array}{l}\text { Time, } \\
\text { min }\end{array}$ & $\begin{array}{c}\text { T-steam } \\
{ }^{\circ} \mathrm{C}\end{array}$ & $\begin{array}{l}\mathrm{T1}, \\
{ }^{\circ} \mathrm{C}\end{array}$ & $\begin{array}{l}\mathrm{T} 2, \\
{ }^{\circ} \mathrm{C}\end{array}$ & $\begin{array}{l}\mathrm{T} 3, \\
{ }^{\circ} \mathrm{C}\end{array}$ & $\begin{array}{l}\mathrm{T} 4, \\
{ }^{\circ} \mathrm{C}\end{array}$ & $\begin{array}{l}\mathrm{T} 5, \\
{ }^{\circ} \mathrm{C}\end{array}$ & $\begin{array}{l}\text { Pinj, } \\
\text { psig }\end{array}$ & $\begin{array}{c}\text { Pout, } \\
\text { psig }\end{array}$ & $\begin{array}{l}\text { Qinj, } \\
\text { g/min }\end{array}$ & $\begin{array}{c}\mathrm{Vw} \\
\mathrm{ml} / \mathrm{min}\end{array}$ \\
\hline 225.0470 & 227.60 & 221.92 & 208.16 & 207.89 & 208.73 & 208.62 & 255.28 & 253.53 & 0.0001 & 5.3522 \\
\hline 225.5478 & 227.58 & 221.78 & 208.20 & 207.91 & 208.70 & 208.63 & 255.74 & 253.68 & 0.0001 & 5.3865 \\
\hline 226.0477 & 227.60 & 221.37 & 208.25 & 207.98 & 208.79 & 208.68 & 256.18 & 254.21 & 0.0004 & 5.3563 \\
\hline 226.5483 & 228.08 & 221.66 & 208.29 & 208.00 & 208.81 & 208.72 & 256.10 & 254.77 & 0.0004 & 5.4357 \\
\hline 227.0482 & 228.23 & 222.38 & 208.34 & 208.02 & 208.85 & 208.74 & 256.55 & 254.18 & 0.0005 & 5.3871 \\
\hline 227.5470 & 230.02 & 223.67 & 208.36 & 208.08 & 208.88 & 208.78 & 256.48 & 254.67 & 0.0005 & 5.5498 \\
\hline 228.0478 & 228.52 & 223.06 & 208.39 & 208.10 & 208.89 & 208.78 & 256.79 & 255.10 & 0.0006 & 5.5414 \\
\hline 228.5477 & 228.74 & 221.99 & 208.46 & 208.15 & 208.94 & 208.85 & 257.24 & 254.94 & 0.0001 & 5.4265 \\
\hline 229.0483 & 228.18 & 223.44 & 208.53 & 208.21 & 209.00 & 208.87 & 257.81 & 255.58 & 0.0000 & 5.4533 \\
\hline 229.5482 & 228.72 & 223.98 & 208.53 & 208.21 & 209.02 & 208.91 & 257.98 & 255.81 & 0.0006 & 5.6171 \\
\hline 230.0480 & 228.89 & 223.19 & 208.61 & 208.31 & 209.07 & 208.97 & 257.91 & 255.66 & 0.0004 & 5.5951 \\
\hline 230.5478 & 229.69 & 222.71 & 208.65 & 208.32 & 209.11 & 209.00 & 258.13 & 255.87 & 0.0005 & 5.4239 \\
\hline 231.0468 & 229.09 & 224.09 & 208.67 & 208.34 & 209.15 & 209.01 & 258.37 & 255.24 & 0.0004 & 5.5074 \\
\hline 231.5483 & 229.00 & 223.58 & 208.72 & 208.40 & 209.19 & 209.06 & 258.55 & 255.55 & 0.0004 & 5.5478 \\
\hline 232.0473 & 230.15 & 224.01 & 208.74 & 208.42 & 209.21 & 209.08 & 258.93 & 255.57 & 0.0004 & 5.4782 \\
\hline 232.5480 & 229.90 & 224.40 & 208.72 & 208.44 & 209.26 & 209.24 & 258.97 & 258.27 & 0.0004 & 5.3325 \\
\hline 233.0478 & 228.47 & 223.19 & 208.73 & 208.32 & 209.10 & 208.92 & 258.19 & 255.01 & 0.0003 & 5.5892 \\
\hline 233.5487 & 229.67 & 222.94 & 208.75 & 208.46 & 209.25 & 209.18 & 259.22 & 256.53 & 0.0004 & 5.4976 \\
\hline 234.0485 & 229.96 & 223.23 & 208.89 & 208.62 & 209.43 & 209.36 & 259.78 & 257.44 & 0.0008 & 5.4795 \\
\hline 234.5473 & 229.83 & 223.69 & 209.02 & 208.73 & 209.52 & 209.43 & 260.39 & 257.87 & 0.0005 & 5.4897 \\
\hline 235.0480 & 229.16 & 223.11 & 209.09 & 208.81 & 209.61 & 209.52 & 260.93 & 258.22 & 0.0002 & 5.4954 \\
\hline 235.5478 & 229.66 & 223.70 & 209.13 & 208.65 & 209.42 & 209.15 & 260.42 & 255.80 & 0.0002 & 5.4311 \\
\hline 236.0487 & 228.86 & 223.54 & 208.85 & 208.43 & 209.22 & 209.04 & 258.97 & 255.45 & 0.0008 & 5.4550 \\
\hline 236.5467 & 230.61 & 224.85 & 208.74 & 208.40 & 209.19 & 209.08 & 258.58 & 255.72 & 0.0002 & 5.5215 \\
\hline 237.0473 & 229.67 & 224.35 & 208.73 & 208.42 & 209.21 & 209.10 & 258.71 & 255.70 & 0.0006 & 5.4379 \\
\hline 237.5482 & 229.01 & 221.92 & 208.73 & 208.41 & 209.18 & 209.09 & 258.65 & 256.53 & 0.0000 & 5.4394 \\
\hline 238.0480 & 228.77 & 223.92 & 208.75 & 208.43 & 209.21 & 209.09 & 258.78 & 255.35 & 0.0007 & 5.4884 \\
\hline 238.5477 & 228.38 & 222.82 & 208.75 & 208.43 & 209.21 & 209.07 & 258.74 & 255.85 & 0.0007 & 5.4853 \\
\hline 239.0467 & 230.32 & 223.10 & 208.75 & 208.45 & 209.25 & 209.11 & 258.75 & 256.17 & 0.0004 & 5.4581 \\
\hline 239.5483 & 231.36 & 224.84 & 208.74 & 208.41 & 209.22 & 209.11 & 258.62 & 256.07 & 0.0005 & 5.4278 \\
\hline 240.0482 & 229.52 & 224.47 & 208.72 & 208.41 & 209.26 & 209.13 & 258.32 & 255.77 & 0.0003 & 5.5517 \\
\hline 240.5480 & 229.23 & 222.54 & 208.69 & 208.40 & 209.20 & 209.10 & 258.24 & 256.31 & 0.0004 & 5.4580 \\
\hline 241.0478 & 228.93 & 223.29 & 208.71 & 208.42 & 209.22 & 209.12 & 258.71 & 255.31 & 0.0004 & 5.4402 \\
\hline 241.5477 & 229.42 & 224.39 & 208.80 & 208.47 & 209.26 & 209.16 & 259.61 & 255.77 & 0.0004 & 5.4505 \\
\hline 242.0483 & 228.97 & 223.31 & 208.98 & 208.62 & 209.35 & 209.23 & 260.89 & 256.24 & 0.0010 & 5.3722 \\
\hline 242.5482 & 229.44 & 225.11 & 209.18 & 208.75 & 209.45 & 209.32 & 262.09 & 256.30 & 0.0006 & 5.4634 \\
\hline 243.0470 & 230.23 & 226.68 & 209.29 & 208.84 & 209.54 & 209.38 & 262.25 & 256.95 & 0.0003 & 5.5427 \\
\hline 243.5478 & 231.13 & 227.29 & 209.24 & 208.81 & 209.54 & 209.36 & 261.34 & 256.49 & 0.0002 & 5.4283 \\
\hline 244.0477 & 230.88 & 228.05 & 209.04 & 208.68 & 209.45 & 209.27 & 260.36 & 256.33 & 0.0006 & 5.3558 \\
\hline 244.5483 & 229.80 & 225.94 & 208.92 & 208.56 & 209.36 & 209.22 & 259.38 & 256.44 & 0.0002 & 5.3776 \\
\hline 245.0482 & 230.77 & 212.78 & 211.30 & 210.15 & 210.51 & 210.31 & 273.20 & 263.76 & 0.0007 & 5.5945 \\
\hline 245.5480 & 231.60 & 212.88 & 211.39 & 210.17 & 210.52 & 210.30 & 273.35 & 263.24 & 0.0004 & 5.5752 \\
\hline 246.0478 & 231.60 & 212.88 & 211.38 & 210.18 & 210.48 & 210.27 & 273.37 & 262.92 & 0.0003 & 5.5735 \\
\hline 246.5477 & 228.47 & 212.62 & 211.43 & 210.18 & 210.50 & 210.31 & 273.88 & 263.67 & 0.0006 & 5.6004 \\
\hline 247.0483 & 228.91 & 212.49 & 211.42 & 210.26 & 210.60 & 210.40 & 273.37 & 264.23 & 0.0002 & 5.5806 \\
\hline 247.5473 & 231.47 & 212.64 & 211.44 & 210.28 & 210.64 & 210.42 & 273.35 & 264.25 & 0.0004 & 5.5628 \\
\hline 248.0480 & 233.89 & 212.72 & 211.45 & 210.25 & 210.57 & 210.34 & 273.39 & 263.39 & 0.0004 & 5.5453 \\
\hline 248.5478 & 232.10 & 212.81 & 211.43 & 210.23 & 210.59 & 210.39 & 273.42 & 263.91 & 0.0005 & 5.5201 \\
\hline 249.0467 & 231.73 & 212.83 & 211.44 & 210.27 & 210.58 & 210.36 & 273.24 & 263.34 & 0.0000 & 5.5063 \\
\hline 249.5475 & 230.88 & 212.87 & 211.44 & 210.20 & 210.49 & 210.29 & 273.73 & 262.94 & 0.0004 & 5.5489 \\
\hline
\end{tabular}


TABLE B3.- CONTINUED.

\begin{tabular}{|c|c|c|c|c|c|c|c|c|c|c|}
\hline $\begin{array}{l}\text { Time, } \\
\text { min }\end{array}$ & $\begin{array}{c}\text { T-steam } \\
{ }^{\circ} \mathrm{C}\end{array}$ & $\begin{array}{l}\mathrm{T1}, \\
{ }^{\circ} \mathrm{C}\end{array}$ & $\begin{array}{l}\mathrm{T} 2, \\
{ }^{\circ} \mathrm{C}\end{array}$ & $\begin{array}{l}\mathrm{T} 3, \\
{ }^{\circ} \mathrm{C}\end{array}$ & $\begin{array}{l}\mathrm{T} 4, \\
{ }^{\circ} \mathrm{C}\end{array}$ & $\begin{array}{l}\mathrm{T} 5, \\
{ }^{\circ} \mathrm{C}\end{array}$ & $\begin{array}{l}\text { Pinj, } \\
\text { psig }\end{array}$ & $\begin{array}{c}\text { Pout, } \\
\text { psig }\end{array}$ & $\begin{array}{l}\text { Qinj, } \\
\text { g/min }\end{array}$ & $\begin{array}{c}\mathrm{Vw} \\
\mathrm{ml} / \mathrm{min}\end{array}$ \\
\hline 250.0492 & 231.02 & 213.07 & 211.39 & 210.17 & 210.49 & 210.24 & 273.27 & 262.76 & 0.0005 & 5.5565 \\
\hline 250.5472 & 230.58 & 213.02 & 211.32 & 210.14 & 210.48 & 210.23 & 272.70 & 263.05 & 0.0004 & 5.5953 \\
\hline 251.0470 & 230.40 & 212.86 & 211.32 & 210.11 & 210.43 & 210.25 & 272.94 & 263.17 & 0.0002 & 5.5893 \\
\hline 251.5477 & 230.57 & 212.63 & 211.26 & 210.09 & 210.41 & 210.22 & 272.57 & 263.28 & 0.0007 & 5.5928 \\
\hline 252.0485 & 230.72 & 212.64 & 211.31 & 210.11 & 210.47 & 210.29 & 272.99 & 263.48 & 0.0001 & 5.5652 \\
\hline 252.5482 & 231.35 & 212.50 & 211.28 & 210.15 & 210.53 & 210.35 & 272.80 & 263.31 & 0.0002 & 5.5712 \\
\hline 253.0480 & 230.74 & 212.52 & 211.28 & 210.16 & 210.51 & 210.32 & 272.60 & 263.43 & 0.0004 & 5.5246 \\
\hline 253.5488 & 231.44 & 212.47 & 211.31 & 210.16 & 210.52 & 210.36 & 272.86 & 263.48 & 0.0002 & 5.5234 \\
\hline 254.0477 & 232.09 & 212.51 & 211.36 & 210.18 & 210.56 & 210.36 & 273.37 & 263.86 & 0.0007 & 5.5461 \\
\hline 254.5475 & 231.91 & 212.51 & 211.37 & 210.20 & 210.54 & 210.36 & 272.88 & 263.64 & 0.0001 & 5.5620 \\
\hline 255.0473 & 231.35 & 212.59 & 211.35 & 210.19 & 210.55 & 210.38 & 272.82 & 263.30 & 0.0004 & 5.5622 \\
\hline 255.5482 & 230.65 & 212.82 & 211.39 & 210.21 & 210.55 & 210.39 & 273.12 & 263.78 & 0.0004 & 5.5535 \\
\hline 256.0480 & 230.55 & 212.79 & 211.41 & 210.21 & 210.55 & 210.36 & 273.04 & 263.44 & 0.0005 & 5.5749 \\
\hline 256.5477 & 227.43 & 212.42 & 211.45 & 210.23 & 210.56 & 210.38 & 273.69 & 263.06 & 0.0007 & 5.5879 \\
\hline 257.0467 & 228.30 & 212.33 & 211.40 & 210.06 & 209.97 & 209.22 & 273.43 & 250.72 & 0.0001 & 3.1443 \\
\hline
\end{tabular}


TABLE B4. PRESSURE AND TEMPERATURE DATA FOR RUN 6 (PROPANE:STEAM = 5:100).

\begin{tabular}{|c|c|c|c|c|c|c|c|c|c|c|}
\hline $\begin{array}{c}\text { Time, } \\
\text { min }\end{array}$ & $\begin{array}{c}\text { T-steam } \\
{ }^{\circ} \mathrm{C}\end{array}$ & $\begin{array}{l}\mathrm{T} 1, \\
{ }^{\circ} \mathrm{C}\end{array}$ & $\begin{array}{l}\mathrm{T} 2, \\
{ }^{\circ} \mathrm{C}\end{array}$ & $\begin{array}{l}\mathrm{T} 3, \\
{ }^{\circ} \mathrm{C}\end{array}$ & $\begin{array}{l}\mathrm{T} 4, \\
{ }^{\circ} \mathrm{C}\end{array}$ & $\begin{array}{l}\mathrm{T} 5, \\
{ }^{\circ} \mathrm{C}\end{array}$ & $\begin{array}{l}\text { Pinj, } \\
\text { psig }\end{array}$ & $\begin{array}{l}\text { Pout, } \\
\text { psig }\end{array}$ & $\begin{array}{l}\text { Qinj, } \\
\text { g/min }\end{array}$ & $\begin{array}{c}\text { Vw } \\
\mathrm{ml} / \mathrm{min}\end{array}$ \\
\hline 0.0503 & 50.77 & 48.31 & 52.29 & 54.33 & 53.78 & 49.93 & 259.65 & 261.27 & 0.2756 & 5.5010 \\
\hline 0.5447 & 57.30 & 48.81 & 52.32 & 54.32 & 53.78 & 49.94 & 263.01 & 263.18 & 0.2716 & 5.4737 \\
\hline 1.0453 & 82.72 & 52.84 & 52.32 & 54.30 & 53.77 & 49.96 & 263.29 & 262.87 & 0.2766 & 5.5007 \\
\hline 1.5452 & 139.56 & 59.09 & 52.33 & 54.21 & 53.74 & 50.04 & 262.69 & 262.83 & 0.2760 & 5.5306 \\
\hline 2.0450 & 200.51 & 74.11 & 52.30 & 54.07 & 53.69 & 49.99 & 263.09 & 263.24 & 0.2762 & 5.5392 \\
\hline 2.5448 & 209.59 & 156.71 & 52.32 & 54.15 & 53.67 & 50.07 & 263.12 & 262.94 & 0.2755 & 5.5114 \\
\hline 3.0455 & 213.28 & 203.44 & 52.31 & 54.25 & 53.81 & 50.12 & 263.67 & 261.95 & 0.2756 & 5.4922 \\
\hline 3.5453 & 216.62 & 206.65 & 52.28 & 54.25 & 53.89 & 50.16 & 263.39 & 262.37 & 0.2756 & 5.5264 \\
\hline 4.0452 & 225.02 & 206.98 & 52.26 & 54.28 & 53.94 & 50.20 & 260.75 & 261.49 & 0.2763 & 5.5296 \\
\hline 4.5442 & 237.24 & 208.02 & 52.23 & 54.25 & 53.92 & 50.15 & 261.32 & 261.53 & 0.2761 & 5.4929 \\
\hline 5.0448 & 249.68 & 210.03 & 52.23 & 54.23 & 53.95 & 50.18 & 264.56 & 261.70 & 0.2757 & 5.4955 \\
\hline 5.5447 & 256.01 & 210.70 & 52.26 & 54.22 & 53.95 & 50.20 & 263.17 & 261.59 & 0.2759 & 5.5136 \\
\hline 6.0453 & 253.71 & 212.49 & 52.28 & 54.24 & 53.98 & 50.21 & 266.68 & 261.69 & 0.2758 & 5.5128 \\
\hline 6.5452 & 250.70 & 213.82 & 52.34 & 54.23 & 53.98 & 50.23 & 263.16 & 261.27 & 0.2765 & 5.4696 \\
\hline 7.0450 & 242.69 & 214.66 & 52.48 & 54.23 & 54.00 & 50.25 & 266.32 & 262.10 & 0.2760 & 5.5192 \\
\hline 7.5448 & 236.85 & 216.07 & 52.56 & 54.22 & 53.99 & 50.24 & 267.91 & 260.92 & 0.2761 & 5.5279 \\
\hline 8.0457 & 231.73 & 213.10 & 52.68 & 54.22 & 54.03 & 50.28 & 274.60 & 261.80 & 0.2755 & 5.5073 \\
\hline 8.5453 & 226.51 & 213.52 & 52.76 & 54.21 & 54.04 & 50.29 & 273.74 & 261.27 & 0.2761 & 5.4869 \\
\hline 9.0452 & 221.32 & 212.07 & 52.88 & 54.18 & 54.02 & 50.29 & 276.13 & 261.40 & 0.2763 & 5.5207 \\
\hline 9.5450 & 219.79 & 211.86 & 53.02 & 54.14 & 54.03 & 50.28 & 275.00 & 261.38 & 0.2767 & 5.5420 \\
\hline 10.0458 & 222.84 & 212.47 & 53.18 & 54.15 & 54.04 & 50.28 & 282.79 & 261.53 & 0.2763 & 5.4916 \\
\hline 10.5457 & 234.30 & 212.69 & 53.39 & 54.15 & 54.02 & 50.29 & 284.67 & 261.54 & 0.2756 & 5.4883 \\
\hline 11.0455 & 240.59 & 214.52 & 53.63 & 54.16 & 54.03 & 50.28 & 286.34 & 261.50 & 0.2767 & 5.5174 \\
\hline 11.5453 & 242.93 & 215.37 & 53.90 & 54.16 & 54.03 & 50.30 & 291.26 & 261.50 & 0.2766 & 5.5246 \\
\hline 12.0442 & 238.44 & 216.14 & 54.19 & 54.17 & 54.05 & 50.30 & 291.79 & 261.56 & 0.2759 & 5.4732 \\
\hline 12.5448 & 235.46 & 216.04 & 54.46 & 54.17 & 54.04 & 50.31 & 294.95 & 261.36 & 0.2771 & 5.4967 \\
\hline 13.0447 & 232.30 & 216.42 & 54.79 & 54.20 & 54.08 & 50.31 & 293.63 & 261.37 & 0.2755 & 5.5660 \\
\hline 13.5445 & 227.24 & 215.82 & 55.13 & 54.18 & 54.09 & 50.32 & 297.64 & 261.49 & 0.2762 & 5.5188 \\
\hline 14.0443 & 224.29 & 215.30 & 55.61 & 54.17 & 54.15 & 50.30 & 299.78 & 261.59 & 0.2757 & 5.4960 \\
\hline 14.5452 & 228.11 & 215.33 & 56.00 & 54.17 & 54.19 & 50.33 & 300.91 & 261.62 & 0.2759 & 5.5242 \\
\hline 15.0450 & 233.18 & 216.71 & 56.33 & 54.16 & 54.22 & 50.30 & 298.70 & 261.59 & 0.2766 & 5.5421 \\
\hline 15.5465 & 238.86 & 216.59 & 56.84 & 54.16 & 54.22 & 50.30 & 300.56 & 261.45 & 0.2756 & 5.5179 \\
\hline 16.0455 & 236.14 & 216.72 & 57.46 & 54.15 & 54.23 & 50.30 & 307.25 & 261.54 & 0.2760 & 5.4869 \\
\hline 16.5443 & 232.94 & 217.81 & 58.18 & 54.17 & 54.23 & 50.35 & 308.86 & 261.48 & 0.2764 & 5.4921 \\
\hline 17.0452 & 233.79 & 217.35 & 58.87 & 54.18 & 54.24 & 50.35 & 311.35 & 261.47 & 0.2763 & 5.5274 \\
\hline 17.5450 & 234.67 & 218.56 & 59.56 & 54.18 & 54.26 & 50.45 & 311.55 & 261.25 & 0.2764 & 5.4902 \\
\hline 18.0457 & 235.16 & 218.15 & 60.36 & 54.11 & 54.26 & 50.49 & 310.92 & 261.31 & 0.2761 & 5.4762 \\
\hline 18.5455 & 237.67 & 218.01 & 61.16 & 54.12 & 54.27 & 50.48 & 314.86 & 261.26 & 0.2755 & 5.4960 \\
\hline 19.0453 & 235.74 & 219.37 & 62.07 & 54.12 & 54.27 & 50.49 & 317.98 & 261.24 & 0.2764 & 5.5006 \\
\hline 19.5452 & 233.61 & 219.07 & 62.95 & 54.11 & 54.28 & 50.49 & 321.62 & 261.17 & 0.2761 & 5.4739 \\
\hline 20.0450 & 231.43 & 219.72 & 63.91 & 54.11 & 54.30 & 50.51 & 323.91 & 261.19 & 0.2757 & 5.4955 \\
\hline 20.5448 & 226.76 & 218.67 & 64.90 & 54.11 & 54.30 & 50.54 & 326.36 & 261.17 & 0.2761 & 5.5275 \\
\hline 21.0447 & 228.70 & 218.85 & 65.89 & 54.14 & 54.33 & 50.56 & 329.42 & 261.32 & 0.2762 & 5.5151 \\
\hline 21.5453 & 228.29 & 219.34 & 67.04 & 54.12 & 54.31 & 50.58 & 333.67 & 261.65 & 0.7086 & 5.4908 \\
\hline 22.0452 & 233.43 & 218.45 & 67.56 & 54.13 & 54.32 & 50.61 & 329.75 & 261.70 & 0.7085 & 5.5235 \\
\hline 22.5450 & 233.26 & 218.83 & 68.65 & 54.15 & 54.36 & 50.63 & 332.40 & 261.90 & 0.7085 & 5.5263 \\
\hline 23.0448 & 231.99 & 219.37 & 69.41 & 54.17 & 54.36 & 50.65 & 331.03 & 261.63 & 0.7085 & 5.4932 \\
\hline 23.5455 & 230.67 & 219.53 & 70.21 & 54.21 & 54.37 & 50.71 & 331.07 & 262.43 & 0.7085 & 5.4863 \\
\hline 24.0453 & 228.59 & 219.34 & 71.06 & 54.22 & 54.37 & 50.73 & 328.21 & 261.87 & 0.1816 & 5.5296 \\
\hline 24.5462 & 228.79 & 219.49 & 72.10 & 54.22 & 54.37 & 50.76 & 328.17 & 262.56 & 0.1207 & 5.5305 \\
\hline
\end{tabular}


TABLE B4.- CONTINUED.

\begin{tabular}{|c|c|c|c|c|c|c|c|c|c|c|}
\hline $\begin{array}{l}\text { Time, } \\
\text { min }\end{array}$ & $\begin{array}{c}\text { T-steam } \\
{ }^{\circ} \mathrm{C}\end{array}$ & $\begin{array}{l}\mathrm{T} 1, \\
{ }^{\circ} \mathrm{C}\end{array}$ & $\begin{array}{l}\mathrm{T} 2, \\
{ }^{\circ} \mathrm{C}\end{array}$ & $\begin{array}{l}\mathrm{T} 3, \\
{ }^{\circ} \mathrm{C}\end{array}$ & $\begin{array}{l}\mathrm{T} 4, \\
{ }^{\circ} \mathrm{C}\end{array}$ & $\begin{array}{l}\mathrm{T} 5, \\
{ }^{\circ} \mathrm{C}\end{array}$ & $\begin{array}{l}\text { Pinj, } \\
\text { psig }\end{array}$ & $\begin{array}{l}\text { Pout, } \\
\text { psig }\end{array}$ & $\begin{array}{l}\text { Qinj, } \\
\text { g/min }\end{array}$ & $\begin{array}{c}\mathrm{Vw} \\
\mathrm{ml} / \mathrm{min}\end{array}$ \\
\hline 25.0450 & 229.93 & 220.01 & 73.10 & 54.24 & 54.40 & 50.80 & 328.29 & 262.58 & 0.7085 & 5.4931 \\
\hline 25.5458 & 229.16 & 220.25 & 74.16 & 54.25 & 54.40 & 50.84 & 328.56 & 260.57 & 0.0717 & 5.4737 \\
\hline 26.0465 & 229.45 & 220.25 & 75.25 & 54.25 & 54.39 & 50.85 & 328.02 & 262.62 & 0.2877 & 5.5171 \\
\hline 26.5453 & 228.77 & 220.32 & 76.57 & 54.26 & 54.43 & 50.93 & 327.36 & 262.63 & 0.4451 & 5.5270 \\
\hline 27.0452 & 228.72 & 220.11 & 77.68 & 54.28 & 54.41 & 50.93 & 326.03 & 261.46 & 0.3045 & 5.4829 \\
\hline 27.5460 & 228.53 & 220.01 & 78.90 & 54.30 & 54.44 & 50.97 & 324.34 & 261.00 & 0.4162 & 5.4870 \\
\hline 28.0448 & 228.51 & 219.83 & 80.09 & 54.30 & 54.44 & 51.01 & 322.90 & 262.29 & 0.3987 & 5.5207 \\
\hline 28.5457 & 228.45 & 219.69 & 81.48 & 54.37 & 54.46 & 51.04 & 321.64 & 262.62 & 0.3519 & 5.4889 \\
\hline 29.0455 & 229.61 & 219.55 & 82.77 & 54.39 & 54.47 & 51.06 & 320.30 & 261.60 & 0.3058 & 5.4755 \\
\hline 29.5443 & 230.62 & 219.63 & 84.25 & 54.45 & 54.47 & 51.12 & 322.68 & 255.40 & 0.2727 & 5.5271 \\
\hline 30.0450 & 229.30 & 219.51 & 85.97 & 54.47 & 54.45 & 51.12 & 321.63 & 256.63 & 0.2731 & 5.5407 \\
\hline 30.5448 & 228.93 & 219.56 & 87.68 & 54.53 & 54.48 & 51.16 & 322.57 & 258.55 & 0.2678 & 5.4842 \\
\hline 31.0457 & 229.16 & 219.69 & 89.23 & 54.61 & 54.48 & 51.23 & 322.55 & 259.19 & 0.2729 & 5.5032 \\
\hline 31.5455 & 228.93 & 219.72 & 90.80 & 54.65 & 54.48 & 51.27 & 324.55 & 257.69 & 0.2599 & 5.5207 \\
\hline 32.0453 & 231.01 & 219.77 & 92.51 & 54.75 & 54.49 & 51.31 & 325.09 & 258.21 & 0.2735 & 5.5299 \\
\hline 32.5452 & 229.24 & 220.01 & 94.28 & 54.83 & 54.51 & 51.35 & 326.37 & 258.97 & 0.2759 & 5.4933 \\
\hline 33.0450 & 228.92 & 220.19 & 96.01 & 54.91 & 54.51 & 51.39 & 328.02 & 255.49 & 0.2760 & 5.5078 \\
\hline 33.5457 & 229.45 & 220.35 & 97.72 & 55.01 & 54.52 & 51.45 & 328.50 & 257.73 & 0.2757 & 5.5259 \\
\hline 34.0455 & 230.24 & 220.63 & 99.38 & 55.11 & 54.54 & 51.49 & 330.76 & 258.47 & 0.2758 & 5.5206 \\
\hline 34.5453 & 229.40 & 220.91 & 101.20 & 55.19 & 54.58 & 51.55 & 335.00 & 257.11 & 0.2751 & 5.4827 \\
\hline 35.0452 & 233.36 & 220.88 & 103.18 & 55.32 & 54.58 & 51.61 & 339.24 & 255.46 & 0.2759 & 5.5001 \\
\hline 35.5450 & 232.75 & 220.76 & 104.81 & 55.42 & 54.59 & 51.64 & 341.72 & 255.99 & 0.2757 & 5.5445 \\
\hline 36.0448 & 235.23 & 220.89 & 106.53 & 55.50 & 54.59 & 51.66 & 343.74 & 256.50 & 0.2759 & 5.4807 \\
\hline 36.5455 & 233.87 & 221.02 & 108.66 & 55.62 & 54.63 & 51.72 & 346.53 & 258.51 & 0.2759 & 5.4712 \\
\hline 37.0453 & 235.83 & 220.97 & 111.11 & 55.74 & 54.67 & 51.78 & 345.82 & 258.90 & 0.2760 & 5.5149 \\
\hline 37.5452 & 233.36 & 220.93 & 113.78 & 55.85 & 54.69 & 51.82 & 345.34 & 257.99 & 0.2760 & 5.5450 \\
\hline 38.0450 & 236.39 & 220.86 & 116.76 & 55.99 & 54.70 & 51.84 & 348.03 & 258.37 & 0.2762 & 5.4976 \\
\hline 38.5448 & 234.99 & 220.67 & 120.05 & 56.12 & 54.72 & 51.92 & 346.90 & 256.21 & 0.2755 & 5.5050 \\
\hline 39.0455 & 232.90 & 220.64 & 123.65 & 56.28 & 54.76 & 51.98 & 345.31 & 257.56 & 0.2767 & 5.5250 \\
\hline 39.5453 & 232.33 & 220.64 & 127.34 & 56.45 & 54.76 & 52.00 & 345.24 & 257.06 & 0.2753 & 5.5380 \\
\hline 40.0452 & 233.59 & 220.27 & 130.89 & 56.68 & 54.80 & 52.06 & 345.99 & 256.25 & 0.2736 & 5.5017 \\
\hline 40.5450 & 232.68 & 220.25 & 134.47 & 56.90 & 54.82 & 52.11 & 344.78 & 258.21 & 0.2555 & 5.4829 \\
\hline 41.0448 & 236.85 & 220.33 & 137.86 & 57.14 & 54.85 & 52.15 & 344.88 & 258.25 & 0.2370 & 5.5286 \\
\hline 41.5455 & 234.58 & 220.58 & 141.49 & 57.41 & 54.85 & 52.17 & 345.32 & 258.44 & 0.1410 & 5.5368 \\
\hline 42.0445 & 232.29 & 220.44 & 144.64 & 57.72 & 54.85 & 52.19 & 343.98 & 257.73 & 0.2939 & 5.4963 \\
\hline 42.5443 & 231.74 & 220.32 & 147.88 & 58.01 & 54.87 & 52.23 & 343.92 & 256.80 & 0.2731 & 5.5028 \\
\hline 43.0450 & 231.11 & 220.32 & 150.75 & 58.32 & 54.90 & 52.22 & 351.39 & 257.04 & 0.7087 & 5.5446 \\
\hline 43.5448 & 233.37 & 219.91 & 155.00 & 58.68 & 54.97 & 52.25 & 347.00 & 256.53 & 0.2741 & 5.5367 \\
\hline 44.0457 & 235.86 & 220.22 & 157.63 & 59.08 & 55.00 & 52.30 & 349.41 & 258.07 & 0.2763 & 5.5097 \\
\hline 44.5453 & 233.34 & 220.33 & 160.53 & 59.54 & 55.08 & 52.34 & 350.13 & 258.35 & 0.2763 & 5.4852 \\
\hline 45.0452 & 237.03 & 219.22 & 162.48 & 59.99 & 55.12 & 52.38 & 346.82 & 258.05 & 0.2753 & 5.5497 \\
\hline 45.5450 & 232.15 & 220.46 & 164.22 & 60.47 & 55.12 & 52.42 & 347.09 & 260.03 & 0.2750 & 5.5275 \\
\hline 46.0440 & 232.97 & 220.42 & 166.22 & 60.97 & 55.14 & 52.46 & 344.69 & 258.71 & 0.2755 & 5.4842 \\
\hline 46.5447 & 232.04 & 220.73 & 168.37 & 61.48 & 55.16 & 52.48 & 344.50 & 257.76 & 0.2757 & 5.5000 \\
\hline 47.0445 & 232.05 & 220.66 & 170.87 & 61.97 & 55.15 & 52.50 & 346.43 & 258.77 & 0.2752 & 5.5175 \\
\hline 47.5453 & 231.89 & 220.63 & 173.23 & 62.54 & 55.17 & 52.58 & 348.32 & 260.28 & 0.2752 & 5.5171 \\
\hline 48.0450 & 231.66 & 220.56 & 175.21 & 63.15 & 55.19 & 52.64 & 348.09 & 262.50 & 0.2752 & 5.4943 \\
\hline 48.5440 & 231.84 & 220.79 & 177.06 & 63.80 & 55.23 & 52.72 & 350.92 & 262.64 & 0.2753 & 5.5201 \\
\hline 49.0457 & 232.02 & 221.14 & 178.70 & 64.50 & 55.27 & 52.84 & 352.57 & 262.86 & 0.2741 & 5.5192 \\
\hline 49.5445 & 232.09 & 220.96 & 180.43 & 65.22 & 55.29 & 52.91 & 352.56 & 262.93 & 0.2753 & 5.5156 \\
\hline
\end{tabular}


TABLE B4.- CONTINUED.

\begin{tabular}{|c|c|c|c|c|c|c|c|c|c|c|}
\hline $\begin{array}{l}\text { Time, } \\
\text { min }\end{array}$ & $\begin{array}{c}\text { T-steam } \\
{ }^{\circ} \mathrm{C}\end{array}$ & $\begin{array}{l}\mathrm{T} 1, \\
{ }^{\circ} \mathrm{C}\end{array}$ & $\begin{array}{l}\mathrm{T} 2, \\
{ }^{\circ} \mathrm{C}\end{array}$ & $\begin{array}{l}\mathrm{T} 3, \\
{ }^{\circ} \mathrm{C}\end{array}$ & $\begin{array}{l}\mathrm{T} 4, \\
{ }^{\circ} \mathrm{C}\end{array}$ & $\begin{array}{l}\mathrm{T} 5, \\
{ }^{\circ} \mathrm{C}\end{array}$ & $\begin{array}{l}\text { Pinj, } \\
\text { psig }\end{array}$ & $\begin{array}{c}\text { Pout, } \\
\text { psig }\end{array}$ & $\begin{array}{l}\text { Qinj, } \\
\text { g/min }\end{array}$ & $\begin{array}{c}\mathrm{Vw} \\
\mathrm{ml} / \mathrm{min}\end{array}$ \\
\hline 50.0453 & 231.17 & 220.78 & 182.01 & 65.98 & 55.33 & 52.98 & 352.36 & 262.99 & 0.2755 & 5.4876 \\
\hline 50.5452 & 231.83 & 220.46 & 184.13 & 66.77 & 55.37 & 53.00 & 350.63 & 261.92 & 0.2742 & 5.5084 \\
\hline 51.0450 & 232.08 & 220.48 & 186.21 & 67.63 & 55.40 & 53.04 & 349.61 & 261.86 & 0.2748 & 5.5407 \\
\hline 51.5457 & 230.69 & 220.45 & 188.33 & 68.46 & 55.47 & 53.08 & 347.52 & 262.08 & 0.2746 & 5.4974 \\
\hline 52.0463 & 231.78 & 219.91 & 190.29 & 69.31 & 55.51 & 53.12 & 344.42 & 262.30 & 0.2750 & 5.4853 \\
\hline 52.5453 & 231.41 & 219.97 & 192.44 & 70.20 & 55.57 & 53.18 & 342.71 & 262.74 & 0.2762 & 5.5191 \\
\hline 53.0452 & 230.14 & 219.45 & 194.47 & 71.09 & 55.63 & 53.26 & 340.02 & 262.89 & 0.2712 & 5.5321 \\
\hline 53.5450 & 232.22 & 219.17 & 196.92 & 71.95 & 55.71 & 53.32 & 337.99 & 263.07 & 0.2855 & 5.4585 \\
\hline 54.0448 & 231.70 & 219.14 & 199.02 & 72.88 & 55.77 & 53.37 & 335.15 & 261.74 & 0.2661 & 5.4905 \\
\hline 54.5455 & 230.58 & 218.51 & 201.01 & 73.82 & 55.87 & 53.45 & 332.02 & 256.71 & 0.2745 & 5.5278 \\
\hline 55.0453 & 230.49 & 218.16 & 203.23 & 74.73 & 55.95 & 53.48 & 325.88 & 261.95 & 0.2745 & 5.5229 \\
\hline 55.5452 & 228.97 & 218.09 & 205.01 & 75.67 & 56.04 & 53.50 & 322.67 & 262.53 & 0.2753 & 5.4898 \\
\hline 56.0450 & 228.92 & 217.79 & 206.85 & 76.63 & 56.14 & 53.50 & 320.52 & 262.22 & 0.2758 & 5.5229 \\
\hline 56.5448 & 228.37 & 217.47 & 208.72 & 77.61 & 56.24 & 53.50 & 320.78 & 263.17 & 0.2749 & 5.5402 \\
\hline 57.0447 & 231.40 & 217.43 & 210.40 & 78.62 & 56.34 & 53.49 & 321.52 & 262.74 & 0.2752 & 5.5193 \\
\hline 57.5453 & 231.26 & 217.27 & 211.82 & 79.69 & 56.47 & 53.49 & 320.35 & 261.36 & 0.2750 & 5.4730 \\
\hline 58.0452 & 230.29 & 217.13 & 212.62 & 80.80 & 56.57 & 53.51 & 318.08 & 260.57 & 0.2748 & 5.5154 \\
\hline 58.5442 & 230.83 & 217.01 & 213.22 & 81.91 & 56.71 & 53.51 & 318.13 & 261.75 & 0.2750 & 5.5410 \\
\hline 59.0438 & 230.90 & 216.82 & 213.54 & 83.01 & 56.80 & 53.53 & 317.54 & 262.18 & 0.2739 & 5.5270 \\
\hline 59.5447 & 231.05 & 216.60 & 213.61 & 84.17 & 56.94 & 53.54 & 317.36 & 262.45 & 0.2752 & 5.4975 \\
\hline 60.0453 & 231.01 & 216.52 & 213.60 & 85.39 & 57.09 & 53.57 & 315.55 & 263.01 & 0.2749 & 5.5118 \\
\hline 60.5452 & 231.61 & 216.14 & 213.51 & 86.68 & 57.21 & 53.60 & 315.20 & 262.99 & 0.2736 & 5.5348 \\
\hline 61.0450 & 231.61 & 216.22 & 213.44 & 88.01 & 57.36 & 53.62 & 312.93 & 262.88 & 0.2747 & 5.4981 \\
\hline 61.5448 & 231.02 & 216.18 & 213.37 & 89.37 & 57.50 & 53.62 & 312.98 & 260.72 & 0.2760 & 5.4861 \\
\hline 62.0447 & 231.13 & 216.28 & 213.30 & 90.83 & 57.69 & 53.66 & 311.47 & 260.58 & 0.2747 & 5.4965 \\
\hline 62.5445 & 232.62 & 216.55 & 213.29 & 92.36 & 57.88 & 53.72 & 311.45 & 259.22 & 0.2757 & 5.5130 \\
\hline 63.0452 & 234.25 & 216.19 & 213.20 & 93.86 & 58.09 & 53.70 & 309.88 & 258.71 & 0.7087 & 5.5095 \\
\hline 63.5450 & 232.05 & 216.46 & 213.15 & 95.41 & 58.32 & 53.76 & 309.57 & 261.89 & 0.2611 & 5.4903 \\
\hline 64.0448 & 230.44 & 215.98 & 213.13 & 96.99 & 58.55 & 53.78 & 307.54 & 261.74 & 0.2787 & 5.5210 \\
\hline 64.5447 & 231.20 & 215.46 & 213.15 & 98.62 & 58.84 & 53.80 & 306.54 & 259.16 & 0.2763 & 5.5258 \\
\hline 65.0455 & 233.70 & 215.48 & 212.98 & 100.28 & 59.16 & 53.83 & 302.03 & 259.63 & 0.2745 & 5.4872 \\
\hline 65.5453 & 232.35 & 216.15 & 212.73 & 101.93 & 59.49 & 53.85 & 302.45 & 258.89 & 0.2765 & 5.5038 \\
\hline 66.0450 & 229.70 & 216.65 & 212.71 & 103.60 & 59.78 & 53.85 & 300.41 & 258.33 & 0.2758 & 5.5088 \\
\hline 66.5448 & 229.48 & 215.95 & 213.38 & 105.43 & 60.14 & 53.83 & 301.19 & 259.87 & 0.2758 & 5.4837 \\
\hline 67.0457 & 231.21 & 216.40 & 213.70 & 107.53 & 60.56 & 53.86 & 304.14 & 259.35 & 0.2759 & 5.4800 \\
\hline 67.5455 & 232.32 & 216.94 & 213.72 & 109.95 & 61.07 & 53.84 & 305.56 & 258.63 & 0.2758 & 5.5406 \\
\hline 68.0453 & 232.25 & 216.80 & 213.70 & 112.41 & 61.72 & 53.88 & 306.14 & 261.31 & 0.2753 & 5.5319 \\
\hline 68.5442 & 232.70 & 216.66 & 213.69 & 114.95 & 62.44 & 53.90 & 307.42 & 261.49 & 0.2755 & 5.5151 \\
\hline 69.0440 & 232.18 & 217.15 & 213.71 & 117.67 & 63.14 & 53.90 & 306.43 & 261.96 & 0.2754 & 5.4852 \\
\hline 69.5457 & 231.77 & 216.54 & 213.60 & 120.62 & 63.82 & 53.94 & 306.15 & 261.77 & 0.2747 & 5.5119 \\
\hline 70.0455 & 232.83 & 216.94 & 213.48 & 123.74 & 64.49 & 53.92 & 305.24 & 261.69 & 0.2763 & 5.5382 \\
\hline 70.5453 & 231.13 & 216.63 & 213.38 & 126.89 & 65.13 & 53.97 & 305.31 & 261.98 & 0.2756 & 5.4684 \\
\hline 71.0452 & 231.90 & 216.67 & 213.38 & 130.16 & 65.78 & 54.01 & 304.31 & 261.92 & 0.2749 & 5.4906 \\
\hline 71.5450 & 231.94 & 217.14 & 213.38 & 133.56 & 66.42 & 54.03 & 302.26 & 262.61 & 0.2749 & 5.5333 \\
\hline 72.0467 & 232.25 & 216.64 & 213.36 & 136.86 & 67.06 & 54.03 & 303.33 & 262.52 & 0.2749 & 5.5404 \\
\hline 72.5447 & 231.91 & 216.89 & 213.38 & 140.33 & 67.69 & 54.05 & 303.05 & 262.89 & 0.2742 & 5.4957 \\
\hline 73.0443 & 231.43 & 217.09 & 213.40 & 144.18 & 68.31 & 54.09 & 304.83 & 262.90 & 0.2752 & 5.5030 \\
\hline 73.5452 & 231.61 & 216.97 & 213.57 & 148.30 & 68.97 & 54.13 & 305.14 & 260.74 & 0.2758 & 5.5455 \\
\hline 74.0450 & 232.18 & 216.90 & 213.55 & 152.79 & 69.68 & 54.17 & 303.89 & 261.26 & 0.2757 & 5.5310 \\
\hline 74.5438 & 231.54 & 216.72 & 213.55 & 157.62 & 70.36 & 54.23 & 304.88 & 261.39 & 0.2749 & 5.4803 \\
\hline
\end{tabular}


TABLE B4.- CONTINUED.

\begin{tabular}{|c|c|c|c|c|c|c|c|c|c|c|}
\hline $\begin{array}{l}\text { Time, } \\
\text { min }\end{array}$ & $\begin{array}{c}\text { T-steam } \\
{ }^{\circ} \mathrm{C}\end{array}$ & $\begin{array}{l}\mathrm{T} 1, \\
{ }^{\circ} \mathrm{C}\end{array}$ & $\begin{array}{l}\mathrm{T} 2, \\
{ }^{\circ} \mathrm{C}\end{array}$ & $\begin{array}{l}\mathrm{T} 3, \\
{ }^{\circ} \mathrm{C}\end{array}$ & $\begin{array}{l}\mathrm{T} 4, \\
{ }^{\circ} \mathrm{C}\end{array}$ & $\begin{array}{l}\mathrm{T} 5, \\
{ }^{\circ} \mathrm{C}\end{array}$ & $\begin{array}{l}\text { Pinj, } \\
\text { psig }\end{array}$ & $\begin{array}{l}\text { Pout, } \\
\text { psig }\end{array}$ & $\begin{array}{l}\text { Qinj, } \\
\text { g/min }\end{array}$ & $\begin{array}{c}\mathrm{Vw} \\
\mathrm{ml} / \mathrm{min}\end{array}$ \\
\hline 75.0447 & 230.38 & 216.76 & 213.66 & 162.49 & 70.98 & 54.27 & 305.67 & 261.68 & 0.2750 & 5.4999 \\
\hline 75.5453 & 231.40 & 216.19 & 213.63 & 167.72 & 71.58 & 54.33 & 305.95 & 261.98 & 0.2749 & 5.5039 \\
\hline 76.0462 & 231.05 & 216.80 & 213.63 & 173.50 & 72.22 & 54.39 & 305.33 & 261.91 & 0.2755 & 5.4998 \\
\hline 76.5450 & 231.35 & 217.01 & 213.67 & 179.52 & 72.87 & 54.43 & 306.62 & 261.75 & 0.2752 & 5.4913 \\
\hline 77.0438 & 231.75 & 216.77 & 213.67 & 184.99 & 73.51 & 54.49 & 305.58 & 262.09 & 0.2754 & 5.5432 \\
\hline 77.5455 & 230.94 & 217.14 & 213.74 & 190.02 & 74.09 & 54.57 & 306.89 & 262.32 & 0.2755 & 5.5249 \\
\hline 78.0453 & 230.44 & 216.95 & 213.78 & 194.18 & 74.73 & 54.64 & 305.56 & 262.32 & 0.2753 & 5.4889 \\
\hline 78.5452 & 232.00 & 216.41 & 213.76 & 197.40 & 75.34 & 54.70 & 307.96 & 262.43 & 0.2750 & 5.5015 \\
\hline 79.0450 & 231.72 & 216.47 & 213.80 & 200.22 & 75.92 & 54.78 & 305.16 & 262.70 & 0.2752 & 5.5389 \\
\hline 79.5448 & 231.15 & 216.52 & 213.80 & 202.69 & 76.49 & 54.88 & 305.09 & 262.42 & 0.2755 & 5.5120 \\
\hline 80.0457 & 230.11 & 216.58 & 213.73 & 204.55 & 77.01 & 54.96 & 304.20 & 262.57 & 0.2701 & 5.4742 \\
\hline 80.5445 & 231.31 & 216.29 & 213.57 & 205.99 & 77.50 & 55.05 & 302.38 & 262.61 & 0.2750 & 5.5062 \\
\hline 81.0452 & 231.10 & 216.78 & 213.45 & 207.17 & 77.97 & 55.11 & 303.20 & 260.20 & 0.2755 & 5.5267 \\
\hline 81.5450 & 230.85 & 216.46 & 213.38 & 208.09 & 78.52 & 55.21 & 303.09 & 260.56 & 0.2746 & 5.5151 \\
\hline 82.0448 & 232.16 & 216.55 & 213.40 & 208.62 & 79.03 & 55.27 & 301.82 & 259.92 & 0.2746 & 5.4808 \\
\hline 82.5457 & 231.18 & 215.71 & 213.31 & 208.89 & 79.44 & 55.35 & 301.35 & 260.37 & 0.2748 & 5.5113 \\
\hline 83.0455 & 232.54 & 215.91 & 213.05 & 209.29 & 79.84 & 55.42 & 297.85 & 261.05 & 0.2751 & 5.5358 \\
\hline 83.5453 & 231.11 & 216.22 & 212.91 & 209.60 & 80.18 & 55.52 & 298.45 & 261.04 & 0.2747 & 5.5061 \\
\hline 84.0450 & 229.84 & 216.09 & 212.87 & 209.79 & 80.57 & 55.62 & 298.61 & 258.59 & 0.2742 & 5.5042 \\
\hline 84.5448 & 229.25 & 215.90 & 212.86 & 209.96 & 80.99 & 55.71 & 300.30 & 261.00 & 0.2759 & 5.5106 \\
\hline 85.0457 & 230.15 & 215.51 & 213.06 & 210.10 & 81.42 & 55.87 & 301.31 & 259.13 & 0.2754 & 5.5341 \\
\hline 85.5455 & 230.47 & 216.19 & 213.18 & 210.26 & 81.95 & 56.00 & 299.75 & 260.60 & 0.2755 & 5.5003 \\
\hline 86.0453 & 229.99 & 216.71 & 213.33 & 210.43 & 82.49 & 56.21 & 299.98 & 259.97 & 0.2758 & 5.4845 \\
\hline 86.5452 & 229.94 & 217.30 & 213.60 & 210.61 & 83.09 & 56.39 & 302.53 & 260.67 & 0.2755 & 5.5176 \\
\hline 87.0450 & 230.32 & 217.41 & 213.83 & 210.93 & 83.77 & 56.58 & 300.96 & 260.30 & 0.2754 & 5.5389 \\
\hline 87.5457 & 230.39 & 216.80 & 213.78 & 211.26 & 84.57 & 56.81 & 301.92 & 258.83 & 0.2752 & 5.4976 \\
\hline 88.0455 & 230.20 & 216.97 & 213.75 & 211.46 & 85.55 & 57.04 & 300.15 & 261.25 & 0.2756 & 5.4950 \\
\hline 88.5443 & 231.06 & 216.29 & 213.71 & 211.51 & 86.74 & 57.29 & 299.58 & 260.74 & 0.2756 & 5.5231 \\
\hline 89.0452 & 230.49 & 215.93 & 213.50 & 211.48 & 88.14 & 57.55 & 296.75 & 261.50 & 0.2750 & 5.5461 \\
\hline 89.5450 & 231.40 & 216.24 & 213.32 & 211.41 & 89.82 & 57.76 & 295.12 & 260.96 & 0.2736 & 5.4933 \\
\hline 90.0457 & 230.11 & 216.44 & 213.07 & 211.27 & 91.91 & 57.99 & 294.18 & 259.85 & 0.2756 & 5.4769 \\
\hline 90.5447 & 229.15 & 215.20 & 212.77 & 211.11 & 94.31 & 58.21 & 291.56 & 260.75 & 0.2737 & 5.5289 \\
\hline 91.0443 & 230.65 & 214.62 & 212.41 & 210.91 & 97.19 & 58.42 & 289.48 & 260.90 & 0.2761 & 5.5554 \\
\hline 91.5442 & 230.14 & 214.71 & 212.00 & 210.66 & 100.55 & 58.61 & 288.61 & 260.85 & 0.5224 & 5.5324 \\
\hline 92.0450 & 230.21 & 214.05 & 211.63 & 210.41 & 104.57 & 58.76 & 287.05 & 260.93 & 0.4493 & 5.5169 \\
\hline 92.5448 & 230.78 & 213.98 & 211.31 & 210.15 & 109.40 & 58.93 & 285.41 & 261.67 & 0.2822 & 5.5326 \\
\hline 93.0465 & 228.99 & 213.74 & 211.04 & 209.88 & 114.86 & 59.07 & 283.95 & 261.55 & 0.2464 & 5.5466 \\
\hline 93.5453 & 229.84 & 213.26 & 210.83 & 209.61 & 120.60 & 59.20 & 283.09 & 261.00 & 0.2663 & 5.5177 \\
\hline 94.0443 & 225.79 & 214.07 & 210.83 & 209.45 & 127.23 & 59.37 & 285.14 & 261.86 & 0.2614 & 5.4827 \\
\hline 94.5442 & 228.93 & 214.27 & 211.07 & 209.35 & 134.85 & 59.53 & 286.48 & 261.53 & 0.2781 & 5.5024 \\
\hline 95.0448 & 230.42 & 214.49 & 211.18 & 209.37 & 142.90 & 59.74 & 285.66 & 261.29 & 0.2562 & 5.5204 \\
\hline 95.5447 & 229.56 & 215.31 & 211.55 & 209.55 & 151.80 & 59.97 & 290.17 & 261.25 & 0.3173 & 5.4880 \\
\hline 96.0453 & 229.88 & 216.23 & 212.00 & 209.78 & 162.26 & 60.24 & 289.20 & 261.69 & 0.2653 & 5.4913 \\
\hline 96.5443 & 229.03 & 216.96 & 212.58 & 210.11 & 171.27 & 60.54 & 291.00 & 261.47 & 0.2577 & 5.5281 \\
\hline 97.0450 & 230.46 & 216.07 & 212.76 & 210.52 & 177.35 & 60.83 & 288.80 & 261.14 & 0.2496 & 5.5377 \\
\hline 97.5440 & 230.82 & 216.05 & 212.49 & 210.81 & 182.09 & 61.06 & 287.56 & 260.63 & 0.3234 & 5.5163 \\
\hline 98.0457 & 230.12 & 215.91 & 212.40 & 210.88 & 186.78 & 61.32 & 285.91 & 261.75 & 0.2799 & 5.4883 \\
\hline 98.5453 & 229.93 & 214.98 & 212.32 & 210.87 & 190.13 & 61.67 & 287.13 & 261.81 & 0.2598 & 5.5016 \\
\hline 99.0452 & 228.80 & 215.25 & 212.12 & 210.87 & 192.73 & 61.97 & 285.26 & 261.83 & 0.2596 & 5.5337 \\
\hline 99.5442 & 229.88 & 214.82 & 211.98 & 210.82 & 195.28 & 62.26 & 285.43 & 261.18 & 0.3226 & 5.5124 \\
\hline
\end{tabular}


TABLE B4.- CONTINUED.

\begin{tabular}{|c|c|c|c|c|c|c|c|c|c|c|}
\hline $\begin{array}{l}\text { Time, } \\
\text { min }\end{array}$ & $\begin{array}{c}\text { T-steam } \\
{ }^{\circ} \mathrm{C}\end{array}$ & $\begin{array}{l}\mathrm{T} 1, \\
{ }^{\circ} \mathrm{C}\end{array}$ & $\begin{array}{l}\mathrm{T} 2, \\
{ }^{\circ} \mathrm{C}\end{array}$ & $\begin{array}{l}\mathrm{T} 3, \\
{ }^{\circ} \mathrm{C}\end{array}$ & $\begin{array}{l}\mathrm{T4}, \\
{ }^{\circ} \mathrm{C}\end{array}$ & $\begin{array}{l}\mathrm{T} 5, \\
{ }^{\circ} \mathrm{C}\end{array}$ & $\begin{array}{l}\text { Pinj, } \\
\text { psig }\end{array}$ & $\begin{array}{l}\text { Pout, } \\
\text { psig }\end{array}$ & $\begin{array}{l}\text { Qinj, } \\
\text { g/min }\end{array}$ & $\begin{array}{c}\text { Vw } \\
\mathrm{ml} / \mathrm{min}\end{array}$ \\
\hline 100.0448 & 231.33 & 214.63 & 211.91 & 210.66 & 197.86 & 62.58 & 284.14 & 261.84 & 0.3184 & 5.4969 \\
\hline 100.5447 & 230.08 & 214.94 & 211.75 & 210.55 & 199.72 & 62.90 & 285.27 & 261.59 & 0.2620 & 5.5023 \\
\hline 101.0455 & 230.64 & 214.74 & 211.61 & 210.43 & 201.42 & 63.23 & 283.74 & 261.91 & 0.2512 & 5.5344 \\
\hline 101.5443 & 231.19 & 214.42 & 211.40 & 210.25 & 202.66 & 63.53 & 283.54 & 261.26 & 0.3140 & 5.5250 \\
\hline 102.0450 & 229.17 & 213.62 & 211.15 & 210.04 & 203.47 & 63.80 & 281.89 & 261.08 & 0.3177 & 5.4646 \\
\hline 102.5448 & 229.51 & 213.17 & 210.93 & 209.82 & 204.26 & 63.99 & 280.33 & 261.27 & 0.2523 & 5.5107 \\
\hline 103.0457 & 231.32 & 212.49 & 210.70 & 209.61 & 204.76 & 64.18 & 278.11 & 261.18 & 0.2499 & 5.5451 \\
\hline 103.5455 & 231.02 & 212.92 & 210.42 & 209.43 & 205.17 & 64.39 & 276.86 & 262.07 & 0.2536 & 5.5104 \\
\hline 104.0453 & 231.24 & 212.93 & 210.17 & 209.22 & 205.36 & 64.52 & 275.86 & 260.30 & 0.3099 & 5.4992 \\
\hline 104.5452 & 229.79 & 213.88 & 210.13 & 209.03 & 205.52 & 64.66 & 275.38 & 260.93 & 0.3666 & 5.5209 \\
\hline 105.0440 & 228.39 & 213.98 & 210.15 & 208.87 & 205.61 & 64.81 & 278.40 & 261.59 & 0.2936 & 5.5597 \\
\hline 105.5457 & 229.58 & 213.90 & 210.76 & 208.94 & 205.97 & 65.09 & 277.33 & 261.74 & 0.2109 & 5.5236 \\
\hline 106.0455 & 230.06 & 213.99 & 210.89 & 209.23 & 206.15 & 65.38 & 279.15 & 261.25 & 0.2236 & 5.4846 \\
\hline 106.5453 & 229.08 & 214.08 & 210.84 & 209.53 & 206.44 & 65.76 & 281.63 & 261.70 & 0.2242 & 5.5056 \\
\hline 107.0442 & 228.99 & 215.60 & 211.27 & 209.61 & 207.17 & 66.16 & 281.79 & 261.55 & 0.2803 & 5.5332 \\
\hline 107.5450 & 228.62 & 216.02 & 211.88 & 209.86 & 207.91 & 66.69 & 283.36 & 260.99 & 0.2565 & 5.5292 \\
\hline 108.0457 & 229.50 & 216.14 & 212.31 & 210.34 & 208.23 & 67.35 & 283.43 & 261.52 & 0.2733 & 5.4937 \\
\hline 108.5465 & 229.37 & 215.77 & 212.35 & 210.76 & 208.83 & 67.90 & 282.65 & 261.41 & 0.2755 & 5.5123 \\
\hline 109.0462 & 229.61 & 215.34 & 212.10 & 210.89 & 209.56 & 68.45 & 282.78 & 261.61 & 0.2817 & 5.5363 \\
\hline 109.5452 & 231.54 & 215.43 & 212.14 & 210.90 & 210.01 & 69.36 & 281.47 & 261.56 & 0.2771 & 5.5190 \\
\hline 110.0450 & 230.99 & 214.92 & 211.89 & 210.87 & 210.12 & 70.58 & 280.70 & 260.84 & 0.2752 & 5.4681 \\
\hline 110.5448 & 229.50 & 215.15 & 211.68 & 210.77 & 210.18 & 71.70 & 279.49 & 259.34 & 0.2755 & 5.5140 \\
\hline 111.0455 & 231.91 & 215.53 & 212.04 & 210.68 & 210.21 & 72.77 & 280.39 & 261.57 & 0.2833 & 5.5234 \\
\hline 111.5453 & 229.90 & 215.05 & 211.72 & 210.73 & 210.18 & 73.79 & 280.01 & 261.60 & 0.2754 & 5.5135 \\
\hline 112.0452 & 229.28 & 214.30 & 211.65 & 210.75 & 210.34 & 74.77 & 281.63 & 261.69 & 0.2756 & 5.4916 \\
\hline 112.5440 & 229.24 & 213.67 & 211.45 & 210.70 & 210.50 & 76.22 & 282.74 & 262.03 & 0.2757 & 5.5275 \\
\hline 113.0438 & 230.82 & 213.46 & 211.06 & 210.56 & 210.56 & 78.11 & 281.03 & 261.90 & 0.2755 & 5.5363 \\
\hline 113.5455 & 230.31 & 213.34 & 210.67 & 210.33 & 210.49 & 80.18 & 281.92 & 262.21 & 0.2739 & 5.5159 \\
\hline 114.0453 & 231.38 & 213.27 & 210.35 & 209.96 & 210.26 & 82.43 & 284.69 & 262.24 & 0.2757 & 5.4922 \\
\hline 114.5452 & 232.92 & 213.75 & 210.12 & 209.58 & 209.96 & 84.73 & 283.02 & 262.71 & 0.2761 & 5.5071 \\
\hline 115.0450 & 230.85 & 213.16 & 209.89 & 209.19 & 209.58 & 87.01 & 281.45 & 262.75 & 0.2756 & 5.5300 \\
\hline 115.5448 & 230.08 & 213.38 & 209.64 & 208.85 & 209.21 & 89.32 & 281.57 & 262.89 & 0.2757 & 5.5146 \\
\hline 116.0455 & 230.03 & 213.97 & 209.67 & 208.60 & 208.91 & 92.43 & 280.12 & 260.84 & 0.2758 & 5.4806 \\
\hline 116.5453 & 229.60 & 213.31 & 209.96 & 208.30 & 208.48 & 95.84 & 278.97 & 260.59 & 0.2748 & 5.5214 \\
\hline 117.0452 & 229.31 & 213.81 & 209.70 & 208.37 & 208.19 & 98.47 & 280.98 & 261.02 & 0.2757 & 5.5061 \\
\hline 117.5450 & 230.68 & 214.92 & 209.81 & 208.37 & 208.00 & 101.55 & 279.72 & 261.24 & 0.2742 & 5.4585 \\
\hline 118.0448 & 230.60 & 215.34 & 209.74 & 208.36 & 208.00 & 104.13 & 276.54 & 257.92 & 0.2764 & 5.4928 \\
\hline 118.5447 & 228.55 & 214.96 & 209.49 & 208.25 & 207.89 & 107.75 & 275.05 & 259.61 & 0.2474 & 5.5360 \\
\hline 119.0445 & 229.68 & 213.91 & 209.29 & 208.15 & 207.91 & 108.73 & 275.73 & 262.36 & 0.2708 & 5.5252 \\
\hline 119.5453 & 231.08 & 213.73 & 209.11 & 208.13 & 207.97 & 108.97 & 272.25 & 261.98 & 0.2763 & 5.5079 \\
\hline 120.0460 & 230.65 & 213.34 & 209.01 & 208.01 & 207.97 & 109.56 & 271.53 & 261.25 & 0.2759 & 5.4947 \\
\hline 120.5448 & 228.48 & 212.71 & 208.89 & 207.94 & 207.92 & 110.17 & 271.55 & 261.84 & 0.2764 & 5.5461 \\
\hline 121.0447 & 227.86 & 212.86 & 208.99 & 207.85 & 207.87 & 110.65 & 274.87 & 261.22 & 0.2756 & 5.5146 \\
\hline 121.5455 & 228.31 & 213.90 & 209.57 & 207.89 & 207.85 & 112.90 & 273.06 & 260.05 & 0.2739 & 5.4827 \\
\hline 122.0453 & 229.74 & 214.21 & 209.73 & 208.14 & 207.82 & 114.89 & 274.33 & 262.42 & 0.2755 & 5.4898 \\
\hline 122.5452 & 229.60 & 212.94 & 209.59 & 208.59 & 207.87 & 116.97 & 276.02 & 262.38 & 0.2750 & 5.5274 \\
\hline 123.0450 & 230.34 & 213.74 & 209.50 & 208.64 & 208.11 & 119.96 & 275.17 & 262.55 & 0.2753 & 5.5459 \\
\hline 123.5457 & 228.48 & 213.12 & 209.54 & 208.54 & 208.45 & 123.39 & 276.79 & 262.72 & 0.2752 & 5.5088 \\
\hline 124.0455 & 229.84 & 212.92 & 209.65 & 208.43 & 208.50 & 127.20 & 276.55 & 262.88 & 0.2755 & 5.4988 \\
\hline 124.5453 & 229.64 & 213.62 & 209.83 & 208.49 & 208.51 & 131.64 & 276.66 & 263.07 & 0.2754 & 5.5161 \\
\hline
\end{tabular}


TABLE B4.- CONTINUED.

\begin{tabular}{|c|c|c|c|c|c|c|c|c|c|c|}
\hline $\begin{array}{c}\text { Time, } \\
\text { min }\end{array}$ & $\begin{array}{c}\text { T-steam } \\
{ }^{0} \mathrm{C}\end{array}$ & $\begin{array}{l}\mathrm{T} 1, \\
{ }^{\circ} \mathrm{C}\end{array}$ & $\begin{array}{l}\mathrm{T} 2, \\
{ }^{\circ} \mathrm{C}\end{array}$ & $\begin{array}{l}\mathrm{T} 3, \\
{ }^{\circ} \mathrm{C}\end{array}$ & $\begin{array}{l}\mathrm{T} 4, \\
{ }^{\circ} \mathrm{C}\end{array}$ & $\begin{array}{l}\mathrm{T} 5, \\
{ }^{\circ} \mathrm{C}\end{array}$ & $\begin{array}{l}\text { Pinj, } \\
\text { psig }\end{array}$ & $\begin{array}{l}\text { Pout, } \\
\text { psig }\end{array}$ & $\begin{array}{l}\text { Qinj, } \\
\text { g/min }\end{array}$ & $\begin{array}{c}\text { Vw } \\
\mathrm{ml} / \mathrm{min}\end{array}$ \\
\hline 125.0452 & 229.61 & 214.27 & 209.89 & 208.58 & 208.42 & 138.43 & 278.17 & 261.25 & 0.2755 & 5.5095 \\
\hline 125.5450 & 229.74 & 214.24 & 209.78 & 208.62 & 208.37 & 147.15 & 276.96 & 260.70 & 0.2748 & 5.4951 \\
\hline 126.0457 & 229.42 & 214.15 & 209.60 & 208.57 & 208.40 & 155.26 & 277.02 & 261.44 & 0.2749 & 5.5080 \\
\hline 126.5455 & 229.24 & 214.17 & 209.53 & 208.46 & 208.42 & 161.96 & 277.56 & 261.59 & 0.2750 & 5.5516 \\
\hline 127.0453 & 228.04 & 213.27 & 209.44 & 208.33 & 208.37 & 167.98 & 276.02 & 261.44 & 0.2750 & 5.5519 \\
\hline 127.5452 & 230.17 & 213.42 & 209.29 & 208.25 & 208.27 & 172.78 & 274.44 & 261.37 & 0.2750 & 5.5031 \\
\hline 128.0450 & 229.80 & 213.37 & 209.13 & 208.14 & 208.16 & 177.21 & 274.87 & 260.68 & 0.2750 & 5.4943 \\
\hline 128.5457 & 230.52 & 213.07 & 208.95 & 208.00 & 208.07 & 180.77 & 273.74 & 260.88 & 0.2754 & 5.5116 \\
\hline 129.0455 & 229.79 & 213.23 & 208.81 & 207.88 & 207.99 & 184.34 & 273.71 & 261.59 & 0.2750 & 5.5146 \\
\hline 129.5453 & 230.29 & 212.68 & 208.86 & 207.74 & 207.86 & 187.25 & 272.23 & 259.93 & 0.2754 & 5.4914 \\
\hline 130.0452 & 230.38 & 212.91 & 208.74 & 207.61 & 207.74 & 188.83 & 273.49 & 261.77 & 0.2756 & 5.4956 \\
\hline 130.5450 & 229.47 & 213.47 & 208.71 & 207.65 & 207.69 & 190.62 & 274.51 & 261.70 & 0.2690 & 5.5350 \\
\hline 131.0438 & 229.92 & 214.18 & 208.87 & 207.58 & 207.60 & 192.58 & 273.87 & 260.69 & 0.2754 & 5.5440 \\
\hline 131.5455 & 228.81 & 213.69 & 208.92 & 207.53 & 207.51 & 194.17 & 272.26 & 262.08 & 0.2753 & 5.4963 \\
\hline 132.0445 & 229.44 & 213.53 & 208.77 & 207.64 & 207.48 & 194.59 & 271.40 & 262.24 & 0.7089 & 5.4971 \\
\hline 132.5443 & 229.33 & 213.17 & 208.73 & 207.69 & 207.44 & 195.13 & 272.79 & 260.93 & 0.6741 & 5.5127 \\
\hline 133.0450 & 229.87 & 213.16 & 208.72 & 207.55 & 207.42 & 196.54 & 270.05 & 262.09 & 0.6035 & 5.5239 \\
\hline 133.5448 & 230.70 & 213.21 & 208.67 & 207.57 & 207.52 & 196.24 & 269.88 & 262.44 & 0.5530 & 5.4878 \\
\hline 134.0447 & 229.11 & 212.80 & 208.63 & 207.56 & 207.49 & 195.85 & 268.75 & 262.39 & 0.5201 & 5.4818 \\
\hline 134.5453 & 229.74 & 213.16 & 208.78 & 207.52 & 207.44 & 195.30 & 268.15 & 262.35 & 0.4827 & 5.5093 \\
\hline 135.0452 & 229.42 & 213.15 & 209.03 & 207.47 & 207.40 & 194.53 & 266.98 & 261.93 & 0.4370 & 5.5072 \\
\hline 135.5450 & 230.31 & 213.20 & 209.00 & 207.55 & 207.31 & 193.45 & 265.63 & 261.38 & 0.3893 & 5.5025 \\
\hline 136.0448 & 229.65 & 213.10 & 209.30 & 207.75 & 207.26 & 192.11 & 265.69 & 261.18 & 0.3436 & 5.5155 \\
\hline 136.5447 & 230.35 & 213.51 & 209.72 & 208.11 & 207.25 & 191.02 & 266.66 & 261.04 & 0.2970 & 5.5449 \\
\hline 137.0445 & 231.77 & 214.17 & 209.84 & 208.72 & 207.27 & 189.97 & 266.64 & 258.64 & 0.2649 & 5.5356 \\
\hline 137.5453 & 230.75 & 214.37 & 209.86 & 208.84 & 207.54 & 191.44 & 266.60 & 258.05 & 0.2605 & 5.4913 \\
\hline 138.0442 & 229.71 & 213.37 & 209.85 & 208.92 & 208.68 & 191.42 & 267.14 & 258.09 & 0.2669 & 5.4939 \\
\hline 138.5440 & 229.84 & 214.64 & 210.08 & 209.08 & 209.15 & 190.94 & 268.51 & 258.05 & 0.2555 & 5.5640 \\
\hline 139.0457 & 231.65 & 215.00 & 210.15 & 209.17 & 209.33 & 191.01 & 268.09 & 257.84 & 0.2760 & 5.5124 \\
\hline 139.5445 & 231.33 & 215.24 & 210.17 & 209.26 & 209.49 & 190.91 & 268.36 & 258.35 & 0.2765 & 5.4732 \\
\hline 140.0462 & 230.04 & 214.10 & 210.26 & 209.41 & 209.76 & 190.50 & 269.41 & 259.10 & 0.2750 & 5.5264 \\
\hline 140.5452 & 230.06 & 215.73 & 210.91 & 209.89 & 210.14 & 190.73 & 275.77 & 261.87 & 0.2756 & 5.5390 \\
\hline 141.0450 & 232.89 & 214.87 & 210.77 & 210.36 & 210.77 & 193.76 & 276.22 & 263.39 & 0.2746 & 5.5140 \\
\hline 141.5457 & 231.66 & 214.35 & 210.40 & 210.29 & 210.95 & 205.69 & 279.76 & 263.48 & 0.2752 & 5.4901 \\
\hline 142.0455 & 232.42 & 214.23 & 209.92 & 209.65 & 210.33 & 208.79 & 279.08 & 262.24 & 0.2759 & 5.4811 \\
\hline 142.5453 & 231.02 & 214.28 & 209.42 & 208.92 & 209.56 & 208.70 & 278.49 & 261.19 & 0.2748 & 5.5366 \\
\hline 143.0452 & 230.56 & 213.82 & 209.02 & 208.27 & 208.92 & 208.31 & 274.12 & 260.92 & 0.2759 & 5.5237 \\
\hline 143.5450 & 229.86 & 213.82 & 208.69 & 207.88 & 208.47 & 207.99 & 273.58 & 260.99 & 0.2757 & 5.4938 \\
\hline 144.0457 & 230.38 & 214.20 & 208.56 & 207.54 & 208.04 & 207.67 & 272.92 & 261.17 & 0.2761 & 5.5049 \\
\hline 144.5455 & 231.15 & 213.95 & 208.28 & 207.39 & 207.80 & 207.46 & 269.98 & 260.39 & 0.2753 & 5.5217 \\
\hline 145.0453 & 230.44 & 214.46 & 207.91 & 207.26 & 207.62 & 207.23 & 271.21 & 260.16 & 0.2731 & 5.5274 \\
\hline 145.5442 & 230.34 & 213.17 & 207.82 & 207.00 & 207.41 & 206.96 & 269.69 & 259.37 & 0.2759 & 5.4866 \\
\hline 146.0450 & 230.64 & 213.10 & 207.70 & 206.75 & 207.25 & 206.80 & 269.21 & 259.59 & 0.2752 & 5.4966 \\
\hline 146.5448 & 231.15 & 212.67 & 207.72 & 206.70 & 207.13 & 206.71 & 268.14 & 259.38 & 0.2746 & 5.5287 \\
\hline 147.0455 & 230.45 & 212.89 & 207.70 & 206.72 & 207.04 & 206.66 & 266.83 & 261.30 & 0.2748 & 5.5491 \\
\hline 147.5453 & 229.20 & 213.00 & 207.72 & 206.68 & 206.90 & 206.47 & 268.62 & 259.97 & 0.2761 & 5.5010 \\
\hline 148.0452 & 230.65 & 212.72 & 207.79 & 206.74 & 206.90 & 206.40 & 266.82 & 261.88 & 0.7082 & 5.4835 \\
\hline 148.5440 & 230.69 & 212.85 & 207.82 & 206.78 & 206.88 & 206.28 & 268.61 & 261.42 & 0.7089 & 5.5349 \\
\hline 149.0448 & 231.23 & 212.61 & 207.82 & 206.74 & 206.85 & 206.14 & 267.38 & 261.22 & 0.7089 & 5.5142 \\
\hline 149.5455 & 231.14 & 212.63 & 207.61 & 206.76 & 206.80 & 205.99 & 268.02 & 260.30 & 0.7089 & 5.4883 \\
\hline
\end{tabular}


TABLE B4.- CONTINUED.

\begin{tabular}{|c|c|c|c|c|c|c|c|c|c|c|}
\hline $\begin{array}{l}\text { Time, } \\
\text { min }\end{array}$ & $\begin{array}{c}\text { T-steam } \\
{ }^{\circ} \mathrm{C}\end{array}$ & $\begin{array}{l}\mathrm{T} 1, \\
{ }^{\circ} \mathrm{C}\end{array}$ & $\begin{array}{l}\mathrm{T} 2, \\
{ }^{\circ} \mathrm{C}\end{array}$ & $\begin{array}{l}\mathrm{T} 3, \\
{ }^{\circ} \mathrm{C}\end{array}$ & $\begin{array}{l}\mathrm{T} 4, \\
{ }^{\circ} \mathrm{C}\end{array}$ & $\begin{array}{l}\mathrm{T} 5, \\
{ }^{\circ} \mathrm{C}\end{array}$ & $\begin{array}{l}\text { Pinj, } \\
\text { psig }\end{array}$ & $\begin{array}{l}\text { Pout, } \\
\text { psig }\end{array}$ & $\begin{array}{l}\text { Qinj, } \\
\text { g/min }\end{array}$ & $\begin{array}{c}\mathrm{Vw} \\
\mathrm{ml} / \mathrm{min}\end{array}$ \\
\hline 150.0453 & 233.04 & 212.83 & 207.43 & 206.73 & 206.82 & 205.91 & 265.17 & 261.31 & 0.7089 & 5.4981 \\
\hline 150.5452 & 230.93 & 213.10 & 207.27 & 206.68 & 206.84 & 205.82 & 264.34 & 260.61 & 0.7089 & 5.5453 \\
\hline 151.0442 & 229.90 & 213.18 & 207.56 & 206.52 & 206.73 & 205.66 & 263.86 & 257.76 & 0.7089 & 5.5489 \\
\hline 151.5440 & 230.18 & 213.41 & 208.51 & 206.31 & 206.65 & 205.52 & 263.11 & 259.26 & 0.7089 & 5.5279 \\
\hline 152.0455 & 231.01 & 213.83 & 208.73 & 206.35 & 206.63 & 205.34 & 262.50 & 260.18 & 0.7089 & 5.5051 \\
\hline 152.5453 & 230.17 & 213.43 & 209.07 & 206.99 & 206.58 & 205.20 & 263.22 & 259.83 & 0.7089 & 5.5130 \\
\hline 153.0443 & 230.33 & 213.71 & 209.30 & 208.09 & 206.53 & 205.01 & 263.76 & 260.78 & 0.3743 & 5.5289 \\
\hline 153.5460 & 229.96 & 213.78 & 209.45 & 208.66 & 206.48 & 204.78 & 264.83 & 260.34 & 0.0835 & 5.5202 \\
\hline 154.0448 & 230.45 & 213.19 & 209.59 & 208.91 & 206.39 & 204.55 & 265.40 & 261.64 & 0.4481 & 5.5108 \\
\hline 154.5457 & 229.14 & 213.18 & 209.72 & 208.97 & 207.13 & 204.44 & 265.42 & 261.39 & 0.4510 & 5.5236 \\
\hline 155.0455 & 230.13 & 213.79 & 209.69 & 209.04 & 209.04 & 204.25 & 265.71 & 261.48 & 0.2803 & 5.5408 \\
\hline 155.5453 & 231.55 & 213.88 & 209.78 & 209.24 & 209.64 & 203.89 & 266.83 & 261.88 & 0.2600 & 5.5434 \\
\hline 156.0450 & 232.30 & 214.56 & 209.92 & 209.33 & 209.84 & 203.66 & 267.10 & 261.93 & 0.6457 & 5.4764 \\
\hline 156.5458 & 231.41 & 214.31 & 210.03 & 209.39 & 209.89 & 203.48 & 267.97 & 261.94 & 0.1507 & 5.4920 \\
\hline 157.0465 & 232.29 & 214.35 & 210.14 & 209.47 & 209.95 & 203.29 & 268.60 & 262.20 & 0.0350 & 5.5449 \\
\hline 157.5455 & 231.73 & 214.80 & 210.20 & 209.50 & 210.00 & 203.11 & 268.37 & 261.88 & 0.1877 & 5.5389 \\
\hline 158.0443 & 231.41 & 214.61 & 210.24 & 209.54 & 210.04 & 202.95 & 268.38 & 261.89 & 0.5007 & 5.5095 \\
\hline 158.5452 & 231.31 & 214.72 & 210.24 & 209.56 & 210.10 & 203.51 & 268.30 & 261.91 & 0.7088 & 5.5076 \\
\hline 159.0440 & 231.53 & 214.97 & 210.26 & 209.53 & 210.00 & 208.46 & 268.94 & 259.21 & 0.5666 & 5.5375 \\
\hline 159.5447 & 231.35 & 214.95 & 210.21 & 209.46 & 209.92 & 209.23 & 268.05 & 259.55 & 0.3953 & 5.5314 \\
\hline 160.0455 & 231.94 & 214.88 & 210.14 & 209.44 & 209.98 & 209.52 & 267.62 & 261.04 & 0.2653 & 5.4837 \\
\hline 160.5443 & 231.75 & 215.26 & 210.14 & 209.56 & 210.13 & 209.71 & 268.10 & 262.30 & 0.2670 & 5.5080 \\
\hline 161.0452 & 232.27 & 215.18 & 210.27 & 209.65 & 210.24 & 209.83 & 268.61 & 262.56 & 0.2686 & 5.5443 \\
\hline 161.5440 & 229.62 & 214.96 & 210.40 & 209.76 & 210.29 & 209.79 & 270.09 & 260.06 & 0.2805 & 5.5390 \\
\hline 162.0457 & 229.05 & 214.20 & 210.39 & 209.62 & 210.10 & 209.63 & 268.99 & 260.44 & 0.2170 & 5.5197 \\
\hline 162.5455 & 231.26 & 214.70 & 210.30 & 209.62 & 210.14 & 209.73 & 268.64 & 260.02 & 0.2700 & 5.4877 \\
\hline 163.0443 & 232.19 & 215.33 & 210.28 & 209.60 & 210.12 & 209.71 & 268.67 & 259.85 & 0.2743 & 5.5055 \\
\hline 163.5452 & 231.42 & 215.87 & 210.30 & 209.57 & 210.09 & 209.70 & 268.60 & 259.51 & 0.2741 & 5.5091 \\
\hline 164.0440 & 229.71 & 215.46 & 210.36 & 209.61 & 210.13 & 209.75 & 269.13 & 260.63 & 0.2749 & 5.4898 \\
\hline 164.5438 & 229.94 & 215.25 & 210.40 & 209.68 & 210.18 & 209.81 & 269.72 & 261.15 & 0.2733 & 5.5009 \\
\hline 165.0455 & 231.59 & 215.47 & 210.53 & 209.79 & 210.29 & 209.94 & 270.08 & 261.80 & 0.2585 & 5.5054 \\
\hline 165.5453 & 230.92 & 215.68 & 210.87 & 210.03 & 210.44 & 210.06 & 274.43 & 262.07 & 0.2362 & 5.5493 \\
\hline 166.0443 & 231.58 & 215.79 & 210.58 & 210.12 & 210.64 & 210.23 & 276.86 & 260.71 & 0.2136 & 5.5438 \\
\hline 166.5450 & 231.28 & 215.54 & 210.09 & 209.44 & 209.96 & 209.58 & 275.23 & 261.15 & 0.2078 & 5.4935 \\
\hline 167.0448 & 232.14 & 215.12 & 209.64 & 209.03 & 209.73 & 209.53 & 272.78 & 262.90 & 0.1892 & 5.4883 \\
\hline 167.5447 & 230.82 & 214.44 & 209.13 & 208.64 & 209.30 & 208.96 & 275.67 & 257.68 & 0.1805 & 5.5172 \\
\hline 168.0453 & 231.84 & 214.91 & 208.91 & 208.11 & 208.81 & 208.65 & 268.50 & 261.56 & 0.1697 & 5.5358 \\
\hline 168.5452 & 229.59 & 214.12 & 208.84 & 207.93 & 208.49 & 208.16 & 270.42 & 257.53 & 0.1653 & 5.4964 \\
\hline 169.0450 & 230.74 & 213.39 & 208.54 & 207.74 & 208.17 & 208.01 & 266.44 & 259.93 & 0.1594 & 5.5109 \\
\hline 169.5448 & 232.15 & 213.88 & 208.38 & 207.79 & 208.19 & 207.97 & 266.75 & 261.10 & 0.1464 & 5.5350 \\
\hline 170.0455 & 229.88 & 213.43 & 208.49 & 207.67 & 208.14 & 207.72 & 268.93 & 259.69 & 0.1358 & 5.5281 \\
\hline 170.5463 & 231.01 & 213.40 & 208.62 & 207.58 & 208.07 & 207.73 & 265.75 & 259.56 & 0.1310 & 5.5188 \\
\hline 171.0470 & 229.55 & 213.11 & 208.59 & 207.73 & 208.03 & 207.78 & 268.96 & 261.49 & 0.1254 & 5.5026 \\
\hline 171.5460 & 229.39 & 213.67 & 208.97 & 208.00 & 208.16 & 207.82 & 267.29 & 262.36 & 0.1218 & 5.5238 \\
\hline 172.0458 & 229.75 & 213.66 & 208.52 & 207.81 & 207.95 & 207.38 & 264.57 & 258.69 & 0.1183 & 5.5269 \\
\hline 172.5465 & 230.23 & 213.86 & 208.86 & 207.90 & 208.22 & 207.54 & 266.63 & 261.23 & 0.1117 & 5.5165 \\
\hline 173.0463 & 230.13 & 212.91 & 208.94 & 208.04 & 208.45 & 207.65 & 266.55 & 262.11 & 0.1097 & 5.5108 \\
\hline 173.5470 & 229.51 & 213.32 & 209.03 & 208.39 & 208.55 & 207.74 & 268.15 & 262.68 & 0.1040 & 5.5082 \\
\hline 174.0460 & 232.02 & 213.95 & 209.37 & 208.44 & 208.64 & 207.85 & 268.04 & 262.79 & 0.1015 & 5.5408 \\
\hline 174.5458 & 230.57 & 213.99 & 209.05 & 208.37 & 208.50 & 207.64 & 266.40 & 257.48 & 0.0953 & 5.5128 \\
\hline
\end{tabular}


TABLE B4.- CONTINUED.

\begin{tabular}{|c|c|c|c|c|c|c|c|c|c|c|}
\hline $\begin{array}{c}\text { Time, } \\
\text { min }\end{array}$ & $\begin{array}{c}\text { T-steam } \\
{ }^{0} \mathrm{C}\end{array}$ & $\begin{array}{l}\mathrm{T} 1, \\
{ }^{\circ} \mathrm{C}\end{array}$ & $\begin{array}{l}\mathrm{T} 2, \\
{ }^{\circ} \mathrm{C}\end{array}$ & $\begin{array}{l}\mathrm{T} 3, \\
{ }^{\circ} \mathrm{C}\end{array}$ & $\begin{array}{l}\mathrm{T} 4, \\
{ }^{\circ} \mathrm{C}\end{array}$ & $\begin{array}{l}\mathrm{T} 5, \\
{ }^{\circ} \mathrm{C}\end{array}$ & $\begin{array}{l}\text { Pinj, } \\
\text { psig }\end{array}$ & $\begin{array}{l}\text { Pout, } \\
\text { psig }\end{array}$ & $\begin{array}{l}\text { Qinj, } \\
\text { g/min }\end{array}$ & $\begin{array}{c}\text { Vw } \\
\mathrm{ml} / \mathrm{min}\end{array}$ \\
\hline 175.0465 & 230.07 & 213.37 & 209.06 & 208.02 & 208.43 & 207.71 & 266.60 & 256.81 & 0.0921 & 5.4943 \\
\hline 175.5463 & 232.47 & 214.41 & 208.97 & 208.18 & 208.41 & 207.88 & 264.05 & 257.70 & 0.0896 & 5.5298 \\
\hline 176.0472 & 231.81 & 213.71 & 208.56 & 208.25 & 208.61 & 208.02 & 263.93 & 257.63 & 0.0768 & 5.5553 \\
\hline 176.5452 & 230.18 & 213.63 & 208.56 & 208.04 & 208.76 & 208.10 & 264.09 & 259.30 & 0.2172 & 5.5307 \\
\hline 177.0450 & 228.86 & 213.33 & 208.99 & 207.96 & 208.73 & 208.26 & 263.91 & 259.11 & 0.2701 & 5.4983 \\
\hline 177.5447 & 228.88 & 213.72 & 209.55 & 208.37 & 208.66 & 208.39 & 271.17 & 258.50 & 0.2737 & 5.4955 \\
\hline 178.0455 & 231.11 & 214.98 & 209.54 & 209.02 & 209.05 & 208.48 & 269.73 & 259.84 & 0.3526 & 5.5071 \\
\hline 178.5453 & 230.53 & 214.17 & 209.04 & 208.86 & 209.50 & 208.84 & 272.40 & 261.12 & 0.2553 & 5.5210 \\
\hline 179.0452 & 231.67 & 214.80 & 208.93 & 208.43 & 209.27 & 209.13 & 270.80 & 261.59 & 0.2339 & 5.4865 \\
\hline 179.5450 & 230.67 & 214.02 & 208.38 & 208.20 & 208.97 & 208.95 & 271.72 & 262.84 & 0.3158 & 5.4974 \\
\hline 180.0457 & 229.70 & 213.27 & 208.17 & 207.92 & 208.76 & 208.71 & 270.87 & 263.01 & 0.2697 & 5.5386 \\
\hline 180.5465 & 230.49 & 212.99 & 208.26 & 207.58 & 208.51 & 208.46 & 270.52 & 262.71 & 0.2297 & 5.5453 \\
\hline 181.0443 & 230.92 & 214.33 & 208.35 & 207.17 & 207.87 & 207.69 & 267.37 & 257.02 & 0.2762 & 5.5063 \\
\hline 181.5442 & 230.64 & 214.53 & 208.05 & 206.92 & 207.28 & 207.19 & 264.69 & 256.43 & 0.2493 & 5.4916 \\
\hline 182.0450 & 230.32 & 213.75 & 207.68 & 206.94 & 207.09 & 206.89 & 265.05 & 256.33 & 0.2619 & 5.5152 \\
\hline 182.5448 & 229.79 & 212.26 & 207.56 & 206.80 & 207.16 & 206.71 & 264.29 & 256.18 & 0.3102 & 5.5150 \\
\hline 183.0447 & 229.61 & 213.07 & 207.42 & 206.72 & 207.18 & 206.74 & 263.49 & 256.69 & 0.2754 & 5.4752 \\
\hline 183.5453 & 230.38 & 213.99 & 207.42 & 206.63 & 207.15 & 206.77 & 263.46 & 256.68 & 0.2754 & 5.5020 \\
\hline 184.0452 & 230.35 & 213.18 & 207.44 & 206.56 & 207.06 & 206.74 & 263.35 & 256.59 & 0.2748 & 5.5102 \\
\hline 184.5450 & 230.12 & 212.88 & 207.42 & 206.56 & 206.99 & 206.71 & 263.73 & 256.60 & 0.2752 & 5.5738 \\
\hline 185.0448 & 230.05 & 213.26 & 207.42 & 206.57 & 206.92 & 206.60 & 263.95 & 256.62 & 0.2755 & 5.5311 \\
\hline 185.5455 & 229.82 & 212.29 & 207.52 & 206.57 & 206.91 & 206.57 & 264.28 & 256.78 & 0.2742 & 5.4861 \\
\hline 186.0453 & 229.57 & 212.66 & 207.54 & 206.61 & 206.93 & 206.50 & 264.56 & 256.85 & 0.2771 & 5.5163 \\
\hline 186.5452 & 229.39 & 213.34 & 207.52 & 206.68 & 206.93 & 206.50 & 263.82 & 257.19 & 0.2743 & 5.5182 \\
\hline 187.0450 & 230.26 & 212.75 & 207.49 & 206.70 & 206.97 & 206.45 & 262.66 & 256.99 & 0.2749 & 5.4785 \\
\hline 187.5448 & 229.24 & 212.77 & 207.44 & 206.69 & 207.03 & 206.45 & 263.41 & 257.01 & 0.2762 & 5.4940 \\
\hline 188.0455 & 229.54 & 212.43 & 207.58 & 206.67 & 207.06 & 206.49 & 263.94 & 256.76 & 0.2760 & 5.5232 \\
\hline 188.5453 & 229.58 & 213.08 & 207.50 & 206.73 & 207.07 & 206.53 & 262.96 & 257.24 & 0.2744 & 5.5228 \\
\hline 189.0452 & 229.48 & 212.58 & 207.61 & 206.73 & 207.03 & 206.55 & 262.77 & 256.54 & 0.2742 & 5.5034 \\
\hline 189.5450 & 228.55 & 212.55 & 207.63 & 206.75 & 207.09 & 206.57 & 263.52 & 256.87 & 0.2740 & 5.4956 \\
\hline 190.0448 & 229.21 & 212.08 & 207.58 & 206.79 & 207.09 & 206.57 & 263.87 & 256.38 & 0.2753 & 5.5389 \\
\hline 190.5457 & 229.52 & 212.23 & 207.51 & 206.79 & 207.15 & 206.59 & 262.51 & 256.83 & 0.2788 & 5.5410 \\
\hline 191.0455 & 229.09 & 212.88 & 207.53 & 206.76 & 207.17 & 206.61 & 263.99 & 256.40 & 0.2736 & 5.5140 \\
\hline 191.5462 & 229.95 & 213.24 & 207.64 & 206.72 & 207.15 & 206.63 & 262.13 & 257.01 & 0.2758 & 5.4938 \\
\hline 192.0450 & 228.95 & 212.34 & 207.57 & 206.78 & 207.15 & 206.69 & 262.65 & 256.79 & 0.2760 & 5.5004 \\
\hline 192.5448 & 228.76 & 213.01 & 207.51 & 206.83 & 207.12 & 206.71 & 261.77 & 256.80 & 0.2749 & 5.5138 \\
\hline 193.0457 & 226.78 & 213.78 & 207.93 & 206.80 & 207.14 & 206.68 & 264.42 & 257.16 & 0.2760 & 5.4998 \\
\hline 193.5455 & 229.18 & 213.57 & 208.47 & 206.98 & 207.21 & 206.66 & 262.43 & 257.15 & 0.2745 & 5.4937 \\
\hline 194.0453 & 229.39 & 213.18 & 207.97 & 207.63 & 207.22 & 206.64 & 264.70 & 256.98 & 0.2742 & 5.5346 \\
\hline 194.5452 & 228.61 & 213.23 & 208.04 & 207.51 & 207.67 & 206.68 & 262.97 & 257.35 & 0.2761 & 5.5514 \\
\hline 195.0450 & 230.47 & 213.50 & 208.30 & 207.33 & 207.96 & 206.74 & 263.27 & 257.18 & 0.2759 & 5.5568 \\
\hline 195.5457 & 229.33 & 212.93 & 208.30 & 207.49 & 207.87 & 207.03 & 263.71 & 257.12 & 0.2747 & 5.5151 \\
\hline 196.0455 & 229.92 & 213.80 & 208.32 & 207.59 & 207.89 & 207.37 & 263.37 & 256.69 & 0.2754 & 5.4843 \\
\hline 196.5453 & 229.75 & 214.41 & 208.38 & 207.62 & 208.04 & 207.50 & 263.80 & 257.35 & 0.2754 & 5.5123 \\
\hline 197.0452 & 229.28 & 214.46 & 208.18 & 207.57 & 208.06 & 207.59 & 263.33 & 257.63 & 0.2743 & 5.5099 \\
\hline 197.5450 & 228.64 & 214.14 & 208.11 & 207.50 & 208.06 & 207.70 & 264.72 & 257.74 & 0.2753 & 5.4735 \\
\hline 198.0457 & 228.25 & 214.02 & 207.95 & 207.38 & 208.01 & 207.72 & 264.48 & 257.22 & 0.2879 & 5.5119 \\
\hline 198.5465 & 228.11 & 213.77 & 207.97 & 207.25 & 207.88 & 207.65 & 264.17 & 257.40 & 0.2501 & 5.5168 \\
\hline 199.0453 & 227.80 & 213.52 & 208.01 & 207.11 & 207.74 & 207.54 & 263.90 & 257.55 & 0.2748 & 5.5199 \\
\hline 199.5442 & 227.88 & 213.76 & 207.87 & 207.19 & 207.62 & 207.47 & 264.05 & 257.54 & 0.2972 & 5.4971 \\
\hline
\end{tabular}


TABLE B4.- CONTINUED.

\begin{tabular}{|c|c|c|c|c|c|c|c|c|c|c|}
\hline $\begin{array}{c}\text { Time, } \\
\text { min }\end{array}$ & $\begin{array}{c}\text { T-steam } \\
{ }^{\circ} \mathrm{C}\end{array}$ & $\begin{array}{l}\mathrm{T} 1, \\
{ }^{\circ} \mathrm{C}\end{array}$ & $\begin{array}{l}\mathrm{T} 2, \\
{ }^{\circ} \mathrm{C}\end{array}$ & $\begin{array}{l}\mathrm{T} 3, \\
{ }^{\circ} \mathrm{C}\end{array}$ & $\begin{array}{l}\mathrm{T} 4, \\
{ }^{\circ} \mathrm{C}\end{array}$ & $\begin{array}{l}\mathrm{T} 5, \\
{ }^{\circ} \mathrm{C}\end{array}$ & $\begin{array}{l}\text { Pinj, } \\
\text { psig }\end{array}$ & $\begin{array}{l}\text { Pout, } \\
\text { psig }\end{array}$ & $\begin{array}{l}\text { Qinj, } \\
\text { g/min }\end{array}$ & $\begin{array}{c}\mathrm{Vw} \\
\mathrm{ml} / \mathrm{min}\end{array}$ \\
\hline 200.0450 & 229.04 & 213.08 & 207.78 & 207.17 & 207.58 & 207.37 & 262.74 & 257.59 & 0.2379 & 5.4853 \\
\hline 200.5448 & 227.95 & 212.99 & 207.75 & 207.05 & 207.57 & 207.24 & 261.92 & 257.35 & 0.2080 & 5.5148 \\
\hline 201.0455 & 227.27 & 214.39 & 208.36 & 207.05 & 207.61 & 207.25 & 262.27 & 257.15 & 0.2748 & 5.4981 \\
\hline 201.5453 & 227.31 & 213.91 & 208.48 & 207.23 & 207.55 & 207.18 & 262.25 & 258.06 & 0.2631 & 5.4658 \\
\hline 202.0452 & 226.47 & 213.16 & 208.47 & 207.72 & 207.54 & 207.16 & 262.99 & 257.23 & 0.2752 & 5.5053 \\
\hline 202.5450 & 225.96 & 214.09 & 208.81 & 207.84 & 207.59 & 207.13 & 262.52 & 258.32 & 0.2752 & 5.5434 \\
\hline 203.0448 & 226.32 & 214.15 & 209.06 & 208.08 & 207.97 & 207.09 & 262.97 & 257.64 & 0.2757 & 5.5101 \\
\hline 203.5455 & 226.73 & 214.58 & 209.26 & 208.46 & 208.29 & 207.04 & 263.56 & 257.73 & 0.2756 & 5.4907 \\
\hline 204.0453 & 226.60 & 214.99 & 209.46 & 208.76 & 208.82 & 207.03 & 264.15 & 257.48 & 0.2757 & 5.4970 \\
\hline 204.5452 & 226.75 & 214.22 & 209.78 & 209.00 & 209.32 & 207.12 & 267.55 & 257.64 & 0.2748 & 5.5132 \\
\hline 205.0450 & 228.33 & 214.63 & 209.46 & 209.11 & 209.61 & 208.41 & 267.79 & 258.66 & 0.2750 & 5.5114 \\
\hline 205.5448 & 226.86 & 213.64 & 209.05 & 208.73 & 209.52 & 209.29 & 268.86 & 258.68 & 0.2762 & 5.4878 \\
\hline 206.0455 & 229.53 & 213.26 & 208.95 & 208.34 & 209.18 & 209.11 & 268.01 & 260.03 & 0.2756 & 5.5058 \\
\hline 206.5453 & 229.68 & 214.16 & 208.77 & 208.21 & 208.90 & 208.86 & 268.36 & 259.76 & 0.2761 & 5.5316 \\
\hline 207.0480 & 229.31 & 225.61 & 209.39 & 208.93 & 209.63 & 209.39 & 262.02 & 257.08 & 0.0002 & 5.5039 \\
\hline 207.5478 & 229.96 & 225.27 & 209.07 & 208.59 & 209.32 & 209.07 & 260.07 & 256.45 & 0.0003 & 5.4928 \\
\hline 208.0467 & 230.14 & 225.08 & 208.86 & 208.45 & 209.20 & 209.04 & 259.25 & 256.08 & 0.0003 & 5.5038 \\
\hline 208.5483 & 229.73 & 224.18 & 208.74 & 208.38 & 209.17 & 209.02 & 258.55 & 256.41 & 0.0000 & 5.5442 \\
\hline 209.0473 & 229.36 & 222.75 & 208.74 & 208.44 & 209.24 & 209.12 & 258.80 & 256.20 & 0.0012 & 5.5137 \\
\hline 209.5480 & 229.32 & 222.45 & 208.76 & 208.42 & 209.21 & 209.08 & 258.58 & 256.86 & 0.0002 & 5.4846 \\
\hline 210.0478 & 228.05 & 222.68 & 208.76 & 208.44 & 209.24 & 209.10 & 258.77 & 255.71 & 0.0006 & 5.4985 \\
\hline 210.5477 & 228.13 & 223.01 & 208.76 & 208.44 & 209.26 & 209.12 & 258.83 & 257.05 & 0.0006 & 5.5276 \\
\hline 211.0483 & 229.08 & 222.71 & 208.80 & 208.50 & 209.30 & 209.19 & 258.80 & 257.97 & 0.0006 & 5.4921 \\
\hline 211.5482 & 228.26 & 222.37 & 208.82 & 208.55 & 209.38 & 209.27 & 259.24 & 258.19 & 0.0005 & 5.4592 \\
\hline 212.0480 & 228.67 & 222.17 & 208.89 & 208.64 & 209.43 & 209.32 & 259.90 & 256.68 & 0.0002 & 5.4933 \\
\hline 212.5478 & 228.98 & 221.94 & 208.95 & 208.66 & 209.49 & 209.34 & 259.76 & 257.80 & 0.0007 & 5.5274 \\
\hline 213.0477 & 229.12 & 222.16 & 208.75 & 208.41 & 209.22 & 209.09 & 258.60 & 255.80 & 0.0005 & 5.5008 \\
\hline 213.5475 & 229.47 & 221.80 & 208.72 & 208.51 & 209.33 & 209.12 & 259.00 & 258.18 & 0.0009 & 5.4825 \\
\hline 214.0482 & 228.27 & 222.11 & 208.94 & 208.69 & 209.51 & 209.39 & 259.71 & 258.16 & 0.0004 & 5.4858 \\
\hline 214.5480 & 228.45 & 222.18 & 208.76 & 208.33 & 209.14 & 208.83 & 257.53 & 254.48 & 0.0004 & 5.5287 \\
\hline 215.0478 & 228.34 & 221.29 & 208.44 & 208.12 & 208.96 & 208.80 & 256.41 & 254.74 & 0.0002 & 5.5285 \\
\hline 215.5468 & 228.43 & 220.67 & 208.34 & 208.05 & 208.86 & 208.73 & 256.09 & 254.33 & 0.0007 & 5.4851 \\
\hline 216.0475 & 228.49 & 220.81 & 208.25 & 207.96 & 208.80 & 208.69 & 255.95 & 255.39 & 0.0000 & 5.4729 \\
\hline 216.5483 & 227.97 & 221.53 & 208.29 & 208.03 & 208.88 & 208.79 & 256.30 & 254.49 & 0.0003 & 5.5007 \\
\hline 217.0480 & 226.71 & 222.37 & 208.56 & 208.24 & 208.99 & 208.88 & 257.89 & 255.48 & 0.0008 & 5.4896 \\
\hline 217.5478 & 227.60 & 221.64 & 208.65 & 208.35 & 209.11 & 209.04 & 258.79 & 256.22 & 0.0005 & 5.4701 \\
\hline 218.0468 & 229.31 & 223.22 & 208.77 & 208.42 & 209.19 & 209.04 & 259.11 & 255.83 & 0.0005 & 5.5371 \\
\hline 218.5475 & 230.27 & 222.29 & 208.80 & 208.47 & 209.24 & 209.12 & 258.98 & 256.07 & 0.0007 & 5.5258 \\
\hline 219.0483 & 228.81 & 222.65 & 208.76 & 208.40 & 209.19 & 209.07 & 258.84 & 255.79 & 0.0000 & 5.4804 \\
\hline 219.5482 & 228.70 & 223.33 & 208.75 & 208.37 & 209.16 & 209.00 & 259.04 & 255.65 & 0.0000 & 5.4884 \\
\hline 220.0488 & 228.88 & 223.30 & 208.77 & 208.41 & 209.16 & 209.00 & 258.97 & 255.28 & 0.0000 & 5.4962 \\
\hline 220.5477 & 229.31 & 223.17 & 208.80 & 208.44 & 209.23 & 209.09 & 259.37 & 256.04 & 0.0006 & 5.4575 \\
\hline 221.0485 & 228.83 & 224.96 & 208.86 & 208.46 & 209.22 & 209.07 & 259.47 & 256.37 & 0.0004 & 5.4106 \\
\hline 221.5483 & 229.53 & 225.61 & 208.84 & 208.47 & 209.22 & 209.08 & 259.38 & 256.40 & 0.0002 & 5.4142 \\
\hline 222.0482 & 229.18 & 224.07 & 208.84 & 208.47 & 209.22 & 209.06 & 259.13 & 255.83 & 0.0003 & 5.4235 \\
\hline 222.5480 & 228.96 & 223.52 & 208.77 & 208.42 & 209.20 & 209.10 & 258.66 & 255.75 & 0.0005 & 5.3977 \\
\hline 223.0478 & 229.86 & 223.74 & 208.70 & 208.35 & 209.17 & 209.03 & 258.09 & 255.89 & 0.0005 & 5.3805 \\
\hline 223.5477 & 229.42 & 224.96 & 208.58 & 208.26 & 209.10 & 208.98 & 257.53 & 255.87 & 0.0000 & 5.3764 \\
\hline 224.0483 & 228.61 & 223.38 & 208.53 & 208.26 & 209.10 & 208.98 & 257.22 & 255.46 & 0.0002 & 5.3664 \\
\hline
\end{tabular}


TABLE B4.- CONTINUED.

\begin{tabular}{|c|c|c|c|c|c|c|c|c|c|c|}
\hline $\begin{array}{l}\text { Time, } \\
\text { min }\end{array}$ & $\begin{array}{c}\text { T-steam } \\
{ }^{\circ} \mathrm{C}\end{array}$ & $\begin{array}{l}\mathrm{T} 1, \\
{ }^{\circ} \mathrm{C}\end{array}$ & $\begin{array}{l}\mathrm{T} 2, \\
{ }^{\circ} \mathrm{C}\end{array}$ & $\begin{array}{l}\mathrm{T} 3, \\
{ }^{\circ} \mathrm{C}\end{array}$ & $\begin{array}{l}\mathrm{T} 4, \\
{ }^{\circ} \mathrm{C}\end{array}$ & $\begin{array}{l}\mathrm{T} 5, \\
{ }^{\circ} \mathrm{C}\end{array}$ & $\begin{array}{l}\text { Pinj, } \\
\text { psig }\end{array}$ & $\begin{array}{l}\text { Pout, } \\
\text { psig }\end{array}$ & $\begin{array}{l}\text { Qinj, } \\
\text { g/min }\end{array}$ & $\begin{array}{c}\mathrm{Vw} \\
\mathrm{ml} / \mathrm{min}\end{array}$ \\
\hline 224.5482 & 227.54 & 223.35 & 208.35 & 208.01 & 208.85 & 208.66 & 255.83 & 253.18 & 0.0005 & 5.3153 \\
\hline 225.0470 & 227.60 & 221.92 & 208.16 & 207.89 & 208.73 & 208.62 & 255.28 & 253.53 & 0.0001 & 5.3522 \\
\hline 225.5478 & 227.58 & 221.78 & 208.20 & 207.91 & 208.70 & 208.63 & 255.74 & 253.68 & 0.0001 & 5.3865 \\
\hline 226.0477 & 227.60 & 221.37 & 208.25 & 207.98 & 208.79 & 208.68 & 256.18 & 254.21 & 0.0001 & 5.3563 \\
\hline 226.5483 & 228.08 & 221.66 & 208.29 & 208.00 & 208.81 & 208.72 & 256.10 & 254.77 & 0.0001 & 5.4357 \\
\hline 227.0482 & 228.23 & 222.38 & 208.34 & 208.02 & 208.85 & 208.74 & 256.55 & 254.18 & 0.0005 & 5.3871 \\
\hline 227.5470 & 230.02 & 223.67 & 208.36 & 208.08 & 208.88 & 208.78 & 256.48 & 254.67 & 0.0005 & 5.5498 \\
\hline 228.0478 & 228.52 & 223.06 & 208.39 & 208.10 & 208.89 & 208.78 & 256.79 & 255.10 & 0.0006 & 5.5414 \\
\hline 228.5477 & 228.74 & 221.99 & 208.46 & 208.15 & 208.94 & 208.85 & 257.24 & 254.94 & 0.0001 & 5.4265 \\
\hline 229.0483 & 228.18 & 223.44 & 208.53 & 208.21 & 209.00 & 208.87 & 257.81 & 255.58 & 0.0000 & 5.4533 \\
\hline 229.5482 & 228.72 & 223.98 & 208.53 & 208.21 & 209.02 & 208.91 & 257.98 & 255.81 & 0.0006 & 5.6171 \\
\hline 230.0480 & 228.89 & 223.19 & 208.61 & 208.31 & 209.07 & 208.97 & 257.91 & 255.66 & 0.0006 & 5.5951 \\
\hline 230.5478 & 229.69 & 222.71 & 208.65 & 208.32 & 209.11 & 209.00 & 258.13 & 255.87 & 0.0006 & 5.4239 \\
\hline 231.0468 & 229.09 & 224.09 & 208.67 & 208.34 & 209.15 & 209.01 & 258.37 & 255.24 & 0.0006 & 5.5074 \\
\hline 231.5483 & 229.00 & 223.58 & 208.72 & 208.40 & 209.19 & 209.06 & 258.55 & 255.55 & 0.0006 & 5.5478 \\
\hline 232.0473 & 230.15 & 224.01 & 208.74 & 208.42 & 209.21 & 209.08 & 258.93 & 255.57 & 0.0006 & 5.4782 \\
\hline 232.5480 & 229.90 & 224.40 & 208.72 & 208.44 & 209.26 & 209.24 & 258.97 & 258.27 & 0.0004 & 5.3325 \\
\hline 233.0478 & 228.47 & 223.19 & 208.73 & 208.32 & 209.10 & 208.92 & 258.19 & 255.01 & 0.0003 & 5.5892 \\
\hline 233.5487 & 229.67 & 222.94 & 208.75 & 208.46 & 209.25 & 209.18 & 259.22 & 256.53 & 0.0004 & 5.4976 \\
\hline 234.0485 & 229.96 & 223.23 & 208.89 & 208.62 & 209.43 & 209.36 & 259.78 & 257.44 & 0.0008 & 5.4795 \\
\hline 234.5473 & 229.83 & 223.69 & 209.02 & 208.73 & 209.52 & 209.43 & 260.39 & 257.87 & 0.0005 & 5.4897 \\
\hline 235.0480 & 229.16 & 223.11 & 209.09 & 208.81 & 209.61 & 209.52 & 260.93 & 258.22 & 0.0002 & 5.4954 \\
\hline 235.5478 & 229.66 & 223.70 & 209.13 & 208.65 & 209.42 & 209.15 & 260.42 & 255.80 & 0.0002 & 5.4311 \\
\hline 236.0487 & 228.86 & 223.54 & 208.85 & 208.43 & 209.22 & 209.04 & 258.97 & 255.45 & 0.0008 & 5.4550 \\
\hline 236.5467 & 230.61 & 224.85 & 208.74 & 208.40 & 209.19 & 209.08 & 258.58 & 255.72 & 0.0002 & 5.5215 \\
\hline 237.0473 & 229.67 & 224.35 & 208.73 & 208.42 & 209.21 & 209.10 & 258.71 & 255.70 & 0.0006 & 5.4379 \\
\hline 237.5482 & 229.01 & 221.92 & 208.73 & 208.41 & 209.18 & 209.09 & 258.65 & 256.53 & 0.0000 & 5.4394 \\
\hline 238.0480 & 228.77 & 223.92 & 208.75 & 208.43 & 209.21 & 209.09 & 258.78 & 255.35 & 0.0007 & 5.4884 \\
\hline 238.5477 & 228.38 & 222.82 & 208.75 & 208.43 & 209.21 & 209.07 & 258.74 & 255.85 & 0.0007 & 5.4853 \\
\hline 239.0467 & 230.32 & 223.10 & 208.75 & 208.45 & 209.25 & 209.11 & 258.75 & 256.17 & 0.0007 & 5.4581 \\
\hline 239.5483 & 231.36 & 224.84 & 208.74 & 208.41 & 209.22 & 209.11 & 258.62 & 256.07 & 0.0007 & 5.4278 \\
\hline 240.0482 & 229.52 & 224.47 & 208.72 & 208.41 & 209.26 & 209.13 & 258.32 & 255.77 & 0.0003 & 5.5517 \\
\hline 240.5480 & 229.23 & 222.54 & 208.69 & 208.40 & 209.20 & 209.10 & 258.24 & 256.31 & 0.0003 & 5.4580 \\
\hline 241.0478 & 228.93 & 223.29 & 208.71 & 208.42 & 209.22 & 209.12 & 258.71 & 255.31 & 0.0003 & 5.4402 \\
\hline 241.5477 & 229.42 & 224.39 & 208.80 & 208.47 & 209.26 & 209.16 & 259.61 & 255.77 & 0.0003 & 5.4505 \\
\hline 242.0483 & 228.97 & 223.31 & 208.98 & 208.62 & 209.35 & 209.23 & 260.89 & 256.24 & 0.0010 & 5.3722 \\
\hline 242.5482 & 229.44 & 225.11 & 209.18 & 208.75 & 209.45 & 209.32 & 262.09 & 256.30 & 0.0006 & 5.4634 \\
\hline 243.0470 & 230.23 & 226.68 & 209.29 & 208.84 & 209.54 & 209.38 & 262.25 & 256.95 & 0.0003 & 5.5427 \\
\hline 243.5478 & 231.13 & 227.29 & 209.24 & 208.81 & 209.54 & 209.36 & 261.34 & 256.49 & 0.0002 & 5.4283 \\
\hline 244.0477 & 230.88 & 228.05 & 209.04 & 208.68 & 209.45 & 209.27 & 260.36 & 256.33 & 0.0006 & 5.3558 \\
\hline 244.5483 & 229.80 & 225.94 & 208.92 & 208.56 & 209.36 & 209.22 & 259.38 & 256.44 & 0.0002 & 5.3776 \\
\hline 245.0482 & 230.77 & 212.78 & 211.30 & 210.15 & 210.51 & 210.31 & 273.20 & 263.76 & 0.0007 & 5.5945 \\
\hline 245.5480 & 231.60 & 212.88 & 211.39 & 210.17 & 210.52 & 210.30 & 273.35 & 263.24 & 0.0007 & 5.5752 \\
\hline 246.0478 & 231.60 & 212.88 & 211.38 & 210.18 & 210.48 & 210.27 & 273.37 & 262.92 & 0.0003 & 5.5735 \\
\hline 246.5477 & 228.47 & 212.62 & 211.43 & 210.18 & 210.50 & 210.31 & 273.88 & 263.67 & 0.0006 & 5.6004 \\
\hline 247.0483 & 228.91 & 212.49 & 211.42 & 210.26 & 210.60 & 210.40 & 273.37 & 264.23 & 0.0002 & 5.5806 \\
\hline 247.5473 & 231.47 & 212.64 & 211.44 & 210.28 & 210.64 & 210.42 & 273.35 & 264.25 & 0.0002 & 5.5628 \\
\hline 248.0480 & 233.89 & 212.72 & 211.45 & 210.25 & 210.57 & 210.34 & 273.39 & 263.39 & 0.0000 & 5.5453 \\
\hline 248.5478 & 232.10 & 212.81 & 211.43 & 210.23 & 210.59 & 210.39 & 273.42 & 263.91 & 0.0005 & 5.5201 \\
\hline 249.0467 & 231.73 & 212.83 & 211.44 & 210.27 & 210.58 & 210.36 & 273.24 & 263.34 & 0.0000 & 5.5063 \\
\hline 249.5475 & 230.88 & 212.87 & 211.44 & 210.20 & 210.49 & 210.29 & 273.73 & 262.94 & 0.0000 & 5.5489 \\
\hline
\end{tabular}


TABLE B4.- CONTINUED.

\begin{tabular}{|c|c|c|c|c|c|c|c|c|c|c|}
\hline $\begin{array}{l}\text { Time, } \\
\text { min }\end{array}$ & $\begin{array}{c}\text { T-steam } \\
{ }^{\circ} \mathrm{C}\end{array}$ & $\begin{array}{l}\mathrm{T} 1, \\
{ }^{\circ} \mathrm{C}\end{array}$ & $\begin{array}{l}\mathrm{T} 2, \\
{ }^{\circ} \mathrm{C}\end{array}$ & $\begin{array}{l}\mathrm{T} 3, \\
{ }^{\circ} \mathrm{C}\end{array}$ & $\begin{array}{l}\mathrm{T} 4, \\
{ }^{\circ} \mathrm{C}\end{array}$ & $\begin{array}{l}\mathrm{T} 5, \\
{ }^{\circ} \mathrm{C}\end{array}$ & $\begin{array}{l}\text { Pinj, } \\
\text { psig }\end{array}$ & $\begin{array}{l}\text { Pout, } \\
\text { psig }\end{array}$ & $\begin{array}{l}\text { Qinj, } \\
\text { g/min }\end{array}$ & $\begin{array}{c}\mathrm{Vw} \\
\mathrm{ml} / \mathrm{min}\end{array}$ \\
\hline 250.0492 & 231.02 & 213.07 & 211.39 & 210.17 & 210.49 & 210.24 & 273.27 & 262.76 & 0.0005 & 5.5565 \\
\hline 250.5472 & 230.58 & 213.02 & 211.32 & 210.14 & 210.48 & 210.23 & 272.70 & 263.05 & 0.0004 & 5.5953 \\
\hline 251.0470 & 230.40 & 212.86 & 211.32 & 210.11 & 210.43 & 210.25 & 272.94 & 263.17 & 0.0002 & 5.5893 \\
\hline 251.5477 & 230.57 & 212.63 & 211.26 & 210.09 & 210.41 & 210.22 & 272.57 & 263.28 & 0.0007 & 5.5928 \\
\hline 252.0485 & 230.72 & 212.64 & 211.31 & 210.11 & 210.47 & 210.29 & 272.99 & 263.48 & 0.0001 & 5.5652 \\
\hline 252.5482 & 231.35 & 212.50 & 211.28 & 210.15 & 210.53 & 210.35 & 272.80 & 263.31 & 0.0002 & 5.5712 \\
\hline 253.0480 & 230.74 & 212.52 & 211.28 & 210.16 & 210.51 & 210.32 & 272.60 & 263.43 & 0.0002 & 5.5246 \\
\hline 253.5488 & 231.44 & 212.47 & 211.31 & 210.16 & 210.52 & 210.36 & 272.86 & 263.48 & 0.0002 & 5.5234 \\
\hline 254.0477 & 232.09 & 212.51 & 211.36 & 210.18 & 210.56 & 210.36 & 273.37 & 263.86 & 0.0007 & 5.5461 \\
\hline 254.5475 & 231.91 & 212.51 & 211.37 & 210.20 & 210.54 & 210.36 & 272.88 & 263.64 & 0.0001 & 5.5620 \\
\hline 255.0473 & 231.35 & 212.59 & 211.35 & 210.19 & 210.55 & 210.38 & 272.82 & 263.30 & 0.0000 & 5.5622 \\
\hline 255.5482 & 230.65 & 212.82 & 211.39 & 210.21 & 210.55 & 210.39 & 273.12 & 263.78 & 0.0004 & 5.5535 \\
\hline 256.0480 & 230.55 & 212.79 & 211.41 & 210.21 & 210.55 & 210.36 & 273.04 & 263.44 & 0.0005 & 5.5749 \\
\hline 256.5477 & 227.43 & 212.42 & 211.45 & 210.23 & 210.56 & 210.38 & 273.69 & 263.06 & 0.0007 & 5.5879 \\
\hline 257.0467 & 228.30 & 212.33 & 211.40 & 210.06 & 209.97 & 209.22 & 273.43 & 250.72 & 0.0001 & 3.1443 \\
\hline
\end{tabular}


TABLE B5. PRESSURE AND TEMPERATURE DATA FOR RUN 7 (PETROLEUM DISTILLATE:STEAM = 5:100).

\begin{tabular}{|c|c|c|c|c|c|c|c|c|c|c|}
\hline $\begin{array}{c}\text { Time, } \\
\text { min }\end{array}$ & $\begin{array}{c}\text { T-steam } \\
{ }^{\circ} \mathrm{C}\end{array}$ & $\begin{array}{l}\mathrm{T} 1, \\
{ }^{\circ} \mathrm{C}\end{array}$ & $\begin{array}{l}\mathrm{T} 2, \\
{ }^{\circ} \mathrm{C}\end{array}$ & $\begin{array}{l}\mathrm{T} 3, \\
{ }^{\circ} \mathrm{C}\end{array}$ & $\begin{array}{l}\mathrm{T} 4, \\
{ }^{\circ} \mathrm{C}\end{array}$ & $\begin{array}{l}\mathrm{T} 5, \\
{ }^{\circ} \mathrm{C}\end{array}$ & $\begin{array}{l}\text { Pinj, } \\
\text { psig }\end{array}$ & $\begin{array}{l}\text { Pout, } \\
\text { psig }\end{array}$ & $\begin{array}{l}\text { Qinj, } \\
\text { g/min }\end{array}$ & $\begin{array}{c}\text { Vw } \\
\mathrm{ml} / \mathrm{min}\end{array}$ \\
\hline 0.0530 & 50.91 & 48.62 & 52.60 & 54.96 & 54.31 & 50.18 & 270.44 & 261.21 & 0.0000 & 5.5097 \\
\hline 0.5473 & 58.98 & 52.71 & 52.58 & 54.96 & 54.33 & 50.20 & 268.15 & 261.32 & 0.0000 & 5.5440 \\
\hline 1.0480 & 68.01 & 61.98 & 52.60 & 54.94 & 54.31 & 50.20 & 260.61 & 261.36 & 0.0003 & 5.5006 \\
\hline 1.5478 & 97.45 & 79.98 & 52.58 & 54.90 & 54.30 & 50.22 & 260.50 & 261.26 & 0.0003 & 5.4906 \\
\hline 2.0468 & 168.34 & 97.43 & 52.58 & 54.79 & 54.20 & 50.26 & 260.93 & 261.33 & 0.0006 & 5.5337 \\
\hline 2.5475 & 203.32 & 171.13 & 52.59 & 54.87 & 54.26 & 50.36 & 265.14 & 261.75 & 0.0004 & 5.5381 \\
\hline 3.0483 & 208.03 & 202.29 & 53.98 & 54.85 & 54.36 & 50.38 & 265.92 & 261.02 & 0.0004 & 5.4981 \\
\hline 3.5482 & 209.48 & 204.94 & 55.15 & 54.83 & 54.41 & 50.40 & 269.77 & 260.92 & 0.0004 & 5.5028 \\
\hline 4.0480 & 211.08 & 206.19 & 56.24 & 54.81 & 54.43 & 50.41 & 273.95 & 261.07 & 0.0007 & 5.5213 \\
\hline 4.5468 & 213.33 & 208.09 & 56.64 & 54.79 & 54.45 & 50.44 & 276.00 & 260.89 & 0.0003 & 5.5361 \\
\hline 5.0475 & 215.79 & 210.61 & 56.56 & 54.78 & 54.47 & 50.46 & 277.95 & 260.88 & 0.0005 & 5.4972 \\
\hline 5.5473 & 217.57 & 213.46 & 56.22 & 54.72 & 54.49 & 50.48 & 278.65 & 260.87 & 0.0005 & 5.4972 \\
\hline 6.0482 & 218.02 & 214.39 & 55.80 & 54.70 & 54.49 & 50.50 & 279.17 & 260.85 & 0.0004 & 5.5408 \\
\hline 6.5480 & 217.11 & 214.57 & 55.43 & 54.68 & 54.51 & 50.52 & 279.88 & 260.85 & 0.0002 & 5.5250 \\
\hline 7.0478 & 216.72 & 214.48 & 55.16 & 54.70 & 54.53 & 50.52 & 281.20 & 260.84 & 0.0003 & 5.4863 \\
\hline 7.5477 & 218.19 & 214.97 & 55.26 & 54.69 & 54.53 & 50.54 & 293.51 & 260.93 & 0.0001 & 5.5186 \\
\hline 8.0483 & 220.69 & 216.00 & 55.45 & 54.69 & 54.57 & 50.54 & 297.46 & 260.89 & 0.0006 & 5.5468 \\
\hline 8.5482 & 223.22 & 217.29 & 55.66 & 54.67 & 54.57 & 50.56 & 303.00 & 260.88 & 0.0000 & 5.5213 \\
\hline 9.0480 & 225.08 & 219.42 & 55.92 & 54.69 & 54.63 & 50.56 & 308.62 & 260.91 & 0.0000 & 5.4908 \\
\hline 9.5478 & 226.48 & 220.87 & 56.25 & 54.67 & 54.67 & 50.56 & 306.70 & 260.83 & 0.0004 & 5.5321 \\
\hline 10.0477 & 226.50 & 221.04 & 56.70 & 54.67 & 54.69 & 50.56 & 308.29 & 260.83 & 0.0005 & 5.5376 \\
\hline 10.5483 & 227.86 & 222.02 & 57.25 & 54.65 & 54.71 & 50.58 & 310.22 & 260.80 & 0.0004 & 5.5077 \\
\hline 11.0492 & 228.47 & 223.13 & 57.82 & 54.65 & 54.73 & 50.58 & 313.13 & 260.80 & 0.0000 & 5.4947 \\
\hline 11.5480 & 230.06 & 223.42 & 58.59 & 54.64 & 54.73 & 50.66 & 318.22 & 260.82 & 0.0004 & 5.5394 \\
\hline 12.0478 & 228.11 & 223.65 & 59.44 & 54.62 & 54.75 & 50.66 & 318.86 & 260.81 & 0.0009 & 5.5360 \\
\hline 12.5477 & 223.94 & 218.64 & 59.88 & 54.60 & 54.75 & 50.66 & 314.41 & 260.80 & 0.0006 & 5.4888 \\
\hline 13.0483 & 223.26 & 218.00 & 60.84 & 54.58 & 54.75 & 50.68 & 313.54 & 260.78 & 0.0003 & 5.5042 \\
\hline 13.5473 & 223.71 & 218.07 & 62.38 & 54.56 & 54.75 & 50.68 & 314.92 & 260.77 & 0.0007 & 5.5396 \\
\hline 14.0480 & 229.82 & 219.08 & 63.91 & 54.53 & 54.79 & 50.72 & 315.37 & 260.79 & 0.0007 & 5.5247 \\
\hline 14.5478 & 245.57 & 221.82 & 64.52 & 54.49 & 54.79 & 50.72 & 313.50 & 260.86 & 0.0007 & 5.4849 \\
\hline 15.0468 & 254.74 & 231.12 & 63.97 & 54.47 & 54.83 & 50.74 & 310.16 & 260.77 & 0.0000 & 5.5252 \\
\hline 15.5475 & 259.30 & 238.20 & 63.61 & 54.47 & 54.85 & 50.78 & 308.04 & 260.73 & 0.0003 & 5.5519 \\
\hline 16.0482 & 260.24 & 241.25 & 63.58 & 54.46 & 54.84 & 50.80 & 304.80 & 260.71 & 0.0002 & 5.4928 \\
\hline 16.5480 & 258.12 & 241.54 & 63.90 & 54.46 & 54.86 & 50.84 & 305.11 & 260.72 & 0.0005 & 5.5073 \\
\hline 17.0470 & 251.19 & 241.07 & 64.34 & 54.46 & 54.86 & 50.88 & 306.56 & 261.27 & 0.0005 & 5.5362 \\
\hline 17.5477 & 236.34 & 239.50 & 64.83 & 54.46 & 54.88 & 50.92 & 307.03 & 259.11 & 0.0002 & 5.5274 \\
\hline 18.0485 & 229.77 & 235.72 & 65.38 & 54.46 & 54.90 & 50.94 & 307.11 & 259.59 & 0.0004 & 5.4811 \\
\hline 18.5473 & 222.67 & 224.74 & 65.91 & 54.46 & 54.86 & 50.96 & 306.95 & 258.09 & 0.0004 & 5.4794 \\
\hline 19.0480 & 220.72 & 217.55 & 66.46 & 54.46 & 54.86 & 50.96 & 304.50 & 257.05 & 0.0000 & 5.5302 \\
\hline 19.5478 & 220.06 & 216.53 & 67.10 & 54.45 & 54.89 & 51.00 & 303.38 & 258.02 & 0.0000 & 5.5093 \\
\hline 20.0487 & 220.18 & 216.43 & 67.90 & 54.43 & 54.91 & 51.04 & 304.42 & 259.01 & 0.0005 & 5.4805 \\
\hline 20.5475 & 220.60 & 216.75 & 68.75 & 54.43 & 54.91 & 51.06 & 307.00 & 258.84 & 0.0003 & 5.5283 \\
\hline 21.0483 & 221.17 & 217.29 & 69.63 & 54.43 & 54.93 & 51.10 & 309.70 & 261.45 & 0.0003 & 5.5241 \\
\hline 21.5482 & 221.62 & 217.70 & 70.45 & 54.42 & 54.95 & 51.12 & 312.00 & 261.62 & 0.0003 & 5.4516 \\
\hline 22.0470 & 222.11 & 218.08 & 71.30 & 54.42 & 54.93 & 51.15 & 313.29 & 261.73 & 0.0003 & 5.4856 \\
\hline 22.5477 & 222.48 & 218.44 & 72.15 & 54.40 & 54.94 & 51.17 & 314.43 & 261.49 & 0.0000 & 5.5058 \\
\hline 23.0475 & 222.74 & 218.69 & 72.96 & 54.41 & 54.96 & 51.19 & 314.75 & 261.58 & 0.0000 & 5.4798 \\
\hline 23.5483 & 222.81 & 218.66 & 73.81 & 54.39 & 54.96 & 51.21 & 315.33 & 261.57 & 0.0000 & 5.5216 \\
\hline 24.0482 & 223.08 & 218.86 & 74.77 & 54.41 & 54.98 & 51.23 & 315.56 & 261.57 & 0.0000 & 5.5700 \\
\hline 24.5470 & 223.16 & 219.06 & 75.78 & 54.41 & 54.98 & 51.25 & 316.23 & 261.54 & 0.0000 & 5.5482 \\
\hline
\end{tabular}


TABLE B5.- CONTINUED.

\begin{tabular}{|c|c|c|c|c|c|c|c|c|c|c|}
\hline $\begin{array}{l}\text { Time, } \\
\text { min }\end{array}$ & $\begin{array}{c}\text { T-steam } \\
{ }^{\circ} \mathrm{C}\end{array}$ & $\begin{array}{l}\mathrm{T} 1, \\
{ }^{\circ} \mathrm{C}\end{array}$ & $\begin{array}{l}\mathrm{T} 2, \\
{ }^{\circ} \mathrm{C}\end{array}$ & $\begin{array}{l}\mathrm{T} 3, \\
{ }^{\circ} \mathrm{C}\end{array}$ & $\begin{array}{l}\mathrm{T} 4, \\
{ }^{\circ} \mathrm{C}\end{array}$ & $\begin{array}{l}\mathrm{T} 5, \\
{ }^{\circ} \mathrm{C}\end{array}$ & $\begin{array}{l}\text { Pinj, } \\
\text { psig }\end{array}$ & $\begin{array}{c}\text { Pout, } \\
\text { psig }\end{array}$ & $\begin{array}{l}\text { Qinj, } \\
\text { g/min }\end{array}$ & $\begin{array}{c}\mathrm{Vw} \\
\mathrm{ml} / \mathrm{min}\end{array}$ \\
\hline 25.0478 & 223.50 & 219.40 & 76.87 & 54.39 & 55.00 & 51.31 & 316.27 & 261.52 & 0.0001 & 5.5069 \\
\hline 25.5477 & 223.63 & 219.31 & 78.02 & 54.42 & 54.99 & 51.33 & 316.00 & 261.49 & 0.0001 & 5.5136 \\
\hline 26.0483 & 224.24 & 219.44 & 79.13 & 54.42 & 54.99 & 51.36 & 316.12 & 261.44 & 0.0003 & 5.5249 \\
\hline 26.5482 & 224.31 & 219.91 & 80.31 & 54.42 & 55.01 & 51.40 & 317.64 & 261.42 & 0.0003 & 5.5491 \\
\hline 27.0470 & 225.55 & 220.18 & 81.76 & 54.42 & 55.01 & 51.42 & 318.51 & 261.42 & 0.0005 & 5.5343 \\
\hline 27.5478 & 226.23 & 221.58 & 83.22 & 54.43 & 55.03 & 51.48 & 320.73 & 261.39 & 0.0003 & 5.5023 \\
\hline 28.0477 & 225.59 & 221.99 & 84.85 & 54.43 & 55.04 & 51.52 & 321.52 & 261.24 & 0.0003 & 5.5228 \\
\hline 28.5483 & 227.08 & 222.33 & 86.38 & 54.45 & 55.06 & 51.56 & 321.72 & 261.24 & 0.0003 & 5.5430 \\
\hline 29.0482 & 229.21 & 225.72 & 88.12 & 54.49 & 55.08 & 51.62 & 321.23 & 261.24 & 0.0001 & 5.5296 \\
\hline 29.5480 & 232.11 & 231.74 & 89.80 & 54.53 & 55.08 & 51.66 & 320.79 & 261.21 & 0.0001 & 5.4966 \\
\hline 30.0478 & 228.19 & 231.08 & 91.54 & 54.54 & 55.11 & 51.72 & 320.97 & 261.23 & 0.0001 & 5.5171 \\
\hline 30.5485 & 226.82 & 227.11 & 93.14 & 54.58 & 55.11 & 51.74 & 320.84 & 261.19 & 0.0004 & 5.5319 \\
\hline 31.0483 & 226.46 & 222.71 & 94.95 & 54.62 & 55.09 & 51.78 & 320.57 & 261.21 & 0.0003 & 5.5509 \\
\hline 31.5473 & 229.42 & 221.89 & 96.77 & 54.68 & 55.10 & 51.81 & 319.80 & 261.21 & 0.0003 & 5.5012 \\
\hline 32.0480 & 231.77 & 226.74 & 98.83 & 54.74 & 55.12 & 51.89 & 320.54 & 261.17 & 0.0003 & 5.4997 \\
\hline 32.5478 & 232.80 & 233.15 & 101.11 & 54.80 & 55.14 & 51.93 & 320.38 & 261.19 & 0.0001 & 5.5176 \\
\hline 33.0477 & 234.25 & 235.92 & 103.76 & 54.90 & 55.15 & 51.99 & 319.28 & 261.15 & 0.0006 & 5.5387 \\
\hline 33.5483 & 228.88 & 235.88 & 107.04 & 55.00 & 55.17 & 52.05 & 318.68 & 260.42 & 0.0006 & 5.5242 \\
\hline 34.0482 & 229.83 & 234.08 & 110.75 & 55.12 & 55.17 & 52.11 & 318.38 & 262.53 & 0.0006 & 5.4683 \\
\hline 34.5480 & 230.82 & 234.01 & 114.97 & 55.25 & 55.19 & 52.19 & 318.27 & 263.46 & 0.0003 & 5.5386 \\
\hline 35.0478 & 228.55 & 235.09 & 120.08 & 55.41 & 55.18 & 52.25 & 318.84 & 263.23 & 0.0003 & 5.5401 \\
\hline 35.5477 & 228.84 & 234.57 & 126.54 & 55.56 & 55.18 & 52.33 & 317.48 & 263.00 & 0.0001 & 5.5138 \\
\hline 36.0485 & 231.44 & 235.58 & 136.83 & 55.76 & 55.17 & 52.41 & 316.78 & 262.03 & 0.0001 & 5.4935 \\
\hline 36.5473 & 232.64 & 236.67 & 148.28 & 55.97 & 55.19 & 52.49 & 315.86 & 255.07 & 0.0001 & 5.5204 \\
\hline 37.0480 & 231.59 & 237.09 & 163.84 & 56.16 & 55.21 & 52.53 & 314.19 & 248.94 & 0.0002 & 5.5310 \\
\hline 37.5478 & 228.77 & 236.65 & 180.79 & 56.38 & 55.22 & 52.56 & 312.99 & 249.87 & 0.0002 & 5.5004 \\
\hline 38.0477 & 228.99 & 235.86 & 192.90 & 56.59 & 55.24 & 52.60 & 312.25 & 250.28 & 0.0002 & 5.4874 \\
\hline 38.5475 & 229.97 & 236.35 & 201.50 & 56.82 & 55.26 & 52.62 & 311.49 & 251.54 & 0.0002 & 5.5267 \\
\hline 39.0483 & 230.68 & 236.71 & 206.51 & 57.04 & 55.27 & 52.64 & 311.36 & 251.26 & 0.0002 & 5.4834 \\
\hline 39.5482 & 230.72 & 236.41 & 209.51 & 57.29 & 55.29 & 52.69 & 310.66 & 251.99 & 0.0002 & 5.4811 \\
\hline 40.0488 & 229.29 & 236.52 & 211.44 & 57.54 & 55.30 & 52.71 & 310.66 & 252.71 & 0.0002 & 5.5308 \\
\hline 40.5477 & 227.70 & 235.13 & 212.93 & 57.82 & 55.32 & 52.75 & 310.77 & 252.28 & 0.0002 & 5.5523 \\
\hline 41.0467 & 227.72 & 234.45 & 214.11 & 58.11 & 55.34 & 52.79 & 311.44 & 253.02 & 0.0002 & 5.5335 \\
\hline 41.5473 & 229.28 & 234.99 & 214.98 & 58.44 & 55.36 & 52.84 & 311.79 & 253.75 & 0.0002 & 5.5039 \\
\hline 42.0482 & 227.35 & 235.62 & 215.66 & 58.77 & 55.35 & 52.88 & 312.33 & 253.54 & 0.0002 & 5.5142 \\
\hline 42.5480 & 228.14 & 235.13 & 216.22 & 59.09 & 55.37 & 52.92 & 312.79 & 253.32 & 0.0002 & 5.5501 \\
\hline 43.0478 & 229.13 & 235.85 & 216.58 & 59.46 & 55.38 & 52.94 & 313.31 & 253.54 & 0.0002 & 5.5411 \\
\hline 43.5477 & 229.60 & 236.69 & 216.78 & 59.79 & 55.38 & 52.99 & 313.59 & 254.17 & 0.0002 & 5.4973 \\
\hline 44.0483 & 229.50 & 236.95 & 216.99 & 60.17 & 55.41 & 53.05 & 314.24 & 254.16 & 0.0002 & 5.5297 \\
\hline 44.5482 & 229.50 & 235.81 & 217.15 & 60.55 & 55.41 & 53.09 & 314.07 & 253.58 & 0.0002 & 5.5291 \\
\hline 45.0480 & 230.94 & 236.22 & 217.24 & 60.92 & 55.41 & 53.11 & 314.38 & 254.31 & 0.0002 & 5.5216 \\
\hline 45.5478 & 229.90 & 236.66 & 217.43 & 61.34 & 55.44 & 53.17 & 315.08 & 253.80 & 0.0002 & 5.5072 \\
\hline 46.0477 & 226.94 & 235.91 & 217.56 & 61.76 & 55.46 & 53.18 & 315.62 & 254.41 & 0.0002 & 5.5049 \\
\hline 46.5483 & 228.25 & 235.16 & 217.74 & 62.26 & 55.47 & 53.22 & 316.56 & 254.97 & 0.0002 & 5.5248 \\
\hline 47.0482 & 230.15 & 235.61 & 217.83 & 62.75 & 55.47 & 53.27 & 316.81 & 254.80 & 0.0002 & 5.5194 \\
\hline 47.5480 & 229.55 & 236.89 & 217.98 & 63.27 & 55.49 & 53.29 & 317.39 & 254.59 & 0.0002 & 5.4521 \\
\hline 48.0478 & 228.96 & 236.79 & 218.04 & 63.84 & 55.52 & 53.31 & 317.18 & 254.53 & 0.0002 & 5.4921 \\
\hline 48.5477 & 226.74 & 236.29 & 218.13 & 64.43 & 55.52 & 53.36 & 318.02 & 255.06 & 0.0002 & 5.5455 \\
\hline 49.0483 & 226.44 & 234.66 & 218.26 & 65.10 & 55.55 & 53.36 & 318.31 & 255.61 & 0.0002 & 5.5107 \\
\hline 49.5473 & 227.68 & 235.13 & 218.36 & 65.78 & 55.57 & 53.38 & 319.10 & 255.25 & 0.0002 & 5.4960 \\
\hline
\end{tabular}


TABLE B5.- CONTINUED.

\begin{tabular}{|c|c|c|c|c|c|c|c|c|c|c|}
\hline $\begin{array}{c}\text { Time, } \\
\text { min }\end{array}$ & $\begin{array}{c}\text { T-steam } \\
{ }^{\circ} \mathrm{C}\end{array}$ & $\begin{array}{l}\mathrm{T} 1, \\
{ }^{\circ} \mathrm{C}\end{array}$ & $\begin{array}{l}\mathrm{T} 2, \\
{ }^{\circ} \mathrm{C}\end{array}$ & $\begin{array}{l}\mathrm{T} 3, \\
{ }^{\circ} \mathrm{C}\end{array}$ & $\begin{array}{l}\mathrm{T} 4, \\
{ }^{\circ} \mathrm{C}\end{array}$ & $\begin{array}{l}\mathrm{T} 5, \\
{ }^{\circ} \mathrm{C}\end{array}$ & $\begin{array}{l}\text { Pinj, } \\
\text { psig }\end{array}$ & $\begin{array}{c}\text { Pout, } \\
\text { psig }\end{array}$ & $\begin{array}{l}\text { Qinj, } \\
\text { g/min }\end{array}$ & $\begin{array}{c}\mathrm{Vw} \\
\mathrm{ml} / \mathrm{min}\end{array}$ \\
\hline 50.0480 & 228.78 & 236.46 & 218.42 & 66.52 & 55.58 & 53.39 & 319.15 & 255.55 & 0.0002 & 5.5452 \\
\hline 50.5478 & 227.59 & 237.11 & 218.49 & 67.34 & 55.60 & 53.39 & 319.68 & 255.38 & 0.0001 & 5.5304 \\
\hline 51.0477 & 226.98 & 236.04 & 218.53 & 68.21 & 55.62 & 53.40 & 319.65 & 256.52 & 0.0001 & 5.5268 \\
\hline 51.5483 & 225.95 & 235.63 & 218.61 & 69.16 & 55.65 & 53.42 & 320.24 & 256.14 & 0.0001 & 5.4962 \\
\hline 52.0482 & 228.53 & 236.30 & 218.70 & 70.18 & 55.67 & 53.43 & 320.55 & 256.40 & 0.0001 & 5.5157 \\
\hline 52.5480 & 228.32 & 238.12 & 218.80 & 71.26 & 55.69 & 53.45 & 321.26 & 255.39 & 0.0001 & 5.5293 \\
\hline 53.0478 & 227.97 & 237.84 & 218.82 & 72.43 & 55.72 & 53.48 & 320.76 & 255.30 & 0.0000 & 5.5107 \\
\hline 53.5477 & 225.13 & 235.69 & 218.92 & 73.68 & 55.74 & 53.46 & 321.55 & 256.04 & 0.0000 & 5.5042 \\
\hline 54.0475 & 224.59 & 224.77 & 218.99 & 75.00 & 55.73 & 53.47 & 322.15 & 255.72 & 0.0000 & 5.5009 \\
\hline 54.5483 & 224.70 & 221.43 & 219.09 & 76.38 & 55.77 & 53.47 & 322.67 & 255.65 & 0.0000 & 5.5164 \\
\hline 55.0480 & 225.61 & 220.47 & 219.13 & 77.84 & 55.76 & 53.48 & 322.49 & 255.15 & 0.0004 & 5.4749 \\
\hline 55.5478 & 227.13 & 223.44 & 219.19 & 79.41 & 55.80 & 53.50 & 322.98 & 255.76 & 0.0000 & 5.4940 \\
\hline 56.0468 & 230.49 & 229.73 & 219.19 & 81.08 & 55.85 & 53.51 & 322.87 & 256.29 & 0.0003 & 5.5415 \\
\hline 56.5475 & 233.44 & 235.42 & 219.21 & 82.84 & 55.87 & 53.53 & 323.05 & 255.55 & 0.0003 & 5.5307 \\
\hline 57.0483 & 235.08 & 240.00 & 219.25 & 84.81 & 55.93 & 53.53 & 323.08 & 256.49 & 0.0004 & 5.4911 \\
\hline 57.5482 & 228.60 & 241.64 & 219.29 & 86.92 & 55.97 & 53.54 & 323.20 & 257.00 & 0.0002 & 5.4958 \\
\hline 58.0470 & 225.74 & 236.11 & 219.32 & 89.26 & 56.04 & 53.55 & 323.55 & 256.48 & 0.0002 & 5.5478 \\
\hline 58.5477 & 224.76 & 224.15 & 219.27 & 91.84 & 56.08 & 53.57 & 322.97 & 255.49 & 0.0002 & 5.5481 \\
\hline 59.0475 & 224.62 & 220.63 & 219.24 & 94.67 & 56.12 & 53.59 & 323.08 & 255.83 & 0.0002 & 5.5011 \\
\hline 59.5483 & 224.72 & 220.48 & 219.26 & 97.68 & 56.18 & 53.60 & 322.97 & 256.38 & 0.0002 & 5.4912 \\
\hline 60.0482 & 225.24 & 220.84 & 219.28 & 100.95 & 56.25 & 53.62 & 323.17 & 256.42 & 0.0002 & 5.5088 \\
\hline 60.5480 & 226.73 & 222.42 & 219.30 & 104.47 & 56.31 & 53.63 & 323.37 & 256.75 & 0.0002 & 5.5252 \\
\hline 61.0478 & 230.10 & 225.61 & 219.36 & 108.39 & 56.37 & 53.67 & 323.40 & 257.12 & 0.0000 & 5.4925 \\
\hline 61.5477 & 229.25 & 231.92 & 219.49 & 112.85 & 56.43 & 53.68 & 324.52 & 256.07 & 0.0000 & 5.4923 \\
\hline 62.0483 & 230.61 & 236.31 & 219.55 & 117.68 & 56.48 & 53.70 & 324.70 & 256.13 & 0.0000 & 5.5017 \\
\hline 62.5482 & 231.07 & 238.84 & 219.56 & 122.58 & 56.56 & 53.73 & 324.69 & 256.05 & 0.0000 & 5.4757 \\
\hline 63.0470 & 226.38 & 238.81 & 219.58 & 127.87 & 56.64 & 53.75 & 324.97 & 255.89 & 0.0000 & 5.4823 \\
\hline 63.5478 & 224.86 & 227.01 & 219.62 & 133.57 & 56.70 & 53.74 & 325.28 & 257.14 & 0.0000 & 5.5320 \\
\hline 64.0477 & 224.87 & 221.54 & 219.59 & 139.45 & 56.78 & 53.74 & 324.88 & 256.97 & 0.0000 & 5.5210 \\
\hline 64.5483 & 225.03 & 221.28 & 219.57 & 145.87 & 56.88 & 53.78 & 324.50 & 257.32 & 0.0000 & 5.4833 \\
\hline 65.0482 & 225.52 & 221.66 & 219.54 & 153.26 & 56.98 & 53.79 & 324.62 & 256.92 & 0.0000 & 5.4976 \\
\hline 65.5472 & 226.82 & 222.97 & 219.57 & 161.18 & 57.12 & 53.79 & 324.67 & 256.17 & 0.0000 & 5.5480 \\
\hline 66.0478 & 228.00 & 227.05 & 219.59 & 170.22 & 57.22 & 53.82 & 324.76 & 257.28 & 0.0000 & 5.5125 \\
\hline 66.5477 & 229.12 & 232.48 & 219.60 & 179.06 & 57.40 & 53.86 & 325.10 & 257.26 & 0.0000 & 5.4953 \\
\hline 67.0483 & 229.57 & 237.43 & 219.67 & 189.66 & 57.53 & 53.87 & 325.38 & 256.30 & 0.0000 & 5.5127 \\
\hline 67.5482 & 227.80 & 239.46 & 219.68 & 199.70 & 57.69 & 53.87 & 325.31 & 256.61 & 0.0000 & 5.5520 \\
\hline 68.0472 & 227.09 & 239.18 & 219.63 & 207.40 & 57.87 & 53.90 & 325.05 & 257.26 & 0.0000 & 5.5140 \\
\hline 68.5478 & 226.49 & 239.31 & 219.70 & 212.01 & 58.02 & 53.92 & 325.61 & 256.86 & 0.0004 & 5.4829 \\
\hline 69.0477 & 226.73 & 238.56 & 219.73 & 214.32 & 58.26 & 53.93 & 325.80 & 256.45 & 0.0002 & 5.5244 \\
\hline 69.5483 & 228.00 & 238.25 & 219.71 & 215.69 & 58.47 & 53.97 & 325.26 & 257.05 & 0.0002 & 5.5316 \\
\hline 70.0482 & 234.08 & 240.76 & 219.68 & 216.55 & 58.70 & 53.97 & 325.13 & 256.75 & 0.0000 & 5.5167 \\
\hline 70.5472 & 237.50 & 244.35 & 219.64 & 217.24 & 58.96 & 54.02 & 324.60 & 258.10 & 0.0001 & 5.4958 \\
\hline 71.0478 & 228.61 & 245.74 & 219.62 & 217.69 & 59.21 & 54.06 & 324.40 & 257.33 & 0.0007 & 5.5080 \\
\hline 71.5477 & 226.64 & 242.42 & 219.55 & 217.93 & 59.50 & 54.08 & 324.28 & 257.80 & 0.0003 & 5.5253 \\
\hline 72.0485 & 225.70 & 239.17 & 219.56 & 218.13 & 59.77 & 54.11 & 324.26 & 258.62 & 0.0003 & 5.5218 \\
\hline 72.5473 & 225.97 & 236.99 & 219.51 & 218.26 & 60.10 & 54.12 & 323.89 & 258.66 & 0.0003 & 5.4827 \\
\hline 73.0480 & 226.68 & 236.26 & 219.52 & 218.39 & 60.46 & 54.14 & 324.04 & 258.29 & 0.0002 & 5.5307 \\
\hline 73.5478 & 230.01 & 236.81 & 219.47 & 218.39 & 60.81 & 54.16 & 323.51 & 258.61 & 0.0010 & 5.5409 \\
\hline 74.0468 & 232.04 & 240.50 & 219.44 & 218.43 & 61.19 & 54.19 & 323.38 & 259.34 & 0.0010 & 5.4706 \\
\hline 74.5475 & 235.45 & 244.59 & 219.41 & 218.42 & 61.63 & 54.19 & 323.13 & 259.56 & 0.0010 & 5.4938 \\
\hline
\end{tabular}


TABLE B5.- CONTINUED.

\begin{tabular}{|c|c|c|c|c|c|c|c|c|c|c|}
\hline $\begin{array}{l}\text { Time, } \\
\text { min }\end{array}$ & $\begin{array}{c}\text { T-steam } \\
{ }^{\circ} \mathrm{C}\end{array}$ & $\begin{array}{l}\mathrm{T} 1, \\
{ }^{\circ} \mathrm{C}\end{array}$ & $\begin{array}{l}\mathrm{T} 2, \\
{ }^{\circ} \mathrm{C}\end{array}$ & $\begin{array}{l}\mathrm{T} 3, \\
{ }^{\circ} \mathrm{C}\end{array}$ & $\begin{array}{l}\mathrm{T} 4, \\
{ }^{\circ} \mathrm{C}\end{array}$ & $\begin{array}{l}\mathrm{T} 5, \\
{ }^{\circ} \mathrm{C}\end{array}$ & $\begin{array}{l}\text { Pinj, } \\
\text { psig }\end{array}$ & $\begin{array}{l}\text { Pout, } \\
\text { psig }\end{array}$ & $\begin{array}{l}\text { Qinj, } \\
\text { g/min }\end{array}$ & $\begin{array}{c}\mathrm{Vw} \\
\mathrm{ml} / \mathrm{min}\end{array}$ \\
\hline 75.0483 & 234.07 & 246.67 & 219.34 & 218.39 & 62.05 & 54.22 & 322.60 & 259.57 & 0.0000 & 5.5525 \\
\hline 75.5482 & 227.81 & 246.23 & 219.27 & 218.36 & 62.51 & 54.22 & 322.09 & 258.92 & 0.0000 & 5.5592 \\
\hline 76.0480 & 225.50 & 241.57 & 219.19 & 218.31 & 62.97 & 54.23 & 321.30 & 258.90 & 0.0000 & 5.5087 \\
\hline 76.5477 & 224.61 & 235.11 & 219.10 & 218.26 & 63.49 & 54.23 & 320.97 & 259.27 & 0.0000 & 5.5093 \\
\hline 77.0467 & 225.10 & 229.99 & 219.04 & 218.23 & 64.04 & 54.24 & 320.61 & 258.73 & 0.0002 & 5.5302 \\
\hline 77.5483 & 228.06 & 229.24 & 218.95 & 218.16 & 64.65 & 54.24 & 320.15 & 258.21 & 0.0000 & 5.5337 \\
\hline 78.0482 & 229.68 & 232.40 & 218.92 & 218.13 & 65.30 & 54.27 & 319.63 & 258.68 & 0.0000 & 5.5129 \\
\hline 78.5480 & 230.95 & 237.12 & 218.89 & 218.12 & 66.02 & 54.31 & 319.77 & 258.73 & 0.0000 & 5.4853 \\
\hline 79.0468 & 236.40 & 241.87 & 218.86 & 218.12 & 66.82 & 54.30 & 319.63 & 259.53 & 0.0000 & 5.5050 \\
\hline 79.5477 & 236.75 & 244.87 & 218.81 & 218.08 & 67.69 & 54.30 & 318.91 & 260.25 & 0.0001 & 5.5044 \\
\hline 80.0483 & 236.99 & 245.68 & 218.67 & 217.94 & 68.64 & 54.31 & 317.92 & 258.97 & 0.0001 & 5.4612 \\
\hline 80.5472 & 231.31 & 245.20 & 218.57 & 217.85 & 69.70 & 54.33 & 317.21 & 258.60 & 0.0001 & 5.5301 \\
\hline 81.0480 & 226.18 & 242.73 & 218.47 & 217.77 & 70.86 & 54.36 & 316.96 & 258.99 & 0.0001 & 5.5353 \\
\hline 81.5478 & 224.34 & 238.56 & 218.38 & 217.72 & 72.12 & 54.36 & 316.25 & 258.74 & 0.0005 & 5.5246 \\
\hline 82.0477 & 226.10 & 235.61 & 218.28 & 217.62 & 73.50 & 54.37 & 315.41 & 258.86 & 0.0005 & 5.4888 \\
\hline 82.5483 & 227.42 & 236.49 & 218.21 & 217.55 & 74.97 & 54.37 & 315.27 & 258.70 & 0.0005 & 5.4916 \\
\hline 83.0482 & 233.42 & 239.24 & 218.13 & 217.49 & 76.58 & 54.40 & 314.47 & 258.71 & 0.0000 & 5.5288 \\
\hline 83.5480 & 238.73 & 242.85 & 218.06 & 217.42 & 78.29 & 54.40 & 314.32 & 259.57 & 0.0005 & 5.5412 \\
\hline 84.0478 & 240.69 & 244.63 & 217.96 & 217.32 & 80.13 & 54.45 & 313.63 & 259.26 & 0.0000 & 5.4916 \\
\hline 84.5477 & 240.39 & 244.71 & 217.84 & 217.23 & 82.14 & 54.47 & 312.58 & 259.68 & 0.0004 & 5.4917 \\
\hline 85.0483 & 238.38 & 243.73 & 217.70 & 217.08 & 84.33 & 54.53 & 311.74 & 258.92 & 0.0004 & 5.5155 \\
\hline 85.5473 & 230.60 & 242.44 & 217.62 & 217.01 & 86.73 & 54.56 & 311.24 & 259.37 & 0.0004 & 5.5184 \\
\hline 86.0472 & 228.27 & 239.81 & 217.50 & 216.89 & 89.35 & 54.60 & 310.54 & 259.71 & 0.0004 & 5.4604 \\
\hline 86.5478 & 225.40 & 237.88 & 217.43 & 216.82 & 92.29 & 54.66 & 310.04 & 259.59 & 0.0000 & 5.4762 \\
\hline 87.0477 & 227.55 & 238.19 & 217.33 & 216.74 & 95.61 & 54.73 & 309.31 & 258.56 & 0.0000 & 5.5110 \\
\hline 87.5483 & 230.62 & 239.90 & 217.23 & 216.64 & 99.32 & 54.77 & 308.65 & 259.55 & 0.0000 & 5.4699 \\
\hline 88.0482 & 230.67 & 240.98 & 217.11 & 216.52 & 103.48 & 54.85 & 308.02 & 259.53 & 0.0000 & 5.5165 \\
\hline 88.5480 & 231.99 & 241.12 & 216.99 & 216.43 & 108.08 & 54.91 & 307.25 & 259.89 & 0.0000 & 5.5480 \\
\hline 89.0478 & 229.40 & 240.88 & 216.85 & 216.31 & 113.17 & 54.99 & 306.20 & 259.04 & 0.0001 & 5.5630 \\
\hline 89.5468 & 227.38 & 240.26 & 216.75 & 216.21 & 118.91 & 55.05 & 305.68 & 259.96 & 0.0002 & 5.5047 \\
\hline 90.0485 & 226.59 & 239.90 & 216.62 & 216.09 & 125.09 & 55.12 & 304.93 & 258.73 & 0.0002 & 5.4996 \\
\hline 90.5483 & 228.26 & 239.94 & 216.50 & 215.99 & 131.64 & 55.20 & 304.26 & 260.03 & 0.0002 & 5.5238 \\
\hline 91.0472 & 230.38 & 240.38 & 216.40 & 215.88 & 138.53 & 55.30 & 303.54 & 259.76 & 0.0000 & 5.5252 \\
\hline 91.5478 & 227.92 & 240.71 & 216.30 & 215.78 & 145.69 & 55.40 & 303.29 & 260.00 & 0.0002 & 5.5150 \\
\hline 92.0468 & 226.60 & 238.72 & 216.23 & 215.73 & 153.64 & 55.48 & 302.46 & 258.60 & 0.0002 & 5.4903 \\
\hline 92.5475 & 228.89 & 238.82 & 216.11 & 215.61 & 161.90 & 55.60 & 301.70 & 257.96 & 0.0003 & 5.5136 \\
\hline 93.0483 & 232.39 & 240.74 & 216.01 & 215.53 & 169.97 & 55.74 & 301.00 & 259.26 & 0.0002 & 5.5220 \\
\hline 93.5482 & 233.56 & 241.57 & 215.89 & 215.39 & 178.64 & 55.86 & 300.18 & 259.03 & 0.0000 & 5.4810 \\
\hline 94.0480 & 233.64 & 241.52 & 215.75 & 215.25 & 186.54 & 56.01 & 299.40 & 259.30 & 0.0000 & 5.4891 \\
\hline 94.5477 & 232.91 & 241.29 & 215.61 & 215.11 & 193.43 & 56.15 & 298.43 & 258.98 & 0.0006 & 5.5282 \\
\hline 95.0475 & 230.30 & 241.21 & 215.48 & 214.97 & 199.08 & 56.35 & 297.67 & 258.84 & 0.0003 & 5.5085 \\
\hline 95.5483 & 228.22 & 240.96 & 215.34 & 214.85 & 203.02 & 56.54 & 297.06 & 259.44 & 0.0008 & 5.4672 \\
\hline 96.0472 & 229.55 & 240.73 & 215.23 & 214.75 & 206.32 & 56.74 & 296.24 & 259.47 & 0.0008 & 5.5002 \\
\hline 96.5480 & 228.52 & 240.29 & 215.17 & 214.68 & 208.80 & 56.95 & 295.85 & 259.52 & 0.0008 & 5.5352 \\
\hline 97.0478 & 229.80 & 240.42 & 215.06 & 214.58 & 210.73 & 57.22 & 295.17 & 259.23 & 0.0008 & 5.5068 \\
\hline 97.5485 & 230.68 & 240.77 & 214.91 & 214.43 & 211.97 & 57.49 & 294.23 & 259.16 & 0.0008 & 5.4936 \\
\hline 98.0483 & 231.81 & 240.75 & 214.72 & 214.25 & 212.70 & 57.76 & 292.83 & 260.71 & 0.0008 & 5.5168 \\
\hline 98.5472 & 231.80 & 240.70 & 214.51 & 214.06 & 213.06 & 58.11 & 291.65 & 259.14 & 0.0002 & 5.5424 \\
\hline 99.0480 & 230.59 & 240.37 & 214.26 & 213.78 & 213.24 & 58.44 & 289.75 & 257.94 & 0.0002 & 5.5212 \\
\hline 99.5478 & 229.87 & 240.09 & 213.96 & 213.50 & 213.28 & 58.84 & 288.24 & 259.46 & 0.0007 & 5.5016 \\
\hline
\end{tabular}


TABLE B5.- CONTINUED.

\begin{tabular}{|c|c|c|c|c|c|c|c|c|c|c|}
\hline $\begin{array}{l}\text { Time, } \\
\text { min }\end{array}$ & $\begin{array}{c}\text { T-steam } \\
{ }^{\circ} \mathrm{C}\end{array}$ & $\begin{array}{l}\mathrm{T} 1, \\
{ }^{\circ} \mathrm{C}\end{array}$ & $\begin{array}{l}\mathrm{T} 2, \\
{ }^{\circ} \mathrm{C}\end{array}$ & $\begin{array}{l}\mathrm{T} 3, \\
{ }^{\circ} \mathrm{C}\end{array}$ & $\begin{array}{l}\mathrm{T} 4, \\
{ }^{\circ} \mathrm{C}\end{array}$ & $\begin{array}{l}\mathrm{T} 5, \\
{ }^{\circ} \mathrm{C}\end{array}$ & $\begin{array}{l}\text { Pinj, } \\
\text { psig }\end{array}$ & $\begin{array}{l}\text { Pout, } \\
\text { psig }\end{array}$ & $\begin{array}{l}\text { Qinj, } \\
\text { g/min }\end{array}$ & $\begin{array}{c}\mathrm{Vw} \\
\mathrm{ml} / \mathrm{min}\end{array}$ \\
\hline 100.0485 & 226.67 & 239.88 & 213.73 & 213.29 & 213.25 & 59.27 & 286.95 & 258.60 & 0.0002 & 5.5011 \\
\hline 100.5475 & 227.81 & 239.77 & 213.56 & 213.11 & 213.22 & 59.73 & 285.95 & 257.49 & 0.0002 & 5.5252 \\
\hline 101.0482 & 227.65 & 240.03 & 213.44 & 213.01 & 213.19 & 60.26 & 285.62 & 259.79 & 0.0003 & 5.4865 \\
\hline 101.5480 & 223.16 & 239.82 & 213.46 & 213.01 & 213.23 & 60.83 & 285.77 & 259.66 & 0.0002 & 5.4952 \\
\hline 102.0468 & 225.94 & 239.37 & 213.47 & 213.04 & 213.31 & 61.48 & 286.09 & 258.91 & 0.0002 & 5.5208 \\
\hline 102.5477 & 230.60 & 240.90 & 213.54 & 213.10 & 213.42 & 62.19 & 286.51 & 260.19 & 0.0001 & 5.5308 \\
\hline 103.0483 & 234.26 & 242.91 & 213.62 & 213.21 & 213.53 & 62.99 & 287.04 & 261.64 & 0.0001 & 5.5195 \\
\hline 103.5482 & 235.79 & 243.89 & 213.66 & 213.21 & 213.59 & 63.86 & 287.16 & 258.68 & 0.0002 & 5.5050 \\
\hline 104.0480 & 235.34 & 243.91 & 213.63 & 213.18 & 213.59 & 64.87 & 286.84 & 259.89 & 0.0001 & 5.5159 \\
\hline 104.5478 & 235.06 & 243.25 & 213.56 & 213.11 & 213.56 & 65.91 & 286.23 & 259.89 & 0.0009 & 5.5060 \\
\hline 105.0477 & 233.17 & 242.47 & 213.44 & 213.01 & 213.48 & 67.07 & 285.56 & 259.24 & 0.0009 & 5.5065 \\
\hline 105.5483 & 233.30 & 241.70 & 213.30 & 212.87 & 213.37 & 68.32 & 284.65 & 259.27 & 0.0004 & 5.4953 \\
\hline 106.0482 & 232.53 & 240.67 & 213.13 & 212.72 & 213.24 & 69.65 & 283.41 & 260.39 & 0.0002 & 5.5147 \\
\hline 106.5480 & 225.43 & 240.03 & 212.97 & 212.56 & 213.11 & 71.01 & 282.45 & 259.86 & 0.0006 & 5.5284 \\
\hline 107.0478 & 224.43 & 238.51 & 212.73 & 212.37 & 212.92 & 72.44 & 280.90 & 259.72 & 0.0006 & 5.4849 \\
\hline 107.5477 & 227.67 & 237.54 & 212.48 & 212.10 & 212.71 & 73.91 & 279.30 & 260.19 & 0.0006 & 5.5060 \\
\hline 108.0485 & 230.58 & 237.19 & 212.20 & 211.84 & 212.47 & 75.42 & 277.66 & 260.57 & 0.0006 & 5.5335 \\
\hline 108.5483 & 230.64 & 237.37 & 211.95 & 211.59 & 212.24 & 76.95 & 276.49 & 260.02 & 0.0006 & 5.5309 \\
\hline 109.0490 & 230.95 & 237.31 & 211.72 & 211.37 & 212.05 & 78.49 & 275.07 & 260.18 & 0.0006 & 5.4930 \\
\hline 109.5478 & 231.42 & 237.04 & 211.53 & 211.19 & 211.85 & 80.05 & 274.09 & 260.08 & 0.0006 & 5.4908 \\
\hline 110.0487 & 228.14 & 239.98 & 211.68 & 211.34 & 211.91 & 81.70 & 276.09 & 259.71 & 0.0006 & 5.5455 \\
\hline 110.5475 & 228.52 & 240.81 & 211.88 & 211.50 & 212.11 & 83.37 & 276.86 & 260.06 & 0.0006 & 5.5185 \\
\hline 111.0483 & 229.14 & 241.21 & 212.06 & 211.71 & 212.28 & 85.11 & 278.11 & 260.02 & 0.0004 & 5.4810 \\
\hline 111.5482 & 229.00 & 241.31 & 212.23 & 211.89 & 212.48 & 86.97 & 279.01 & 260.44 & 0.0004 & 5.5190 \\
\hline 112.0470 & 229.59 & 241.38 & 212.38 & 212.02 & 212.63 & 88.86 & 279.75 & 262.71 & 0.0001 & 5.5443 \\
\hline 112.5477 & 229.62 & 241.30 & 212.47 & 212.10 & 212.70 & 90.85 & 280.02 & 261.35 & 0.0006 & 5.5142 \\
\hline 113.0475 & 229.10 & 241.00 & 212.47 & 212.12 & 212.73 & 92.96 & 279.89 & 258.52 & 0.0006 & 5.4842 \\
\hline 113.5483 & 225.22 & 240.37 & 212.37 & 212.01 & 212.66 & 95.33 & 278.92 & 259.52 & 0.0006 & 5.5087 \\
\hline 114.0482 & 228.18 & 239.57 & 212.21 & 211.86 & 212.52 & 97.87 & 277.87 & 259.11 & 0.0002 & 5.5319 \\
\hline 114.5470 & 230.17 & 239.18 & 212.04 & 211.68 & 212.36 & 100.66 & 276.82 & 259.17 & 0.0004 & 5.4762 \\
\hline 115.0468 & 230.95 & 239.13 & 211.88 & 211.53 & 212.22 & 103.74 & 275.91 & 261.10 & 0.0004 & 5.4833 \\
\hline 115.5477 & 230.36 & 239.60 & 211.78 & 211.44 & 212.12 & 107.14 & 275.81 & 260.60 & 0.0004 & 5.5448 \\
\hline 116.0483 & 230.49 & 240.50 & 211.80 & 211.45 & 212.12 & 110.82 & 276.15 & 259.83 & 0.0004 & 5.5414 \\
\hline 116.5472 & 229.47 & 241.35 & 211.93 & 211.58 & 212.18 & 114.83 & 277.11 & 259.82 & 0.0004 & 5.5068 \\
\hline 117.0480 & 230.61 & 241.89 & 212.10 & 211.74 & 212.35 & 119.36 & 278.37 & 260.72 & 0.0002 & 5.4915 \\
\hline 117.5478 & 231.69 & 242.33 & 212.32 & 211.96 & 212.53 & 124.73 & 279.79 & 262.61 & 0.0002 & 5.5352 \\
\hline 118.0477 & 233.00 & 242.73 & 212.55 & 212.18 & 212.75 & 131.57 & 281.16 & 262.60 & 0.0002 & 5.5298 \\
\hline 118.5483 & 233.72 & 243.09 & 212.76 & 212.36 & 212.95 & 140.33 & 282.14 & 262.17 & 0.0002 & 5.4804 \\
\hline 119.0482 & 234.21 & 243.08 & 212.80 & 212.40 & 212.97 & 151.67 & 281.85 & 259.71 & 0.0002 & 5.4797 \\
\hline 119.5472 & 234.87 & 242.67 & 212.62 & 212.21 & 212.82 & 163.70 & 280.33 & 260.91 & 0.0002 & 5.5262 \\
\hline 120.0478 & 235.52 & 242.39 & 212.36 & 211.95 & 212.57 & 173.87 & 278.72 & 260.15 & 0.0000 & 5.5407 \\
\hline 120.5477 & 235.67 & 242.12 & 212.08 & 211.66 & 212.33 & 183.50 & 276.98 & 261.16 & 0.0001 & 5.4973 \\
\hline 121.0483 & 235.92 & 241.79 & 211.79 & 211.36 & 212.04 & 191.47 & 275.27 & 260.58 & 0.0001 & 5.5117 \\
\hline 121.5482 & 235.82 & 241.43 & 211.49 & 211.10 & 211.80 & 197.03 & 273.71 & 261.04 & 0.0002 & 5.5250 \\
\hline 122.0480 & 235.59 & 241.13 & 211.25 & 210.85 & 211.55 & 201.55 & 272.09 & 260.10 & 0.0003 & 5.5325 \\
\hline 122.5478 & 235.67 & 240.65 & 210.98 & 210.61 & 211.31 & 204.86 & 270.53 & 261.33 & 0.0002 & 5.4696 \\
\hline 123.0477 & 235.62 & 239.92 & 210.68 & 210.34 & 211.08 & 206.60 & 269.04 & 260.23 & 0.0002 & 5.4941 \\
\hline 123.5483 & 235.77 & 239.03 & 210.42 & 210.08 & 210.85 & 207.58 & 267.39 & 261.05 & 0.0004 & 5.5199 \\
\hline 124.0482 & 235.77 & 238.16 & 210.16 & 209.85 & 210.62 & 207.88 & 265.99 & 260.40 & 0.0000 & 5.4989 \\
\hline 124.5480 & 236.17 & 237.98 & 209.96 & 209.66 & 210.41 & 208.00 & 265.21 & 260.15 & 0.0004 & 5.4677 \\
\hline
\end{tabular}


TABLE B5.- CONTINUED.

\begin{tabular}{|c|c|c|c|c|c|c|c|c|c|c|}
\hline $\begin{array}{l}\text { Time, } \\
\text { min }\end{array}$ & $\begin{array}{c}\text { T-steam } \\
{ }^{\circ} \mathrm{C}\end{array}$ & $\begin{array}{l}\mathrm{T} 1, \\
{ }^{\circ} \mathrm{C}\end{array}$ & $\begin{array}{l}\mathrm{T} 2, \\
{ }^{\circ} \mathrm{C}\end{array}$ & $\begin{array}{l}\mathrm{T} 3, \\
{ }^{\circ} \mathrm{C}\end{array}$ & $\begin{array}{l}\mathrm{T} 4, \\
{ }^{\circ} \mathrm{C}\end{array}$ & $\begin{array}{l}\mathrm{T} 5, \\
{ }^{\circ} \mathrm{C}\end{array}$ & $\begin{array}{l}\text { Pinj, } \\
\text { psig }\end{array}$ & $\begin{array}{l}\text { Pout, } \\
\text { psig }\end{array}$ & $\begin{array}{l}\text { Qinj, } \\
\text { g/min }\end{array}$ & $\begin{array}{c}\mathrm{Vw} \\
\mathrm{ml} / \mathrm{min}\end{array}$ \\
\hline 125.0478 & 235.87 & 238.00 & 209.83 & 209.52 & 210.31 & 208.04 & 264.27 & 260.75 & 0.0001 & 5.5356 \\
\hline 125.5477 & 234.94 & 238.37 & 209.74 & 209.42 & 210.19 & 208.17 & 264.08 & 260.13 & 0.0001 & 5.5285 \\
\hline 126.0485 & 232.07 & 239.91 & 209.83 & 209.49 & 210.19 & 208.37 & 265.66 & 261.20 & 0.0002 & 5.4878 \\
\hline 126.5483 & 231.35 & 241.47 & 210.09 & 209.77 & 210.45 & 208.87 & 266.72 & 260.29 & 0.0003 & 5.5038 \\
\hline 127.0480 & 224.55 & 240.19 & 210.36 & 210.02 & 210.70 & 209.29 & 268.25 & 261.37 & 0.0001 & 5.5177 \\
\hline 127.5478 & 228.87 & 239.93 & 210.49 & 210.15 & 210.83 & 209.61 & 268.76 & 261.78 & 0.0002 & 5.5364 \\
\hline 128.0477 & 232.50 & 241.36 & 210.55 & 210.21 & 210.89 & 209.80 & 269.04 & 261.45 & 0.0002 & 5.4872 \\
\hline 128.5475 & 234.47 & 242.10 & 210.54 & 210.20 & 210.89 & 209.95 & 268.87 & 263.37 & 0.0002 & 5.5026 \\
\hline 129.0483 & 235.80 & 242.14 & 210.49 & 210.11 & 210.85 & 209.95 & 268.22 & 261.03 & 0.0003 & 5.5329 \\
\hline 129.5472 & 237.11 & 241.74 & 210.37 & 210.03 & 210.76 & 209.96 & 267.48 & 261.30 & 0.0003 & 5.4993 \\
\hline 130.0480 & 236.88 & 241.33 & 210.23 & 209.91 & 210.66 & 209.91 & 266.62 & 263.03 & 0.0003 & 5.4865 \\
\hline 130.5477 & 236.32 & 240.63 & 210.07 & 209.77 & 210.54 & 209.84 & 265.82 & 262.42 & 0.0003 & 5.5243 \\
\hline 131.0475 & 236.45 & 240.05 & 209.95 & 209.65 & 210.44 & 209.79 & 265.09 & 261.49 & 0.0006 & 5.5578 \\
\hline 131.5483 & 236.51 & 239.37 & 209.83 & 209.54 & 210.33 & 209.72 & 264.53 & 260.28 & 0.0002 & 5.5184 \\
\hline 132.0500 & 235.90 & 240.04 & 209.84 & 209.55 & 210.32 & 209.75 & 265.15 & 261.31 & 0.0002 & 5.4919 \\
\hline 132.5470 & 234.78 & 241.94 & 210.07 & 209.77 & 210.47 & 209.95 & 266.65 & 263.26 & 0.0001 & 5.5026 \\
\hline 133.0478 & 232.92 & 242.29 & 210.27 & 209.97 & 210.67 & 210.11 & 267.84 & 262.87 & 0.0003 & 5.5320 \\
\hline 133.5477 & 232.17 & 242.51 & 210.44 & 210.08 & 210.71 & 209.92 & 268.42 & 258.68 & 0.0003 & 5.5024 \\
\hline 134.0492 & 233.21 & 242.76 & 210.35 & 209.94 & 210.57 & 209.84 & 267.87 & 259.90 & 0.0006 & 5.4717 \\
\hline 134.5472 & 231.50 & 243.04 & 210.30 & 209.91 & 210.52 & 209.82 & 267.55 & 260.26 & 0.0002 & 5.5211 \\
\hline 135.0480 & 233.22 & 242.90 & 210.24 & 209.83 & 210.47 & 209.85 & 266.99 & 260.18 & 0.0002 & 5.5610 \\
\hline 135.5478 & 234.68 & 242.72 & 210.14 & 209.74 & 210.42 & 209.85 & 266.28 & 260.06 & 0.0000 & 5.5482 \\
\hline 136.0477 & 235.71 & 242.46 & 209.96 & 209.60 & 210.32 & 209.78 & 265.27 & 261.01 & 0.0002 & 5.4959 \\
\hline 136.5493 & 235.91 & 241.98 & 209.81 & 209.48 & 210.23 & 209.75 & 264.37 & 261.33 & 0.0002 & 5.4899 \\
\hline 137.0482 & 236.13 & 241.07 & 209.65 & 209.35 & 210.15 & 209.70 & 263.37 & 260.14 & 0.0002 & 5.5326 \\
\hline 137.5480 & 236.49 & 239.95 & 209.51 & 209.24 & 210.05 & 209.65 & 262.72 & 261.53 & 0.0003 & 5.5118 \\
\hline 138.0487 & 237.84 & 238.52 & 209.39 & 209.12 & 209.96 & 209.57 & 261.83 & 259.77 & 0.0000 & 5.4627 \\
\hline 138.5467 & 237.23 & 237.66 & 209.27 & 209.02 & 209.88 & 209.52 & 261.45 & 259.81 & 0.0000 & 5.5087 \\
\hline 139.0475 & 235.84 & 236.45 & 209.22 & 208.97 & 209.81 & 209.47 & 261.17 & 260.62 & 0.0002 & 5.5334 \\
\hline 139.5482 & 229.32 & 235.90 & 209.24 & 208.99 & 209.80 & 209.47 & 262.72 & 260.17 & 0.0002 & 5.5058 \\
\hline 140.0490 & 226.03 & 236.64 & 209.48 & 209.19 & 209.91 & 209.46 & 263.05 & 258.70 & 0.0002 & 5.4944 \\
\hline 140.5478 & 225.31 & 237.38 & 209.63 & 209.31 & 210.02 & 209.65 & 264.36 & 260.25 & 0.0002 & 5.5181 \\
\hline 141.0477 & 227.67 & 238.72 & 209.92 & 209.60 & 210.22 & 209.79 & 266.49 & 261.23 & 0.0002 & 5.5428 \\
\hline 141.5483 & 229.72 & 239.39 & 210.30 & 209.91 & 210.50 & 209.98 & 268.58 & 260.77 & 0.0002 & 5.5204 \\
\hline 142.0482 & 233.16 & 240.88 & 210.48 & 210.07 & 210.64 & 209.96 & 268.80 & 259.85 & 0.0001 & 5.4811 \\
\hline 142.5480 & 236.66 & 241.75 & 210.33 & 209.93 & 210.59 & 210.02 & 267.42 & 260.86 & 0.0001 & 5.5236 \\
\hline 143.0478 & 238.58 & 241.70 & 210.10 & 209.74 & 210.46 & 209.90 & 266.02 & 260.19 & 0.0001 & 5.5355 \\
\hline 143.5477 & 239.25 & 241.40 & 209.83 & 209.44 & 210.14 & 209.53 & 264.11 & 258.52 & 0.0001 & 5.4888 \\
\hline 144.0485 & 234.15 & 240.79 & 209.55 & 209.23 & 209.98 & 209.57 & 262.95 & 259.53 & 0.0001 & 5.4871 \\
\hline 144.5483 & 234.13 & 239.24 & 209.43 & 209.15 & 209.93 & 209.59 & 262.57 & 259.82 & 0.0005 & 5.5075 \\
\hline 145.0480 & 232.56 & 237.54 & 209.36 & 209.06 & 209.85 & 209.44 & 262.04 & 257.97 & 0.0002 & 5.5100 \\
\hline 145.5478 & 231.78 & 237.08 & 209.23 & 208.94 & 209.73 & 209.35 & 261.33 & 258.51 & 0.0002 & 5.5031 \\
\hline 146.0477 & 232.21 & 236.98 & 209.20 & 208.94 & 209.75 & 209.50 & 261.39 & 260.09 & 0.0002 & 5.5140 \\
\hline 146.5475 & 230.14 & 236.95 & 209.17 & 208.86 & 209.63 & 209.24 & 260.72 & 257.90 & 0.0006 & 5.5373 \\
\hline 147.0483 & 231.60 & 236.40 & 209.03 & 208.78 & 209.58 & 209.28 & 260.17 & 259.00 & 0.0007 & 5.5400 \\
\hline 147.5482 & 233.37 & 235.61 & 208.98 & 208.73 & 209.57 & 209.28 & 259.79 & 258.46 & 0.0007 & 5.4682 \\
\hline 148.0480 & 229.60 & 234.72 & 208.93 & 208.69 & 209.54 & 209.30 & 259.57 & 259.05 & 0.0007 & 5.4959 \\
\hline 148.5477 & 232.13 & 233.94 & 208.91 & 208.68 & 209.54 & 209.27 & 259.62 & 258.54 & 0.0007 & 5.5393 \\
\hline 149.0475 & 225.26 & 234.69 & 208.95 & 208.72 & 209.55 & 209.28 & 259.86 & 259.43 & 0.0007 & 5.5115 \\
\hline 149.5483 & 226.25 & 234.18 & 208.98 & 208.76 & 209.60 & 209.35 & 260.15 & 259.02 & 0.0007 & 5.5130 \\
\hline
\end{tabular}


TABLE B5.- CONTINUED.

\begin{tabular}{|c|c|c|c|c|c|c|c|c|c|c|}
\hline $\begin{array}{l}\text { Time, } \\
\text { min }\end{array}$ & $\begin{array}{c}\text { T-steam } \\
{ }^{\circ} \mathrm{C}\end{array}$ & $\begin{array}{l}\mathrm{T} 1, \\
{ }^{\circ} \mathrm{C}\end{array}$ & $\begin{array}{l}\mathrm{T} 2, \\
{ }^{\circ} \mathrm{C}\end{array}$ & $\begin{array}{l}\mathrm{T} 3, \\
{ }^{\circ} \mathrm{C}\end{array}$ & $\begin{array}{l}\mathrm{T} 4, \\
{ }^{\circ} \mathrm{C}\end{array}$ & $\begin{array}{l}\mathrm{T} 5, \\
{ }^{\circ} \mathrm{C}\end{array}$ & $\begin{array}{l}\text { Pinj, } \\
\text { psig }\end{array}$ & $\begin{array}{l}\text { Pout, } \\
\text { psig }\end{array}$ & $\begin{array}{l}\text { Qinj, } \\
\text { g/min }\end{array}$ & $\begin{array}{c}\mathrm{Vw} \\
\mathrm{ml} / \mathrm{min}\end{array}$ \\
\hline 150.0490 & 226.15 & 235.05 & 209.11 & 208.87 & 209.68 & 209.43 & 261.24 & 260.31 & 0.0001 & 5.5114 \\
\hline 150.5480 & 224.56 & 236.25 & 209.36 & 209.11 & 209.86 & 209.61 & 262.89 & 260.00 & 0.0001 & 5.5213 \\
\hline 151.0478 & 228.61 & 236.95 & 209.64 & 209.37 & 210.12 & 209.83 & 264.41 & 261.36 & 0.0001 & 5.5293 \\
\hline 151.5467 & 228.50 & 237.93 & 209.93 & 209.66 & 210.36 & 210.09 & 266.06 & 262.38 & 0.0001 & 5.4982 \\
\hline 152.0483 & 230.66 & 238.49 & 210.15 & 209.86 & 210.56 & 210.22 & 267.05 & 262.61 & 0.0001 & 5.5105 \\
\hline 152.5482 & 234.44 & 239.30 & 210.13 & 209.76 & 210.44 & 209.88 & 266.42 & 260.38 & 0.0001 & 5.5348 \\
\hline 153.0480 & 235.97 & 239.86 & 210.01 & 209.67 & 210.39 & 209.98 & 265.91 & 261.69 & 0.0001 & 5.5253 \\
\hline 153.5468 & 235.81 & 239.02 & 209.96 & 209.68 & 210.45 & 210.14 & 265.54 & 262.81 & 0.0001 & 5.4825 \\
\hline 154.0477 & 237.52 & 238.33 & 209.90 & 209.61 & 210.40 & 210.00 & 264.86 & 263.04 & 0.0001 & 5.5531 \\
\hline 154.5483 & 235.45 & 237.20 & 209.78 & 209.47 & 210.28 & 209.83 & 263.91 & 259.65 & 0.0001 & 5.5431 \\
\hline 155.0482 & 238.12 & 236.51 & 209.39 & 209.04 & 209.83 & 209.40 & 261.50 & 259.00 & 0.0001 & 5.5150 \\
\hline 155.5470 & 238.82 & 236.55 & 209.16 & 208.89 & 209.71 & 209.39 & 260.72 & 258.61 & 0.0001 & 5.4800 \\
\hline 156.0478 & 239.19 & 236.46 & 209.05 & 208.80 & 209.63 & 209.36 & 260.26 & 259.25 & 0.0001 & 5.4999 \\
\hline 156.5477 & 238.96 & 236.41 & 209.02 & 208.81 & 209.61 & 209.36 & 260.53 & 259.31 & 0.0001 & 5.5444 \\
\hline 157.0483 & 237.46 & 237.49 & 209.17 & 208.94 & 209.73 & 209.48 & 261.58 & 259.40 & 0.0001 & 5.5117 \\
\hline 157.5473 & 229.62 & 237.35 & 209.39 & 209.12 & 209.89 & 209.62 & 263.07 & 260.36 & 0.0001 & 5.4936 \\
\hline 158.0480 & 227.94 & 235.84 & 209.63 & 209.34 & 210.06 & 209.74 & 264.35 & 260.61 & 0.0001 & 5.5264 \\
\hline 158.5478 & 224.92 & 233.42 & 209.90 & 209.58 & 210.26 & 209.94 & 265.87 & 261.53 & 0.0001 & 5.5193 \\
\hline 159.0485 & 232.42 & 233.27 & 210.01 & 209.64 & 210.28 & 209.71 & 266.06 & 259.29 & 0.0000 & 5.4683 \\
\hline 159.5483 & 239.90 & 237.25 & 209.93 & 209.52 & 210.20 & 209.75 & 265.42 & 260.14 & 0.0000 & 5.4878 \\
\hline 160.0482 & 243.15 & 240.16 & 209.85 & 209.49 & 210.18 & 209.76 & 264.81 & 259.81 & 0.0000 & 5.5532 \\
\hline 160.5480 & 243.57 & 240.91 & 209.72 & 209.40 & 210.15 & 209.78 & 264.18 & 260.37 & 0.0000 & 5.5355 \\
\hline 161.0478 & 239.41 & 239.95 & 209.62 & 209.33 & 210.12 & 209.80 & 263.61 & 260.28 & 0.0000 & 5.5040 \\
\hline 161.5477 & 240.22 & 238.06 & 209.54 & 209.27 & 210.07 & 209.75 & 263.04 & 260.03 & 0.0000 & 5.4949 \\
\hline 162.0485 & 235.43 & 235.87 & 209.45 & 209.20 & 210.02 & 209.74 & 262.52 & 260.07 & 0.0000 & 5.5109 \\
\hline 162.5473 & 234.50 & 232.71 & 209.42 & 209.19 & 210.05 & 209.83 & 262.46 & 260.52 & 0.0000 & 5.5331 \\
\hline 163.0480 & 229.49 & 229.78 & 209.41 & 209.23 & 210.14 & 209.80 & 262.60 & 259.79 & 0.0000 & 5.4636 \\
\hline 163.5478 & 225.00 & 226.20 & 209.39 & 209.22 & 210.07 & 209.84 & 262.36 & 260.91 & 0.0000 & 5.4804 \\
\hline 164.0468 & 225.65 & 223.65 & 209.38 & 209.20 & 210.06 & 209.88 & 262.37 & 261.02 & 0.0001 & 5.5277 \\
\hline 164.5475 & 220.80 & 223.79 & 209.42 & 209.22 & 210.07 & 209.85 & 262.64 & 260.78 & 0.0001 & 5.5388 \\
\hline 165.0483 & 220.16 & 226.61 & 209.68 & 209.44 & 210.20 & 209.96 & 264.59 & 262.32 & 0.0001 & 5.4867 \\
\hline 165.5482 & 222.32 & 227.38 & 209.95 & 209.74 & 210.49 & 210.27 & 266.21 & 262.95 & 0.0001 & 5.4998 \\
\hline 166.0480 & 221.87 & 228.41 & 210.15 & 209.88 & 210.62 & 210.33 & 267.16 & 262.94 & 0.0001 & 5.5167 \\
\hline 166.5468 & 221.59 & 228.22 & 210.21 & 209.92 & 210.68 & 210.34 & 267.10 & 262.63 & 0.0001 & 5.5136 \\
\hline 167.0475 & 224.05 & 228.26 & 210.16 & 209.89 & 210.66 & 210.29 & 266.64 & 261.38 & 0.0001 & 5.4782 \\
\hline 167.5483 & 224.36 & 228.41 & 209.92 & 209.56 & 210.27 & 209.76 & 264.56 & 258.96 & 0.0001 & 5.5320 \\
\hline 168.0482 & 227.16 & 228.89 & 209.60 & 209.29 & 210.05 & 209.69 & 263.12 & 260.20 & 0.0001 & 5.5245 \\
\hline 168.5488 & 231.76 & 230.99 & 209.50 & 209.21 & 210.02 & 209.71 & 262.85 & 259.19 & 0.0001 & 5.4831 \\
\hline 169.0468 & 237.75 & 234.63 & 209.50 & 209.21 & 210.04 & 209.77 & 262.91 & 259.63 & 0.0001 & 5.5001 \\
\hline 169.5477 & 242.02 & 238.26 & 209.45 & 209.15 & 209.94 & 209.63 & 262.62 & 260.60 & 0.0001 & 5.5363 \\
\hline 170.0483 & 242.85 & 240.16 & 209.49 & 209.22 & 210.01 & 209.80 & 263.27 & 259.67 & 0.0001 & 5.5374 \\
\hline 170.5482 & 242.34 & 240.78 & 209.52 & 209.21 & 210.00 & 209.71 & 263.12 & 260.54 & 0.0001 & 5.4760 \\
\hline 171.0480 & 240.96 & 240.14 & 209.58 & 209.31 & 210.06 & 209.66 & 263.59 & 259.64 & 0.0001 & 5.4801 \\
\hline 171.5468 & 235.99 & 238.96 & 209.62 & 209.35 & 210.12 & 209.83 & 263.94 & 259.64 & 0.0004 & 5.5275 \\
\hline 172.0477 & 233.71 & 236.69 & 209.68 & 209.41 & 210.16 & 209.87 & 264.21 & 260.49 & 0.0004 & 5.4894 \\
\hline 172.5483 & 228.20 & 233.97 & 209.75 & 209.48 & 210.25 & 209.95 & 264.53 & 261.24 & 0.0004 & 5.4838 \\
\hline 173.0482 & 226.95 & 230.59 & 209.79 & 209.54 & 210.33 & 210.02 & 264.62 & 260.74 & 0.0004 & 5.5033 \\
\hline 173.5470 & 226.39 & 228.00 & 209.72 & 209.51 & 210.30 & 210.03 & 264.35 & 261.16 & 0.0004 & 5.5293 \\
\hline 174.0478 & 228.66 & 227.52 & 209.76 & 209.53 & 210.34 & 210.05 & 264.47 & 261.46 & 0.0004 & 5.4808 \\
\hline 174.5477 & 230.76 & 227.97 & 209.70 & 209.46 & 210.27 & 209.97 & 264.05 & 260.66 & 0.0004 & 5.4943 \\
\hline
\end{tabular}


TABLE B5.- CONTINUED.

\begin{tabular}{|c|c|c|c|c|c|c|c|c|c|c|}
\hline $\begin{array}{l}\text { Time, } \\
\text { min }\end{array}$ & $\begin{array}{c}\text { T-steam } \\
{ }^{\circ} \mathrm{C}\end{array}$ & $\begin{array}{l}\mathrm{T} 1, \\
{ }^{\circ} \mathrm{C}\end{array}$ & $\begin{array}{l}\mathrm{T} 2, \\
{ }^{\circ} \mathrm{C}\end{array}$ & $\begin{array}{l}\mathrm{T} 3, \\
{ }^{\circ} \mathrm{C}\end{array}$ & $\begin{array}{l}\mathrm{T} 4, \\
{ }^{\circ} \mathrm{C}\end{array}$ & $\begin{array}{l}\mathrm{T} 5, \\
{ }^{\circ} \mathrm{C}\end{array}$ & $\begin{array}{l}\text { Pinj, } \\
\text { psig }\end{array}$ & $\begin{array}{l}\text { Pout, } \\
\text { psig }\end{array}$ & $\begin{array}{l}\text { Qinj, } \\
\text { g/min }\end{array}$ & $\begin{array}{c}\mathrm{Vw} \\
\mathrm{ml} / \mathrm{min}\end{array}$ \\
\hline 175.0483 & 227.65 & 227.10 & 209.67 & 209.43 & 210.24 & 210.03 & 263.86 & 261.23 & 0.0000 & 5.5254 \\
\hline 175.5482 & 228.98 & 226.42 & 209.76 & 209.53 & 210.33 & 210.03 & 265.26 & 260.46 & 0.0001 & 5.4891 \\
\hline 176.0480 & 236.17 & 229.47 & 209.93 & 209.62 & 210.38 & 210.02 & 265.61 & 260.13 & 0.0001 & 5.4510 \\
\hline 176.5488 & 239.26 & 232.45 & 209.86 & 209.57 & 210.36 & 210.13 & 265.25 & 261.57 & 0.0001 & 5.4825 \\
\hline 177.0468 & 236.72 & 233.87 & 209.88 & 209.60 & 210.38 & 210.12 & 265.30 & 262.27 & 0.0001 & 5.5074 \\
\hline 177.5475 & 235.63 & 233.68 & 209.89 & 209.58 & 210.34 & 209.98 & 265.12 & 260.79 & 0.0001 & 5.4848 \\
\hline 178.0482 & 232.75 & 232.11 & 209.80 & 209.55 & 210.34 & 210.07 & 264.76 & 260.92 & 0.0001 & 5.4949 \\
\hline 178.5480 & 233.31 & 230.09 & 209.72 & 209.47 & 210.27 & 209.97 & 264.22 & 260.50 & 0.0001 & 5.5298 \\
\hline 179.0478 & 228.98 & 228.91 & 209.74 & 209.53 & 210.35 & 210.15 & 264.63 & 261.50 & 0.0001 & 5.4859 \\
\hline 179.5468 & 230.58 & 228.18 & 209.59 & 209.19 & 209.93 & 209.16 & 262.57 & 255.83 & 0.0001 & 5.4756 \\
\hline 180.0493 & 230.28 & 229.05 & 209.07 & 208.68 & 209.41 & 208.91 & 260.11 & 255.68 & 0.0001 & 5.5096 \\
\hline 180.5483 & 229.84 & 229.61 & 208.86 & 208.54 & 209.29 & 208.95 & 259.71 & 255.96 & 0.0001 & 5.4979 \\
\hline 181.0480 & 231.22 & 229.25 & 208.92 & 208.64 & 209.37 & 209.08 & 260.28 & 256.72 & 0.0001 & 5.4556 \\
\hline 181.5470 & 232.48 & 229.83 & 209.07 & 208.80 & 209.54 & 209.29 & 261.32 & 258.11 & 0.0001 & 5.4970 \\
\hline 182.0477 & 237.02 & 231.47 & 209.25 & 209.00 & 209.74 & 209.49 & 262.45 & 258.61 & 0.0001 & 5.5312 \\
\hline 182.5475 & 236.34 & 232.28 & 209.42 & 209.13 & 209.90 & 209.63 & 262.98 & 259.55 & 0.0001 & 5.4780 \\
\hline 183.0483 & 232.08 & 231.06 & 209.37 & 209.07 & 209.84 & 209.48 & 262.47 & 259.13 & 0.0001 & 5.4887 \\
\hline 183.5482 & 234.36 & 229.55 & 209.27 & 209.02 & 209.80 & 209.52 & 261.83 & 258.51 & 0.0001 & 5.5190 \\
\hline 184.0480 & 234.35 & 229.48 & 209.17 & 208.92 & 209.74 & 209.45 & 261.16 & 258.23 & 0.0001 & 5.4862 \\
\hline 184.5477 & 236.70 & 229.38 & 209.06 & 208.85 & 209.71 & 209.49 & 260.64 & 258.82 & 0.0001 & 5.4800 \\
\hline 185.0475 & 236.15 & 228.63 & 209.00 & 208.78 & 209.66 & 209.43 & 260.18 & 258.21 & 0.0001 & 5.5414 \\
\hline 185.5483 & 229.16 & 227.11 & 208.88 & 208.66 & 209.52 & 209.29 & 259.49 & 258.12 & 0.0001 & 5.5252 \\
\hline 186.0472 & 232.24 & 225.72 & 208.79 & 208.61 & 209.49 & 209.29 & 259.22 & 257.95 & 0.0001 & 5.4938 \\
\hline 186.5480 & 232.78 & 226.64 & 208.76 & 208.60 & 209.46 & 209.28 & 259.16 & 257.91 & 0.0001 & 5.4893 \\
\hline 187.0478 & 233.67 & 227.49 & 208.77 & 208.59 & 209.47 & 209.27 & 259.38 & 257.83 & 0.0001 & 5.5153 \\
\hline 187.5467 & 232.78 & 229.18 & 209.06 & 208.84 & 209.61 & 209.42 & 261.65 & 258.42 & 0.0001 & 5.4903 \\
\hline 188.0483 & 232.89 & 229.66 & 209.32 & 209.06 & 209.83 & 209.60 & 262.44 & 258.89 & 0.0001 & 5.4889 \\
\hline 188.5482 & 231.67 & 230.14 & 209.43 & 209.18 & 209.95 & 209.71 & 263.39 & 260.58 & 0.0001 & 5.5135 \\
\hline 189.0470 & 233.42 & 230.37 & 209.52 & 209.29 & 210.06 & 209.86 & 263.82 & 260.56 & 0.0001 & 5.5166 \\
\hline 189.5478 & 233.08 & 230.83 & 209.67 & 209.46 & 210.23 & 209.97 & 264.61 & 260.21 & 0.0001 & 5.5140 \\
\hline 190.0485 & 233.68 & 230.12 & 209.71 & 209.48 & 210.28 & 210.07 & 264.40 & 261.53 & 0.0001 & 5.4745 \\
\hline 190.5483 & 233.29 & 229.17 & 209.75 & 209.55 & 210.38 & 210.18 & 264.79 & 261.94 & 0.0001 & 5.4914 \\
\hline 191.0482 & 228.23 & 228.96 & 209.70 & 209.44 & 210.22 & 209.81 & 264.70 & 258.58 & 0.0001 & 5.5189 \\
\hline 191.5480 & 230.02 & 223.20 & 209.40 & 209.10 & 209.91 & 209.57 & 262.09 & 258.93 & 0.0001 & 5.4867 \\
\hline 192.0478 & 235.96 & 223.01 & 209.25 & 209.02 & 209.84 & 209.57 & 261.72 & 258.54 & 0.0001 & 5.4855 \\
\hline 192.5477 & 240.17 & 226.86 & 209.06 & 208.70 & 209.47 & 208.74 & 259.95 & 253.32 & 0.0001 & 5.5611 \\
\hline 193.0483 & 239.87 & 231.02 & 208.52 & 208.20 & 208.99 & 208.67 & 257.49 & 255.76 & 0.0001 & 5.5177 \\
\hline 193.5473 & 240.90 & 232.93 & 208.47 & 208.24 & 209.03 & 208.83 & 257.88 & 256.45 & 0.0001 & 5.4661 \\
\hline 194.0480 & 237.37 & 233.61 & 208.62 & 208.39 & 209.16 & 208.95 & 258.97 & 256.72 & 0.0001 & 5.4737 \\
\hline 194.5478 & 233.17 & 232.35 & 208.79 & 208.54 & 209.31 & 209.08 & 259.89 & 256.80 & 0.0001 & 5.5271 \\
\hline 195.0477 & 231.17 & 230.81 & 208.92 & 208.67 & 209.44 & 209.21 & 260.46 & 257.26 & 0.0001 & 5.4835 \\
\hline 195.5483 & 230.44 & 229.60 & 209.02 & 208.78 & 209.57 & 209.32 & 261.08 & 258.02 & 0.0001 & 5.4871 \\
\hline 196.0482 & 233.67 & 229.19 & 208.99 & 208.70 & 209.49 & 209.15 & 260.19 & 257.05 & 0.0001 & 5.5056 \\
\hline 196.5472 & 233.53 & 229.68 & 208.85 & 208.58 & 209.40 & 209.13 & 259.35 & 257.53 & 0.0001 & 5.5296 \\
\hline 197.0478 & 233.07 & 228.90 & 208.75 & 208.50 & 209.34 & 209.03 & 258.68 & 256.21 & 0.0001 & 5.4836 \\
\hline 197.5477 & 232.18 & 228.42 & 208.61 & 208.39 & 209.23 & 209.00 & 258.21 & 256.19 & 0.0001 & 5.4871 \\
\hline 198.0485 & 234.07 & 227.53 & 208.50 & 208.31 & 209.15 & 208.99 & 257.72 & 257.51 & 0.0001 & 5.4978 \\
\hline 198.5473 & 233.52 & 227.25 & 208.47 & 208.28 & 209.14 & 208.90 & 257.57 & 255.99 & 0.0001 & 5.4763 \\
\hline 199.0480 & 231.87 & 227.81 & 208.50 & 208.30 & 209.16 & 208.98 & 258.30 & 256.28 & 0.0001 & 5.4754 \\
\hline 199.5478 & 233.33 & 229.03 & 208.84 & 208.63 & 209.40 & 209.20 & 260.22 & 256.88 & 0.0001 & 5.5446 \\
\hline
\end{tabular}


TABLE B5.- CONTINUED.

\begin{tabular}{|c|c|c|c|c|c|c|c|c|c|c|}
\hline $\begin{array}{c}\text { Time, } \\
\text { min }\end{array}$ & $\begin{array}{c}\text { T-steam } \\
{ }^{\circ} \mathrm{C}\end{array}$ & $\begin{array}{l}\mathrm{T} 1, \\
{ }^{\circ} \mathrm{C}\end{array}$ & $\begin{array}{l}\mathrm{T} 2, \\
{ }^{\circ} \mathrm{C}\end{array}$ & $\begin{array}{l}\mathrm{T} 3, \\
{ }^{\circ} \mathrm{C}\end{array}$ & $\begin{array}{l}\mathrm{T} 4, \\
{ }^{\circ} \mathrm{C}\end{array}$ & $\begin{array}{l}\mathrm{T} 5, \\
{ }^{\circ} \mathrm{C}\end{array}$ & $\begin{array}{l}\text { Pinj, } \\
\text { psig }\end{array}$ & $\begin{array}{c}\text { Pout, } \\
\text { psig }\end{array}$ & $\begin{array}{l}\text { Qinj, } \\
\text { g/min }\end{array}$ & $\begin{array}{c}\mathrm{Vw} \\
\mathrm{ml} / \mathrm{min}\end{array}$ \\
\hline 200.0477 & 233.41 & 229.68 & 208.99 & 208.76 & 209.55 & 209.30 & 260.84 & 257.41 & 0.0001 & 5.5326 \\
\hline 200.5475 & 236.80 & 231.10 & 209.10 & 208.87 & 209.66 & 209.43 & 261.55 & 258.95 & 0.0001 & 5.4814 \\
\hline 201.0483 & 235.48 & 231.63 & 209.22 & 208.98 & 209.79 & 209.56 & 262.00 & 259.44 & 0.0005 & 5.4868 \\
\hline 201.5482 & 233.01 & 231.29 & 209.26 & 209.04 & 209.85 & 209.63 & 262.05 & 259.15 & 0.0005 & 5.5063 \\
\hline 202.0480 & 234.34 & 230.24 & 209.23 & 209.01 & 209.84 & 209.66 & 261.79 & 260.21 & 0.0005 & 5.5178 \\
\hline 202.5477 & 230.25 & 229.58 & 209.21 & 208.96 & 209.80 & 209.54 & 261.55 & 258.57 & 0.0005 & 5.4866 \\
\hline 203.0475 & 232.52 & 227.96 & 209.00 & 208.77 & 209.61 & 209.33 & 260.34 & 257.26 & 0.0005 & 5.4923 \\
\hline 203.5473 & 231.24 & 228.29 & 208.89 & 208.65 & 209.49 & 209.24 & 259.72 & 257.34 & 0.0005 & 5.5226 \\
\hline 204.0472 & 232.48 & 228.51 & 208.89 & 208.71 & 209.55 & 209.43 & 260.32 & 258.97 & 0.0005 & 5.5432 \\
\hline 204.5480 & 231.25 & 228.55 & 209.13 & 208.93 & 209.75 & 209.59 & 261.79 & 258.77 & 0.0005 & 5.4689 \\
\hline 205.0478 & 230.52 & 228.50 & 209.37 & 209.15 & 209.94 & 209.71 & 263.20 & 258.73 & 0.0005 & 5.4753 \\
\hline 205.5477 & 234.06 & 229.13 & 209.51 & 209.26 & 210.02 & 209.78 & 263.83 & 260.52 & 0.0005 & 5.5340 \\
\hline 206.0483 & 234.49 & 230.85 & 209.68 & 209.47 & 210.22 & 210.00 & 265.05 & 260.48 & 0.0005 & 5.5360 \\
\hline 206.5482 & 234.98 & 231.61 & 209.83 & 209.58 & 210.33 & 210.12 & 265.73 & 261.71 & 0.0005 & 5.4740 \\
\hline 207.0470 & 235.61 & 231.73 & 209.96 & 209.67 & 210.43 & 210.09 & 266.24 & 260.59 & 0.0005 & 5.4850 \\
\hline 207.5478 & 233.36 & 231.95 & 209.97 & 209.70 & 210.45 & 210.20 & 266.46 & 261.87 & 0.0005 & 5.5408 \\
\hline 208.0477 & 231.09 & 230.50 & 210.01 & 209.74 & 210.47 & 209.97 & 266.33 & 258.08 & 0.0005 & 5.5207 \\
\hline 208.5475 & 232.56 & 230.13 & 209.67 & 209.30 & 210.05 & 209.55 & 263.49 & 257.90 & 0.0005 & 5.4541 \\
\hline 209.0482 & 235.76 & 229.99 & 209.32 & 209.03 & 209.86 & 209.55 & 262.02 & 258.01 & 0.0005 & 5.4949 \\
\hline 209.5480 & 238.65 & 230.35 & 209.18 & 208.95 & 209.81 & 209.59 & 261.54 & 258.49 & 0.0005 & 5.5145 \\
\hline 210.0487 & 238.65 & 230.36 & 209.13 & 208.92 & 209.78 & 209.58 & 261.10 & 258.86 & 0.0005 & 5.4949 \\
\hline 210.5477 & 236.24 & 229.52 & 209.07 & 208.89 & 209.78 & 209.64 & 261.22 & 259.45 & 0.0005 & 5.4947 \\
\hline 211.0475 & 230.80 & 227.72 & 209.03 & 208.89 & 209.77 & 209.63 & 260.71 & 259.04 & 0.0005 & 5.5295 \\
\hline 211.5492 & 229.84 & 226.58 & 209.04 & 208.88 & 209.76 & 209.60 & 260.96 & 259.00 & 0.0005 & 5.5127 \\
\hline 212.0480 & 230.86 & 225.80 & 209.08 & 208.90 & 209.76 & 209.60 & 261.08 & 259.26 & 0.0005 & 5.4778 \\
\hline 212.5470 & 232.34 & 226.41 & 209.16 & 209.02 & 209.84 & 209.73 & 262.47 & 259.85 & 0.0005 & 5.4904 \\
\hline 213.0477 & 232.36 & 229.09 & 209.47 & 209.27 & 210.08 & 209.92 & 263.88 & 260.28 & 0.0005 & 5.5365 \\
\hline 213.5483 & 234.43 & 229.74 & 209.55 & 209.31 & 210.12 & 209.87 & 264.12 & 259.75 & 0.0005 & 5.5312 \\
\hline 214.0482 & 236.12 & 230.37 & 209.57 & 209.34 & 210.14 & 209.91 & 263.93 & 260.49 & 0.0005 & 5.5064 \\
\hline 214.5472 & 234.92 & 230.89 & 209.68 & 209.45 & 210.22 & 209.84 & 264.44 & 259.75 & 0.0005 & 5.4929 \\
\hline 215.0478 & 232.19 & 230.27 & 209.56 & 209.33 & 210.13 & 209.85 & 263.94 & 259.69 & 0.0005 & 5.5337 \\
\hline 215.5468 & 231.76 & 228.86 & 209.41 & 209.10 & 209.89 & 209.48 & 262.28 & 257.61 & 0.0005 & 5.4990 \\
\hline 216.0485 & 233.04 & 229.06 & 209.14 & 208.86 & 209.68 & 209.39 & 261.14 & 258.01 & 0.0005 & 5.4873 \\
\hline 216.5483 & 236.27 & 229.82 & 209.04 & 208.83 & 209.63 & 209.42 & 260.81 & 257.28 & 0.0005 & 5.4789 \\
\hline 217.0480 & 234.34 & 230.60 & 209.03 & 208.79 & 209.64 & 209.42 & 260.94 & 257.80 & 0.0005 & 5.5374 \\
\hline 217.5470 & 232.12 & 230.37 & 209.03 & 208.82 & 209.64 & 209.46 & 261.13 & 258.57 & 0.0005 & 5.5248 \\
\hline 218.0477 & 231.68 & 229.87 & 209.07 & 208.86 & 209.70 & 209.49 & 261.27 & 258.58 & 0.0005 & 5.4868 \\
\hline 218.5467 & 232.56 & 229.77 & 209.17 & 208.95 & 209.78 & 209.56 & 261.84 & 258.88 & 0.0005 & 5.4947 \\
\hline 219.0473 & 233.13 & 229.74 & 209.21 & 209.01 & 209.80 & 209.59 & 262.02 & 258.72 & 0.0005 & 5.5357 \\
\hline 219.5472 & 227.19 & 228.39 & 209.29 & 209.07 & 209.86 & 209.65 & 262.44 & 258.54 & 0.0005 & 5.5210 \\
\hline 220.0480 & 230.83 & 226.85 & 209.29 & 209.10 & 209.90 & 209.65 & 262.55 & 258.17 & 0.0005 & 5.4884 \\
\hline 220.5477 & 237.39 & 228.84 & 209.32 & 209.09 & 209.91 & 209.66 & 262.55 & 259.12 & 0.0005 & 5.4944 \\
\hline 221.0475 & 237.88 & 231.48 & 209.36 & 209.14 & 209.95 & 209.72 & 262.84 & 259.48 & 0.0005 & 5.5212 \\
\hline 221.5483 & 239.39 & 232.13 & 209.36 & 209.15 & 209.97 & 209.77 & 262.72 & 259.27 & 0.0005 & 5.5328 \\
\hline 222.0482 & 238.75 & 232.15 & 209.33 & 209.14 & 209.96 & 209.78 & 262.67 & 259.22 & 0.0005 & 5.4798 \\
\hline 222.5480 & 232.25 & 230.55 & 209.37 & 209.18 & 210.00 & 209.80 & 262.93 & 259.37 & 0.0005 & 5.4934 \\
\hline 223.0478 & 229.43 & 227.74 & 209.43 & 209.25 & 210.08 & 209.88 & 263.48 & 260.25 & 0.0005 & 5.5172 \\
\hline 223.5485 & 226.92 & 226.19 & 209.63 & 209.44 & 210.21 & 209.97 & 264.87 & 260.50 & 0.0005 & 5.4870 \\
\hline 224.0483 & 230.26 & 225.85 & 209.69 & 209.46 & 210.23 & 210.00 & 264.79 & 259.87 & 0.0005 & 5.4809 \\
\hline 224.5490 & 231.39 & 226.84 & 209.77 & 209.54 & 210.36 & 210.15 & 265.30 & 261.27 & 0.0005 & 5.5207 \\
\hline
\end{tabular}


TABLE B5.- CONTINUED.

\begin{tabular}{|c|c|c|c|c|c|c|c|c|c|c|}
\hline $\begin{array}{l}\text { Time, } \\
\text { min }\end{array}$ & $\begin{array}{c}\text { T-steam } \\
{ }^{\circ} \mathrm{C}\end{array}$ & $\begin{array}{l}\mathrm{T} 1, \\
{ }^{\circ} \mathrm{C}\end{array}$ & $\begin{array}{l}\mathrm{T} 2, \\
{ }^{\circ} \mathrm{C}\end{array}$ & $\begin{array}{l}\mathrm{T} 3, \\
{ }^{\circ} \mathrm{C}\end{array}$ & $\begin{array}{l}\mathrm{T} 4, \\
{ }^{\circ} \mathrm{C}\end{array}$ & $\begin{array}{l}\mathrm{T} 5, \\
{ }^{\circ} \mathrm{C}\end{array}$ & $\begin{array}{l}\text { Pinj, } \\
\text { psig }\end{array}$ & $\begin{array}{l}\text { Pout, } \\
\text { psig }\end{array}$ & $\begin{array}{l}\text { Qinj, } \\
\text { g/min }\end{array}$ & $\begin{array}{c}\mathrm{Vw} \\
\mathrm{ml} / \mathrm{min}\end{array}$ \\
\hline 225.0480 & 232.36 & 227.14 & 209.78 & 209.52 & 210.31 & 209.97 & 264.79 & 259.62 & 0.0002 & 5.5343 \\
\hline 225.5468 & 235.47 & 227.96 & 209.62 & 209.37 & 210.19 & 209.91 & 264.00 & 259.57 & 0.0002 & 5.4993 \\
\hline 226.0477 & 233.00 & 228.63 & 209.52 & 209.28 & 210.12 & 209.89 & 263.49 & 259.29 & 0.0002 & 5.4849 \\
\hline 226.5483 & 231.23 & 227.40 & 209.43 & 209.26 & 210.10 & 209.97 & 263.07 & 260.80 & 0.0002 & 5.5117 \\
\hline 227.0482 & 229.52 & 225.19 & 209.44 & 209.28 & 210.14 & 209.98 & 263.23 & 259.90 & 0.0002 & 5.5162 \\
\hline 227.5480 & 232.87 & 223.49 & 209.43 & 209.27 & 210.14 & 210.00 & 263.03 & 261.11 & 0.0002 & 5.5048 \\
\hline 228.0478 & 230.64 & 224.82 & 209.57 & 209.38 & 210.20 & 210.08 & 264.54 & 261.37 & 0.0002 & 5.5123 \\
\hline 228.5477 & 231.74 & 226.26 & 209.76 & 209.53 & 210.33 & 210.08 & 265.61 & 261.69 & 0.0002 & 5.5295 \\
\hline 229.0483 & 233.16 & 227.27 & 209.96 & 209.76 & 210.55 & 210.30 & 266.69 & 259.81 & 0.0002 & 5.5347 \\
\hline 229.5482 & 233.99 & 228.19 & 210.02 & 209.79 & 210.57 & 210.36 & 266.96 & 262.41 & 0.0002 & 5.4915 \\
\hline 230.0490 & 232.72 & 228.53 & 210.04 & 209.81 & 210.58 & 210.29 & 266.69 & 261.86 & 0.0002 & 5.4747 \\
\hline 230.5478 & 232.58 & 227.53 & 209.89 & 209.62 & 210.44 & 210.15 & 265.46 & 261.12 & 0.0002 & 5.5375 \\
\hline 231.0477 & 232.39 & 227.16 & 209.80 & 209.59 & 210.39 & 210.18 & 265.16 & 260.81 & 0.0002 & 5.5453 \\
\hline 231.5483 & 230.18 & 226.83 & 209.72 & 209.50 & 210.36 & 210.13 & 264.64 & 260.77 & 0.0002 & 5.4952 \\
\hline 232.0482 & 230.27 & 225.42 & 209.51 & 209.26 & 210.10 & 209.81 & 263.23 & 259.39 & 0.0002 & 5.4990 \\
\hline 232.5480 & 231.12 & 224.76 & 209.37 & 209.17 & 210.05 & 209.87 & 262.91 & 259.76 & 0.0002 & 5.5150 \\
\hline 233.0470 & 231.71 & 224.96 & 209.39 & 209.18 & 210.04 & 209.82 & 262.97 & 259.74 & 0.0002 & 5.5252 \\
\hline 233.5477 & 232.17 & 226.22 & 209.49 & 209.29 & 210.11 & 209.97 & 264.47 & 261.09 & 0.0002 & 5.4758 \\
\hline 234.0485 & 231.99 & 227.14 & 209.78 & 209.56 & 210.37 & 210.21 & 265.68 & 261.51 & 0.0002 & 5.5015 \\
\hline 234.5473 & 231.37 & 228.00 & 209.89 & 209.64 & 210.41 & 210.16 & 266.48 & 261.22 & 0.0002 & 5.5195 \\
\hline 235.0480 & 228.78 & 222.01 & 210.04 & 209.81 & 210.54 & 210.29 & 266.98 & 262.23 & 0.0002 & 5.5423 \\
\hline 235.5478 & 233.17 & 222.23 & 210.06 & 209.83 & 210.60 & 210.37 & 266.86 & 261.72 & 0.0002 & 5.4898 \\
\hline 236.0477 & 236.54 & 225.87 & 210.00 & 209.76 & 210.57 & 210.32 & 266.35 & 261.40 & 0.0002 & 5.4988 \\
\hline 236.5475 & 236.14 & 227.54 & 209.88 & 209.64 & 210.43 & 210.06 & 265.25 & 259.40 & 0.0002 & 5.5346 \\
\hline 237.0492 & 235.53 & 227.60 & 209.58 & 209.33 & 210.15 & 209.83 & 263.38 & 259.12 & 0.0002 & 5.5479 \\
\hline 237.5472 & 237.17 & 226.19 & 209.35 & 209.15 & 210.01 & 209.85 & 262.46 & 259.49 & 0.0002 & 5.4937 \\
\hline 238.0480 & 234.83 & 225.52 & 209.32 & 209.14 & 210.00 & 209.86 & 262.61 & 260.49 & 0.0002 & 5.4947 \\
\hline 238.5477 & 234.15 & 225.07 & 209.32 & 209.15 & 210.02 & 209.84 & 262.40 & 259.33 & 0.0002 & 5.5263 \\
\hline 239.0475 & 235.27 & 225.35 & 209.31 & 209.13 & 210.03 & 209.88 & 262.65 & 260.22 & 0.0002 & 5.5206 \\
\hline 239.5483 & 231.03 & 225.98 & 209.55 & 209.37 & 210.18 & 210.03 & 264.75 & 260.14 & 0.0002 & 5.4765 \\
\hline 240.0490 & 228.42 & 225.29 & 209.84 & 209.63 & 210.40 & 210.25 & 266.19 & 262.17 & 0.0002 & 5.4871 \\
\hline 240.5488 & 231.07 & 224.74 & 210.04 & 209.83 & 210.60 & 210.42 & 267.23 & 262.21 & 0.0002 & 5.5337 \\
\hline 241.0478 & 233.27 & 226.07 & 210.14 & 209.92 & 210.73 & 210.48 & 267.47 & 262.46 & 0.0002 & 5.5180 \\
\hline 241.5477 & 233.24 & 227.36 & 210.14 & 209.93 & 210.73 & 210.48 & 267.13 & 262.39 & 0.0002 & 5.4927 \\
\hline 242.0473 & 236.57 & 227.60 & 209.99 & 209.66 & 210.44 & 209.68 & 265.26 & 256.54 & 0.0002 & 5.4862 \\
\hline 242.5482 & 232.85 & 227.69 & 209.31 & 208.99 & 209.81 & 209.49 & 261.70 & 258.15 & 0.0002 & 5.5286 \\
\hline 243.0470 & 232.36 & 226.32 & 209.23 & 209.14 & 210.00 & 209.28 & 263.11 & 262.10 & 0.0002 & 0.3457 \\
\hline
\end{tabular}


TABLE B6. PRESSURE AND TEMPERATURE DATA FOR RUN 8 (PETROLEUM DISTILLATE:STEAM = 5:100).

\begin{tabular}{|c|c|c|c|c|c|c|c|c|c|c|}
\hline $\begin{array}{c}\text { Time, } \\
\text { min }\end{array}$ & $\begin{array}{c}\text { T-steam } \\
{ }^{\circ} \mathrm{C}\end{array}$ & $\begin{array}{l}\mathrm{T} 1, \\
{ }^{\circ} \mathrm{C}\end{array}$ & $\begin{array}{l}\mathrm{T} 2, \\
{ }^{\circ} \mathrm{C}\end{array}$ & $\begin{array}{l}\mathrm{T} 3, \\
{ }^{\circ} \mathrm{C}\end{array}$ & $\begin{array}{l}\mathrm{T} 4, \\
{ }^{\circ} \mathrm{C}\end{array}$ & $\begin{array}{l}\mathrm{T} 5, \\
{ }^{\circ} \mathrm{C}\end{array}$ & $\begin{array}{l}\text { Pinj, } \\
\text { psig }\end{array}$ & $\begin{array}{l}\text { Pout, } \\
\text { Psig }\end{array}$ & $\begin{array}{l}\text { Qinj, } \\
\text { g/min }\end{array}$ & $\begin{array}{c}\text { Vw } \\
\mathrm{ml} / \mathrm{min}\end{array}$ \\
\hline 0.0540 & 152.68 & 55.98 & 51.62 & 53.26 & 52.80 & 48.76 & 258.12 & 260.44 & 0.0010 & 5.5658 \\
\hline 0.5483 & 201.09 & 111.52 & 51.59 & 53.21 & 52.70 & 48.74 & 256.93 & 259.90 & 0.0015 & 5.5138 \\
\hline 1.0480 & 208.10 & 192.56 & 51.55 & 53.34 & 52.79 & 48.79 & 256.99 & 259.88 & 0.0015 & 5.5198 \\
\hline 1.5470 & 211.47 & 202.24 & 51.56 & 53.37 & 52.84 & 48.82 & 257.88 & 260.27 & 0.0011 & 5.5610 \\
\hline 2.0477 & 215.05 & 205.17 & 51.54 & 53.42 & 52.91 & 48.87 & 256.19 & 259.17 & 0.0016 & 5.5764 \\
\hline 2.5475 & 219.03 & 205.54 & 51.53 & 53.38 & 52.94 & 48.86 & 257.81 & 259.74 & 0.0010 & 5.5619 \\
\hline 3.0483 & 222.55 & 206.43 & 51.54 & 53.33 & 52.97 & 48.89 & 260.57 & 259.37 & 0.0009 & 5.5328 \\
\hline 3.5482 & 225.37 & 207.73 & 54.87 & 53.35 & 52.97 & 48.91 & 263.02 & 259.20 & 0.0015 & 5.5274 \\
\hline 4.0480 & 225.98 & 208.74 & 58.91 & 53.36 & 52.96 & 48.92 & 264.14 & 259.16 & 0.0008 & 5.5733 \\
\hline 4.5468 & 227.94 & 209.51 & 60.30 & 53.33 & 52.97 & 48.91 & 266.86 & 259.16 & 0.0009 & 5.5729 \\
\hline 5.0475 & 230.08 & 210.39 & 60.16 & 53.33 & 52.97 & 48.92 & 269.26 & 259.14 & 0.0016 & 5.5478 \\
\hline 5.5483 & 232.23 & 211.39 & 59.53 & 53.26 & 52.98 & 48.94 & 273.74 & 259.20 & 0.0017 & 5.5103 \\
\hline 6.0472 & 233.94 & 213.12 & 58.73 & 53.18 & 52.99 & 48.93 & 275.46 & 259.16 & 0.0016 & 5.5407 \\
\hline 6.5480 & 234.70 & 213.56 & 57.60 & 53.17 & 52.96 & 48.92 & 276.41 & 259.16 & 0.0010 & 5.5643 \\
\hline 7.0478 & 236.77 & 215.49 & 56.70 & 53.21 & 52.98 & 48.94 & 276.86 & 259.11 & 0.0013 & 5.5645 \\
\hline 7.5477 & 237.65 & 217.02 & 56.11 & 53.22 & 53.01 & 48.93 & 279.54 & 259.17 & 0.0012 & 5.5417 \\
\hline 8.0475 & 235.17 & 217.03 & 55.83 & 53.23 & 53.00 & 48.94 & 285.87 & 259.36 & 0.0013 & 5.5219 \\
\hline 8.5482 & 232.89 & 215.32 & 55.96 & 53.22 & 53.03 & 48.95 & 293.11 & 259.43 & 0.0011 & 5.5291 \\
\hline 9.0480 & 234.13 & 213.62 & 56.45 & 53.23 & 53.10 & 48.99 & 300.52 & 259.50 & 0.0016 & 5.5780 \\
\hline 9.5468 & 233.32 & 213.40 & 57.07 & 53.25 & 53.21 & 49.00 & 300.62 & 259.50 & 0.0013 & 5.5643 \\
\hline 10.0477 & 233.21 & 213.32 & 57.71 & 53.24 & 53.26 & 49.01 & 298.66 & 259.46 & 0.0013 & 5.5147 \\
\hline 10.5483 & 235.98 & 214.30 & 58.27 & 53.23 & 53.31 & 49.00 & 298.67 & 259.38 & 0.0011 & 5.5093 \\
\hline 11.0482 & 237.24 & 215.72 & 58.74 & 53.23 & 53.32 & 49.04 & 300.22 & 259.40 & 0.0012 & 5.5559 \\
\hline 11.5480 & 236.84 & 215.59 & 59.03 & 53.24 & 53.33 & 49.03 & 296.47 & 259.36 & 0.0008 & 5.5774 \\
\hline 12.0468 & 233.11 & 214.35 & 59.16 & 53.25 & 53.33 & 49.04 & 294.20 & 259.28 & 0.0018 & 5.5251 \\
\hline 12.5477 & 232.39 & 212.90 & 59.51 & 53.26 & 53.34 & 49.09 & 295.06 & 259.28 & 0.0015 & 5.5209 \\
\hline 13.0483 & 227.89 & 213.43 & 60.02 & 53.26 & 53.35 & 49.12 & 293.60 & 259.22 & 0.0016 & 5.5293 \\
\hline 13.5482 & 221.81 & 214.40 & 60.13 & 53.27 & 53.37 & 49.14 & 288.73 & 259.26 & 0.0012 & 5.5527 \\
\hline 14.0472 & 223.85 & 214.56 & 60.65 & 53.26 & 53.40 & 49.19 & 290.56 & 259.27 & 0.0008 & 5.5696 \\
\hline 14.5478 & 245.41 & 215.11 & 61.42 & 53.26 & 53.41 & 49.22 & 290.90 & 259.23 & 0.0011 & 5.5227 \\
\hline 15.0477 & 261.87 & 216.44 & 62.21 & 53.25 & 53.42 & 49.27 & 290.85 & 259.16 & 0.0008 & 5.5203 \\
\hline 15.5483 & 266.45 & 218.48 & 63.15 & 53.22 & 53.42 & 49.26 & 292.38 & 259.13 & 0.0003 & 5.5359 \\
\hline 16.0482 & 271.43 & 219.74 & 63.80 & 53.22 & 53.39 & 49.31 & 292.46 & 259.39 & 0.0009 & 5.5556 \\
\hline 16.5480 & 270.72 & 219.22 & 64.48 & 53.21 & 53.42 & 49.36 & 293.41 & 258.07 & 0.0013 & 5.5370 \\
\hline 17.0470 & 268.68 & 219.77 & 65.26 & 53.23 & 53.42 & 49.42 & 294.14 & 259.61 & 0.0010 & 5.5140 \\
\hline 17.5477 & 264.86 & 218.76 & 66.05 & 53.24 & 53.43 & 49.47 & 295.16 & 258.42 & 0.0010 & 5.5270 \\
\hline 18.0485 & 261.00 & 218.93 & 67.01 & 53.23 & 53.42 & 49.50 & 295.31 & 258.33 & 0.0006 & 5.5422 \\
\hline 18.5492 & 256.67 & 219.84 & 68.06 & 53.26 & 53.45 & 49.53 & 296.44 & 258.35 & 0.0010 & 5.5490 \\
\hline 19.0472 & 249.99 & 219.28 & 69.05 & 53.30 & 53.45 & 49.58 & 296.62 & 258.14 & 0.0012 & 5.5147 \\
\hline 19.5478 & 244.28 & 218.92 & 70.05 & 53.29 & 53.46 & 49.61 & 293.98 & 258.49 & 0.0010 & 5.5094 \\
\hline 20.0487 & 236.26 & 218.60 & 71.00 & 53.30 & 53.48 & 49.65 & 292.07 & 257.95 & 0.0003 & 5.5248 \\
\hline 20.5475 & 231.04 & 217.04 & 71.99 & 53.36 & 53.49 & 49.68 & 290.70 & 257.94 & 0.0009 & 5.5628 \\
\hline 21.0483 & 221.03 & 215.86 & 73.08 & 53.39 & 53.46 & 49.71 & 290.84 & 257.91 & 0.0007 & 5.5653 \\
\hline 21.5482 & 220.13 & 214.98 & 74.32 & 53.42 & 53.50 & 49.73 & 291.56 & 258.65 & 0.0008 & 5.5115 \\
\hline 22.0480 & 221.41 & 215.19 & 75.61 & 53.45 & 53.51 & 49.78 & 293.00 & 257.49 & 0.0016 & 5.5259 \\
\hline 22.5468 & 224.43 & 215.63 & 76.84 & 53.51 & 53.51 & 49.83 & 293.93 & 258.29 & 0.0010 & 5.5362 \\
\hline 23.0467 & 229.73 & 216.29 & 78.17 & 53.56 & 53.54 & 49.87 & 295.86 & 257.78 & 0.0000 & 5.5729 \\
\hline 23.5483 & 234.67 & 217.19 & 79.46 & 53.59 & 53.53 & 49.92 & 298.17 & 257.36 & 0.0008 & 5.5539 \\
\hline 24.0482 & 235.16 & 218.59 & 80.86 & 53.66 & 53.57 & 49.95 & 300.53 & 259.78 & 0.0011 & 5.5127 \\
\hline 24.5480 & 239.64 & 220.37 & 82.16 & 53.73 & 53.58 & 50.02 & 300.59 & 259.91 & 0.0007 & 5.5281 \\
\hline
\end{tabular}


TABLE B6.- CONTINUED.

\begin{tabular}{|c|c|c|c|c|c|c|c|c|c|c|}
\hline $\begin{array}{c}\text { Time, } \\
\text { min }\end{array}$ & $\begin{array}{c}\text { T-steam } \\
{ }^{\circ} \mathrm{C}\end{array}$ & $\begin{array}{l}\mathrm{T} 1, \\
{ }^{\circ} \mathrm{C}\end{array}$ & $\begin{array}{l}\mathrm{T} 2, \\
{ }^{\circ} \mathrm{C}\end{array}$ & $\begin{array}{l}\mathrm{T} 3, \\
{ }^{\circ} \mathrm{C}\end{array}$ & $\begin{array}{l}\mathrm{T} 4, \\
{ }^{\circ} \mathrm{C}\end{array}$ & $\begin{array}{l}\mathrm{T} 5, \\
{ }^{\circ} \mathrm{C}\end{array}$ & $\begin{array}{l}\text { Pinj, } \\
\text { psig }\end{array}$ & $\begin{array}{c}\text { Pout, } \\
\text { psig }\end{array}$ & $\begin{array}{l}\text { Qinj, } \\
\text { g/min }\end{array}$ & $\begin{array}{c}\mathrm{Vw} \\
\mathrm{ml} / \mathrm{min}\end{array}$ \\
\hline 25.0468 & 242.54 & 220.92 & 83.62 & 53.83 & 53.58 & 50.06 & 301.55 & 259.62 & 0.0007 & 5.5637 \\
\hline 25.5477 & 242.59 & 221.83 & 85.20 & 53.94 & 53.59 & 50.11 & 301.63 & 259.96 & 0.0004 & 5.5635 \\
\hline 26.0483 & 235.04 & 221.16 & 87.05 & 54.03 & 53.61 & 50.16 & 301.17 & 259.74 & 0.0008 & 5.5289 \\
\hline 26.5482 & 231.69 & 220.12 & 89.13 & 54.14 & 53.60 & 50.20 & 300.80 & 259.72 & 0.0001 & 5.5126 \\
\hline 27.0480 & 223.90 & 217.65 & 91.50 & 54.25 & 53.62 & 50.23 & 299.77 & 259.77 & 0.0003 & 5.5254 \\
\hline 27.5468 & 221.89 & 216.77 & 94.17 & 54.42 & 53.64 & 50.29 & 298.71 & 259.86 & 0.0013 & 5.5625 \\
\hline 28.0477 & 222.37 & 216.39 & 97.12 & 54.54 & 53.65 & 50.32 & 298.50 & 259.28 & 0.0005 & 5.5477 \\
\hline 28.5483 & 227.57 & 216.51 & 100.31 & 54.71 & 53.67 & 50.35 & 297.76 & 259.60 & 0.0003 & 5.5236 \\
\hline 29.0492 & 233.78 & 217.57 & 103.73 & 54.90 & 53.66 & 50.41 & 297.98 & 259.89 & 0.0004 & 5.5078 \\
\hline 29.5472 & 240.70 & 219.46 & 107.14 & 55.07 & 53.70 & 50.44 & 296.84 & 259.98 & 0.0004 & 5.5537 \\
\hline 30.0478 & 248.03 & 221.53 & 110.87 & 55.29 & 53.70 & 50.50 & 295.81 & 256.99 & 0.0003 & 5.5636 \\
\hline 30.5477 & 249.19 & 222.10 & 114.80 & 55.54 & 53.73 & 50.53 & 296.57 & 259.38 & 0.0007 & 5.5408 \\
\hline 31.0483 & 249.07 & 222.37 & 118.82 & 55.80 & 53.73 & 50.57 & 295.42 & 259.78 & 0.0005 & 5.5092 \\
\hline 31.5482 & 246.12 & 220.22 & 122.91 & 56.10 & 53.73 & 50.59 & 294.10 & 259.51 & 0.0004 & 5.5184 \\
\hline 32.0472 & 241.98 & 219.82 & 127.02 & 56.44 & 53.73 & 50.62 & 293.04 & 257.23 & 0.0006 & 5.5621 \\
\hline 32.5478 & 236.30 & 218.48 & 131.19 & 56.86 & 53.74 & 50.64 & 292.42 & 259.46 & 0.0007 & 5.5505 \\
\hline 33.0477 & 228.70 & 217.53 & 135.29 & 57.35 & 53.78 & 50.69 & 291.70 & 259.47 & 0.0005 & 5.5285 \\
\hline 33.5483 & 222.16 & 216.24 & 139.25 & 57.88 & 53.80 & 50.73 & 290.94 & 259.77 & 0.0003 & 5.5001 \\
\hline 34.0473 & 219.34 & 214.90 & 143.03 & 58.49 & 53.81 & 50.77 & 290.21 & 257.57 & 0.0001 & 5.5321 \\
\hline 34.5480 & 219.26 & 214.68 & 146.56 & 59.09 & 53.83 & 50.77 & 289.98 & 260.25 & 0.0001 & 5.5853 \\
\hline 35.0478 & 220.03 & 214.80 & 149.97 & 59.81 & 53.89 & 50.80 & 290.77 & 257.43 & 0.0003 & 5.5493 \\
\hline 35.5468 & 223.22 & 215.25 & 153.23 & 60.57 & 53.91 & 50.86 & 291.04 & 259.86 & 0.0002 & 5.5171 \\
\hline 36.0493 & 227.83 & 215.82 & 156.35 & 61.34 & 53.94 & 50.90 & 291.37 & 258.99 & 0.0003 & 5.5164 \\
\hline 36.5483 & 235.75 & 216.40 & 159.37 & 62.18 & 54.00 & 50.92 & 292.24 & 257.40 & 0.0003 & 5.5561 \\
\hline 37.0480 & 243.92 & 218.92 & 162.33 & 63.05 & 54.02 & 50.95 & 294.57 & 259.92 & 0.0002 & 5.5587 \\
\hline 37.5478 & 247.12 & 220.08 & 165.09 & 63.97 & 54.04 & 50.95 & 295.67 & 259.18 & 0.0001 & 5.5373 \\
\hline 38.0477 & 246.92 & 220.08 & 167.81 & 64.94 & 54.07 & 50.97 & 295.95 & 259.42 & 0.0001 & 5.5309 \\
\hline 38.5475 & 242.18 & 219.92 & 170.36 & 65.96 & 54.11 & 51.03 & 295.73 & 259.65 & 0.0003 & 5.5308 \\
\hline 39.0483 & 238.15 & 218.67 & 172.79 & 67.00 & 54.17 & 51.07 & 295.53 & 258.97 & 0.0003 & 5.5520 \\
\hline 39.5482 & 233.31 & 217.70 & 175.04 & 68.06 & 54.23 & 51.11 & 294.42 & 260.01 & 0.0003 & 5.5796 \\
\hline 40.0480 & 224.47 & 216.72 & 176.98 & 69.15 & 54.29 & 51.13 & 293.37 & 258.83 & 0.0003 & 5.5599 \\
\hline 40.5477 & 221.07 & 215.58 & 178.63 & 70.28 & 54.32 & 51.17 & 293.34 & 259.43 & 0.0006 & 5.5341 \\
\hline 41.0475 & 219.87 & 215.18 & 180.11 & 71.43 & 54.40 & 51.19 & 293.16 & 259.66 & 0.0003 & 5.5364 \\
\hline 41.5483 & 219.98 & 215.16 & 181.51 & 72.64 & 54.46 & 51.23 & 293.41 & 260.11 & 0.0003 & 5.5216 \\
\hline 42.0482 & 221.23 & 215.35 & 182.80 & 73.88 & 54.52 & 51.25 & 294.35 & 258.88 & 0.0003 & 5.5680 \\
\hline 42.5470 & 226.35 & 215.92 & 184.15 & 75.14 & 54.62 & 51.29 & 295.53 & 259.08 & 0.0001 & 5.5516 \\
\hline 43.0478 & 233.20 & 216.91 & 185.41 & 76.46 & 54.69 & 51.33 & 296.48 & 259.02 & 0.0003 & 5.5325 \\
\hline 43.5467 & 240.90 & 217.52 & 186.61 & 77.79 & 54.77 & 51.35 & 296.73 & 258.14 & 0.0003 & 5.5072 \\
\hline 44.0473 & 249.08 & 220.49 & 187.74 & 79.14 & 54.87 & 51.37 & 296.99 & 259.84 & 0.0002 & 5.5400 \\
\hline 44.5482 & 252.26 & 222.07 & 188.84 & 80.51 & 54.97 & 51.39 & 297.24 & 259.78 & 0.0003 & 5.5765 \\
\hline 45.0488 & 252.05 & 222.50 & 189.90 & 81.96 & 55.06 & 51.41 & 297.81 & 257.34 & 0.0003 & 5.5880 \\
\hline 45.5478 & 248.75 & 221.95 & 190.90 & 83.44 & 55.14 & 51.43 & 298.01 & 257.18 & 0.0003 & 5.5076 \\
\hline 46.0477 & 245.72 & 220.62 & 191.84 & 84.97 & 55.24 & 51.45 & 298.84 & 259.65 & 0.0003 & 5.5112 \\
\hline 46.5483 & 241.31 & 220.05 & 192.75 & 86.54 & 55.38 & 51.48 & 298.54 & 259.63 & 0.0003 & 5.5473 \\
\hline 47.0482 & 233.64 & 218.41 & 193.54 & 88.17 & 55.49 & 51.50 & 298.86 & 260.06 & 0.0002 & 5.5534 \\
\hline 47.5480 & 226.25 & 217.37 & 194.39 & 89.87 & 55.61 & 51.52 & 299.17 & 259.56 & 0.0002 & 5.5610 \\
\hline 48.0478 & 222.85 & 216.80 & 195.23 & 91.58 & 55.74 & 51.54 & 299.26 & 259.80 & 0.0003 & 5.5133 \\
\hline 48.5467 & 221.96 & 216.50 & 195.99 & 93.43 & 55.88 & 51.58 & 299.18 & 260.01 & 0.0004 & 5.5142 \\
\hline 49.0475 & 223.70 & 216.70 & 196.76 & 95.31 & 56.04 & 51.60 & 299.60 & 259.82 & 0.0001 & 5.5615 \\
\hline 49.5482 & 229.72 & 217.55 & 197.55 & 97.28 & 56.19 & 51.63 & 301.17 & 259.49 & 0.0003 & 5.5679 \\
\hline
\end{tabular}


TABLE B6.- CONTINUED.

\begin{tabular}{|c|c|c|c|c|c|c|c|c|c|c|}
\hline $\begin{array}{l}\text { Time, } \\
\text { min }\end{array}$ & $\begin{array}{c}\text { T-steam } \\
{ }^{\circ} \mathrm{C}\end{array}$ & $\begin{array}{l}\mathrm{T} 1, \\
{ }^{\circ} \mathrm{C}\end{array}$ & $\begin{array}{l}\mathrm{T} 2, \\
{ }^{\circ} \mathrm{C}\end{array}$ & $\begin{array}{l}\mathrm{T} 3, \\
{ }^{\circ} \mathrm{C}\end{array}$ & $\begin{array}{l}\mathrm{T} 4, \\
{ }^{\circ} \mathrm{C}\end{array}$ & $\begin{array}{l}\mathrm{T} 5, \\
{ }^{\circ} \mathrm{C}\end{array}$ & $\begin{array}{l}\text { Pinj, } \\
\text { psig }\end{array}$ & $\begin{array}{l}\text { Pout, } \\
\text { psig }\end{array}$ & $\begin{array}{l}\text { Qinj, } \\
\text { g/min }\end{array}$ & $\begin{array}{c}\text { Vw } \\
\mathrm{ml} / \mathrm{min}\end{array}$ \\
\hline 50.0480 & 235.79 & 218.16 & 198.40 & 99.45 & 56.36 & 51.65 & 302.04 & 259.78 & 0.0001 & 5.5516 \\
\hline 50.5478 & 243.50 & 219.72 & 199.14 & 101.79 & 56.54 & 51.69 & 303.52 & 259.60 & 0.0003 & 5.5277 \\
\hline 51.0477 & 248.01 & 221.28 & 199.87 & 104.33 & 56.73 & 51.71 & 303.18 & 256.77 & 0.0001 & 5.5178 \\
\hline 51.5483 & 244.26 & 221.77 & 200.61 & 107.20 & 56.93 & 51.76 & 304.15 & 259.90 & 0.0000 & 5.5411 \\
\hline 52.0473 & 242.44 & 221.31 & 201.46 & 110.32 & 57.12 & 51.76 & 303.80 & 259.04 & 0.0003 & 5.5647 \\
\hline 52.5480 & 239.43 & 220.20 & 202.36 & 113.67 & 57.34 & 51.80 & 304.00 & 259.36 & 0.0003 & 5.5503 \\
\hline 53.0478 & 235.03 & 219.33 & 203.33 & 117.28 & 57.53 & 51.83 & 303.78 & 259.35 & 0.0003 & 5.5171 \\
\hline 53.5477 & 227.39 & 218.85 & 204.15 & 121.10 & 57.76 & 51.87 & 303.22 & 259.51 & 0.0008 & 5.5103 \\
\hline 54.0485 & 223.09 & 217.54 & 205.02 & 125.15 & 58.01 & 51.91 & 304.13 & 259.12 & 0.0003 & 5.5392 \\
\hline 54.5483 & 221.54 & 216.92 & 205.90 & 129.25 & 58.26 & 51.93 & 302.60 & 257.84 & 0.0003 & 5.5585 \\
\hline 55.0490 & 221.49 & 216.80 & 206.60 & 133.23 & 58.52 & 51.98 & 302.49 & 259.81 & 0.0003 & 5.5422 \\
\hline 55.5470 & 222.79 & 216.92 & 207.41 & 136.90 & 58.77 & 52.02 & 302.52 & 259.58 & 0.0003 & 5.5001 \\
\hline 56.0477 & 227.39 & 217.31 & 208.60 & 140.70 & 59.06 & 52.04 & 302.90 & 259.59 & 0.0000 & 5.5229 \\
\hline 56.5475 & 233.48 & 217.89 & 209.84 & 144.68 & 59.35 & 52.07 & 304.22 & 258.20 & 0.0003 & 5.5652 \\
\hline 57.0483 & 240.67 & 219.04 & 210.70 & 148.48 & 59.67 & 52.11 & 305.51 & 259.84 & 0.0001 & 5.5576 \\
\hline 57.5482 & 247.92 & 221.21 & 211.58 & 152.16 & 60.00 & 52.15 & 305.83 & 259.36 & 0.0003 & 5.5365 \\
\hline 58.0480 & 249.88 & 222.83 & 212.47 & 155.92 & 60.35 & 52.20 & 305.99 & 257.63 & 0.0003 & 5.5169 \\
\hline 58.5477 & 247.88 & 222.83 & 213.42 & 159.56 & 60.69 & 52.24 & 307.83 & 259.79 & 0.0003 & 5.5260 \\
\hline 59.0475 & 242.34 & 222.14 & 214.16 & 162.94 & 61.08 & 52.30 & 306.74 & 259.59 & 0.0003 & 5.5598 \\
\hline 59.5473 & 239.17 & 220.55 & 214.45 & 165.86 & 61.44 & 52.33 & 306.07 & 258.26 & 0.0003 & 5.5595 \\
\hline 60.0482 & 232.62 & 219.79 & 214.67 & 168.68 & 61.82 & 52.37 & 305.80 & 259.69 & 0.0001 & 5.5381 \\
\hline 60.5480 & 226.81 & 218.48 & 214.98 & 171.38 & 62.23 & 52.43 & 305.85 & 259.83 & 0.0003 & 5.5123 \\
\hline 61.0468 & 222.63 & 217.47 & 215.07 & 173.62 & 62.63 & 52.48 & 305.86 & 259.38 & 0.0002 & 5.5376 \\
\hline 61.5477 & 223.01 & 217.47 & 215.36 & 176.06 & 63.07 & 52.52 & 305.80 & 259.87 & 0.0003 & 5.5607 \\
\hline 62.0483 & 225.55 & 217.82 & 215.56 & 178.24 & 63.53 & 52.56 & 306.74 & 257.17 & 0.0003 & 5.5475 \\
\hline 62.5482 & 228.72 & 218.34 & 215.80 & 180.19 & 63.99 & 52.65 & 307.71 & 259.62 & 0.0003 & 5.5157 \\
\hline 63.0480 & 236.18 & 218.58 & 215.95 & 182.04 & 64.51 & 52.71 & 307.51 & 259.67 & 0.0003 & 5.5211 \\
\hline 63.5487 & 243.11 & 220.93 & 216.17 & 184.31 & 65.00 & 52.77 & 308.56 & 259.86 & 0.0003 & 5.5462 \\
\hline 64.0477 & 252.03 & 225.63 & 216.28 & 186.56 & 65.54 & 52.83 & 308.51 & 259.41 & 0.0002 & 5.5527 \\
\hline 64.5483 & 256.75 & 227.82 & 216.34 & 188.91 & 66.07 & 52.90 & 308.74 & 259.56 & 0.0003 & 5.5510 \\
\hline 65.0482 & 257.71 & 228.45 & 216.46 & 190.89 & 66.65 & 52.96 & 309.14 & 259.80 & 0.0003 & 5.5032 \\
\hline 65.5480 & 256.39 & 228.15 & 216.43 & 192.60 & 67.22 & 53.04 & 308.51 & 259.15 & 0.0001 & 5.5228 \\
\hline 66.0478 & 252.75 & 227.69 & 216.50 & 194.24 & 67.81 & 53.11 & 309.16 & 259.20 & 0.0003 & 5.5597 \\
\hline 66.5477 & 247.23 & 226.25 & 216.40 & 195.69 & 68.44 & 53.19 & 308.28 & 259.56 & 0.0003 & 5.5465 \\
\hline 67.0483 & 241.09 & 224.57 & 216.46 & 197.29 & 69.09 & 53.27 & 308.49 & 259.57 & 0.0003 & 5.5224 \\
\hline 67.5473 & 229.43 & 222.41 & 216.49 & 198.37 & 69.77 & 53.33 & 308.35 & 259.61 & 0.0003 & 5.5074 \\
\hline 68.0472 & 224.74 & 219.28 & 216.44 & 199.92 & 70.46 & 53.42 & 307.41 & 259.65 & 0.0003 & 5.5340 \\
\hline 68.5478 & 222.89 & 217.89 & 216.36 & 201.11 & 71.18 & 53.48 & 307.17 & 257.69 & 0.0004 & 5.5462 \\
\hline 69.0477 & 223.59 & 217.97 & 216.33 & 201.94 & 71.96 & 53.56 & 306.78 & 259.81 & 0.0004 & 5.5565 \\
\hline 69.5483 & 226.82 & 218.00 & 216.44 & 203.05 & 72.75 & 53.65 & 306.93 & 257.89 & 0.0003 & 5.5075 \\
\hline 70.0482 & 233.88 & 218.92 & 216.41 & 203.65 & 73.59 & 53.75 & 306.93 & 259.81 & 0.0002 & 5.5041 \\
\hline 70.5480 & 239.09 & 220.78 & 216.47 & 204.32 & 74.46 & 53.83 & 307.33 & 259.52 & 0.0003 & 5.5545 \\
\hline 71.0478 & 246.67 & 222.22 & 216.53 & 204.86 & 75.39 & 53.93 & 307.34 & 259.39 & 0.0004 & 5.5591 \\
\hline 71.5477 & 252.50 & 226.36 & 216.59 & 205.98 & 76.31 & 54.05 & 307.56 & 259.59 & 0.0002 & 5.5300 \\
\hline 72.0485 & 254.21 & 227.39 & 216.54 & 206.50 & 77.31 & 54.14 & 306.88 & 258.94 & 0.0003 & 5.5149 \\
\hline 72.5473 & 253.39 & 227.50 & 216.51 & 208.05 & 78.32 & 54.26 & 306.89 & 259.63 & 0.0003 & 5.5279 \\
\hline 73.0480 & 250.47 & 227.08 & 216.50 & 208.63 & 79.37 & 54.36 & 306.96 & 257.69 & 0.0003 & 5.5682 \\
\hline 73.5478 & 246.51 & 226.01 & 216.44 & 208.94 & 80.46 & 54.48 & 306.40 & 256.81 & 0.0004 & 5.5288 \\
\hline 74.0477 & 239.85 & 224.55 & 216.42 & 209.20 & 81.61 & 54.62 & 306.00 & 259.21 & 0.0004 & 5.5046 \\
\hline 74.5475 & 231.45 & 222.73 & 216.34 & 209.47 & 82.82 & 54.74 & 305.67 & 258.84 & 0.0003 & 5.5059 \\
\hline
\end{tabular}


TABLE B6.- CONTINUED.

\begin{tabular}{|c|c|c|c|c|c|c|c|c|c|c|}
\hline $\begin{array}{l}\text { Time, } \\
\text { min }\end{array}$ & $\begin{array}{c}\text { T-steam } \\
{ }^{\circ} \mathrm{C}\end{array}$ & $\begin{array}{l}\mathrm{T} 1, \\
{ }^{\circ} \mathrm{C}\end{array}$ & $\begin{array}{l}\mathrm{T} 2, \\
{ }^{\circ} \mathrm{C}\end{array}$ & $\begin{array}{l}\mathrm{T} 3, \\
{ }^{\circ} \mathrm{C}\end{array}$ & $\begin{array}{l}\mathrm{T} 4, \\
{ }^{\circ} \mathrm{C}\end{array}$ & $\begin{array}{l}\mathrm{T} 5, \\
{ }^{\circ} \mathrm{C}\end{array}$ & $\begin{array}{l}\text { Pinj, } \\
\text { psig }\end{array}$ & $\begin{array}{l}\text { Pout, } \\
\text { psig }\end{array}$ & $\begin{array}{l}\text { Qinj, } \\
\text { g/min }\end{array}$ & $\begin{array}{c}\mathrm{Vw} \\
\mathrm{ml} / \mathrm{min}\end{array}$ \\
\hline 75.0483 & 224.23 & 219.23 & 216.37 & 210.07 & 84.12 & 54.88 & 305.04 & 259.17 & 0.0003 & 5.5251 \\
\hline 75.5482 & 222.37 & 217.48 & 216.29 & 210.56 & 85.45 & 55.02 & 304.36 & 257.27 & 0.0003 & 5.5404 \\
\hline 76.0480 & 223.22 & 217.47 & 216.22 & 210.51 & 86.84 & 55.16 & 304.43 & 259.52 & 0.0003 & 5.5367 \\
\hline 76.5468 & 227.92 & 217.55 & 216.21 & 210.91 & 88.32 & 55.34 & 304.02 & 259.52 & 0.0003 & 5.4882 \\
\hline 77.0475 & 234.21 & 218.24 & 216.13 & 211.21 & 89.87 & 55.50 & 303.42 & 259.54 & 0.0003 & 5.5189 \\
\hline 77.5483 & 236.67 & 219.37 & 216.22 & 211.45 & 91.54 & 55.68 & 304.38 & 257.07 & 0.0003 & 5.5421 \\
\hline 78.0472 & 243.43 & 223.69 & 216.28 & 211.77 & 93.31 & 55.88 & 304.29 & 259.55 & 0.0005 & 5.5539 \\
\hline 78.5480 & 251.15 & 226.10 & 216.26 & 211.91 & 95.20 & 56.06 & 303.83 & 259.25 & 0.0003 & 5.5137 \\
\hline 79.0478 & 253.12 & 227.04 & 216.21 & 212.07 & 97.19 & 56.25 & 303.74 & 259.69 & 0.0003 & 5.5055 \\
\hline 79.5467 & 252.73 & 227.21 & 216.23 & 212.33 & 99.29 & 56.47 & 303.73 & 259.28 & 0.0002 & 5.5461 \\
\hline 80.0483 & 250.66 & 226.32 & 216.14 & 212.32 & 101.58 & 56.69 & 302.88 & 259.21 & 0.0001 & 5.5654 \\
\hline 80.5482 & 245.91 & 225.56 & 216.11 & 212.47 & 104.00 & 56.94 & 302.46 & 259.05 & 0.0003 & 5.5236 \\
\hline 81.0480 & 241.20 & 224.15 & 216.04 & 212.59 & 106.61 & 57.17 & 302.07 & 258.24 & 0.0003 & 5.5101 \\
\hline 81.5478 & 233.61 & 221.49 & 215.96 & 212.76 & 109.40 & 57.43 & 301.57 & 259.70 & 0.0003 & 5.5275 \\
\hline 82.0477 & 224.91 & 219.22 & 215.86 & 212.76 & 112.34 & 57.68 & 300.80 & 259.66 & 0.0003 & 5.5506 \\
\hline 82.5483 & 222.04 & 217.07 & 215.80 & 212.82 & 115.47 & 57.96 & 300.17 & 258.97 & 0.0003 & 5.5478 \\
\hline 83.0482 & 221.98 & 216.64 & 215.71 & 212.80 & 118.68 & 58.23 & 299.82 & 259.68 & 0.0003 & 5.5177 \\
\hline 83.5472 & 223.54 & 216.83 & 215.74 & 212.91 & 121.87 & 58.52 & 300.50 & 258.71 & 0.0000 & 5.5125 \\
\hline 84.0468 & 230.62 & 217.23 & 215.74 & 213.02 & 125.25 & 58.85 & 299.87 & 258.95 & 0.0003 & 5.5497 \\
\hline 84.5477 & 237.95 & 218.20 & 215.72 & 212.96 & 128.60 & 59.16 & 299.61 & 259.41 & 0.0003 & 5.5509 \\
\hline 85.0483 & 246.35 & 222.01 & 215.72 & 212.99 & 131.99 & 59.48 & 299.77 & 259.38 & 0.0002 & 5.5332 \\
\hline 85.5492 & 254.68 & 225.93 & 215.71 & 213.14 & 135.40 & 59.85 & 299.51 & 259.35 & 0.0003 & 5.5050 \\
\hline 86.0472 & 258.66 & 228.07 & 215.67 & 213.18 & 138.78 & 60.21 & 299.41 & 259.24 & 0.0003 & 5.5178 \\
\hline 86.5478 & 258.87 & 229.17 & 215.62 & 213.15 & 142.15 & 60.58 & 298.73 & 259.44 & 0.0004 & 5.5367 \\
\hline 87.0477 & 257.11 & 229.14 & 215.52 & 213.21 & 145.42 & 60.97 & 298.31 & 259.48 & 0.0003 & 5.5363 \\
\hline 87.5483 & 250.73 & 228.34 & 215.46 & 213.15 & 148.53 & 61.37 & 297.79 & 259.36 & 0.0003 & 5.5108 \\
\hline 88.0473 & 247.83 & 227.06 & 215.34 & 213.16 & 151.54 & 61.82 & 296.94 & 259.38 & 0.0003 & 5.5173 \\
\hline 88.5480 & 242.96 & 225.73 & 215.26 & 213.09 & 154.26 & 62.26 & 296.43 & 259.30 & 0.0003 & 5.5518 \\
\hline 89.0478 & 234.62 & 223.80 & 215.25 & 213.08 & 156.80 & 62.72 & 296.16 & 259.31 & 0.0003 & 5.5502 \\
\hline 89.5477 & 225.47 & 220.84 & 215.16 & 213.07 & 159.12 & 63.22 & 295.71 & 259.28 & 0.0003 & 5.5151 \\
\hline 90.0485 & 221.65 & 217.36 & 215.07 & 213.04 & 161.31 & 63.74 & 294.83 & 259.14 & 0.0003 & 5.4956 \\
\hline 90.5473 & 220.57 & 215.84 & 214.95 & 212.94 & 163.45 & 64.26 & 294.34 & 259.12 & 0.0002 & 5.5451 \\
\hline 91.0480 & 221.04 & 215.64 & 214.92 & 212.90 & 165.47 & 64.80 & 294.27 & 259.20 & 0.0003 & 5.5320 \\
\hline 91.5478 & 224.66 & 215.63 & 214.87 & 212.91 & 167.37 & 65.37 & 293.85 & 259.10 & 0.0003 & 5.5159 \\
\hline 92.0477 & 230.99 & 216.63 & 214.81 & 212.84 & 169.20 & 65.98 & 293.37 & 259.10 & 0.0004 & 5.5035 \\
\hline 92.5467 & 238.09 & 218.25 & 214.82 & 212.88 & 171.00 & 66.60 & 293.32 & 259.06 & 0.0003 & 5.5297 \\
\hline 93.0473 & 247.26 & 221.98 & 214.81 & 212.89 & 172.60 & 67.23 & 293.43 & 259.01 & 0.0001 & 5.5391 \\
\hline 93.5482 & 255.51 & 226.09 & 214.76 & 212.86 & 174.15 & 67.88 & 292.96 & 259.01 & 0.0007 & 5.5341 \\
\hline 94.0480 & 260.37 & 229.21 & 214.73 & 212.86 & 175.72 & 68.57 & 293.08 & 258.96 & 0.0003 & 5.4975 \\
\hline 94.5477 & 261.54 & 230.89 & 214.67 & 212.81 & 177.26 & 69.25 & 292.57 & 258.90 & 0.0003 & 5.5034 \\
\hline 95.0475 & 260.13 & 231.49 & 214.62 & 212.78 & 178.77 & 69.99 & 292.23 & 258.93 & 0.0003 & 5.5389 \\
\hline 95.5483 & 257.66 & 231.10 & 214.52 & 212.74 & 180.27 & 70.76 & 291.23 & 258.92 & 0.0003 & 5.5454 \\
\hline 96.0490 & 253.97 & 229.89 & 214.35 & 212.60 & 181.63 & 71.54 & 290.47 & 258.89 & 0.0001 & 5.4982 \\
\hline 96.5470 & 247.93 & 228.45 & 214.20 & 212.50 & 182.94 & 72.34 & 289.33 & 258.86 & 0.0003 & 5.5087 \\
\hline 97.0478 & 242.39 & 226.95 & 214.17 & 212.45 & 184.15 & 73.15 & 289.77 & 258.82 & 0.0000 & 5.5372 \\
\hline 97.5477 & 232.80 & 225.51 & 214.22 & 212.46 & 185.38 & 74.03 & 289.91 & 258.75 & 0.0003 & 5.5459 \\
\hline 98.0473 & 226.07 & 222.94 & 214.21 & 212.49 & 186.86 & 74.92 & 289.70 & 258.72 & 0.0003 & 5.5244 \\
\hline 98.5482 & 223.14 & 219.47 & 214.21 & 212.50 & 188.43 & 75.86 & 289.82 & 258.77 & 0.0002 & 5.5035 \\
\hline 99.0480 & 225.32 & 218.87 & 214.18 & 212.50 & 190.25 & 76.83 & 289.37 & 258.68 & 0.0003 & 5.5273 \\
\hline 99.5468 & 230.27 & 221.12 & 214.12 & 212.44 & 192.78 & 77.82 & 288.97 & $258.77 \mathrm{~S}$ & 0.0002 & 5.5478 \\
\hline
\end{tabular}


TABLE B6.- CONTINUED.

\begin{tabular}{|c|c|c|c|c|c|c|c|c|c|c|}
\hline $\begin{array}{l}\text { Time, } \\
\text { min }\end{array}$ & $\begin{array}{c}\text { T-steam } \\
{ }^{\circ} \mathrm{C}\end{array}$ & $\begin{array}{l}\mathrm{T} 1, \\
{ }^{\circ} \mathrm{C}\end{array}$ & $\begin{array}{l}\mathrm{T} 2, \\
{ }^{\circ} \mathrm{C}\end{array}$ & $\begin{array}{l}\mathrm{T} 3, \\
{ }^{\circ} \mathrm{C}\end{array}$ & $\begin{array}{l}\mathrm{T} 4, \\
{ }^{\circ} \mathrm{C}\end{array}$ & $\begin{array}{l}\mathrm{T} 5, \\
{ }^{\circ} \mathrm{C}\end{array}$ & $\begin{array}{l}\text { Pinj, } \\
\text { psig }\end{array}$ & $\begin{array}{l}\text { Pout, } \\
\text { psig }\end{array}$ & $\begin{array}{l}\text { Qinj, } \\
\text { g/min }\end{array}$ & $\begin{array}{c}\mathrm{Vw} \\
\mathrm{ml} / \mathrm{min}\end{array}$ \\
\hline 100.0477 & 237.73 & 223.35 & 214.01 & 212.36 & 195.17 & 78.84 & 287.92 & 258.70 & 0.0003 & 5.5229 \\
\hline 100.5483 & 244.92 & 225.84 & 213.89 & 212.26 & 197.65 & 79.91 & 286.97 & 258.67 & 0.0003 & 5.4991 \\
\hline 101.0482 & 250.56 & 228.00 & 213.68 & 212.11 & 199.33 & 80.99 & 285.67 & 258.56 & 0.0000 & 5.5339 \\
\hline 101.5480 & 251.99 & 228.87 & 213.48 & 211.92 & 201.11 & 82.08 & 284.41 & 258.52 & 0.0003 & 5.5446 \\
\hline 102.0478 & 250.85 & 229.00 & 213.34 & 211.80 & 202.28 & 83.20 & 283.98 & 258.59 & 0.0003 & 5.5266 \\
\hline 102.5477 & 248.13 & 228.79 & 213.29 & 211.72 & 203.00 & 84.36 & 284.23 & 258.58 & 0.0002 & 5.5142 \\
\hline 103.0483 & 244.17 & 228.48 & 213.37 & 211.78 & 204.10 & 85.55 & 285.21 & 258.50 & 0.0003 & 5.5278 \\
\hline 103.5482 & 238.37 & 227.57 & 213.54 & 211.89 & 205.24 & 86.81 & 286.28 & 258.55 & 0.0003 & 5.5482 \\
\hline 104.0480 & 229.44 & 225.54 & 213.80 & 212.10 & 206.50 & 88.12 & 287.84 & 258.61 & 0.0002 & 5.5257 \\
\hline 104.5470 & 224.28 & 223.04 & 214.00 & 212.29 & 208.05 & 89.51 & 288.71 & 258.63 & 0.0001 & 5.4989 \\
\hline 105.0477 & 221.64 & 219.18 & 214.14 & 212.42 & 209.13 & 90.95 & 289.41 & 258.61 & 0.0009 & 5.5041 \\
\hline 105.5483 & 224.13 & 217.24 & 214.16 & 212.50 & 209.67 & 92.50 & 289.18 & 258.58 & 0.0002 & 5.5486 \\
\hline 106.0482 & 229.37 & 217.88 & 214.10 & 212.47 & 210.00 & 94.17 & 288.30 & 258.57 & 0.0000 & 5.5404 \\
\hline 106.5480 & 231.13 & 220.89 & 214.00 & 212.37 & 210.28 & 96.00 & 287.38 & 258.49 & 0.0001 & 5.4912 \\
\hline 107.0470 & 240.97 & 223.01 & 213.72 & 212.18 & 210.30 & 98.05 & 285.63 & 258.44 & 0.0003 & 5.5120 \\
\hline 107.5477 & 249.80 & 225.67 & 213.42 & 211.94 & 210.26 & 100.33 & 283.71 & 258.47 & 0.0003 & 5.5272 \\
\hline 108.0485 & 254.71 & 227.95 & 213.11 & 211.68 & 210.14 & 102.86 & 281.73 & 258.33 & 0.0003 & 5.5389 \\
\hline 108.5483 & 255.85 & 228.85 & 212.76 & 211.40 & 210.08 & 105.50 & 279.64 & 258.34 & 0.0003 & 5.5150 \\
\hline 109.0472 & 255.13 & 228.38 & 212.38 & 211.09 & 209.92 & 108.11 & 277.42 & 258.35 & 0.0003 & 5.5009 \\
\hline 109.5478 & 253.25 & 227.27 & 211.99 & 210.79 & 209.75 & 110.64 & 275.15 & 258.65 & 0.0004 & 5.5281 \\
\hline 110.0487 & 250.46 & 226.03 & 211.66 & 210.50 & 209.66 & 112.93 & 273.37 & 258.70 & 0.0003 & 5.5520 \\
\hline 110.5475 & 246.61 & 224.55 & 211.36 & 210.25 & 209.50 & 115.02 & 271.95 & 258.56 & 0.0001 & 5.5194 \\
\hline 111.0483 & 240.95 & 223.18 & 211.23 & 210.10 & 209.38 & 116.94 & 271.23 & 258.42 & 0.0003 & 5.5044 \\
\hline 111.5482 & 232.18 & 222.17 & 211.32 & 210.09 & 209.36 & 118.58 & 273.44 & 257.20 & 0.0004 & 5.5219 \\
\hline 112.0470 & 224.15 & 220.60 & 211.73 & 210.37 & 209.58 & 120.17 & 275.50 & 259.19 & 0.0006 & 5.5388 \\
\hline 112.5477 & 219.95 & 218.10 & 212.00 & 210.62 & 209.80 & 121.84 & 276.88 & 257.04 & 0.0001 & 5.5477 \\
\hline 113.0475 & 219.83 & 215.07 & 212.24 & 210.83 & 209.95 & 123.52 & 278.24 & 258.70 & 0.0003 & 5.4981 \\
\hline 113.5483 & 225.62 & 215.61 & 212.36 & 210.96 & 210.16 & 125.36 & 278.39 & 258.39 & 0.0003 & 5.5021 \\
\hline 114.0482 & 232.25 & 217.37 & 212.35 & 210.99 & 210.22 & 127.43 & 277.92 & 258.84 & 0.0003 & 5.5246 \\
\hline 114.5470 & 239.14 & 220.28 & 212.26 & 210.94 & 210.26 & 129.72 & 277.32 & 258.69 & 0.0001 & 5.5406 \\
\hline 115.0478 & 241.82 & 221.36 & 212.07 & 210.82 & 210.21 & 132.30 & 275.67 & 257.56 & 0.0002 & 5.5225 \\
\hline 115.5477 & 242.61 & 221.71 & 211.85 & 210.65 & 210.13 & 135.01 & 274.62 & 257.67 & 0.0005 & 5.4923 \\
\hline 116.0483 & 241.39 & 221.27 & 211.63 & 210.46 & 209.98 & 137.75 & 273.17 & 258.58 & 0.0003 & 5.5166 \\
\hline 116.5482 & 235.66 & 220.17 & 211.42 & 210.27 & 209.88 & 140.32 & 272.24 & 258.34 & 0.0003 & 5.5496 \\
\hline 117.0480 & 231.37 & 218.05 & 211.30 & 210.16 & 209.80 & 142.74 & 271.67 & 256.54 & 0.0003 & 5.5386 \\
\hline 117.5478 & 225.84 & 217.43 & 211.58 & 210.27 & 209.81 & 145.05 & 274.40 & 258.91 & 0.0004 & 5.4761 \\
\hline 118.0477 & 224.35 & 217.80 & 211.78 & 210.44 & 209.94 & 147.42 & 275.47 & 258.81 & 0.0003 & 5.5165 \\
\hline 118.5483 & 228.06 & 219.32 & 212.01 & 210.63 & 210.11 & 149.82 & 276.93 & 259.10 & 0.0008 & 5.5452 \\
\hline 119.0473 & 233.60 & 222.54 & 212.28 & 210.85 & 210.30 & 152.38 & 278.44 & 258.90 & 0.0003 & 5.5458 \\
\hline 119.5480 & 239.52 & 225.32 & 212.51 & 211.04 & 210.45 & 155.30 & 279.53 & 258.79 & 0.0001 & 5.4986 \\
\hline 120.0478 & 246.70 & 228.19 & 212.62 & 211.17 & 210.60 & 158.56 & 279.87 & 257.14 & 0.0003 & 5.4972 \\
\hline 120.5477 & 248.07 & 229.11 & 212.57 & 211.16 & 210.53 & 162.86 & 279.27 & 257.20 & 0.0007 & 5.5135 \\
\hline 121.0483 & 246.95 & 228.53 & 212.40 & 211.01 & 210.45 & 167.35 & 277.82 & 258.17 & 0.0002 & 5.5448 \\
\hline 121.5473 & 244.68 & 227.17 & 212.12 & 210.80 & 210.33 & 171.21 & 276.01 & 255.34 & 0.0003 & 5.5126 \\
\hline 122.0480 & 238.91 & 225.43 & 211.81 & 210.57 & 210.16 & 174.54 & 274.07 & 255.70 & 0.0007 & 5.5056 \\
\hline 122.5488 & 234.41 & 222.95 & 211.48 & 210.28 & 209.97 & 177.43 & 272.06 & 256.03 & 0.0003 & 5.5386 \\
\hline 123.0468 & 232.43 & 220.23 & 211.11 & 210.00 & 209.80 & 179.72 & 269.96 & 256.38 & 0.0003 & 5.5659 \\
\hline 123.5483 & 228.07 & 216.93 & 210.79 & 209.74 & 209.61 & 181.52 & 268.09 & 254.40 & 0.0003 & 5.5248 \\
\hline 124.0492 & 221.20 & 214.74 & 210.51 & 209.51 & 209.40 & 183.03 & 266.66 & 254.80 & 0.0004 & 5.4910 \\
\hline 124.5480 & 217.43 & 212.11 & 210.45 & 209.38 & 209.29 & 184.03 & 267.80 & 255.67 & 0.0001 & 5.5180 \\
\hline
\end{tabular}


TABLE B6.- CONTINUED.

\begin{tabular}{|c|c|c|c|c|c|c|c|c|c|c|}
\hline $\begin{array}{l}\text { Time, } \\
\text { min }\end{array}$ & $\begin{array}{c}\text { T-steam } \\
{ }^{\circ} \mathrm{C}\end{array}$ & $\begin{array}{l}\mathrm{T} 1, \\
{ }^{\circ} \mathrm{C}\end{array}$ & $\begin{array}{l}\mathrm{T} 2, \\
{ }^{\circ} \mathrm{C}\end{array}$ & $\begin{array}{l}\mathrm{T} 3, \\
{ }^{\circ} \mathrm{C}\end{array}$ & $\begin{array}{l}\mathrm{T} 4, \\
{ }^{\circ} \mathrm{C}\end{array}$ & $\begin{array}{l}\mathrm{T} 5, \\
{ }^{\circ} \mathrm{C}\end{array}$ & $\begin{array}{l}\text { Pinj, } \\
\text { psig }\end{array}$ & $\begin{array}{l}\text { Pout, } \\
\text { psig }\end{array}$ & $\begin{array}{l}\text { Qinj, } \\
\text { g/min }\end{array}$ & $\begin{array}{c}\mathrm{Vw} \\
\mathrm{ml} / \mathrm{min}\end{array}$ \\
\hline 125.0470 & 219.30 & 211.66 & 210.69 & 209.51 & 209.33 & 184.99 & 268.98 & 254.63 & 0.0003 & 5.5389 \\
\hline 125.5477 & 224.00 & 212.85 & 210.95 & 209.70 & 209.46 & 186.11 & 270.81 & 256.43 & 0.0007 & 5.5377 \\
\hline 126.0485 & 231.79 & 216.15 & 211.19 & 209.88 & 209.65 & 187.93 & 271.89 & 255.80 & 0.0005 & 5.4846 \\
\hline 126.5492 & 240.31 & 220.14 & 211.34 & 210.05 & 209.80 & 190.21 & 272.44 & 256.43 & 0.0002 & 5.4959 \\
\hline 127.0480 & 248.49 & 224.05 & 211.40 & 210.13 & 209.90 & 191.92 & 272.41 & 256.36 & 0.0003 & 5.5271 \\
\hline 127.5488 & 256.99 & 228.00 & 211.34 & 210.14 & 209.92 & 194.45 & 271.87 & 255.40 & 0.0005 & 5.5477 \\
\hline 128.0477 & 263.59 & 231.09 & 211.20 & 210.04 & 209.86 & 196.31 & 270.88 & 256.04 & 0.0003 & 5.5194 \\
\hline 128.5475 & 265.80 & 232.85 & 211.03 & 209.88 & 209.78 & 197.78 & 269.95 & 256.47 & 0.0003 & 5.4895 \\
\hline 129.0483 & 265.23 & 233.50 & 210.87 & 209.80 & 209.73 & 198.88 & 269.33 & 255.29 & 0.0003 & 5.5176 \\
\hline 129.5482 & 263.15 & 233.70 & 210.85 & 209.72 & 209.66 & 199.80 & 269.53 & 258.42 & 0.0003 & 5.5463 \\
\hline 130.0480 & 259.94 & 233.89 & 210.89 & 209.76 & 209.69 & 200.65 & 269.96 & 255.79 & 0.0006 & 5.5298 \\
\hline 130.5468 & 256.03 & 233.59 & 210.99 & 209.82 & 209.72 & 201.93 & 270.69 & 257.38 & 0.0001 & 5.5087 \\
\hline 131.0475 & 250.94 & 233.26 & 211.17 & 209.96 & 209.81 & 202.98 & 272.09 & 255.91 & 0.0002 & 5.5294 \\
\hline 131.5483 & 244.24 & 232.26 & 211.39 & 210.12 & 209.91 & 204.04 & 273.43 & 257.20 & 0.0003 & 5.5441 \\
\hline 132.0482 & 235.41 & 229.85 & 211.62 & 210.31 & 210.02 & 205.33 & 274.73 & 257.90 & 0.0003 & 5.5506 \\
\hline 132.5480 & 230.58 & 224.69 & 211.82 & 210.50 & 210.14 & 205.86 & 275.61 & 258.57 & 0.0006 & 5.5026 \\
\hline 133.0478 & 225.59 & 220.11 & 211.97 & 210.63 & 210.25 & 206.40 & 276.45 & 258.97 & 0.0006 & 5.5071 \\
\hline 133.5485 & 222.72 & 217.92 & 212.07 & 210.69 & 210.26 & 206.72 & 276.66 & 256.01 & 0.0000 & 5.5417 \\
\hline 134.0483 & 226.77 & 216.39 & 212.02 & 210.66 & 210.21 & 207.01 & 275.89 & 256.50 & 0.0001 & 5.5554 \\
\hline 134.5482 & 231.40 & 217.43 & 211.88 & 210.54 & 210.15 & 207.30 & 274.85 & 256.90 & 0.0000 & 5.5177 \\
\hline 135.0470 & 236.76 & 219.48 & 211.66 & 210.41 & 210.09 & 207.56 & 273.50 & 256.23 & 0.0003 & 5.5015 \\
\hline 135.5478 & 247.18 & 222.64 & 211.42 & 210.22 & 210.00 & 207.59 & 271.93 & 256.03 & 0.0003 & 5.5265 \\
\hline 136.0477 & 254.17 & 225.78 & 211.12 & 209.99 & 209.87 & 207.67 & 270.16 & 256.13 & 0.0004 & 5.5329 \\
\hline 136.5483 & 256.82 & 227.22 & 210.81 & 209.77 & 209.75 & 207.69 & 268.35 & 257.43 & 0.0000 & 5.5582 \\
\hline 137.0473 & 256.66 & 227.31 & 210.51 & 209.51 & 209.58 & 207.65 & 266.76 & 256.56 & 0.0001 & 5.5037 \\
\hline 137.5480 & 254.78 & 227.25 & 210.30 & 209.33 & 209.46 & 207.72 & 265.76 & 257.22 & 0.0003 & 5.5025 \\
\hline 138.0468 & 250.27 & 226.47 & 210.08 & 209.20 & 209.38 & 207.64 & 264.84 & 256.35 & 0.0003 & 5.5359 \\
\hline 138.5477 & 246.36 & 225.87 & 210.01 & 209.08 & 209.22 & 207.56 & 264.70 & 254.58 & 0.0002 & 5.5481 \\
\hline 139.0483 & 240.11 & 225.97 & 210.47 & 209.32 & 209.29 & 207.57 & 268.24 & 255.73 & 0.0004 & 5.5073 \\
\hline 139.5482 & 232.20 & 222.52 & 210.65 & 209.49 & 209.43 & 207.79 & 269.24 & 255.58 & 0.0003 & 5.5059 \\
\hline 140.0480 & 227.84 & 219.61 & 210.98 & 209.75 & 209.62 & 207.97 & 271.59 & 256.16 & 0.0004 & 5.5249 \\
\hline 140.5470 & 224.61 & 217.36 & 211.44 & 210.09 & 209.86 & 208.11 & 274.21 & 258.37 & 0.0004 & 5.5828 \\
\hline 141.0477 & 225.26 & 216.15 & 211.84 & 210.42 & 210.06 & 208.20 & 276.10 & 257.97 & 0.0003 & 5.5308 \\
\hline 141.5483 & 230.17 & 217.73 & 212.00 & 210.61 & 210.22 & 208.37 & 276.22 & 258.71 & 0.0003 & 5.5166 \\
\hline 142.0482 & 237.40 & 219.06 & 211.89 & 210.62 & 210.26 & 208.34 & 275.07 & 256.14 & 0.0003 & 5.5063 \\
\hline 142.5472 & 240.36 & 221.09 & 211.71 & 210.48 & 210.21 & 208.44 & 273.83 & 256.35 & 0.0000 & 5.5423 \\
\hline 143.0478 & 241.66 & 220.40 & 211.54 & 210.36 & 210.18 & 208.61 & 272.82 & 257.25 & 0.0002 & 5.5497 \\
\hline 143.5477 & 240.55 & 220.68 & 211.32 & 210.23 & 210.03 & 208.26 & 271.58 & 254.37 & 0.0003 & 5.5152 \\
\hline 144.0493 & 238.73 & 220.13 & 211.07 & 209.95 & 209.82 & 208.34 & 270.04 & 255.62 & 0.0001 & 5.4877 \\
\hline 144.5473 & 239.89 & 219.67 & 210.81 & 209.78 & 209.70 & 208.25 & 268.68 & 254.87 & 0.0003 & 5.5130 \\
\hline 145.0480 & 230.17 & 217.64 & 210.59 & 209.59 & 209.59 & 208.35 & 267.32 & 256.79 & 0.0003 & 5.5240 \\
\hline 145.5470 & 228.83 & 215.23 & 210.33 & 209.41 & 209.54 & 208.43 & 265.71 & 256.62 & 0.0003 & 5.5207 \\
\hline 146.0477 & 224.19 & 212.79 & 210.14 & 209.28 & 209.42 & 208.17 & 264.43 & 254.56 & 0.0003 & 5.4810 \\
\hline 146.5475 & 225.05 & 211.47 & 209.86 & 209.05 & 209.25 & 208.23 & 263.07 & 254.95 & 0.0003 & 5.5190 \\
\hline 147.0483 & 223.72 & 211.65 & 209.74 & 208.95 & 209.18 & 208.27 & 262.72 & 255.97 & 0.0003 & 5.5446 \\
\hline 147.5482 & 234.02 & 212.81 & 209.69 & 208.90 & 209.21 & 208.35 & 262.64 & 256.19 & 0.0003 & 5.5287 \\
\hline 148.0480 & 234.69 & 214.46 & 209.81 & 208.93 & 209.23 & 208.41 & 265.68 & 256.50 & 0.0005 & 5.4967 \\
\hline 148.5468 & 244.80 & 218.76 & 210.39 & 209.35 & 209.47 & 208.56 & 267.88 & 256.23 & 0.0003 & 5.4972 \\
\hline 149.0475 & 246.84 & 222.46 & 210.63 & 209.61 & 209.68 & 208.64 & 268.39 & 257.47 & 0.0003 & 5.5194 \\
\hline 149.5483 & 243.26 & 223.30 & 210.67 & 209.69 & 209.74 & 208.56 & 268.73 & 255.63 & 0.0003 & 5.5392 \\
\hline
\end{tabular}


TABLE B6.- CONTINUED.

\begin{tabular}{|c|c|c|c|c|c|c|c|c|c|c|}
\hline $\begin{array}{l}\text { Time, } \\
\text { min }\end{array}$ & $\begin{array}{c}\text { T-steam } \\
{ }^{\circ} \mathrm{C}\end{array}$ & $\begin{array}{l}\mathrm{T} 1, \\
{ }^{\circ} \mathrm{C}\end{array}$ & $\begin{array}{l}\mathrm{T} 2, \\
{ }^{\circ} \mathrm{C}\end{array}$ & $\begin{array}{l}\mathrm{T} 3, \\
{ }^{\circ} \mathrm{C}\end{array}$ & $\begin{array}{l}\mathrm{T} 4, \\
{ }^{\circ} \mathrm{C}\end{array}$ & $\begin{array}{l}\mathrm{T} 5, \\
{ }^{\circ} \mathrm{C}\end{array}$ & $\begin{array}{l}\text { Pinj, } \\
\text { psig }\end{array}$ & $\begin{array}{l}\text { Pout, } \\
\text { psig }\end{array}$ & $\begin{array}{l}\text { Qinj, } \\
\text { g/min }\end{array}$ & $\begin{array}{c}\mathrm{Vw} \\
\mathrm{ml} / \mathrm{min}\end{array}$ \\
\hline 150.0490 & 240.99 & 221.82 & 210.66 & 209.66 & 209.67 & 208.55 & 268.30 & 256.64 & 0.0003 & 5.5125 \\
\hline 150.5480 & 234.42 & 220.58 & 210.61 & 209.63 & 209.73 & 208.75 & 268.00 & 257.20 & 0.0003 & 5.4907 \\
\hline 151.0468 & 232.15 & 218.85 & 210.53 & 209.62 & 209.72 & 208.72 & 267.38 & 256.62 & 0.0003 & 5.5316 \\
\hline 151.5477 & 226.53 & 215.96 & 210.43 & 209.55 & 209.75 & 208.78 & 266.98 & 255.59 & 0.0003 & 5.5477 \\
\hline 152.0483 & 223.25 & 213.73 & 210.45 & 209.54 & 209.61 & 208.56 & 267.35 & 255.73 & 0.0003 & 5.4984 \\
\hline 152.5472 & 223.42 & 212.89 & 210.50 & 209.51 & 209.60 & 208.63 & 267.61 & 257.10 & 0.0005 & 5.5004 \\
\hline 153.0480 & 228.31 & 213.33 & 210.61 & 209.61 & 209.70 & 208.71 & 268.49 & 256.92 & 0.0003 & 5.5308 \\
\hline 153.5487 & 237.81 & 215.31 & 210.78 & 209.74 & 209.79 & 208.84 & 269.87 & 257.43 & 0.0004 & 5.5532 \\
\hline 154.0477 & 240.76 & 217.71 & 210.98 & 209.91 & 209.92 & 208.98 & 270.81 & 258.51 & 0.0001 & 5.5211 \\
\hline 154.5483 & 251.25 & 221.48 & 211.13 & 210.06 & 210.09 & 209.14 & 271.40 & 259.01 & 0.0007 & 5.5009 \\
\hline 155.0482 & 255.91 & 225.70 & 211.23 & 210.10 & 209.92 & 208.58 & 271.75 & 255.06 & 0.0001 & 5.5049 \\
\hline 155.5480 & 256.03 & 227.79 & 211.14 & 210.00 & 209.87 & 208.71 & 270.99 & 255.72 & 0.0003 & 5.5408 \\
\hline 156.0478 & 250.09 & 228.19 & 211.04 & 209.93 & 209.81 & 208.59 & 270.56 & 255.30 & 0.0003 & 5.5285 \\
\hline 156.5477 & 249.31 & 226.82 & 210.85 & 209.78 & 209.67 & 208.45 & 269.12 & 254.52 & 0.0001 & 5.4973 \\
\hline 157.0483 & 245.26 & 223.67 & 210.66 & 209.63 & 209.59 & 208.48 & 268.00 & 254.74 & 0.0003 & 5.4992 \\
\hline 157.5482 & 242.70 & 223.00 & 210.45 & 209.51 & 209.54 & 208.56 & 266.74 & 255.46 & 0.0004 & 5.5347 \\
\hline 158.0480 & 234.84 & 220.14 & 210.34 & 209.41 & 209.51 & 208.62 & 266.29 & 255.29 & 0.0003 & 5.5475 \\
\hline 158.5478 & 229.23 & 217.52 & 210.31 & 209.38 & 209.52 & 208.62 & 266.52 & 255.42 & 0.0001 & 5.4986 \\
\hline 159.0477 & 227.19 & 216.34 & 210.35 & 209.40 & 209.49 & 208.60 & 266.89 & 256.54 & 0.0002 & 5.5097 \\
\hline 159.5475 & 232.84 & 216.69 & 210.57 & 209.51 & 209.55 & 208.65 & 268.51 & 255.47 & 0.0003 & 5.5293 \\
\hline 160.0482 & 239.03 & 218.70 & 210.81 & 209.68 & 209.68 & 208.79 & 269.78 & 256.74 & 0.0003 & 5.5466 \\
\hline 160.5480 & 237.78 & 220.89 & 211.01 & 209.88 & 209.85 & 208.86 & 270.84 & 256.03 & 0.0003 & 5.5238 \\
\hline 161.0488 & 243.71 & 222.74 & 211.07 & 209.96 & 209.84 & 208.69 & 270.79 & 254.84 & 0.0003 & 5.4990 \\
\hline 161.5477 & 239.19 & 222.62 & 211.04 & 209.93 & 209.83 & 208.77 & 270.59 & 256.69 & 0.0003 & 5.5226 \\
\hline 162.0493 & 240.32 & 222.05 & 210.94 & 209.90 & 209.83 & 208.83 & 269.78 & 256.79 & 0.0003 & 5.5274 \\
\hline 162.5473 & 235.49 & 221.20 & 210.84 & 209.84 & 209.84 & 208.84 & 269.21 & 255.79 & 0.0003 & 5.5085 \\
\hline 163.0480 & 231.97 & 218.97 & 210.70 & 209.74 & 209.72 & 208.73 & 268.30 & 255.42 & 0.0004 & 5.4828 \\
\hline 163.5478 & 225.24 & 216.01 & 210.57 & 209.64 & 209.65 & 208.67 & 267.62 & 255.81 & 0.0003 & 5.5017 \\
\hline 164.0477 & 221.90 & 213.72 & 210.45 & 209.54 & 209.62 & 208.71 & 267.14 & 256.00 & 0.0005 & 5.5186 \\
\hline 164.5467 & 221.69 & 212.92 & 210.49 & 209.52 & 209.61 & 208.81 & 268.45 & 256.53 & 0.0001 & 5.5199 \\
\hline 165.0473 & 226.98 & 213.86 & 210.61 & 209.62 & 209.67 & 208.87 & 268.05 & 256.02 & 0.0000 & 5.5072 \\
\hline 165.5482 & 231.77 & 215.39 & 210.61 & 209.62 & 209.61 & 208.61 & 268.13 & 257.87 & 0.0005 & 5.4963 \\
\hline 166.0470 & 239.73 & 217.33 & 210.63 & 209.65 & 209.70 & 208.93 & 268.24 & 256.84 & 0.0001 & 5.5279 \\
\hline 166.5477 & 250.39 & 221.41 & 210.64 & 209.71 & 209.85 & 209.14 & 268.38 & 258.19 & 0.0003 & 5.5477 \\
\hline 167.0475 & 254.32 & 224.68 & 210.72 & 209.79 & 209.93 & 209.02 & 268.91 & 255.76 & 0.0003 & 5.5158 \\
\hline 167.5483 & 247.78 & 226.42 & 210.78 & 209.74 & 209.74 & 208.79 & 269.52 & 256.54 & 0.0003 & 5.4920 \\
\hline 168.0472 & 250.69 & 226.50 & 210.80 & 209.76 & 209.78 & 208.87 & 269.57 & 256.28 & 0.0006 & 5.5127 \\
\hline 168.5480 & 246.16 & 226.61 & 210.90 & 209.84 & 209.84 & 208.95 & 270.33 & 256.29 & 0.0003 & 5.5274 \\
\hline 169.0487 & 243.80 & 224.38 & 210.97 & 209.92 & 209.92 & 209.02 & 270.73 & 256.64 & 0.0001 & 5.5229 \\
\hline 169.5467 & 238.35 & 224.06 & 211.09 & 210.01 & 210.00 & 209.10 & 271.08 & 258.00 & 0.0003 & 5.4909 \\
\hline 170.0483 & 234.83 & 221.42 & 211.09 & 210.09 & 210.07 & 209.20 & 270.96 & 258.53 & 0.0003 & 5.5022 \\
\hline 170.5472 & 231.36 & 219.53 & 211.08 & 210.11 & 210.19 & 209.40 & 270.67 & 259.40 & 0.0003 & 5.5506 \\
\hline 171.0480 & 226.41 & 216.51 & 210.98 & 210.09 & 210.21 & 209.44 & 270.08 & 259.21 & 0.0003 & 5.5165 \\
\hline 171.5478 & 222.71 & 214.31 & 210.84 & 209.93 & 209.86 & 208.55 & 268.79 & 254.00 & 0.0003 & 5.4926 \\
\hline 172.0477 & 220.37 & 212.67 & 210.56 & 209.51 & 209.34 & 208.25 & 266.91 & 253.13 & 0.0003 & 5.5108 \\
\hline 172.5483 & 217.55 & 211.48 & 210.25 & 209.22 & 209.14 & 208.15 & 265.32 & 253.35 & 0.0003 & 5.5446 \\
\hline 173.0482 & 218.97 & 211.49 & 210.04 & 209.07 & 209.09 & 208.34 & 264.54 & 253.29 & 0.0003 & 5.5491 \\
\hline 173.5470 & 226.53 & 212.17 & 210.11 & 209.08 & 209.11 & 208.34 & 265.62 & 253.76 & 0.0003 & 5.5131 \\
\hline 174.0468 & 233.86 & 214.40 & 210.26 & 209.19 & 209.19 & 208.38 & 266.27 & 253.94 & 0.0005 & 5.4922 \\
\hline 174.5477 & 238.11 & 216.53 & 210.41 & 209.30 & 209.27 & 208.46 & 267.40 & 254.56 & 0.0000 & 5.5305 \\
\hline
\end{tabular}


TABLE B6.- CONTINUED.

\begin{tabular}{|c|c|c|c|c|c|c|c|c|c|c|}
\hline $\begin{array}{l}\text { Time, } \\
\text { min }\end{array}$ & $\begin{array}{c}\text { T-steam } \\
{ }^{\circ} \mathrm{C}\end{array}$ & $\begin{array}{l}\mathrm{T} 1, \\
{ }^{\circ} \mathrm{C}\end{array}$ & $\begin{array}{l}\mathrm{T} 2, \\
{ }^{\circ} \mathrm{C}\end{array}$ & $\begin{array}{l}\mathrm{T} 3, \\
{ }^{\circ} \mathrm{C}\end{array}$ & $\begin{array}{l}\mathrm{T} 4, \\
{ }^{\circ} \mathrm{C}\end{array}$ & $\begin{array}{l}\mathrm{T} 5, \\
{ }^{\circ} \mathrm{C}\end{array}$ & $\begin{array}{l}\text { Pinj, } \\
\text { psig }\end{array}$ & $\begin{array}{l}\text { Pout, } \\
\text { psig }\end{array}$ & $\begin{array}{l}\text { Qinj, } \\
\text { g/min }\end{array}$ & $\begin{array}{c}\mathrm{Vw} \\
\mathrm{ml} / \mathrm{min}\end{array}$ \\
\hline 175.0483 & 237.02 & 218.47 & 210.51 & 209.40 & 209.33 & 208.47 & 267.91 & 254.26 & 0.0000 & 5.5345 \\
\hline 175.5482 & 239.75 & 218.57 & 210.53 & 209.46 & 209.40 & 208.56 & 267.62 & 255.42 & 0.0004 & 5.5035 \\
\hline 176.0472 & 235.28 & 217.89 & 210.48 & 209.44 & 209.43 & 208.57 & 267.22 & 254.42 & 0.0003 & 5.5045 \\
\hline 176.5478 & 231.56 & 216.82 & 210.38 & 209.40 & 209.43 & 208.59 & 266.41 & 254.11 & 0.0003 & 5.5274 \\
\hline 177.0477 & 229.97 & 215.09 & 210.24 & 209.31 & 209.40 & 208.62 & 265.69 & 254.70 & 0.0003 & 5.5475 \\
\hline 177.5483 & 225.25 & 213.60 & 210.14 & 209.25 & 209.32 & 208.50 & 265.22 & 253.95 & 0.0005 & 5.5140 \\
\hline 178.0482 & 221.46 & 212.39 & 210.02 & 209.15 & 209.31 & 208.64 & 264.92 & 255.27 & 0.0003 & 5.4849 \\
\hline 178.5480 & 221.61 & 211.93 & 210.03 & 209.15 & 209.28 & 208.62 & 264.89 & 254.24 & 0.0003 & 5.5174 \\
\hline 179.0470 & 223.30 & 212.38 & 210.09 & 209.19 & 209.30 & 208.62 & 265.46 & 255.02 & 0.0003 & 5.5424 \\
\hline 179.5477 & 229.89 & 213.32 & 210.20 & 209.25 & 209.36 & 208.66 & 265.78 & 253.93 & 0.0003 & 5.5240 \\
\hline 180.0485 & 243.19 & 215.74 & 210.23 & 209.29 & 209.40 & 208.70 & 266.14 & 254.25 & 0.0003 & 5.5080 \\
\hline 180.5483 & 249.38 & 219.05 & 210.28 & 209.32 & 209.43 & 208.69 & 266.24 & 254.20 & 0.0002 & 5.4988 \\
\hline 181.0480 & 246.50 & 220.89 & 210.31 & 209.34 & 209.41 & 208.70 & 266.50 & 254.31 & 0.0003 & 5.5391 \\
\hline 181.5488 & 249.07 & 222.18 & 210.35 & 209.36 & 209.45 & 208.70 & 267.16 & 255.37 & 0.0003 & 5.5418 \\
\hline 182.0477 & 244.34 & 222.92 & 210.46 & 209.46 & 209.53 & 208.82 & 267.60 & 255.54 & 0.0001 & 5.4808 \\
\hline 182.5475 & 239.29 & 222.42 & 210.45 & 209.50 & 209.56 & 208.77 & 267.55 & 254.01 & 0.0006 & 5.4975 \\
\hline 183.0483 & 239.31 & 220.83 & 210.47 & 209.47 & 209.52 & 208.76 & 267.31 & 255.24 & 0.0002 & 5.5253 \\
\hline 183.5490 & 232.98 & 219.26 & 210.42 & 209.48 & 209.55 & 208.83 & 267.27 & 256.11 & 0.0005 & 5.5369 \\
\hline 184.0480 & 229.60 & 217.50 & 210.41 & 209.48 & 209.59 & 208.87 & 267.03 & 255.15 & 0.0003 & 5.4910 \\
\hline 184.5477 & 224.97 & 214.98 & 210.40 & 209.49 & 209.59 & 208.90 & 267.07 & 255.98 & 0.0004 & 5.4937 \\
\hline 185.0467 & 222.04 & 213.61 & 210.39 & 209.49 & 209.60 & 208.88 & 266.97 & 255.57 & 0.0002 & 5.5404 \\
\hline 185.5473 & 219.52 & 212.32 & 210.36 & 209.50 & 209.61 & 208.75 & 266.79 & 254.11 & 0.0000 & 5.5459 \\
\hline 186.0482 & 217.20 & 211.29 & 210.34 & 209.41 & 209.50 & 208.75 & 266.53 & 254.57 & 0.0003 & 5.5066 \\
\hline 186.5480 & 217.28 & 211.10 & 210.30 & 209.40 & 209.51 & 208.85 & 266.38 & 255.29 & 0.0000 & 5.4982 \\
\hline 187.0478 & 219.70 & 211.22 & 210.32 & 209.43 & 209.55 & 208.89 & 266.46 & 256.46 & 0.0004 & 5.5282 \\
\hline 187.5467 & 225.56 & 211.94 & 210.29 & 209.41 & 209.56 & 208.87 & 266.24 & 255.21 & 0.0004 & 5.5629 \\
\hline 188.0483 & 233.51 & 213.05 & 210.26 & 209.38 & 209.56 & 208.92 & 266.24 & 256.29 & 0.0002 & 5.5144 \\
\hline 188.5482 & 239.77 & 215.24 & 210.34 & 209.44 & 209.59 & 208.96 & 266.93 & 256.17 & 0.0003 & 5.4687 \\
\hline 189.0480 & 250.23 & 218.52 & 210.41 & 209.50 & 209.64 & 209.00 & 267.45 & 256.48 & 0.0003 & 5.5212 \\
\hline 189.5478 & 255.07 & 222.23 & 210.51 & 209.58 & 209.70 & 209.02 & 267.81 & 256.19 & 0.0001 & 5.5543 \\
\hline 190.0477 & 255.65 & 225.19 & 210.55 & 209.60 & 209.71 & 209.01 & 268.23 & 256.13 & 0.0003 & 5.5201 \\
\hline 190.5483 & 253.80 & 226.86 & 210.65 & 209.66 & 209.72 & 208.98 & 268.65 & 255.96 & 0.0003 & 5.4746 \\
\hline 191.0473 & 246.29 & 226.72 & 210.69 & 209.68 & 209.72 & 208.95 & 269.08 & 256.77 & 0.0004 & 5.5091 \\
\hline 191.5480 & 247.07 & 226.39 & 210.75 & 209.73 & 209.78 & 209.01 & 269.31 & 255.44 & 0.0003 & 5.5354 \\
\hline 192.0478 & 241.76 & 223.96 & 210.77 & 209.79 & 209.82 & 209.05 & 269.31 & 256.63 & 0.0003 & 5.5053 \\
\hline 192.5467 & 236.96 & 222.64 & 210.77 & 209.79 & 209.84 & 209.11 & 269.38 & 256.88 & 0.0003 & 5.4942 \\
\hline 193.0475 & 231.94 & 220.44 & 210.78 & 209.83 & 209.87 & 209.10 & 269.49 & 256.29 & 0.0003 & 5.5128 \\
\hline 193.5482 & 227.18 & 218.64 & 210.79 & 209.84 & 209.89 & 209.10 & 269.37 & 256.17 & 0.0004 & 5.5480 \\
\hline 194.0480 & 228.60 & 216.82 & 210.76 & 209.82 & 209.86 & 209.13 & 269.05 & 256.77 & 0.0002 & 5.5374 \\
\hline 194.5478 & 233.19 & 217.19 & 210.71 & 209.79 & 209.90 & 209.15 & 268.69 & 256.53 & 0.0003 & 5.4948 \\
\hline 195.0468 & 238.84 & 219.87 & 210.64 & 209.73 & 209.89 & 209.19 & 268.45 & 257.51 & 0.0003 & 5.4994 \\
\hline 195.5483 & 241.80 & 222.69 & 210.66 & 209.75 & 209.89 & 209.25 & 269.02 & 257.76 & 0.0003 & 5.5276 \\
\hline 196.0482 & 250.74 & 223.84 & 210.72 & 209.83 & 209.94 & 209.26 & 268.76 & 256.83 & 0.0003 & 5.5391 \\
\hline 196.5480 & 254.46 & 226.87 & 210.66 & 209.78 & 209.92 & 209.26 & 268.48 & 256.95 & 0.0003 & 5.4963 \\
\hline 197.0470 & 253.91 & 228.40 & 210.61 & 209.77 & 209.94 & 209.14 & 268.42 & 256.15 & 0.0000 & 5.4970 \\
\hline 197.5477 & 247.74 & 228.28 & 210.67 & 209.74 & 209.84 & 209.16 & 269.04 & 257.18 & 0.0001 & 5.5184 \\
\hline 198.0485 & 248.03 & 227.75 & 210.73 & 209.79 & 209.92 & 209.22 & 269.32 & 257.07 & 0.0003 & 5.5406 \\
\hline 198.5483 & 241.44 & 227.21 & 210.89 & 209.92 & 209.98 & 209.33 & 270.96 & 257.89 & 0.0001 & 5.5007 \\
\hline 199.0480 & 235.74 & 225.46 & 211.06 & 210.05 & 210.09 & 209.34 & 271.61 & 258.11 & 0.0001 & 5.4748 \\
\hline 199.5478 & 231.13 & 223.34 & 211.17 & 210.19 & 210.19 & 209.45 & 272.39 & 258.57 & 0.0003 & 5.5402 \\
\hline
\end{tabular}


TABLE B6.- CONTINUED.

\begin{tabular}{|c|c|c|c|c|c|c|c|c|c|c|}
\hline $\begin{array}{l}\text { Time, } \\
\text { min }\end{array}$ & $\begin{array}{c}\text { T-steam } \\
{ }^{\circ} \mathrm{C}\end{array}$ & $\begin{array}{l}\mathrm{T} 1, \\
{ }^{\circ} \mathrm{C}\end{array}$ & $\begin{array}{l}\mathrm{T} 2, \\
{ }^{\circ} \mathrm{C}\end{array}$ & $\begin{array}{l}\mathrm{T} 3, \\
{ }^{\circ} \mathrm{C}\end{array}$ & $\begin{array}{l}\mathrm{T} 4, \\
{ }^{\circ} \mathrm{C}\end{array}$ & $\begin{array}{l}\mathrm{T} 5, \\
{ }^{\circ} \mathrm{C}\end{array}$ & $\begin{array}{l}\text { Pinj, } \\
\text { psig }\end{array}$ & $\begin{array}{c}\text { Pout, } \\
\text { psig }\end{array}$ & $\begin{array}{l}\text { Qinj, } \\
\text { g/min }\end{array}$ & $\begin{array}{c}\mathrm{Vw} \\
\mathrm{ml} / \mathrm{min}\end{array}$ \\
\hline 200.0477 & 225.91 & 220.12 & 211.30 & 210.30 & 210.28 & 209.47 & 272.80 & 257.82 & 0.0003 & 5.5421 \\
\hline 200.5475 & 226.90 & 217.68 & 211.31 & 210.25 & 210.13 & 209.14 & 272.31 & 256.51 & 0.0003 & 5.4962 \\
\hline 201.0483 & 231.45 & 218.54 & 211.17 & 210.11 & 210.01 & 209.07 & 271.08 & 256.09 & 0.0001 & 5.4882 \\
\hline 201.5482 & 237.54 & 219.92 & 210.96 & 209.97 & 209.94 & 209.01 & 269.91 & 255.42 & 0.0001 & 5.5157 \\
\hline 202.0470 & 241.54 & 219.88 & 210.77 & 209.82 & 209.83 & 209.03 & 268.71 & 255.26 & 0.0002 & 5.5408 \\
\hline 202.5477 & 240.44 & 220.09 & 210.52 & 209.66 & 209.77 & 209.04 & 267.43 & 255.40 & 0.0004 & 5.5143 \\
\hline 203.0475 & 238.65 & 220.98 & 210.35 & 209.54 & 209.70 & 209.04 & 266.66 & 256.09 & 0.0003 & 5.4705 \\
\hline 203.5483 & 234.12 & 220.50 & 210.33 & 209.49 & 209.64 & 208.97 & 267.30 & 257.23 & 0.0005 & 5.5134 \\
\hline 204.0472 & 232.05 & 219.48 & 210.48 & 209.61 & 209.73 & 209.12 & 268.34 & 256.79 & 0.0003 & 5.5525 \\
\hline 204.5470 & 231.00 & 217.97 & 210.56 & 209.65 & 209.75 & 209.11 & 268.01 & 256.54 & 0.0003 & 5.5259 \\
\hline 205.0468 & 224.68 & 216.02 & 210.55 & 209.69 & 209.83 & 209.15 & 268.08 & 256.08 & 0.0003 & 5.5016 \\
\hline 205.5477 & 223.06 & 213.95 & 210.57 & 209.67 & 209.78 & 209.03 & 267.97 & 256.18 & 0.0003 & 5.5239 \\
\hline 206.0483 & 222.39 & 213.55 & 210.54 & 209.63 & 209.72 & 209.04 & 267.77 & 255.72 & 0.0003 & 5.5240 \\
\hline 206.5482 & 225.70 & 214.07 & 210.51 & 209.61 & 209.74 & 209.09 & 267.64 & 256.47 & 0.0003 & 5.5391 \\
\hline 207.0480 & 231.42 & 215.16 & 210.51 & 209.64 & 209.74 & 209.08 & 267.92 & 256.98 & 0.0003 & 5.4917 \\
\hline 207.5478 & 243.55 & 217.50 & 210.52 & 209.64 & 209.77 & 209.07 & 267.59 & 256.03 & 0.0000 & 5.5122 \\
\hline 208.0477 & 245.93 & 220.06 & 210.47 & 209.61 & 209.79 & 209.13 & 267.48 & 255.78 & 0.0003 & 5.5317 \\
\hline 208.5475 & 252.18 & 221.98 & 210.42 & 209.60 & 209.76 & 209.10 & 267.41 & 256.10 & 0.0001 & 5.5603 \\
\hline 209.0482 & 250.55 & 224.72 & 210.46 & 209.60 & 209.76 & 209.06 & 268.44 & 255.64 & 0.0003 & 5.5275 \\
\hline 209.5480 & 248.51 & 224.98 & 210.65 & 209.68 & 209.77 & 209.14 & 269.47 & 256.98 & 0.0001 & 5.4927 \\
\hline 210.0478 & 242.31 & 225.54 & 210.85 & 209.84 & 209.88 & 209.15 & 270.16 & 255.58 & 0.0003 & 5.5051 \\
\hline 210.5477 & 236.66 & 223.99 & 210.85 & 209.88 & 209.94 & 209.17 & 269.97 & 256.34 & 0.0003 & 5.5340 \\
\hline 211.0483 & 235.22 & 222.47 & 210.78 & 209.87 & 209.94 & 209.19 & 269.46 & 255.74 & 0.0003 & 5.5389 \\
\hline 211.5482 & 232.48 & 220.47 & 210.70 & 209.82 & 209.93 & 209.16 & 268.68 & 256.77 & 0.0004 & 5.4863 \\
\hline 212.0480 & 229.23 & 218.54 & 210.58 & 209.74 & 209.88 & 209.18 & 267.99 & 257.21 & 0.0006 & 5.4912 \\
\hline 212.5478 & 234.95 & 218.85 & 210.46 & 209.65 & 209.85 & 209.20 & 267.25 & 256.67 & 0.0003 & 5.5352 \\
\hline 213.0468 & 238.12 & 220.43 & 210.39 & 209.57 & 209.76 & 209.10 & 267.21 & 255.99 & 0.0007 & 5.5555 \\
\hline 213.5475 & 249.53 & 224.23 & 210.65 & 209.72 & 209.84 & 209.18 & 269.18 & 256.39 & 0.0004 & 5.5055 \\
\hline 214.0482 & 251.85 & 227.81 & 210.78 & 209.81 & 209.88 & 209.13 & 269.70 & 255.56 & 0.0008 & 5.4849 \\
\hline 214.5480 & 250.20 & 229.72 & 210.85 & 209.87 & 209.92 & 209.17 & 270.25 & 255.77 & 0.0003 & 5.5038 \\
\hline 215.0478 & 252.56 & 230.10 & 210.89 & 209.91 & 209.96 & 209.18 & 270.28 & 255.88 & 0.0000 & 5.5529 \\
\hline 215.5477 & 246.43 & 230.05 & 210.88 & 209.91 & 209.99 & 209.23 & 270.24 & 256.90 & 0.0002 & 5.5334 \\
\hline 216.0485 & 246.00 & 228.41 & 210.87 & 209.94 & 210.03 & 209.26 & 269.88 & 256.61 & 0.0003 & 5.5186 \\
\hline 216.5483 & 240.86 & 226.46 & 210.80 & 209.91 & 209.99 & 209.26 & 269.25 & 256.73 & 0.0000 & 5.5014 \\
\hline 217.0480 & 235.92 & 224.42 & 210.68 & 209.82 & 209.95 & 209.27 & 268.61 & 256.72 & 0.0003 & 5.5141 \\
\hline 217.5478 & 231.45 & 222.37 & 210.59 & 209.77 & 209.91 & 209.23 & 268.06 & 257.15 & 0.0003 & 5.5210 \\
\hline 218.0477 & 226.98 & 219.39 & 210.58 & 209.72 & 209.88 & 209.27 & 268.48 & 257.68 & 0.0001 & 5.5126 \\
\hline 218.5467 & 224.53 & 216.69 & 210.52 & 209.69 & 209.89 & 209.28 & 267.84 & 257.30 & 0.0004 & 5.4916 \\
\hline 219.0483 & 220.68 & 213.88 & 210.47 & 209.68 & 209.89 & 209.28 & 267.15 & 256.44 & 0.0003 & 5.5276 \\
\hline 219.5482 & 220.44 & 212.74 & 210.45 & 209.65 & 209.84 & 209.18 & 267.61 & 256.21 & 0.0003 & 5.5398 \\
\hline 220.0480 & 226.89 & 213.43 & 210.44 & 209.63 & 209.85 & 209.31 & 267.20 & 256.98 & 0.0004 & 5.4959 \\
\hline 220.5477 & 230.17 & 214.94 & 210.50 & 209.67 & 209.91 & 209.30 & 267.69 & 257.42 & 0.0001 & 5.4908 \\
\hline 221.0475 & 238.73 & 217.16 & 210.56 & 209.71 & 209.91 & 209.27 & 268.49 & 256.37 & 0.0003 & 5.5238 \\
\hline 221.5483 & 248.17 & 219.81 & 210.65 & 209.77 & 209.95 & 209.31 & 268.63 & 256.69 & 0.0002 & 5.5556 \\
\hline 222.0482 & 251.05 & 223.93 & 210.67 & 209.81 & 209.94 & 209.21 & 269.03 & 256.49 & 0.0004 & 5.5141 \\
\hline 222.5470 & 255.74 & 227.52 & 210.73 & 209.82 & 210.00 & 209.41 & 269.61 & 257.37 & 0.0003 & 5.4975 \\
\hline 223.0478 & 249.44 & 229.46 & 210.81 & 209.91 & 210.02 & 209.36 & 269.75 & 257.62 & 0.0003 & 5.5156 \\
\hline 223.5477 & 252.89 & 229.32 & 210.77 & 209.92 & 210.02 & 209.36 & 269.46 & 257.24 & 0.0006 & 5.5435 \\
\hline 224.0492 & 251.06 & 228.94 & 210.76 & 209.87 & 210.03 & 209.31 & 269.31 & 256.78 & 0.0006 & 5.5384 \\
\hline 224.5472 & 244.59 & 227.79 & 210.76 & 209.89 & 210.03 & 209.32 & 269.91 & 256.44 & 0.0003 & 5.4862 \\
\hline
\end{tabular}


TABLE B6.- CONTINUED.

\begin{tabular}{|c|c|c|c|c|c|c|c|c|c|c|}
\hline $\begin{array}{c}\text { Time, } \\
\text { min }\end{array}$ & $\begin{array}{c}\text { T-steam } \\
{ }^{\circ} \mathrm{C}\end{array}$ & $\begin{array}{l}\mathrm{T} 1, \\
{ }^{\circ} \mathrm{C}\end{array}$ & $\begin{array}{l}\mathrm{T} 2, \\
{ }^{\circ} \mathrm{C}\end{array}$ & $\begin{array}{l}\mathrm{T} 3, \\
{ }^{\circ} \mathrm{C}\end{array}$ & $\begin{array}{l}\mathrm{T} 4, \\
{ }^{\circ} \mathrm{C}\end{array}$ & $\begin{array}{l}\mathrm{T} 5, \\
{ }^{\circ} \mathrm{C}\end{array}$ & $\begin{array}{l}\text { Pinj, } \\
\text { psig }\end{array}$ & $\begin{array}{c}\text { Pout, } \\
\text { psig }\end{array}$ & $\begin{array}{l}\text { Qinj, } \\
\text { g/min }\end{array}$ & $\begin{array}{c}\mathrm{Vw} \\
\mathrm{ml} / \mathrm{min}\end{array}$ \\
\hline 225.0480 & 238.41 & 226.14 & 226.14 & 209.91 & 210.03 & 209.34 & 269.53 & 257.48 & 0.0002 & 5.4903 \\
\hline 225.5487 & 235.94 & 223.75 & 223.75 & 209.90 & 210.04 & 209.38 & 268.96 & 256.86 & 0.0005 & 5.5359 \\
\hline 226.0477 & 229.18 & 221.61 & 221.61 & 209.88 & 210.03 & 209.36 & 269.89 & 257.43 & 0.0003 & 5.5183 \\
\hline 226.5475 & 228.55 & 219.12 & 219.12 & 209.98 & 210.07 & 209.39 & 270.29 & 256.80 & 0.0003 & 5.4989 \\
\hline 227.0482 & 223.74 & 216.17 & 216.17 & 210.02 & 210.07 & 209.35 & 270.46 & 257.35 & 0.0003 & 5.4951 \\
\hline 227.5480 & 223.33 & 215.28 & 215.28 & 210.02 & 210.09 & 209.40 & 270.37 & 257.41 & 0.0003 & 5.5045 \\
\hline 228.0468 & 228.25 & 215.77 & 215.77 & 210.03 & 210.11 & 209.36 & 269.88 & 256.94 & 0.0003 & 5.5355 \\
\hline 228.5467 & 234.84 & 217.39 & 217.39 & 209.96 & 210.05 & 209.26 & 269.24 & 256.85 & 0.0003 & 5.4903 \\
\hline 229.0475 & 242.03 & 219.61 & 219.61 & 209.85 & 210.01 & 209.34 & 268.53 & 256.83 & 0.0003 & 5.4963 \\
\hline 229.5482 & 239.85 & 221.65 & 221.65 & 209.80 & 210.00 & 209.36 & 268.47 & 256.60 & 0.0000 & 5.5278 \\
\hline 230.0480 & 242.43 & 221.55 & 221.55 & 209.77 & 209.99 & 209.34 & 267.94 & 256.62 & 0.0003 & 5.5606 \\
\hline 230.5478 & 241.86 & 221.20 & 221.20 & 209.72 & 209.94 & 209.28 & 267.62 & 256.53 & 0.0004 & 5.5207 \\
\hline 231.0477 & 239.39 & 221.97 & 221.97 & 209.89 & 210.03 & 209.42 & 270.71 & 257.71 & 0.0006 & 5.4887 \\
\hline 231.5483 & 234.54 & 221.24 & 221.24 & 210.05 & 210.11 & 209.39 & 271.16 & 257.53 & 0.0003 & 5.4923 \\
\hline 232.0482 & 229.26 & 219.53 & 219.53 & 210.20 & 210.20 & 209.45 & 272.72 & 257.42 & 0.0003 & 5.5400 \\
\hline 232.5480 & 227.62 & 218.13 & 218.13 & 210.40 & 210.33 & 209.52 & 274.81 & 258.14 & 0.0004 & 5.5217 \\
\hline 233.0470 & 226.18 & 216.55 & 216.55 & 210.68 & 210.51 & 209.62 & 276.41 & 258.27 & 0.0003 & 5.4930 \\
\hline 233.5477 & 227.66 & 216.21 & 216.21 & 210.86 & 210.64 & 209.66 & 277.02 & 258.31 & 0.0004 & 5.4925 \\
\hline 234.0485 & 230.73 & 217.00 & 217.00 & 210.84 & 210.65 & 209.57 & 276.11 & 257.87 & 0.0004 & 5.5209 \\
\hline 234.5483 & 238.90 & 218.49 & 218.49 & 210.70 & 210.58 & 209.59 & 274.46 & 257.98 & 0.0003 & 5.5346 \\
\hline 235.0472 & 243.60 & 220.80 & 220.80 & 210.51 & 210.48 & 209.55 & 272.96 & 257.79 & 0.0001 & 5.4972 \\
\hline 235.5478 & 249.33 & 224.15 & 224.15 & 210.34 & 210.37 & 209.51 & 271.92 & 257.57 & 0.0003 & 5.4940 \\
\hline 236.0477 & 258.63 & 227.74 & 227.74 & 210.20 & 210.29 & 209.50 & 270.84 & 257.45 & 0.0004 & 5.5229 \\
\hline 236.5467 & 261.37 & 230.16 & 230.16 & 210.06 & 210.20 & 209.47 & 269.93 & 257.61 & 0.0000 & 5.5472 \\
\hline 237.0473 & 260.98 & 231.11 & 231.11 & 209.94 & 210.15 & 209.45 & 269.15 & 257.31 & 0.0003 & 5.5013 \\
\hline 237.5482 & 258.81 & 231.06 & 231.06 & 209.83 & 210.08 & 209.46 & 268.25 & 257.87 & 0.0001 & 5.4886 \\
\hline 238.0480 & 252.78 & 230.24 & 230.24 & 209.71 & 210.02 & 209.43 & 267.38 & 257.11 & 0.0003 & 5.5209 \\
\hline 238.5468 & 249.46 & 228.19 & 228.19 & 209.59 & 209.95 & 209.41 & 265.85 & 257.63 & 0.0001 & 5.5296 \\
\hline 239.0475 & 247.78 & 226.45 & 226.45 & 209.45 & 209.84 & 209.36 & 264.76 & 257.15 & 0.0009 & 5.5218 \\
\hline 239.5483 & 242.00 & 224.04 & 224.04 & 209.33 & 209.79 & 209.42 & 264.46 & 257.72 & 0.0005 & 5.4968 \\
\hline 240.0490 & 233.34 & 222.41 & 222.41 & 209.32 & 209.76 & 209.37 & 264.09 & 257.22 & 0.0002 & 5.5021 \\
\hline 240.5480 & 230.78 & 220.11 & 220.11 & 209.25 & 209.71 & 209.30 & 263.85 & 257.24 & 0.0000 & 5.5560 \\
\hline 241.0487 & 229.51 & 219.02 & 219.02 & 209.32 & 209.75 & 209.38 & 266.66 & 257.25 & 0.0006 & 5.5195 \\
\hline 241.5477 & 232.25 & 221.78 & 221.78 & 209.68 & 209.95 & 209.51 & 268.76 & 257.92 & 0.0004 & 5.4839 \\
\hline 242.0483 & 238.33 & 223.63 & 223.63 & 209.92 & 210.10 & 209.47 & 270.02 & 257.71 & 0.0003 & 5.5106 \\
\hline 242.5482 & 246.65 & 226.93 & 226.93 & 210.05 & 210.21 & 209.55 & 271.02 & 257.98 & 0.0005 & 5.5424 \\
\hline 243.0470 & 250.33 & 228.76 & 228.76 & 210.12 & 210.25 & 209.59 & 270.57 & 258.00 & 0.0003 & 5.5144 \\
\hline 243.5478 & 253.07 & 229.49 & 229.49 & 210.06 & 210.27 & 209.59 & 269.79 & 258.36 & 0.0002 & 5.4775 \\
\hline 244.0477 & 246.91 & 228.74 & 228.74 & 209.99 & 210.19 & 209.54 & 268.99 & 257.91 & 0.0004 & 5.4913 \\
\hline 244.5483 & 247.82 & 228.16 & 228.16 & 209.87 & 210.14 & 209.53 & 268.55 & 257.91 & 0.0005 & 5.5330 \\
\hline 245.0473 & 242.98 & 226.66 & 226.66 & 209.82 & 210.07 & 209.48 & 268.11 & 257.70 & 0.0003 & 5.5385 \\
\hline 245.5480 & 234.58 & 223.87 & 223.87 & 209.78 & 210.05 & 209.46 & 267.57 & 257.65 & 0.0003 & 5.4990 \\
\hline 246.0478 & 232.22 & 221.56 & 221.56 & 209.68 & 210.00 & 209.45 & 267.10 & 257.53 & 0.0003 & 5.4992 \\
\hline 246.5477 & 228.66 & 219.15 & 219.15 & 209.74 & 209.99 & 209.42 & 267.77 & 257.62 & 0.0002 & 5.5256 \\
\hline 247.0483 & 224.70 & 217.18 & 217.18 & 209.74 & 210.01 & 209.44 & 268.35 & 257.32 & 0.0004 & 5.5494 \\
\hline
\end{tabular}




\section{APPENDIX C}

\section{PRODUCTION DATA}

TABLE C1. PRODUCTION DATA FOR RUN 3.

\begin{tabular}{|c|c|c|c|c|c|c|c|c|c|c|}
\hline $\begin{array}{l}\text { Sample } \\
\text { Number }\end{array}$ & $\begin{array}{c}\text { Sampling } \\
\text { Time, } \\
\text { min }\end{array}$ & $\begin{array}{l}\text { Vol. } \\
\text { Oil, } \\
\mathrm{cm}^{3}\end{array}$ & $\begin{array}{c}\text { Vol. } \\
\text { Water, } \\
\text { cm }^{3}\end{array}$ & $\begin{array}{c}\text { Vol. } \\
\text { Total, } \\
\text { cm }^{3}\end{array}$ & $\begin{array}{c}\text { Cum. } \\
\text { Oil, } \\
\mathrm{cm}^{3}\end{array}$ & $\begin{array}{c}\text { Cum. } \\
\text { Water, } \\
\text { cm }^{3}\end{array}$ & $\begin{array}{c}\text { Oil } \\
\text { Rate, } \\
\mathrm{cm}^{3}\end{array}$ & $\begin{array}{c}\text { Water } \\
\text { Rate, } \\
\mathrm{cm}^{3}\end{array}$ & $\begin{array}{c}\text { Pore } \\
\text { Vol. } \\
\text { Injected, } \\
\text { fraction }\end{array}$ & $\begin{array}{c}\text { Oil } \\
\text { Recovery, } \\
\%\end{array}$ \\
\hline 1 & 17 & 2 & 8 & 10 & 2 & 8 & 0.6667 & 2.6667 & 0.0826 & 0.0021 \\
\hline 2 & 20 & 4 & 9 & 13 & 6 & 17 & 1.3333 & 3.0000 & 0.0971 & 0.0062 \\
\hline 3 & 23 & 5 & 9 & 14 & 11 & 26 & 1.6667 & 3.0000 & 0.1117 & 0.0114 \\
\hline 4 & 26 & 7 & 9.5 & 16.5 & 18 & 35.5 & 2.3333 & 3.1667 & 0.1263 & 0.0187 \\
\hline 5 & 29 & 8 & 8.5 & 16.5 & 26 & 44 & 2.6667 & 2.8333 & 0.1408 & 0.0270 \\
\hline 6 & 32 & 8 & 10 & 18 & 34 & 54 & 2.6667 & 3.3333 & 0.1554 & 0.0353 \\
\hline 7 & 35 & 9 & 12 & 21 & 43 & 66 & 3.0000 & 4.0000 & 0.1700 & 0.0446 \\
\hline 8 & 38 & 9 & 11 & 20 & 52 & 77 & 3.0000 & 3.6667 & 0.1845 & 0.0540 \\
\hline 9 & 41 & 11 & 14 & 25 & 63 & 91 & 3.6667 & 4.6667 & 0.1991 & 0.0654 \\
\hline 10 & 44 & 11 & 18 & 29 & 74 & 109 & 3.6667 & 6.0000 & 0.2137 & 0.0768 \\
\hline 11 & 47 & 12 & 15 & 27 & 86 & 124 & 4.0000 & 5.0000 & 0.2282 & 0.0893 \\
\hline 12 & 50 & 16 & 16 & 32 & 102 & 140 & 5.3333 & 5.3333 & 0.2428 & 0.1059 \\
\hline 13 & 53 & 17 & 17 & 34 & 119 & 157 & 5.6667 & 5.6667 & 0.2574 & 0.1235 \\
\hline 14 & 56 & 20 & 18 & 38 & 139 & 175 & 6.6667 & 6.0000 & 0.2719 & 0.1443 \\
\hline 15 & 59 & 24 & 17 & 41 & 163 & 192 & 8.0000 & 5.6667 & 0.2865 & 0.1692 \\
\hline 16 & 62 & 35 & 14 & 49 & 198 & 206 & 11.6667 & 4.6667 & 0.3011 & 0.2055 \\
\hline 17 & 65 & 45 & 15 & 60 & 243 & 221 & 15.0000 & 5.0000 & 0.3156 & 0.2522 \\
\hline 18 & 68 & 25 & 16 & 41 & 268 & 237 & 8.3333 & 5.3333 & 0.3302 & 0.2782 \\
\hline 19 & 71 & 20 & 16 & 36 & 288 & 253 & 6.6667 & 5.3333 & 0.3448 & 0.2989 \\
\hline 20 & 74 & 14 & 15 & 29 & 302 & 268 & 4.6667 & 5.0000 & 0.3593 & 0.3135 \\
\hline 21 & 77 & 12 & 14 & 26 & 314 & 282 & 4.0000 & 4.6667 & 0.3739 & 0.3259 \\
\hline 22 & 80 & 8 & 16 & 24 & 322 & 298 & 2.6667 & 5.3333 & 0.3885 & 0.3342 \\
\hline 23 & 83 & 6 & 17 & 23 & 328 & 315 & 2.0000 & 5.6667 & 0.4030 & 0.3405 \\
\hline 24 & 86 & 6 & 15 & 21 & 334 & 330 & 2.0000 & 5.0000 & 0.4176 & 0.3467 \\
\hline 25 & 89 & 2 & 15 & 17 & 336 & 345 & 0.6667 & 5.0000 & 0.4322 & 0.3488 \\
\hline 26 & 92 & 1 & 14 & 15 & 337 & 359 & 0.3333 & 4.6667 & 0.4467 & 0.3498 \\
\hline 27 & 95 & 1 & 16 & 17 & 338 & 375 & 0.3333 & 5.3333 & 0.4613 & 0.3508 \\
\hline 28 & 98 & 2 & 15 & 17 & 340 & 390 & 0.6667 & 5.0000 & 0.4759 & 0.3529 \\
\hline 29 & 101 & 1 & 17 & 18 & 341 & 407 & 0.3333 & 5.6667 & 0.4905 & 0.3540 \\
\hline 30 & 104 & 1 & 15 & 16 & 342 & 422 & 0.3333 & 5.0000 & 0.5050 & 0.3550 \\
\hline 31 & 107 & 2 & 14 & 16 & 344 & 436 & 0.6667 & 4.6667 & 0.5196 & 0.3571 \\
\hline 32 & 110 & 1 & 14 & 15 & 345 & 450 & 0.3333 & 4.6667 & 0.5342 & 0.3581 \\
\hline 33 & 113 & 1.5 & 14 & 15.5 & 346.5 & 464 & 0.5000 & 4.6667 & 0.5487 & 0.3597 \\
\hline 34 & 116 & 2 & 13 & 15 & 348.5 & 477 & 0.6667 & 4.3333 & 0.5633 & 0.3617 \\
\hline 35 & 119 & 2 & 13 & 15 & 350.5 & 490 & 0.5000 & 3.2500 & 0.5779 & 0.3638 \\
\hline 36 & 123 & 2 & 17 & 19 & 352.5 & 507 & 0.5000 & 4.2500 & 0.5973 & 0.3659 \\
\hline 37 & 127 & 2 & 15 & 17 & 354.5 & 522 & 0.5000 & 3.7500 & 0.6167 & 0.3680 \\
\hline 38 & 131 & 2 & 15 & 17 & 356.5 & 537 & 0.5000 & 3.7500 & 0.6361 & 0.3700 \\
\hline 39 & 135 & 4 & 17 & 21 & 360.5 & 554 & 1.0000 & 4.2500 & 0.6556 & 0.3742 \\
\hline 40 & 139 & 9 & 15 & 24 & 369.5 & 569 & 2.2500 & 3.7500 & 0.6750 & 0.3835 \\
\hline
\end{tabular}


TABLE C1. -CONTINUED.

\begin{tabular}{|c|c|c|c|c|c|c|c|c|c|c|}
\hline $\begin{array}{c}\text { Sample } \\
\text { Number }\end{array}$ & $\begin{array}{c}\text { Sampling } \\
\text { Time, } \\
\text { min }\end{array}$ & $\begin{array}{l}\text { Vol. } \\
\text { Oil, } \\
\mathrm{cm}^{3}\end{array}$ & $\begin{array}{c}\text { Vol. } \\
\text { Water, } \\
\text { cm }^{3}\end{array}$ & $\begin{array}{c}\text { Vol. } \\
\text { Total, } \\
\text { cm }^{3}\end{array}$ & $\begin{array}{c}\text { Cum. } \\
\text { Oil, } \\
\text { cm }^{3}\end{array}$ & $\begin{array}{c}\text { Cum. } \\
\text { Water, } \\
\text { cm }^{3}\end{array}$ & $\begin{array}{c}\text { Oil } \\
\text { Rate, } \\
\text { cm }^{3}\end{array}$ & $\begin{array}{l}\text { Water } \\
\text { Rate, } \\
\text { cm }^{3}\end{array}$ & $\begin{array}{c}\text { Pore } \\
\text { Vol. } \\
\text { Injected, } \\
\text { fraction } \\
\end{array}$ & $\begin{array}{c}\text { Oil } \\
\text { Recovery, } \\
\%\end{array}$ \\
\hline 41 & 143 & 15 & 16 & 31 & 384.5 & 585 & 5.0000 & 5.3333 & 0.6944 & 0.3991 \\
\hline 42 & 146 & 8 & 16 & 24 & 392.5 & 601 & 1.6000 & 3.2000 & 0.7090 & 0.4074 \\
\hline 43 & 151 & 6 & 17 & 23 & 398.5 & 618 & 1.2000 & 3.4000 & 0.7333 & 0.4136 \\
\hline 44 & 156 & 3 & 30 & 33 & 401.5 & 648 & 0.5000 & 5.0000 & 0.7575 & 0.4168 \\
\hline 45 & 162 & 2 & 35 & 37 & 403.5 & 683 & 0.3333 & 5.8333 & 0.7867 & 0.4188 \\
\hline 46 & 168 & 1 & 32 & 33 & 404.5 & 715 & 0.1667 & 5.3333 & 0.8158 & 0.4199 \\
\hline 47 & 174 & 1 & 33 & 34 & 405.5 & 748 & 0.1667 & 5.5000 & 0.8449 & 0.4209 \\
\hline 48 & 180 & 0.5 & 36 & 36.5 & 406 & 784 & 0.0833 & 6.0000 & 0.8741 & 0.4214 \\
\hline 49 & 186 & 0.5 & 32 & 32.5 & 406.5 & 816 & 0.0833 & 5.3333 & 0.9032 & 0.4219 \\
\hline 50 & 192 & 1 & 31 & 32 & 407.5 & 847 & 0.1667 & 5.1667 & 0.9323 & 0.4230 \\
\hline 51 & 198 & 0 & 33 & 33 & 407.5 & 880 & 0.0000 & 5.5000 & 0.9615 & 0.4230 \\
\hline 52 & 204 & 0 & 34 & 34 & 407.5 & 914 & 0.0000 & 5.6667 & 0.9906 & 0.4230 \\
\hline 53 & 210 & 0 & 35 & 35 & 407.5 & 949 & 0.0000 & 5.8333 & 1.0198 & 0.4230 \\
\hline 54 & 216 & 0 & 33 & 33 & 407.5 & 982 & 0.0000 & 5.5000 & 1.0489 & 0.4230 \\
\hline
\end{tabular}


TABLE C2. PRODUCTION DATA FOR RUN 4.

\begin{tabular}{|c|c|c|c|c|c|c|c|c|c|c|}
\hline $\begin{array}{l}\text { Sample } \\
\text { Number }\end{array}$ & $\begin{array}{c}\text { Sampling } \\
\text { Time, } \\
\text { min }\end{array}$ & $\begin{array}{l}\text { Vol. } \\
\text { Oil, } \\
\mathrm{cm}^{3}\end{array}$ & $\begin{array}{c}\text { Vol. } \\
\text { Water, } \\
\mathrm{cm}^{3}\end{array}$ & $\begin{array}{c}\text { Vol. } \\
\text { Total, } \\
\text { cm }^{3}\end{array}$ & $\begin{array}{c}\text { Cum. } \\
\text { Oil, } \\
\mathrm{cm}^{3}\end{array}$ & $\begin{array}{c}\text { Cum. } \\
\text { Water, } \\
\mathrm{cm}^{3}\end{array}$ & $\begin{array}{c}\text { Oil } \\
\text { Rate, } \\
\mathrm{cm}^{3}\end{array}$ & $\begin{array}{c}\text { Water } \\
\text { Rate, } \\
\mathrm{cm}^{3}\end{array}$ & $\begin{array}{c}\text { Pore } \\
\text { Vol. } \\
\text { Injected, } \\
\text { fraction }\end{array}$ & $\begin{array}{c}\text { Oil } \\
\text { Recovery, } \\
\%\end{array}$ \\
\hline 1 & 21 & 0 & 20 & 20 & 0 & 20 & 0.0000 & 6.6667 & 0.1020 & 0.0000 \\
\hline 2 & 24 & 1 & 18 & 19 & 1 & 38 & 0.3333 & 6.0000 & 0.1165 & 0.0010 \\
\hline 3 & 27 & 2 & 19 & 21 & 3 & 57 & 0.6667 & 6.3333 & 0.1311 & 0.0031 \\
\hline 4 & 30 & 1 & 20 & 21 & 4 & 77 & 0.3333 & 6.6667 & 0.1457 & 0.0042 \\
\hline 5 & 33 & 2 & 10 & 12 & 6 & 87 & 0.6667 & 3.3333 & 0.1602 & 0.0062 \\
\hline 6 & 36 & 2 & 9 & 11 & 8 & 96 & 0.6667 & 3.0000 & 0.1748 & 0.0083 \\
\hline 7 & 39 & 1.5 & 17 & 18.5 & 9.5 & 113 & 0.5000 & 5.6667 & 0.1894 & 0.0099 \\
\hline 8 & 42 & 1.5 & 21 & 22.5 & 11 & 134 & 0.5000 & 7.0000 & 0.2040 & 0.0114 \\
\hline 9 & 45 & 2 & 16 & 18 & 13 & 150 & 0.6667 & 5.3333 & 0.2185 & 0.0135 \\
\hline 10 & 48 & 3 & 15 & 18 & 16 & 165 & 1.0000 & 5.0000 & 0.2331 & 0.0166 \\
\hline 11 & 51 & 2 & 18 & 20 & 18 & 183 & 0.6667 & 6.0000 & 0.2477 & 0.0187 \\
\hline 12 & 54 & 1.5 & 20 & 21.5 & 19.5 & 203 & 0.5000 & 6.6667 & 0.2622 & 0.0202 \\
\hline 13 & 57 & 1.5 & 19 & 20.5 & 21 & 222 & 0.5000 & 6.3333 & 0.2768 & 0.0218 \\
\hline 14 & 60 & 2 & 18 & 20 & 23 & 240 & 0.6667 & 6.0000 & 0.2914 & 0.0239 \\
\hline 15 & 63 & 1 & 21 & 22 & 24 & 261 & 0.3333 & 7.0000 & 0.3059 & 0.0249 \\
\hline 16 & 66 & 2 & 20 & 22 & 26 & 281 & 0.6667 & 6.6667 & 0.3205 & 0.0270 \\
\hline 17 & 69 & 2 & 20 & 22 & 28 & 301 & 0.6667 & 6.6667 & 0.3351 & 0.0291 \\
\hline 18 & 72 & 2 & 17 & 19 & 30 & 318 & 0.6667 & 5.6667 & 0.3496 & 0.0311 \\
\hline 19 & 75 & 1 & 17 & 18 & 31 & 335 & 0.3333 & 5.6667 & 0.3642 & 0.0322 \\
\hline 20 & 78 & 1 & 19.5 & 20.5 & 32 & 354.5 & 0.3333 & 6.5000 & 0.3788 & 0.0332 \\
\hline 21 & 81 & 3 & 21 & 24 & 35 & 375.5 & 1.0000 & 7.0000 & 0.3933 & 0.0363 \\
\hline 22 & 84 & 1 & 22 & 23 & 36 & 397.5 & 0.3333 & 7.3333 & 0.4079 & 0.0374 \\
\hline 23 & 87 & 3 & 16 & 19 & 39 & 413.5 & 1.0000 & 5.3333 & 0.4225 & 0.0405 \\
\hline 24 & 90 & 2 & 19 & 21 & 41 & 432.5 & 0.6667 & 6.3333 & 0.4370 & 0.0426 \\
\hline 25 & 93 & 2 & 15 & 17 & 43 & 447.5 & 0.6667 & 5.0000 & 0.4516 & 0.0446 \\
\hline 26 & 96 & 3 & 13.5 & 16.5 & 46 & 461 & 1.0000 & 4.5000 & 0.4662 & 0.0477 \\
\hline 27 & 99 & 2 & 18 & 20 & 48 & 479 & 0.6667 & 6.0000 & 0.4807 & 0.0498 \\
\hline 28 & 102 & 2 & 18 & 20 & 50 & 497 & 0.6667 & 6.0000 & 0.4953 & 0.0519 \\
\hline 29 & 105 & 1.5 & 11.5 & 13 & 51.5 & 508.5 & 0.5000 & 3.8333 & 0.5099 & 0.0535 \\
\hline 30 & 108 & 2 & 21 & 23 & 53.5 & 529.5 & 0.6667 & 7.0000 & 0.5244 & 0.0555 \\
\hline 31 & 111 & 3 & 10 & 13 & 56.5 & 539.5 & 1.0000 & 3.3333 & 0.5390 & 0.0586 \\
\hline 32 & 114 & 2 & 12 & 14 & 58.5 & 551.5 & 0.6667 & 4.0000 & 0.5536 & 0.0607 \\
\hline 33 & 117 & 1.5 & 8 & 9.5 & 60 & 559.5 & 0.5000 & 2.6667 & 0.5681 & 0.0623 \\
\hline 34 & 120 & 2 & 11 & 13 & 62 & 570.5 & 0.6667 & 3.6667 & 0.5827 & 0.0644 \\
\hline 35 & 123 & 2.5 & 7 & 9.5 & 64.5 & 577.5 & 0.8333 & 2.3333 & 0.5973 & 0.0670 \\
\hline 36 & 126 & 3 & 7 & 10 & 67.5 & 584.5 & 1.0000 & 2.3333 & 0.6119 & 0.0701 \\
\hline 37 & 129 & 3.5 & 12 & 15.5 & 71 & 596.5 & 1.1667 & 4.0000 & 0.6264 & 0.0737 \\
\hline 38 & 132 & 3 & 13 & 16 & 74 & 609.5 & 1.0000 & 4.3333 & 0.6410 & 0.0768 \\
\hline 39 & 135 & 3 & 14 & 17 & 77 & 623.5 & 1.0000 & 4.6667 & 0.6556 & 0.0799 \\
\hline 40 & 138 & 3.5 & 12.5 & 16 & 80.5 & 636 & 1.1667 & 4.1667 & 0.6701 & 0.0836 \\
\hline 41 & 141 & 3.5 & 10 & 13.5 & 84 & 646 & 1.1667 & 3.3333 & 0.6847 & 0.0872 \\
\hline 42 & 144 & 2.5 & 10 & 12.5 & 86.5 & 656 & 0.8333 & 3.3333 & 0.6993 & 0.0898 \\
\hline 43 & 147 & 3 & 11 & 14 & 89.5 & 667 & 1.0000 & 3.6667 & 0.7138 & 0.0929 \\
\hline 44 & 150 & 3 & 11 & 14 & 92.5 & 678 & 1.0000 & 3.6667 & 0.7284 & 0.0960 \\
\hline 45 & 153 & 2.5 & 11 & 13.5 & 95 & 689 & 0.8333 & 3.6667 & 0.7430 & 0.0986 \\
\hline 46 & 156 & 3 & 12 & 15 & 98 & 701 & 1.0000 & 4.0000 & 0.7575 & 0.1017 \\
\hline 47 & 159 & 2 & 12 & 14 & 100 & 713 & 0.6667 & 4.0000 & 0.7721 & 0.1038 \\
\hline 48 & 162 & 3 & 11 & 14 & 103 & 724 & 1.0000 & 3.6667 & 0.7867 & 0.1069 \\
\hline 49 & 165 & 3 & 11 & 14 & 106 & 735 & 1.0000 & 3.6667 & 0.8012 & 0.1100 \\
\hline 50 & 168 & 2 & 10 & 12 & 108 & 745 & 0.6667 & 3.3333 & 0.8158 & 0.1121 \\
\hline
\end{tabular}


TABLE C2. -CONTINUED.

\begin{tabular}{|c|c|c|c|c|c|c|c|c|c|c|}
\hline $\begin{array}{l}\text { Sample } \\
\text { Number }\end{array}$ & $\begin{array}{c}\text { Sampling } \\
\text { Time, } \\
\text { min }\end{array}$ & $\begin{array}{l}\text { Vol. } \\
\text { Oil, } \\
\mathrm{cm}^{3}\end{array}$ & $\begin{array}{c}\text { Vol. } \\
\text { Water, } \\
\mathrm{cm}^{3}\end{array}$ & $\begin{array}{c}\text { Vol. } \\
\text { Total, } \\
\text { cm }^{3}\end{array}$ & $\begin{array}{l}\text { Cum. } \\
\text { Oil, } \\
\mathrm{cm}^{3}\end{array}$ & $\begin{array}{c}\text { Cum. } \\
\text { Water, } \\
\mathrm{cm}^{3}\end{array}$ & $\begin{array}{c}\text { Oil } \\
\text { Rate, } \\
\mathrm{cm}^{3}\end{array}$ & $\begin{array}{c}\text { Water } \\
\text { Rate, } \\
\mathrm{cm}^{3}\end{array}$ & $\begin{array}{c}\text { Pore } \\
\text { Vol. } \\
\text { Injected, } \\
\text { fraction }\end{array}$ & $\begin{array}{c}\text { Oil } \\
\text { Recovery, } \\
\%\end{array}$ \\
\hline 51 & 171 & 2.5 & 10 & 12.5 & 110.5 & 755 & 0.8333 & 3.3333 & 0.8304 & 0.1147 \\
\hline 52 & 174 & 7 & 10 & 17 & 117.5 & 765 & 2.3333 & 3.3333 & 0.8449 & 0.1220 \\
\hline 53 & 177 & 15 & 10 & 25 & 132.5 & 775 & 5.0000 & 3.3333 & 0.8595 & 0.1375 \\
\hline 54 & 180 & 21 & 10 & 31 & 153.5 & 785 & 7.0000 & 3.3333 & 0.8741 & 0.1593 \\
\hline 55 & 183 & 25 & 8 & 33 & 178.5 & 793 & 8.3333 & 2.6667 & 0.8886 & 0.1853 \\
\hline 56 & 186 & 20 & 6 & 26 & 198.5 & 799 & 6.6667 & 2.0000 & 0.9032 & 0.2060 \\
\hline 57 & 189 & 29 & 5 & 34 & 227.5 & 804 & 9.6667 & 1.6667 & 0.9178 & 0.2361 \\
\hline 58 & 192 & 45 & 4.5 & 49.5 & 272.5 & 808.5 & 15.0000 & 1.5000 & 0.9323 & 0.2829 \\
\hline 59 & 195 & 32 & 4 & 36 & 304.5 & 812.5 & 10.6667 & 1.3333 & 0.9469 & 0.3161 \\
\hline 60 & 198 & 18 & 4 & 22 & 322.5 & 816.5 & 6.0000 & 1.3333 & 0.9615 & 0.3348 \\
\hline 61 & 201 & 9 & 3.5 & 12.5 & 331.5 & 820 & 3.0000 & 1.1667 & 0.9760 & 0.3441 \\
\hline 62 & 204 & 6 & 10 & 16 & 337.5 & 830 & 2.0000 & 3.3333 & 0.9906 & 0.3503 \\
\hline 63 & 207 & 3 & 14 & 17 & 340.5 & 844 & 1.0000 & 4.6667 & 1.0052 & 0.3534 \\
\hline 64 & 210 & 3.5 & 16 & 19.5 & 344 & 860 & 1.1667 & 5.3333 & 1.0198 & 0.3571 \\
\hline 65 & 213 & 2 & 15 & 17 & 346 & 875 & 0.6667 & 5.0000 & 1.0343 & 0.3591 \\
\hline 66 & 216 & 7 & 16 & 23 & 353 & 891 & 2.3333 & 5.3333 & 1.0489 & 0.3664 \\
\hline 67 & 219 & 10 & 15 & 25 & 363 & 906 & 3.3333 & 5.0000 & 1.0635 & 0.3768 \\
\hline 68 & 222 & 4 & 15 & 19 & 367 & 921 & 1.3333 & 5.0000 & 1.0780 & 0.3809 \\
\hline 69 & 225 & 3 & 15 & 18 & 370 & 936 & 1.0000 & 5.0000 & 1.0926 & 0.3841 \\
\hline 70 & 228 & 3 & 16 & 19 & 373 & 952 & 1.0000 & 5.3333 & 1.1072 & 0.3872 \\
\hline 71 & 231 & 2 & 16 & 18 & 375 & 968 & 0.6667 & 5.3333 & 1.1217 & 0.3893 \\
\hline 72 & 234 & 2.5 & 9 & 11.5 & 377.5 & 977 & 0.8333 & 3.0000 & 1.1363 & 0.3918 \\
\hline 73 & 237 & 3 & 7 & 10 & 380.5 & 984 & 1.0000 & 2.3333 & 1.1509 & 0.3950 \\
\hline 74 & 240 & 3.5 & 11 & 14.5 & 384 & 995 & 1.1667 & 3.6667 & 1.1654 & 0.3986 \\
\hline 75 & 243 & 3 & 14 & 17 & 387 & 1009 & 1.0000 & 4.6667 & 1.1800 & 0.4017 \\
\hline 76 & 246 & 2 & 16 & 18 & 389 & 1025 & 0.6667 & 5.3333 & 1.1946 & 0.4038 \\
\hline 77 & 249 & 2 & 16 & 18 & 391 & 1041 & 0.6667 & 5.3333 & 1.2091 & 0.4059 \\
\hline 78 & 252 & 1 & 17 & 18 & 392 & 1058 & 0.3333 & 5.6667 & 1.2237 & 0.4069 \\
\hline 79 & 255 & 1 & 14 & 15 & 393 & 1072 & 0.3333 & 4.6667 & 1.2383 & 0.4079 \\
\hline 80 & 258 & 0.5 & 14 & 14.5 & 393.5 & 1086 & 0.1667 & 4.6667 & 1.2528 & 0.4085 \\
\hline 81 & 261 & 0.5 & 15 & 15.5 & 394 & 1101 & 0.1667 & 5.0000 & 1.2674 & 0.4090 \\
\hline 82 & 264 & 0 & 16 & 16 & 394 & 1117 & 0.0000 & 5.3333 & 1.2820 & 0.4090 \\
\hline 83 & 267 & 0 & 15 & 15 & 394 & 1132 & 0.0000 & 5.0000 & 1.2965 & 0.4090 \\
\hline 84 & 270 & 0 & 15 & 15 & 394 & 1147 & 0.0000 & 5.0000 & 1.3111 & 0.4090 \\
\hline 85 & 273 & 0 & 15 & 15 & 394 & 1162 & 0.0000 & 5.0000 & 1.3257 & 0.4090 \\
\hline
\end{tabular}


TABLE C3. PRODUCTION DATA FOR RUN 5.

\begin{tabular}{|c|c|c|c|c|c|c|c|c|c|c|}
\hline $\begin{array}{l}\text { Sample } \\
\text { Number }\end{array}$ & $\begin{array}{c}\text { Sampling } \\
\text { Time, } \\
\text { min }\end{array}$ & $\begin{array}{l}\text { Vol. } \\
\text { Oil, } \\
\mathrm{cm}^{3}\end{array}$ & $\begin{array}{c}\text { Vol. } \\
\text { Water, } \\
\text { cm }^{3}\end{array}$ & $\begin{array}{c}\text { Vol. } \\
\text { Total, } \\
\mathrm{cm}^{3}\end{array}$ & $\begin{array}{c}\text { Cum. } \\
\text { Oil, } \\
\mathrm{cm}^{3}\end{array}$ & $\begin{array}{c}\text { Cum. } \\
\text { Water, } \\
\text { cm }^{3}\end{array}$ & $\begin{array}{c}\text { Oil } \\
\text { Rate, } \\
\mathrm{cm}^{3}\end{array}$ & $\begin{array}{c}\text { Water } \\
\text { Rate, } \\
\mathrm{cm}^{3}\end{array}$ & $\begin{array}{c}\text { Pore } \\
\text { Vol. } \\
\text { Injected, } \\
\text { fraction }\end{array}$ & $\begin{array}{c}\text { Oil } \\
\text { Recovery, } \\
\%\end{array}$ \\
\hline 1 & 21 & 0 & 36 & 36 & 0 & 36 & 0.0000 & 12.0000 & 0.1020 & 0.0000 \\
\hline 2 & 24 & 0 & 24 & 24 & 0 & 60 & 0.0000 & 8.0000 & 0.1165 & 0.0000 \\
\hline 3 & 27 & 0.5 & 26 & 26.5 & 0.5 & 86 & 0.1667 & 8.6667 & 0.1311 & 0.0005 \\
\hline 4 & 30 & 2 & 25 & 27 & 2.5 & 111 & 0.6667 & 8.3333 & 0.1457 & 0.0026 \\
\hline 5 & 33 & 4 & 23 & 27 & 6.5 & 134 & 1.3333 & 7.6667 & 0.1602 & 0.0067 \\
\hline 6 & 36 & 4 & 21 & 25 & 10.5 & 155 & 1.3333 & 7.0000 & 0.1748 & 0.0109 \\
\hline 7 & 39 & 4 & 22 & 26 & 14.5 & 177 & 1.3333 & 7.3333 & 0.1894 & 0.0151 \\
\hline 8 & 42 & 3 & 23 & 26 & 17.5 & 200 & 1.0000 & 7.6667 & 0.2040 & 0.0182 \\
\hline 9 & 45 & 4 & 22 & 26 & 21.5 & 222 & 1.3333 & 7.3333 & 0.2185 & 0.0223 \\
\hline 10 & 48 & 3 & 21 & 24 & 24.5 & 243 & 1.0000 & 7.0000 & 0.2331 & 0.0254 \\
\hline 11 & 51 & 2 & 22.5 & 24.5 & 26.5 & 265.5 & 0.6667 & 7.5000 & 0.2477 & 0.0275 \\
\hline 12 & 54 & 2 & 21.5 & 23.5 & 28.5 & 287 & 0.6667 & 7.1667 & 0.2622 & 0.0296 \\
\hline 13 & 57 & 2 & 23 & 25 & 30.5 & 310 & 0.6667 & 7.6667 & 0.2768 & 0.0317 \\
\hline 14 & 60 & 2 & 18 & 20 & 32.5 & 328 & 0.6667 & 6.0000 & 0.2914 & 0.0337 \\
\hline 15 & 63 & 3 & 15 & 18 & 35.5 & 343 & 1.0000 & 5.0000 & 0.3059 & 0.0368 \\
\hline 16 & 66 & 3 & 16 & 19 & 38.5 & 359 & 1.0000 & 5.3333 & 0.3205 & 0.0400 \\
\hline 17 & 69 & 3.5 & 16.5 & 20 & 42 & 375.5 & 1.1667 & 5.5000 & 0.3351 & 0.0436 \\
\hline 18 & 72 & 3.5 & 16 & 19.5 & 45.5 & 391.5 & 1.1667 & 5.3333 & 0.3496 & 0.0472 \\
\hline 19 & 75 & 3 & 16.5 & 19.5 & 48.5 & 408 & 1.0000 & 5.5000 & 0.3642 & 0.0503 \\
\hline 20 & 78 & 3 & 17.5 & 20.5 & 51.5 & 425.5 & 1.0000 & 5.8333 & 0.3788 & 0.0535 \\
\hline 21 & 81 & 2.5 & 17 & 19.5 & 54 & 442.5 & 0.8333 & 5.6667 & 0.3933 & 0.0561 \\
\hline 22 & 84 & 2.5 & 16 & 18.5 & 56.5 & 458.5 & 0.8333 & 5.3333 & 0.4079 & 0.0586 \\
\hline 23 & 87 & 2 & 17 & 19 & 58.5 & 475.5 & 0.6667 & 5.6667 & 0.4225 & 0.0607 \\
\hline 24 & 90 & 2 & 16.5 & 18.5 & 60.5 & 492 & 0.6667 & 5.5000 & 0.4370 & 0.0628 \\
\hline 25 & 93 & 2 & 15.5 & 17.5 & 62.5 & 507.5 & 0.6667 & 5.1667 & 0.4516 & 0.0649 \\
\hline 26 & 96 & 2.5 & 17 & 19.5 & 65 & 524.5 & 0.8333 & 5.6667 & 0.4662 & 0.0675 \\
\hline 27 & 99 & 1.5 & 17.5 & 19 & 66.5 & 542 & 0.5000 & 5.8333 & 0.4807 & 0.0690 \\
\hline 28 & 102 & 2 & 17.5 & 19.5 & 68.5 & 559.5 & 0.6667 & 5.8333 & 0.4953 & 0.0711 \\
\hline 29 & 105 & 2 & 18 & 20 & 70.5 & 577.5 & 0.6667 & 6.0000 & 0.5099 & 0.0732 \\
\hline 30 & 108 & 2.5 & 18.5 & 21 & 73 & 596 & 0.8333 & 6.1667 & 0.5244 & 0.0758 \\
\hline 31 & 111 & 2.5 & 19 & 21.5 & 75.5 & 615 & 0.8333 & 6.3333 & 0.5390 & 0.0784 \\
\hline 32 & 114 & 2 & 18.5 & 20.5 & 77.5 & 633.5 & 0.6667 & 6.1667 & 0.5536 & 0.0804 \\
\hline 33 & 117 & 1.5 & 18 & 19.5 & 79 & 651.5 & 0.5000 & 6.0000 & 0.5681 & 0.0820 \\
\hline 34 & 120 & 1.5 & 17.5 & 19 & 80.5 & 669 & 0.5000 & 5.8333 & 0.5827 & 0.0836 \\
\hline 35 & 123 & 2 & 17 & 19 & 82.5 & 686 & 0.6667 & 5.6667 & 0.5973 & 0.0856 \\
\hline 36 & 126 & 2 & 17.5 & 19.5 & 84.5 & 703.5 & 0.6667 & 5.8333 & 0.6119 & 0.0877 \\
\hline 37 & 129 & 2 & 17 & 19 & 86.5 & 720.5 & 0.6667 & 5.6667 & 0.6264 & 0.0898 \\
\hline 38 & 132 & 1 & 18 & 19 & 87.5 & 738.5 & 0.3333 & 6.0000 & 0.6410 & 0.0908 \\
\hline 39 & 135 & 1.5 & 16.5 & 18 & 89 & 755 & 0.5000 & 5.5000 & 0.6556 & 0.0924 \\
\hline 40 & 138 & 1 & 17 & 18 & 90 & 772 & 0.3333 & 5.6667 & 0.6701 & 0.0934 \\
\hline 41 & 141 & 1.5 & 18 & 19.5 & 91.5 & 790 & 0.5000 & 6.0000 & 0.6847 & 0.0950 \\
\hline 42 & 144 & 2 & 18.5 & 20.5 & 93.5 & 808.5 & 0.6667 & 6.1667 & 0.6993 & 0.0971 \\
\hline 43 & 147 & 1.5 & 16 & 17.5 & 95 & 824.5 & 0.5000 & 5.3333 & 0.7138 & 0.0986 \\
\hline 44 & 150 & 2 & 14 & 16 & 97 & 838.5 & 0.6667 & 4.6667 & 0.7284 & 0.1007 \\
\hline 45 & 153 & 1.5 & 12.5 & 14 & 98.5 & 851 & 0.5000 & 4.1667 & 0.7430 & 0.1022 \\
\hline 46 & 156 & 1.5 & 10 & 11.5 & 100 & 861 & 0.5000 & 3.3333 & 0.7575 & 0.1038 \\
\hline 47 & 159 & 2 & 8 & 10 & 102 & 869 & 0.6667 & 2.6667 & 0.7721 & 0.1059 \\
\hline 48 & 162 & 1.5 & 6 & 7.5 & 103.5 & 875 & 0.5000 & 2.0000 & 0.7867 & 0.1074 \\
\hline 49 & 165 & 2 & 13 & 15 & 105.5 & 888 & 0.6667 & 4.3333 & 0.8012 & 0.1095 \\
\hline 50 & 168 & 1.5 & 12 & 13.5 & 107 & 900 & 0.5000 & 4.0000 & 0.8158 & 0.1111 \\
\hline
\end{tabular}


TABLE C3. -CONTINUED.

\begin{tabular}{|c|c|c|c|c|c|c|c|c|c|c|}
\hline $\begin{array}{c}\text { Sample } \\
\text { Number }\end{array}$ & $\begin{array}{c}\text { Sampling } \\
\text { Time, } \\
\text { min }\end{array}$ & $\begin{array}{l}\text { Vol. } \\
\text { Oil, } \\
\text { cm }^{3}\end{array}$ & $\begin{array}{c}\text { Vol. } \\
\text { Water, } \\
\mathrm{cm}^{3}\end{array}$ & $\begin{array}{c}\text { Vol. } \\
\text { Total, } \\
\text { cm }^{3}\end{array}$ & $\begin{array}{c}\text { Cum. } \\
\text { Oil, } \\
\text { cm }^{3}\end{array}$ & $\begin{array}{c}\text { Cum. } \\
\text { Water, } \\
\text { cm }^{3}\end{array}$ & $\begin{array}{c}\text { Oil } \\
\text { Rate, } \\
\mathrm{cm}^{3}\end{array}$ & $\begin{array}{c}\text { Water } \\
\text { Rate, } \\
\text { cm }^{3}\end{array}$ & $\begin{array}{c}\text { Pore } \\
\text { Vol. } \\
\text { Injected, } \\
\text { fraction }\end{array}$ & $\begin{array}{c}\text { Oil } \\
\text { Recovery, } \\
\%\end{array}$ \\
\hline 51 & 171 & 1.5 & 6 & 7.5 & 108.5 & 906 & 0.5000 & 2.0000 & 0.8304 & 0.1126 \\
\hline 52 & 174 & 2 & 4 & 6 & 110.5 & 910 & 0.6667 & 1.3333 & 0.8449 & 0.1147 \\
\hline 53 & 177 & 1.5 & 4.5 & 6 & 112 & 914.5 & 0.5000 & 1.5000 & 0.8595 & 0.1163 \\
\hline 54 & 180 & 7 & 3 & 10 & 119 & 917.5 & 2.3333 & 1.0000 & 0.8741 & 0.1235 \\
\hline 55 & 183 & 21 & 3.4 & 24.4 & 140 & 920.9 & 7.0000 & 1.1333 & 0.8886 & 0.1453 \\
\hline 56 & 186 & 45 & 1 & 46 & 185 & 921.9 & 15.0000 & 0.3333 & 0.9032 & 0.1920 \\
\hline 57 & 189 & 32 & 2 & 34 & 217 & 923.9 & 10.6667 & 0.6667 & 0.9178 & 0.2252 \\
\hline 58 & 192 & 18 & 3 & 21 & 235 & 926.9 & 6.0000 & 1.0000 & 0.9323 & 0.2439 \\
\hline 59 & 195 & 14 & 4 & 18 & 249 & 930.9 & 4.6667 & 1.3333 & 0.9469 & 0.2585 \\
\hline 60 & 198 & 22 & 5 & 27 & 271 & 935.9 & 7.3333 & 1.6667 & 0.9615 & 0.2813 \\
\hline 61 & 201 & 18 & 4 & 22 & 289 & 939.9 & 6.0000 & 1.3333 & 0.9760 & 0.3000 \\
\hline 62 & 204 & 15 & 3 & 18 & 304 & 942.9 & 5.0000 & 1.0000 & 0.9906 & 0.3156 \\
\hline 63 & 207 & 14 & 5 & 19 & 318 & 947.9 & 4.6667 & 1.6667 & 1.0052 & 0.3301 \\
\hline 64 & 210 & 8 & 6 & 14 & 326 & 953.9 & 2.6667 & 2.0000 & 1.0198 & 0.3384 \\
\hline 65 & 213 & 4 & 3 & 7 & 330 & 956.9 & 1.3333 & 1.0000 & 1.0343 & 0.3425 \\
\hline 66 & 216 & 4 & 4 & 8 & 334 & 960.9 & 1.3333 & 1.3333 & 1.0489 & 0.3467 \\
\hline 67 & 219 & 3 & 18 & 21 & 337 & 978.9 & 1.0000 & 6.0000 & 1.0635 & 0.3498 \\
\hline 68 & 222 & 3.5 & 16.5 & 20 & 340.5 & 995.4 & 1.1667 & 5.5000 & 1.0780 & 0.3534 \\
\hline 69 & 225 & 10 & 5 & 15 & 350.5 & 1000.4 & 3.3333 & 1.6667 & 1.0926 & 0.3638 \\
\hline 70 & 228 & 15 & 4 & 19 & 365.5 & 1004.4 & 5.0000 & 1.3333 & 1.1072 & 0.3794 \\
\hline 71 & 231 & 8 & 10 & 18 & 373.5 & 1014.4 & 2.6667 & 3.3333 & 1.1217 & 0.3877 \\
\hline 72 & 234 & 5 & 18 & 23 & 378.5 & 1032.4 & 1.6667 & 6.0000 & 1.1363 & 0.3929 \\
\hline 73 & 237 & 4 & 17.5 & 21.5 & 382.5 & 1049.9 & 1.3333 & 5.8333 & 1.1509 & 0.3970 \\
\hline 74 & 240 & 2 & 17 & 19 & 384.5 & 1066.9 & 0.6667 & 5.6667 & 1.1654 & 0.3991 \\
\hline 75 & 243 & 1.5 & 18.5 & 20 & 386 & 1085.4 & 0.5000 & 6.1667 & 1.1800 & 0.4007 \\
\hline 76 & 246 & 1.5 & 18 & 19.5 & 387.5 & 1103.4 & 0.5000 & 6.0000 & 1.1946 & 0.4022 \\
\hline 77 & 249 & 1 & 17.5 & 18.5 & 388.5 & 1120.9 & 0.3333 & 5.8333 & 1.2091 & 0.4033 \\
\hline 78 & 252 & 1 & 18 & 19 & 389.5 & 1138.9 & 0.3333 & 6.0000 & 1.2237 & 0.4043 \\
\hline 79 & 255 & 0.5 & 19 & 19.5 & 390 & 1157.9 & 0.1667 & 6.3333 & 1.2383 & 0.4048 \\
\hline 80 & 258 & 0.5 & 16.5 & 17 & 390.5 & 1174.4 & 0.1667 & 5.5000 & 1.2528 & 0.4053 \\
\hline 81 & 261 & 0 & 17 & 17 & 390.5 & 1191.4 & 0.0000 & 5.6667 & 1.2674 & 0.4053 \\
\hline 82 & 264 & 0 & 18 & 18 & 390.5 & 1209.4 & 0.0000 & 6.0000 & 1.2820 & 0.4053 \\
\hline 83 & 267 & 0 & 17.5 & 17.5 & 390.5 & 1226.9 & 0.0000 & 5.8333 & 1.2965 & 0.4053 \\
\hline 84 & 270 & 0 & 18 & 18 & 390.5 & 1244.9 & 0.0000 & 6.0000 & 1.3111 & 0.4053 \\
\hline 85 & 273 & 0 & 16.5 & 16.5 & 390.5 & 1261.4 & 0.0000 & 5.5000 & 1.3257 & 0.4053 \\
\hline
\end{tabular}


TABLE C4. PRODUCTION DATA FOR RUN 6.

\begin{tabular}{|c|c|c|c|c|c|c|c|c|c|c|}
\hline $\begin{array}{l}\text { Sample } \\
\text { Number }\end{array}$ & $\begin{array}{c}\text { Sampling } \\
\text { Time, } \\
\text { min }\end{array}$ & $\begin{array}{l}\text { Vol. } \\
\text { Oil, } \\
\mathrm{cm}^{3}\end{array}$ & $\begin{array}{c}\text { Vol. } \\
\text { Water, } \\
\mathrm{cm}^{3}\end{array}$ & $\begin{array}{c}\text { Vol. } \\
\text { Total, } \\
\mathrm{cm}^{3}\end{array}$ & $\begin{array}{c}\text { Cum. } \\
\text { Oil, } \\
\mathrm{cm}^{3}\end{array}$ & $\begin{array}{c}\text { Cum. } \\
\text { Water, } \\
\mathrm{cm}^{3}\end{array}$ & $\begin{array}{c}\text { Oil } \\
\text { Rate, } \\
\mathrm{cm}^{3}\end{array}$ & $\begin{array}{c}\text { Water } \\
\text { Rate, } \\
\mathrm{cm}^{3}\end{array}$ & $\begin{array}{c}\text { Pore } \\
\text { Vol. } \\
\text { Injected, } \\
\text { fraction } \\
\end{array}$ & $\begin{array}{c}\text { Oil } \\
\text { Recovery, } \\
\%\end{array}$ \\
\hline 1 & 14 & 0 & 10 & 10 & 0 & 0 & 0.0000 & 3.3333 & 0.0680 & 0.0000 \\
\hline 2 & 17 & 2 & 13 & 15 & 2 & 13 & 0.6667 & 4.3333 & 0.0826 & 0.0021 \\
\hline 3 & 20 & 3 & 13.5 & 16.5 & 5 & 26.5 & 1.0000 & 4.5000 & 0.0971 & 0.0052 \\
\hline 4 & 23 & 4 & 12.5 & 16.5 & 9 & 39 & 1.3333 & 4.1667 & 0.1117 & 0.0093 \\
\hline 5 & 26 & 5 & 12 & 17 & 14 & 51 & 1.6667 & 4.0000 & 0.1263 & 0.0145 \\
\hline 6 & 29 & 6.5 & 13 & 19.5 & 20.5 & 64 & 2.1667 & 4.3333 & 0.1408 & 0.0213 \\
\hline 7 & 32 & 7 & 15 & 22 & 27.5 & 79 & 2.3333 & 5.0000 & 0.1554 & 0.0285 \\
\hline 8 & 35 & 7.5 & 16 & 23.5 & 35 & 95 & 2.5000 & 5.3333 & 0.1700 & 0.0363 \\
\hline 9 & 38 & 9 & 17 & 26 & 44 & 112 & 3.0000 & 5.6667 & 0.1845 & 0.0457 \\
\hline 10 & 41 & 9 & 17 & 26 & 53 & 129 & 3.0000 & 5.6667 & 0.1991 & 0.0550 \\
\hline 11 & 44 & 10 & 17 & 27 & 63 & 146 & 3.3333 & 5.6667 & 0.2137 & 0.0654 \\
\hline 12 & 47 & 11 & 16 & 27 & 74 & 162 & 3.6667 & 5.3333 & 0.2282 & 0.0768 \\
\hline 13 & 50 & 13 & 15 & 28 & 87 & 177 & 4.3333 & 5.0000 & 0.2428 & 0.0903 \\
\hline 14 & 53 & 13 & 15.5 & 28.5 & 100 & 192.5 & 4.3333 & 5.1667 & 0.2574 & 0.1038 \\
\hline 15 & 56 & 14 & 16 & 30 & 114 & 208.5 & 4.6667 & 5.3333 & 0.2719 & 0.1183 \\
\hline 16 & 59 & 20 & 15 & 35 & 134 & 223.5 & 6.6667 & 5.0000 & 0.2865 & 0.1391 \\
\hline 17 & 62 & 46 & 4 & 50 & 180 & 227.5 & 15.3333 & 1.3333 & 0.3011 & 0.1868 \\
\hline 18 & 65 & 29 & 9 & 38 & 209 & 236.5 & 9.6667 & 3.0000 & 0.3156 & 0.2169 \\
\hline 19 & 68 & 25 & 12 & 37 & 234 & 248.5 & 8.3333 & 4.0000 & 0.3302 & 0.2429 \\
\hline 20 & 71 & 18 & 16 & 34 & 252 & 264.5 & 6.0000 & 5.3333 & 0.3448 & 0.2616 \\
\hline 21 & 74 & 15 & 17 & 32 & 267 & 281.5 & 5.0000 & 5.6667 & 0.3593 & 0.2771 \\
\hline 22 & 77 & 13 & 16 & 29 & 280 & 297.5 & 4.3333 & 5.3333 & 0.3739 & 0.2906 \\
\hline 23 & 80 & 11 & 15 & 26 & 291 & 312.5 & 3.6667 & 5.0000 & 0.3885 & 0.3021 \\
\hline 24 & 83 & 8 & 14 & 22 & 299 & 326.5 & 2.6667 & 4.6667 & 0.4030 & 0.3104 \\
\hline 25 & 86 & 7.5 & 12 & 19.5 & 306.5 & 338.5 & 2.5000 & 4.0000 & 0.4176 & 0.3181 \\
\hline 26 & 89 & 5 & 11.5 & 16.5 & 311.5 & 350 & 1.6667 & 3.8333 & 0.4322 & 0.3233 \\
\hline 27 & 92 & 5 & 11.5 & 16.5 & 316.5 & 361.5 & 1.6667 & 3.8333 & 0.4467 & 0.3285 \\
\hline 28 & 95 & 5 & 11.5 & 16.5 & 321.5 & 373 & 1.6667 & 3.8333 & 0.4613 & 0.3337 \\
\hline 29 & 98 & 3 & 13.5 & 16.5 & 324.5 & 386.5 & 1.0000 & 4.5000 & 0.4759 & 0.3368 \\
\hline 30 & 101 & 3.5 & 14.5 & 18 & 328 & 401 & 1.1667 & 4.8333 & 0.4905 & 0.3405 \\
\hline 31 & 104 & 3.5 & 14.5 & 18 & 331.5 & 415.5 & 1.1667 & 4.8333 & 0.5050 & 0.3441 \\
\hline 32 & 107 & 2.5 & 14 & 16.5 & 334 & 429.5 & 0.8333 & 4.6667 & 0.5196 & 0.3467 \\
\hline 32 & 110 & 3 & 13.5 & 16.5 & 337 & 443 & 1.0000 & 4.5000 & 0.5342 & 0.3498 \\
\hline 33 & 113 & 3 & 13.5 & 16.5 & 340 & 456.5 & 1.0000 & 4.5000 & 0.5487 & 0.3529 \\
\hline 34 & 116 & 2.5 & 14 & 16.5 & 342.5 & 470.5 & 0.8333 & 4.6667 & 0.5633 & 0.3555 \\
\hline 35 & 119 & 3.5 & 13 & 16.5 & 346 & 483.5 & 0.8750 & 3.2500 & 0.5779 & 0.3591 \\
\hline 36 & 123 & 3 & 17 & 20 & 349 & 500.5 & 0.7500 & 4.2500 & 0.5973 & 0.3623 \\
\hline 37 & 127 & 4 & 16 & 20 & 353 & 516.5 & 1.0000 & 4.0000 & 0.6167 & 0.3664 \\
\hline 38 & 131 & 9 & 17 & 26 & 362 & 533.5 & 2.2500 & 4.2500 & 0.6361 & 0.3758 \\
\hline 39 & 135 & 17 & 15 & 32 & 379 & 548.5 & 4.2500 & 3.7500 & 0.6556 & 0.3934 \\
\hline 40 & 139 & 9 & 16 & 25 & 388 & 564.5 & 2.2500 & 4.0000 & 0.6750 & 0.4027 \\
\hline
\end{tabular}


TABLE C4. -CONTINUED.

\begin{tabular}{|c|c|c|c|c|c|c|c|c|c|c|}
\hline $\begin{array}{l}\text { Sample } \\
\text { Number }\end{array}$ & $\begin{array}{c}\text { Sampling } \\
\text { Time, } \\
\text { min }\end{array}$ & $\begin{array}{l}\text { Vol. } \\
\text { Oil, } \\
\text { cm }^{3}\end{array}$ & $\begin{array}{c}\text { Vol. } \\
\text { Water, } \\
\text { cm }^{3}\end{array}$ & $\begin{array}{c}\text { Vol. } \\
\text { Total, } \\
\text { cm }^{3}\end{array}$ & $\begin{array}{c}\text { Cum. } \\
\text { Oil, } \\
\mathrm{cm}^{3}\end{array}$ & $\begin{array}{c}\text { Cum. } \\
\text { Water, } \\
\mathbf{c m}^{3}\end{array}$ & $\begin{array}{c}\text { Oil } \\
\text { Rate, } \\
\mathbf{c m}^{3}\end{array}$ & $\begin{array}{c}\text { Water } \\
\text { Rate, } \\
\mathrm{cm}^{3}\end{array}$ & $\begin{array}{c}\text { Pore } \\
\text { Vol. } \\
\text { Injected, } \\
\text { fraction } \\
\end{array}$ & $\begin{array}{c}\text { Oil } \\
\text { Recovery, } \\
\%\end{array}$ \\
\hline 41 & 143 & 4 & 15 & 19 & 392 & 579.5 & 1.3333 & 5.0000 & 0.6944 & 0.4069 \\
\hline 42 & 146 & 3 & 13.5 & 16.5 & 395 & 593 & 0.6000 & 2.7000 & 0.7090 & 0.4100 \\
\hline 43 & 151 & 2.5 & 25.5 & 28 & 397.5 & 618.5 & 0.5000 & 5.1000 & 0.7333 & 0.4126 \\
\hline 44 & 156 & 1.5 & 26.5 & 28 & 399 & 645 & 0.2500 & 4.4167 & 0.7575 & 0.4142 \\
\hline 45 & 162 & 1.5 & 31 & 32.5 & 400.5 & 676 & 0.2500 & 5.1667 & 0.7867 & 0.4157 \\
\hline 46 & 168 & 1 & 30 & 31 & 401.5 & 706 & 0.1667 & 5.0000 & 0.8158 & 0.4168 \\
\hline 47 & 174 & 1 & 33 & 34 & 402.5 & 739 & 0.1667 & 5.5000 & 0.8449 & 0.4178 \\
\hline 48 & 180 & 0.5 & 32.5 & 33 & 403 & 771.5 & 0.0833 & 5.4167 & 0.8741 & 0.4183 \\
\hline 49 & 186 & 0.5 & 32.5 & 33 & 403.5 & 804 & 0.0833 & 5.4167 & 0.9032 & 0.4188 \\
\hline 50 & 192 & 0 & 33 & 33 & 403.5 & 837 & 0.0000 & 5.5000 & 0.9323 & 0.4188 \\
\hline 51 & 198 & 0 & 35 & 35 & 403.5 & 872 & 0.0000 & 5.8333 & 0.9615 & 0.4188 \\
\hline 52 & 204 & 0 & 34 & 34 & 403.5 & 906 & 0.0000 & 5.6667 & 0.9906 & 0.4188 \\
\hline 53 & 210 & 0 & 34.5 & 34.5 & 403.5 & 940.5 & 0.0000 & 5.7500 & 1.0198 & 0.4188 \\
\hline 54 & 216 & 0 & 33.5 & 33.5 & 403.5 & 974 & 0.0000 & 5.5833 & 1.0489 & 0.41 \\
\hline
\end{tabular}


TABLE C5. PRODUCTION DATA FOR RUN 7.

\begin{tabular}{|c|c|c|c|c|c|c|c|c|c|c|}
\hline $\begin{array}{l}\text { Sample } \\
\text { Number }\end{array}$ & $\begin{array}{c}\text { Sampling } \\
\text { Time, } \\
\text { min }\end{array}$ & $\begin{array}{l}\text { Vol. } \\
\text { Oil, } \\
\mathrm{cm}^{3}\end{array}$ & $\begin{array}{c}\text { Vol. } \\
\text { Water, } \\
\mathrm{cm}^{3}\end{array}$ & $\begin{array}{c}\text { Vol. } \\
\text { Total, } \\
\mathrm{cm}^{3}\end{array}$ & $\begin{array}{c}\text { Cum. } \\
\text { Oil, } \\
\mathrm{cm}^{3}\end{array}$ & $\begin{array}{c}\text { Cum. } \\
\text { Water, } \\
\mathrm{cm}^{3}\end{array}$ & $\begin{array}{c}\text { Oil } \\
\text { Rate, } \\
\mathrm{cm}^{3}\end{array}$ & $\begin{array}{c}\text { Water } \\
\text { Rate, } \\
\mathrm{cm}^{3}\end{array}$ & $\begin{array}{c}\text { Pore } \\
\text { Vol. } \\
\text { Injected, } \\
\text { fraction }\end{array}$ & $\begin{array}{c}\text { Oil } \\
\text { Recovery, } \\
\%\end{array}$ \\
\hline 1 & 15 & 14.5 & 4.5 & 19 & 14.5 & 4.5 & 14.5000 & 4.5000 & 0.0728 & 0.0151 \\
\hline 2 & 16 & 19 & 2 & 21 & 33.5 & 6.5 & 19.0000 & 2.0000 & 0.0777 & 0.0348 \\
\hline 3 & 17 & 18.5 & 6.5 & 25 & 52 & 13 & 1.8500 & 0.6500 & 0.0826 & 0.0540 \\
\hline 4 & 27 & 14.5 & 17.5 & 32 & 66.5 & 30.5 & 2.9000 & 3.5000 & 0.1311 & 0.0690 \\
\hline 5 & 32 & 7 & 17.5 & 24.5 & 73.5 & 48 & 3.5000 & 8.7500 & 0.1554 & 0.0763 \\
\hline 6 & 34 & 8.5 & 15 & 23.5 & 82 & 63 & 4.2500 & 7.5000 & 0.1651 & 0.0851 \\
\hline 7 & 36 & 15 & 17 & 32 & 97 & 80 & 7.5000 & 8.5000 & 0.1748 & 0.1007 \\
\hline 8 & 38 & 13 & 17 & 30 & 110 & 97 & 4.3333 & 5.6667 & 0.1845 & 0.1142 \\
\hline 9 & 41 & 18 & 19.5 & 37.5 & 128 & 116.5 & 4.5000 & 4.8750 & 0.1991 & 0.1329 \\
\hline 10 & 45 & 18 & 18 & 36 & 146 & 134.5 & 3.6000 & 3.6000 & 0.2185 & 0.1515 \\
\hline 11 & 50 & 19 & 15 & 34 & 165 & 149.5 & 3.8000 & 3.0000 & 0.2428 & 0.1713 \\
\hline 12 & 55 & 19 & 16 & 35 & 184 & 165.5 & 4.7500 & 4.0000 & 0.2671 & 0.1910 \\
\hline 13 & 59 & 20 & 15 & 35 & 204 & 180.5 & 5.0000 & 3.7500 & 0.2865 & 0.2118 \\
\hline 14 & 63 & 24 & 16 & 40 & 228 & 196.5 & 6.0000 & 4.0000 & 0.3059 & 0.2367 \\
\hline 15 & 67 & 23 & 16 & 39 & 251 & 212.5 & 5.7500 & 4.0000 & 0.3253 & 0.2605 \\
\hline 16 & 71 & 19 & 17 & 36 & 270 & 229.5 & 6.3333 & 5.6667 & 0.3448 & 0.2803 \\
\hline 17 & 74 & 23 & 17 & 40 & 293 & 246.5 & 5.7500 & 4.2500 & 0.3593 & 0.3041 \\
\hline 18 & 78 & 21 & 19 & 40 & 314 & 265.5 & 5.2500 & 4.7500 & 0.3788 & 0.3259 \\
\hline 19 & 82 & 15 & 18 & 33 & 329 & 283.5 & 5.0000 & 6.0000 & 0.3982 & 0.3415 \\
\hline 20 & 85 & 19 & 20 & 39 & 348 & 303.5 & 4.7500 & 5.0000 & 0.4128 & 0.3612 \\
\hline 21 & 89 & 11 & 19 & 30 & 359 & 322.5 & 3.6667 & 6.3333 & 0.4322 & 0.3726 \\
\hline 22 & 92 & 11 & 19 & 30 & 370 & 341.5 & 2.7500 & 4.7500 & 0.4467 & 0.3841 \\
\hline 23 & 96 & 3.5 & 20 & 23.5 & 373.5 & 361.5 & 1.1667 & 6.6667 & 0.4662 & 0.3877 \\
\hline 24 & 99 & 5 & 19 & 24 & 378.5 & 380.5 & 1.6667 & 6.3333 & 0.4807 & 0.3929 \\
\hline 25 & 102 & 5 & 17 & 22 & 383.5 & 397.5 & 1.2500 & 4.2500 & 0.4953 & 0.3981 \\
\hline 26 & 106 & 12 & 15 & 27 & 395.5 & 412.5 & 1.5000 & 1.8750 & 0.5147 & 0.4105 \\
\hline 27 & 114 & 4 & 18 & 22 & 399.5 & 430.5 & 1.3333 & 6.0000 & 0.5536 & 0.4147 \\
\hline 28 & 117 & 8 & 1 & 9 & 407.5 & 431.5 & 2.0000 & 0.2500 & 0.5681 & 0.4230 \\
\hline 29 & 121 & 15 & 1 & 16 & 422.5 & 432.5 & 7.5000 & 0.5000 & 0.5876 & 0.4386 \\
\hline 30 & 123 & 4 & 9 & 13 & 426.5 & 441.5 & 0.8000 & 1.8000 & 0.5973 & 0.4427 \\
\hline 31 & 128 & 4 & 16 & 20 & 430.5 & 457.5 & 0.5714 & 2.2857 & 0.6216 & 0.4469 \\
\hline 32 & 135 & 2 & 15 & 17 & 432.5 & 472.5 & 0.4000 & 3.0000 & 0.6556 & 0.4489 \\
\hline 33 & 140 & 1.5 & 16 & 17.5 & 434 & 488.5 & 0.3000 & 3.2000 & 0.6798 & 0.4505 \\
\hline 34 & 145 & 1.5 & 15 & 16.5 & 435.5 & 503.5 & 0.2143 & 2.1429 & 0.7041 & 0.4520 \\
\hline 35 & 152 & 1 & 17 & 18 & 436.5 & 520.5 & 0.1667 & 2.8333 & 0.7381 & 0.4531 \\
\hline 36 & 158 & 1 & 17 & 18 & 437.5 & 537.5 & 0.1667 & 2.8333 & 0.7672 & 0.4541 \\
\hline 37 & 164 & 1 & 16 & 17 & 438.5 & 553.5 & 0.1667 & 2.6667 & 0.7964 & 0.4552 \\
\hline 38 & 170 & 0.5 & 17 & 17.5 & 439 & 570.5 & 0.0625 & 2.1250 & 0.8255 & 0.4557 \\
\hline 39 & 178 & 0.5 & 18 & 18.5 & 439.5 & 588.5 & 0.2500 & 9.0000 & 0.8644 & 0.4562 \\
\hline 40 & 180 & 1 & 17 & 18 & 440.5 & 605.5 & 0.1000 & 1.7000 & 0.8741 & 0.4572 \\
\hline 41 & 190 & 0.5 & 26.5 & 27 & 441 & 632 & 0.0833 & 4.4167 & 0.9226 & 0.4578 \\
\hline 42 & 196 & 0.5 & 19 & 19.5 & 441.5 & 651 & 0.0556 & 2.1111 & 0.9518 & 0.4583 \\
\hline 43 & 205 & 0 & 31 & 31 & 441.5 & 682 & 0.0000 & 3.8750 & 0.9955 & 0.4583 \\
\hline 44 & 213 & 0 & 33 & 33 & 441.5 & 715 & 0.0000 & 4.7143 & 1.0343 & 0.4583 \\
\hline 45 & 220 & 0 & 31.5 & 31.5 & 441.5 & 746.5 & 0.0000 & 3.5000 & 1.0683 & 0.4583 \\
\hline 46 & 229 & 0 & 36.5 & 36.5 & 441.5 & 783 & 0.0000 & 5.2143 & 1.1120 & 0.4583 \\
\hline 47 & 236 & 0 & 26.5 & 26.5 & 441.5 & 809.5 & 0.0000 & 3.7857 & 1.1460 & 0.4583 \\
\hline
\end{tabular}


TABLE C6. PRODUCTION DATA FOR RUN 8.

\begin{tabular}{|c|c|c|c|c|c|c|c|c|c|c|}
\hline $\begin{array}{l}\text { Sample } \\
\text { Number }\end{array}$ & $\begin{array}{c}\text { Sampling } \\
\text { Time, } \\
\text { min }\end{array}$ & $\begin{array}{l}\text { Vol. } \\
\text { Oil, } \\
\mathrm{cm}^{3}\end{array}$ & $\begin{array}{c}\text { Vol. } \\
\text { Water, } \\
\mathrm{cm}^{3}\end{array}$ & $\begin{array}{c}\text { Vol. } \\
\text { Total, } \\
\mathrm{cm}^{3}\end{array}$ & $\begin{array}{c}\text { Cum. } \\
\text { Oil, } \\
\mathrm{cm}^{3}\end{array}$ & $\begin{array}{c}\text { Cum. } \\
\text { Water, } \\
\mathrm{cm}^{3}\end{array}$ & $\begin{array}{c}\text { Oil } \\
\text { Rate, } \\
\mathrm{cm}^{3}\end{array}$ & $\begin{array}{c}\text { Water } \\
\text { Rate, } \\
\mathrm{cm}^{3}\end{array}$ & $\begin{array}{c}\text { Pore } \\
\text { Vol. } \\
\text { Injected, } \\
\text { fraction }\end{array}$ & $\begin{array}{c}\text { Oil } \\
\text { Recovery, } \\
\%\end{array}$ \\
\hline 1 & 15 & 16 & 0.25 & 16.25 & 16 & 0.25 & 16.0000 & 0.2500 & 0.0728 & 0.0166 \\
\hline 2 & 16 & 14 & 0.5 & 14.5 & 30 & 0.75 & 14.0000 & 0.5000 & 0.0777 & 0.0311 \\
\hline 3 & 17 & 28 & 4 & 32 & 58 & 4.75 & 2.8000 & 0.4000 & 0.0826 & 0.0602 \\
\hline 4 & 27 & 20 & 10 & 30 & 78 & 14.75 & 4.0000 & 2.0000 & 0.1311 & 0.0810 \\
\hline 5 & 32 & 9 & 10 & 19 & 87 & 24.75 & 4.5000 & 5.0000 & 0.1554 & 0.0903 \\
\hline 6 & 34 & 9 & 6 & 15 & 96 & 30.75 & 4.5000 & 3.0000 & 0.1651 & 0.0996 \\
\hline 7 & 36 & 9 & 6 & 15 & 105 & 36.75 & 4.5000 & 3.0000 & 0.1748 & 0.1090 \\
\hline 8 & 38 & 9 & 6 & 15 & 114 & 42.75 & 3.0000 & 2.0000 & 0.1845 & 0.1183 \\
\hline 9 & 41 & 14 & 10 & 24 & 128 & 52.75 & 3.5000 & 2.5000 & 0.1991 & 0.1329 \\
\hline 10 & 45 & 14 & 24 & 38 & 142 & 76.75 & 2.8000 & 4.8000 & 0.2185 & 0.1474 \\
\hline 11 & 50 & 16 & 22 & 38 & 158 & 98.75 & 3.2000 & 4.4000 & 0.2428 & 0.1640 \\
\hline 12 & 55 & 16 & 20 & 36 & 174 & 118.75 & 4.0000 & 5.0000 & 0.2671 & 0.1806 \\
\hline 13 & 59 & 16 & 22 & 38 & 190 & 140.75 & 4.0000 & 5.5000 & 0.2865 & 0.1972 \\
\hline 14 & 63 & 17 & 16 & 33 & 207 & 156.75 & 4.2500 & 4.0000 & 0.3059 & 0.2149 \\
\hline 15 & 67 & 16.5 & 16 & 32.5 & 223.5 & 172.75 & 4.1250 & 4.0000 & 0.3253 & 0.2320 \\
\hline 16 & 71 & 10 & 15 & 25 & 233.5 & 187.75 & 3.3333 & 5.0000 & 0.3448 & 0.2424 \\
\hline 17 & 74 & 16 & 15 & 31 & 249.5 & 202.75 & 4.0000 & 3.7500 & 0.3593 & 0.2590 \\
\hline 18 & 78 & 16 & 13 & 29 & 265.5 & 215.75 & 4.0000 & 3.2500 & 0.3788 & 0.2756 \\
\hline 19 & 82 & 10 & 11 & 21 & 275.5 & 226.75 & 3.3333 & 3.6667 & 0.3982 & 0.2860 \\
\hline 20 & 85 & 15 & 11 & 26 & 290.5 & 237.75 & 3.7500 & 2.7500 & 0.4128 & 0.3015 \\
\hline 21 & 89 & 7 & 10 & 17 & 297.5 & 247.75 & 2.3333 & 3.3333 & 0.4322 & 0.3088 \\
\hline 22 & 92 & 14 & 9 & 23 & 311.5 & 256.75 & 3.5000 & 2.2500 & 0.4467 & 0.3233 \\
\hline 23 & 96 & 11 & 8 & 19 & 322.5 & 264.75 & 3.6667 & 2.6667 & 0.4662 & 0.3348 \\
\hline 24 & 99 & 11 & 10 & 21 & 333.5 & 274.75 & 3.6667 & 3.3333 & 0.4807 & 0.3462 \\
\hline 25 & 102 & 12 & 20 & 32 & 345.5 & 294.75 & 3.0000 & 5.0000 & 0.4953 & 0.3586 \\
\hline 26 & 106 & 20 & 30 & 50 & 365.5 & 324.75 & 2.5000 & 3.7500 & 0.5147 & 0.3794 \\
\hline 27 & 114 & 40 & 6 & 46 & 405.5 & 330.75 & 13.3333 & 2.0000 & 0.5536 & 0.4209 \\
\hline 28 & 117 & 25 & 6 & 31 & 430.5 & 336.75 & 6.2500 & 1.5000 & 0.5681 & 0.4469 \\
\hline 29 & 121 & 6 & 10 & 16 & 436.5 & 346.75 & 3.0000 & 5.0000 & 0.5876 & 0.4531 \\
\hline 30 & 123 & 3 & 19 & 22 & 439.5 & 365.75 & 0.6000 & 3.8000 & 0.5973 & 0.4562 \\
\hline 31 & 128 & 2 & 21 & 23 & 441.5 & 386.75 & 0.2857 & 3.0000 & 0.6216 & 0.4583 \\
\hline 32 & 135 & 2 & 20 & 22 & 443.5 & 406.75 & 0.4000 & 4.0000 & 0.6556 & 0.4604 \\
\hline 33 & 140 & 1 & 20 & 21 & 444.5 & 426.75 & 0.2000 & 4.0000 & 0.6798 & 0.4614 \\
\hline 34 & 145 & 1 & 20 & 21 & 445.5 & 446.75 & 0.1429 & 2.8571 & 0.7041 & 0.4624 \\
\hline 35 & 152 & 1 & 18 & 19 & 446.5 & 464.75 & 0.1667 & 3.0000 & 0.7381 & 0.4635 \\
\hline 36 & 158 & 1 & 19 & 20 & 447.5 & 483.75 & 0.1667 & 3.1667 & 0.7672 & 0.4645 \\
\hline 37 & 164 & 1 & 20 & 21 & 448.5 & 503.75 & 0.1667 & 3.3333 & 0.7964 & 0.4655 \\
\hline 38 & 170 & 0.5 & 19 & 19.5 & 449 & 522.75 & 0.0625 & 2.3750 & 0.8255 & 0.4661 \\
\hline 39 & 178 & 0.5 & 10 & 10.5 & 449.5 & 532.75 & 0.2500 & 5.0000 & 0.8644 & 0.4666 \\
\hline 40 & 180 & 0.5 & 25 & 25.5 & 450 & 557.75 & 0.0500 & 2.5000 & 0.8741 & 0.4671 \\
\hline 41 & 190 & 0.5 & 25 & 25.5 & 450.5 & 582.75 & 0.0833 & 4.1667 & 0.9226 & 0.4676 \\
\hline 42 & 196 & 0 & 24 & 24 & 450.5 & 606.75 & 0.0000 & 2.6667 & 0.9518 & 0.4676 \\
\hline 43 & 205 & 0 & 28 & 28 & 450.5 & 634.75 & 0.0000 & 3.5000 & 0.9955 & 0.4676 \\
\hline 44 & 213 & 0 & 28 & 28 & 450.5 & 662.75 & 0.0000 & 4.0000 & 1.0343 & 0.4676 \\
\hline 45 & 220 & 0 & 28 & 28 & 450.5 & 690.75 & 0.0000 & 3.1111 & 1.0683 & 0.4676 \\
\hline 46 & 229 & 0 & 28 & 28 & 450.5 & 718.75 & 0.0000 & 4.0000 & 1.1120 & 0.4676 \\
\hline
\end{tabular}




\section{APPENDIX D}

\section{DENSITY AND VISCOSITY DATA}

TABLE D1. DENSITY AND VISCOSITY (AT $\left.50^{\circ} \mathrm{C}\right)$ OF THE PRODUCED OIL FOR RUN 3

\begin{tabular}{|c|c|c|c|}
\hline $\begin{array}{c}\text { Time, } \\
\text { Min }\end{array}$ & $\begin{array}{c}\text { Sample } \\
\text { Number }\end{array}$ & $\begin{array}{c}\text { Density, } \\
{ }^{\mathbf{0} A P I}\end{array}$ & $\begin{array}{c}\text { Viscosity, } \\
\text { cp }\end{array}$ \\
\hline 17 & 14 & 11 & 3644 \\
53 & 17 & 11.5 & 3164 \\
65 & 20 & 11.5 & 3100 \\
77 & 23 & 12 & 3238 \\
83 & 26 & 12.5 & 2914 \\
92 & 29 & 12.5 & 2685 \\
104 & 32 & 13 & 2768 \\
113 & 35 & 13 & 2890 \\
119 & 38 & 13.5 & 2778 \\
135 & 41 & 13 & 2578 \\
146 & 43 & 13.5 & 2486 \\
168 & 46 & 13.5 & 2386 \\
222 & 49 & 13 & 2316 \\
\hline
\end{tabular}

Table D2. Density and viscosity (at $50^{\circ} \mathrm{C}$ ) of the produced oil for run 4

\begin{tabular}{|c|c|c|c|}
\hline $\begin{array}{c}\text { Time, } \\
\text { Min }\end{array}$ & $\begin{array}{c}\text { Sample } \\
\text { Number }\end{array}$ & $\begin{array}{c}\text { Density, } \\
{ }^{\mathbf{o}} \text { API }\end{array}$ & $\begin{array}{c}\text { Viscosity, } \\
\text { cp }\end{array}$ \\
\hline & & & \\
18 & 1 & 11 & 3500 \\
36 & 5 & 11.5 & 3200 \\
51 & 9 & 11.5 & 3250 \\
69 & 13 & 12 & 3200 \\
90 & 17 & 12 & 3350 \\
111 & 21 & 12.5 & 3100 \\
135 & 25 & 12.5 & 2950 \\
153 & 29 & 12.5 & 2900 \\
171 & 33 & 13 & 2850 \\
186 & 37 & 13.5 & 2700 \\
204 & 41 & 13 & 2600 \\
225 & 45 & 13.5 & 2650 \\
240 & 49 & 13 & 2500 \\
258 & 53 & 13 & 2550 \\
273 & 57 & 13.5 & 2500 \\
\hline
\end{tabular}

TABLE D3. DENSITY AND VISCOSITY (AT 50 ${ }^{\circ} \mathrm{C}$ ) OF THE PRODUCED OIL FOR RUN 7

\begin{tabular}{|c|c|c|c|}
\hline $\begin{array}{c}\text { Time, } \\
\text { Min }\end{array}$ & $\begin{array}{c}\text { Sample } \\
\text { Number }\end{array}$ & $\begin{array}{c}\text { Viscosity, } \\
\text { cp }\end{array}$ & $\begin{array}{c}\text { Density, } \\
{ }^{\mathbf{0}} \text { API }\end{array}$ \\
\hline & & 2908 & 12.5 \\
15 & 1 & 2866 & 12.5 \\
36 & 4 & 2706 & 14 \\
59 & 7 & 2904 & 13 \\
85 & 10 & 2812 & 13.5 \\
117 & 13 & 2680 & 14.5 \\
145 & 20 & 2420 & 15 \\
180 & 30 & 2106 & 14.5 \\
236 & 40 & & \\
\hline
\end{tabular}


APPENDIX E

\title{
VISUAL BASIC SOURCE CODE FOR STEAM FRONT ADVANCEMENT AND CUMULATIVE OIL PRODUCTION CALCULATION BASED ON ONE DIMENSIONAL ANALYTICAL MODEL
}

\author{
Public Type data3Col \\ logtime As Variant: Pinj As Variant: Ts As Variant: Pout As Variant \\ End Type \\ Public LogData() As data3Col \\ Public fileToOpen As Variant \\ Public Type FieldUnit \\ Dlogtime As Double: Pinj As Double: KelTs As Double \\ FahTs As Double: TabHs As Double: Psat As Double \\ Tsat As Double: phase As String: fTabHs As Double \\ HsRate As Double: xconv As Double: dx As Double \\ x As Double: Np As Double: int_delP As Double \\ End Type \\ Public EqInput() As FieldUnit \\ Public Type data2Col \\ stime As Variant: cumoil As Variant \\ End Type \\ Public ProdData() As data2Col \\ Public Type dataTherm \\ ttime As Variant: propagation As Variant \\ End Type \\ Public ThermData() As dataTherm \\ Public Type Shifted \\ Dtime As Double: Mtime As Double: x As Double: Np As Double \\ End Type \\ Public Plot() As Shifted \\ Public Type fill_up \\ Dtime As Double: Mtime As Double: VoidLength As Double \\ End Type \\ Public FillUp As fill_up \\ Dim xaxis() As Double, yaxis() As Double \\ Public arEnd As Double, arProd As Double \\ Sub ModelingAcc() \\ Dim i As Integer, iw As Double \\ Dim j As Integer \\ Dim rad As Double, Lcell As Double, Tcell As Double \\ Dim Co As Double, por As Double, voidL As Double \\ Dim mratio As Double, tfu_total As Double, Mr As Double \\ Dim U As Double, tmod As Double \\ Const PI As Double = 3.14159265358979 \\ With Sheets("Main") \\ rad = .Range("F11"): Lcell = .Range("F12") \\ Tcell = .Range("F13"): Co = .Range("F17") \\ por = .Range("F22"): So = .Range("F23")
}




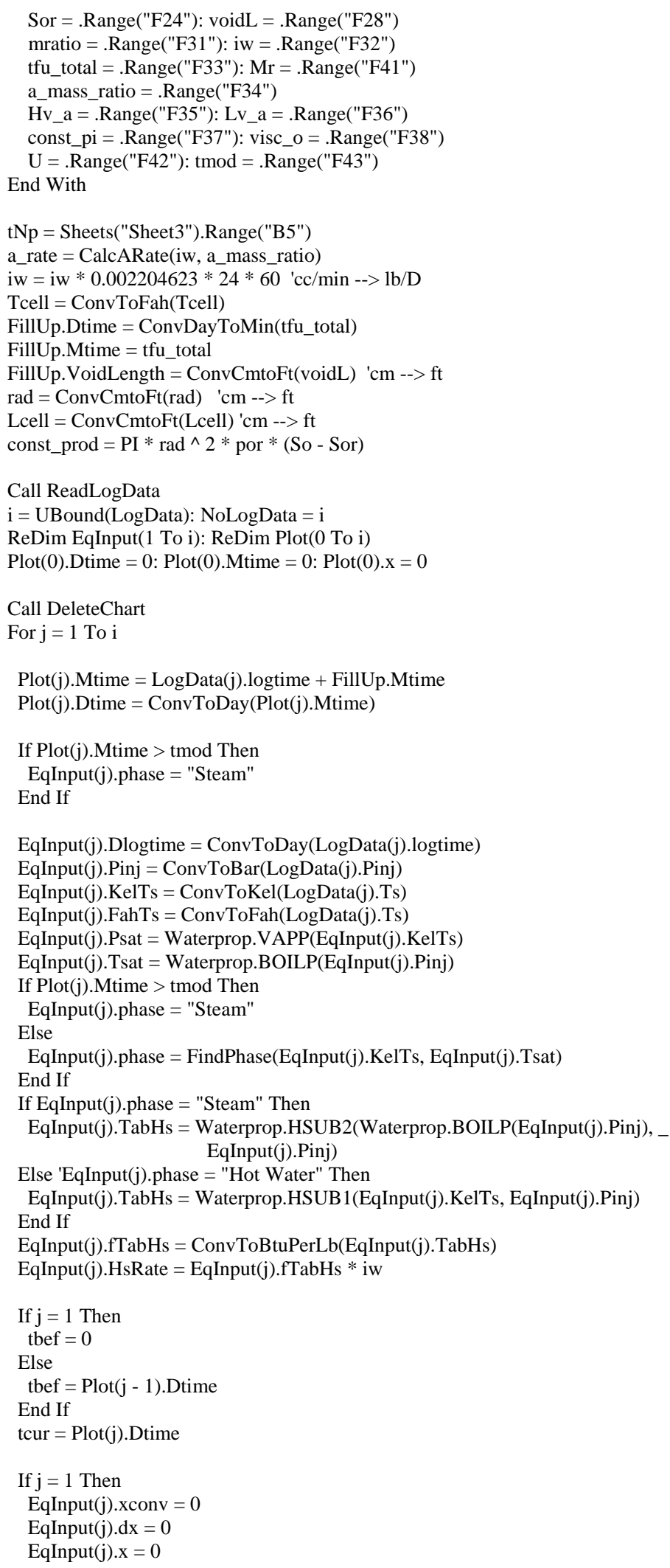


EqInput(j).Np $=0$

EqInput(j).int_delP $=0$

Else

TabTs = Waterprop.BOILP(EqInput(j).Pinj)

TabTs $=$ ConvKelToFah $($ TabTs $)$

EqInput(j).xconv = calc_x_conv(Co, mratio, iw, TabTs, Tcell, tcur, tbef $)$

EqInput(j).dx = calc_dx(EqInput(j).HsRate, EqInput(j - 1).Dlogtime, _

EqInput(j).Dlogtime, rad, EqInput( $\mathrm{j}-1) . \mathrm{x}$,

FillUp.VoidLength, U, EqInput(j).FahTs, Tcell, _ Mr, EqInput(j).xconv)

EqInput(j).x $=$ EqInput( $\mathrm{j}-1) \cdot \mathrm{x}+$ EqInput(j).dx

'To encounter discontinuities

If EqInput( $\mathrm{j}) \cdot \mathrm{x}<0$ Then

EqInput(j).x $=0$

End If

'EqInput(j).Np = const_prod * EqInput(j).x

EqInput(j).int_delP = calc_int_delP(Plot(j - 1).Mtime, Plot(j).Mtime, LogData(j).Pout, LogData(j).Pinj, EqInput(j - 1).int_delP)

EqInput(j).Np = calc_Np(const_pi, visc_o, EqInput(j).int_delP)

If EqInput(j). $\mathrm{Np}<0$ Then

EqInput(j).Np $=0$

End If

'catch the proper maximum $\mathrm{Np}$

If EqInput(j).x $<$ Lcell Then

flat $=\mathrm{j}$

End If

'adjust steam front according to length of cell

If EqInput(j). $x$ > Lcell Then

EqInput(j).x = Lcell

' EqInput(j).Np = EqInput(flat).Np

End If

Plot(j).x = ConvFtToCm(EqInput(j).x

$\operatorname{Plot}(\mathrm{j}) \cdot \mathrm{Np}=$ EqInput $(\mathrm{j}) \cdot \mathrm{Np}$

'catch the array number at the last Np according to production data If Plot(j).Mtime < tNp Then

arEnd $=\mathrm{j}$

End If

End If

Next

Sheets("Sheet3").Range("A6:B6").ClearContents

Sheets("Sheet3").Range("A6") = "Last array at last production time: "

Sheets("Sheet3").Range("B6") = arEnd

arProd = Sheets("Sheet3").Range("B2")

'Send steam front model calculated data to sheet

ReDim xaxis(0 To NoLogData): ReDim yaxis(0 To NoLogData)

For $\mathrm{j}=0$ To NoLogData

$\operatorname{xaxis}(\mathrm{j})=\operatorname{Plot}(\mathrm{j}) \cdot$ Mtime: $\operatorname{yaxis}(\mathrm{j})=\operatorname{Plot}(\mathrm{j}) \cdot \mathrm{x}$

Next

Call WriteToSheetXModel(xaxis, yaxis)

'Send Np model calculated data to sheet

ReDim xaxis(0 To NoLogData): ReDim yaxis(0 To NoLogData)

For $\mathrm{j}=0$ To NoLogData

$\operatorname{xaxis}(\mathrm{j})=\operatorname{Plot}(\mathrm{j}) \cdot$ Mtime: $\operatorname{yaxis}(\mathrm{j})=\operatorname{Plot}(\mathrm{j}) \cdot \mathrm{Np}$ 


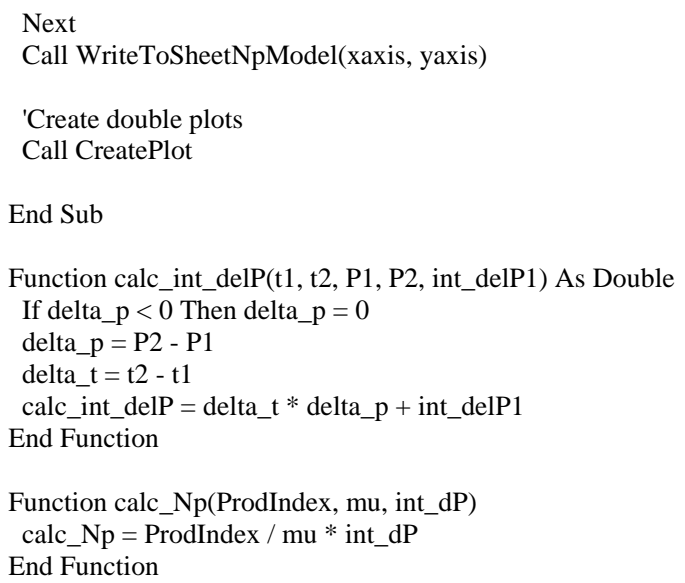




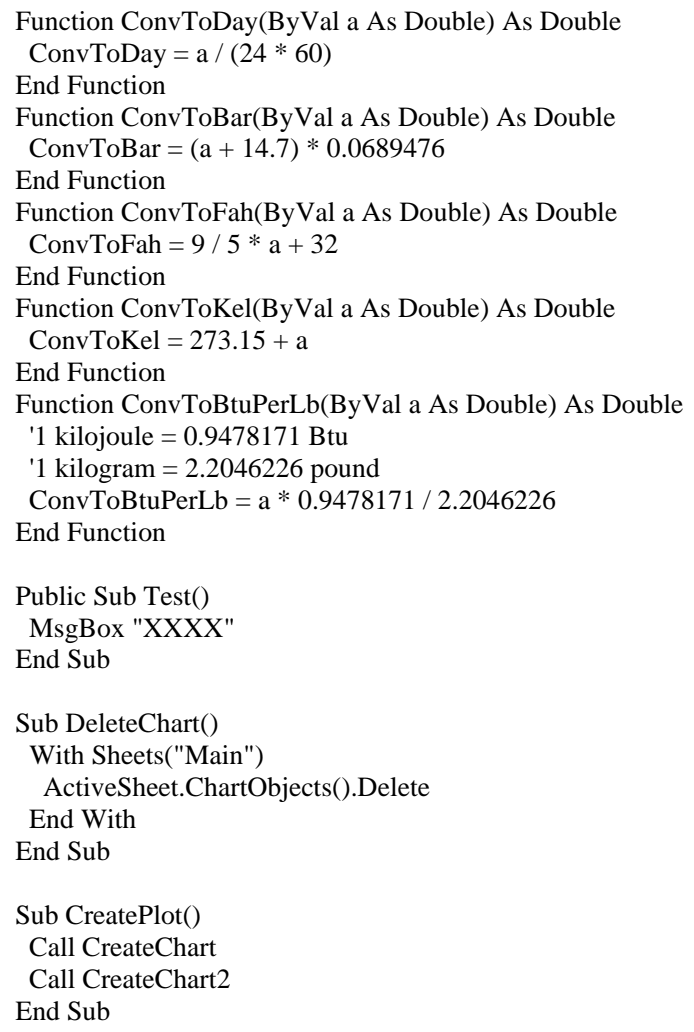

Public Sub OpenDataExample()

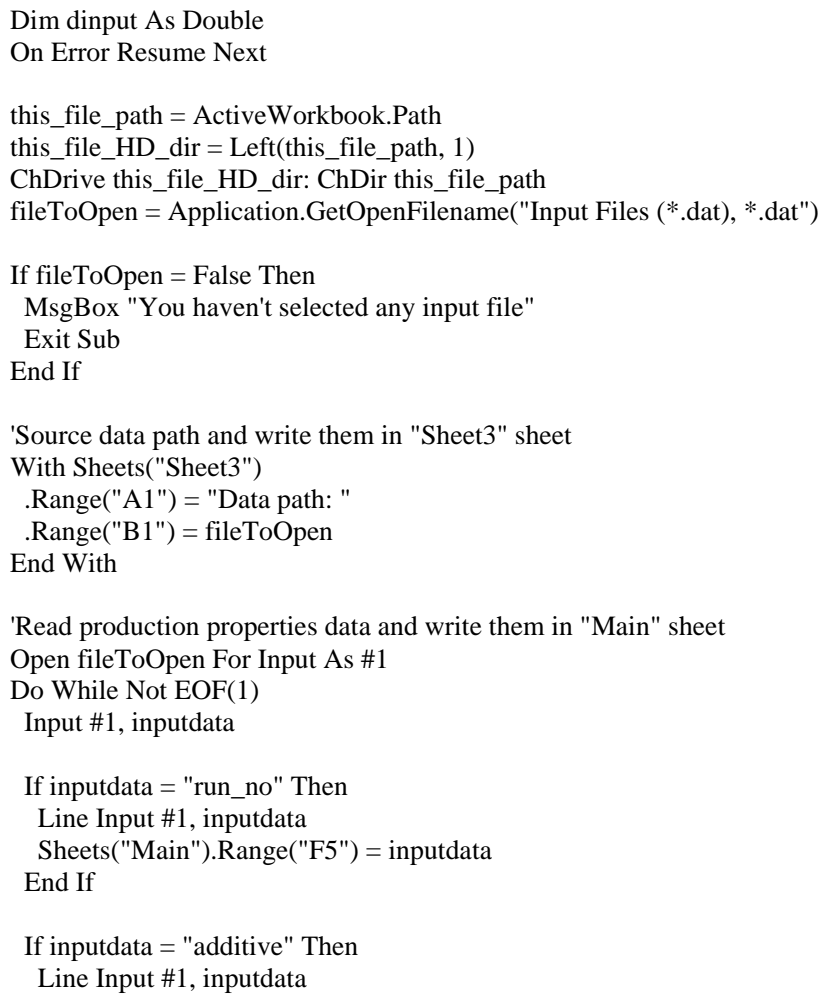


Sheets("Main").Range("F6") = inputdata

End If

If inputdata $=$ "researcher" Then

Input \#1, inputdata

Sheets("Main").Range("F7") = inputdata

End If

If inputdata = "cell_radius" Then

Input \#1, inputdata

dinput $=\mathrm{CDbl}($ inputdata $)$

Sheets("Main").Range("F11") = dinput

End If

If inputdata = "cell_length" Then

Input \#1, inputdata

dinput $=\mathrm{CDbl}($ inputdata $)$

Sheets("Main").Range("F12") = dinput

End If

If inputdata = "tcell" Then

Input \#1, inputdata

dinput $=\mathrm{CDbl}($ inputdata $)$

Sheets("Main").Range("F13") = dinput

End If

If inputdata $=$ "Cs" Then

Input \#1, inputdata

dinput $=\mathrm{CDbl}($ inputdata $)$

Sheets("Main").Range("F16") = dinput

End If

If inputdata $=$ "Co" Then

Input \#1, inputdata

dinput $=\mathrm{CDbl}($ inputdata $)$

Sheets("Main").Range("F17") = dinput

End If

If inputdata = "Cw" Then

Input \#1, inputdata

dinput $=\mathrm{CDbl}($ inputdata $)$

Sheets("Main").Range("F18") = dinput

End If

If inputdata = "dens_sand" Then

Input \#1, inputdata

dinput $=$ CDbl(inputdata $)$

Sheets("Main").Range("F19") = dinput

End If

If inputdata = "dens_oil" Then

Input \#1, inputdata

dinput $=\mathrm{CDbl}($ inputdata $)$

Sheets("Main").Range("F20") = dinput End If

If inputdata = "dens_water" Then

Input \#1, inputdata

dinput $=$ CDbl(inputdata)

Sheets("Main").Range("F21") = dinput

End If

If inputdata = "por" Then

Input \#1, inputdata

dinput $=$ CDbl(inputdata) 
Sheets("Main").Range("F22") = dinput

End If

If inputdata = "Soi" Then

Input \#1, inputdata

dinput $=\mathrm{CDbl}($ inputdata $)$

Sheets("Main").Range("F23") = dinput

End If

If inputdata $=$ "Sor" Then

Input \#1, inputdata

dinput $=\mathrm{CDbl}($ inputdata $)$

Sheets("Main").Range("F24") = dinput

End If

If inputdata = "Sgi" Then

Input \#1, inputdata

dinput $=$ CDbl(inputdata)

Sheets("Main").Range("F25") = dinput

End If

If inputdata = "Swc" Then

Input \#1, inputdata

dinput $=$ CDbl(inputdata)

Sheets("Main").Range("F26") = dinput

End If

If inputdata = "porvol" Then

Input \#1, inputdata

dinput $=$ CDbl(inputdata)

Sheets("Main").Range("F27") = dinput

End If

If inputdata = "void_length" Then

Input \#1, inputdata

dinput $=\mathrm{CDbl}($ inputdata $)$

Sheets("Main").Range("F28") = dinput

End If

If inputdata = "o_w_ratio" Then

Input \#1, inputdata

dinput $=\mathrm{CDbl}($ inputdata $)$

Sheets("Main").Range("F31") = dinput

End If

If inputdata $=$ "iw" Then

Input \#1, inputdata

dinput $=\mathrm{CDbl}($ inputdata $)$

Sheets("Main").Range("F32") = dinput

End If

If inputdata = "tfu_total" Then

Input \#1, inputdata

dinput $=$ CDbl(inputdata)

Sheets("Main").Range("F33") = dinput

End If

If inputdata = "ratio" Then

Input \#1, inputdata

dinput $=$ CDbl(inputdata $)$

Sheets("Main").Range("F34") = dinput

End If

If inputdata = "Hv_enthalphy" Then

Input \#1, inputdata 


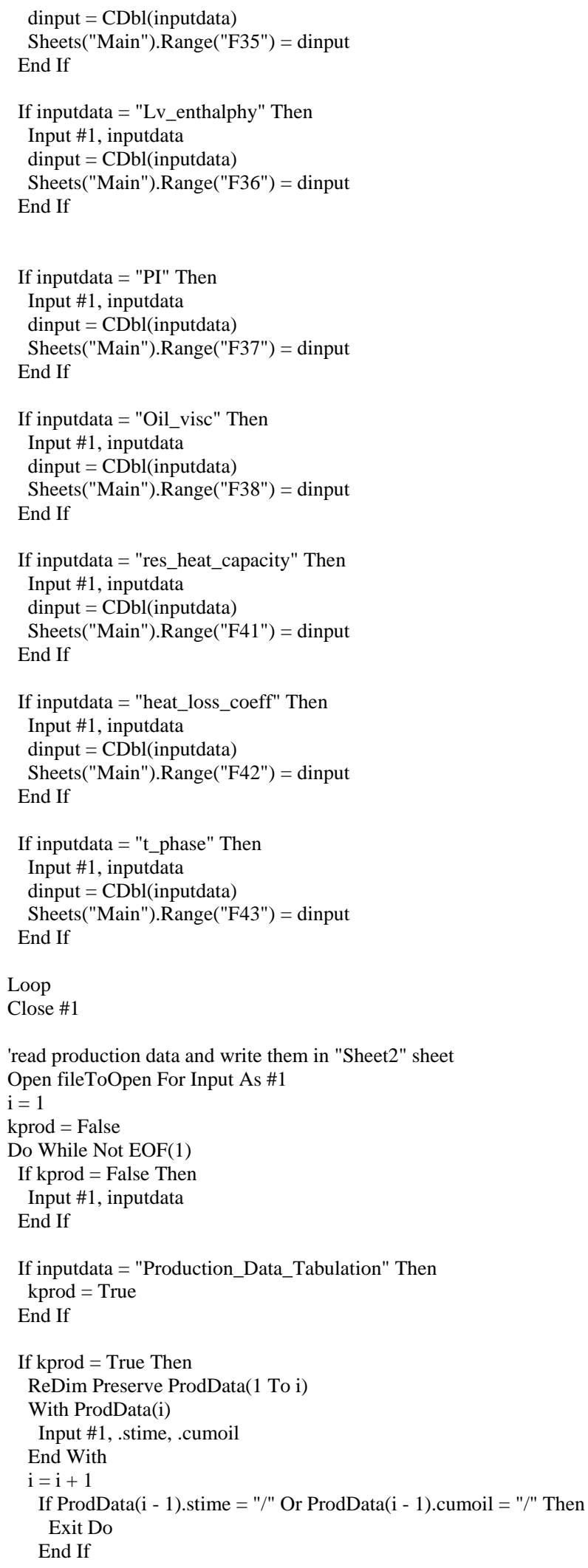




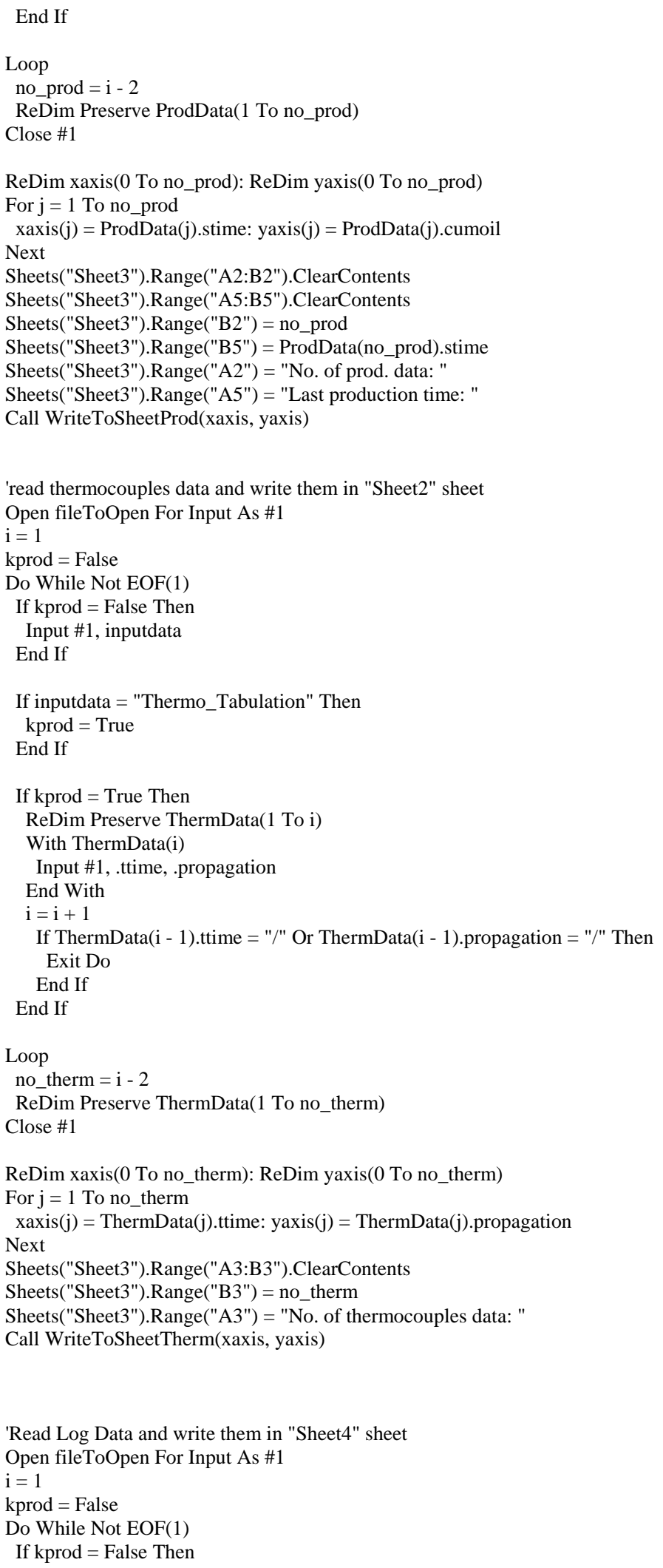




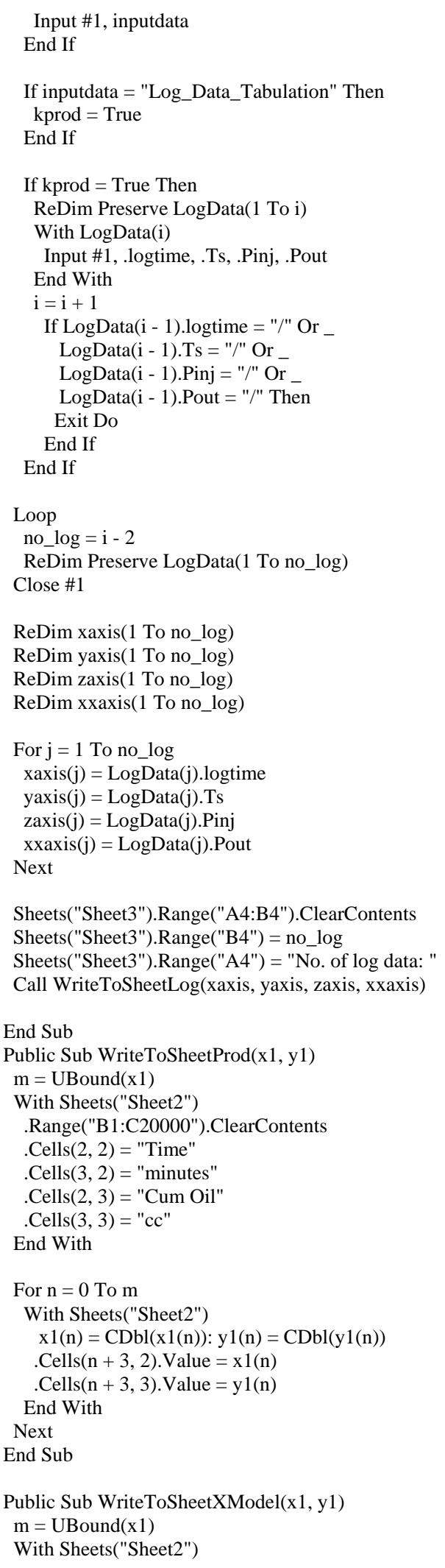




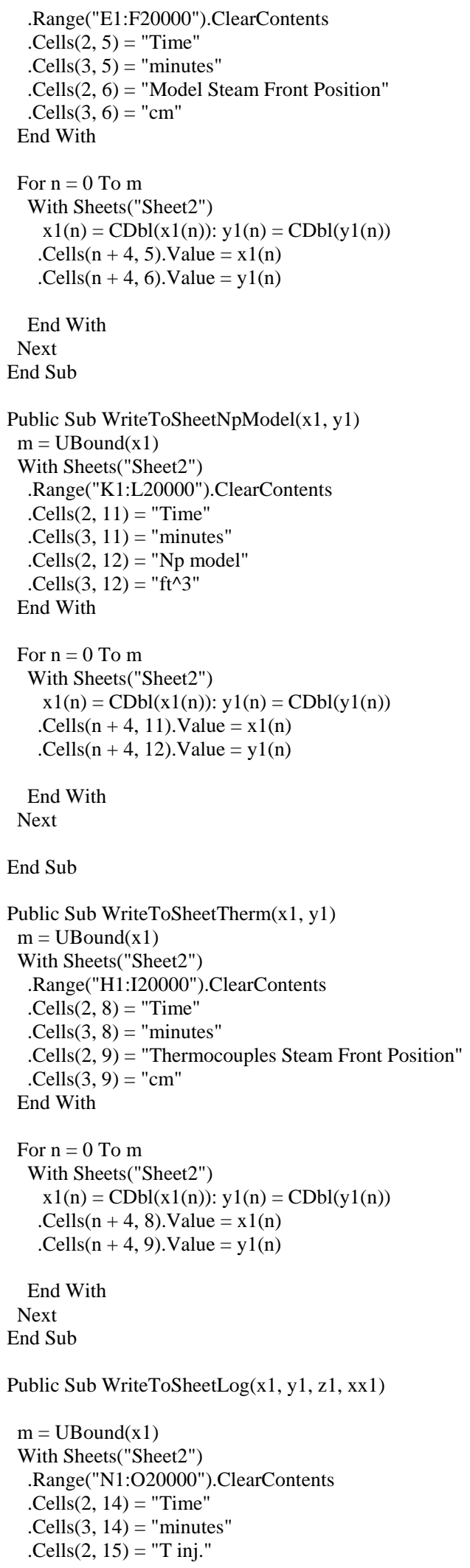




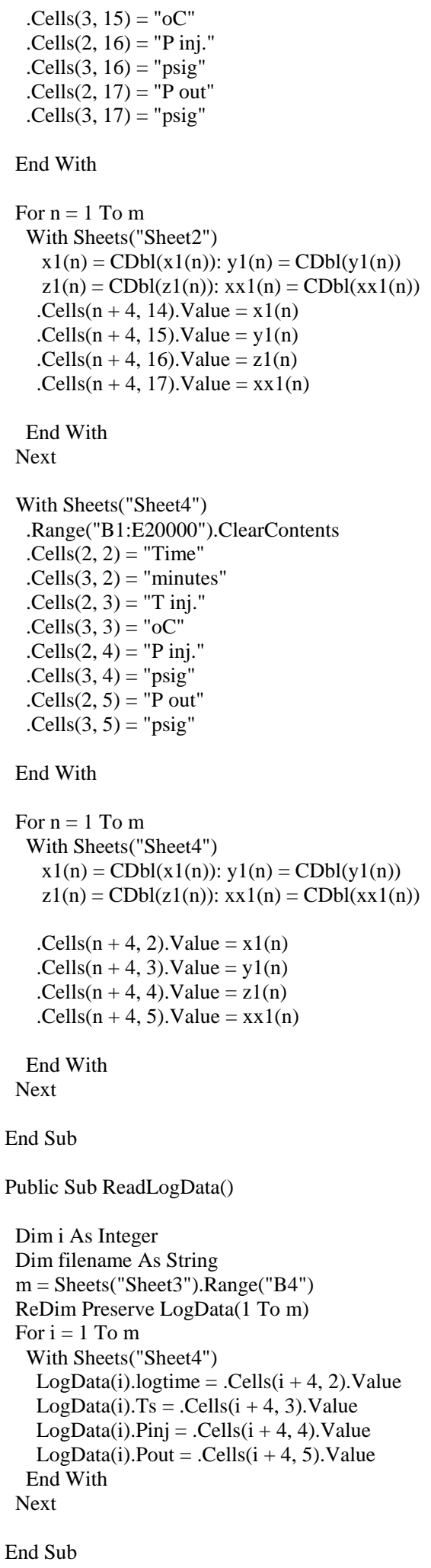

Public Sub ErrorHandling() 


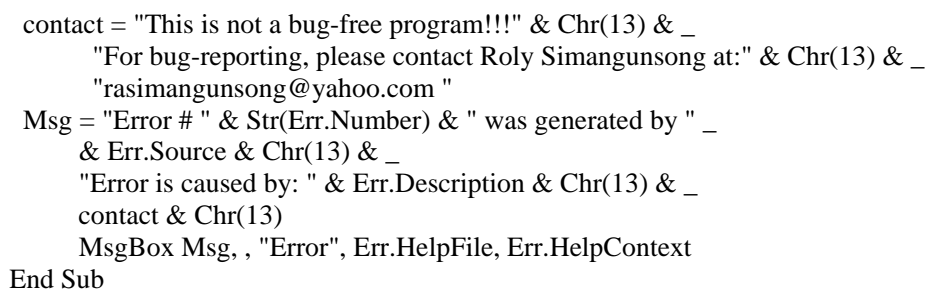




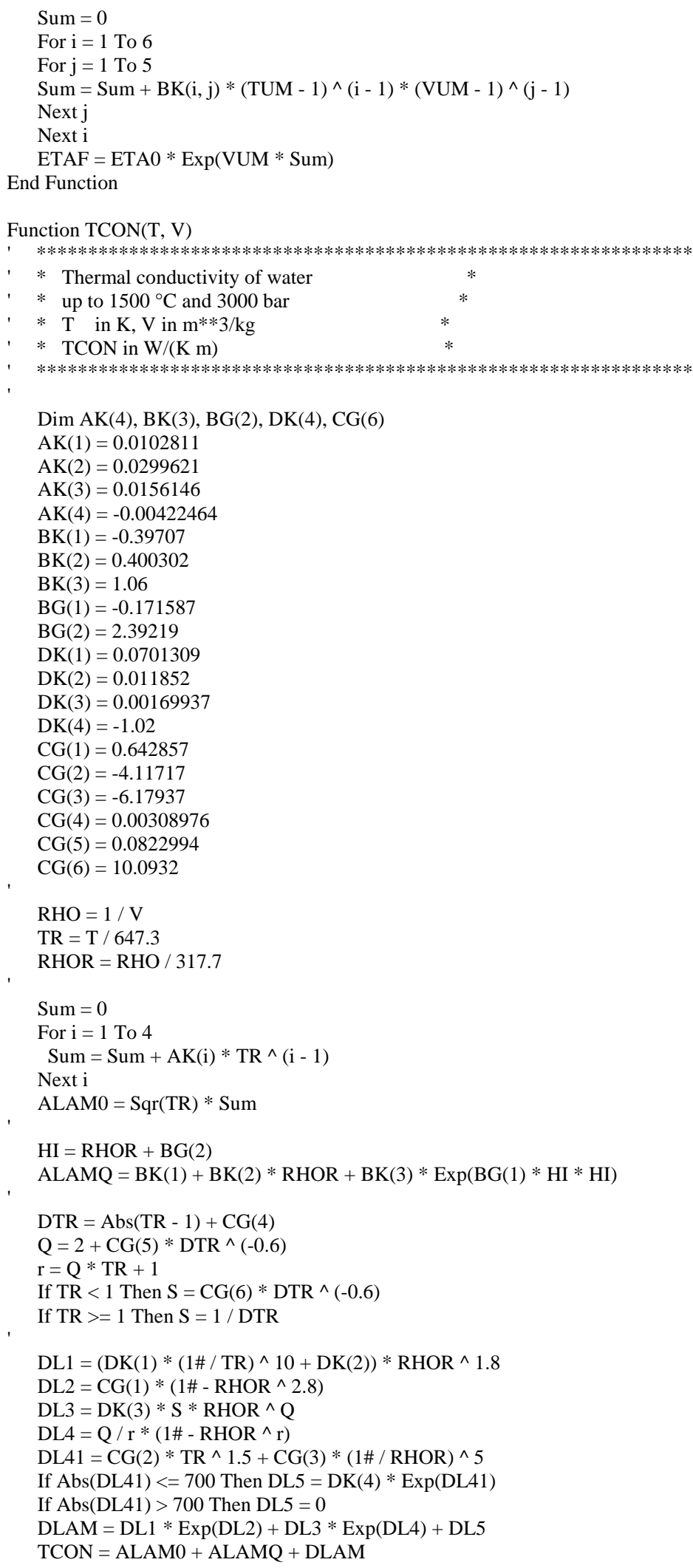


End Function

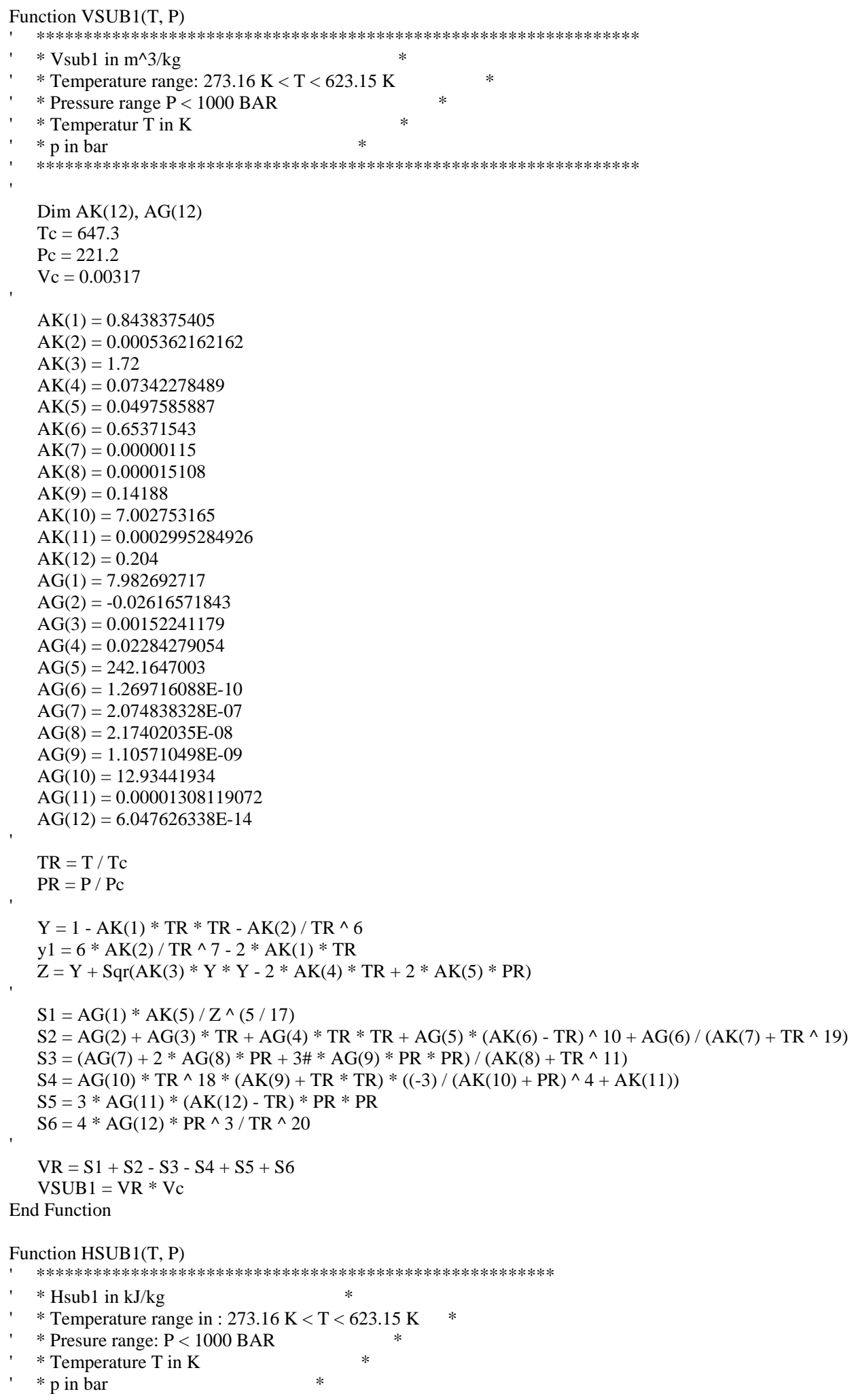




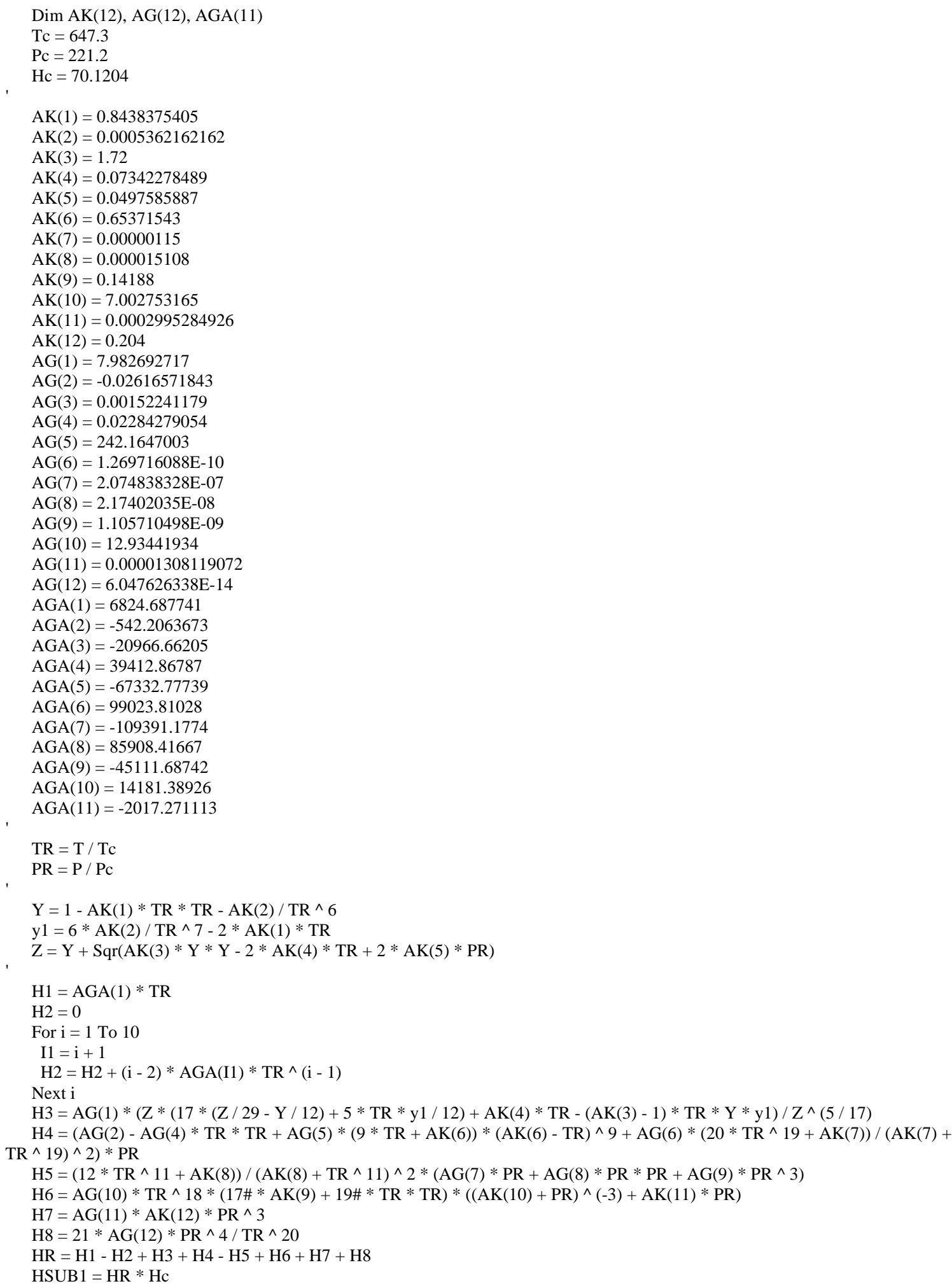




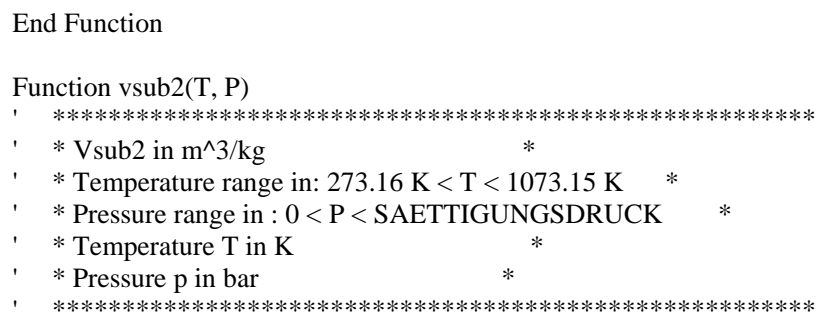

Dim NA(8), NZ(8, 3), NL(3), NX(3, 2), BG(8, 3), BK(3, 2), B9(7)

$\mathrm{Tc}=647.3$

Pc $=221.2$

$\mathrm{Vc}=0.00317$

$\mathrm{NA}(1)=2$

$\mathrm{NA}(2)=3$

$\mathrm{NA}(3)=2$

$\mathrm{NA}(4)=2$

$\mathrm{NA}(5)=3$

$\mathrm{NA}(6)=2$

$\mathrm{NA}(7)=2$

$\mathrm{NA}(8)=2$

$\mathrm{NZ}(1,1)=13$

$\mathrm{NZ}(2,1)=18$

$\mathrm{NZ}(3,1)=18$

$\mathrm{NZ}(4,1)=25$

$\mathrm{NZ}(5,1)=32$

$\mathrm{NZ}(6,1)=12$

$\mathrm{NZ}(7,1)=24$

$\mathrm{NZ}(8,1)=24$

$\mathrm{NZ}(1,2)=3$

$\mathrm{NZ}(2,2)=2$

$\mathrm{NZ}(3,2)=10$

$\mathrm{NZ}(4,2)=14$

$\mathrm{NZ}(5,2)=28$

$\mathrm{NZ}(6,2)=11$

$\operatorname{NZ}(7,2)=18$

$\mathrm{NZ}(8,2)=14$

$\mathrm{NZ}(1,3)=0$

$\mathrm{NZ}(2,3)=1$

$\mathrm{NZ}(3,3)=0$

$\mathrm{NZ}(4,3)=0$

$\mathrm{NZ}(5,3)=24$

$\mathrm{NZ}(6,3)=0$

$\mathrm{NZ}(7,3)=0$

$\mathrm{NZ}(8,3)=0$

$\mathrm{NL}(1)=1$

$\mathrm{NL}(2)=1$

$\mathrm{NL}(3)=2$

$\mathrm{NX}(1,1)=14$

$\mathrm{NX}(2,1)=19$

$\mathrm{NX}(3,1)=54$

$\mathrm{NX}(1,2)=0$

$\mathrm{NX}(2,2)=0$

$\mathrm{NX}(3,2)=27$

$\mathrm{BG}(1,1)=0.06670375918$

$\mathrm{BG}(2,1)=0.08390104328$

$\mathrm{BG}(3,1)=0.4520918904$

$\operatorname{BG}(4,1)=-0.5975336707$

$\mathrm{BG}(5,1)=0.5958051609$

$\mathrm{BG}(6,1)=0.119061027$

$\operatorname{BG}(7,1)=0.1683998803$

$\mathrm{BG}(8,1)=0.006552390126$

$\mathrm{BG}(1,2)=1.388983801$ 
$\mathrm{BG}(2,2)=0.02614670893$

$\mathrm{BG}(3,2)=0.1069036614$

$\mathrm{BG}(4,2)=-0.08847535804$

$\operatorname{BG}(5,2)=-0.5159303373$

$\operatorname{BG}(6,2)=-0.09867174132$

$\operatorname{BG}(7,2)=-0.05809438001$

$\mathrm{BG}(8,2)=0.0005710218649$

$\mathrm{BG}(1,3)=0$

$\operatorname{BG}(2,3)=-0.03373439453$

$\mathrm{BG}(3,3)=0$

$\mathrm{BG}(4,3)=0$

$\mathrm{BG}(5,3)=0.2075021122$

$\mathrm{BG}(6,3)=0$

$\operatorname{BG}(7,3)=0$

$\mathrm{BG}(8,3)=0$

$\operatorname{BK}(1,1)=0.4006073948$

$\mathrm{BK}(2,1)=0.08636081627$

$\operatorname{BK}(3,1)=-0.8532322921$

$\mathrm{BK}(1,2)=0$

$\mathrm{BK}(2,2)=0$

$\mathrm{BK}(3,2)=0.3460208861$

$\mathrm{B} 9(1)=193.6587558$

$\mathrm{B} 9(2)=-1388.522425$

$\mathrm{B} 9(3)=4126.607219$

B9(4) $=-6508.211677$

$\mathrm{B} 9(5)=5745.984054$

$\mathrm{B} 9(6)=-2693.088365$

$\mathrm{B} 9(7)=523.5718623$

RI1 = 4.260321148

$\mathrm{BKA}=0.7633333333$

$\mathrm{TR}=\mathrm{T} / \mathrm{TC}$

$\mathrm{PR}=\mathrm{P} / \mathrm{PC}$

$\mathrm{x}=\operatorname{Exp}(\mathrm{BKA} *(1-\mathrm{TR}))$

$\mathrm{PRL}=\mathrm{BL}(\mathrm{TR})$

DPRL $=$ DBL $(T R)$

$\mathrm{S} 1=\mathrm{RI} 1 * \mathrm{TR} / \mathrm{PR}$

$\mathrm{S} 2=0$

For $\mathrm{i}=1$ To 5

$\mathrm{S} 2 \mathrm{~A}=0$

$\mathrm{NAB}=\mathrm{NA}(\mathrm{i})$

For IA $=1$ To NAB

$\mathrm{S} 2 \mathrm{~A}=\mathrm{S} 2 \mathrm{~A}+\mathrm{BG}(\mathrm{i}, \mathrm{IA}) * \mathrm{x} \wedge \mathrm{NZ}(\mathrm{i}, \mathrm{IA})$

Next IA

$\mathrm{S} 2=\mathrm{S} 2+\mathrm{i} * \mathrm{PR} \wedge(\mathrm{i}-1) * \mathrm{~S} 2 \mathrm{~A}$

Next i

S3 $=0$

For $\mathrm{i}=6$ To 8

$\mathrm{LAM}=\mathrm{i}-5$

$\mathrm{S} 3 \mathrm{~A}=0$

$\mathrm{NAB}=\mathrm{NA}(\mathrm{i})$

For IA $=1$ To NAB

$\mathrm{S} 3 \mathrm{~A}=\mathrm{S} 3 \mathrm{~A}+\mathrm{BG}(\mathrm{i}, \mathrm{IA}) * \mathrm{x} \wedge \mathrm{NZ}(\mathrm{i}, \mathrm{IA})$

Next IA

$\mathrm{S} 3 Z A E=(i-2) * P R \wedge(1-i) * S 3 A$

$\mathrm{S} 3 \mathrm{~B}=0$

NAC $=$ NL $(L A M)$

For IB $=1$ To NAC

S3B $=$ S3B + BK(LAM, IB $) * x^{\wedge}$ NX(LAM, IB $)$

Next IB

$\mathrm{S} 3 \mathrm{HI}=\mathrm{PR} \wedge(2-\mathrm{i})+\mathrm{S} 3 \mathrm{~B}$

S3NEN $=$ S3HI $*$ S3HI 


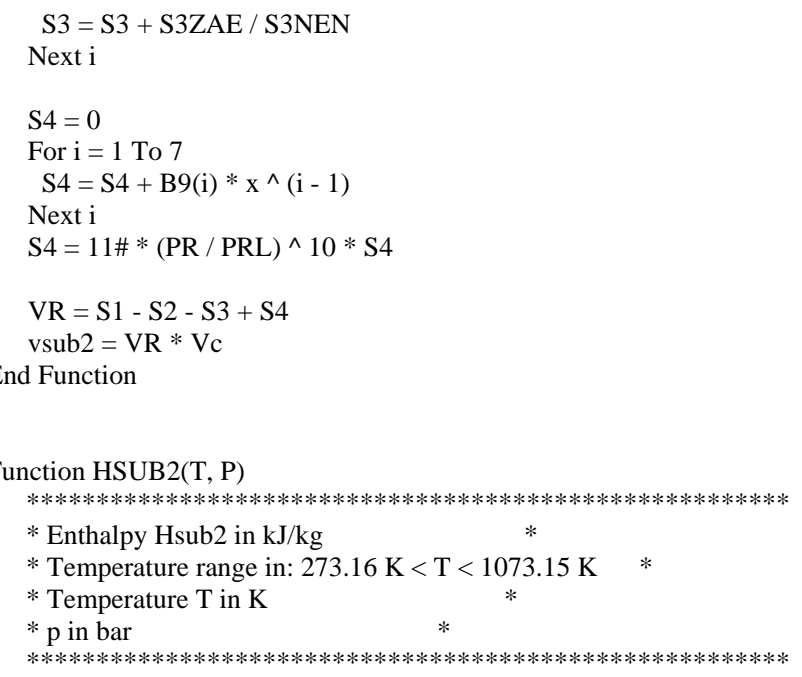

Dim NA(8), NZ(8, 3), NL(3), NX(3, 2), BGA(6), BG(8, 3), BK(3, 2), B9(7)

$\mathrm{Tc}=647.3$

Pc $=221.2$

Hc $=70.1204$

$\mathrm{NA}(1)=2$

$\mathrm{NA}(2)=3$

$\mathrm{NA}(3)=2$

$\mathrm{NA}(4)=2$

$\mathrm{NA}(5)=3$

$\mathrm{NA}(6)=2$

$\mathrm{NA}(7)=2$

$\mathrm{NA}(8)=2$

$\mathrm{NZ}(1,1)=13$

$N Z(2,1)=18$

$\operatorname{NZ}(3,1)=18$

$\mathrm{NZ}(4,1)=25$

$\mathrm{NZ}(5,1)=32$

$N Z(6,1)=12$

$\operatorname{NZ}(7,1)=24$

$\mathrm{NZ}(8,1)=24$

$\mathrm{NZ}(1,2)=3$

$\operatorname{NZ}(2,2)=2$

$\mathrm{NZ}(3,2)=10$

$\mathrm{NZ}(4,2)=14$

$N Z(5,2)=28$

$\operatorname{NZ}(6,2)=11$

$\operatorname{NZ}(7,2)=18$

$\mathrm{NZ}(8,2)=14$

$\mathrm{NZ}(1,3)=0$

$\mathrm{NZ}(2,3)=1$

$\mathrm{NZ}(3,3)=0$

$\mathrm{NZ}(4,3)=0$

$N Z(5,3)=24$

$\mathrm{NZ}(6,3)=0$

$\mathrm{NZ}(7,3)=0$

$\mathrm{NZ}(8,3)=0$

$\mathrm{NL}(1)=1$

$\mathrm{NL}(2)=1$

$\mathrm{NL}(3)=2$

$\mathrm{NX}(1,1)=14$

$\mathrm{NX}(2,1)=19$

$\mathrm{NX}(3,1)=54$

$\mathrm{NX}(1,2)=0$ 


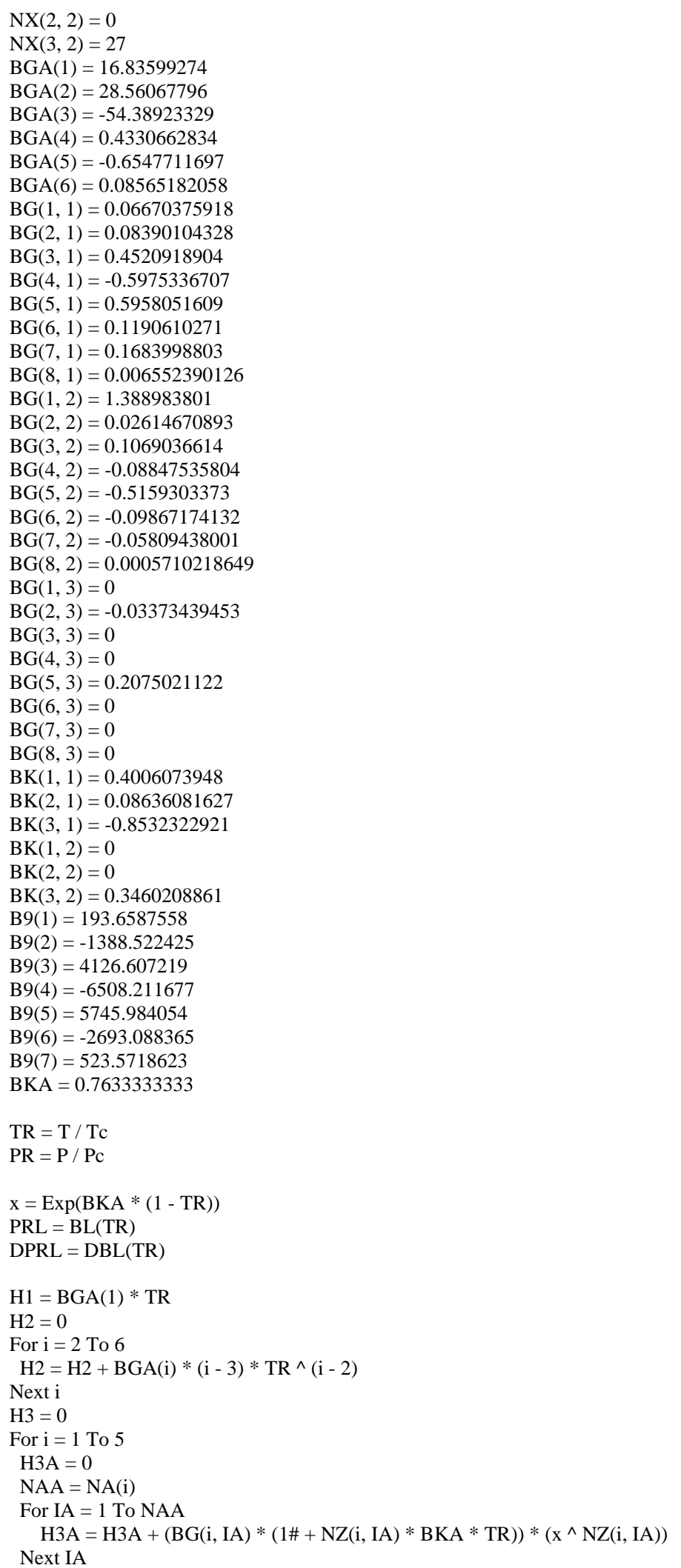




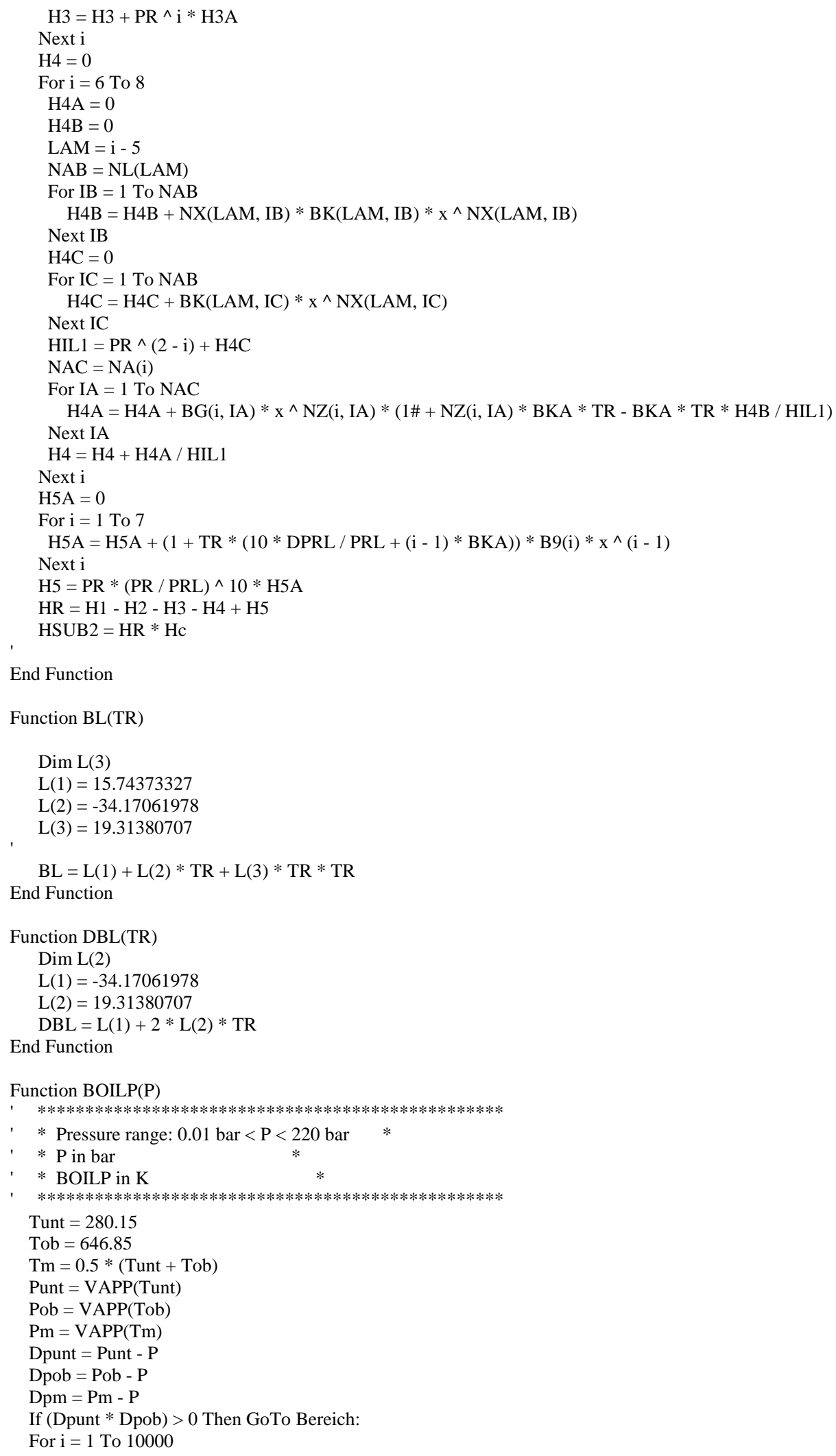




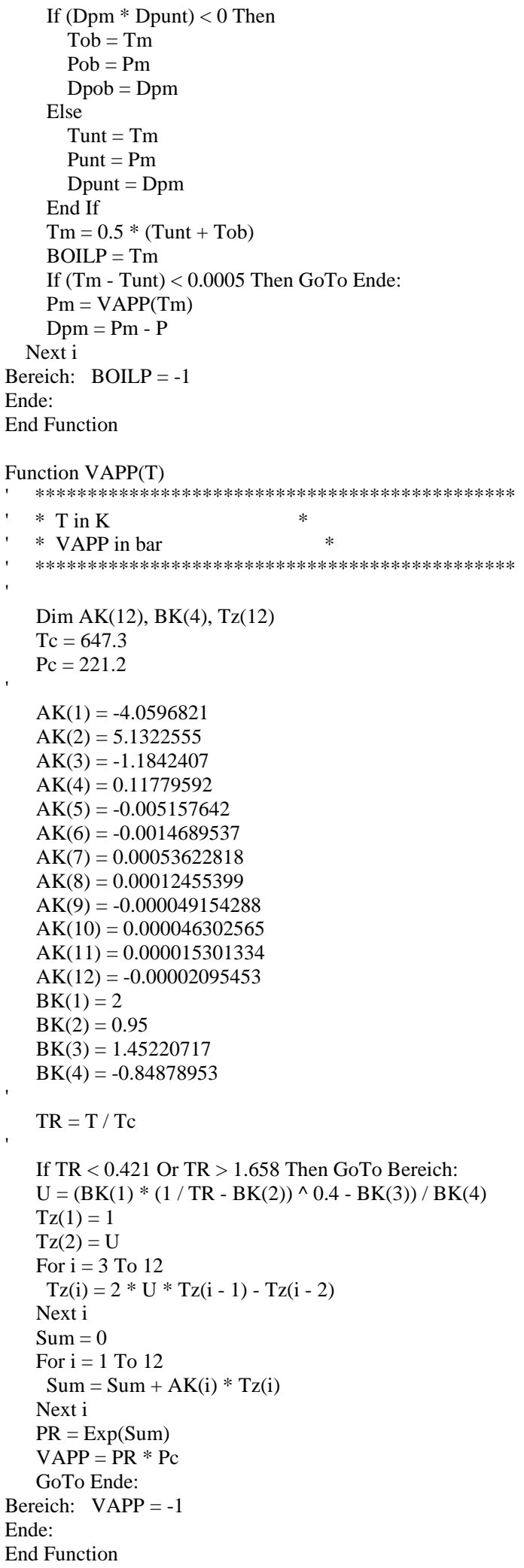




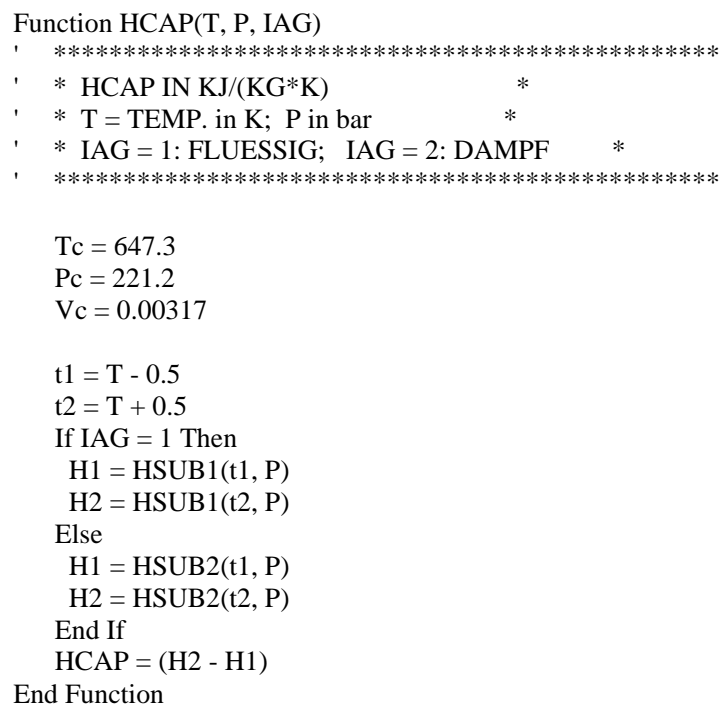

\section{Public Sub OpenDataExample()}

Dim dinput As Double

On Error Resume Next

this_file_path = ActiveWorkbook.Path

this_file_HD_dir = Left(this_file_path, 1)

ChDrive this_file_HD_dir: ChDir this_file_path

fileToOpen = Application.GetOpenFilename("Input Files (*.dat), *.dat")

If fileToOpen $=$ False Then

MsgBox "You haven't selected any input file"

Exit Sub

End If

'Source data path and write them in "Sheet3" sheet

With Sheets("Sheet3")

.Range("A1") = "Data path: "

.Range("B1") = fileToOpen

End With

'Read production properties data and write them in "Main" sheet

Open fileToOpen For Input As \#1

Do While Not EOF(1)

Input \#1, inputdata

If inputdata = "run_no" Then

Line Input \#1, inputdata

Sheets("Main").Range("F5") = inputdata

End If

If inputdata $=$ "additive" Then

Line Input \#1, inputdata

Sheets("Main").Range("F6") = inputdata

End If

If inputdata $=$ "researcher" Then

Input \#1, inputdata

Sheets("Main").Range("F7") = inputdata

End If

If inputdata = "cell_radius" Then 


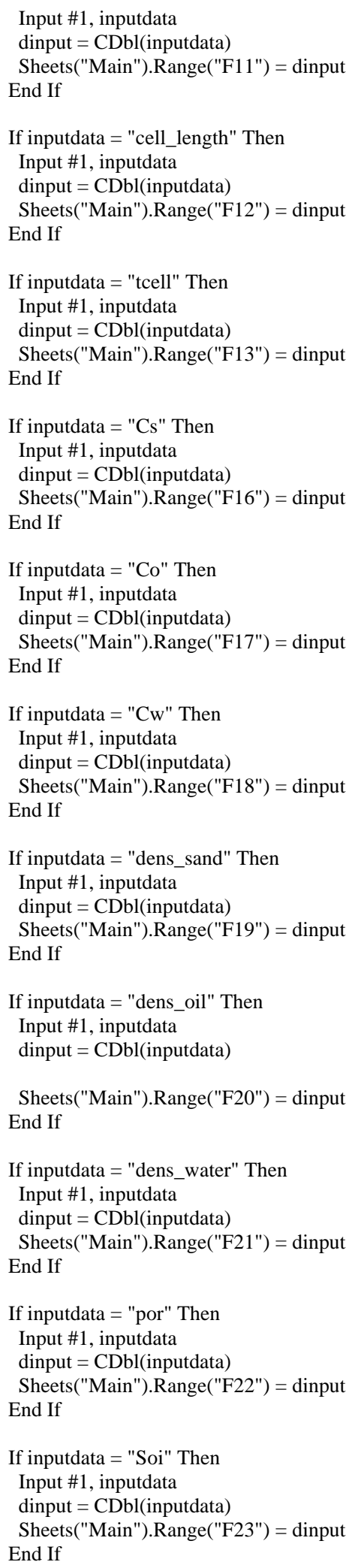




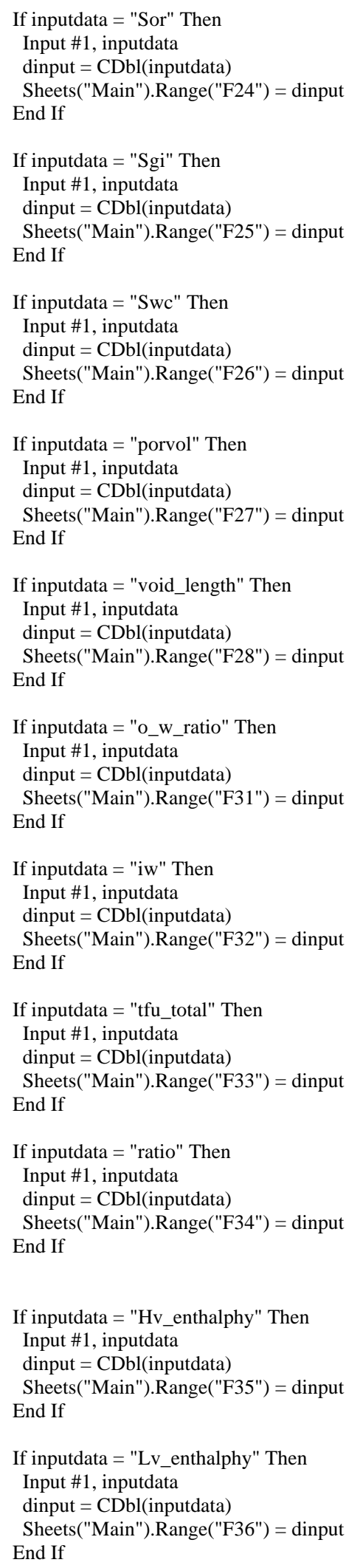




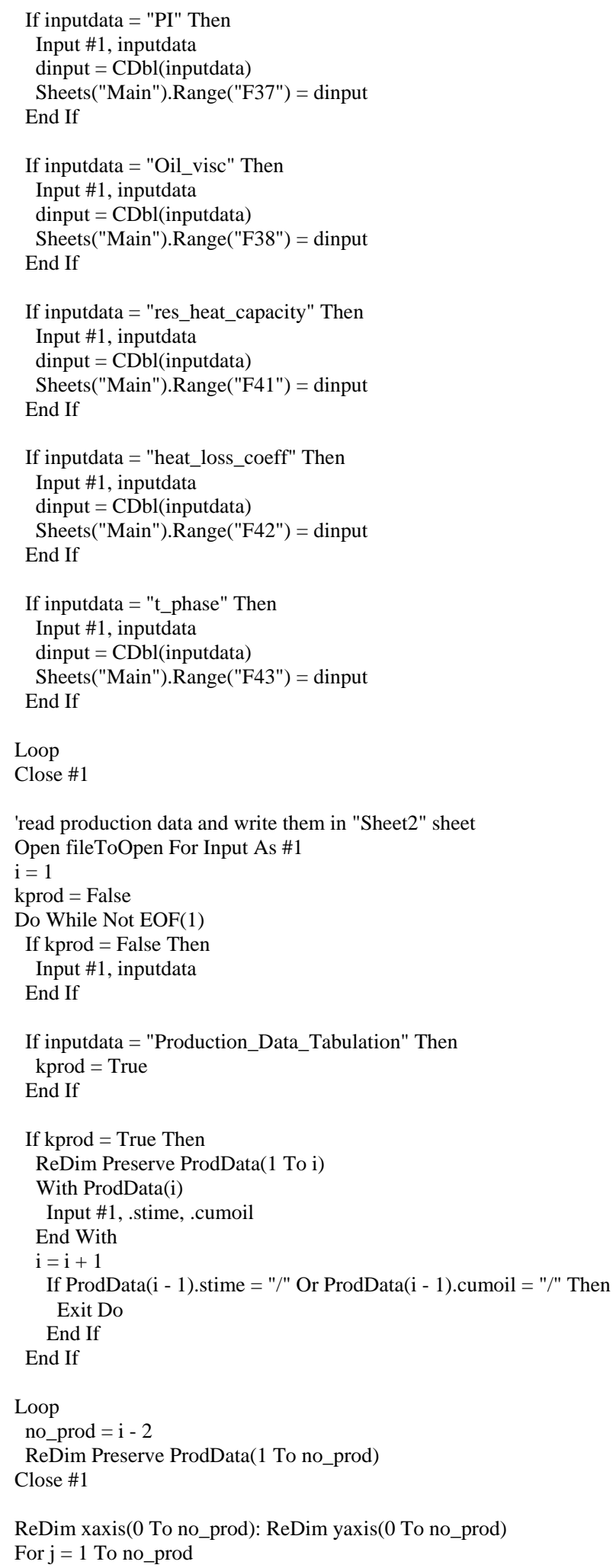




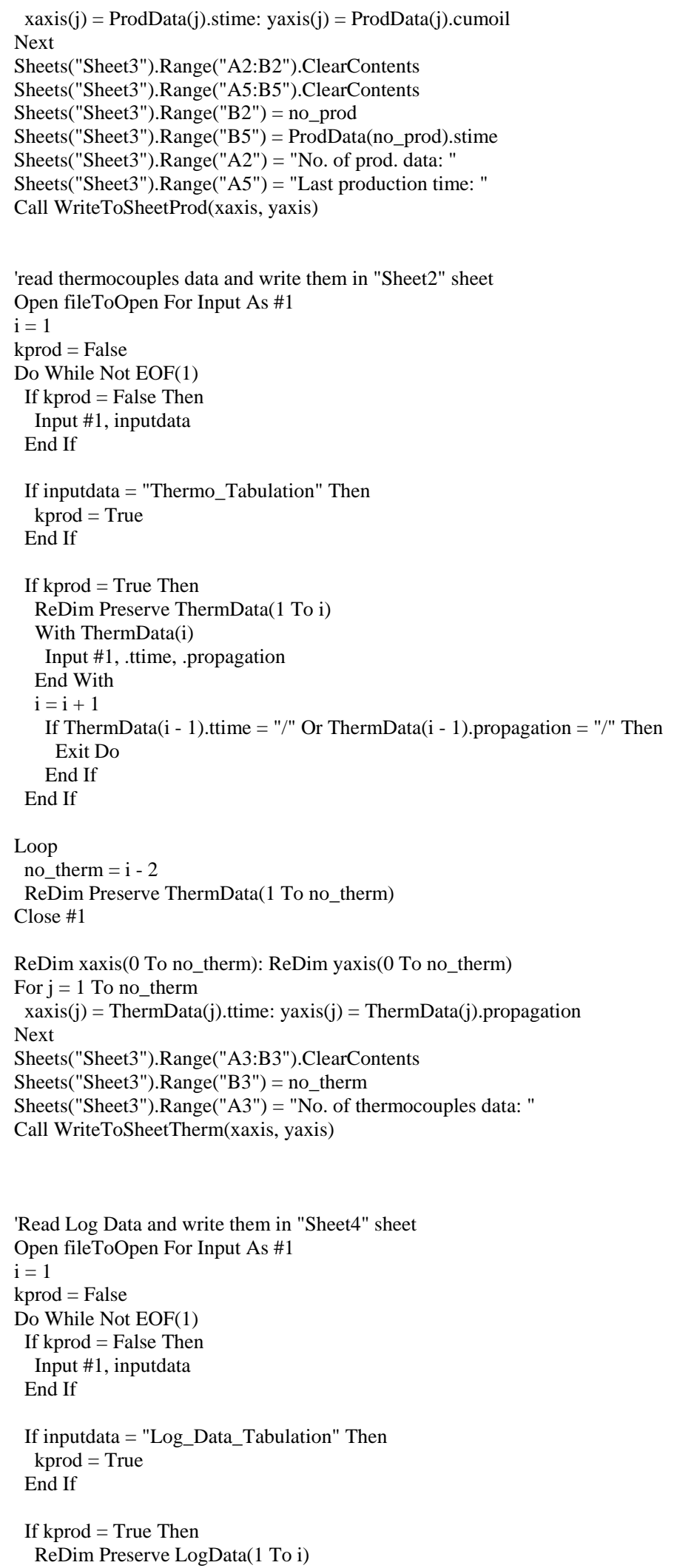




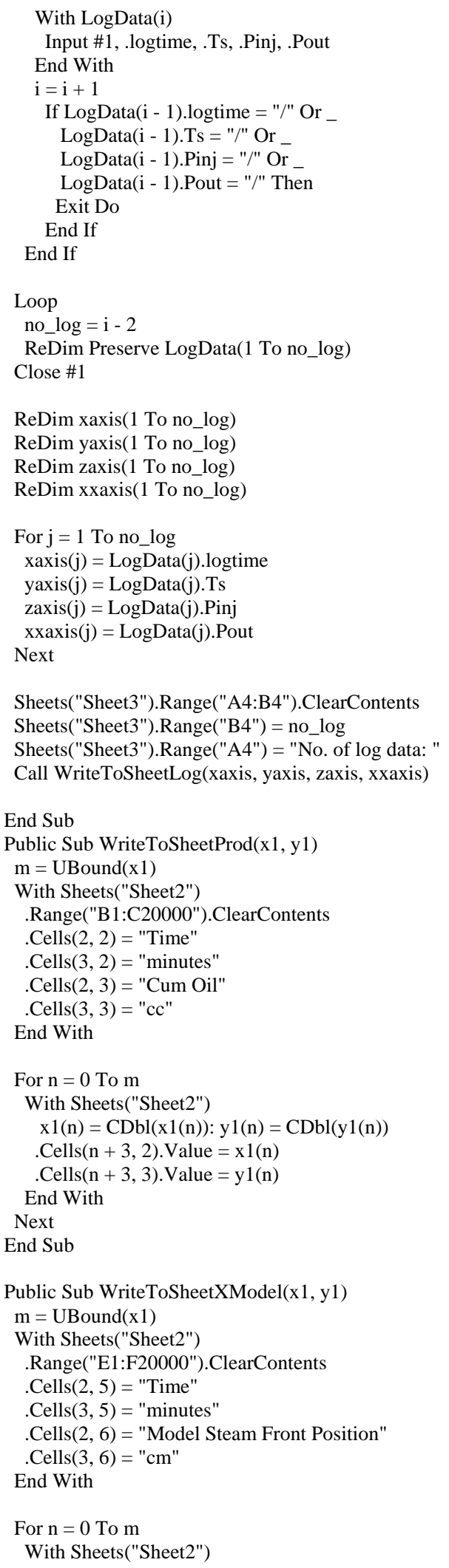




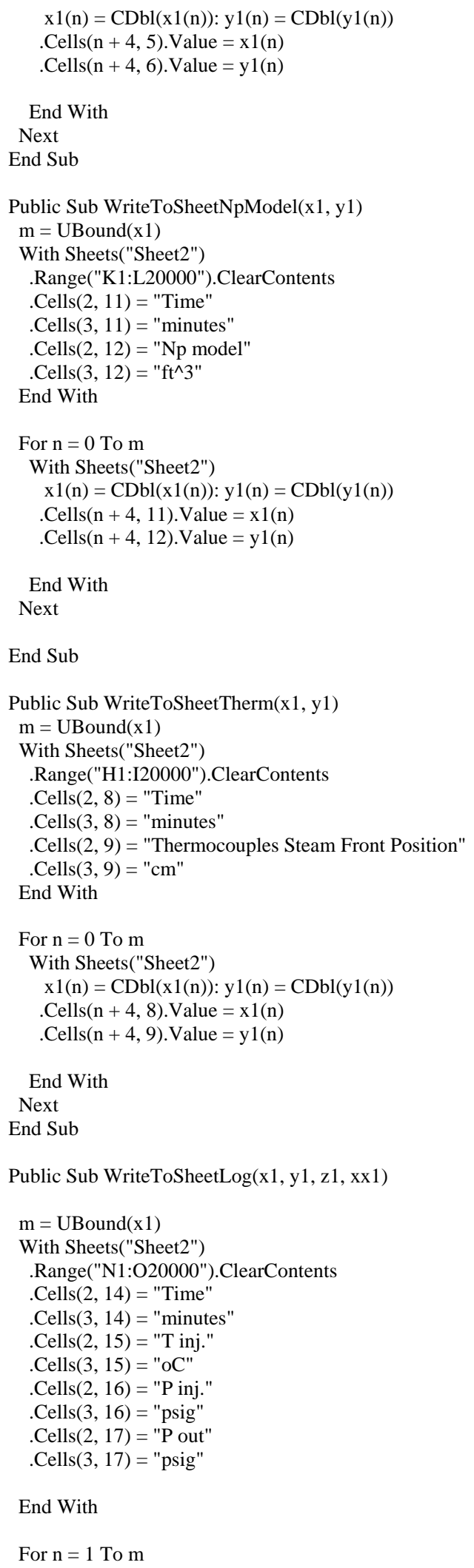




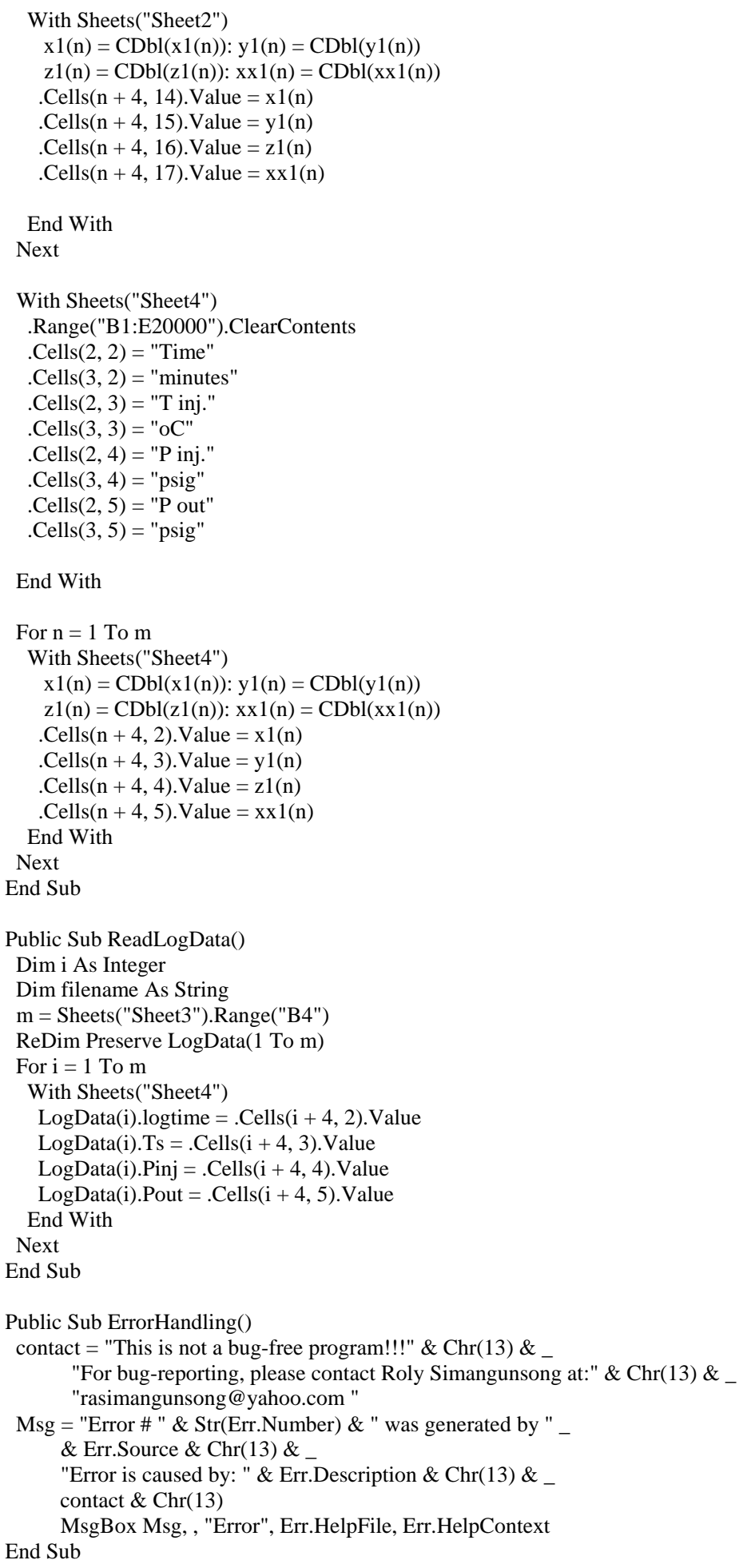


APPENDIX F

\section{DATA INPUT FOR STEAM FRONT ADVANCEMENT AND CUMULATIVE OIL PRODUCTION BASED ON ONE DIMENSIONAL ANAL YTICAL MODEL PROGRAM}

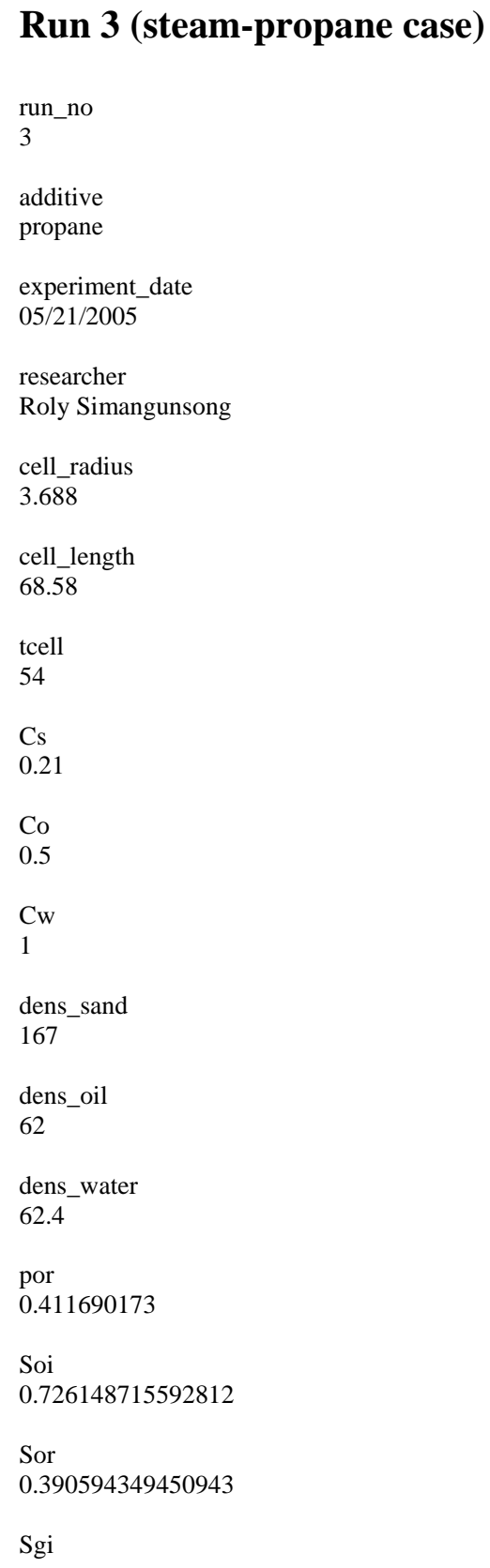




\begin{tabular}{|c|c|}
\hline \multicolumn{2}{|c|}{0.0936861640173443} \\
\hline \multicolumn{2}{|c|}{$\begin{array}{l}\text { Swc } \\
0.16516512\end{array}$} \\
\hline \multicolumn{2}{|c|}{ porvol } \\
\hline \multicolumn{2}{|c|}{$\begin{array}{l}\text { void_length } \\
2.58\end{array}$} \\
\hline o_w_ratio & 0.2 \\
\hline iw & 5.5 \\
\hline \multicolumn{2}{|c|}{ tfu_total } \\
\hline \multicolumn{2}{|c|}{ ratio } \\
\hline \multicolumn{2}{|c|}{0.358262377} \\
\hline \multicolumn{2}{|c|}{$\begin{array}{l}\text { Lv_enthalphy } \\
182.8470859\end{array}$} \\
\hline \multicolumn{2}{|c|}{$\begin{array}{l}\mathrm{PI} \\
7.4\end{array}$} \\
\hline \multicolumn{2}{|c|}{ Oil_visc } \\
\hline \multicolumn{2}{|c|}{$\begin{array}{l}\text { res_heat_capacity } \\
28\end{array}$} \\
\hline \multicolumn{2}{|c|}{$\begin{array}{l}\text { heat_loss_coeff } \\
1\end{array}$} \\
\hline \multicolumn{2}{|c|}{ t_phase } \\
\hline \multicolumn{2}{|c|}{$\begin{array}{l}\text {-- Time, minutes Cumulative Oil, cc } \\
\text { Production_Data_Tabulation }\end{array}$} \\
\hline 0 & 0 \\
\hline 2 & 0 \\
\hline 5 & 0 \\
\hline 8 & 0 \\
\hline 11 & 0 \\
\hline 14 & 0 \\
\hline 17 & 2 \\
\hline 20 & 5 \\
\hline 23 & 9 \\
\hline 26 & 14 \\
\hline 29 & 20.5 \\
\hline 32 & 27.5 \\
\hline 35 & 35 \\
\hline 38 & 44 \\
\hline 41 & 53 \\
\hline 44 & 63 \\
\hline 47 & 74 \\
\hline 50 & 87 \\
\hline 53 & 100 \\
\hline 56 & 114 \\
\hline
\end{tabular}




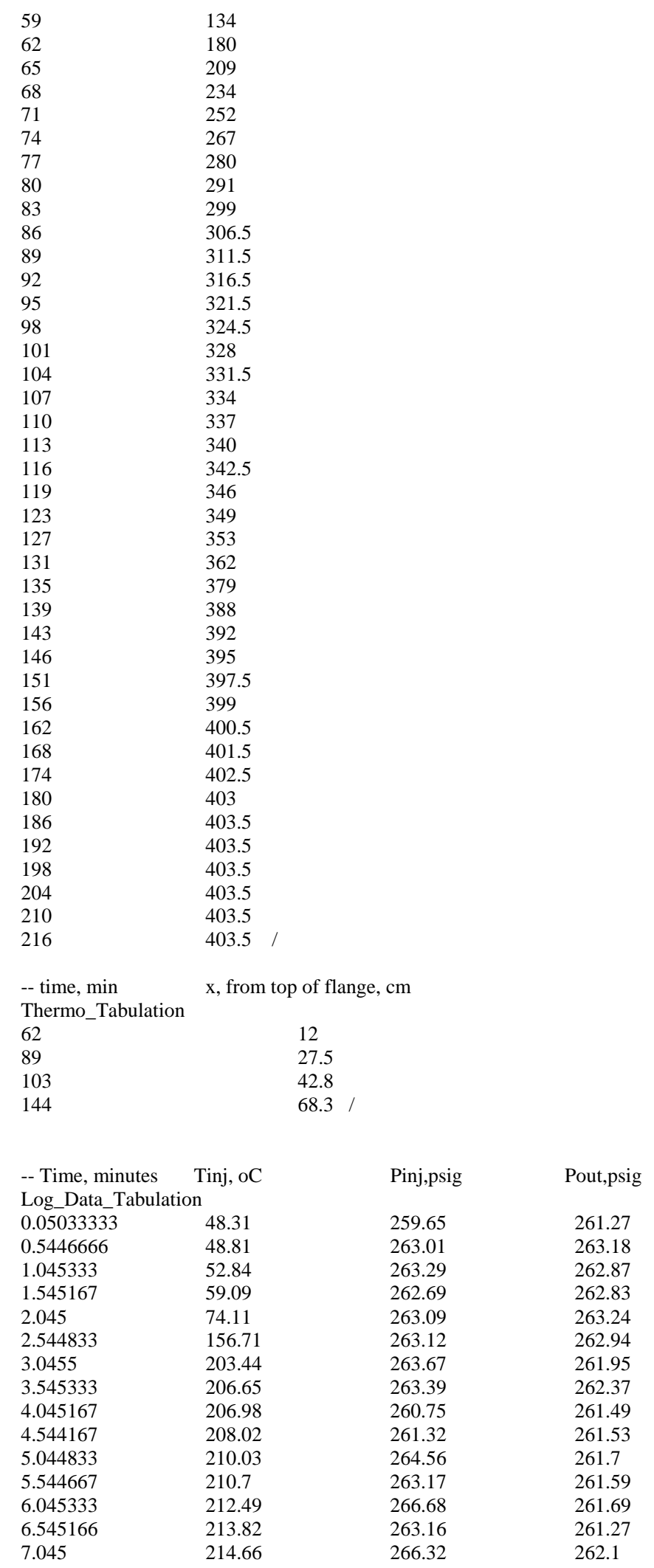




\begin{tabular}{|c|c|c|c|}
\hline 7.544833 & 216.07 & 267.91 & 260.92 \\
\hline 8.045667 & 213.1 & 274.6 & 261.8 \\
\hline 8.545333 & 213.52 & 273.74 & 261.27 \\
\hline 9.045167 & 212.07 & 276.13 & 261.4 \\
\hline 9.545 & 211.86 & 275 & 261.38 \\
\hline 10.04583 & 212.47 & 282.79 & 261.53 \\
\hline 10.54567 & 212.69 & 284.67 & 261.54 \\
\hline 11.0455 & 214.52 & 286.34 & 261.5 \\
\hline 11.54533 & 215.37 & 291.26 & 261.5 \\
\hline 12.04417 & 216.14 & 291.79 & 261.56 \\
\hline 12.54483 & 216.04 & 294.95 & 261.36 \\
\hline 13.04467 & 216.42 & 293.63 & 261.37 \\
\hline 13.5445 & 215.82 & 297.64 & 261.49 \\
\hline 14.04433 & 215.3 & 299.78 & 261.59 \\
\hline 14.54517 & 215.33 & 300.91 & 261.62 \\
\hline 15.045 & 216.71 & 298.7 & 261.59 \\
\hline 15.5465 & 216.59 & 300.56 & 261.45 \\
\hline 16.0455 & 216.72 & 307.25 & 261.54 \\
\hline 16.54433 & 217.81 & 308.86 & 261.48 \\
\hline 17.04517 & 217.35 & 311.35 & 261.47 \\
\hline 17.545 & 218.56 & 311.55 & 261.25 \\
\hline 18.04567 & 218.15 & 310.92 & 261.31 \\
\hline 18.5455 & 218.01 & 314.86 & 261.26 \\
\hline 19.04533 & 219.37 & 317.98 & 261.24 \\
\hline 19.54517 & 219.07 & 321.62 & 261.17 \\
\hline 20.045 & 219.72 & 323.91 & 261.19 \\
\hline 20.54483 & 218.67 & 326.36 & 261.17 \\
\hline 21.04467 & 218.85 & 329.42 & 261.32 \\
\hline 21.54533 & 219.34 & 333.67 & 261.65 \\
\hline 22.04517 & 218.45 & 329.75 & 261.7 \\
\hline 22.545 & 218.83 & 332.4 & 261.9 \\
\hline 23.04483 & 219.37 & 331.03 & 261.63 \\
\hline 23.5455 & 219.53 & 331.07 & 262.43 \\
\hline 24.04533 & 219.34 & 328.21 & 261.87 \\
\hline 24.54617 & 219.49 & 328.17 & 262.56 \\
\hline 25.045 & 220.01 & 328.29 & 262.58 \\
\hline 25.54583 & 220.25 & 328.56 & 260.57 \\
\hline 26.0465 & 220.25 & 328.02 & 262.62 \\
\hline 26.54533 & 220.32 & 327.36 & 262.63 \\
\hline 27.04517 & 220.11 & 326.03 & 261.46 \\
\hline 27.546 & 220.01 & 324.34 & 261 \\
\hline 28.04483 & 219.83 & 322.9 & 262.29 \\
\hline 28.54567 & 219.69 & 321.64 & 262.62 \\
\hline 29.0455 & 219.55 & 320.3 & 261.6 \\
\hline 29.54433 & 219.63 & 322.68 & 255.4 \\
\hline 30.045 & 219.51 & 321.63 & 256.63 \\
\hline 30.54483 & 219.56 & 322.57 & 258.55 \\
\hline 31.04567 & 219.69 & 322.55 & 259.19 \\
\hline 31.5455 & 219.72 & 324.55 & 257.69 \\
\hline 32.04533 & 219.77 & 325.09 & 258.21 \\
\hline 32.54517 & 220.01 & 326.37 & 258.97 \\
\hline 33.045 & 220.19 & 328.02 & 255.49 \\
\hline 33.54567 & 220.35 & 328.5 & 257.73 \\
\hline 34.0455 & 220.63 & 330.76 & 258.47 \\
\hline 34.54533 & 220.91 & 335 & 257.11 \\
\hline 35.04517 & 220.88 & 339.24 & 255.46 \\
\hline 35.545 & 220.76 & 341.72 & 255.99 \\
\hline 36.04483 & 220.89 & 343.74 & 256.5 \\
\hline 36.5455 & 221.02 & 346.53 & 258.51 \\
\hline 37.04533 & 220.97 & 345.82 & 258.9 \\
\hline 37.54517 & 220.93 & 345.34 & 257.99 \\
\hline 38.045 & 220.86 & 348.03 & 258.37 \\
\hline 38.54483 & 220.67 & 346.9 & 256.21 \\
\hline 39.0455 & 220.64 & 345.31 & 257.56 \\
\hline 39.54533 & 220.64 & 345.24 & 257.06 \\
\hline 40.04517 & 220.27 & 345.99 & 256.25 \\
\hline
\end{tabular}




\begin{tabular}{|c|c|c|c|}
\hline 40.545 & 220.25 & 344.78 & 258.21 \\
\hline 41.04483 & 220.33 & 344.88 & 258.25 \\
\hline 41.5455 & 220.58 & 345.32 & 258.44 \\
\hline 42.0445 & 220.44 & 343.98 & 257.73 \\
\hline 42.54433 & 220.32 & 343.92 & 256.8 \\
\hline 43.045 & 220.32 & 351.39 & 257.04 \\
\hline 43.54483 & 219.91 & 347 & 256.53 \\
\hline 44.04567 & 220.22 & 349.41 & 258.07 \\
\hline 44.54533 & 220.33 & 350.13 & 258.35 \\
\hline 45.04517 & 219.22 & 346.82 & 258.05 \\
\hline 45.545 & 220.46 & 347.09 & 260.03 \\
\hline 46.044 & 220.42 & 344.69 & 258.71 \\
\hline 46.54467 & 220.73 & 344.5 & 257.76 \\
\hline 47.0445 & 220.66 & 346.43 & 258.77 \\
\hline 47.54533 & 220.63 & 348.32 & 260.28 \\
\hline 48.045 & 220.56 & 348.09 & 262.5 \\
\hline 48.544 & 220.79 & 350.92 & 262.64 \\
\hline 49.04567 & 221.14 & 352.57 & 262.86 \\
\hline 49.5445 & 220.96 & 352.56 & 262.93 \\
\hline 50.04533 & 220.78 & 352.36 & 262.99 \\
\hline 50.54517 & 220.46 & 350.63 & 261.92 \\
\hline 51.045 & 220.48 & 349.61 & 261.86 \\
\hline 51.54567 & 220.45 & 347.52 & 262.08 \\
\hline 52.04633 & 219.91 & 344.42 & 262.3 \\
\hline 52.54533 & 219.97 & 342.71 & 262.74 \\
\hline 53.04517 & 219.45 & 340.02 & 262.89 \\
\hline 53.545 & 219.17 & 337.99 & 263.07 \\
\hline 54.04483 & 219.14 & 335.15 & 261.74 \\
\hline 54.5455 & 218.51 & 332.02 & 256.71 \\
\hline 55.04533 & 218.16 & 325.88 & 261.95 \\
\hline 55.54517 & 218.09 & 322.67 & 262.53 \\
\hline 56.045 & 217.79 & 320.52 & 262.22 \\
\hline 56.54483 & 217.47 & 320.78 & 263.17 \\
\hline 57.04467 & 217.43 & 321.52 & 262.74 \\
\hline 57.54533 & 217.27 & 320.35 & 261.36 \\
\hline 58.04517 & 217.13 & 318.08 & 260.57 \\
\hline 58.54417 & 217.01 & 318.13 & 261.75 \\
\hline 59.04383 & 216.82 & 317.54 & 262.18 \\
\hline 59.54467 & 216.6 & 317.36 & 262.45 \\
\hline 60.04533 & 216.52 & 315.55 & 263.01 \\
\hline 60.54517 & 216.14 & 315.2 & 262.99 \\
\hline 61.045 & 216.22 & 312.93 & 262.88 \\
\hline 61.54483 & 216.18 & 312.98 & 260.72 \\
\hline 62.04467 & 216.28 & 311.47 & 260.58 \\
\hline 62.5445 & 216.55 & 311.45 & 259.22 \\
\hline 63.04517 & 216.19 & 309.88 & 258.71 \\
\hline 63.545 & 216.46 & 309.57 & 261.89 \\
\hline 64.04483 & 215.98 & 307.54 & 261.74 \\
\hline 64.54466 & 215.46 & 306.54 & 259.16 \\
\hline 65.0455 & 215.48 & 302.03 & 259.63 \\
\hline 65.54533 & 216.15 & 302.45 & 258.89 \\
\hline 66.045 & 216.65 & 300.41 & 258.33 \\
\hline 66.54483 & 215.95 & 301.19 & 259.87 \\
\hline 67.04567 & 216.4 & 304.14 & 259.35 \\
\hline 67.5455 & 216.94 & 305.56 & 258.63 \\
\hline 68.04533 & 216.8 & 306.14 & 261.31 \\
\hline 68.54417 & 216.66 & 307.42 & 261.49 \\
\hline 69.044 & 217.15 & 306.43 & 261.96 \\
\hline 69.54567 & 216.54 & 306.15 & 261.77 \\
\hline 70.0455 & 216.94 & 305.24 & 261.69 \\
\hline 70.54533 & 216.63 & 305.31 & 261.98 \\
\hline 71.04517 & 216.67 & 304.31 & 261.92 \\
\hline 71.54501 & 217.14 & 302.26 & 262.61 \\
\hline 72.04666 & 216.64 & 303.33 & 262.52 \\
\hline 72.54467 & 216.89 & 303.05 & 262.89 \\
\hline 73.04433 & 217.09 & 304.83 & 262.9 \\
\hline
\end{tabular}




\begin{tabular}{|c|c|c|c|}
\hline 73.54517 & 216.97 & 305.14 & 260.74 \\
\hline 74.04501 & 216.9 & 303.89 & 261.26 \\
\hline 74.54383 & 216.72 & 304.88 & 261.39 \\
\hline 75.04467 & 216.76 & 305.67 & 261.68 \\
\hline 75.54533 & 216.19 & 305.95 & 261.98 \\
\hline 76.04617 & 216.8 & 305.33 & 261.91 \\
\hline 76.54501 & 217.01 & 306.62 & 261.75 \\
\hline 77.04383 & 216.77 & 305.58 & 262.09 \\
\hline 77.5455 & 217.14 & 306.89 & 262.32 \\
\hline 78.04533 & 216.95 & 305.56 & 262.32 \\
\hline 78.54517 & 216.41 & 307.96 & 262.43 \\
\hline 79.04501 & 216.47 & 305.16 & 262.7 \\
\hline 79.54483 & 216.52 & 305.09 & 262.42 \\
\hline 80.04567 & 216.58 & 304.2 & 262.57 \\
\hline 80.5445 & 216.29 & 302.38 & 262.61 \\
\hline 81.04517 & 216.78 & 303.2 & 260.2 \\
\hline 81.54501 & 216.46 & 303.09 & 260.56 \\
\hline 82.04483 & 216.55 & 301.82 & 259.92 \\
\hline 82.54567 & 215.71 & 301.35 & 260.37 \\
\hline 83.0455 & 215.91 & 297.85 & 261.05 \\
\hline 83.54533 & 216.22 & 298.45 & 261.04 \\
\hline 84.04501 & 216.09 & 298.61 & 258.59 \\
\hline 84.54483 & 215.9 & 300.3 & 261 \\
\hline 85.04567 & 215.51 & 301.31 & 259.13 \\
\hline 85.5455 & 216.19 & 299.75 & 260.6 \\
\hline 86.04533 & 216.71 & 299.98 & 259.97 \\
\hline 86.54517 & 217.3 & 302.53 & 260.67 \\
\hline 87.04501 & 217.41 & 300.96 & 260.3 \\
\hline 87.54567 & 216.8 & 301.92 & 258.83 \\
\hline 88.0455 & 216.97 & 300.15 & 261.25 \\
\hline 88.54433 & 216.29 & 299.58 & 260.74 \\
\hline 89.04517 & 215.93 & 296.75 & 261.5 \\
\hline 89.54501 & 216.24 & 295.12 & 260.96 \\
\hline 90.04567 & 216.44 & 294.18 & 259.85 \\
\hline 90.54467 & 215.2 & 291.56 & 260.75 \\
\hline 91.04433 & 214.62 & 289.48 & 260.9 \\
\hline 91.54417 & 214.71 & 288.61 & 260.85 \\
\hline 92.04501 & 214.05 & 287.05 & 260.93 \\
\hline 92.54483 & 213.98 & 285.41 & 261.67 \\
\hline 93.0465 & 213.74 & 283.95 & 261.55 \\
\hline 93.54533 & 213.26 & 283.09 & 261 \\
\hline 94.04433 & 214.07 & 285.14 & 261.86 \\
\hline 94.54417 & 214.27 & 286.48 & 261.53 \\
\hline 95.04483 & 214.49 & 285.66 & 261.29 \\
\hline 95.54467 & 215.31 & 290.17 & 261.25 \\
\hline 96.04533 & 216.23 & 289.2 & 261.69 \\
\hline 96.54433 & 216.96 & 291 & 261.47 \\
\hline 97.04501 & 216.07 & 288.8 & 261.14 \\
\hline 97.544 & 216.05 & 287.56 & 260.63 \\
\hline 98.04567 & 215.91 & 285.91 & 261.75 \\
\hline 98.54533 & 214.98 & 287.13 & 261.81 \\
\hline 99.04517 & 215.25 & 285.26 & 261.83 \\
\hline 99.54417 & 214.82 & 285.43 & 261.18 \\
\hline 100.0448 & 214.63 & 284.14 & 261.84 \\
\hline 100.5447 & 214.94 & 285.27 & 261.59 \\
\hline 101.0455 & 214.74 & 283.74 & 261.91 \\
\hline 101.5443 & 214.42 & 283.54 & 261.26 \\
\hline 102.045 & 213.62 & 281.89 & 261.08 \\
\hline 102.5448 & 213.17 & 280.33 & 261.27 \\
\hline 103.0457 & 212.49 & 278.11 & 261.18 \\
\hline 103.5455 & 212.92 & 276.86 & 262.07 \\
\hline 104.0453 & 212.93 & 275.86 & 260.3 \\
\hline 104.5452 & 213.88 & 275.38 & 260.93 \\
\hline 105.044 & 213.98 & 278.4 & 261.59 \\
\hline 105.5457 & 213.9 & 277.33 & 261.74 \\
\hline 106.0455 & 213.99 & 279.15 & 261.25 \\
\hline
\end{tabular}




\begin{tabular}{|c|c|c|c|}
\hline 106.5453 & 214.08 & 281.63 & 261.7 \\
\hline 107.0442 & 215.6 & 281.79 & 261.55 \\
\hline 107.545 & 216.02 & 283.36 & 260.99 \\
\hline 108.0457 & 216.14 & 283.43 & 261.52 \\
\hline 108.5465 & 215.77 & 282.65 & 261.41 \\
\hline 109.0462 & 215.34 & 282.78 & 261.61 \\
\hline 109.5452 & 215.43 & 281.47 & 261.56 \\
\hline 110.045 & 214.92 & 280.7 & 260.84 \\
\hline 110.5448 & 215.15 & 279.49 & 259.34 \\
\hline 111.0455 & 215.53 & 280.39 & 261.57 \\
\hline 111.5453 & 215.05 & 280.01 & 261.6 \\
\hline 112.0452 & 214.3 & 281.63 & 261.69 \\
\hline 112.544 & 213.67 & 282.74 & 262.03 \\
\hline 113.0438 & 213.46 & 281.03 & 261.9 \\
\hline 113.5455 & 213.34 & 281.92 & 262.21 \\
\hline 114.0453 & 213.27 & 284.69 & 262.24 \\
\hline 114.5452 & 213.75 & 283.02 & 262.71 \\
\hline 115.045 & 213.16 & 281.45 & 262.75 \\
\hline 115.5448 & 213.38 & 281.57 & 262.89 \\
\hline 116.0455 & 213.97 & 280.12 & 260.84 \\
\hline 116.5453 & 213.31 & 278.97 & 260.59 \\
\hline 117.0452 & 213.81 & 280.98 & 261.02 \\
\hline 117.545 & 214.92 & 279.72 & 261.24 \\
\hline 118.0448 & 215.34 & 276.54 & 257.92 \\
\hline 118.5447 & 214.96 & 275.05 & 259.61 \\
\hline 119.0445 & 213.91 & 275.73 & 262.36 \\
\hline 119.5453 & 213.73 & 272.25 & 261.98 \\
\hline 120.046 & 213.34 & 271.53 & 261.25 \\
\hline 120.5448 & 212.71 & 271.55 & 261.84 \\
\hline 121.0447 & 212.86 & 274.87 & 261.22 \\
\hline 121.5455 & 213.9 & 273.06 & 260.05 \\
\hline 122.0453 & 214.21 & 274.33 & 262.42 \\
\hline 122.5452 & 212.94 & 276.02 & 262.38 \\
\hline 123.045 & 213.74 & 275.17 & 262.55 \\
\hline 123.5457 & 213.12 & 276.79 & 262.72 \\
\hline 124.0455 & 212.92 & 276.55 & 262.88 \\
\hline 124.5453 & 213.62 & 276.66 & 263.07 \\
\hline 125.0452 & 214.27 & 278.17 & 261.25 \\
\hline 125.545 & 214.24 & 276.96 & 260.7 \\
\hline 126.0457 & 214.15 & 277.02 & 261.44 \\
\hline 126.5455 & 214.17 & 277.56 & 261.59 \\
\hline 127.0453 & 213.27 & 276.02 & 261.44 \\
\hline 127.5452 & 213.42 & 274.44 & 261.37 \\
\hline 128.045 & 213.37 & 274.87 & 260.68 \\
\hline 128.5457 & 213.07 & 273.74 & 260.88 \\
\hline 129.0455 & 213.23 & 273.71 & 261.59 \\
\hline 129.5453 & 212.68 & 272.23 & 259.93 \\
\hline 130.0452 & 212.91 & 273.49 & 261.77 \\
\hline 130.545 & 213.47 & 274.51 & 261.7 \\
\hline 131.0438 & 214.18 & 273.87 & 260.69 \\
\hline 131.5455 & 213.69 & 272.26 & 262.08 \\
\hline 132.0445 & 213.53 & 271.4 & 262.24 \\
\hline 132.5443 & 213.17 & 272.79 & 260.93 \\
\hline 133.045 & 213.16 & 270.05 & 262.09 \\
\hline 133.5448 & 213.21 & 269.88 & 262.44 \\
\hline 134.0447 & 212.8 & 268.75 & 262.39 \\
\hline 134.5453 & 213.16 & 268.15 & 262.35 \\
\hline 135.0452 & 213.15 & 266.98 & 261.93 \\
\hline 135.545 & 213.2 & 265.63 & 261.38 \\
\hline 136.0448 & 213.1 & 265.69 & 261.18 \\
\hline 136.5447 & 213.51 & 266.66 & 261.04 \\
\hline 137.0445 & 214.17 & 266.64 & 258.64 \\
\hline 137.5453 & 214.37 & 266.6 & 258.05 \\
\hline 138.0442 & 213.37 & 267.14 & 258.09 \\
\hline 138.544 & 214.64 & 268.51 & 258.05 \\
\hline 139.0457 & 215 & 268.09 & 257.84 \\
\hline
\end{tabular}




\begin{tabular}{|c|c|c|c|}
\hline 139.5445 & 215.24 & 268.36 & 258.35 \\
\hline 140.0462 & 214.1 & 269.41 & 259.1 \\
\hline 140.5452 & 215.73 & 275.77 & 261.87 \\
\hline 141.045 & 214.87 & 276.22 & 263.39 \\
\hline 141.5457 & 214.35 & 279.76 & 263.48 \\
\hline 142.0455 & 214.23 & 279.08 & 262.24 \\
\hline 142.5453 & 214.28 & 278.49 & 261.19 \\
\hline 143.0452 & 213.82 & 274.12 & 260.92 \\
\hline 143.545 & 213.82 & 273.58 & 260.99 \\
\hline 144.0457 & 214.2 & 272.92 & 261.17 \\
\hline 144.5455 & 213.95 & 269.98 & 260.39 \\
\hline 145.0453 & 214.46 & 271.21 & 260.16 \\
\hline 145.5442 & 213.17 & 269.69 & 259.37 \\
\hline 146.045 & 213.1 & 269.21 & 259.59 \\
\hline 146.5448 & 212.67 & 268.14 & 259.38 \\
\hline 147.0455 & 212.89 & 266.83 & 261.3 \\
\hline 147.5453 & 213 & 268.62 & 259.97 \\
\hline 148.0452 & 212.72 & 266.82 & 261.88 \\
\hline 148.544 & 212.85 & 268.61 & 261.42 \\
\hline 149.0448 & 212.61 & 267.38 & 261.22 \\
\hline 149.5455 & 212.63 & 268.02 & 260.3 \\
\hline 150.0453 & 212.83 & 265.17 & 261.31 \\
\hline 150.5452 & 213.1 & 264.34 & 260.61 \\
\hline 151.0442 & 213.18 & 263.86 & 257.76 \\
\hline 151.544 & 213.41 & 263.11 & 259.26 \\
\hline 152.0455 & 213.83 & 262.5 & 260.18 \\
\hline 152.5453 & 213.43 & 263.22 & 259.83 \\
\hline 153.0443 & 213.71 & 263.76 & 260.78 \\
\hline 153.546 & 213.78 & 264.83 & 260.34 \\
\hline 154.0448 & 213.19 & 265.4 & 261.64 \\
\hline 154.5457 & 213.18 & 265.42 & 261.39 \\
\hline 155.0455 & 213.79 & 265.71 & 261.48 \\
\hline 155.5453 & 213.88 & 266.83 & 261.88 \\
\hline 156.045 & 214.56 & 267.1 & 261.93 \\
\hline 156.5458 & 214.31 & 267.97 & 261.94 \\
\hline 157.0465 & 214.35 & 268.6 & 262.2 \\
\hline 157.5455 & 214.8 & 268.37 & 261.88 \\
\hline 158.0443 & 214.61 & 268.38 & 261.89 \\
\hline 158.5452 & 214.72 & 268.3 & 261.91 \\
\hline 159.044 & 214.97 & 268.94 & 259.21 \\
\hline 159.5447 & 214.95 & 268.05 & 259.55 \\
\hline 160.0455 & 214.88 & 267.62 & 261.04 \\
\hline 160.5443 & 215.26 & 268.1 & 262.3 \\
\hline 161.0452 & 215.18 & 268.61 & 262.56 \\
\hline 161.544 & 214.96 & 270.09 & 260.06 \\
\hline 162.0457 & 214.2 & 268.99 & 260.44 \\
\hline 162.5455 & 214.7 & 268.64 & 260.02 \\
\hline 163.0443 & 215.33 & 268.67 & 259.85 \\
\hline 163.5452 & 215.87 & 268.6 & 259.51 \\
\hline 164.044 & 215.46 & 269.13 & 260.63 \\
\hline 164.5438 & 215.25 & 269.72 & 261.15 \\
\hline 165.0455 & 215.47 & 270.08 & 261.8 \\
\hline 165.5453 & 215.68 & 274.43 & 262.07 \\
\hline 166.0443 & 215.79 & 276.86 & 260.71 \\
\hline 166.545 & 215.54 & 275.23 & 261.15 \\
\hline 167.0448 & 215.12 & 272.78 & 262.9 \\
\hline 167.5447 & 214.44 & 275.67 & 257.68 \\
\hline 168.0453 & 214.91 & 268.5 & 261.56 \\
\hline 168.5452 & 214.12 & 270.42 & 257.53 \\
\hline 169.045 & 213.39 & 266.44 & 259.93 \\
\hline 169.5448 & 213.88 & 266.75 & 261.1 \\
\hline 170.0455 & 213.43 & 268.93 & 259.69 \\
\hline 170.5463 & 213.4 & 265.75 & 259.56 \\
\hline 171.047 & 213.11 & 268.96 & 261.49 \\
\hline 171.546 & 213.67 & 267.29 & 262.36 \\
\hline 172.0458 & 213.66 & 264.57 & 258.69 \\
\hline
\end{tabular}




\begin{tabular}{|c|c|c|c|}
\hline 172.5465 & 213.86 & 266.63 & 261.23 \\
\hline 173.0463 & 212.91 & 266.55 & 262.11 \\
\hline 173.547 & 213.32 & 268.15 & 262.68 \\
\hline 174.046 & 213.95 & 268.04 & 262.79 \\
\hline 174.5458 & 213.99 & 266.4 & 257.48 \\
\hline 175.0465 & 213.37 & 266.6 & 256.81 \\
\hline 175.5463 & 214.41 & 264.05 & 257.7 \\
\hline 176.0472 & 213.71 & 263.93 & 257.63 \\
\hline 176.5452 & 213.63 & 264.09 & 259.3 \\
\hline 177.045 & 213.33 & 263.91 & 259.11 \\
\hline 177.5447 & 213.72 & 271.17 & 258.5 \\
\hline 178.0455 & 214.98 & 269.73 & 259.84 \\
\hline 178.5453 & 214.17 & 272.4 & 261.12 \\
\hline 179.0452 & 214.8 & 270.8 & 261.59 \\
\hline 179.545 & 214.02 & 271.72 & 262.84 \\
\hline 180.0457 & 213.27 & 270.87 & 263.01 \\
\hline 180.5465 & 212.99 & 270.52 & 262.71 \\
\hline 181.0443 & 214.33 & 267.37 & 257.02 \\
\hline 181.5442 & 214.53 & 264.69 & 256.43 \\
\hline 182.045 & 213.75 & 265.05 & 256.33 \\
\hline 182.5448 & 212.26 & 264.29 & 256.18 \\
\hline 183.0447 & 213.07 & 263.49 & 256.69 \\
\hline 183.5453 & 213.99 & 263.46 & 256.68 \\
\hline 184.0452 & 213.18 & 263.35 & 256.59 \\
\hline 184.545 & 212.88 & 263.73 & 256.6 \\
\hline 185.0448 & 213.26 & 263.95 & 256.62 \\
\hline 185.5455 & 212.29 & 264.28 & 256.78 \\
\hline 186.0453 & 212.66 & 264.56 & 256.85 \\
\hline 186.5452 & 213.34 & 263.82 & 257.19 \\
\hline 187.045 & 212.75 & 262.66 & 256.99 \\
\hline 187.5448 & 212.77 & 263.41 & 257.01 \\
\hline 188.0455 & 212.43 & 263.94 & 256.76 \\
\hline 188.5453 & 213.08 & 262.96 & 257.24 \\
\hline 189.0452 & 212.58 & 262.77 & 256.54 \\
\hline 189.545 & 212.55 & 263.52 & 256.87 \\
\hline 190.0448 & 212.08 & 263.87 & 256.38 \\
\hline 190.5457 & 212.23 & 262.51 & 256.83 \\
\hline 191.0455 & 212.88 & 263.99 & 256.4 \\
\hline 191.5462 & 213.24 & 262.13 & 257.01 \\
\hline 192.045 & 212.34 & 262.65 & 256.79 \\
\hline 192.5448 & 213.01 & 261.77 & 256.8 \\
\hline 193.0457 & 213.78 & 264.42 & 257.16 \\
\hline 193.5455 & 213.57 & 262.43 & 257.15 \\
\hline 194.0453 & 213.18 & 264.7 & 256.98 \\
\hline 194.5452 & 213.23 & 262.97 & 257.35 \\
\hline 195.045 & 213.5 & 263.27 & 257.18 \\
\hline 195.5457 & 212.93 & 263.71 & 257.12 \\
\hline 196.0455 & 213.8 & 263.37 & 256.69 \\
\hline 196.5453 & 214.41 & 263.8 & 257.35 \\
\hline 197.0452 & 214.46 & 263.33 & 257.63 \\
\hline 197.545 & 214.14 & 264.72 & 257.74 \\
\hline 198.0457 & 214.02 & 264.48 & 257.22 \\
\hline 198.5465 & 213.77 & 264.17 & 257.4 \\
\hline 199.0453 & 213.52 & 263.9 & 257.55 \\
\hline 199.5442 & 213.76 & 264.05 & 257.54 \\
\hline 200.045 & 213.08 & 262.74 & 257.59 \\
\hline 200.5448 & 212.99 & 261.92 & 257.35 \\
\hline 201.0455 & 214.39 & 262.27 & 257.15 \\
\hline 201.5453 & 213.91 & 262.25 & 258.06 \\
\hline 202.0452 & 213.16 & 262.99 & 257.23 \\
\hline 202.545 & 214.09 & 262.52 & 258.32 \\
\hline 203.0448 & 214.15 & 262.97 & 257.64 \\
\hline 203.5455 & 214.58 & 263.56 & 257.73 \\
\hline 204.0453 & 214.99 & 264.15 & 257.48 \\
\hline 204.5452 & 214.22 & 267.55 & 257.64 \\
\hline 205.045 & 214.63 & 267.79 & 258.66 \\
\hline
\end{tabular}




\begin{tabular}{|c|c|c|c|}
\hline 205.5448 & 213.64 & 268.86 & 258.68 \\
\hline 206.0455 & 213.26 & 268.01 & 260.03 \\
\hline 206.5453 & 214.16 & 268.36 & 259.76 \\
\hline 207.048 & 225.61 & 262.02 & 257.08 \\
\hline 207.5478 & 225.27 & 260.07 & 256.45 \\
\hline 208.0467 & 225.08 & 259.25 & 256.08 \\
\hline 208.5483 & 224.18 & 258.55 & 256.41 \\
\hline 209.0473 & 222.75 & 258.8 & 256.2 \\
\hline 209.548 & 222.45 & 258.58 & 256.86 \\
\hline 210.0478 & 222.68 & 258.77 & 255.71 \\
\hline 210.5477 & 223.01 & 258.83 & 257.05 \\
\hline 211.0483 & 222.71 & 258.8 & 257.97 \\
\hline 211.5482 & 222.37 & 259.24 & 258.19 \\
\hline 212.048 & 222.17 & 259.9 & 256.68 \\
\hline 212.5478 & 221.94 & 259.76 & 257.8 \\
\hline 213.0477 & 222.16 & 258.6 & 255.8 \\
\hline 213.5475 & 221.8 & 259 & 258.18 \\
\hline 214.0482 & 222.11 & 259.71 & 258.16 \\
\hline 214.548 & 222.18 & 257.53 & 254.48 \\
\hline 215.0478 & 221.29 & 256.41 & 254.74 \\
\hline 215.5468 & 220.67 & 256.09 & 254.33 \\
\hline 216.0475 & 220.81 & 255.95 & 255.39 \\
\hline 216.5483 & 221.53 & 256.3 & 254.49 \\
\hline 217.048 & 222.37 & 257.89 & 255.48 \\
\hline 217.5478 & 221.64 & 258.79 & 256.22 \\
\hline 218.0468 & 223.22 & 259.11 & 255.83 \\
\hline 218.5475 & 222.29 & 258.98 & 256.07 \\
\hline 219.0483 & 222.65 & 258.84 & 255.79 \\
\hline 219.5482 & 223.33 & 259.04 & 255.65 \\
\hline 220.0488 & 223.3 & 258.97 & 255.28 \\
\hline 220.5477 & 223.17 & 259.37 & 256.04 \\
\hline 221.0485 & 224.96 & 259.47 & 256.37 \\
\hline 221.5483 & 225.61 & 259.38 & 256.4 \\
\hline 222.0482 & 224.07 & 259.13 & 255.83 \\
\hline 222.548 & 223.52 & 258.66 & 255.75 \\
\hline 223.0478 & 223.74 & 258.09 & 255.89 \\
\hline 223.5477 & 224.96 & 257.53 & 255.87 \\
\hline 224.0483 & 223.38 & 257.22 & 255.46 \\
\hline 224.5482 & 223.35 & 255.83 & 253.18 \\
\hline 225.047 & 221.92 & 255.28 & 253.53 \\
\hline 225.5478 & 221.78 & 255.74 & 253.68 \\
\hline 226.0477 & 221.37 & 256.18 & 254.21 \\
\hline 226.5483 & 221.66 & 256.1 & 254.77 \\
\hline 227.0482 & 222.38 & 256.55 & 254.18 \\
\hline 227.547 & 223.67 & 256.48 & 254.67 \\
\hline 228.0478 & 223.06 & 256.79 & 255.1 \\
\hline 228.5477 & 221.99 & 257.24 & 254.94 \\
\hline 229.0483 & 223.44 & 257.81 & 255.58 \\
\hline 229.5482 & 223.98 & 257.98 & 255.81 \\
\hline 230.048 & 223.19 & 257.91 & 255.66 \\
\hline 230.5478 & 222.71 & 258.13 & 255.87 \\
\hline 231.0468 & 224.09 & 258.37 & 255.24 \\
\hline 231.5483 & 223.58 & 258.55 & 255.55 \\
\hline 232.0473 & 224.01 & 258.93 & 255.57 \\
\hline 232.548 & 224.4 & 258.97 & 258.27 \\
\hline 233.0478 & 223.19 & 258.19 & 255.01 \\
\hline 233.5487 & 222.94 & 259.22 & 256.53 \\
\hline 234.0485 & 223.23 & 259.78 & 257.44 \\
\hline 234.5473 & 223.69 & 260.39 & 257.87 \\
\hline 235.048 & 223.11 & 260.93 & 258.22 \\
\hline 235.5478 & 223.7 & 260.42 & 255.8 \\
\hline 236.0487 & 223.54 & 258.97 & 255.45 \\
\hline 236.5467 & 224.85 & 258.58 & 255.72 \\
\hline 237.0473 & 224.35 & 258.71 & 255.7 \\
\hline 237.5482 & 221.92 & 258.65 & 256.53 \\
\hline 238.048 & 223.92 & 258.78 & 255.35 \\
\hline
\end{tabular}




\begin{tabular}{|c|c|c|c|}
\hline 238.5477 & 222.82 & 258.74 & 255.85 \\
\hline 239.0467 & 223.1 & 258.75 & 256.17 \\
\hline 239.5483 & 224.84 & 258.62 & 256.07 \\
\hline 240.0482 & 224.47 & 258.32 & 255.77 \\
\hline 240.548 & 222.54 & 258.24 & 256.31 \\
\hline 241.0478 & 223.29 & 258.71 & 255.31 \\
\hline 241.5477 & 224.39 & 259.61 & 255.77 \\
\hline 242.0483 & 223.31 & 260.89 & 256.24 \\
\hline 242.5482 & 225.11 & 262.09 & 256.3 \\
\hline 243.047 & 226.68 & 262.25 & 256.95 \\
\hline 243.5478 & 227.29 & 261.34 & 256.49 \\
\hline 244.0477 & 228.05 & 260.36 & 256.33 \\
\hline 244.5483 & 225.94 & 259.38 & 256.44 \\
\hline 245.0482 & 212.78 & 273.2 & 263.76 \\
\hline 245.548 & 212.88 & 273.35 & 263.24 \\
\hline 246.0478 & 212.88 & 273.37 & 262.92 \\
\hline 246.5477 & 212.62 & 273.88 & 263.67 \\
\hline 247.0483 & 212.49 & 273.37 & 264.23 \\
\hline 247.5473 & 212.64 & 273.35 & 264.25 \\
\hline 248.048 & 212.72 & 273.39 & 263.39 \\
\hline 248.5478 & 212.81 & 273.42 & 263.91 \\
\hline 249.0467 & 212.83 & 273.24 & 263.34 \\
\hline 249.5475 & 212.87 & 273.73 & 262.94 \\
\hline 250.0492 & 213.07 & 273.27 & 262.76 \\
\hline 250.5472 & 213.02 & 272.7 & 263.05 \\
\hline 251.047 & 212.86 & 272.94 & 263.17 \\
\hline 251.5477 & 212.63 & 272.57 & 263.28 \\
\hline 252.0485 & 212.64 & 272.99 & 263.48 \\
\hline 252.5482 & 212.5 & 272.8 & 263.31 \\
\hline 253.048 & 212.52 & 272.6 & 263.43 \\
\hline 253.5488 & 212.47 & 272.86 & 263.48 \\
\hline 254.0477 & 212.51 & 273.37 & 263.86 \\
\hline 254.5475 & 212.51 & 272.88 & 263.64 \\
\hline 255.0473 & 212.59 & 272.82 & 263.3 \\
\hline 255.5482 & 212.82 & 273.12 & 263.78 \\
\hline 256.048 & 212.79 & 273.04 & 263.44 \\
\hline 256.5477 & 212.42 & 273.69 & 263.06 \\
\hline 257.0467 & 212.33 & 273.43 & 250.72 \\
\hline
\end{tabular}

\section{Run 4 (pure steam case)}

run_no
4
additive
none
experiment_date
$05 / 20 / 2005$
researcher
Roly Simangunsong
cell_radius
3.688
cell_length
68.58
tcell
54
Cs
0.21




Co
0.5
Cw
1
dens_sand
167
dens_oil
62
dens_water
62.4
por
0.411690173
Soi
0.741148716
Sor
0.415
res_heat_capacity
34.33456123
heat_loss_coeff
10
0.093686164
PI
7.8
Oil_visc
0.16516512
Lv_enthalphy
0
porvol
1162.444515
void_length
2.58
Hv_enthalphy
0
0.2
iw
5.5
44.8

10 


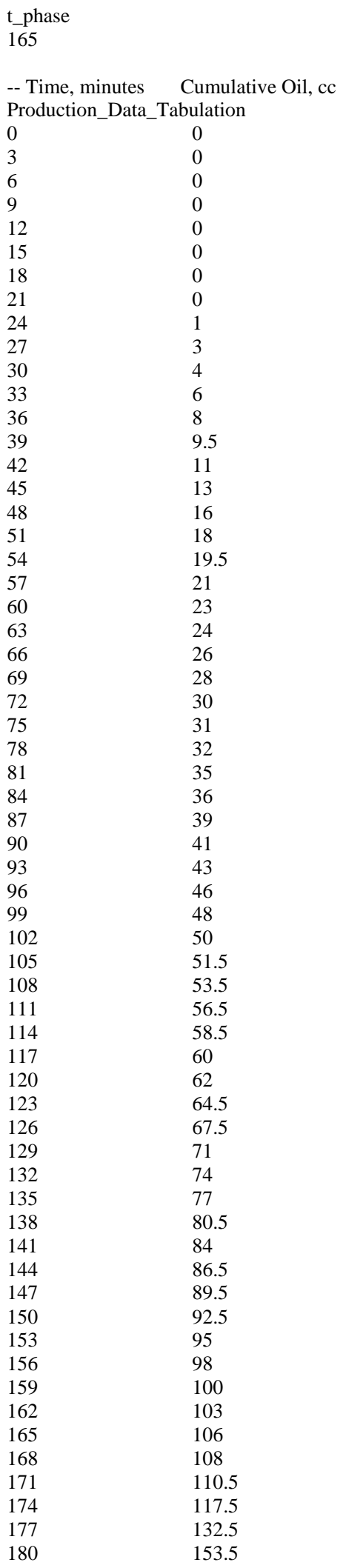




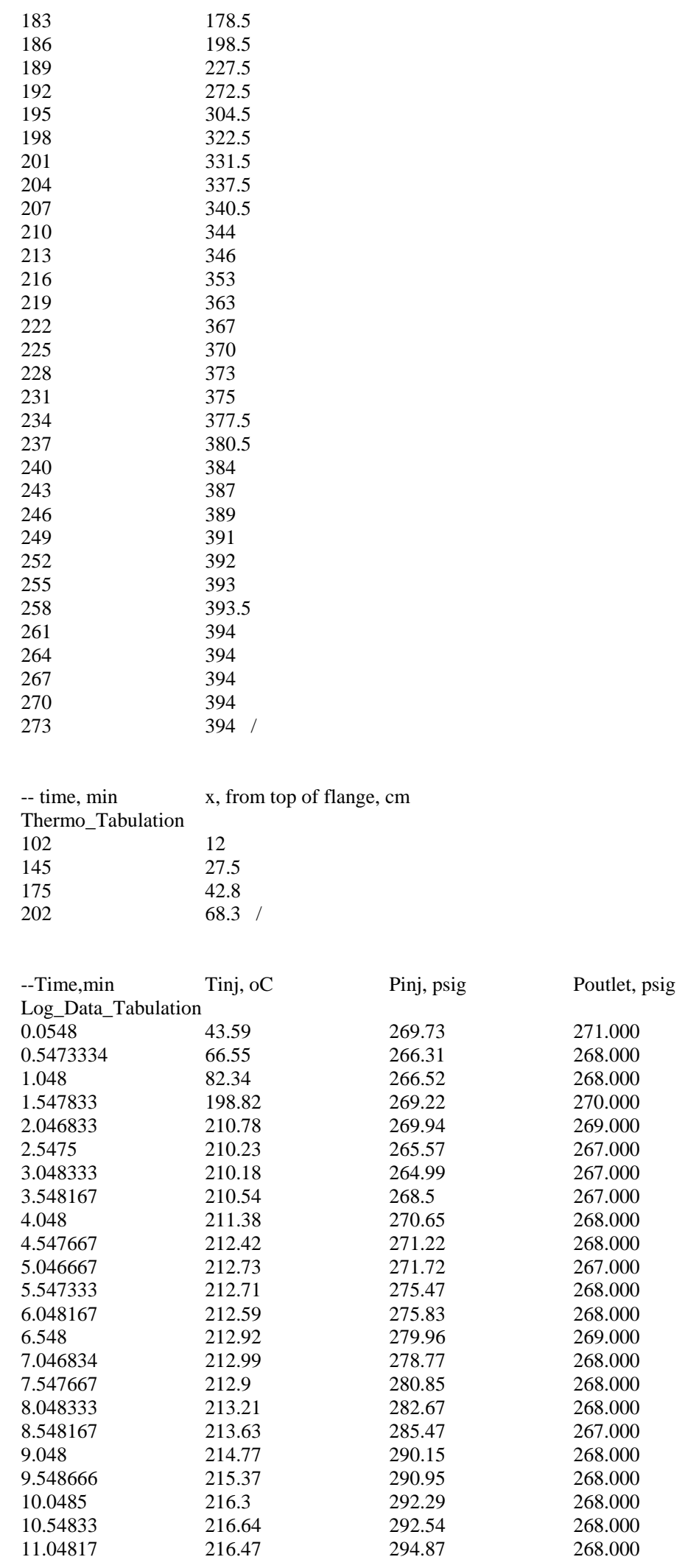




\begin{tabular}{|c|c|c|c|}
\hline 11.548 & 215.65 & 295.64 & 268.000 \\
\hline 12.04783 & 215.38 & 295.21 & 268.000 \\
\hline 12.54667 & 215.46 & 297.05 & 268.000 \\
\hline 13.0475 & 215.76 & 298.28 & 268.000 \\
\hline 13.54817 & 216.09 & 300.71 & 268.000 \\
\hline 14.048 & 216.41 & 302.67 & 268.000 \\
\hline 14.547 & 216.76 & 305.39 & 268.000 \\
\hline 15.04767 & 217.12 & 307.51 & 268.000 \\
\hline 15.5475 & 217.39 & 309.1 & 268.000 \\
\hline 16.04817 & 217.71 & 310.31 & 268.000 \\
\hline 16.548 & 218.04 & 313.22 & 268.000 \\
\hline 17.04883 & 218.42 & 315.49 & 268.000 \\
\hline 17.54767 & 218.87 & 318.7 & 268.000 \\
\hline 18.0485 & 219.39 & 321.22 & 265.000 \\
\hline 18.54833 & 219.48 & 322 & 268.000 \\
\hline 19.049 & 219.39 & 321.34 & 265.000 \\
\hline 19.547 & 219.4 & 322.08 & 269.000 \\
\hline 20.04767 & 219.61 & 323.2 & 269.000 \\
\hline 20.5475 & 219.88 & 324.41 & 266.000 \\
\hline 21.04833 & 220.05 & 325.87 & 268.000 \\
\hline 21.54817 & 220.23 & 327.75 & 269.000 \\
\hline 22.04883 & 220.45 & 328.88 & 269.000 \\
\hline 22.54683 & 220.67 & 329.97 & 269.000 \\
\hline 23.0475 & 220.85 & 331.58 & 269.000 \\
\hline 23.54833 & 221.03 & 332.53 & 269.000 \\
\hline 24.049 & 221.3 & 334.21 & 269.000 \\
\hline 24.547 & 221.43 & 334.75 & 245.000 \\
\hline 25.04783 & 221.46 & 334.12 & 269.000 \\
\hline 25.54767 & 221.34 & 333.77 & 266.000 \\
\hline 26.04833 & 221.47 & 335.11 & 269.000 \\
\hline 26.54817 & 221.62 & 336.09 & 269.000 \\
\hline 27.048 & 221.69 & 336.51 & 268.000 \\
\hline 27.54783 & 221.82 & 337.51 & 269.000 \\
\hline 28.04767 & 221.95 & 338.25 & 269.000 \\
\hline 28.54833 & 221.95 & 338.59 & 269.000 \\
\hline 29.04817 & 222.06 & 338.61 & 269.000 \\
\hline 29.54717 & 222.08 & 338.43 & 269.000 \\
\hline 30.04783 & 222.08 & 338.02 & 268.000 \\
\hline 30.54767 & 222.07 & 338.86 & 267.000 \\
\hline 31.04833 & 222.05 & 338.65 & 269.000 \\
\hline 31.54817 & 222.11 & 339.12 & 269.000 \\
\hline 32.048 & 222.24 & 339.15 & 268.000 \\
\hline 32.54783 & 222.27 & 339.2 & 268.000 \\
\hline 33.04766 & 222.38 & 340.46 & 268.000 \\
\hline 33.54833 & 222.44 & 341.39 & 263.000 \\
\hline 34.04817 & 222.51 & 341.66 & 269.000 \\
\hline 34.54717 & 222.57 & 341.63 & 269.000 \\
\hline 35.04784 & 222.64 & 341.94 & 268.000 \\
\hline 35.54767 & 222.63 & 342.69 & 268.000 \\
\hline 36.0485 & 222.79 & 343.45 & 268.000 \\
\hline 36.54833 & 222.88 & 344.03 & 269.000 \\
\hline 37.048 & 222.91 & 343.16 & 266.000 \\
\hline 37.54784 & 222.87 & 343.65 & 268.000 \\
\hline 38.04767 & 222.77 & 343.07 & 268.000 \\
\hline 38.5475 & 222.84 & 344.32 & 266.000 \\
\hline 39.04833 & 222.92 & 344.47 & 258.000 \\
\hline 39.54816 & 222.92 & 344.47 & 262.000 \\
\hline 40.048 & 223.03 & 344.07 & 261.000 \\
\hline 40.54683 & 222.9 & 343.34 & 264.000 \\
\hline 41.0475 & 222.89 & 344.05 & 262.000 \\
\hline 41.54833 & 222.95 & 344.59 & 262.000 \\
\hline 42.04816 & 222.98 & 344.8 & 263.000 \\
\hline 42.547 & 223.04 & 345.3 & 259.000 \\
\hline 43.04784 & 223.02 & 345.23 & 254.000 \\
\hline 43.54767 & 222.95 & 343.45 & 261.000 \\
\hline 44.04917 & 222.9 & 343.5 & 258.000 \\
\hline
\end{tabular}




\begin{tabular}{|c|c|c|c|}
\hline 44.54717 & 222.89 & 343.79 & 262.000 \\
\hline 45.048 & 222.89 & 343.82 & 262.000 \\
\hline 45.54784 & 222.93 & 344.52 & 262.000 \\
\hline 46.04767 & 223.09 & 345.03 & 265.000 \\
\hline 46.5475 & 223.14 & 345.45 & 266.000 \\
\hline 47.04816 & 223.18 & 346.39 & 261.000 \\
\hline 47.548 & 223.19 & 346.17 & 257.000 \\
\hline 48.04784 & 223.21 & 346.19 & 248.000 \\
\hline 48.54767 & 223.19 & 344.94 & 259.000 \\
\hline 49.0475 & 223 & 343.6 & 258.000 \\
\hline 49.54816 & 222.91 & 343.88 & 258.000 \\
\hline 50.049 & 222.87 & 343.65 & 264.000 \\
\hline 50.54784 & 222.88 & 344.33 & 265.000 \\
\hline 51.04683 & 222.9 & 344.27 & 265.000 \\
\hline 51.5475 & 223.13 & 345.24 & 263.000 \\
\hline 52.04816 & 223.15 & 345.54 & 263.000 \\
\hline 52.548 & 223.14 & 345.76 & 269.000 \\
\hline 53.047 & 223.16 & 346.16 & 263.000 \\
\hline 53.54767 & 223.25 & 346.77 & 266.000 \\
\hline 54.0485 & 223.3 & 347.12 & 269.000 \\
\hline 54.54833 & 223.34 & 347.51 & 260.080 \\
\hline 55.04717 & 223.36 & 347.48 & 264.000 \\
\hline 55.54784 & 223.29 & 347.08 & 265.000 \\
\hline 56.04866 & 223.28 & 347.13 & 258.000 \\
\hline 56.5475 & 223.24 & 346.93 & 263.000 \\
\hline 57.04734 & 223.17 & 346.06 & 268.000 \\
\hline 57.54816 & 223.27 & 346.34 & 266.000 \\
\hline 58.048 & 223.23 & 346.5 & 268.000 \\
\hline 58.54767 & 223.27 & 346.7 & 267.000 \\
\hline 59.0475 & 223.18 & 346.58 & 267.000 \\
\hline 59.54833 & 223.33 & 347.16 & 257.000 \\
\hline 60.04816 & 223.19 & 345.51 & 263.000 \\
\hline 60.548 & 223.12 & 345.3 & 262.000 \\
\hline 61.04683 & 223.05 & 345.01 & 264.000 \\
\hline 61.54767 & 223.05 & 345.2 & 265.000 \\
\hline 62.04833 & 223 & 345.33 & 263.000 \\
\hline 62.54816 & 223.09 & 345.28 & 263.000 \\
\hline 63.047 & 223.13 & 345.71 & 263.000 \\
\hline 63.54784 & 223.11 & 345.68 & 265.000 \\
\hline 64.04767 & 223.17 & 346.03 & 266.000 \\
\hline 64.54933 & 223.24 & 346.37 & 269.000 \\
\hline 65.04733 & 223.31 & 346.44 & 258.000 \\
\hline 65.548 & 223.17 & 345.66 & 259.000 \\
\hline 66.04867 & 223.09 & 345.18 & 256.000 \\
\hline 66.54667 & 223.03 & 344.63 & 265.000 \\
\hline 67.0475 & 223 & 344.48 & 268.000 \\
\hline 67.54816 & 223.06 & 344.98 & 265.000 \\
\hline 68.048 & 223.08 & 345.11 & 263.000 \\
\hline 68.547 & 223.11 & 345.15 & 264.000 \\
\hline 69.04766 & 223.12 & 345.75 & 266.000 \\
\hline 69.54833 & 223.21 & 345.87 & 268.000 \\
\hline 70.04817 & 223.26 & 346.09 & 267.000 \\
\hline 70.548 & 223.21 & 345.87 & 265.000 \\
\hline 71.04784 & 223.18 & 346 & 268.000 \\
\hline 71.54684 & 223.22 & 346.13 & 269.000 \\
\hline 72.0475 & 223.25 & 346.57 & 267.000 \\
\hline 72.54833 & 223.29 & 347.14 & 263.000 \\
\hline 73.04716 & 223.29 & 346.58 & 263.000 \\
\hline 73.54784 & 223.37 & 346.53 & 261.000 \\
\hline 74.04867 & 223.24 & 346.25 & 260.000 \\
\hline 74.5475 & 223.21 & 346.15 & 263.000 \\
\hline 75.04833 & 223.19 & 346.05 & 265.000 \\
\hline 75.54817 & 223.23 & 345.98 & 258.000 \\
\hline 76.048 & 223.3 & 346.11 & 258.000 \\
\hline 76.54684 & 223.2 & 345.91 & 261.000 \\
\hline 77.0475 & 223.15 & 345.81 & 263.000 \\
\hline
\end{tabular}




\begin{tabular}{|c|c|c|c|}
\hline 77.54833 & 223.18 & 346.15 & 266.000 \\
\hline 78.04817 & 223.26 & 346.06 & 260.000 \\
\hline 78.547 & 223.21 & 345.68 & 251.000 \\
\hline 79.04784 & 223.12 & 345.04 & 253.000 \\
\hline 79.54766 & 223.09 & 345.09 & 252.000 \\
\hline 80.04833 & 223.16 & 344.7 & 251.000 \\
\hline 80.54817 & 223.04 & 344.43 & 267.000 \\
\hline 81.048 & 222.98 & 344.57 & 263.000 \\
\hline 81.54684 & 222.97 & 344.64 & 265.000 \\
\hline 82.04766 & 223.1 & 344.85 & 264.000 \\
\hline 82.54833 & 223.11 & 344.93 & 261.000 \\
\hline 83.04817 & 223.1 & 345.44 & 263.000 \\
\hline 83.548 & 223.12 & 345.79 & 263.000 \\
\hline 84.04784 & 223.17 & 345.89 & 262.000 \\
\hline 84.54666 & 223.19 & 346.15 & 260.000 \\
\hline 85.04933 & 223.26 & 346.15 & 261.000 \\
\hline 85.54817 & 223.23 & 346.36 & 264.000 \\
\hline 86.048 & 223.25 & 346.51 & 262.000 \\
\hline 86.54784 & 223.24 & 346.74 & 259.000 \\
\hline 87.04684 & 223.24 & 346.56 & 259.000 \\
\hline 87.5475 & 223.29 & 346.5 & 261.000 \\
\hline 88.04817 & 223.33 & 347.03 & 262.000 \\
\hline 88.548 & 223.29 & 347.04 & 263.000 \\
\hline 89.047 & 223.26 & 346.36 & 263.000 \\
\hline 89.54766 & 223.3 & 347.23 & 260.000 \\
\hline 90.0485 & 223.37 & 347.68 & 261.000 \\
\hline 90.54833 & 223.53 & 348.78 & 268.000 \\
\hline 91.048 & 223.62 & 349.68 & 266.000 \\
\hline 91.547 & 223.73 & 350.27 & 265.000 \\
\hline 92.04766 & 223.77 & 350.5 & 263.000 \\
\hline 92.5485 & 223.88 & 351.2 & 263.000 \\
\hline 93.04833 & 223.97 & 351.3 & 262.000 \\
\hline 93.54716 & 223.9 & 350.61 & 253.000 \\
\hline 94.048 & 223.79 & 350.21 & 255.000 \\
\hline 94.54766 & 223.72 & 349.68 & 262.000 \\
\hline 95.04666 & 223.69 & 349.69 & 263.000 \\
\hline 95.54733 & 223.69 & 349.6 & 260.000 \\
\hline 96.04817 & 223.73 & 349.62 & 265.000 \\
\hline 96.548 & 223.73 & 349.69 & 264.000 \\
\hline 97.04784 & 223.68 & 349.89 & 265.000 \\
\hline 97.54766 & 223.71 & 350.01 & 266.000 \\
\hline 98.04833 & 223.77 & 349.99 & 266.000 \\
\hline 98.54817 & 223.75 & 350.06 & 263.000 \\
\hline 99.048 & 223.75 & 350.01 & 262.000 \\
\hline 99.54784 & 223.68 & 349.59 & 262.000 \\
\hline 100.0477 & 223.67 & 349.63 & 262.000 \\
\hline 100.5483 & 223.69 & 349.48 & 266.000 \\
\hline 101.0482 & 223.62 & 349.23 & 262.000 \\
\hline 101.548 & 223.6 & 349.16 & 268.000 \\
\hline 102.0478 & 223.62 & 349.2 & 262.000 \\
\hline 102.5477 & 223.6 & 349.03 & 266.000 \\
\hline 103.0483 & 223.62 & 349.32 & 264.000 \\
\hline 103.5492 & 223.59 & 348.91 & 258.000 \\
\hline 104.0472 & 223.61 & 349.14 & 261.000 \\
\hline 104.5478 & 223.57 & 348.84 & 262.000 \\
\hline 105.0477 & 223.59 & 348.82 & 263.000 \\
\hline 105.5493 & 223.57 & 348.84 & 262.000 \\
\hline 106.0473 & 223.59 & 349.24 & 264.000 \\
\hline 106.548 & 223.56 & 348.82 & 258.000 \\
\hline 107.0478 & 223.54 & 348.84 & 263.000 \\
\hline 107.5468 & 223.54 & 348.86 & 258.000 \\
\hline 108.0475 & 223.58 & 348.73 & 258.000 \\
\hline 108.5483 & 223.55 & 348.44 & 259.000 \\
\hline 109.048 & 223.49 & 348.53 & 260.000 \\
\hline 109.547 & 223.57 & 348.31 & 264.000 \\
\hline 110.0487 & 223.58 & 348.43 & 267.000 \\
\hline
\end{tabular}




\begin{tabular}{|c|c|c|c|}
\hline 110.5475 & 223.55 & 348.52 & 262.000 \\
\hline 111.0483 & 223.53 & 348.55 & 256.000 \\
\hline 111.5482 & 223.44 & 348.33 & 257.000 \\
\hline 112.047 & 223.48 & 348.08 & 260.000 \\
\hline 112.5477 & 223.5 & 347.61 & 253.000 \\
\hline 113.0475 & 223.38 & 346.95 & 259.000 \\
\hline 113.5483 & 223.32 & 347.3 & 261.000 \\
\hline 114.0472 & 223.29 & 347 & 262.000 \\
\hline 114.548 & 223.33 & 346.85 & 262.000 \\
\hline 115.0478 & 223.31 & 347.1 & 266.000 \\
\hline 115.5467 & 223.31 & 347.16 & 256.000 \\
\hline 116.0473 & 223.33 & 346.59 & 263.000 \\
\hline 116.5482 & 223.33 & 346.95 & 264.000 \\
\hline 117.048 & 223.35 & 347.35 & 260.000 \\
\hline 117.5468 & 223.35 & 347.11 & 249.000 \\
\hline 118.0477 & 223.32 & 346.52 & 255.000 \\
\hline 118.5483 & 223.21 & 345.97 & 259.000 \\
\hline 119.0482 & 223.3 & 346.38 & 252.000 \\
\hline 119.5472 & 223.3 & 346.35 & 257.000 \\
\hline 120.0478 & 223.27 & 346.34 & 261.000 \\
\hline 120.5477 & 223.23 & 346.11 & 257.000 \\
\hline 121.0483 & 223.16 & 345.53 & 261.000 \\
\hline 121.5482 & 223.18 & 345.72 & 264.000 \\
\hline 122.0472 & 223.23 & 345.98 & 262.000 \\
\hline 122.5478 & 223.25 & 346 & 264.000 \\
\hline 123.0468 & 223.16 & 345.6 & 266.000 \\
\hline 123.5475 & 223.18 & 346.06 & 262.000 \\
\hline 124.0482 & 223.22 & 345.94 & 260.000 \\
\hline 124.548 & 223.22 & 345.98 & 263.000 \\
\hline 125.0478 & 223.24 & 345.82 & 263.000 \\
\hline 125.5477 & 223.19 & 346.08 & 259.000 \\
\hline 126.0475 & 223.17 & 345.63 & 261.000 \\
\hline 126.5483 & 223.15 & 345.79 & 264.000 \\
\hline 127.048 & 223.15 & 345.72 & 263.000 \\
\hline 127.547 & 223.15 & 345.54 & 264.000 \\
\hline 128.0477 & 223.19 & 345.82 & 251.000 \\
\hline 128.5485 & 223.1 & 345.02 & 258.000 \\
\hline 129.0483 & 223.03 & 344.84 & 257.000 \\
\hline 129.5482 & 222.94 & 344.35 & 259.000 \\
\hline 130.048 & 222.96 & 344.17 & 262.000 \\
\hline 130.5477 & 222.96 & 343.94 & 254.000 \\
\hline 131.0475 & 222.82 & 342.88 & 265.000 \\
\hline 131.5483 & 222.8 & 342.64 & 264.000 \\
\hline 132.0482 & 222.75 & 342.91 & 261.000 \\
\hline 132.547 & 222.76 & 342.96 & 267.000 \\
\hline 133.0478 & 222.82 & 343.11 & 266.000 \\
\hline 133.5477 & 222.89 & 343.5 & 264.000 \\
\hline 134.0483 & 222.91 & 343.66 & 266.000 \\
\hline 134.5482 & 222.87 & 343.24 & 265.000 \\
\hline 135.047 & 222.87 & 343.17 & 262.000 \\
\hline 135.5478 & 222.86 & 343.25 & 264.000 \\
\hline 136.0485 & 222.78 & 343.26 & 264.000 \\
\hline 136.5483 & 222.73 & 343.1 & 267.000 \\
\hline 137.0473 & 222.75 & 342.88 & 269.000 \\
\hline 137.548 & 222.81 & 343.28 & 268.000 \\
\hline 138.0468 & 222.88 & 343.34 & 264.000 \\
\hline 138.5477 & 222.91 & 343.48 & 268.000 \\
\hline 139.0483 & 222.93 & 343.78 & 262.000 \\
\hline 139.5482 & 222.89 & 343.94 & 262.000 \\
\hline 140.048 & 222.84 & 343.43 & 253.000 \\
\hline 140.5478 & 222.81 & 342.67 & 260.000 \\
\hline 141.0468 & 222.74 & 341.97 & 261.000 \\
\hline 141.5483 & 222.61 & 341.76 & 261.000 \\
\hline 142.0482 & 222.56 & 341.36 & 236.000 \\
\hline 142.548 & 222.32 & 338.02 & 254.000 \\
\hline 143.0478 & 222.22 & 337.6 & 256.000 \\
\hline
\end{tabular}




\begin{tabular}{|c|c|c|c|}
\hline 143.5477 & 222.11 & 337.3 & 260.000 \\
\hline 144.0493 & 222.06 & 336.99 & 264.000 \\
\hline 144.5473 & 221.97 & 336.95 & 267.000 \\
\hline 145.048 & 221.93 & 336.87 & 266.000 \\
\hline 145.5488 & 221.93 & 337.19 & 267.000 \\
\hline 146.0468 & 221.91 & 337.37 & 265.000 \\
\hline 146.5475 & 222.06 & 337.16 & 262.000 \\
\hline 147.0483 & 222.06 & 337.28 & 264.000 \\
\hline 147.5482 & 222.02 & 337.4 & 269.000 \\
\hline 148.048 & 222.11 & 337.33 & 269.000 \\
\hline 148.5477 & 222.1 & 337.28 & 268.000 \\
\hline 149.0475 & 222.04 & 337.34 & 269.000 \\
\hline 149.5492 & 222.01 & 337.42 & 268.000 \\
\hline 150.049 & 221.97 & 337.13 & 268.000 \\
\hline 150.548 & 221.94 & 337.25 & 269.000 \\
\hline 151.0478 & 222.04 & 337.11 & 264.000 \\
\hline 151.5477 & 221.97 & 336.75 & 268.000 \\
\hline 152.0483 & 221.92 & 336.18 & 269.000 \\
\hline 152.5482 & 221.87 & 336.78 & 267.000 \\
\hline 153.047 & 221.81 & 336.68 & 269.000 \\
\hline 153.5478 & 221.83 & 336.52 & 268.000 \\
\hline 154.0467 & 221.83 & 336.34 & 268.000 \\
\hline 154.5483 & 221.85 & 335.95 & 268.000 \\
\hline 155.0482 & 221.8 & 335.61 & 268.000 \\
\hline 155.547 & 221.72 & 335.46 & 267.000 \\
\hline 156.0478 & 221.76 & 335.22 & 268.000 \\
\hline 156.5477 & 221.69 & 334.78 & 267.000 \\
\hline 157.0493 & 221.6 & 334.59 & 268.000 \\
\hline 157.5473 & 221.57 & 334.32 & 268.000 \\
\hline 158.048 & 221.55 & 333.98 & 268.000 \\
\hline 158.5488 & 221.51 & 333.67 & 266.000 \\
\hline 159.0468 & 221.44 & 333.56 & 268.000 \\
\hline 159.5475 & 221.42 & 333.03 & 267.000 \\
\hline 160.0482 & 221.42 & 332.77 & 268.000 \\
\hline 160.548 & 221.34 & 332.45 & 269.000 \\
\hline 161.0478 & 221.23 & 331.93 & 268.000 \\
\hline 161.5477 & 221.19 & 331.61 & 268.000 \\
\hline 162.0475 & 221.12 & 331.46 & 268.000 \\
\hline 162.5483 & 221.18 & 330.79 & 268.000 \\
\hline 163.048 & 221.19 & 331.56 & 269.000 \\
\hline 163.5478 & 221.18 & 330.56 & 268.000 \\
\hline 164.0477 & 221.11 & 330.08 & 268.000 \\
\hline 164.5467 & 221.04 & 330.24 & 269.000 \\
\hline 165.0473 & 220.93 & 329.94 & 269.000 \\
\hline 165.5482 & 221.11 & 329.63 & 268.000 \\
\hline 166.048 & 220.98 & 329.18 & 268.000 \\
\hline 166.5468 & 220.84 & 328.75 & 268.000 \\
\hline 167.0475 & 220.75 & 328.3 & 267.000 \\
\hline 167.5483 & 220.77 & 327.45 & 267.000 \\
\hline 168.0482 & 220.75 & 327.37 & 268.000 \\
\hline 168.547 & 220.65 & 326.92 & 267.000 \\
\hline 169.0478 & 220.59 & 326.56 & 269.000 \\
\hline 169.5477 & 220.6 & 326.24 & 265.000 \\
\hline 170.0492 & 220.43 & 325.85 & 263.000 \\
\hline 170.5482 & 220.36 & 324.83 & 267.000 \\
\hline 171.048 & 220.26 & 323.28 & 262.000 \\
\hline 171.5478 & 220.21 & 322.8 & 265.000 \\
\hline 172.0477 & 219.99 & 321.46 & 267.000 \\
\hline 172.5493 & 219.96 & 321.89 & 267.000 \\
\hline 173.0473 & 219.9 & 320.86 & 267.000 \\
\hline 173.548 & 219.87 & 320.31 & 268.000 \\
\hline 174.0487 & 219.82 & 319.49 & 267.000 \\
\hline 174.5467 & 219.83 & 318.91 & 267.000 \\
\hline 175.0475 & 219.73 & 318.41 & 268.000 \\
\hline 175.5482 & 219.53 & 318.19 & 269.000 \\
\hline 176.048 & 219.3 & 317.63 & 268.000 \\
\hline
\end{tabular}




\begin{tabular}{|c|c|c|c|}
\hline 176.547 & 219.21 & 316.98 & 269.000 \\
\hline 177.0477 & 219.3 & 316.26 & 268.000 \\
\hline 177.5483 & 219.29 & 315.43 & 266.000 \\
\hline 178.0482 & 219.29 & 314.81 & 263.000 \\
\hline 178.5472 & 219.2 & 313.44 & 258.000 \\
\hline 179.0478 & 219.22 & 311.92 & 257.000 \\
\hline 179.5487 & 219.36 & 308.95 & 264.000 \\
\hline 180.0485 & 219.51 & 307.03 & 267.000 \\
\hline 180.5483 & 218.92 & 305.88 & 269.000 \\
\hline 181.0472 & 218.69 & 305.01 & 268.000 \\
\hline 181.5478 & 218.33 & 304.42 & 268.000 \\
\hline 182.0477 & 217.92 & 303.88 & 268.000 \\
\hline 182.5467 & 218.03 & 303.31 & 268.000 \\
\hline 183.0473 & 217.87 & 303.07 & 268.000 \\
\hline 183.5482 & 217.66 & 301.96 & 268.000 \\
\hline 184.048 & 217.46 & 301.34 & 268.000 \\
\hline 184.5468 & 217.3 & 300.47 & 268.000 \\
\hline 185.0475 & 217.12 & 300.31 & 268.000 \\
\hline 185.5483 & 217.11 & 299.6 & 268.000 \\
\hline 186.0482 & 217.45 & 299.54 & 268.000 \\
\hline 186.547 & 217.5 & 298.88 & 267.000 \\
\hline 187.0478 & 217.35 & 298.05 & 265.000 \\
\hline 187.5485 & 216.92 & 296.97 & 266.000 \\
\hline 188.0483 & 216.72 & 295.98 & 266.000 \\
\hline 188.5472 & 216.51 & 294.91 & 268.000 \\
\hline 189.048 & 216.48 & 294.26 & 264.000 \\
\hline 189.5478 & 216.39 & 293.53 & 263.000 \\
\hline 190.0485 & 216.37 & 292.61 & 263.000 \\
\hline 190.5483 & 216.11 & 291.66 & 263.000 \\
\hline 191.0492 & 215.93 & 290.57 & 267.000 \\
\hline 191.547 & 215.75 & 290.03 & 268.000 \\
\hline 192.0478 & 215.74 & 289.33 & 268.000 \\
\hline 192.5477 & 215.56 & 288.95 & 268.000 \\
\hline 193.0493 & 215.99 & 288.61 & 268.000 \\
\hline 193.5473 & 216.16 & 288.18 & 268.000 \\
\hline 194.048 & 216.09 & 288.01 & 268.000 \\
\hline 194.5478 & 215.84 & 287.85 & 267.000 \\
\hline 195.0468 & 215.41 & 287.61 & 266.000 \\
\hline 195.5483 & 214.7 & 286.94 & 266.000 \\
\hline 196.0473 & 214.47 & 286.13 & 266.000 \\
\hline 196.548 & 214.44 & 285.73 & 266.000 \\
\hline 197.0478 & 214.47 & 285.28 & 266.000 \\
\hline 197.5468 & 214.49 & 284.94 & 266.000 \\
\hline 198.0485 & 214.3 & 284.78 & 266.000 \\
\hline 198.5483 & 214.28 & 284.17 & 266.000 \\
\hline 199.048 & 214.54 & 283.58 & 266.000 \\
\hline 199.5478 & 214.45 & 283.24 & 266.000 \\
\hline 200.0477 & 214.29 & 282.92 & 266.000 \\
\hline 200.5475 & 214.31 & 282.74 & 266.000 \\
\hline 201.0483 & 214.15 & 282.32 & 266.000 \\
\hline 201.5472 & 214.08 & 282.25 & 266.000 \\
\hline 202.048 & 214.05 & 281.76 & 266.000 \\
\hline 202.5468 & 214 & 281.47 & 266.000 \\
\hline 203.0475 & 213.95 & 281.38 & 266.000 \\
\hline 203.5483 & 213.92 & 281.05 & 266.000 \\
\hline 204.0482 & 214.03 & 281.09 & 266.000 \\
\hline 204.548 & 213.89 & 280.94 & 266.000 \\
\hline 205.0478 & 214.03 & 280.54 & 266.000 \\
\hline 205.5467 & 214.27 & 280.32 & 266.000 \\
\hline 206.0483 & 214.14 & 280.04 & 266.000 \\
\hline 206.5472 & 213.93 & 279.65 & 266.000 \\
\hline 207.048 & 213.67 & 279.47 & 266.000 \\
\hline 207.5478 & 213.69 & 279.48 & 266.000 \\
\hline 208.0467 & 213.57 & 279.2 & 265.000 \\
\hline 208.5483 & 213.52 & 278.95 & 265.000 \\
\hline 209.0482 & 213.86 & 278.84 & 266.000 \\
\hline
\end{tabular}




\begin{tabular}{|c|c|c|c|}
\hline 209.548 & 214.13 & 278.3 & 266.000 \\
\hline 210.0478 & 213.94 & 277.83 & 266.000 \\
\hline 210.5477 & 213.65 & 277.82 & 266.000 \\
\hline 211.0475 & 213.55 & 277.67 & 266.000 \\
\hline 211.5482 & 213.37 & 277.51 & 266.000 \\
\hline 212.0472 & 213.3 & 277.13 & 264.000 \\
\hline 212.5478 & 213.17 & 276.74 & 266.000 \\
\hline 213.0477 & 213.2 & 276.08 & 266.000 \\
\hline 213.5483 & 213.06 & 275.81 & 262.000 \\
\hline 214.0473 & 213.3 & 275.35 & 262.000 \\
\hline 214.549 & 213.07 & 274.48 & 261.000 \\
\hline 215.047 & 213.27 & 273.78 & 261.000 \\
\hline 215.5477 & 213.08 & 273.31 & 261.000 \\
\hline 216.0485 & 212.65 & 272.99 & 262.000 \\
\hline 216.5483 & 212.63 & 272.28 & 261.000 \\
\hline 217.048 & 212.76 & 271.66 & 262.000 \\
\hline 217.5478 & 212.75 & 271.07 & 260.000 \\
\hline 218.0468 & 212.64 & 270.61 & 260.000 \\
\hline 218.5475 & 212.56 & 269.96 & 262.000 \\
\hline 219.0483 & 212.46 & 269.8 & 260.000 \\
\hline 219.5482 & 212.3 & 269.57 & 260.000 \\
\hline 220.0488 & 212.43 & 269.21 & 261.000 \\
\hline 220.5477 & 212.34 & 268.83 & 259.000 \\
\hline 221.0475 & 212.34 & 268.59 & 260.000 \\
\hline 221.5483 & 212.13 & 268.58 & 260.000 \\
\hline 222.0482 & 212.08 & 268.62 & 260.000 \\
\hline 222.547 & 212.05 & 268.48 & 261.000 \\
\hline 223.0478 & 212.02 & 268.72 & 260.000 \\
\hline 223.5477 & 212.07 & 268.7 & 261.000 \\
\hline 224.0492 & 211.97 & 268.74 & 261.000 \\
\hline 224.5472 & 211.87 & 268.5 & 260.000 \\
\hline 225.048 & 212.16 & 268.58 & 263.000 \\
\hline 225.5478 & 212.04 & 269.02 & 260.000 \\
\hline 226.0477 & 212.02 & 269.25 & 260.000 \\
\hline 226.5483 & 211.99 & 269.08 & 263.000 \\
\hline 227.0482 & 212.19 & 269.51 & 260.000 \\
\hline 227.548 & 212.27 & 269.77 & 262.000 \\
\hline 228.0478 & 212.23 & 269.71 & 261.000 \\
\hline 228.5467 & 212.29 & 269.83 & 261.000 \\
\hline 229.0475 & 212.33 & 269.2 & 261.000 \\
\hline 229.5482 & 212.07 & 269.34 & 260.000 \\
\hline 230.048 & 212.1 & 270.07 & 260.000 \\
\hline 230.5488 & 211.98 & 269.25 & 261.000 \\
\hline 231.0477 & 211.95 & 269.88 & 263.000 \\
\hline 231.5483 & 212.15 & 270.81 & 263.000 \\
\hline 232.0482 & 212.51 & 270.73 & 262.000 \\
\hline 232.548 & 212.39 & 271.55 & 262.000 \\
\hline 233.0478 & 212.72 & 271.04 & 262.000 \\
\hline 233.5477 & 213.06 & 271.02 & 263.000 \\
\hline 234.0485 & 213.06 & 270.97 & 262.000 \\
\hline 234.5483 & 212.89 & 270.76 & 262.000 \\
\hline 235.048 & 212.84 & 271.26 & 262.000 \\
\hline 235.5478 & 212.73 & 271.1 & 262.000 \\
\hline 236.0477 & 212.76 & 271.42 & 263.000 \\
\hline 236.5475 & 212.76 & 271.8 & 263.000 \\
\hline 237.0483 & 212.71 & 271.98 & 263.000 \\
\hline 237.5482 & 212.69 & 272.11 & 263.000 \\
\hline 238.048 & 212.64 & 271.97 & 263.000 \\
\hline 238.5468 & 212.52 & 272.1 & 262.000 \\
\hline 239.0475 & 212.35 & 272.57 & 263.000 \\
\hline 239.5483 & 212.35 & 272.08 & 262.000 \\
\hline 240.0482 & 212.46 & 271.81 & 262.000 \\
\hline 240.548 & 212.55 & 271.93 & 262.000 \\
\hline 241.0478 & 212.65 & 271.99 & 263.000 \\
\hline 241.5477 & 212.67 & 272.02 & 263.000 \\
\hline 242.0483 & 212.6 & 272.25 & 263.000 \\
\hline
\end{tabular}




\begin{tabular}{|c|c|c|c|}
\hline 242.5482 & 212.6 & 272.42 & 263.000 \\
\hline 243.048 & 212.79 & 272.66 & 263.000 \\
\hline 243.5478 & 212.72 & 272.38 & 263.000 \\
\hline 244.0485 & 212.9 & 272.77 & 263.000 \\
\hline 244.5475 & 212.91 & 272.5 & 264.000 \\
\hline 245.0482 & 212.78 & 273.2 & 264.000 \\
\hline 245.548 & 212.88 & 273.35 & 263.000 \\
\hline 246.0478 & 212.88 & 273.37 & 263.000 \\
\hline 246.5477 & 212.62 & 273.88 & 264.000 \\
\hline 247.0483 & 212.49 & 273.37 & 264.000 \\
\hline 247.5473 & 212.64 & 273.35 & 264.000 \\
\hline 248.048 & 212.72 & 273.39 & 263.000 \\
\hline 248.5478 & 212.81 & 273.42 & 264.000 \\
\hline 249.0467 & 212.83 & 273.24 & 263.000 \\
\hline 249.5475 & 212.87 & 273.73 & 263.000 \\
\hline 250.0492 & 213.07 & 273.27 & 263.000 \\
\hline 250.5472 & 213.02 & 272.7 & 263.000 \\
\hline 251.047 & 212.86 & 272.94 & 263.000 \\
\hline 251.5477 & 212.63 & 272.57 & 263.000 \\
\hline 252.0485 & 212.64 & 272.99 & 263.000 \\
\hline 252.5482 & 212.5 & 272.8 & 263.000 \\
\hline 253.048 & 212.52 & 272.6 & 263.000 \\
\hline 253.5488 & 212.47 & 272.86 & 263.000 \\
\hline 254.0477 & 212.51 & 273.37 & 264.000 \\
\hline 254.5475 & 212.51 & 272.88 & 264.000 \\
\hline 255.0473 & 212.59 & 272.82 & 263.000 \\
\hline 255.5482 & 212.82 & 273.12 & 264.000 \\
\hline 256.048 & 212.79 & 273.04 & 263.000 \\
\hline 256.5477 & 212.42 & 273.69 & 263.000 \\
\hline 257.0467 & 212.33 & 273.43 & 251.000 \\
\hline
\end{tabular}

\section{Run 5 (pure steam case)}

run_no
5
additive
none
experiment_date
$05 / 21 / 2005$
researcher
Roly Simangunsong
cell_radius
3.688
cell_length
68.58
tcell
54
Cs
0.21
Co
0.5
Cw
1
dens_sand


dens_oil

62

dens_water

62.4

por

0.411690173

Soi

0.741148716

Sor

0.415

Sgi

0.0936861640173443

Swc

0.16516512

porvol

1162.444515

void_length

2.58

o_w_ratio

0.2

iw

5.5

tfu_total

44.8

ratio

0

Hv_enthalphy

0

Lv_enthalphy

0

PI

7.8

Oil_visc

276

res_heat_capacity

34.33456123

heat_loss_coeff

10

t_phase

163

-- Time, minutes Cumulative Oil, cc Production_Data_Tabulation

0

3

0 


\begin{tabular}{|c|c|}
\hline 6 & 0 \\
\hline 9 & 0 \\
\hline 12 & 0 \\
\hline 15 & 0 \\
\hline 18 & 0 \\
\hline 21 & 0 \\
\hline 24 & 0 \\
\hline 27 & 0.5 \\
\hline 30 & 2.5 \\
\hline 33 & 6.5 \\
\hline 36 & 10.5 \\
\hline 39 & 14.5 \\
\hline 42 & 17.5 \\
\hline 45 & 21.5 \\
\hline 48 & 24.5 \\
\hline 51 & 26.5 \\
\hline 54 & 28.5 \\
\hline 57 & 30.5 \\
\hline 60 & 32.5 \\
\hline 63 & 35.5 \\
\hline 66 & 38.5 \\
\hline 69 & 42 \\
\hline 72 & 45.5 \\
\hline 75 & 48.5 \\
\hline 78 & 51.5 \\
\hline 81 & 54 \\
\hline 84 & 56.5 \\
\hline 87 & 58.5 \\
\hline 90 & 60.5 \\
\hline 93 & 62.5 \\
\hline 96 & 65 \\
\hline 99 & 66.5 \\
\hline 102 & 68.5 \\
\hline 105 & 70.5 \\
\hline 108 & 73 \\
\hline 111 & 75.5 \\
\hline 114 & 77.5 \\
\hline 117 & 79 \\
\hline 120 & 80.5 \\
\hline 123 & 82.5 \\
\hline 126 & 84.5 \\
\hline 129 & 86.5 \\
\hline 132 & 87.5 \\
\hline 135 & 89 \\
\hline 138 & 90 \\
\hline 141 & 91.5 \\
\hline 144 & 93.5 \\
\hline 147 & 95 \\
\hline 150 & 97 \\
\hline 153 & 98.5 \\
\hline 156 & 100 \\
\hline 159 & 102 \\
\hline 162 & 103.5 \\
\hline 165 & 105.5 \\
\hline 168 & 107 \\
\hline 171 & 108.5 \\
\hline 174 & 110.5 \\
\hline 177 & 112 \\
\hline 180 & 119 \\
\hline 183 & 140 \\
\hline 186 & 185 \\
\hline 189 & 217 \\
\hline 192 & 235 \\
\hline 195 & 249 \\
\hline 198 & 271 \\
\hline 201 & 289 \\
\hline
\end{tabular}




$\begin{array}{ll}204 & 304 \\ 207 & 318 \\ 210 & 326 \\ 213 & 330 \\ 216 & 334 \\ 219 & 337 \\ 222 & 340.5 \\ 225 & 350.5 \\ 228 & 365.5 \\ 231 & 373.5 \\ 234 & 378.5 \\ 237 & 382.5 \\ 240 & 384.5 \\ 243 & 386 \\ 246 & 387.5 \\ 249 & 388.5 \\ 252 & 389.5 \\ 255 & 390 \\ 258 & 390.5 \\ 261 & 390.5 \\ 264 & 390.5 \\ 267 & 390.5 \\ 270 & 390.5 \\ 273 & 390.5\end{array}$

$\begin{array}{ll}-- \text { time, min } & \mathrm{x} \text {, from top of flange, } \mathrm{cm} \\ \text { Thermo_Tabulation } & \\ 102 & 12 \\ 145 & 27.5 \\ 175 & 42.8 \\ 202 & 68.3 \quad\end{array}$

-- Time, minutes Pinj,psig Tinj, oC Log_Data_Tabulation

$\begin{array}{llll}0.054 & 58.3 & 267.23 & 268.56 \\ 0.5483333 & 75.46 & 267.75 & 269.04 \\ 1.048 & 71.54 & 267.1 & 268.39 \\ 1.547833 & 72.18 & 267.29 & 268.24 \\ 2.047667 & 85.05 & 267.75 & 268.41 \\ 2.5475 & 118.91 & 268.57 & 268.19 \\ 3.048333 & 207.16 & 272.45 & 269.01 \\ 3.549 & 210.39 & 266.01 & 266.39 \\ 4.048 & 210.18 & 264.28 & 265.04 \\ 4.547667 & 210.42 & 266.13 & 265.49 \\ 5.0475 & 210.71 & 268.86 & 267.07 \\ 5.548333 & 210.93 & 270.65 & 267.73 \\ 6.049 & 211.56 & 272.4 & 268.29 \\ 6.548 & 212.36 & 271.39 & 266.74 \\ 7.047833 & 212.56 & 269.19 & 263.86 \\ 7.547667 & 212.04 & 268.54 & 265.43 \\ 8.048333 & 211.67 & 270.82 & 266.67 \\ 8.548167 & 212.11 & 276.66 & 267.82 \\ 9.047 & 213.01 & 279.14 & 267.66 \\ 9.547833 & 213.95 & 281.32 & 267.67 \\ 10.04767 & 215.04 & 284.66 & 267.6 \\ 10.54833 & 215.59 & 287.83 & 267.55 \\ 11.04817 & 216.42 & 289.38 & 267.38 \\ 11.548 & 216.26 & 290.31 & 267.18 \\ 12.04783 & 216.63 & 292.74 & 267.07 \\ 12.54767 & 216.15 & 293.95 & 266.68 \\ 13.0475 & 215.72 & 295.21 & 266.84 \\ 13.54817 & 215.84 & 296.34 & 266.81 \\ 14.048 & 215.75 & 297.56 & 266.85 \\ 14.547 & 215.9 & 298.5 & \\ 15.04767 & 216.23 & 300.91 & \end{array}$




\begin{tabular}{|c|c|c|c|}
\hline 15.54833 & 216.44 & 302.25 & 266.73 \\
\hline 16.04817 & 216.69 & 304.47 & 266.7 \\
\hline 16.549 & 216.93 & 306.03 & 266.9 \\
\hline 17.047 & 217.22 & 308.07 & 267.15 \\
\hline 17.54767 & 217.53 & 308.99 & 258.96 \\
\hline 18.0485 & 217.85 & 311.35 & 258.35 \\
\hline 18.54833 & 218.05 & 313.19 & 258.26 \\
\hline 19.048 & 218.27 & 314.53 & 258.19 \\
\hline 19.547 & 218.53 & 316.14 & 258.78 \\
\hline 20.04767 & 218.78 & 317.97 & 258.77 \\
\hline 20.5475 & 219.01 & 319.51 & 258.11 \\
\hline 21.04833 & 219.28 & 321.06 & 259.16 \\
\hline 21.54817 & 219.52 & 322.73 & 259.04 \\
\hline 22.048 & 219.72 & 324.02 & 258.11 \\
\hline 22.54867 & 220.02 & 326.13 & 258.88 \\
\hline 23.0475 & 220.26 & 327.84 & 259.03 \\
\hline 23.54833 & 220.59 & 330.01 & 257.12 \\
\hline 24.04717 & 220.86 & 331.59 & 258.95 \\
\hline 24.548 & 221.1 & 333.14 & 259.15 \\
\hline 25.04683 & 221.36 & 334.96 & 259.04 \\
\hline 25.54767 & 221.65 & 336.36 & 259.05 \\
\hline 26.04833 & 221.8 & 338.17 & 257.75 \\
\hline 26.54817 & 222.03 & 339.05 & 259.04 \\
\hline 27.048 & 222.25 & 340.49 & 258.94 \\
\hline 27.54783 & 222.4 & 341.89 & 258.86 \\
\hline 28.04767 & 222.53 & 342.23 & 258.06 \\
\hline 28.54833 & 222.64 & 343.61 & 259.06 \\
\hline 29.04733 & 222.78 & 343.92 & 259.07 \\
\hline 29.548 & 222.86 & 344.36 & 259.01 \\
\hline 30.04783 & 222.77 & 344.38 & 258.96 \\
\hline 30.54767 & 222.87 & 345.2 & 258.88 \\
\hline 31.04833 & 223.04 & 346.11 & 258.97 \\
\hline 31.54733 & 223.14 & 346.89 & 258.8 \\
\hline 32.048 & 223.25 & 347.72 & 259.04 \\
\hline 32.54783 & 223.31 & 347.27 & 258.95 \\
\hline 33.04683 & 223.21 & 347.33 & 258.9 \\
\hline 33.5475 & 223.29 & 348.55 & 258.94 \\
\hline 34.04817 & 223.37 & 349.09 & 258.02 \\
\hline 34.548 & 223.47 & 349.54 & 258.92 \\
\hline 35.047 & 223.53 & 350.05 & 258.9 \\
\hline 35.54767 & 223.64 & 351.06 & 258.79 \\
\hline 36.0485 & 223.79 & 352.06 & 258.75 \\
\hline 36.54833 & 223.89 & 352.49 & 258.88 \\
\hline 37.048 & 223.9 & 352.87 & 258.85 \\
\hline 37.54784 & 224 & 353.13 & 258.86 \\
\hline 38.04767 & 223.99 & 353.44 & 258.87 \\
\hline 38.5475 & 224.1 & 353.91 & 258.84 \\
\hline 39.04833 & 224.15 & 354.25 & 258.8 \\
\hline 39.54816 & 224.17 & 354.41 & 258.75 \\
\hline 40.048 & 224.25 & 355.15 & 257.82 \\
\hline 40.54767 & 224.29 & 355.43 & 258.75 \\
\hline 41.04667 & 224.34 & 355.63 & 258.8 \\
\hline 41.54833 & 224.38 & 355.87 & 258.28 \\
\hline 42.04816 & 224.35 & 356.01 & 258.82 \\
\hline 42.548 & 224.29 & 355.6 & 258.87 \\
\hline 43.04784 & 224.32 & 355.98 & 258.66 \\
\hline 43.54767 & 224.4 & 356.06 & 258.78 \\
\hline 44.04833 & 224.33 & 354.98 & 258.76 \\
\hline 44.54816 & 224.29 & 356.88 & 258.7 \\
\hline 45.047 & 224.56 & 358.37 & 258.7 \\
\hline 45.54784 & 224.66 & 359.1 & 258.84 \\
\hline 46.04767 & 224.76 & 359.27 & 258.78 \\
\hline 46.54833 & 224.86 & 359.81 & 258.72 \\
\hline 47.04734 & 224.9 & 360.7 & 257.12 \\
\hline 47.548 & 225.03 & 361.45 & 258.76 \\
\hline 48.04784 & 225.1 & 361.23 & 258.3 \\
\hline
\end{tabular}




\begin{tabular}{|c|c|c|c|}
\hline 48.54667 & 225.27 & 363.17 & 258.78 \\
\hline 49.0475 & 225.26 & 363 & 258.66 \\
\hline 49.54816 & 225.12 & 361.19 & 258.69 \\
\hline 50.049 & 224.99 & 360.64 & 258.48 \\
\hline 50.54784 & 224.94 & 360.16 & 258.76 \\
\hline 51.04683 & 224.89 & 360.17 & 258.76 \\
\hline 51.5475 & 224.87 & 359.98 & 258.75 \\
\hline 52.04816 & 224.91 & 360.26 & 257.65 \\
\hline 52.549 & 224.95 & 360.69 & 258.75 \\
\hline 53.04784 & 224.98 & 360.84 & 258.72 \\
\hline 53.54767 & 224.97 & 360.8 & 258.59 \\
\hline 54.0485 & 224.98 & 360.66 & 258.62 \\
\hline 54.54833 & 224.93 & 360.61 & 258.63 \\
\hline 55.048 & 224.91 & 359.69 & 258.63 \\
\hline 55.54784 & 224.86 & 359.57 & 258.71 \\
\hline 56.04767 & 224.83 & 359.51 & 258.54 \\
\hline 56.54667 & 224.82 & 359.39 & 258.62 \\
\hline 57.04734 & 224.87 & 359.72 & 258.58 \\
\hline 57.54816 & 224.89 & 360.05 & 258.61 \\
\hline 58.048 & 224.92 & 360.33 & 258.67 \\
\hline 58.54683 & 224.91 & 360.14 & 258.66 \\
\hline 59.0475 & 224.9 & 359.8 & 258.46 \\
\hline 59.54833 & 224.93 & 359.93 & 258.61 \\
\hline 60.049 & 224.92 & 359.86 & 256.53 \\
\hline 60.548 & 224.85 & 359.61 & 258.64 \\
\hline 61.04683 & 224.81 & 358.86 & 258.08 \\
\hline 61.54767 & 224.8 & 358.95 & 258.65 \\
\hline 62.04833 & 224.73 & 358.28 & 258.55 \\
\hline 62.54816 & 224.67 & 358.37 & 253.61 \\
\hline 63.047 & 224.7 & 357.93 & 255.04 \\
\hline 63.54784 & 224.61 & 357.24 & 254.36 \\
\hline 64.04767 & 224.57 & 357.21 & 254.93 \\
\hline 64.54833 & 224.58 & 357.03 & 254.85 \\
\hline 65.04816 & 224.51 & 356.47 & 253.66 \\
\hline 65.548 & 224.48 & 356.34 & 256.2 \\
\hline 66.04784 & 224.45 & 355.66 & 257.2 \\
\hline 66.54767 & 224.43 & 355.65 & 249.63 \\
\hline 67.04833 & 224.38 & 355.44 & 255.83 \\
\hline 67.54816 & 224.3 & 354.6 & 257.63 \\
\hline 68.048 & 224.22 & 354.03 & 253.84 \\
\hline 68.547 & 224.14 & 353.31 & 253.18 \\
\hline 69.04766 & 224 & 352.21 & 247.27 \\
\hline 69.54833 & 223.9 & 351.43 & 249.58 \\
\hline 70.04917 & 223.89 & 351.55 & 248.98 \\
\hline 70.548 & 223.86 & 351.05 & 252.12 \\
\hline 71.047 & 223.8 & 350.41 & 257.69 \\
\hline 71.54766 & 223.75 & 349.95 & 257.62 \\
\hline 72.0485 & 223.74 & 349.98 & 254.77 \\
\hline 72.54833 & 223.64 & 349.64 & 258.74 \\
\hline 73.04716 & 223.61 & 349.95 & 258.56 \\
\hline 73.54784 & 223.72 & 350.3 & 258.73 \\
\hline 74.04766 & 223.77 & 350.08 & 258.75 \\
\hline 74.5475 & 223.7 & 350.28 & 258.71 \\
\hline 75.04833 & 223.69 & 349.96 & 258.62 \\
\hline 75.54817 & 223.66 & 349.57 & 257.07 \\
\hline 76.048 & 223.76 & 350.57 & 257.68 \\
\hline 76.54766 & 223.82 & 350.15 & 255.85 \\
\hline 77.0475 & 223.66 & 350.26 & 255.49 \\
\hline 77.54833 & 223.74 & 350.82 & 258.99 \\
\hline 78.04817 & 223.77 & 350.16 & 256.51 \\
\hline 78.548 & 223.92 & 350.09 & 256.85 \\
\hline 79.04784 & 223.78 & 349.75 & 254.49 \\
\hline 79.54666 & 223.61 & 348.93 & 254.99 \\
\hline 80.04833 & 223.59 & 348.39 & 256.47 \\
\hline 80.54817 & 223.64 & 347.82 & 256.63 \\
\hline 81.047 & 223.5 & 347.5 & 258.75 \\
\hline
\end{tabular}




\begin{tabular}{|c|c|c|c|}
\hline 81.54784 & 223.49 & 347.57 & 258.74 \\
\hline 82.04766 & 223.44 & 347.34 & 256.34 \\
\hline 82.54833 & 223.32 & 346.64 & 256.19 \\
\hline 83.04733 & 223.36 & 346.33 & 258.69 \\
\hline 83.548 & 223.31 & 345.79 & 258.66 \\
\hline 84.04684 & 223.18 & 345.69 & 258.71 \\
\hline 84.54766 & 223.13 & 345.47 & 258.71 \\
\hline 85.04833 & 223.1 & 345.02 & 258.66 \\
\hline 85.54817 & 223.19 & 344.73 & 258.72 \\
\hline 86.049 & 223.02 & 344.55 & 258.67 \\
\hline 86.547 & 222.92 & 343.91 & 258.62 \\
\hline 87.04766 & 222.89 & 343.47 & 258.6 \\
\hline 87.54833 & 223.07 & 343.66 & 258.15 \\
\hline 88.04817 & 222.95 & 343.47 & 258.58 \\
\hline 88.54716 & 222.87 & 343.34 & 258.6 \\
\hline 89.04784 & 222.91 & 343.32 & 258.64 \\
\hline 89.54766 & 222.9 & 343.18 & 258.65 \\
\hline 90.04933 & 222.8 & 342.95 & 258.58 \\
\hline 90.54833 & 222.89 & 342.6 & 258.61 \\
\hline 91.04716 & 222.86 & 342.22 & 258.58 \\
\hline 91.54784 & 222.76 & 341.49 & 258.66 \\
\hline 92.04766 & 222.73 & 340.86 & 258.54 \\
\hline 92.54666 & 222.61 & 340.56 & 258.52 \\
\hline 93.04733 & 222.66 & 340.23 & 258.6 \\
\hline 93.54817 & 222.78 & 339.63 & 258.55 \\
\hline 94.048 & 222.59 & 339.51 & 258.49 \\
\hline 94.54684 & 222.5 & 339.12 & 258.44 \\
\hline 95.0475 & 222.45 & 338.84 & 258.43 \\
\hline 95.54833 & 222.33 & 338.39 & 258.56 \\
\hline 96.04817 & 222.43 & 338.41 & 258.52 \\
\hline 96.547 & 222.16 & 337.98 & 258.48 \\
\hline 97.04784 & 222.18 & 337.36 & 258.55 \\
\hline 97.54766 & 222.21 & 336.99 & 258.59 \\
\hline 98.04833 & 222.12 & 336.57 & 258.54 \\
\hline 98.54817 & 222.13 & 336.31 & 258.49 \\
\hline 99.048 & 222.06 & 336.11 & 258.47 \\
\hline 99.54784 & 222.03 & 335.78 & 258.48 \\
\hline 100.0477 & 222.09 & 335.55 & 258.45 \\
\hline 100.5483 & 221.98 & 335.39 & 257.98 \\
\hline 101.0482 & 221.83 & 335.03 & 257.72 \\
\hline 101.548 & 222.06 & 335.02 & 258.49 \\
\hline 102.0478 & 222.03 & 334.93 & 257.33 \\
\hline 102.5477 & 221.96 & 334.85 & 258.51 \\
\hline 103.0483 & 221.95 & 334.39 & 256.78 \\
\hline 103.5482 & 221.85 & 334.32 & 258.27 \\
\hline 104.048 & 221.81 & 334.33 & 256.98 \\
\hline 104.5478 & 221.78 & 334.06 & 258.49 \\
\hline 105.0477 & 221.65 & 334.06 & 258.47 \\
\hline 105.5483 & 221.79 & 333.85 & 258.23 \\
\hline 106.0482 & 221.81 & 333.87 & 258.49 \\
\hline 106.5472 & 221.89 & 334.1 & 258.46 \\
\hline 107.0478 & 221.75 & 334.19 & 258.44 \\
\hline 107.5468 & 221.58 & 334.4 & 258.41 \\
\hline 108.0485 & 221.79 & 334.53 & 258.47 \\
\hline 108.5483 & 221.69 & 334.49 & 258.45 \\
\hline 109.048 & 221.75 & 334.94 & 258.45 \\
\hline 109.5478 & 221.7 & 334.83 & 258.39 \\
\hline 110.0477 & 221.65 & 334.97 & 258.57 \\
\hline 110.5475 & 221.87 & 335.48 & 258.42 \\
\hline 111.0483 & 221.85 & 335.52 & 258.37 \\
\hline 111.5482 & 221.94 & 335.8 & 258.35 \\
\hline 112.047 & 221.95 & 336.18 & 258.32 \\
\hline 112.5477 & 221.99 & 336.54 & 258.36 \\
\hline 113.0475 & 221.99 & 337.16 & 258.37 \\
\hline 113.5483 & 222.08 & 337.77 & 258.3 \\
\hline 114.049 & 222.12 & 337.99 & 258.45 \\
\hline
\end{tabular}




\begin{tabular}{|c|c|c|c|}
\hline 114.5488 & 222.18 & 338.27 & 257.09 \\
\hline 115.0478 & 222.27 & 338.48 & 258.33 \\
\hline 115.5477 & 222.33 & 338.76 & 256.49 \\
\hline 116.0483 & 222.35 & 338.81 & 258.41 \\
\hline 116.5482 & 222.43 & 338.85 & 257.92 \\
\hline 117.048 & 222.3 & 338.68 & 258.35 \\
\hline 117.5478 & 222.25 & 338.64 & 258.37 \\
\hline 118.0477 & 222.31 & 338.66 & 258.41 \\
\hline 118.5483 & 222.35 & 338.93 & 258.45 \\
\hline 119.0482 & 222.25 & 339.07 & 252.87 \\
\hline 119.5472 & 222.23 & 338.89 & 249.65 \\
\hline 120.0487 & 222.22 & 338.84 & 252.23 \\
\hline 120.5477 & 222.17 & 338.93 & 252.89 \\
\hline 121.0493 & 222.12 & 338.84 & 252.06 \\
\hline 121.5473 & 222.35 & 339.16 & 253.85 \\
\hline 122.048 & 222.3 & 338.99 & 250.31 \\
\hline 122.5478 & 222.34 & 338.87 & 253.09 \\
\hline 123.0477 & 222.34 & 338.72 & 255.56 \\
\hline 123.5483 & 222.42 & 338.81 & 254.89 \\
\hline 124.0473 & 222.26 & 338.75 & 253.02 \\
\hline 124.548 & 222.41 & 338.82 & 254.9 \\
\hline 125.0478 & 222.46 & 338.91 & 254.02 \\
\hline 125.5477 & 222.32 & 338.56 & 254.31 \\
\hline 126.0485 & 222.24 & 338.42 & 254.78 \\
\hline 126.5483 & 222.24 & 338.17 & 256.45 \\
\hline 127.048 & 222.19 & 338.06 & 255.1 \\
\hline 127.547 & 222.12 & 338.01 & 257.21 \\
\hline 128.0477 & 222.18 & 338.01 & 254.65 \\
\hline 128.5475 & 222.25 & 337.76 & 257.41 \\
\hline 129.0483 & 222.11 & 337.48 & 257.26 \\
\hline 129.5482 & 222.1 & 337.44 & 258.26 \\
\hline 130.048 & 222.15 & 337.05 & 258.37 \\
\hline 130.5477 & 222.12 & 337.1 & 255.63 \\
\hline 131.0467 & 222.14 & 336.95 & 255.82 \\
\hline 131.5483 & 222.04 & 336.72 & 254.56 \\
\hline 132.0472 & 222.02 & 336.37 & 254.83 \\
\hline 132.548 & 221.97 & 335.89 & 254.64 \\
\hline 133.0478 & 221.81 & 335.71 & 256.57 \\
\hline 133.5467 & 221.73 & 335.5 & 255.88 \\
\hline 134.0473 & 221.73 & 334.83 & 255.63 \\
\hline 134.5482 & 221.79 & 334.46 & 257.28 \\
\hline 135.048 & 221.75 & 334.56 & 255.95 \\
\hline 135.5487 & 221.77 & 334.2 & 255.9 \\
\hline 136.0477 & 221.86 & 334.2 & 253.96 \\
\hline 136.5483 & 221.65 & 334.08 & 255.35 \\
\hline 137.0473 & 221.69 & 333.7 & 255.38 \\
\hline 137.548 & 221.71 & 333.5 & 254.9 \\
\hline 138.0478 & 221.62 & 333.21 & 256.16 \\
\hline 138.5467 & 221.57 & 333.14 & 255.99 \\
\hline 139.0483 & 221.63 & 332.85 & 255.82 \\
\hline 139.5473 & 221.5 & 332.78 & 256.15 \\
\hline 140.048 & 221.54 & 332.23 & 256.23 \\
\hline 140.547 & 221.42 & 331.94 & 254.87 \\
\hline 141.0477 & 221.33 & 331.78 & 256.75 \\
\hline 141.5483 & 221.6 & 331.59 & 256.88 \\
\hline 142.0482 & 221.51 & 331.53 & 255.61 \\
\hline 142.548 & 221.48 & 331.56 & 256.56 \\
\hline 143.0478 & 221.41 & 331.68 & 255.76 \\
\hline 143.5477 & 221.4 & 331.64 & 255.38 \\
\hline 144.0485 & 221.34 & 331.43 & 255.24 \\
\hline 144.5483 & 221.24 & 331.16 & 255.68 \\
\hline 145.048 & 221.35 & 331.08 & 257.72 \\
\hline 145.547 & 221.21 & 330.76 & 258.02 \\
\hline 146.0477 & 221.12 & 330.68 & 258.6 \\
\hline 146.5475 & 221.09 & 330.06 & 258.56 \\
\hline 147.0483 & 221.09 & 329.73 & 258.63 \\
\hline
\end{tabular}




\begin{tabular}{|c|c|c|c|}
\hline 147.5482 & 221.23 & 329.8 & 258.65 \\
\hline 148.047 & 221.27 & 329.1 & 258.6 \\
\hline 148.5477 & 221.22 & 329.26 & 258.64 \\
\hline 149.0475 & 221.2 & 329.3 & 258.47 \\
\hline 149.5483 & 221.13 & 329.24 & 258.53 \\
\hline 150.0482 & 220.98 & 329.31 & 258.52 \\
\hline 150.547 & 221.07 & 329.24 & 258.55 \\
\hline 151.0478 & 221.05 & 329.27 & 258.46 \\
\hline 151.5477 & 220.84 & 328.62 & 258.5 \\
\hline 152.0483 & 220.93 & 328.61 & 258.51 \\
\hline 152.5482 & 220.91 & 328.36 & 258.44 \\
\hline 153.047 & 220.9 & 328.11 & 258.51 \\
\hline 153.5478 & 220.92 & 327.85 & 258.45 \\
\hline 154.0477 & 220.76 & 327.74 & 256.51 \\
\hline 154.5483 & 220.81 & 327.55 & 258.45 \\
\hline 155.0482 & 220.76 & 327.3 & 258.41 \\
\hline 155.547 & 220.57 & 327.1 & 258.37 \\
\hline 156.0478 & 220.61 & 326.53 & 258.44 \\
\hline 156.5477 & 220.59 & 326.38 & 258.44 \\
\hline 157.0483 & 220.73 & 325.66 & 257.78 \\
\hline 157.5482 & 220.61 & 325.12 & 258.43 \\
\hline 158.048 & 220.45 & 324.73 & 258.43 \\
\hline 158.547 & 220.47 & 324.27 & 258.42 \\
\hline 159.0477 & 220.33 & 323.67 & 258.37 \\
\hline 159.5483 & 220.31 & 323.42 & 258.23 \\
\hline 160.0482 & 220.33 & 322.97 & 258.42 \\
\hline 160.548 & 220.18 & 322.62 & 258.41 \\
\hline 161.0478 & 220.09 & 322.12 & 258.43 \\
\hline 161.5477 & 220.07 & 321.58 & 258.43 \\
\hline 162.0485 & 220.11 & 321.08 & 258.41 \\
\hline 162.5483 & 220.2 & 320.61 & 257.8 \\
\hline 163.048 & 220.08 & 319.64 & 258.41 \\
\hline 163.5478 & 220.15 & 319.28 & 258.4 \\
\hline 164.0477 & 219.97 & 318.94 & 258.38 \\
\hline 164.5475 & 219.96 & 318.27 & 258.41 \\
\hline 165.0483 & 219.8 & 317.97 & 258.42 \\
\hline 165.5472 & 219.87 & 317.75 & 258.35 \\
\hline 166.048 & 219.96 & 317.09 & 258.39 \\
\hline 166.5477 & 220.06 & 316.62 & 258.4 \\
\hline 167.0475 & 219.61 & 315.9 & 258.34 \\
\hline 167.5483 & 219.52 & 315.75 & 258.42 \\
\hline 168.049 & 219.42 & 315.04 & 258.34 \\
\hline 168.548 & 219.36 & 314.53 & 258.39 \\
\hline 169.0468 & 219.19 & 313.45 & 258.31 \\
\hline 169.5477 & 219.26 & 312.81 & 258.3 \\
\hline 170.0492 & 219.44 & 312.31 & 258.34 \\
\hline 170.5482 & 219.48 & 311.31 & 257.97 \\
\hline 171.048 & 219.01 & 310.45 & 258.29 \\
\hline 171.5478 & 219.21 & 309.76 & 258.14 \\
\hline 172.0467 & 219.29 & 308.84 & 258.28 \\
\hline 172.5483 & 219.16 & 308.04 & 256.32 \\
\hline 173.0482 & 218.93 & 307.26 & 257.76 \\
\hline 173.5488 & 219.63 & 306.55 & 258.44 \\
\hline 174.0478 & 219.71 & 305.69 & 257.23 \\
\hline 174.5477 & 219.91 & 305.33 & 258.37 \\
\hline 175.0475 & 219.5 & 304.62 & 258.08 \\
\hline 175.5473 & 219.07 & 303.81 & 255.93 \\
\hline 176.048 & 219.25 & 303.12 & 256.43 \\
\hline 176.5488 & 218.23 & 302.27 & 257.88 \\
\hline 177.0468 & 217.8 & 301.43 & 256.23 \\
\hline 177.5483 & 218.75 & 300.63 & 258.31 \\
\hline 178.0482 & 218.61 & 299.79 & 258.27 \\
\hline 178.548 & 218.61 & 299.02 & 258.28 \\
\hline 179.0478 & 219.08 & 298 & 258.24 \\
\hline 179.5468 & 217.74 & 297.29 & 258.2 \\
\hline 180.0493 & 218.41 & 296.29 & 258.16 \\
\hline
\end{tabular}




\begin{tabular}{|c|c|c|c|}
\hline 180.5483 & 219.18 & 295.66 & 258.13 \\
\hline 181.048 & 219.02 & 294.63 & 258.17 \\
\hline 181.547 & 218.64 & 294.01 & 258.17 \\
\hline 182.0477 & 218 & 293.01 & 258.14 \\
\hline 182.5475 & 218.25 & 292.05 & 258.17 \\
\hline 183.0483 & 217.66 & 291.21 & 258.14 \\
\hline 183.5482 & 218.06 & 290.16 & 258.1 \\
\hline 184.047 & 217.67 & 289.2 & 258.1 \\
\hline 184.5477 & 217.88 & 288.25 & 258.06 \\
\hline 185.0475 & 217.98 & 287.1 & 258.09 \\
\hline 185.5483 & 217.49 & 285.97 & 258.09 \\
\hline 186.0482 & 217.69 & 285.12 & 258.05 \\
\hline 186.548 & 217.1 & 283.94 & 258.08 \\
\hline 187.0468 & 216.82 & 282.75 & 258.03 \\
\hline 187.5477 & 217.7 & 281.62 & 258.02 \\
\hline 188.0483 & 217.14 & 280.48 & 257.97 \\
\hline 188.5482 & 217.11 & 279.24 & 257.96 \\
\hline 189.0488 & 217.29 & 277.89 & 257.9 \\
\hline 189.5478 & 217.08 & 276.61 & 257.91 \\
\hline 190.0477 & 216.35 & 275.29 & 257.88 \\
\hline 190.5483 & 216.9 & 273.71 & 257.87 \\
\hline 191.0482 & 216.85 & 272.28 & 257.88 \\
\hline 191.547 & 217.26 & 270.88 & 257.9 \\
\hline 192.0478 & 216.59 & 268.94 & 257.85 \\
\hline 192.5477 & 216.68 & 267.35 & 257.82 \\
\hline 193.0483 & 216.07 & 265.44 & 257.75 \\
\hline 193.5482 & 216.16 & 264.11 & 257.68 \\
\hline 194.048 & 215.09 & 263.54 & 257.66 \\
\hline 194.5478 & 214.99 & 263.34 & 257.72 \\
\hline 195.0477 & 216.63 & 263.78 & 257.75 \\
\hline 195.5483 & 219.12 & 264.54 & 257.84 \\
\hline 196.0482 & 219.95 & 265.2 & 257.89 \\
\hline 196.548 & 220.18 & 264.97 & 257.9 \\
\hline 197.0478 & 220.09 & 263.8 & 257.72 \\
\hline 197.5477 & 220.24 & 262.52 & 257.5 \\
\hline 198.0485 & 219.61 & 261.48 & 257.74 \\
\hline 198.5483 & 220.57 & 261.02 & 257.75 \\
\hline 199.048 & 218.63 & 260.63 & 257.87 \\
\hline 199.5478 & 219.24 & 260.4 & 257.92 \\
\hline 200.0477 & 218.33 & 260.25 & 257.97 \\
\hline 200.5475 & 218.64 & 260.11 & 257.92 \\
\hline 201.0502 & 217.98 & 259.92 & 257.94 \\
\hline 201.549 & 217.46 & 259.61 & 257.96 \\
\hline 202.048 & 216.41 & 259.17 & 257.86 \\
\hline 202.5468 & 216.39 & 258.69 & 257.83 \\
\hline 203.0475 & 216.93 & 257.9 & 256.9 \\
\hline 203.5483 & 215.57 & 257.28 & 255.21 \\
\hline 204.0482 & 216.04 & 258.28 & 256.67 \\
\hline 204.547 & 217.08 & 259.97 & 257.95 \\
\hline 205.0478 & 219.1 & 261.88 & 258.19 \\
\hline 205.5477 & 222.79 & 264.14 & 258.32 \\
\hline 206.0483 & 227.25 & 265.03 & 258.11 \\
\hline 206.5482 & 227.72 & 263.23 & 257.2 \\
\hline 207.048 & 225.61 & 262.02 & 257.08 \\
\hline 207.5478 & 225.27 & 260.07 & 256.45 \\
\hline 208.0467 & 225.08 & 259.25 & 256.08 \\
\hline 208.5483 & 224.18 & 258.55 & 256.41 \\
\hline 209.0473 & 222.75 & 258.8 & 256.2 \\
\hline 209.548 & 222.45 & 258.58 & 256.86 \\
\hline 210.0478 & 222.68 & 258.77 & 255.71 \\
\hline 210.5477 & 223.01 & 258.83 & 257.05 \\
\hline 211.0483 & 222.71 & 258.8 & 257.97 \\
\hline 211.5482 & 222.37 & 259.24 & 258.19 \\
\hline 212.048 & 222.17 & 259.9 & 256.68 \\
\hline 212.5478 & 221.94 & 259.76 & 257.8 \\
\hline 213.0477 & 222.16 & 258.6 & 255.8 \\
\hline
\end{tabular}




\begin{tabular}{|c|c|c|c|}
\hline 213.5475 & 221.8 & 259 & 258.18 \\
\hline 214.0482 & 222.11 & 259.71 & 258.16 \\
\hline 214.548 & 222.18 & 257.53 & 254.48 \\
\hline 215.0478 & 221.29 & 256.41 & 254.74 \\
\hline 215.5468 & 220.67 & 256.09 & 254.33 \\
\hline 216.0475 & 220.81 & 255.95 & 255.39 \\
\hline 216.5483 & 221.53 & 256.3 & 254.49 \\
\hline 217.048 & 222.37 & 257.89 & 255.48 \\
\hline 217.5478 & 221.64 & 258.79 & 256.22 \\
\hline 218.0468 & 223.22 & 259.11 & 255.83 \\
\hline 218.5475 & 222.29 & 258.98 & 256.07 \\
\hline 219.0483 & 222.65 & 258.84 & 255.79 \\
\hline 219.5482 & 223.33 & 259.04 & 255.65 \\
\hline 220.0488 & 223.3 & 258.97 & 255.28 \\
\hline 220.5477 & 223.17 & 259.37 & 256.04 \\
\hline 221.0485 & 224.96 & 259.47 & 256.37 \\
\hline 221.5483 & 225.61 & 259.38 & 256.4 \\
\hline 222.0482 & 224.07 & 259.13 & 255.83 \\
\hline 222.548 & 223.52 & 258.66 & 255.75 \\
\hline 223.0478 & 223.74 & 258.09 & 255.89 \\
\hline 223.5477 & 224.96 & 257.53 & 255.87 \\
\hline 224.0483 & 223.38 & 257.22 & 255.46 \\
\hline 224.5482 & 223.35 & 255.83 & 253.18 \\
\hline 225.047 & 221.92 & 255.28 & 253.53 \\
\hline 225.5478 & 221.78 & 255.74 & 253.68 \\
\hline 226.0477 & 221.37 & 256.18 & 254.21 \\
\hline 226.5483 & 221.66 & 256.1 & 254.77 \\
\hline 227.0482 & 222.38 & 256.55 & 254.18 \\
\hline 227.547 & 223.67 & 256.48 & 254.67 \\
\hline 228.0478 & 223.06 & 256.79 & 255.1 \\
\hline 228.5477 & 221.99 & 257.24 & 254.94 \\
\hline 229.0483 & 223.44 & 257.81 & 255.58 \\
\hline 229.5482 & 223.98 & 257.98 & 255.81 \\
\hline 230.048 & 223.19 & 257.91 & 255.66 \\
\hline 230.5478 & 222.71 & 258.13 & 255.87 \\
\hline 231.0468 & 224.09 & 258.37 & 255.24 \\
\hline 231.5483 & 223.58 & 258.55 & 255.55 \\
\hline 232.0473 & 224.01 & 258.93 & 255.57 \\
\hline 232.548 & 224.4 & 258.97 & 258.27 \\
\hline 233.0478 & 223.19 & 258.19 & 255.01 \\
\hline 233.5487 & 222.94 & 259.22 & 256.53 \\
\hline 234.0485 & 223.23 & 259.78 & 257.44 \\
\hline 234.5473 & 223.69 & 260.39 & 257.87 \\
\hline 235.048 & 223.11 & 260.93 & 258.22 \\
\hline 235.5478 & 223.7 & 260.42 & 255.8 \\
\hline 236.0487 & 223.54 & 258.97 & 255.45 \\
\hline 236.5467 & 224.85 & 258.58 & 255.72 \\
\hline 237.0473 & 224.35 & 258.71 & 255.7 \\
\hline 237.5482 & 221.92 & 258.65 & 256.53 \\
\hline 238.048 & 223.92 & 258.78 & 255.35 \\
\hline 238.5477 & 222.82 & 258.74 & 255.85 \\
\hline 239.0467 & 223.1 & 258.75 & 256.17 \\
\hline 239.5483 & 224.84 & 258.62 & 256.07 \\
\hline 240.0482 & 224.47 & 258.32 & 255.77 \\
\hline 240.548 & 222.54 & 258.24 & 256.31 \\
\hline 241.0478 & 223.29 & 258.71 & 255.31 \\
\hline 241.5477 & 224.39 & 259.61 & 255.77 \\
\hline 242.0483 & 223.31 & 260.89 & 256.24 \\
\hline 242.5482 & 225.11 & 262.09 & 256.3 \\
\hline 243.047 & 226.68 & 262.25 & 256.95 \\
\hline 243.5478 & 227.29 & 261.34 & 256.49 \\
\hline 244.0477 & 228.05 & 260.36 & 256.33 \\
\hline 244.5483 & 225.94 & 259.38 & 256.44 \\
\hline 245.0482 & 212.78 & 273.2 & 263.76 \\
\hline 245.548 & 212.88 & 273.35 & 263.24 \\
\hline 246.0478 & 212.88 & 273.37 & 262.92 \\
\hline
\end{tabular}




$\begin{array}{llll}246.5477 & 212.62 & 273.88 & 263.67 \\ 247.0483 & 212.49 & 273.37 & 264.23 \\ 247.5473 & 212.64 & 273.35 & 264.25 \\ 248.048 & 212.72 & 273.39 & 263.39 \\ 248.5478 & 212.81 & 273.42 & 263.91 \\ 249.0467 & 212.83 & 273.24 & 263.34 \\ 249.5475 & 212.87 & 273.73 & 262.94 \\ 250.0492 & 213.07 & 273.27 & 262.76 \\ 250.5472 & 213.02 & 272.7 & 263.05 \\ 251.047 & 212.86 & 272.94 & 263.17 \\ 251.5477 & 212.63 & 272.57 & 263.28 \\ 252.0485 & 212.64 & 272.99 & 263.48 \\ 252.5482 & 212.5 & 272.8 & 263.31 \\ 253.048 & 212.52 & 272.6 & 263.43 \\ 253.5488 & 212.47 & 272.86 & 263.48 \\ 254.0477 & 212.51 & 273.37 & 263.86 \\ 254.5475 & 212.51 & 272.88 & 263.64 \\ 255.0473 & 212.59 & 272.82 & 263.3 \\ 255.5482 & 212.82 & 273.12 & 263.78 \\ 256.048 & 212.79 & 273.04 & 263.44 \\ 256.5477 & 212.42 & 273.69 & 263.06 \\ 257.0467 & 212.33 & 273.43 & 250.72\end{array}$

\section{Run 6 (steam-propane case)}

run_no
6
additive
propane
experiment_date
$05 / 21 / 2005$
researcher
Roly Simangunsong
cell_radius
3.688
cell_length
68.58
tcell
54
Cs
0.21
Co
0.5
Cw
1
dens_sand
167
dens_oil
62
dens_water
62.4
por
0.411690173




\begin{tabular}{|c|c|}
\hline \multicolumn{2}{|c|}{$\begin{array}{l}\text { Soi } \\
0.726148715592812\end{array}$} \\
\hline \multicolumn{2}{|c|}{ Sor } \\
\hline \multicolumn{2}{|c|}{ Sgi } \\
\hline \multicolumn{2}{|c|}{0.0936861640173443} \\
\hline \multicolumn{2}{|c|}{ Swc } \\
\hline porvol & 1162.444515 \\
\hline \multicolumn{2}{|c|}{ void_length } \\
\hline \multicolumn{2}{|c|}{ o_w_ratio } \\
\hline $\begin{array}{l}\text { iw } \\
5.5\end{array}$ & \\
\hline tfu_total & $14 . \overline{8}$ \\
\hline \multicolumn{2}{|c|}{0.05} \\
\hline \multicolumn{2}{|c|}{ Hv_enthalphy } \\
\hline 182.8470859 & \\
\hline \multicolumn{2}{|c|}{$\begin{array}{l}\text { res_heat_capacity } \\
28\end{array}$} \\
\hline \multicolumn{2}{|c|}{ heat_loss_coeff } \\
\hline & $\begin{array}{l}\text { t_phase } \\
163\end{array}$ \\
\hline $\begin{array}{l}--\mathrm{T} \\
\text { Pro }\end{array}$ & $\begin{array}{l}\text { Cumulative Oil, cc } \\
\text { abulation }\end{array}$ \\
\hline 0 & 0 \\
\hline 2 & 0 \\
\hline 5 & 0 \\
\hline 8 & 0 \\
\hline 11 & 0 \\
\hline 14 & 0 \\
\hline 17 & 2 \\
\hline 20 & 5 \\
\hline 23 & 9 \\
\hline 26 & 14 \\
\hline 29 & 20.5 \\
\hline 32 & 27.5 \\
\hline 35 & 35 \\
\hline 38 & 44 \\
\hline 41 & 53 \\
\hline 44 & 63 \\
\hline 47 & 74 \\
\hline 50 & 87 \\
\hline
\end{tabular}




\begin{tabular}{|c|c|c|}
\hline 53 & \multicolumn{2}{|l|}{100} \\
\hline 56 & \multicolumn{2}{|l|}{114} \\
\hline 59 & \multicolumn{2}{|l|}{134} \\
\hline 62 & \multicolumn{2}{|l|}{180} \\
\hline 65 & \multicolumn{2}{|l|}{209} \\
\hline 68 & \multicolumn{2}{|l|}{234} \\
\hline 71 & \multicolumn{2}{|l|}{252} \\
\hline 74 & \multicolumn{2}{|l|}{267} \\
\hline 77 & \multicolumn{2}{|l|}{280} \\
\hline 80 & \multicolumn{2}{|l|}{291} \\
\hline 83 & \multicolumn{2}{|l|}{299} \\
\hline 86 & \multicolumn{2}{|l|}{306.5} \\
\hline 89 & \multicolumn{2}{|l|}{311.5} \\
\hline 92 & \multicolumn{2}{|l|}{316.5} \\
\hline 95 & \multicolumn{2}{|l|}{321.5} \\
\hline 98 & \multicolumn{2}{|l|}{324.5} \\
\hline 101 & \multicolumn{2}{|l|}{328} \\
\hline 104 & \multicolumn{2}{|l|}{331.5} \\
\hline 107 & \multicolumn{2}{|l|}{334} \\
\hline 110 & \multicolumn{2}{|l|}{337} \\
\hline 113 & \multicolumn{2}{|l|}{340} \\
\hline 116 & \multicolumn{2}{|l|}{342.5} \\
\hline 119 & \multicolumn{2}{|l|}{346} \\
\hline 123 & \multicolumn{2}{|l|}{349} \\
\hline 127 & 353 & \\
\hline 131 & 362 & \\
\hline 135 & 379 & \\
\hline 139 & 388 & \\
\hline 143 & 392 & \\
\hline 146 & 395 & \\
\hline 151 & 397.5 & \\
\hline 156 & 399 & \\
\hline 162 & 400.5 & \\
\hline 168 & 401.5 & \\
\hline 174 & 402.5 & \\
\hline 180 & 403 & \\
\hline 186 & 403.5 & \\
\hline 192 & 403.5 & \\
\hline 198 & 403.5 & \\
\hline 204 & 403.5 & \\
\hline 210 & 403.5 & \\
\hline 216 & 403.5 & / \\
\hline -- time, min & $\mathrm{x}$, from & $\mathrm{n}$ top of flange, $\mathrm{cm}$ \\
\hline Thermo_Tabulation & & \\
\hline 62 & & 12 \\
\hline 89 & & 27.5 \\
\hline 103 & & 42.8 \\
\hline 144 & & $68.3 /$ \\
\hline
\end{tabular}

\begin{tabular}{|c|c|c|c|}
\hline \multirow{2}{*}{\multicolumn{4}{|c|}{$\begin{array}{l}\text {-- Time, minutes Tinj, oC } \\
\text { Log Data Tabulation }\end{array}$}} \\
\hline & & & \\
\hline 0.05033333 & 48.31 & 259.65 & 261.27 \\
\hline 0.5446666 & 48.81 & 263.01 & 263.18 \\
\hline 1.045333 & 52.84 & 263.29 & 262.87 \\
\hline 1.545167 & 59.09 & 262.69 & 262.83 \\
\hline 2.045 & 74.11 & 263.09 & 263.24 \\
\hline 2.544833 & 156.71 & 263.12 & 262.94 \\
\hline 3.0455 & 203.44 & 263.67 & 261.95 \\
\hline 3.545333 & 206.65 & 263.39 & 262.37 \\
\hline 4.045167 & 206.98 & 260.75 & 261.49 \\
\hline 4.544167 & 208.02 & 261.32 & 261.53 \\
\hline 5.044833 & 210.03 & 264.56 & 261.7 \\
\hline 5.544667 & 210.7 & 263.17 & 261.59 \\
\hline 6.045333 & 212.49 & 266.68 & 261.69 \\
\hline
\end{tabular}




\begin{tabular}{|c|c|c|c|}
\hline 6.545166 & 213.82 & 263.16 & 261.27 \\
\hline 7.045 & 214.66 & 266.32 & 262.1 \\
\hline 7.544833 & 216.07 & 267.91 & 260.92 \\
\hline 8.045667 & 213.1 & 274.6 & 261.8 \\
\hline 8.545333 & 213.52 & 273.74 & 261.27 \\
\hline 9.045167 & 212.07 & 276.13 & 261.4 \\
\hline 9.545 & 211.86 & 275 & 261.38 \\
\hline 10.04583 & 212.47 & 282.79 & 261.53 \\
\hline 10.54567 & 212.69 & 284.67 & 261.54 \\
\hline 11.0455 & 214.52 & 286.34 & 261.5 \\
\hline 11.54533 & 215.37 & 291.26 & 261.5 \\
\hline 12.04417 & 216.14 & 291.79 & 261.56 \\
\hline 12.54483 & 216.04 & 294.95 & 261.36 \\
\hline 13.04467 & 216.42 & 293.63 & 261.37 \\
\hline 13.5445 & 215.82 & 297.64 & 261.49 \\
\hline 14.04433 & 215.3 & 299.78 & 261.59 \\
\hline 14.54517 & 215.33 & 300.91 & 261.62 \\
\hline 15.045 & 216.71 & 298.7 & 261.59 \\
\hline 15.5465 & 216.59 & 300.56 & 261.45 \\
\hline 16.0455 & 216.72 & 307.25 & 261.54 \\
\hline 16.54433 & 217.81 & 308.86 & 261.48 \\
\hline 17.04517 & 217.35 & 311.35 & 261.47 \\
\hline 17.545 & 218.56 & 311.55 & 261.25 \\
\hline 18.04567 & 218.15 & 310.92 & 261.31 \\
\hline 18.5455 & 218.01 & 314.86 & 261.26 \\
\hline 19.04533 & 219.37 & 317.98 & 261.24 \\
\hline 19.54517 & 219.07 & 321.62 & 261.17 \\
\hline 20.045 & 219.72 & 323.91 & 261.19 \\
\hline 20.54483 & 218.67 & 326.36 & 261.17 \\
\hline 21.04467 & 218.85 & 329.42 & 261.32 \\
\hline 21.54533 & 219.34 & 333.67 & 261.65 \\
\hline 22.04517 & 218.45 & 329.75 & 261.7 \\
\hline 22.545 & 218.83 & 332.4 & 261.9 \\
\hline 23.04483 & 219.37 & 331.03 & 261.63 \\
\hline 23.5455 & 219.53 & 331.07 & 262.43 \\
\hline 24.04533 & 219.34 & 328.21 & 261.87 \\
\hline 24.54617 & 219.49 & 328.17 & 262.56 \\
\hline 25.045 & 220.01 & 328.29 & 262.58 \\
\hline 25.54583 & 220.25 & 328.56 & 260.57 \\
\hline 26.0465 & 220.25 & 328.02 & 262.62 \\
\hline 26.54533 & 220.32 & 327.36 & 262.63 \\
\hline 27.04517 & 220.11 & 326.03 & 261.46 \\
\hline 27.546 & 220.01 & 324.34 & 261 \\
\hline 28.04483 & 219.83 & 322.9 & 262.29 \\
\hline 28.54567 & 219.69 & 321.64 & 262.62 \\
\hline 29.0455 & 219.55 & 320.3 & 261.6 \\
\hline 29.54433 & 219.63 & 322.68 & 255.4 \\
\hline 30.045 & 219.51 & 321.63 & 256.63 \\
\hline 30.54483 & 219.56 & 322.57 & 258.55 \\
\hline 31.04567 & 219.69 & 322.55 & 259.19 \\
\hline 31.5455 & 219.72 & 324.55 & 257.69 \\
\hline 32.04533 & 219.77 & 325.09 & 258.21 \\
\hline 32.54517 & 220.01 & 326.37 & 258.97 \\
\hline 33.045 & 220.19 & 328.02 & 255.49 \\
\hline 33.54567 & 220.35 & 328.5 & 257.73 \\
\hline 34.0455 & 220.63 & 330.76 & 258.47 \\
\hline 34.54533 & 220.91 & 335 & 257.11 \\
\hline 35.04517 & 220.88 & 339.24 & 255.46 \\
\hline 35.545 & 220.76 & 341.72 & 255.99 \\
\hline 36.04483 & 220.89 & 343.74 & 256.5 \\
\hline 36.5455 & 221.02 & 346.53 & 258.51 \\
\hline 37.04533 & 220.97 & 345.82 & 258.9 \\
\hline 37.54517 & 220.93 & 345.34 & 257.99 \\
\hline 38.045 & 220.86 & 348.03 & 258.37 \\
\hline 38.54483 & 220.67 & 346.9 & 256.21 \\
\hline 39.0455 & 220.64 & 345.31 & 257.56 \\
\hline
\end{tabular}




\begin{tabular}{|c|c|c|c|}
\hline 39.54533 & 220.64 & 345.24 & 257.06 \\
\hline 40.04517 & 220.27 & 345.99 & 256.25 \\
\hline 40.545 & 220.25 & 344.78 & 258.21 \\
\hline 41.04483 & 220.33 & 344.88 & 258.25 \\
\hline 41.5455 & 220.58 & 345.32 & 258.44 \\
\hline 42.0445 & 220.44 & 343.98 & 257.73 \\
\hline 42.54433 & 220.32 & 343.92 & 256.8 \\
\hline 43.045 & 220.32 & 351.39 & 257.04 \\
\hline 43.54483 & 219.91 & 347 & 256.53 \\
\hline 44.04567 & 220.22 & 349.41 & 258.07 \\
\hline 44.54533 & 220.33 & 350.13 & 258.35 \\
\hline 45.04517 & 219.22 & 346.82 & 258.05 \\
\hline 45.545 & 220.46 & 347.09 & 260.03 \\
\hline 46.044 & 220.42 & 344.69 & 258.71 \\
\hline 46.54467 & 220.73 & 344.5 & 257.76 \\
\hline 47.0445 & 220.66 & 346.43 & 258.77 \\
\hline 47.54533 & 220.63 & 348.32 & 260.28 \\
\hline 48.045 & 220.56 & 348.09 & 262.5 \\
\hline 48.544 & 220.79 & 350.92 & 262.64 \\
\hline 49.04567 & 221.14 & 352.57 & 262.86 \\
\hline 49.5445 & 220.96 & 352.56 & 262.93 \\
\hline 50.04533 & 220.78 & 352.36 & 262.99 \\
\hline 50.54517 & 220.46 & 350.63 & 261.92 \\
\hline 51.045 & 220.48 & 349.61 & 261.86 \\
\hline 51.54567 & 220.45 & 347.52 & 262.08 \\
\hline 52.04633 & 219.91 & 344.42 & 262.3 \\
\hline 52.54533 & 219.97 & 342.71 & 262.74 \\
\hline 53.04517 & 219.45 & 340.02 & 262.89 \\
\hline 53.545 & 219.17 & 337.99 & 263.07 \\
\hline 54.04483 & 219.14 & 335.15 & 261.74 \\
\hline 54.5455 & 218.51 & 332.02 & 256.71 \\
\hline 55.04533 & 218.16 & 325.88 & 261.95 \\
\hline 55.54517 & 218.09 & 322.67 & 262.53 \\
\hline 56.045 & 217.79 & 320.52 & 262.22 \\
\hline 56.54483 & 217.47 & 320.78 & 263.17 \\
\hline 57.04467 & 217.43 & 321.52 & 262.74 \\
\hline 57.54533 & 217.27 & 320.35 & 261.36 \\
\hline 58.04517 & 217.13 & 318.08 & 260.57 \\
\hline 58.54417 & 217.01 & 318.13 & 261.75 \\
\hline 59.04383 & 216.82 & 317.54 & 262.18 \\
\hline 59.54467 & 216.6 & 317.36 & 262.45 \\
\hline 60.04533 & 216.52 & 315.55 & 263.01 \\
\hline 60.54517 & 216.14 & 315.2 & 262.99 \\
\hline 61.045 & 216.22 & 312.93 & 262.88 \\
\hline 61.54483 & 216.18 & 312.98 & 260.72 \\
\hline 62.04467 & 216.28 & 311.47 & 260.58 \\
\hline 62.5445 & 216.55 & 311.45 & 259.22 \\
\hline 63.04517 & 216.19 & 309.88 & 258.71 \\
\hline 63.545 & 216.46 & 309.57 & 261.89 \\
\hline 64.04483 & 215.98 & 307.54 & 261.74 \\
\hline 64.54466 & 215.46 & 306.54 & 259.16 \\
\hline 65.0455 & 215.48 & 302.03 & 259.63 \\
\hline 65.54533 & 216.15 & 302.45 & 258.89 \\
\hline 66.045 & 216.65 & 300.41 & 258.33 \\
\hline 66.54483 & 215.95 & 301.19 & 259.87 \\
\hline 67.04567 & 216.4 & 304.14 & 259.35 \\
\hline 67.5455 & 216.94 & 305.56 & 258.63 \\
\hline 68.04533 & 216.8 & 306.14 & 261.31 \\
\hline 68.54417 & 216.66 & 307.42 & 261.49 \\
\hline 69.044 & 217.15 & 306.43 & 261.96 \\
\hline 69.54567 & 216.54 & 306.15 & 261.77 \\
\hline 70.0455 & 216.94 & 305.24 & 261.69 \\
\hline 70.54533 & 216.63 & 305.31 & 261.98 \\
\hline 71.04517 & 216.67 & 304.31 & 261.92 \\
\hline 71.54501 & 217.14 & 302.26 & 262.61 \\
\hline 72.04666 & 216.64 & 303.33 & 262.52 \\
\hline
\end{tabular}




\begin{tabular}{|c|c|c|c|}
\hline 72.54467 & 216.89 & 303.05 & 262.89 \\
\hline 73.04433 & 217.09 & 304.83 & 262.9 \\
\hline 73.54517 & 216.97 & 305.14 & 260.74 \\
\hline 74.04501 & 216.9 & 303.89 & 261.26 \\
\hline 74.54383 & 216.72 & 304.88 & 261.39 \\
\hline 75.04467 & 216.76 & 305.67 & 261.68 \\
\hline 75.54533 & 216.19 & 305.95 & 261.98 \\
\hline 76.04617 & 216.8 & 305.33 & 261.91 \\
\hline 76.54501 & 217.01 & 306.62 & 261.75 \\
\hline 77.04383 & 216.77 & 305.58 & 262.09 \\
\hline 77.5455 & 217.14 & 306.89 & 262.32 \\
\hline 78.04533 & 216.95 & 305.56 & 262.32 \\
\hline 78.54517 & 216.41 & 307.96 & 262.43 \\
\hline 79.04501 & 216.47 & 305.16 & 262.7 \\
\hline 79.54483 & 216.52 & 305.09 & 262.42 \\
\hline 80.04567 & 216.58 & 304.2 & 262.57 \\
\hline 80.5445 & 216.29 & 302.38 & 262.61 \\
\hline 81.04517 & 216.78 & 303.2 & 260.2 \\
\hline 81.54501 & 216.46 & 303.09 & 260.56 \\
\hline 82.04483 & 216.55 & 301.82 & 259.92 \\
\hline 82.54567 & 215.71 & 301.35 & 260.37 \\
\hline 83.0455 & 215.91 & 297.85 & 261.05 \\
\hline 83.54533 & 216.22 & 298.45 & 261.04 \\
\hline 84.04501 & 216.09 & 298.61 & 258.59 \\
\hline 84.54483 & 215.9 & 300.3 & 261 \\
\hline 85.04567 & 215.51 & 301.31 & 259.13 \\
\hline 85.5455 & 216.19 & 299.75 & 260.6 \\
\hline 86.04533 & 216.71 & 299.98 & 259.97 \\
\hline 86.54517 & 217.3 & 302.53 & 260.67 \\
\hline 87.04501 & 217.41 & 300.96 & 260.3 \\
\hline 87.54567 & 216.8 & 301.92 & 258.83 \\
\hline 88.0455 & 216.97 & 300.15 & 261.25 \\
\hline 88.54433 & 216.29 & 299.58 & 260.74 \\
\hline 89.04517 & 215.93 & 296.75 & 261.5 \\
\hline 89.54501 & 216.24 & 295.12 & 260.96 \\
\hline 90.04567 & 216.44 & 294.18 & 259.85 \\
\hline 90.54467 & 215.2 & 291.56 & 260.75 \\
\hline 91.04433 & 214.62 & 289.48 & 260.9 \\
\hline 91.54417 & 214.71 & 288.61 & 260.85 \\
\hline 92.04501 & 214.05 & 287.05 & 260.93 \\
\hline 92.54483 & 213.98 & 285.41 & 261.67 \\
\hline 93.0465 & 213.74 & 283.95 & 261.55 \\
\hline 93.54533 & 213.26 & 283.09 & 261 \\
\hline 94.04433 & 214.07 & 285.14 & 261.86 \\
\hline 94.54417 & 214.27 & 286.48 & 261.53 \\
\hline 95.04483 & 214.49 & 285.66 & 261.29 \\
\hline 95.54467 & 215.31 & 290.17 & 261.25 \\
\hline 96.04533 & 216.23 & 289.2 & 261.69 \\
\hline 96.54433 & 216.96 & 291 & 261.47 \\
\hline 97.04501 & 216.07 & 288.8 & 261.14 \\
\hline 97.544 & 216.05 & 287.56 & 260.63 \\
\hline 98.04567 & 215.91 & 285.91 & 261.75 \\
\hline 98.54533 & 214.98 & 287.13 & 261.81 \\
\hline 99.04517 & 215.25 & 285.26 & 261.83 \\
\hline 99.54417 & 214.82 & 285.43 & 261.18 \\
\hline 100.0448 & 214.63 & 284.14 & 261.84 \\
\hline 100.5447 & 214.94 & 285.27 & 261.59 \\
\hline 101.0455 & 214.74 & 283.74 & 261.91 \\
\hline 101.5443 & 214.42 & 283.54 & 261.26 \\
\hline 102.045 & 213.62 & 281.89 & 261.08 \\
\hline 102.5448 & 213.17 & 280.33 & 261.27 \\
\hline 103.0457 & 212.49 & 278.11 & 261.18 \\
\hline 103.5455 & 212.92 & 276.86 & 262.07 \\
\hline 104.0453 & 212.93 & 275.86 & 260.3 \\
\hline 104.5452 & 213.88 & 275.38 & 260.93 \\
\hline 105.044 & 213.98 & 278.4 & 261.59 \\
\hline
\end{tabular}




\begin{tabular}{|c|c|c|c|}
\hline 105.5457 & 213.9 & 277.33 & 261.74 \\
\hline 106.0455 & 213.99 & 279.15 & 261.25 \\
\hline 106.5453 & 214.08 & 281.63 & 261.7 \\
\hline 107.0442 & 215.6 & 281.79 & 261.55 \\
\hline 107.545 & 216.02 & 283.36 & 260.99 \\
\hline 108.0457 & 216.14 & 283.43 & 261.52 \\
\hline 108.5465 & 215.77 & 282.65 & 261.41 \\
\hline 109.0462 & 215.34 & 282.78 & 261.61 \\
\hline 109.5452 & 215.43 & 281.47 & 261.56 \\
\hline 110.045 & 214.92 & 280.7 & 260.84 \\
\hline 110.5448 & 215.15 & 279.49 & 259.34 \\
\hline 111.0455 & 215.53 & 280.39 & 261.57 \\
\hline 111.5453 & 215.05 & 280.01 & 261.6 \\
\hline 112.0452 & 214.3 & 281.63 & 261.69 \\
\hline 112.544 & 213.67 & 282.74 & 262.03 \\
\hline 113.0438 & 213.46 & 281.03 & 261.9 \\
\hline 113.5455 & 213.34 & 281.92 & 262.21 \\
\hline 114.0453 & 213.27 & 284.69 & 262.24 \\
\hline 114.5452 & 213.75 & 283.02 & 262.71 \\
\hline 115.045 & 213.16 & 281.45 & 262.75 \\
\hline 115.5448 & 213.38 & 281.57 & 262.89 \\
\hline 116.0455 & 213.97 & 280.12 & 260.84 \\
\hline 116.5453 & 213.31 & 278.97 & 260.59 \\
\hline 117.0452 & 213.81 & 280.98 & 261.02 \\
\hline 117.545 & 214.92 & 279.72 & 261.24 \\
\hline 118.0448 & 215.34 & 276.54 & 257.92 \\
\hline 118.5447 & 214.96 & 275.05 & 259.61 \\
\hline 119.0445 & 213.91 & 275.73 & 262.36 \\
\hline 119.5453 & 213.73 & 272.25 & 261.98 \\
\hline 120.046 & 213.34 & 271.53 & 261.25 \\
\hline 120.5448 & 212.71 & 271.55 & 261.84 \\
\hline 121.0447 & 212.86 & 274.87 & 261.22 \\
\hline 121.5455 & 213.9 & 273.06 & 260.05 \\
\hline 122.0453 & 214.21 & 274.33 & 262.42 \\
\hline 122.5452 & 212.94 & 276.02 & 262.38 \\
\hline 123.045 & 213.74 & 275.17 & 262.55 \\
\hline 123.5457 & 213.12 & 276.79 & 262.72 \\
\hline 124.0455 & 212.92 & 276.55 & 262.88 \\
\hline 124.5453 & 213.62 & 276.66 & 263.07 \\
\hline 125.0452 & 214.27 & 278.17 & 261.25 \\
\hline 125.545 & 214.24 & 276.96 & 260.7 \\
\hline 126.0457 & 214.15 & 277.02 & 261.44 \\
\hline 126.5455 & 214.17 & 277.56 & 261.59 \\
\hline 127.0453 & 213.27 & 276.02 & 261.44 \\
\hline 127.5452 & 213.42 & 274.44 & 261.37 \\
\hline 128.045 & 213.37 & 274.87 & 260.68 \\
\hline 128.5457 & 213.07 & 273.74 & 260.88 \\
\hline 129.0455 & 213.23 & 273.71 & 261.59 \\
\hline 129.5453 & 212.68 & 272.23 & 259.93 \\
\hline 130.0452 & 212.91 & 273.49 & 261.77 \\
\hline 130.545 & 213.47 & 274.51 & 261.7 \\
\hline 131.0438 & 214.18 & 273.87 & 260.69 \\
\hline 131.5455 & 213.69 & 272.26 & 262.08 \\
\hline 132.0445 & 213.53 & 271.4 & 262.24 \\
\hline 132.5443 & 213.17 & 272.79 & 260.93 \\
\hline 133.045 & 213.16 & 270.05 & 262.09 \\
\hline 133.5448 & 213.21 & 269.88 & 262.44 \\
\hline 134.0447 & 212.8 & 268.75 & 262.39 \\
\hline 134.5453 & 213.16 & 268.15 & 262.35 \\
\hline 135.0452 & 213.15 & 266.98 & 261.93 \\
\hline 135.545 & 213.2 & 265.63 & 261.38 \\
\hline 136.0448 & 213.1 & 265.69 & 261.18 \\
\hline 136.5447 & 213.51 & 266.66 & 261.04 \\
\hline 137.0445 & 214.17 & 266.64 & 258.64 \\
\hline 137.5453 & 214.37 & 266.6 & 258.05 \\
\hline 138.0442 & 213.37 & 267.14 & 258.09 \\
\hline
\end{tabular}




\begin{tabular}{|c|c|c|c|}
\hline 138.544 & 214.64 & 268.51 & 258.05 \\
\hline 139.0457 & 215 & 268.09 & 257.84 \\
\hline 139.5445 & 215.24 & 268.36 & 258.35 \\
\hline 140.0462 & 214.1 & 269.41 & 259.1 \\
\hline 140.5452 & 215.73 & 275.77 & 261.87 \\
\hline 141.045 & 214.87 & 276.22 & 263.39 \\
\hline 141.5457 & 214.35 & 279.76 & 263.48 \\
\hline 142.0455 & 214.23 & 279.08 & 262.24 \\
\hline 142.5453 & 214.28 & 278.49 & 261.19 \\
\hline 143.0452 & 213.82 & 274.12 & 260.92 \\
\hline 143.545 & 213.82 & 273.58 & 260.99 \\
\hline 144.0457 & 214.2 & 272.92 & 261.17 \\
\hline 144.5455 & 213.95 & 269.98 & 260.39 \\
\hline 145.0453 & 214.46 & 271.21 & 260.16 \\
\hline 145.5442 & 213.17 & 269.69 & 259.37 \\
\hline 146.045 & 213.1 & 269.21 & 259.59 \\
\hline 146.5448 & 212.67 & 268.14 & 259.38 \\
\hline 147.0455 & 212.89 & 266.83 & 261.3 \\
\hline 147.5453 & 213 & 268.62 & 259.97 \\
\hline 148.0452 & 212.72 & 266.82 & 261.88 \\
\hline 148.544 & 212.85 & 268.61 & 261.42 \\
\hline 149.0448 & 212.61 & 267.38 & 261.22 \\
\hline 149.5455 & 212.63 & 268.02 & 260.3 \\
\hline 150.0453 & 212.83 & 265.17 & 261.31 \\
\hline 150.5452 & 213.1 & 264.34 & 260.61 \\
\hline 151.0442 & 213.18 & 263.86 & 257.76 \\
\hline 151.544 & 213.41 & 263.11 & 259.26 \\
\hline 152.0455 & 213.83 & 262.5 & 260.18 \\
\hline 152.5453 & 213.43 & 263.22 & 259.83 \\
\hline 153.0443 & 213.71 & 263.76 & 260.78 \\
\hline 153.546 & 213.78 & 264.83 & 260.34 \\
\hline 154.0448 & 213.19 & 265.4 & 261.64 \\
\hline 154.5457 & 213.18 & 265.42 & 261.39 \\
\hline 155.0455 & 213.79 & 265.71 & 261.48 \\
\hline 155.5453 & 213.88 & 266.83 & 261.88 \\
\hline 156.045 & 214.56 & 267.1 & 261.93 \\
\hline 156.5458 & 214.31 & 267.97 & 261.94 \\
\hline 157.0465 & 214.35 & 268.6 & 262.2 \\
\hline 157.5455 & 214.8 & 268.37 & 261.88 \\
\hline 158.0443 & 214.61 & 268.38 & 261.89 \\
\hline 158.5452 & 214.72 & 268.3 & 261.91 \\
\hline 159.044 & 214.97 & 268.94 & 259.21 \\
\hline 159.5447 & 214.95 & 268.05 & 259.55 \\
\hline 160.0455 & 214.88 & 267.62 & 261.04 \\
\hline 160.5443 & 215.26 & 268.1 & 262.3 \\
\hline 161.0452 & 215.18 & 268.61 & 262.56 \\
\hline 161.544 & 214.96 & 270.09 & 260.06 \\
\hline 162.0457 & 214.2 & 268.99 & 260.44 \\
\hline 162.5455 & 214.7 & 268.64 & 260.02 \\
\hline 163.0443 & 215.33 & 268.67 & 259.85 \\
\hline 163.5452 & 215.87 & 268.6 & 259.51 \\
\hline 164.044 & 215.46 & 269.13 & 260.63 \\
\hline 164.5438 & 215.25 & 269.72 & 261.15 \\
\hline 165.0455 & 215.47 & 270.08 & 261.8 \\
\hline 165.5453 & 215.68 & 274.43 & 262.07 \\
\hline 166.0443 & 215.79 & 276.86 & 260.71 \\
\hline 166.545 & 215.54 & 275.23 & 261.15 \\
\hline 167.0448 & 215.12 & 272.78 & 262.9 \\
\hline 167.5447 & 214.44 & 275.67 & 257.68 \\
\hline 168.0453 & 214.91 & 268.5 & 261.56 \\
\hline 168.5452 & 214.12 & 270.42 & 257.53 \\
\hline 169.045 & 213.39 & 266.44 & 259.93 \\
\hline 169.5448 & 213.88 & 266.75 & 261.1 \\
\hline 170.0455 & 213.43 & 268.93 & 259.69 \\
\hline 170.5463 & 213.4 & 265.75 & 259.56 \\
\hline 171.047 & 213.11 & 268.96 & 261.49 \\
\hline
\end{tabular}




\begin{tabular}{|c|c|c|c|}
\hline 171.546 & 213.67 & 267.29 & 262.36 \\
\hline 172.0458 & 213.66 & 264.57 & 258.69 \\
\hline 172.5465 & 213.86 & 266.63 & 261.23 \\
\hline 173.0463 & 212.91 & 266.55 & 262.11 \\
\hline 173.547 & 213.32 & 268.15 & 262.68 \\
\hline 174.046 & 213.95 & 268.04 & 262.79 \\
\hline 174.5458 & 213.99 & 266.4 & 257.48 \\
\hline 175.0465 & 213.37 & 266.6 & 256.81 \\
\hline 175.5463 & 214.41 & 264.05 & 257.7 \\
\hline 176.0472 & 213.71 & 263.93 & 257.63 \\
\hline 176.5452 & 213.63 & 264.09 & 259.3 \\
\hline 177.045 & 213.33 & 263.91 & 259.11 \\
\hline 177.5447 & 213.72 & 271.17 & 258.5 \\
\hline 178.0455 & 214.98 & 269.73 & 259.84 \\
\hline 178.5453 & 214.17 & 272.4 & 261.12 \\
\hline 179.0452 & 214.8 & 270.8 & 261.59 \\
\hline 179.545 & 214.02 & 271.72 & 262.84 \\
\hline 180.0457 & 213.27 & 270.87 & 263.01 \\
\hline 180.5465 & 212.99 & 270.52 & 262.71 \\
\hline 181.0443 & 214.33 & 267.37 & 257.02 \\
\hline 181.5442 & 214.53 & 264.69 & 256.43 \\
\hline 182.045 & 213.75 & 265.05 & 256.33 \\
\hline 182.5448 & 212.26 & 264.29 & 256.18 \\
\hline 183.0447 & 213.07 & 263.49 & 256.69 \\
\hline 183.5453 & 213.99 & 263.46 & 256.68 \\
\hline 184.0452 & 213.18 & 263.35 & 256.59 \\
\hline 184.545 & 212.88 & 263.73 & 256.6 \\
\hline 185.0448 & 213.26 & 263.95 & 256.62 \\
\hline 185.5455 & 212.29 & 264.28 & 256.78 \\
\hline 186.0453 & 212.66 & 264.56 & 256.85 \\
\hline 186.5452 & 213.34 & 263.82 & 257.19 \\
\hline 187.045 & 212.75 & 262.66 & 256.99 \\
\hline 187.5448 & 212.77 & 263.41 & 257.01 \\
\hline 188.0455 & 212.43 & 263.94 & 256.76 \\
\hline 188.5453 & 213.08 & 262.96 & 257.24 \\
\hline 189.0452 & 212.58 & 262.77 & 256.54 \\
\hline 189.545 & 212.55 & 263.52 & 256.87 \\
\hline 190.0448 & 212.08 & 263.87 & 256.38 \\
\hline 190.5457 & 212.23 & 262.51 & 256.83 \\
\hline 191.0455 & 212.88 & 263.99 & 256.4 \\
\hline 191.5462 & 213.24 & 262.13 & 257.01 \\
\hline 192.045 & 212.34 & 262.65 & 256.79 \\
\hline 192.5448 & 213.01 & 261.77 & 256.8 \\
\hline 193.0457 & 213.78 & 264.42 & 257.16 \\
\hline 193.5455 & 213.57 & 262.43 & 257.15 \\
\hline 194.0453 & 213.18 & 264.7 & 256.98 \\
\hline 194.5452 & 213.23 & 262.97 & 257.35 \\
\hline 195.045 & 213.5 & 263.27 & 257.18 \\
\hline 195.5457 & 212.93 & 263.71 & 257.12 \\
\hline 196.0455 & 213.8 & 263.37 & 256.69 \\
\hline 196.5453 & 214.41 & 263.8 & 257.35 \\
\hline 197.0452 & 214.46 & 263.33 & 257.63 \\
\hline 197.545 & 214.14 & 264.72 & 257.74 \\
\hline 198.0457 & 214.02 & 264.48 & 257.22 \\
\hline 198.5465 & 213.77 & 264.17 & 257.4 \\
\hline 199.0453 & 213.52 & 263.9 & 257.55 \\
\hline 199.5442 & 213.76 & 264.05 & 257.54 \\
\hline 200.045 & 213.08 & 262.74 & 257.59 \\
\hline 200.5448 & 212.99 & 261.92 & 257.35 \\
\hline 201.0455 & 214.39 & 262.27 & 257.15 \\
\hline 201.5453 & 213.91 & 262.25 & 258.06 \\
\hline 202.0452 & 213.16 & 262.99 & 257.23 \\
\hline 202.545 & 214.09 & 262.52 & 258.32 \\
\hline 203.0448 & 214.15 & 262.97 & 257.64 \\
\hline 203.5455 & 214.58 & 263.56 & 257.73 \\
\hline 204.0453 & 214.99 & 264.15 & 257.48 \\
\hline
\end{tabular}




\begin{tabular}{|c|c|c|c|}
\hline 204.5452 & 214.22 & 267.55 & 257.64 \\
\hline 205.045 & 214.63 & 267.79 & 258.66 \\
\hline 205.5448 & 213.64 & 268.86 & 258.68 \\
\hline 206.0455 & 213.26 & 268.01 & 260.03 \\
\hline 206.5453 & 214.16 & 268.36 & 259.76 \\
\hline 207.048 & 225.61 & 262.02 & 257.08 \\
\hline 207.5478 & 225.27 & 260.07 & 256.45 \\
\hline 208.0467 & 225.08 & 259.25 & 256.08 \\
\hline 208.5483 & 224.18 & 258.55 & 256.41 \\
\hline 209.0473 & 222.75 & 258.8 & 256.2 \\
\hline 209.548 & 222.45 & 258.58 & 256.86 \\
\hline 210.0478 & 222.68 & 258.77 & 255.71 \\
\hline 210.5477 & 223.01 & 258.83 & 257.05 \\
\hline 211.0483 & 222.71 & 258.8 & 257.97 \\
\hline 211.5482 & 222.37 & 259.24 & 258.19 \\
\hline 212.048 & 222.17 & 259.9 & 256.68 \\
\hline 212.5478 & 221.94 & 259.76 & 257.8 \\
\hline 213.0477 & 222.16 & 258.6 & 255.8 \\
\hline 213.5475 & 221.8 & 259 & 258.18 \\
\hline 214.0482 & 222.11 & 259.71 & 258.16 \\
\hline 214.548 & 222.18 & 257.53 & 254.48 \\
\hline 215.0478 & 221.29 & 256.41 & 254.74 \\
\hline 215.5468 & 220.67 & 256.09 & 254.33 \\
\hline 216.0475 & 220.81 & 255.95 & 255.39 \\
\hline 216.5483 & 221.53 & 256.3 & 254.49 \\
\hline 217.048 & 222.37 & 257.89 & 255.48 \\
\hline 217.5478 & 221.64 & 258.79 & 256.22 \\
\hline 218.0468 & 223.22 & 259.11 & 255.83 \\
\hline 218.5475 & 222.29 & 258.98 & 256.07 \\
\hline 219.0483 & 222.65 & 258.84 & 255.79 \\
\hline 219.5482 & 223.33 & 259.04 & 255.65 \\
\hline 220.0488 & 223.3 & 258.97 & 255.28 \\
\hline 220.5477 & 223.17 & 259.37 & 256.04 \\
\hline 221.0485 & 224.96 & 259.47 & 256.37 \\
\hline 221.5483 & 225.61 & 259.38 & 256.4 \\
\hline 222.0482 & 224.07 & 259.13 & 255.83 \\
\hline 222.548 & 223.52 & 258.66 & 255.75 \\
\hline 223.0478 & 223.74 & 258.09 & 255.89 \\
\hline 223.5477 & 224.96 & 257.53 & 255.87 \\
\hline 224.0483 & 223.38 & 257.22 & 255.46 \\
\hline 224.5482 & 223.35 & 255.83 & 253.18 \\
\hline 225.047 & 221.92 & 255.28 & 253.53 \\
\hline 225.5478 & 221.78 & 255.74 & 253.68 \\
\hline 226.0477 & 221.37 & 256.18 & 254.21 \\
\hline 226.5483 & 221.66 & 256.1 & 254.77 \\
\hline 227.0482 & 222.38 & 256.55 & 254.18 \\
\hline 227.547 & 223.67 & 256.48 & 254.67 \\
\hline 228.0478 & 223.06 & 256.79 & 255.1 \\
\hline 228.5477 & 221.99 & 257.24 & 254.94 \\
\hline 229.0483 & 223.44 & 257.81 & 255.58 \\
\hline 229.5482 & 223.98 & 257.98 & 255.81 \\
\hline 230.048 & 223.19 & 257.91 & 255.66 \\
\hline 230.5478 & 222.71 & 258.13 & 255.87 \\
\hline 231.0468 & 224.09 & 258.37 & 255.24 \\
\hline 231.5483 & 223.58 & 258.55 & 255.55 \\
\hline 232.0473 & 224.01 & 258.93 & 255.57 \\
\hline 232.548 & 224.4 & 258.97 & 258.27 \\
\hline 233.0478 & 223.19 & 258.19 & 255.01 \\
\hline 233.5487 & 222.94 & 259.22 & 256.53 \\
\hline 234.0485 & 223.23 & 259.78 & 257.44 \\
\hline 234.5473 & 223.69 & 260.39 & 257.87 \\
\hline 235.048 & 223.11 & 260.93 & 258.22 \\
\hline 235.5478 & 223.7 & 260.42 & 255.8 \\
\hline 236.0487 & 223.54 & 258.97 & 255.45 \\
\hline 236.5467 & 224.85 & 258.58 & 255.72 \\
\hline 237.0473 & 224.35 & 258.71 & 255.7 \\
\hline
\end{tabular}




\begin{tabular}{|c|c|c|c|}
\hline 237.5482 & 221.92 & 258.65 & 256.53 \\
\hline 238.048 & 223.92 & 258.78 & 255.35 \\
\hline 238.5477 & 222.82 & 258.74 & 255.85 \\
\hline 239.0467 & 223.1 & 258.75 & 256.17 \\
\hline 239.5483 & 224.84 & 258.62 & 256.07 \\
\hline 240.0482 & 224.47 & 258.32 & 255.77 \\
\hline 240.548 & 222.54 & 258.24 & 256.31 \\
\hline 241.0478 & 223.29 & 258.71 & 255.31 \\
\hline 241.5477 & 224.39 & 259.61 & 255.77 \\
\hline 242.0483 & 223.31 & 260.89 & 256.24 \\
\hline 242.5482 & 225.11 & 262.09 & 256.3 \\
\hline 243.047 & 226.68 & 262.25 & 256.95 \\
\hline 243.5478 & 227.29 & 261.34 & 256.49 \\
\hline 244.0477 & 228.05 & 260.36 & 256.33 \\
\hline 244.5483 & 225.94 & 259.38 & 256.44 \\
\hline 245.0482 & 212.78 & 273.2 & 263.76 \\
\hline 245.548 & 212.88 & 273.35 & 263.24 \\
\hline 246.0478 & 212.88 & 273.37 & 262.92 \\
\hline 246.5477 & 212.62 & 273.88 & 263.67 \\
\hline 247.0483 & 212.49 & 273.37 & 264.23 \\
\hline 247.5473 & 212.64 & 273.35 & 264.25 \\
\hline 248.048 & 212.72 & 273.39 & 263.39 \\
\hline 248.5478 & 212.81 & 273.42 & 263.91 \\
\hline 249.0467 & 212.83 & 273.24 & 263.34 \\
\hline 249.5475 & 212.87 & 273.73 & 262.94 \\
\hline 250.0492 & 213.07 & 273.27 & 262.76 \\
\hline 250.5472 & 213.02 & 272.7 & 263.05 \\
\hline 251.047 & 212.86 & 272.94 & 263.17 \\
\hline 251.5477 & 212.63 & 272.57 & 263.28 \\
\hline 252.0485 & 212.64 & 272.99 & 263.48 \\
\hline 252.5482 & 212.5 & 272.8 & 263.31 \\
\hline 253.048 & 212.52 & 272.6 & 263.43 \\
\hline 253.5488 & 212.47 & 272.86 & 263.48 \\
\hline 254.0477 & 212.51 & 273.37 & 263.86 \\
\hline 254.5475 & 212.51 & 272.88 & 263.64 \\
\hline 255.0473 & 212.59 & 272.82 & 263.3 \\
\hline 255.5482 & 212.82 & 273.12 & 263.78 \\
\hline 256.048 & 212.79 & 273.04 & 263.44 \\
\hline 256.5477 & 212.42 & 273.69 & 263.06 \\
\hline 257.0467 & 212.33 & 273.43 & 250.72 \\
\hline
\end{tabular}

\section{Run 7 (steam-petroleum distillate case)}

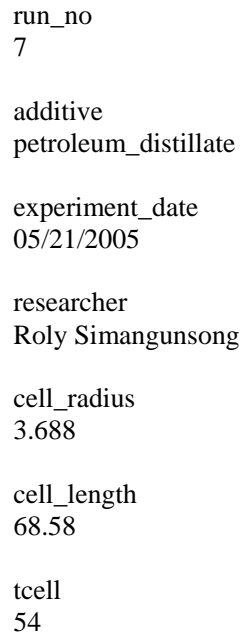




Cs
0.21
Co
0.5
Cw
1
dens_sand
167
dens_oil
62

dens_water
62.4
por
0.411690173

Soi

0.726148715592812

Sor

0.390594349450943

Sgi

0.0936861640173443

Swc

0.16516512

porvol

1162.444515

void_length

2.58

o_w_ratio

0.2

iw

5.5

tfu_total

8

ratio

0.05

Hv_enthalphy

0.5466

Lv_enthalphy

185.466

PI

6.95

Oil_visc

95

res_heat_capacity

34.00 
heat_loss_coeff

15

t_phase

40

-- Time, minutes Cumulative Oil, cc

Production_Data_Tabulation

$\begin{array}{ll}0 & 0 \\ 1 & 0 \\ 3 & 0 \\ 5 & 0 \\ 7 & 0 \\ 9 & 0 \\ 11 & 0 \\ 13 & 0 \\ 15 & 14.5 \\ 16 & 33.5 \\ 17 & 52 \\ 27 & 66.5 \\ 32 & 73.5 \\ 34 & 82 \\ 36 & 97 \\ 38 & 110 \\ 41 & 128 \\ 45 & 146 \\ 50 & 165 \\ 55 & 184 \\ 59 & 204 \\ 63 & 228 \\ 67 & 251 \\ 71 & 270 \\ 74 & 293 \\ 78 & 314 \\ 82 & 329 \\ 85 & 348 \\ 89 & 359 \\ 92 & 370 \\ 96 & 373.5 \\ 99 & 378.5 \\ 102 & 383.5 \\ 106 & 395.5 \\ 114 & 399.5 \\ 117 & 407.5 \\ 121 & 422.5 \\ 123 & 426.5 \\ 128 & 430.5 \\ 135 & 432.5 \\ 140 & 434 \\ 145 & 435.5 \\ 152 & 436.5 \\ 158 & 437.5 \\ 164 & 438.5 \\ 170 & 439 \\ 178 & 439.5 \\ 180 & 440.5 \\ 190 & 441.5 \\ 196 & \\ 205 & \text { from top of flange, cm } \\ 220 & \text { time, min } \\ 229 & \end{array}$

Thermo_Tabulation 


$\begin{array}{ll}43 & 12 \\ 70 & 27.5 \\ 103 & 42.8 \\ 125 & 68.3\end{array}$

\begin{tabular}{|c|c|c|c|}
\hline \multirow{2}{*}{\multicolumn{4}{|c|}{$\begin{array}{l}\text {-- Time, minutes Tinj, oC } \\
\text { Log_Data_Tabulation }\end{array}$}} \\
\hline & & & \\
\hline 0.053 & 48.62 & 270.44 & 261.21 \\
\hline 0.5473 & 52.71 & 268.15 & 261.32 \\
\hline 1.048 & 61.98 & 260.61 & 261.36 \\
\hline 1.5478 & 79.98 & 260.50 & 261.26 \\
\hline 2.0468 & 97.43 & 260.93 & 261.33 \\
\hline 2.5475 & 171.13 & 265.14 & 261.75 \\
\hline 3.0483 & 202.29 & 265.92 & 261.02 \\
\hline 3.5482 & 204.94 & 269.77 & 260.92 \\
\hline 4.048 & 206.19 & 273.95 & 261.07 \\
\hline 4.5468 & 208.09 & 276.00 & 260.89 \\
\hline 5.0475 & 210.61 & 277.95 & 260.88 \\
\hline 5.5473 & 213.46 & 278.65 & 260.87 \\
\hline 6.0482 & 214.39 & 279.17 & 260.85 \\
\hline 6.548 & 214.57 & 279.88 & 260.85 \\
\hline 7.0478 & 214.48 & 281.20 & 260.84 \\
\hline 7.5477 & 214.97 & 293.51 & 260.93 \\
\hline 8.0483 & 216.00 & 297.46 & 260.89 \\
\hline 8.5482 & 217.29 & 303.00 & 260.88 \\
\hline 9.048 & 219.42 & 308.62 & 260.91 \\
\hline 9.5478 & 220.87 & 306.70 & 260.83 \\
\hline 10.0477 & 221.04 & 308.29 & 260.83 \\
\hline 10.5483 & 222.02 & 310.22 & 260.80 \\
\hline 11.0492 & 223.13 & 313.13 & 260.80 \\
\hline 11.548 & 223.42 & 318.22 & 260.82 \\
\hline 12.0478 & 223.65 & 318.86 & 260.81 \\
\hline 12.5477 & 218.64 & 314.41 & 260.80 \\
\hline 13.0483 & 218.00 & 313.54 & 260.78 \\
\hline 13.5473 & 218.07 & 314.92 & 260.77 \\
\hline 14.048 & 219.08 & 315.37 & 260.79 \\
\hline 14.5478 & 221.82 & 313.50 & 260.86 \\
\hline 15.0468 & 231.12 & 310.16 & 260.77 \\
\hline 15.5475 & 238.20 & 308.04 & 260.73 \\
\hline 16.0482 & 241.25 & 304.80 & 260.71 \\
\hline 16.548 & 241.54 & 305.11 & 260.72 \\
\hline 17.047 & 241.07 & 306.56 & 261.27 \\
\hline 17.5477 & 239.50 & 307.03 & 259.11 \\
\hline 18.0485 & 235.72 & 307.11 & 259.59 \\
\hline 18.5473 & 224.74 & 306.95 & 258.09 \\
\hline 19.048 & 217.55 & 304.50 & 257.05 \\
\hline 19.5478 & 216.53 & 303.38 & 258.02 \\
\hline 20.0487 & 216.43 & 304.42 & 259.01 \\
\hline 20.5475 & 216.75 & 307.00 & 258.84 \\
\hline 21.0483 & 217.29 & 309.70 & 261.45 \\
\hline 21.5482 & 217.70 & 312.00 & 261.62 \\
\hline 22.047 & 218.08 & 313.29 & 261.73 \\
\hline 22.5477 & 218.44 & 314.43 & 261.49 \\
\hline 23.0475 & 218.69 & 314.75 & 261.58 \\
\hline 23.5483 & 218.66 & 315.33 & 261.57 \\
\hline 24.0482 & 218.86 & 315.56 & 261.57 \\
\hline 24.547 & 219.06 & 316.23 & 261.54 \\
\hline 25.0478 & 219.40 & 316.27 & 261.52 \\
\hline 25.5477 & 219.31 & 316.00 & 261.49 \\
\hline 26.0483 & 219.44 & 316.12 & 261.44 \\
\hline 26.5482 & 219.91 & 317.64 & 261.42 \\
\hline 27.047 & 220.18 & 318.51 & 261.42 \\
\hline 27.5478 & 221.58 & 320.73 & 261.39 \\
\hline 28.0477 & 221.99 & 321.52 & 261.24 \\
\hline 28.5483 & 222.33 & 321.72 & 261.24 \\
\hline
\end{tabular}




\begin{tabular}{|c|c|c|c|}
\hline 29.0482 & 225.72 & 321.23 & 261.24 \\
\hline 29.548 & 231.74 & 320.79 & 261.21 \\
\hline 30.0478 & 231.08 & 320.97 & 261.23 \\
\hline 30.5485 & 227.11 & 320.84 & 261.19 \\
\hline 31.0483 & 222.71 & 320.57 & 261.21 \\
\hline 31.5473 & 221.89 & 319.80 & 261.21 \\
\hline 32.048 & 226.74 & 320.54 & 261.17 \\
\hline 32.5478 & 233.15 & 320.38 & 261.19 \\
\hline 33.0477 & 235.92 & 319.28 & 261.15 \\
\hline 33.5483 & 235.88 & 318.68 & 260.42 \\
\hline 34.0482 & 234.08 & 318.38 & 262.53 \\
\hline 34.548 & 234.01 & 318.27 & 263.46 \\
\hline 35.0478 & 235.09 & 318.84 & 263.23 \\
\hline 35.5477 & 234.57 & 317.48 & 263.00 \\
\hline 36.0485 & 235.58 & 316.78 & 262.03 \\
\hline 36.5473 & 236.67 & 315.86 & 255.07 \\
\hline 37.048 & 237.09 & 314.19 & 248.94 \\
\hline 37.5478 & 236.65 & 312.99 & 249.87 \\
\hline 38.0477 & 235.86 & 312.25 & 250.28 \\
\hline 38.5475 & 236.35 & 311.49 & 251.54 \\
\hline 39.0483 & 236.71 & 311.36 & 251.26 \\
\hline 39.5482 & 236.41 & 310.66 & 251.99 \\
\hline 40.0488 & 236.52 & 310.66 & 252.71 \\
\hline 40.5477 & 235.13 & 310.77 & 252.28 \\
\hline 41.0467 & 234.45 & 311.44 & 253.02 \\
\hline 41.5473 & 234.99 & 311.79 & 253.75 \\
\hline 42.0482 & 235.62 & 312.33 & 253.54 \\
\hline 42.548 & 235.13 & 312.79 & 253.32 \\
\hline 43.0478 & 235.85 & 313.31 & 253.54 \\
\hline 43.5477 & 236.69 & 313.59 & 254.17 \\
\hline 44.0483 & 236.95 & 314.24 & 254.16 \\
\hline 44.5482 & 235.81 & 314.07 & 253.58 \\
\hline 45.048 & 236.22 & 314.38 & 254.31 \\
\hline 45.5478 & 236.66 & 315.08 & 253.80 \\
\hline 46.0477 & 235.91 & 315.62 & 254.41 \\
\hline 46.5483 & 235.16 & 316.56 & 254.97 \\
\hline 47.0482 & 235.61 & 316.81 & 254.80 \\
\hline 47.548 & 236.89 & 317.39 & 254.59 \\
\hline 48.0478 & 236.79 & 317.18 & 254.53 \\
\hline 48.5477 & 236.29 & 318.02 & 255.06 \\
\hline 49.0483 & 234.66 & 318.31 & 255.61 \\
\hline 49.5473 & 235.13 & 319.10 & 255.25 \\
\hline 50.048 & 236.46 & 319.15 & 255.55 \\
\hline 50.5478 & 237.11 & 319.68 & 255.38 \\
\hline 51.0477 & 236.04 & 319.65 & 256.52 \\
\hline 51.5483 & 235.63 & 320.24 & 256.14 \\
\hline 52.0482 & 236.30 & 320.55 & 256.40 \\
\hline 52.548 & 238.12 & 321.26 & 255.39 \\
\hline 53.0478 & 237.84 & 320.76 & 255.30 \\
\hline 53.5477 & 235.69 & 321.55 & 256.04 \\
\hline 54.0475 & 224.77 & 322.15 & 255.72 \\
\hline 54.5483 & 221.43 & 322.67 & 255.65 \\
\hline 55.048 & 220.47 & 322.49 & 255.15 \\
\hline 55.5478 & 223.44 & 322.98 & 255.76 \\
\hline 56.0468 & 229.73 & 322.87 & 256.29 \\
\hline 56.5475 & 235.42 & 323.05 & 255.55 \\
\hline 57.0483 & 240.00 & 323.08 & 256.49 \\
\hline 57.5482 & 241.64 & 323.20 & 257.00 \\
\hline 58.047 & 236.11 & 323.55 & 256.48 \\
\hline 58.5477 & 224.15 & 322.97 & 255.49 \\
\hline 59.0475 & 220.63 & 323.08 & 255.83 \\
\hline 59.5483 & 220.48 & 322.97 & 256.38 \\
\hline 60.0482 & 220.84 & 323.17 & 256.42 \\
\hline 60.548 & 222.42 & 323.37 & 256.75 \\
\hline 61.0478 & 225.61 & 323.40 & 257.12 \\
\hline 61.5477 & 231.92 & 324.52 & 256.07 \\
\hline
\end{tabular}




\begin{tabular}{|c|c|c|c|}
\hline 62.0483 & 236.31 & 324.70 & 256.13 \\
\hline 62.5482 & 238.84 & 324.69 & 256.05 \\
\hline 63.047 & 238.81 & 324.97 & 255.89 \\
\hline 63.5478 & 227.01 & 325.28 & 257.14 \\
\hline 64.0477 & 221.54 & 324.88 & 256.97 \\
\hline 64.5483 & 221.28 & 324.50 & 257.32 \\
\hline 65.0482 & 221.66 & 324.62 & 256.92 \\
\hline 65.5472 & 222.97 & 324.67 & 256.17 \\
\hline 66.0478 & 227.05 & 324.76 & 257.28 \\
\hline 66.5477 & 232.48 & 325.10 & 257.26 \\
\hline 67.0483 & 237.43 & 325.38 & 256.30 \\
\hline 67.5482 & 239.46 & 325.31 & 256.61 \\
\hline 68.0472 & 239.18 & 325.05 & 257.26 \\
\hline 68.5478 & 239.31 & 325.61 & 256.86 \\
\hline 69.0477 & 238.56 & 325.80 & 256.45 \\
\hline 69.5483 & 238.25 & 325.26 & 257.05 \\
\hline 70.0482 & 240.76 & 325.13 & 256.75 \\
\hline 70.5472 & 244.35 & 324.60 & 258.10 \\
\hline 71.0478 & 245.74 & 324.40 & 257.33 \\
\hline 71.5477 & 242.42 & 324.28 & 257.80 \\
\hline 72.0485 & 239.17 & 324.26 & 258.62 \\
\hline 72.5473 & 236.99 & 323.89 & 258.66 \\
\hline 73.048 & 236.26 & 324.04 & 258.29 \\
\hline 73.5478 & 236.81 & 323.51 & 258.61 \\
\hline 74.0468 & 240.50 & 323.38 & 259.34 \\
\hline 74.5475 & 244.59 & 323.13 & 259.56 \\
\hline 75.0483 & 246.67 & 322.60 & 259.57 \\
\hline 75.5482 & 246.23 & 322.09 & 258.92 \\
\hline 76.048 & 241.57 & 321.30 & 258.90 \\
\hline 76.5477 & 235.11 & 320.97 & 259.27 \\
\hline 77.0467 & 229.99 & 320.61 & 258.73 \\
\hline 77.5483 & 229.24 & 320.15 & 258.21 \\
\hline 78.0482 & 232.40 & 319.63 & 258.68 \\
\hline 78.548 & 237.12 & 319.77 & 258.73 \\
\hline 79.0468 & 241.87 & 319.63 & 259.53 \\
\hline 79.5477 & 244.87 & 318.91 & 260.25 \\
\hline 80.0483 & 245.68 & 317.92 & 258.97 \\
\hline 80.5472 & 245.20 & 317.21 & 258.60 \\
\hline 81.048 & 242.73 & 316.96 & 258.99 \\
\hline 81.5478 & 238.56 & 316.25 & 258.74 \\
\hline 82.0477 & 235.61 & 315.41 & 258.86 \\
\hline 82.5483 & 236.49 & 315.27 & 258.70 \\
\hline 83.0482 & 239.24 & 314.47 & 258.71 \\
\hline 83.548 & 242.85 & 314.32 & 259.57 \\
\hline 84.0478 & 244.63 & 313.63 & 259.26 \\
\hline 84.5477 & 244.71 & 312.58 & 259.68 \\
\hline 85.0483 & 243.73 & 311.74 & 258.92 \\
\hline 85.5473 & 242.44 & 311.24 & 259.37 \\
\hline 86.0472 & 239.81 & 310.54 & 259.71 \\
\hline 86.5478 & 237.88 & 310.04 & 259.59 \\
\hline 87.0477 & 238.19 & 309.31 & 258.56 \\
\hline 87.5483 & 239.90 & 308.65 & 259.55 \\
\hline 88.0482 & 240.98 & 308.02 & 259.53 \\
\hline 88.548 & 241.12 & 307.25 & 259.89 \\
\hline 89.0478 & 240.88 & 306.20 & 259.04 \\
\hline 89.5468 & 240.26 & 305.68 & 259.96 \\
\hline 90.0485 & 239.90 & 304.93 & 258.73 \\
\hline 90.5483 & 239.94 & 304.26 & 260.03 \\
\hline 91.0472 & 240.38 & 303.54 & 259.76 \\
\hline 91.5478 & 240.71 & 303.29 & 260.00 \\
\hline 92.0468 & 238.72 & 302.46 & 258.60 \\
\hline 92.5475 & 238.82 & 301.70 & 257.96 \\
\hline 93.0483 & 240.74 & 301.00 & 259.26 \\
\hline 93.5482 & 241.57 & 300.18 & 259.03 \\
\hline 94.048 & 241.52 & 299.40 & 259.30 \\
\hline 94.5477 & 241.29 & 298.43 & 258.98 \\
\hline
\end{tabular}




\begin{tabular}{|c|c|c|c|}
\hline 95.0475 & 241.21 & 297.67 & 258.84 \\
\hline 95.5483 & 240.96 & 297.06 & 259.44 \\
\hline 96.0472 & 240.73 & 296.24 & 259.47 \\
\hline 96.548 & 240.29 & 295.85 & 259.52 \\
\hline 97.0478 & 240.42 & 295.17 & 259.23 \\
\hline 97.5485 & 240.77 & 294.23 & 259.16 \\
\hline 98.0483 & 240.75 & 292.83 & 260.71 \\
\hline 98.5472 & 240.70 & 291.65 & 259.14 \\
\hline 99.048 & 240.37 & 289.75 & 257.94 \\
\hline 99.5478 & 240.09 & 288.24 & 259.46 \\
\hline 100.0485 & 239.88 & 286.95 & 258.60 \\
\hline 100.5475 & 239.77 & 285.95 & 257.49 \\
\hline 101.0482 & 240.03 & 285.62 & 259.79 \\
\hline 101.548 & 239.82 & 285.77 & 259.66 \\
\hline 102.0468 & 239.37 & 286.09 & 258.91 \\
\hline 102.5477 & 240.90 & 286.51 & 260.19 \\
\hline 103.0483 & 242.91 & 287.04 & 261.64 \\
\hline 103.5482 & 243.89 & 287.16 & 258.68 \\
\hline 104.048 & 243.91 & 286.84 & 259.89 \\
\hline 104.5478 & 243.25 & 286.23 & 259.89 \\
\hline 105.0477 & 242.47 & 285.56 & 259.24 \\
\hline 105.5483 & 241.70 & 284.65 & 259.27 \\
\hline 106.0482 & 240.67 & 283.41 & 260.39 \\
\hline 106.548 & 240.03 & 282.45 & 259.86 \\
\hline 107.0478 & 238.51 & 280.90 & 259.72 \\
\hline 107.5477 & 237.54 & 279.30 & 260.19 \\
\hline 108.0485 & 237.19 & 277.66 & 260.57 \\
\hline 108.5483 & 237.37 & 276.49 & 260.02 \\
\hline 109.049 & 237.31 & 275.07 & 260.18 \\
\hline 109.5478 & 237.04 & 274.09 & 260.08 \\
\hline 110.0487 & 239.98 & 276.09 & 259.71 \\
\hline 110.5475 & 240.81 & 276.86 & 260.06 \\
\hline 111.0483 & 241.21 & 278.11 & 260.02 \\
\hline 111.5482 & 241.31 & 279.01 & 260.44 \\
\hline 112.047 & 241.38 & 279.75 & 262.71 \\
\hline 112.5477 & 241.30 & 280.02 & 261.35 \\
\hline 113.0475 & 241.00 & 279.89 & 258.52 \\
\hline 113.5483 & 240.37 & 278.92 & 259.52 \\
\hline 114.0482 & 239.57 & 277.87 & 259.11 \\
\hline 114.547 & 239.18 & 276.82 & 259.17 \\
\hline 115.0468 & 239.13 & 275.91 & 261.10 \\
\hline 115.5477 & 239.60 & 275.81 & 260.60 \\
\hline 116.0483 & 240.50 & 276.15 & 259.83 \\
\hline 116.5472 & 241.35 & 277.11 & 259.82 \\
\hline 117.048 & 241.89 & 278.37 & 260.72 \\
\hline 117.5478 & 242.33 & 279.79 & 262.61 \\
\hline 118.0477 & 242.73 & 281.16 & 262.60 \\
\hline 118.5483 & 243.09 & 282.14 & 262.17 \\
\hline 119.0482 & 243.08 & 281.85 & 259.71 \\
\hline 119.5472 & 242.67 & 280.33 & 260.91 \\
\hline 120.0478 & 242.39 & 278.72 & 260.15 \\
\hline 120.5477 & 242.12 & 276.98 & 261.16 \\
\hline 121.0483 & 241.79 & 275.27 & 260.58 \\
\hline 121.5482 & 241.43 & 273.71 & 261.04 \\
\hline 122.048 & 241.13 & 272.09 & 260.10 \\
\hline 122.5478 & 240.65 & 270.53 & 261.33 \\
\hline 123.0477 & 239.92 & 269.04 & 260.23 \\
\hline 123.5483 & 239.03 & 267.39 & 261.05 \\
\hline 124.0482 & 238.16 & 265.99 & 260.40 \\
\hline 124.548 & 237.98 & 265.21 & 260.15 \\
\hline 125.0478 & 238.00 & 264.27 & 260.75 \\
\hline 125.5477 & 238.37 & 264.08 & 260.13 \\
\hline 126.0485 & 239.91 & 265.66 & 261.20 \\
\hline 126.5483 & 241.47 & 266.72 & 260.29 \\
\hline 127.048 & 240.19 & 268.25 & 261.37 \\
\hline 127.5478 & 239.93 & 268.76 & 261.78 \\
\hline
\end{tabular}




\begin{tabular}{|c|c|c|c|}
\hline 128.0477 & 241.36 & 269.04 & 261.45 \\
\hline 128.5475 & 242.10 & 268.87 & 263.37 \\
\hline 129.0483 & 242.14 & 268.22 & 261.03 \\
\hline 129.5472 & 241.74 & 267.48 & 261.30 \\
\hline 130.048 & 241.33 & 266.62 & 263.03 \\
\hline 130.5477 & 240.63 & 265.82 & 262.42 \\
\hline 131.0475 & 240.05 & 265.09 & 261.49 \\
\hline 131.5483 & 239.37 & 264.53 & 260.28 \\
\hline 132.05 & 240.04 & 265.15 & 261.31 \\
\hline 132.547 & 241.94 & 266.65 & 263.26 \\
\hline 133.0478 & 242.29 & 267.84 & 262.87 \\
\hline 133.5477 & 242.51 & 268.42 & 258.68 \\
\hline 134.0492 & 242.76 & 267.87 & 259.90 \\
\hline 134.5472 & 243.04 & 267.55 & 260.26 \\
\hline 135.048 & 242.90 & 266.99 & 260.18 \\
\hline 135.5478 & 242.72 & 266.28 & 260.06 \\
\hline 136.0477 & 242.46 & 265.27 & 261.01 \\
\hline 136.5493 & 241.98 & 264.37 & 261.33 \\
\hline 137.0482 & 241.07 & 263.37 & 260.14 \\
\hline 137.548 & 239.95 & 262.72 & 261.53 \\
\hline 138.0487 & 238.52 & 261.83 & 259.77 \\
\hline 138.5467 & 237.66 & 261.45 & 259.81 \\
\hline 139.0475 & 236.45 & 261.17 & 260.62 \\
\hline 139.5482 & 235.90 & 262.72 & 260.17 \\
\hline 140.049 & 236.64 & 263.05 & 258.70 \\
\hline 140.5478 & 237.38 & 264.36 & 260.25 \\
\hline 141.0477 & 238.72 & 266.49 & 261.23 \\
\hline 141.5483 & 239.39 & 268.58 & 260.77 \\
\hline 142.0482 & 240.88 & 268.80 & 259.85 \\
\hline 142.548 & 241.75 & 267.42 & 260.86 \\
\hline 143.0478 & 241.70 & 266.02 & 260.19 \\
\hline 143.5477 & 241.40 & 264.11 & 258.52 \\
\hline 144.0485 & 240.79 & 262.95 & 259.53 \\
\hline 144.5483 & 239.24 & 262.57 & 259.82 \\
\hline 145.048 & 237.54 & 262.04 & 257.97 \\
\hline 145.5478 & 237.08 & 261.33 & 258.51 \\
\hline 146.0477 & 236.98 & 261.39 & 260.09 \\
\hline 146.5475 & 236.95 & 260.72 & 257.90 \\
\hline 147.0483 & 236.40 & 260.17 & 259.00 \\
\hline 147.5482 & 235.61 & 259.79 & 258.46 \\
\hline 148.048 & 234.72 & 259.57 & 259.05 \\
\hline 148.5477 & 233.94 & 259.62 & 258.54 \\
\hline 149.0475 & 234.69 & 259.86 & 259.43 \\
\hline 149.5483 & 234.18 & 260.15 & 259.02 \\
\hline 150.049 & 235.05 & 261.24 & 260.31 \\
\hline 150.548 & 236.25 & 262.89 & 260.00 \\
\hline 151.0478 & 236.95 & 264.41 & 261.36 \\
\hline 151.5467 & 237.93 & 266.06 & 262.38 \\
\hline 152.0483 & 238.49 & 267.05 & 262.61 \\
\hline 152.5482 & 239.30 & 266.42 & 260.38 \\
\hline 153.048 & 239.86 & 265.91 & 261.69 \\
\hline 153.5468 & 239.02 & 265.54 & 262.81 \\
\hline 154.0477 & 238.33 & 264.86 & 263.04 \\
\hline 154.5483 & 237.20 & 263.91 & 259.65 \\
\hline 155.0482 & 236.51 & 261.50 & 259.00 \\
\hline 155.547 & 236.55 & 260.72 & 258.61 \\
\hline 156.0478 & 236.46 & 260.26 & 259.25 \\
\hline 156.5477 & 236.41 & 260.53 & 259.31 \\
\hline 157.0483 & 237.49 & 261.58 & 259.40 \\
\hline 157.5473 & 237.35 & 263.07 & 260.36 \\
\hline 158.048 & 235.84 & 264.35 & 260.61 \\
\hline 158.5478 & 233.42 & 265.87 & 261.53 \\
\hline 159.0485 & 233.27 & 266.06 & 259.29 \\
\hline 159.5483 & 237.25 & 265.42 & 260.14 \\
\hline 160.0482 & 240.16 & 264.81 & 259.81 \\
\hline 160.548 & 240.91 & 264.18 & 260.37 \\
\hline
\end{tabular}




\begin{tabular}{|c|c|c|c|}
\hline 161.0478 & 239.95 & 263.61 & 260.28 \\
\hline 161.5477 & 238.06 & 263.04 & 260.03 \\
\hline 162.0485 & 235.87 & 262.52 & 260.07 \\
\hline 162.5473 & 232.71 & 262.46 & 260.52 \\
\hline 163.048 & 229.78 & 262.60 & 259.79 \\
\hline 163.5478 & 226.20 & 262.36 & 260.91 \\
\hline 164.0468 & 223.65 & 262.37 & 261.02 \\
\hline 164.5475 & 223.79 & 262.64 & 260.78 \\
\hline 165.0483 & 226.61 & 264.59 & 262.32 \\
\hline 165.5482 & 227.38 & 266.21 & 262.95 \\
\hline 166.048 & 228.41 & 267.16 & 262.94 \\
\hline 166.5468 & 228.22 & 267.10 & 262.63 \\
\hline 167.0475 & 228.26 & 266.64 & 261.38 \\
\hline 167.5483 & 228.41 & 264.56 & 258.96 \\
\hline 168.0482 & 228.89 & 263.12 & 260.20 \\
\hline 168.5488 & 230.99 & 262.85 & 259.19 \\
\hline 169.0468 & 234.63 & 262.91 & 259.63 \\
\hline 169.5477 & 238.26 & 262.62 & 260.60 \\
\hline 170.0483 & 240.16 & 263.27 & 259.67 \\
\hline 170.5482 & 240.78 & 263.12 & 260.54 \\
\hline 171.048 & 240.14 & 263.59 & 259.64 \\
\hline 171.5468 & 238.96 & 263.94 & 259.64 \\
\hline 172.0477 & 236.69 & 264.21 & 260.49 \\
\hline 172.5483 & 233.97 & 264.53 & 261.24 \\
\hline 173.0482 & 230.59 & 264.62 & 260.74 \\
\hline 173.547 & 228.00 & 264.35 & 261.16 \\
\hline 174.0478 & 227.52 & 264.47 & 261.46 \\
\hline 174.5477 & 227.97 & 264.05 & 260.66 \\
\hline 175.0483 & 227.10 & 263.86 & 261.23 \\
\hline 175.5482 & 226.42 & 265.26 & 260.46 \\
\hline 176.048 & 229.47 & 265.61 & 260.13 \\
\hline 176.5488 & 232.45 & 265.25 & 261.57 \\
\hline 177.0468 & 233.87 & 265.30 & 262.27 \\
\hline 177.5475 & 233.68 & 265.12 & 260.79 \\
\hline 178.0482 & 232.11 & 264.76 & 260.92 \\
\hline 178.548 & 230.09 & 264.22 & 260.50 \\
\hline 179.0478 & 228.91 & 264.63 & 261.50 \\
\hline 179.5468 & 228.18 & 262.57 & 255.83 \\
\hline 180.0493 & 229.05 & 260.11 & 255.68 \\
\hline 180.5483 & 229.61 & 259.71 & 255.96 \\
\hline 181.048 & 229.25 & 260.28 & 256.72 \\
\hline 181.547 & 229.83 & 261.32 & 258.11 \\
\hline 182.0477 & 231.47 & 262.45 & 258.61 \\
\hline 182.5475 & 232.28 & 262.98 & 259.55 \\
\hline 183.0483 & 231.06 & 262.47 & 259.13 \\
\hline 183.5482 & 229.55 & 261.83 & 258.51 \\
\hline 184.048 & 229.48 & 261.16 & 258.23 \\
\hline 184.5477 & 229.38 & 260.64 & 258.82 \\
\hline 185.0475 & 228.63 & 260.18 & 258.21 \\
\hline 185.5483 & 227.11 & 259.49 & 258.12 \\
\hline 186.0472 & 225.72 & 259.22 & 257.95 \\
\hline 186.548 & 226.64 & 259.16 & 257.91 \\
\hline 187.0478 & 227.49 & 259.38 & 257.83 \\
\hline 187.5467 & 229.18 & 261.65 & 258.42 \\
\hline 188.0483 & 229.66 & 262.44 & 258.89 \\
\hline 188.5482 & 230.14 & 263.39 & 260.58 \\
\hline 189.047 & 230.37 & 263.82 & 260.56 \\
\hline 189.5478 & 230.83 & 264.61 & 260.21 \\
\hline 190.0485 & 230.12 & 264.40 & 261.53 \\
\hline 190.5483 & 229.17 & 264.79 & 261.94 \\
\hline 191.0482 & 228.96 & 264.70 & 258.58 \\
\hline 191.548 & 223.20 & 262.09 & 258.93 \\
\hline 192.0478 & 223.01 & 261.72 & 258.54 \\
\hline 192.5477 & 226.86 & 259.95 & 253.32 \\
\hline 193.0483 & 231.02 & 257.49 & 255.76 \\
\hline 193.5473 & 232.93 & 257.88 & 256.45 \\
\hline
\end{tabular}




\begin{tabular}{|c|c|c|c|}
\hline 194.048 & 233.61 & 258.97 & 256.72 \\
\hline 194.5478 & 232.35 & 259.89 & 256.80 \\
\hline 195.0477 & 230.81 & 260.46 & 257.26 \\
\hline 195.5483 & 229.60 & 261.08 & 258.02 \\
\hline 196.0482 & 229.19 & 260.19 & 257.05 \\
\hline 196.5472 & 229.68 & 259.35 & 257.53 \\
\hline 197.0478 & 228.90 & 258.68 & 256.21 \\
\hline 197.5477 & 228.42 & 258.21 & 256.19 \\
\hline 198.0485 & 227.53 & 257.72 & 257.51 \\
\hline 198.5473 & 227.25 & 257.57 & 255.99 \\
\hline 199.048 & 227.81 & 258.30 & 256.28 \\
\hline 199.5478 & 229.03 & 260.22 & 256.88 \\
\hline 200.0477 & 229.68 & 260.84 & 257.41 \\
\hline 200.5475 & 231.10 & 261.55 & 258.95 \\
\hline 201.0483 & 231.63 & 262.00 & 259.44 \\
\hline 201.5482 & 231.29 & 262.05 & 259.15 \\
\hline 202.048 & 230.24 & 261.79 & 260.21 \\
\hline 202.5477 & 229.58 & 261.55 & 258.57 \\
\hline 203.0475 & 227.96 & 260.34 & 257.26 \\
\hline 203.5473 & 228.29 & 259.72 & 257.34 \\
\hline 204.0472 & 228.51 & 260.32 & 258.97 \\
\hline 204.548 & 228.55 & 261.79 & 258.77 \\
\hline 205.0478 & 228.50 & 263.20 & 258.73 \\
\hline 205.5477 & 229.13 & 263.83 & 260.52 \\
\hline 206.0483 & 230.85 & 265.05 & 260.48 \\
\hline 206.5482 & 231.61 & 265.73 & 261.71 \\
\hline 207.047 & 231.73 & 266.24 & 260.59 \\
\hline 207.5478 & 231.95 & 266.46 & 261.87 \\
\hline 208.0477 & 230.50 & 266.33 & 258.08 \\
\hline 208.5475 & 230.13 & 263.49 & 257.90 \\
\hline 209.0482 & 229.99 & 262.02 & 258.01 \\
\hline 209.548 & 230.35 & 261.54 & 258.49 \\
\hline 210.0487 & 230.36 & 261.10 & 258.86 \\
\hline 210.5477 & 229.52 & 261.22 & 259.45 \\
\hline 211.0475 & 227.72 & 260.71 & 259.04 \\
\hline 211.5492 & 226.58 & 260.96 & 259.00 \\
\hline 212.048 & 225.80 & 261.08 & 259.26 \\
\hline 212.547 & 226.41 & 262.47 & 259.85 \\
\hline 213.0477 & 229.09 & 263.88 & 260.28 \\
\hline 213.5483 & 229.74 & 264.12 & 259.75 \\
\hline 214.0482 & 230.37 & 263.93 & 260.49 \\
\hline 214.5472 & 230.89 & 264.44 & 259.75 \\
\hline 215.0478 & 230.27 & 263.94 & 259.69 \\
\hline 215.5468 & 228.86 & 262.28 & 257.61 \\
\hline 216.0485 & 229.06 & 261.14 & 258.01 \\
\hline 216.5483 & 229.82 & 260.81 & 257.28 \\
\hline 217.048 & 230.60 & 260.94 & 257.80 \\
\hline 217.547 & 230.37 & 261.13 & 258.57 \\
\hline 218.0477 & 229.87 & 261.27 & 258.58 \\
\hline 218.5467 & 229.77 & 261.84 & 258.88 \\
\hline 219.0473 & 229.74 & 262.02 & 258.72 \\
\hline 219.5472 & 228.39 & 262.44 & 258.54 \\
\hline 220.048 & 226.85 & 262.55 & 258.17 \\
\hline 220.5477 & 228.84 & 262.55 & 259.12 \\
\hline 221.0475 & 231.48 & 262.84 & 259.48 \\
\hline 221.5483 & 232.13 & 262.72 & 259.27 \\
\hline 222.0482 & 232.15 & 262.67 & 259.22 \\
\hline 222.548 & 230.55 & 262.93 & 259.37 \\
\hline 223.0478 & 227.74 & 263.48 & 260.25 \\
\hline 223.5485 & 226.19 & 264.87 & 260.50 \\
\hline 224.0483 & 225.85 & 264.79 & 259.87 \\
\hline 224.549 & 226.84 & 265.30 & 261.27 \\
\hline 225.048 & 227.14 & 264.79 & 259.62 \\
\hline 225.5468 & 227.96 & 264.00 & 259.57 \\
\hline 226.0477 & 228.63 & 263.49 & 259.29 \\
\hline 226.5483 & 227.40 & 263.07 & 260.80 \\
\hline
\end{tabular}




$\begin{array}{llll}227.0482 & 225.19 & 263.23 & 259.90 \\ 227.548 & 223.49 & 263.03 & 261.11 \\ 228.0478 & 224.82 & 264.54 & 261.37 \\ 228.5477 & 226.26 & 265.61 & 261.69 \\ 229.0483 & 227.27 & 266.69 & 259.81 \\ 229.5482 & 228.19 & 266.96 & 262.41 \\ 230.049 & 228.53 & 266.69 & 261.86 \\ 230.5478 & 227.53 & 265.46 & 261.12 \\ 231.0477 & 227.16 & 265.16 & 260.81 \\ 231.5483 & 226.83 & 264.64 & 260.77 \\ 232.0482 & 225.42 & 263.23 & 259.39 \\ 232.548 & 224.76 & 262.91 & 259.76 \\ 233.047 & 224.96 & 262.97 & 259.74 \\ 233.5477 & 226.22 & 264.47 & 261.09 \\ 234.0485 & 227.14 & 265.68 & 261.51 \\ 234.5473 & 228.00 & 266.48 & 261.22 \\ 235.048 & 222.01 & 266.98 & 262.23 \\ 235.5478 & 222.23 & 266.86 & 261.72 \\ 236.0477 & 225.87 & 266.35 & 261.40 \\ 236.5475 & 227.54 & 265.25 & 259.40 \\ 237.0492 & 227.60 & 263.38 & 259.12 \\ 237.5472 & 226.19 & 262.46 & 259.49 \\ 238.048 & 225.52 & 262.61 & 260.49 \\ 238.5477 & 225.07 & 262.40 & 259.33 \\ 239.0475 & 225.35 & 262.65 & 260.22 \\ 239.5483 & 225.98 & 264.75 & 260.14 \\ 240.049 & 225.29 & 266.19 & 262.17 \\ 240.5488 & 224.74 & 267.23 & 262.21 \\ 241.0478 & 226.07 & 267.47 & 262.46 \\ 241.5477 & 227.36 & 267.13 & 262.39 \\ 242.0473 & 227.60 & 265.26 & 256.54 \\ 242.5482 & 227.69 & 261.70 & 258.15 \\ 243.047 & 226.32 & 263.11 & 262.10\end{array}$




\section{VITA}

Name:

Roly Simangunsong

Born:

April 2, 1978

Bandung, Indonesia

Parents:

Wesley Simangunsong

and Rospita Simangunsong boru Simanjuntak

Permanent address:

Jalan Bengawan no. 35

Bandung-Indonesia, 40114

Email:

rasimangunsong@yahoo.com

Education:

B.S., Petroleum Engineering (February 2001)

Bandung Institute of Techonology

Bandung, Indonesia

M.S., Petroleum Engineering (August 2005)

Texas A\&M University

College Station, Texas 77843 